\title{
Global Structure Optimization of Pt Clusters Based on the Modified Empirical Potentials, Calibrated using Density Functional Theory
}

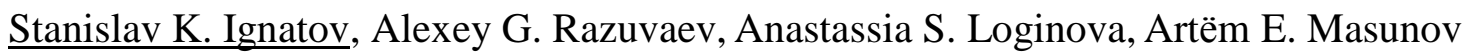

\section{Supplementary Information}

Content:

$1 \quad$ Table S1. Cartesian coordinates energies (Hartrees) of platinum clusters optimized at the PBEO/LANL08 theory level. M is a spin multiplicity.
Page :

S1

clusters

(̊) and binding

S137

clusters

S218

S226

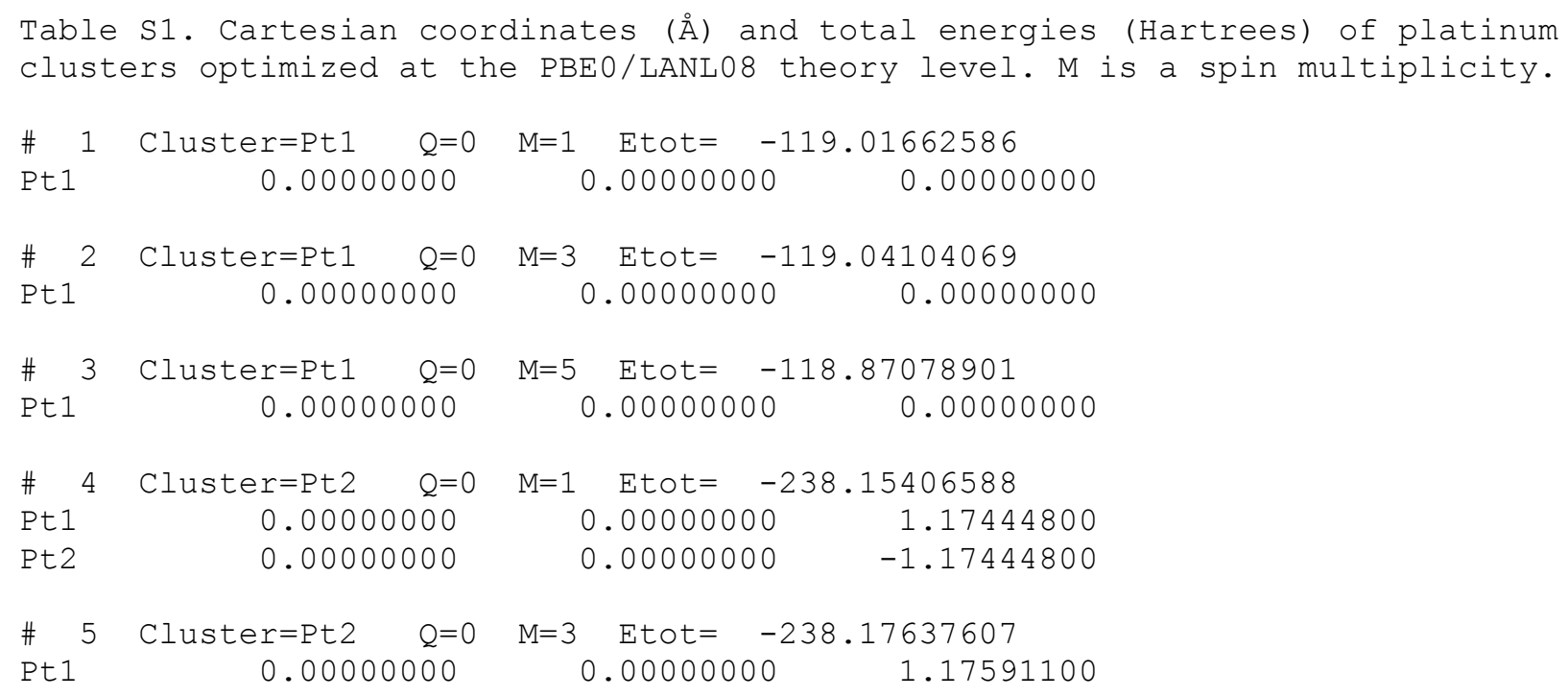




$\begin{array}{lccc}\text { Pt2 } & 0.00000000 & 0.00000000 & -1.17591100 \\ \text { \# } 6 & \text { Cluster=Pt2 } \quad \mathrm{Q}=0 & \mathrm{M}=5 \quad \text { Etot }= & -238.14053873 \\ \text { Pt1 } & 0.00000000 & 0.00000000 & 1.16113900 \\ \text { Pt2 } & 0.00000000 & 0.00000000 & -1.16113900\end{array}$

\# 7 Cluster=Pt3 $Q=0 \quad \mathrm{M}=1 \quad$ Etot $=-357.32918580$

$\begin{array}{lllr}\text { Pt1 } & 0.00000000 & 0.00000000 & 1.42920300 \\ \text { Pt2 } & 0.00000000 & 1.23788800 & -0.71460200\end{array}$

Pt3 0.00000000

$-1.23788800 \quad-0.71460200$

\# 8 Cluster=Pt3 $Q=0 \quad \mathrm{M}=3 \quad$ Etot $=-357.31915433$

$\begin{array}{lllr}\text { Pt1 } & 0.00000000 & 0.00000000 & 1.47565000 \\ \text { Pt2 } & 0.00000000 & 1.24628000 & -0.73782500\end{array}$

Pt3 0.00000000

$-1.24628000 \quad-0.73782500$

\# 9 Cluster $=$ Pt $3 \quad \mathrm{Q}=0 \quad \mathrm{M}=5 \quad$ Etot $=-357.29386155$

$\begin{array}{lllr}\text { Pt1 } & 0.00000000 & 0.00000000 & 1.41587100 \\ \text { Pt2 } & 0.00000000 & 1.33780200 & -0.70793500\end{array}$

$\begin{array}{llll}\text { Pt3 } & 0.00000000 & -1.33780200 & -0.70793500\end{array}$

\# 10 Cluster=Pt4 $Q=0 \quad \mathrm{M}=1 \quad$ Etot $=-476.47998423$

$\begin{array}{llll}\text { Pt1 } & -1.02238600 & -0.05899400 & -1.21646700\end{array}$

$\begin{array}{llll}\text { Pt2 } & -0.86976000 & 0.02651300 & 1.31990000\end{array}$

$\begin{array}{llll}\text { Pt3 } & 0.97245700 & -1.27210700 & -0.01093600\end{array}$

Pt4 0.91968900

\# 11 Cluster=Pt4 $Q=0$

Pt1 $\quad-1.36866100$

Pt2 $\quad 0.00481500$

Pt3 $\quad-0.00444200$

Pt $4 \quad 1.36828800$

$1.30458800 \quad-0.09249700$

$\mathrm{M}=3 \quad \mathrm{Etot}=-476.48630549$

$\begin{array}{rr}0.00371000 & -0.88276800 \\ 1.28803600 & 0.88212100 \\ -1.28674800 & 0.88389000 \\ -0.00499800 & -0.88324300\end{array}$

\# 12 Cluster=Pt $4 \quad \mathrm{Q}=0 \quad \mathrm{M}=5 \quad$ Etot $=-476.48340720$

Pt1 $\quad-0.97737200$

Pt2 $\quad 0.97425800$

Pt3 $\quad 0.97630200$

Pt $4 \quad-0.97318900$

$\begin{array}{ll}M=5 \quad \text { Etot }=-476.48340720 \\ 0.56747300 & -1.11633700 \\ -1.11816300 & -0.56881300 \\ 1.11820800 & 0.56582100 \\ -0.56751900 & 1.11932900\end{array}$

\# 13 Cluster=Pt4 $Q=0 \quad \mathrm{M}=1 \quad$ Etot $=-476.46679497$

Pt1 0.00000000

Pt2 1.74728600

Pt3 $\quad 0.00000000$

Pt4 -1.74728600
1.74728600
0.00000000
0.00000000
0.00000000
$-1.74728600$
0.00000000
0.00000000
0.00000000

\# 14 Cluster=Pt4 $\mathrm{Q}=0$

$\mathrm{M}=3 \quad$ Etot $=-476.45545794$

Pt1 0.00000000

Pt2 1.75911500

Pt3 $\quad 0.00000000$

Pt4 -1.75911500

1.75911500

0.00000000

0.00000000

$-1.75911500$

0.00000000

0.00000000

0.00000000

0.00000000

\# 15 Cluster=Pt4 $Q=0 \quad \mathrm{M}=5 \quad$ Etot $=-476.42350675$

Pt1 0.00000000

Pt2 1.76610700

1.76610700

0.00000000

0.00000000

0.00000000

$-1.76610700$

0.00000000

Pt $4 \quad-1.76610700$

0.00000000

0.00000000

\# 16 Cluster $=$ Pt $4 \quad \mathrm{Q}=0$

$\mathrm{M}=7 \quad$ Etot $=-476.42712040$

Pt1 0.00000000

1.79981800

0.00000000 


\begin{tabular}{|c|c|c|c|}
\hline Pt2 & 1.79981800 & 0.00000000 & 0.00000000 \\
\hline Pt3 & 0.00000000 & -1.79981800 & 0.00000000 \\
\hline Pt 4 & -1.79981800 & 0.00000000 & 0.00000000 \\
\hline \# 17 & Cluster $=$ Pt 4 & \multicolumn{2}{|c|}{$\mathrm{M}=1 \quad$ Etot $=-476.45166107$} \\
\hline Pt1 & 0.91914900 & 0.91914900 & 0.91914900 \\
\hline Pt2 & -0.91914900 & -0.91914900 & 0.91914900 \\
\hline Pt3 & -0.91914900 & 0.91914900 & -0.91914900 \\
\hline Pt 4 & 0.91914900 & -0.91914900 & -0.91914900 \\
\hline$\# 18$ & Cluster $=$ Pt 4 & \multicolumn{2}{|c|}{$\mathrm{M}=3 \quad$ Etot $=-476.48049194$} \\
\hline Pt1 & 0.92023700 & \multirow{2}{*}{$\begin{array}{r}0.92023700 \\
-0.92023700\end{array}$} & $0 \quad 0.92023700$ \\
\hline Pt2 & -0.92023700 & & 0.92023700 \\
\hline t3 & -0.92023700 & 0.92023700 & -0.92023700 \\
\hline$t 4$ & 0.92023700 & -0.92023700 & -0.92023700 \\
\hline \# 19 & \multirow{2}{*}{$\begin{aligned} \text { Cluster } & =\text { Pt } 4 \quad Q=0 \\
& 0.91414500\end{aligned}$} & \multicolumn{2}{|c|}{$\mathrm{M}=5 \quad \mathrm{Etot}=-476.48009163$} \\
\hline Pt1 & & 0.91414500 & $0 \quad 0.91414500$ \\
\hline Pt2 & -0.91414500 & \multirow{2}{*}{$\begin{array}{r}-0.91414500 \\
0.91414500\end{array}$} & 0.91414500 \\
\hline Pt 3 & -0.91414500 & & -0.91414500 \\
\hline Pt 4 & 0.91414500 & -0.91414500 & -0.91414500 \\
\hline \# 20 & \multirow{2}{*}{$\begin{aligned} \text { Cluster } & =\text { Pt } 4 \quad \mathrm{Q}=0 \\
& 0.91559800\end{aligned}$} & \multicolumn{2}{|c|}{$\mathrm{M}=7 \quad \mathrm{E}$ tot $=-476.42600106$} \\
\hline Pt1 & & 0.91559800 & $0 \quad 0.91559800$ \\
\hline Pt2 & -0.91559800 & -0.91559800 & 0.91559800 \\
\hline Pt3 & -0.91559800 & 0.91559800 & -0.91559800 \\
\hline Pt 4 & 0.91559800 & -0.91559800 & -0.91559800 \\
\hline \# 21 & Cluster=Pt5 & $M=1 \quad$ Etot $=-$ & -595.60149369 \\
\hline Pt1 & 0.00000000 & 1.78538100 & -0.40796400 \\
\hline Pt2 & 1.78538100 & 0.00000000 & -0.40796400 \\
\hline Pt3 & 0.00000000 & -1.78538100 & -0.40796400 \\
\hline Pt 4 & -1.78538100 & 0.00000000 & -0.40796400 \\
\hline Pt5 & 0.00000000 & 0.00000000 & 1.63185500 \\
\hline$\# 22$ & Cluster=Pt5 & $\mathrm{M}=3 \quad$ Etot $=-$ & -595.63047733 \\
\hline Pt 1 & 0.00000000 & 1.82731400 & -0.37275000 \\
\hline Pt2 & 1.82731400 & 0.00000000 & -0.37275000 \\
\hline Pt3 & 0.00000000 & -1.82731400 & -0.37275000 \\
\hline Pt 4 & -1.82731400 & 0.00000000 & -0.37275000 \\
\hline Pt5 & 0.00000000 & 0.00000000 & 1.49100000 \\
\hline \# 23 & Cluster $=$ Pt 5 & $M=5 \quad$ Etot $=$ & -595.61547437 \\
\hline Pt1 & 0.00000000 & 1.79793800 & -0.38671200 \\
\hline Pt2 & 1.79793800 & 0.00000000 & -0.38671200 \\
\hline Pt3 & 0.00000000 & -1.79793800 & -0.38671200 \\
\hline Pt 4 & -1.79793800 & 0.00000000 & -0.38671200 \\
\hline Pt 5 & 0.00000000 & 0.00000000 & 1.54684700 \\
\hline \# 24 & Cluster $=$ Pt 5 & $\mathrm{M}=7 \quad$ Etot $=$ & -595.62529490 \\
\hline Pt 1 & 0.00000000 & 1.79740200 & -0.38372300 \\
\hline Pt2 & 1.79740200 & 0.00000000 & -0.38372300 \\
\hline Pt 3 & 0.00000000 & -1.79740200 & -0.38372300 \\
\hline Pt 4 & -1.79740200 & 0.00000000 & -0.38372300 \\
\hline Pt 5 & 0.00000000 & 0.00000000 & 1.53489100 \\
\hline$\# 25$ & Cluster $=$ Pt $5 \quad \mathrm{Q}=0$ & $\mathrm{M}=1 \quad$ Etot $=-$ & -595.62611413 \\
\hline Pt 1 & 0.00000000 & 0.00000000 & 1.56299700 \\
\hline & 1.35359500 & 0.00000000 & -0.78149800 \\
\hline
\end{tabular}




$\begin{array}{lrrr}\text { Pt3 } & -1.35359500 & 0.00000000 & -0.78149800 \\ \text { Pt4 } & 0.00000000 & -2.08299200 & 0.00000000 \\ \text { Pt5 } & 0.00000000 & 2.08299200 & 0.00000000\end{array}$

\# 26 Cluster=Pt5 $\quad \mathrm{Q}=0 \quad \mathrm{M}=3 \quad$ Etot $=\quad-595.62279491$

$\begin{array}{rrrr}\text { Pt1 } & 0.00000000 & 0.00000000 & 1.60201800 \\ \text { Pt2 } & 1.38738800 & 0.00000000 & -0.80100900 \\ \text { Pt3 } & -1.38738800 & 0.00000000 & -0.80100900 \\ \text { Pt4 } & 0.00000000 & -2.02775300 & 0.00000000 \\ \text { Pt5 } & 0.00000000 & 2.02775300 & 0.00000000\end{array}$

$\begin{array}{lrrrr}\# 27 & \text { Cluster=Pt5 } Q=0 & \mathrm{M}=5 \quad \text { Etot }=-595.61361704 \\ \text { Pt1 } & 0.00000000 & 0.00000000 & 1.78126200 \\ \text { Pt2 } & 1.54261800 & 0.00000000 & -0.89063100 \\ \text { Pt3 } & -1.54261800 & 0.00000000 & -0.89063100 \\ \text { Pt4 } & 0.00000000 & -1.80469800 & 0.00000000 \\ \text { Pt5 } & 0.00000000 & 1.80469800 & 0.00000000 \\ & & & \\ \# 28 & \text { Cluster=Pt5 } \mathrm{Q}=0 & \mathrm{M}=7 & \text { Etot }= & -595.62583421 \\ \text { Pt1 } & 0.00000000 & 0.00000000 & 1.58769900 \\ \text { Pt2 } & 1.37498800 & 0.00000000 & -0.79384900 \\ \text { Pt3 } & -1.37498800 & 0.00000000 & -0.79384900 \\ \text { Pt4 } & 0.00000000 & -2.02712300 & 0.00000000 \\ \text { Pt5 } & 0.00000000 & 2.02712300 & 0.00000000\end{array}$

\# 29 Cluster=Pt6 $\quad \mathrm{Q}=0 \quad \mathrm{M}=1 \quad$ Etot $=-714.79093629$

$\begin{array}{llll}\text { Pt1 } & 0.00000000 & 0.00000000 & 1.48463500\end{array}$

$\begin{array}{llll}\text { Pt2 } & 1.28573100 & 0.00000000 & -0.74231700\end{array}$

$\begin{array}{llll}\text { Pt3 } & -1.28573100 & 0.00000000 & -0.74231700\end{array}$

$\begin{array}{llll}\text { Pt4 } & 0.00000000 & -2.54324200 & 1.48463500\end{array}$

$\begin{array}{llll}\text { Pt5 } & 1.28573100 & -2.54324200 & -0.74231700\end{array}$

\# 30 Cluster=Pt6 $\mathrm{Q}=0 \quad \mathrm{M}=3 \quad$ Etot $=-714.79687858$

$\begin{array}{lrrr}\text { Pt1 } & 0.00000000 & 0.00000000 & 1.48446800 \\ \text { Pt2 } & 1.28558700 & 0.00000000 & -0.74223400 \\ \text { Pt3 } & -1.28558700 & 0.00000000 & -0.74223400 \\ \text { Pt4 } & 0.0000000 & -2.54880400 & 1.48446800 \\ \text { Pt5 } & 1.28558700 & -2.54880400 & -0.74223400 \\ \text { Pt6 } & -1.28558700 & -2.54880400 & -0.74223400\end{array}$

\# 31 Cluster=Pt6 $\quad \mathrm{Q}=0 \quad \mathrm{M}=5 \quad$ Etot $=-714.79348149$

$\begin{array}{llll}\text { Pt1 } & 0.00000000 & 0.00000000 & 1.48384100\end{array}$

$\begin{array}{llll}\text { Pt2 } & 1.28504400 & 0.00000000 & -0.74192100\end{array}$

$\begin{array}{llll}\text { Pt3 } & -1.28504400 & 0.00000000 & -0.74192100\end{array}$

$\begin{array}{llll}\text { Pt4 } & 0.00000000 & -2.54834800 & 1.48384100\end{array}$

$\begin{array}{llll}\text { Pt5 } & 1.28504400 & -2.54834800 & -0.74192100\end{array}$

$-2.54834800 \quad-0.74192100$

$\begin{array}{lcccr}\text { \# 32 } & \text { Cluster=Pt6 } Q=0 & M=7 \quad \text { Etot }=-714.78364410 \\ \text { Pt1 } & 0.00000000 & 0.00000000 & 1.49305600 \\ \text { Pt2 } & 1.29302400 & 0.00000000 & -0.74652800 \\ \text { Pt3 } & -1.29302400 & 0.00000000 & -0.74652800 \\ \text { Pt4 } & 0.0000000 & -2.51688000 & 1.49305600 \\ \text { Pt5 } & 1.29302400 & -2.51688000 & -0.74652800 \\ \text { Pt6 } & -1.29302400 & -2.51688000 & -0.74652800\end{array}$

\# 33 Cluster $=$ Pt6 $\quad \mathrm{Q}=0 \quad \mathrm{M}=1 \quad$ Etot $=-714.75587228$

$\begin{array}{llll}\text { Pt1 } & 0.00000000 & 1.86902600 & 0.00000000\end{array}$ 


\begin{tabular}{|c|c|c|c|}
\hline Pt2 & 1.86902600 & 0.00000000 & 0.00000000 \\
\hline Pt3 & 0.00000000 & -1.86902600 & 0.00000000 \\
\hline 4 & -1.86902600 & 0.00000000 & 0.00000000 \\
\hline & 0.00000000 & 0.00000000 & 1.86921900 \\
\hline & 0.00000000 & 0.00000000 & -1.86921900 \\
\hline 34 & luster $=$ Pt 6 & $\mathrm{M}=3 \quad \mathrm{E}$ tot $=$ & -714.76443740 \\
\hline 11 & 0.00000000 & 1.82607500 & 0.00000000 \\
\hline 2 & 1.82607500 & 0.00000000 & 0.00000000 \\
\hline 3 & 0.00000000 & -1.82607500 & 0.00000000 \\
\hline & -1.82607500 & 0.00000000 & 0.00000000 \\
\hline 5 & 0.00000000 & 0.00000000 & 1.93385100 \\
\hline$=6$ & 0.00000000 & 0.000 & -1.93 \\
\hline 35 & luster $=$ Pt 6 & $=5 \quad$ Etot $=$ & 7208977 \\
\hline 1 & 0.00000000 & 1.85346000 & 0.00000000 \\
\hline 2 & 1.85346000 & 0.00000000 & 0.00000000 \\
\hline 3 & 0.00000000 & -1.85346000 & 0.00000000 \\
\hline 4 & -1.85346000 & 0.0 & 00 \\
\hline 55 & 0.00000000 & 0.00 & 00 \\
\hline$=6$ & 0.00000000 & 0.00 & -1 \\
\hline 36 & Cluster $=$ Pt 6 & Etot $=$ & -71 \\
\hline t1 & 0.00000000 & 1.79792900 & 000000 \\
\hline 2 & 1.79792900 & 0.00 & 00 \\
\hline$=3$ & 0.00000000 & -1.79 & 0 . \\
\hline$=4$ & -1.79792900 & 0.00 & 0.00000000 \\
\hline$=5$ & 0.00000000 & 0.00000000 & 2.04593700 \\
\hline-6 & 0.00000000 & 0.00000000 & -2.04593700 \\
\hline 37 & Cluster=Pt 6 & Etot $=$ & 552 \\
\hline t1 & 0.00000000 & 0.00000000 & 1.52794700 \\
\hline$=2$ & 1.32324100 & 0.00000000 & -0.76397400 \\
\hline 3 & -1.32324100 & 0.00000000 & -0.76397400 \\
\hline & -1.32324100 & -2.16046400 & 0.76397400 \\
\hline 5 & 4100 & -2 & 0 . \\
\hline t 6 & 0.00000000 & -2.16046400 & -1 \\
\hline 38 & $e r=\operatorname{Pt} \sigma$ & Etot $=$ & 790 \\
\hline 1 & 0.00000000 & 0.00000000 & 1.56044900 \\
\hline ? & 1.35138900 & 0.00000000 & -0.78022500 \\
\hline & 3900 & 0.0 & -0 . \\
\hline-1 & 138900 & -2 & 0 . \\
\hline 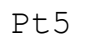 & 38900 & -2.09344800 & 022500 \\
\hline t 6 & 0.00000000 & -2.09344800 & 044900 \\
\hline \# 39 & Cluster=Pt 6 & $\mathrm{M}=5 \quad \mathrm{Etot}=$ & -714.7833 \\
\hline 1 & 0.00000000 & 0.00000000 & 1.52823400 \\
\hline-2 & 348900 & 0.00000000 & -0.76411700 \\
\hline t3 & -1.32348900 & 0.00000000 & -0.76411700 \\
\hline Pt 4 & -1.32348900 & -2.15935900 & 0.76411700 \\
\hline Pt 5 & 1.32348900 & -2.15935900 & 0.76411700 \\
\hline Pt 6 & 0.00000000 & -2.15935900 & -1.52823400 \\
\hline 4 & Cluster $=$ Pt 6 & Etot $=$ & -714.78742730 \\
\hline & 0.00000000 & 0.00000000 & 1.54736600 \\
\hline$\tau 2$ & 1.34005800 & 0.00000000 & -0.77368300 \\
\hline & -1.34005800 & 0.00000000 & -0.77368300 \\
\hline & -1.34005800 & -2.11087800 & 0.7736830 \\
\hline
\end{tabular}




$\begin{array}{lccr}\text { Pt5 } & 1.34005800 & -2.11087800 & 0.77368300 \\ \text { Pt6 } & 0.00000000 & -2.11087800 & -1.54736600 \\ & & & \\ \# 41 & \text { Cluster=Pt7 Q }=0 & \text { M=1 Etot }=-833.92464219 \\ \text { Pt1 } & 0.00000000 & 0.00000000 & 2.23034700 \\ \text { Pt2 } & 2.12118600 & 0.00000000 & 0.68921500 \\ \text { Pt3 } & 1.31096500 & 0.00000000 & -1.80438900 \\ \text { Pt4 } & -1.31096500 & 0.00000000 & -1.80438900 \\ \text { Pt5 } & -2.12118600 & 0.00000000 & 0.68921500 \\ \text { Pt6 } & 0.00000000 & -1.54163400 & 0.00000000 \\ \text { Pt7 } & 0.00000000 & 1.54163400 & 0.00000000\end{array}$

$\begin{array}{lccrr}\# 42 & \text { Cluster=Pt7 } Q=0 & M=3 \quad \text { Etot }=-833.92687688 \\ \text { Pt1 } & 0.0000000 & 0.00000000 & 2.24051700 \\ \text { Pt2 } & 2.13085800 & 0.00000000 & 0.69235800 \\ \text { Pt3 } & 1.31694300 & 0.00000000 & -1.81261600 \\ \text { Pt4 } & -1.31694300 & 0.00000000 & -1.81261600 \\ \text { Pt5 } & -2.13085800 & 0.00000000 & 0.69235800 \\ \text { Pt6 } & 0.00000000 & -1.49486700 & 0.00000000 \\ \text { Pt7 } & 0.00000000 & 1.49486700 & 0.00000000 \\ \text { \#43 } & \text { Cluster=Pt7 } \mathrm{Q}=0 & \mathrm{M}=5 \quad \text { Etot }=-833.93243670 \\ \text { Pt1 } & 0.00000000 & 0.00000000 & 2.25282200 \\ \text { Pt2 } & 2.14256100 & 0.00000000 & 0.69616000 \\ \text { Pt3 } & 1.32417500 & 0.00000000 & -1.82257100 \\ \text { Pt4 } & -1.32417500 & 0.00000000 & -1.82257100 \\ \text { Pt5 } & -2.14256100 & 0.00000000 & 0.69616000 \\ \text { Pt6 } & 0.00000000 & -1.45794600 & 0.00000000 \\ \text { Pt7 } & 0.00000000 & 1.45794600 & 0.00000000\end{array}$

\# 44 Cluster=Pt7 $Q=0 \quad \mathrm{M}=1 \quad$ Etot $=-833.82954875$

$\begin{array}{llll}\text { Pt1 } & 0.00000000 & 0.00000000 & 0.00000000\end{array}$

$\begin{array}{llll}\text { Pt2 } & 0.00000000 & 2.58475000 & 0.00000000\end{array}$

$\begin{array}{llll}\text { Pt3 } & 2.23845900 & 1.29237500 & 0.00000000\end{array}$

$\begin{array}{llll}\text { Pt4 } & 2.23845900 & -1.29237500 & 0.00000000\end{array}$

$\begin{array}{llll}\text { Pt5 } & 0.00000000 & -2.58475000 & 0.00000000\end{array}$

$\begin{array}{llll}\text { Pt } 6 & -2.23845900 & -1.29237500 & 0.00000000\end{array}$

\# 45 Cluster $=$ Pt7 $\quad \mathrm{Q}=0 \quad \mathrm{M}=3 \quad$ Etot $=-833.84278333$

$\begin{array}{llll}\text { Pt2 } & 0.00000000 & 2.56306900 & 0.00000000\end{array}$

$\begin{array}{llll}\text { Pt3 } & 2.21968300 & 1.28153400 & 0.00000000\end{array}$

$\begin{array}{llll}\text { Pt4 } & 2.21968300 & -1.28153400 & 0.00000000\end{array}$

$\begin{array}{llll}\text { Pt5 } & 0.00000000 & -2.56306900 & 0.00000000\end{array}$

$\begin{array}{llll}\text { Pt } 6 & -2.21968300 & -1.28153400 & 0.00000000\end{array}$

$\begin{array}{llll}\text { Pt7 } & -2.21968300 & 1.28153400 & 0.00000000\end{array}$

\# 46 Cluster=Pt7 $Q=0 \quad M=5 \quad$ Etot $=-833.86730595$

$\begin{array}{lrrr}\text { Pt1 } & 0.00000000 & 0.00000000 & 0.00000000 \\ \text { Pt2 } & 0.00000000 & 2.57516200 & 0.00000000 \\ \text { Pt3 } & 2.23015600 & 1.28758100 & 0.00000000 \\ \text { Pt4 } & 2.23015600 & -1.28758100 & 0.00000000 \\ \text { Pt5 } & 0.00000000 & -2.57516200 & 0.00000000 \\ \text { Pt6 } & -2.23015600 & -1.28758100 & 0.00000000 \\ \text { Pt7 } & -2.23015600 & 1.28758100 & 0.00000000\end{array}$

\# 47 Cluster=Pt7 $\quad \mathrm{Q}=0 \quad \mathrm{M}=7 \quad$ Etot $=-833.91097582$

$\begin{array}{llll}\text { Pt1 } & 0.00000000 & 0.00000000 & 0.00000000\end{array}$ 


\begin{tabular}{|c|c|c|c|}
\hline t2 & 0.00000000 & 0.00000000 & 2.59049600 \\
\hline Pt 3 & 2.24343500 & 0.00000000 & 1.29524800 \\
\hline t 4 & 2.24343500 & 0.00000000 & -1.29524800 \\
\hline t5 & 0.00000000 & 0.00000000 & -2.59049600 \\
\hline t 6 & -2.24343500 & 0.00000000 & -1.29524800 \\
\hline t7 & -2.24343500 & 0.00000000 & 1.29524800 \\
\hline 48 & luster $=$ Pt 13 & \multicolumn{2}{|c|}{$\mathrm{M}=1 \quad$ Etot $=-1548.90302842$} \\
\hline t1 & 0.00000000 & 0.00000000 & 0.0000000 \\
\hline t2 & 0.00000000 & -2.64854700 & 0.00000000 \\
\hline t3 & 0.00000000 & 2.64854700 & 0.0000000 \\
\hline t 4 & 0.00000000 & -1.18446600 & 2.36893200 \\
\hline t5 & 2.25298800 & -1.18446600 & 0.73204000 \\
\hline t 6 & 1.39242300 & -1.18446600 & -1.91650600 \\
\hline t 7 & -1.39242300 & -1.18446600 & -1.91650600 \\
\hline t 8 & -2.25298800 & -1.18446600 & 0.73204000 \\
\hline 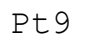 & 0.00000000 & 1.18446600 & -2.36893200 \\
\hline 0 & -2.25298800 & 1.18446600 & 3204 \\
\hline t11 & -1.39242300 & 1.18 & 1.91650 \\
\hline t12 & 1.39242300 & 1.184 & 1.9165 \\
\hline t13 & 2.25298800 & 1.184 & -0 \\
\hline \# 49 & Cluster $=$ Pt1 $3 \quad Q=0$ & \multicolumn{2}{|c|}{$\mathrm{M}=3 \quad \mathrm{E}$ tot $=-1548.90288846$} \\
\hline Pt1 & 0.00000000 & 00000 & 00000 \\
\hline t2 & 0.00000000 & -2.64 & 0 . \\
\hline t3 & 0.00000000 & 2.64508400 & 0.00000000 \\
\hline Pt 4 & 0.00000000 & -1.18291800 & 2.36583500 \\
\hline Pt5 & 2.25004300 & -1.18291800 & 0.73108300 \\
\hline Pt 6 & 1.39060300 & -1.18291800 & -1.91400100 \\
\hline Pt & -1.39060300 & -1.18 & -1.91 \\
\hline Pt 8 & -2.25004300 & -1.182 & 0 . \\
\hline Pt9 & 0.00000000 & 1.18291800 & -2.36583500 \\
\hline Pt10 & -2.25004300 & 1.18291800 & -0.73108300 \\
\hline 5 & -1.39060300 & 1.18291800 & 1.91400100 \\
\hline 1 & 1.39060300 & 1.18291800 & 1.91400100 \\
\hline Pt13 & 2.25004300 & 1.18291800 & -0.73108300 \\
\hline 50 & Iuster $=\operatorname{Pt13} \quad \mathrm{Q}=0$ & \multicolumn{2}{|c|}{$\mathrm{M}=5 \quad$ Etot $=-1548.90592929$} \\
\hline t1 & 0.00000000 & 0.00000000 & 0.00000000 \\
\hline t 2 & 0.00000000 & -2.64268500 & 0.00000000 \\
\hline Pt 3 & 0.00000000 & 2.642 & 0.00000000 \\
\hline$P$ & 0.00000000 & -1.18184500 & 2.3636 \\
\hline Pt & 2.24800200 & $-1 \cdot 1$ & 0 . \\
\hline Pt 6 & 1.38934200 & -1.18184500 & -1.91226500 \\
\hline Pt & -1.38934200 & -1.18184500 & -1.91226500 \\
\hline Pt & -2.24800200 & -1.18184500 & 0.73042000 \\
\hline Pt & 0.00000000 & 34500 & -2.36369000 \\
\hline Pt1 & -2.24800200 & 1.18184500 & -0.73042000 \\
\hline & -1.38934200 & 34500 & 1.91226500 \\
\hline & 1.38934200 & 1.18184500 & 1.91226500 \\
\hline & 2.24800200 & 1.18184500 & -0.730420 \\
\hline \# 51 & Cluster $=$ Pt13 $\quad Q=0$ & \multicolumn{2}{|c|}{$\mathrm{M}=1 \quad$ Etot $=-1548.92867068$} \\
\hline Pt & 0.00000000 & 0.00000000 & 0.00000 \\
\hline Pt2 & 0.00000000 & 0.00000000 & 2.69143100 \\
\hline Pt3 & 2.69143100 & 0.00000000 & 0.00000000 \\
\hline$P t$ & 0.00000000 & 0.00000000 & -2.6914310 \\
\hline$P \tau$ & -2.69143100 & 0.00000000 & 0.00000000 \\
\hline & 1.34571500 & -1.90312900 & 1.34571500 \\
\hline
\end{tabular}




\begin{tabular}{|c|c|c|c|}
\hline t7 & 1.34571500 & 1.90312900 & 1.34571500 \\
\hline Pt 8 & 1.34571500 & -1.90312900 & -1.34571500 \\
\hline t9 & 1.34571500 & 1.90312900 & -1.34571500 \\
\hline t10 & -1.34571500 & -1.90312900 & -1.34571500 \\
\hline t11 & -1.34571500 & 1.90312900 & -1.34571500 \\
\hline E12 & -1.34571500 & -1.90312900 & 1.34571500 \\
\hline t13 & -1.34571500 & 1.90312900 & 1.34571500 \\
\hline 52 & er $=\operatorname{Pt} 13$ & \multicolumn{2}{|c|}{$\mathrm{M}=3 \quad$ Etot $=-1548.92439133$} \\
\hline t1 & 0.00000000 & 0.00000000 & 0.00000000 \\
\hline t2 & 0.00000000 & 0.00000000 & 2.69289400 \\
\hline$=3$ & 2.69289400 & 0.00000000 & 0.00000000 \\
\hline t 4 & 0.00000000 & 0.00000000 & -2.69289400 \\
\hline 25 & -2.69289400 & 0.00000000 & 0.00000000 \\
\hline & 1.34644700 & -1.90416400 & 1.34644700 \\
\hline t 7 & 1.34644700 & 1.90416400 & 1.34644700 \\
\hline & 1.34644700 & -1.90416400 & -1.34644700 \\
\hline & 1.34644700 & 1.90416400 & -1.34 \\
\hline$=10$ & -1.34644700 & -1.9041 & -1 \\
\hline & -1.34644700 & 1.90416400 & -1.34 \\
\hline & -1.34644700 & -1.90416400 & 1.34644700 \\
\hline & -1.34644700 & 1.90416400 & 1.3464470 \\
\hline כנ & Cluster $=\mathrm{Pt} 13$ & \multicolumn{2}{|c|}{$\mathrm{M}=5 \quad$ Etot $=-1548.91818261$} \\
\hline Pt 1 & 0.00000000 & 0.00000000 & 00000 \\
\hline t2 & 0.00000000 & 0.00000000 & 2.69 \\
\hline t & 2.69219300 & 0.00000000 & 0.00000000 \\
\hline 24 & 0.00000000 & 0.00000000 & -2.69219300 \\
\hline & -2.69219300 & 0.00000000 & 0.00000000 \\
\hline $\mathrm{P}$ & 1.34609700 & -1.90366800 & 1. \\
\hline Pt & 1.34609700 & 1.90 & 1 . \\
\hline pt & 1.34609700 & -1.90366800 & -1.34 \\
\hline Pt & 1.34609700 & 1.90366800 & -1.34609700 \\
\hline 10 & -1.34609700 & -1.90366800 & -1.34609700 \\
\hline & -1.34609700 & 1.90366800 & -1.34609700 \\
\hline+ & -1.34 & -1.9 & 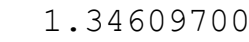 \\
\hline & -1.34609700 & 1.90366800 & 1.3460 \\
\hline 54 & ster $=$ Pt $13 \quad Q=0$ & \multicolumn{2}{|c|}{$\mathrm{M}=1 \quad$ Etot $=-1548.87236842$} \\
\hline Pt 1 & 0.00000000 & 0.00000000 & 0.00000000 \\
\hline & 0.00000000 & 2.48084600 & 0.00000000 \\
\hline 3 & 2.48084600 & 0.00000000 & 0.00000000 \\
\hline & 00000 & -2.48 & \\
\hline & -1.29479200 & 1.29479200 & 2.1321 \\
\hline$P t$ & 1.29479200 & 1.29479200 & 2.13213500 \\
\hline Pt & 1.29479200 & -1.29479200 & 2.13213500 \\
\hline Pt & -1.29479200 & -1.29479200 & 2.13213500 \\
\hline & 1.29479200 & 1.29479200 & -2.13213500 \\
\hline & -1.29479200 & 1.29 & -2.13 \\
\hline & -1.29479200 & -1.29479200 & -2.132 \\
\hline & 1.29479200 & -1.29479200 & -2.13213500 \\
\hline & -2.48084600 & 0.00000000 & 0.00000000 \\
\hline & Cluster $=$ Pt1 $3 \quad Q=0$ & \multicolumn{2}{|c|}{$\mathrm{M}=3 \quad$ Etot $=-1548.88959853$} \\
\hline & 0.00000000 & 0.00000000 & 0.00000 \\
\hline Pt & 0.00000000 & 2.49663400 & 0.0000000 \\
\hline 15 & 2.49663400 & 0.00000000 & 0.0000000 \\
\hline Pt & 0.00000000 & -2.49663400 & 0.00000000 \\
\hline & -1.31060800 & 1.31060800 & $2.093387 \mathrm{C}$ \\
\hline
\end{tabular}




\begin{tabular}{|c|c|c|c|}
\hline Pt 6 & 1.31060800 & 1.31060800 & 2.09338700 \\
\hline Pt 7 & 1.31060800 & -1.31060800 & 2.09338700 \\
\hline Pt 8 & -1.31060800 & -1.31060800 & 2.09338700 \\
\hline-9 & 1.31060800 & 1.31060800 & -2.09338700 \\
\hline$=10$ & -1.31060800 & 1.31060800 & -2.09338700 \\
\hline 11 & -1.31060800 & -1.31060800 & -2.09338700 \\
\hline$=12$ & 1.31060800 & -1.31060800 & -2.09338700 \\
\hline 13 & -2.49663400 & 0.00000000 & 0.00000000 \\
\hline 56 & luster $=$ Pt13 $\quad \mathrm{Q}=0$ & \multicolumn{2}{|c|}{$M=5 \quad$ Etot $=-1548.90181373$} \\
\hline t1 & 0.00000000 & 0.00000000 & 0.00000000 \\
\hline$=2$ & 0.00000000 & 2.48868800 & 0.00000000 \\
\hline 3 & 2.48868800 & 0.00000000 & 0.00000000 \\
\hline 54 & 0.00000000 & -2.48868800 & 0.00000000 \\
\hline$=5$ & -1.33067800 & 1.33067800 & 2.06577200 \\
\hline 6 & 1.33067800 & 1.33067800 & 2.06577200 \\
\hline$=7$ & 1.33067800 & -1.33067800 & 2.06577200 \\
\hline 8 & -1.33067800 & -1.33067800 & 2.06577200 \\
\hline 9 & 1.33067800 & 1.33067800 & -2.06577200 \\
\hline 10 & -1.33067800 & 1.3306 & -2.0 \\
\hline$=11$ & -1.33067800 & -1.33 & -2.0 \\
\hline 12 & 1.33067800 & -1.33067800 & -2.06577200 \\
\hline 13 & -2.48868800 & 0.00000000 & 0.00000000 \\
\hline 57 & Cluster $=$ Pt13 $\quad Q=0$ & \multicolumn{2}{|c|}{$\mathrm{M}=7 \quad$ Etot $=-1548.91756366$} \\
\hline Pt 1 & 0.00000000 & 0.00000000 & 000000 \\
\hline t2 & 0.00000000 & 2.5523 & 00 \\
\hline-3 & 2.55231500 & 0.00000000 & 0000000 \\
\hline t4 & 0.00000000 & -2.55231500 & 0.00000000 \\
\hline$=5$ & -1.35017900 & 1.35017900 & 2.00323700 \\
\hline 6 & 1.35017900 & 1.3501 & 32370 \\
\hline 7 & 1.35017900 & -1.350 & 100 \\
\hline t 8 & -1.35017900 & -1.3501 & 23700 \\
\hline t9 & 1.35017900 & 1.35017900 & -2.00323700 \\
\hline 10 & -1.35017900 & 1.35017900 & -2.00323700 \\
\hline 11 & -1.35017900 & -1.35017900 & -2.00323700 \\
\hline$=12$ & 1.35017900 & -1.35017900 & -2.003237 \\
\hline & -2.55231500 & 0.00000000 & 0.0000 \\
\hline 58 & Cluster $=$ Pt 19 & \multicolumn{2}{|c|}{$\mathrm{M}=1 \quad$ Etot $=-2264.01751766$} \\
\hline Pt 1 & 3.73677000 & 0.00000000 & 0.0000000 \\
\hline Pt2 & 1.93761774 & -1.93761774 & 0.00000000 \\
\hline Pt3 & 1.93761774 & 0.00000000 & -1.93761774 \\
\hline Pt 4 & 1.93761774 & 0.00 & 761774 \\
\hline Pt 5 & 1.93761774 & 1.93761774 & 000000 \\
\hline Pt 6 & 0.00000000 & -3.73677000 & 0.00000000 \\
\hline Pt 7 & 0.00000000 & -1.93761774 & -1.93761774 \\
\hline Pt 8 & 0.00000000 & 0.00000000 & -3.73677000 \\
\hline Pt9 & 0.00000000 & -1.937 & \\
\hline & 0.00000000 & 0.0000 & 000000 \\
\hline & 0.00000000 & 1.93761774 & -1.93761774 \\
\hline & 0.00000000 & 0.00000000 & 3.73677000 \\
\hline Pt13 & 0.00000000 & 1.93761774 & 1.93761774 \\
\hline Pt14 & 0.00000000 & 3.73677000 & 0.00000000 \\
\hline 5 & -3.73677000 & 0.00000000 & 000000 \\
\hline & -1.93761774 & -1.93761774 & 0.00000000 \\
\hline & -1.93761774 & 0.00000000 & -1.9376177 \\
\hline & -1.93761774 & 0.00000000 & 1.9376177 \\
\hline & -1.93761774 & 1.93761774 & 0.0 \\
\hline
\end{tabular}




\begin{tabular}{|c|c|c|c|}
\hline \# 59 & Cluster $=\operatorname{Pt} 19 \quad \mathrm{Q}=0$ & \multicolumn{2}{|c|}{ Etot $=-2264.03355210$} \\
\hline Pt1 & 3.64673422 & 0.00000000 & 0.00000000 \\
\hline Pt2 & 1.97670794 & -1.97670794 & 0.00000000 \\
\hline Pt3 & 1.97670794 & 0.00000000 & -1.97670794 \\
\hline Pt 4 & 1.97670794 & 0.00000000 & 1.97670794 \\
\hline Pt5 & 1.97670794 & 1.97670794 & 0.00000000 \\
\hline Pt 6 & 0.00000000 & -3.64673422 & 0.00000000 \\
\hline Pt7 & 0.00000000 & -1.97670794 & -1.97670794 \\
\hline Pt 8 & 0.00000000 & 0.00000000 & -3.64673422 \\
\hline Pt9 & 0.00000000 & -1.97670794 & 1.97670794 \\
\hline Pt10 & 0.00000000 & 0.00000000 & 0.00000000 \\
\hline Pt11 & 0.00000000 & 1.97670794 & -1.97670794 \\
\hline Pt12 & 0.00000000 & 0.00000000 & 3.64673422 \\
\hline Pt13 & 0.00000000 & 1.97670794 & 1.97670794 \\
\hline Pt14 & 0.00000000 & 3.64673422 & 0.00000000 \\
\hline Pt15 & -3.64673422 & 0.00000000 & 0.00000000 \\
\hline Pt16 & -1.97670794 & -1.97670794 & 0.00000000 \\
\hline Pt17 & -1.97670794 & 0.00000000 & -1.97670794 \\
\hline Pt18 & -1.97670794 & 0.00000000 & 1.97670794 \\
\hline Pt19 & -1.97670794 & 1.97670794 & 0.00000000 \\
\hline 60 & er $=$ Pt19 $Q=0$ & Etot $=-22$ & 2365053 \\
\hline Pt1 & -0.17767700 & 0.44246000 & 3.58267100 \\
\hline Pt2 & 1.94558700 & 0.37223000 & 1.90496300 \\
\hline Pt3 & -0.27713700 & 2.09664700 & 1.63739900 \\
\hline Pt 4 & 0.12660100 & -1.65512100 & 2.08543400 \\
\hline Pt5 & -2.09689000 & 0.03142500 & 1.86882300 \\
\hline Pt 6 & 3.83248700 & 0.47228100 & 0.07221800 \\
\hline Pt7 & 1.83141100 & 2.13435500 & -0.20913300 \\
\hline Pt 8 & -0.35070800 & 3.61491800 & -0.42939800 \\
\hline Pt9 & 2.31311200 & -1.69 & 0.27655200 \\
\hline Pt10 & -0.03176800 & -0.00908000 & -0.01074700 \\
\hline Pt11 & -2.29691500 & 1.80231900 & -0.26842800 \\
\hline Pt12 & 0.41549200 & -3.60950600 & 0.45148500 \\
\hline Pt13 & -1.82185500 & -2.19656500 & 0.18760700 \\
\hline Pt14 & -3.74710100 & -0.40626800 & -0.07783800 \\
\hline Pt15 & 0.06215000 & -0.43 & -3.58501800 \\
\hline Pt16 & 2.10703900 & -0.08887700 & -1.82557100 \\
\hline Pt17 & -0.13833600 & 1.64442200 & -2.06648700 \\
\hline Pt18 & 0.26711500 & -2.11448500 & -1.64630900 \\
\hline Pt19 & -1.96260700 & -0.40487900 & -1.94822300 \\
\hline \# 61 & luster $=$ Pt19 & Etot $=-22$ & 9159 \\
\hline Pt 1 & -0.20630764 & 2.31326318 & 2.36393854 \\
\hline Pt2 & 0.00000000 & 0.00000000 & 3.89183725 \\
\hline Pt3 & 0.00000000 & 0.00000000 & -3.89183725 \\
\hline Pt 4 & 2.13629146 & 0.91104786 & -2.36393854 \\
\hline Pt5 & 1.24692956 & 2.08927785 & 0.00000000 \\
\hline Pt 6 & -2.26379659 & $0.51 \xi-2$ & -2.36393854 \\
\hline Pt 7 & 0.21925950 & -2.42318809 & 0.00000000 \\
\hline Pt 8 & -1.19279560 & -1.99273382 & 2.36393854 \\
\hline Pt9 & 1.52660837 & -1.75020464 & -2.36393854 \\
\hline Pt10 & 0.00000000 & 0.00000000 & -1.22708140 \\
\hline Pt11 & -0.20630764 & 2.31326318 & -2.36393854 \\
\hline Pt12 & -1.60169889 & 1.83152285 & 0.00000000 \\
\hline Pt13 & 2.13629146 & 0.91104786 & 2.36393854 \\
\hline Pt14 & -2.26379659 & 0.51862741 & 2.36393854 \\
\hline Pt15 & -2.23683391 & -0.95733448 & 0.00000000 \\
\hline
\end{tabular}




\begin{tabular}{|c|c|c|c|}
\hline Pt16 & 2.37234374 & -0.54027812 & 0.00000000 \\
\hline Pt17 & 0.00000000 & 0.00000000 & 1.22708140 \\
\hline Pt18 & 1.52660837 & -1.75020464 & 2.36393854 \\
\hline 19 & -1.19279560 & -1.99273382 & -2.36393854 \\
\hline 62 & luster $=\operatorname{Pt19} \quad \mathrm{Q}=0$ & \multicolumn{2}{|c|}{$\mathrm{M}=3 \quad \mathrm{E}$ tot $=-2263.94382920$} \\
\hline t1 & -0.21977262 & 2.44997559 & 2.1633792 \\
\hline 2 & 0.00000000 & 0.00000000 & 3.84139648 \\
\hline$=3$ & 0.00000000 & 0.00000000 & -3.841396 \\
\hline 4 & 2.26215177 & 0.96610028 & -2.163379 \\
\hline & 1.28610730 & 2.15072465 & 0.00000000 \\
\hline & -2.39797872 & 0.54806791 & -2.16337921 \\
\hline & 0.22368156 & -2.49592769 & 0.00000000 \\
\hline$=8$ & -1.26225974 & -2.11125099 & 2.16337921 \\
\hline 9 & 1.61785930 & -1.85289278 & -2.16337921 \\
\hline 10 & 0.00000000 & 0.00000000 & -1.2734006 \\
\hline & -0.21977262 & 2.44997559 & -2.16337921 \\
\hline & -1.64803168 & 1.88777120 & 0.00000000 \\
\hline & 2.26215177 & 0.96610028 & 2.163379 \\
\hline & -2.39797872 & 0.548 & 2 . \\
\hline & -2.30464689 & -0.984 & 0 . \\
\hline 1 & 2.44288970 & -0.55855027 & 0.00000000 \\
\hline 7 & 0.00000000 & 0.00000000 & 1.27340064 \\
\hline & 1.61785930 & -1.85289278 & 2.16337921 \\
\hline & -1.26225974 & -2.11125099 & -2.16337921 \\
\hline 63 & ter $=$ Pt19 & \multicolumn{2}{|c|}{$M=5 \quad E$ tot $=-2263.92023629$} \\
\hline Pt 1 & -0.21250741 & 2.38123160 & 2.287517 \\
\hline ? & 0.00000000 & 0.00000000 & 3.84165338 \\
\hline & 0.00000000 & 0.00000000 & -3.84165338 \\
\hline & 2.19901743 & 0.93794759 & -2.287517 \\
\hline 5 & 1.25930503 & 2.10957035 & 0 . \\
\hline Pt 6 & -2.33035423 & 0.53373447 & -2.2875177 \\
\hline Pt & 0.22117517 & -2.44687919 & 0.00000000 \\
\hline 8 & -1.22773071 & -2.05136555 & 2.28751778 \\
\hline & 1.57157492 & -1.80154811 & -2.2875177 \\
\hline & 0.00000000 & 0.000 & -1.3 \\
\hline & -0.21250741 & 2.381 & -2.2 \\
\hline & -1.61717397 & 1.84956334 & 000 \\
\hline & 2.19901743 & 0.93794759 & 2.28751778 \\
\hline & -2.33035423 & 0.53373447 & 2.28751778 \\
\hline & -2.25877351 & -0.96647734 & 0.00000000 \\
\hline & 2.39546728 & -0.54577716 & 0.00000000 \\
\hline & 0.00000000 & 0 . & 1 . \\
\hline Pt1 & 1.57157492 & -1.80154811 & 3751778 \\
\hline Pt19 & -1.22773071 & -2.05136555 & -2.28751778 \\
\hline \# 64 & Cluster $=$ Pt19 $Q=0$ & \multicolumn{2}{|c|}{$\mathrm{M}=5 \quad \mathrm{Etot}=-2263.99768143$} \\
\hline$P+1$ & 2.28684700 & 2.39770300 & 0.19099 \\
\hline $8+$ & 3.83036300 & -0.14437900 & 0. \\
\hline Pt3 & -4.17330200 & -0.28046100 & -0.0277160 \\
\hline Pt 4 & -1.89750400 & 1.04600500 & -2.52863900 \\
\hline Pt 5 & 0.21735900 & 2.08438800 & -1.45449500 \\
\hline Pt 6 & -1.95405000 & 0.66901000 & 2.62382300 \\
\hline Pt 7 & -0.28717100 & -2.54421800 & -0.1850570 \\
\hline Pt & 2.05052800 & -2.30957800 & 1.14879100 \\
\hline & -2.42729000 & -1.50845900 & -1.48561300 \\
\hline & -1.59459900 & 0.44874300 & 0.0210700 \\
\hline & -1.54100400 & 3.02708400 & 0.173409 \\
\hline
\end{tabular}




\begin{tabular}{|c|c|c|c|}
\hline Pt12 & 0.16830300 & 1.85416800 & 1.71984900 \\
\hline Pt13 & 2.36235500 & 0.65512400 & -2.10502100 \\
\hline Pt14 & 2.30858200 & 0.35263500 & 2.20810800 \\
\hline 15 & -0.04058500 & -1.10851100 & 2.16740000 \\
\hline 1 & -0.00090200 & -0.80397600 & -2.30567300 \\
\hline 1 & 1.02934600 & -0.03239900 & -0.00285800 \\
\hline 1 & 2.11537700 & -2.09079300 & -1.40435000 \\
\hline-1 & -2.45265400 & -1.71208500 & 1.23324800 \\
\hline 65 & uster $=P$ t2 $4 \quad Q=0$ & $=3 \quad$ Etot $=-2 \varepsilon$ & 03682393 \\
\hline Pt 1 & 1.31966259 & 2.71617563 & 1.46770345 \\
\hline 8 & 1.05602894 & 2.03393672 & -1.45230921 \\
\hline 2 & -2.01123472 & -0.27111531 & -1.49658478 \\
\hline 24 & -1.16619216 & 2.10085226 & 0.80599145 \\
\hline$=5$ & 1.85473280 & -0.46517699 & -1.64467858 \\
\hline$=6$ & 3.12833938 & 1.26327005 & -0.01560171 \\
\hline 7 & -2.23856504 & 3.07618630 & -1.30597592 \\
\hline 8 & 2.67653477 & 3.83080358 & -0.44573826 \\
\hline 9 & -1.83997241 & -0.34978561 & 1.89081580 \\
\hline 0 & 1.83434903 & -0.01380177 & 1.91626726 \\
\hline & -0.91900019 & 1.49682979 & -2.90475595 \\
\hline 1 & -0.01958776 & 4.15195951 & -0.49653636 \\
\hline 3 & 0.00372135 & -0.24741197 & 0.12996635 \\
\hline & -0.09787229 & 1.24723858 & 3.09443971 \\
\hline & 0.09167929 & -1.55120390 & 3.24179314 \\
\hline 16 & -1.32580855 & -3.017 & 1.46288889 \\
\hline 1 & 1.13471822 & -2.50642565 & 0.96706324 \\
\hline 1 & -3.46384630 & 1.04788435 & 0.11959008 \\
\hline & -0.14673217 & -1.02332184 & -3.13263296 \\
\hline & -3.37285821 & -1.63109917 & 0.21395335 \\
\hline & -1.21359124 & -2.74646150 & -1.12220664 \\
\hline 2 & -0.00602959 & -4.77015997 & 493045 \\
\hline 23 & 1.32909548 & -3.00162975 & -1.55452357 \\
\hline & 3.39242879 & -1.37042547 & 0.24614078 \\
\hline 66 & Cluster $=\mathrm{Pt} 24$ & $=5 \quad$ Etot $=-28$ & 0027 \\
\hline 1 & 1.87936377 & 2.88571152 & 461674 \\
\hline Pt2 & 0.10623365 & 1.59456642 & 0 . \\
\hline Pt 3 & -1.93757357 & -0.10976071 & -1.60607114 \\
\hline Pt 4 & -1.95518816 & 2.67987340 & 1.23998148 \\
\hline Pt 5 & 1.88955275 & -0.00930849 & -1.66255010 \\
\hline Pt 6 & 3.52835927 & 1.17184960 & 0.01779277 \\
\hline Pt 7 & -2.09975102 & 2.96294396 & -1.35931872 \\
\hline Pt 8 & 2.03579054 & 2.78 & -1.3 \\
\hline & -1.85764472 & -0.12484209 & 297786 \\
\hline r & 1.88346213 & -0.03377890 & 1.64271346 \\
\hline Pt11 & -0.20946893 & 1.58001497 & -2.56392723 \\
\hline Pt12 & -0.04697960 & 4.11639985 & -0.03313398 \\
\hline & 0.00847262 & -1.01780521 & -0.02234915 \\
\hline & -0.01364393 & 1.47490481 & 2.7719321 \\
\hline & 0.06293631 & -1.19791361 & 3.0935535 \\
\hline 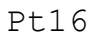 & -1.31229750 & -2.79241388 & 1.35065826 \\
\hline Pt 17 & 1.43461415 & -2.73736413 & 1.30990595 \\
\hline & -3.59556199 & 1.04785687 & -0.02514466 \\
\hline 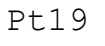 & -0.01236333 & -1.08439447 & -3.0826330 \\
\hline & -3.38111331 & -1.60297536 & 0.00832067 \\
\hline & -1.34654500 & -2.79000536 & -1.36021947 \\
\hline & 0.09983735 & -4.59441266 & -0.0494298 \\
\hline & 1.42405238 & -2.69985725 & -1.4237682 \\
\hline
\end{tabular}




\begin{tabular}{|c|c|c|c|}
\hline Pt24 & 3.41545615 & -1.50020926 & -0.00437366 \\
\hline$\# 67$ & uster $=$ Pt 24 & \multicolumn{2}{|c|}{ Etot $=-2860.01115685$} \\
\hline Pt1 & 2.02327665 & 3.25538402 & 1.75850172 \\
\hline Pt2 & 0.09548345 & 1.62191358 & 0.26868267 \\
\hline Pt 3 & -1.72842259 & -0.41051139 & -1.90198215 \\
\hline Pt4 & -1.92328209 & 2.72524718 & 1.36539630 \\
\hline Pt5 & 1.66452455 & -0.17724074 & -1.94803489 \\
\hline Pt 6 & 2.61455810 & 1.36945580 & -0.03925414 \\
\hline Pt7 & -1.98523485 & 3.30803442 & -1.25610554 \\
\hline Pt 8 & 1.80642549 & 3.27764748 & -1.65633410 \\
\hline Pt9 & -1.82029889 & -0.09216706 & 1.62199967 \\
\hline Pt10 & 1.86078997 & -0.40561406 & 1.74071230 \\
\hline Pt11 & -0.19437584 & 1.65221622 & -2.23692030 \\
\hline Pt12 & 0.14790339 & 4.09225859 & 0.15981452 \\
\hline Pt13 & 0.02203760 & -0.87040728 & -0.07376367 \\
\hline Pt14 & 0.41960858 & 1.48046492 & 2.78659380 \\
\hline Pt15 & -0.10773079 & -1.11968260 & 3.30398497 \\
\hline Pt 16 & -1.21629470 & -2.69842050 & 1.28766056 \\
\hline Pt17 & 1.42760712 & -2.94418616 & 1889 \\
\hline Pt18 & -3.13064645 & 1.0 & 2727 \\
\hline Pt19 & 0.01749967 & -1.63624830 & -3.38865095 \\
\hline Pt20 & -3.34952878 & -1.56055110 & -0.06010422 \\
\hline Pt21 & -1.31219682 & -3.00215646 & -1.37366065 \\
\hline Pt 22 & 0.03357334 & -4.771 & 8563 \\
\hline Pt23 & 1.30275128 & -2.799 & -1.26905730 \\
\hline Pt24 & 3.33197261 & $-1 \cdot 32$ & -0 \\
\hline$\# 68$ & ter $=$ Pt 38 & $=1 \quad$ Etot $=-45$ & 6294 \\
\hline Pt1 & 3.52862470 & 2.26900364 & 319846 \\
\hline Pt2 & 4.11096666 & 0.33482297 & 565588 \\
\hline Pt 3 & 3.27252099 & 0.236 & -2.6 \\
\hline Pt 4 & 3.91809146 & -1.71561502 & -0.93592591 \\
\hline Pt 5 & 1.50150467 & 3.96642630 & -0.52062783 \\
\hline Pt 6 & 2.34445250 & 2.30869622 & 1.43495759 \\
\hline Pt 7 & 2.79305060 & $0.22-3$ & 7981 \\
\hline Pt 8 & 1.34777606 & 2.18 & -2.5 \\
\hline Pt9 & 1.83231844 & 296 & -0.45999465 \\
\hline Pt10 & 2.66722319 & -1.90596595 & 1.39990789 \\
\hline Pt11 & 1.04691409 & 0.00235052 & -4.07242937 \\
\hline Pt12 & 1.69347793 & -2.00894246 & -2.39587097 \\
\hline Pt13 & 2.18826884 & -3.75428485 & -0.47209761 \\
\hline Pt14 & 0.15 & 3.8 & 1.8 \\
\hline Pt15 & 0.84066389 & 691 & 30624 \\
\hline Pt16 & -0.83045775 & 3.60633633 & -1.91394296 \\
\hline Pt17 & -0.13809196 & 1.88086733 & -0.00432606 \\
\hline Pt18 & 0.48607880 & 0.070 & 1.82991896 \\
\hline Pt19 & 1.12684033 & -1.83188247 & 3.58275934 \\
\hline Pt20 & -1.02871556 & 1.665 & -3.70480172 \\
\hline Pt21 & -0.43687862 & $-0.03 \xi$ & -1.80682215 \\
\hline Pt22 & 0.12694079 & -1.85889832 & 0.04735061 \\
\hline Pt23 & 0.67921390 & -3.73399879 & 1.79799861 \\
\hline Pt24 & -0.72213171 & -1.91210737 & -3.59276334 \\
\hline Pt 25 & -0.25424120 & -3.75022714 & -1.70790317 \\
\hline Pt26 & -3.78642698 & 1.51594074 & 0.94600913 \\
\hline Pt27 & -3.17888280 & -0.19362487 & 2.90455716 \\
\hline Pt 28 & -4.28533515 & -0.31383817 & -0.95470905 \\
\hline Pt29 & -3.56810447 & -1.99244388 & 0.9958350 \\
\hline Pt30 & -2.15047142 & 3.53189719 & 0.41415192 \\
\hline
\end{tabular}




\begin{tabular}{|c|c|c|c|}
\hline Pt 31 & -1.61734068 & 1.95508216 & 2.53361606 \\
\hline Pt 32 & -0.82945161 & -0.02069065 & 4.18880675 \\
\hline Pt 33 & -2.69385206 & 1.72191485 & -1.54356355 \\
\hline Pt 34 & -1.81143641 & -0.11782186 & 0.50440194 \\
\hline Pt 35 & -1.33830747 & -2.12048767 & 2.58185962 \\
\hline & -2.89294879 & -0.27849509 & -3.29637566 \\
\hline I & -2.40425124 & -2.13624948 & -1.48270160 \\
\hline-38 & -1.69108437 & -3.80460958 & 0.51711881 \\
\hline$\# 69$ & Cluster $=\mathrm{Pt} 38$ & Etot $=-45$ & .62893548 \\
\hline Pt 1 & 3.39983172 & 2.21233516 & -0.83548937 \\
\hline$t 2$ & 3.96174988 & 0.38279276 & 1.01382820 \\
\hline 7 & 3.30456212 & 0.26400116 & -2.76787821 \\
\hline $\mathrm{P}$ & 3.86365800 & -1.61677947 & -0.86530473 \\
\hline$\Gamma$ & 1.39662421 & 3.90968963 & -0.55265818 \\
\hline$t 6$ & 2.20482031 & 2.32248975 & 1.58743310 \\
\hline 7 & 2.49387247 & 0.15759300 & 3.244 \\
\hline $\mathrm{P}$ & 1.37046147 & 2.11384347 & -2.63272406 \\
\hline 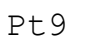 & 1.82691996 & 0.13602821 & -0.50975985 \\
\hline$t 1$ & 2.61834765 & -1.90258025 & 1.49702573 \\
\hline 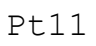 & 1.05652637 & -0.04040367 & -4.18854056 \\
\hline t 1 & 1.69277108 & -1.93171294 & -2.47747087 \\
\hline 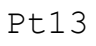 & 2.24606412 & -3.66617702 & -0.54469205 \\
\hline t1 & 0.19812408 & 4.04263394 & 1.87837371 \\
\hline 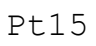 & 0.83558681 & 2.21367035 & 3.82977809 \\
\hline P & -0.78194604 & 3.54272924 & -2.04454089 \\
\hline $\mathrm{P}$ & -0.15136101 & 1.89143192 & -0.06098313 \\
\hline Pt 1 & 0.44201289 & 0.04114163 & 1.7283 \\
\hline $\mathrm{P}$ & 1.09660783 & -2.08297472 & 3.6563 \\
\hline 2 & -1.08139661 & 1.53647005 & -3.75928076 \\
\hline $\mathrm{P}$ & -0.46701545 & -0.04685984 & -1.80297016 \\
\hline Pt 22 & 0.13072039 & -1.88800920 & -0.00519271 \\
\hline $\mathrm{P}$ & 0.70309255 & -3.81600234 & 1.6636 \\
\hline Pt2 & -0.78579530 & -1.89898553 & -3.55997908 \\
\hline $\mathrm{P}$ & -0.18360364 & -3.75101704 & -1.73843601 \\
\hline $\mathrm{P}$ & -3.63971463 & 1.71112834 & 0.9891 \\
\hline $2^{7}$ & -2.98698111 & -0.11135048 & $2.819^{\circ}$ \\
\hline Pt 2 & -4.23238502 & -0.27085494 & -0.8212 \\
\hline $\mathrm{P}$ & -3.54416470 & -2.14242631 & 1.0532 \\
\hline $\mathrm{P}$ & -1.99535158 & 3.66827623 & 0.3254 \\
\hline $\mathrm{P}$ & -1.44834207 & 2.06251272 & 2.53459788 \\
\hline & -0.60848933 & -0.06326073 & 4.05425855 \\
\hline & -2.73842151 & 1.82262960 & -1.56966450 \\
\hline & -1.84949278 & -0.12565471 & 0.44344129 \\
\hline & -1.28974163 & -2.18150788 & 2.51298326 \\
\hline & -2.93159054 & -0.32386169 & -3.16017104 \\
\hline & -2.40781402 & -2.22318408 & -1.41806129 \\
\hline Pt 38 & -1.71874691 & -3.94779432 & 0.48230959 \\
\hline \# 70 & Cluster $=\mathrm{Pt} 38 \quad \mathrm{Q}=0$ & Etot $=-45$ & .62859402 \\
\hline Pt 1 & 3.54635203 & 2.17833318 & -0.94802818 \\
\hline & 4.13280190 & 0.31169929 & 0.88114235 \\
\hline Pt & 3.19589893 & 0.16734443 & -2.77895958 \\
\hline & 3.80173528 & -1.63401860 & -0.91668683 \\
\hline & 1.57628658 & 3.92995657 & -0.53919062 \\
\hline & 2.31961400 & 2.27479011 & 1.42049898 \\
\hline Pt & 2.76026674 & 0.26235623 & 3.13182391 \\
\hline & 1.35642891 & 2.11616813 & -2.51066017 \\
\hline$P$ & 1.82104787 & 0.13544172 & -0.46379846 \\
\hline
\end{tabular}




\begin{tabular}{|c|c|c|c|}
\hline Pt10 & 2.68402211 & -1.87783034 & 1.51830412 \\
\hline Pt11 & 0.90961797 & 0.03939901 & -4.15076572 \\
\hline Pt12 & 1.61949514 & -1.95544727 & -2.47958700 \\
\hline Pt13 & 2.13805344 & -3.64787441 & -0.43640300 \\
\hline Pt14 & 0.17132160 & 3.86739313 & 1.77644947 \\
\hline Pt15 & 0.81547809 & 2.05211366 & 3.62364985 \\
\hline Pt16 & -0.75896533 & 3.72235147 & -1.84789749 \\
\hline Pt17 & -0.16114052 & 1.89404872 & -0.02404403 \\
\hline Pt18 & 0.45326030 & 0.06002790 & 1.80379910 \\
\hline Pt19 & 1.04425387 & -1.82919137 & 3.63427986 \\
\hline Pt20 & -1.06666470 & 1.76637945 & -3.64944286 \\
\hline Pt21 & -0.47304193 & -0.04589459 & -1.80733113 \\
\hline Pt22 & 0.14798837 & -1.88448074 & 0.02738379 \\
\hline Pt23 & 0.76458564 & -3.75246636 & 1.84806633 \\
\hline Pt24 & -0.82374000 & -2.01940702 & -3.62886198 \\
\hline Pt25 & -0.14554250 & -3.82656152 & -1.78885726 \\
\hline Pt26 & -3.79692119 & 1.65045297 & 0.93597041 \\
\hline Pt27 & -3.19104192 & -0.18831257 & 2.76094418 \\
\hline Pt28 & -4.13055540 & -0.30427982 & -0.83264036 \\
\hline Pt29 & -3.54898220 & -2.20359665 & 0.97934066 \\
\hline Pt 30 & -2.11733626 & 3.67080411 & 0.42935922 \\
\hline Pt 31 & -1.61767535 & 1.98339588 & 2.49297481 \\
\hline Pt 32 & -0.90166878 & -0.04522369 & 4.11665733 \\
\hline Pt33 & -2.68536896 & 1.86945893 & -1.51864906 \\
\hline Pt 34 & -1.83253468 & -0.11750853 & 0.46271850 \\
\hline Pt35 & -1.34711094 & -2.13382495 & 2.48409079 \\
\hline Pt36 & -2.76611893 & -0.25712428 & -3.13713674 \\
\hline Pt 37 & -2.32383777 & -2.27992502 & -1.41472837 \\
\hline Pt 38 & -1.57026142 & -3.94894714 & 0.54621520 \\
\hline \# 71 & Cluster $=$ Pt 38 & $=7 \quad$ Etot $=-4$ & .62729666 \\
\hline Pt1 & 3.41054909 & 2.23207637 & -0.88880038 \\
\hline Pt2 & 4.02620263 & 0.37171577 & 0.91163417 \\
\hline Pt3 & 3.23853623 & 0.26491628 & -2.71969946 \\
\hline Pt 4 & 3.92875762 & -1.62897440 & -0.90839709 \\
\hline Pt 5 & 1.35813228 & 3.88873263 & -0.52893467 \\
\hline Pt 6 & 2.23456661 & 2.32482036 & 1.53254538 \\
\hline Pt 7 & 2.65822722 & 0.17362049 & 3.15546248 \\
\hline Pt 8 & 1.30954702 & 2.13018753 & -2.58271139 \\
\hline Pt9 & 1.81223455 & 0.14918232 & -0.45388007 \\
\hline Pt10 & 2.68324040 & -1.91885056 & 1.4260481 \\
\hline Pt11 & 1.01976027 & -0.05151913 & -4.17294329 \\
\hline Pt12 & 1.73398422 & -1.93562397 & -2.41597607 \\
\hline Pt13 & 2.26381034 & -3.72526709 & -0.53387440 \\
\hline Pt14 & 0.19897918 & 3.96410767 & 1.92828343 \\
\hline Pt15 & 0.84154796 & 2.08770579 & 3.78777510 \\
\hline Pt16 & -0.82318017 & 3.65253558 & -2.0854454 \\
\hline Pt17 & -0.15554975 & 1.86162239 & -0.01739291 \\
\hline Pt18 & 0.44745297 & 0.05171555 & 1.80950779 \\
\hline Pt19 & 1.06651113 & -1.95793349 & 3.5443606 \\
\hline Pt20 & -1.07172790 & 1.58734757 & -3.76704991 \\
\hline Pt21 & -0.45184460 & -0.04513566 & -1.79679848 \\
\hline Pt22 & 0.13281237 & -1.84645080 & 0.02580682 \\
\hline Pt23 & 0.68827750 & -3.77192648 & 1.6488829 \\
\hline Pt24 & -0.74119166 & -1.93925610 & -3.5304463 \\
\hline Pt25 & -0.14322435 & -3.75432013 & -1.6649278 \\
\hline Pt26 & -3.64929053 & 1.69198207 & 0.9423566 \\
\hline Pt27 & -3.04872479 & -0.12474772 & 2.8067233 \\
\hline & -4.22238823 & -0.24092260 & -0.8278721 \\
\hline
\end{tabular}




\begin{tabular}{|c|c|c|c|}
\hline Pt29 & -3.63341284 & -2.08669879 & 1.06128770 \\
\hline Pt30 & -1.97123037 & 3.64256827 & 0.30562707 \\
\hline Pt31 & -1.52985288 & 2.05930587 & 2.54636955 \\
\hline Pt32 & -0.74338140 & -0.10042878 & 4.10058217 \\
\hline Pt3. & -2.66195066 & 1.81780265 & -1.57141843 \\
\hline Pt34 & -1.83281041 & -0.12872867 & 0.47327110 \\
\hline Pt35 & -1.36116855 & -2.20944542 & 2.50034118 \\
\hline Pt36 & -2.87363162 & -0.32542586 & -3.13408201 \\
\hline Pt37 & -2.38283685 & -2.24537190 & -1.40929077 \\
\hline Pt 3 & -1.75573203 & -3.91491764 & 0.50307542 \\
\hline \# 72 & Cluster $=$ Pt55 $\quad \mathrm{Q}=0$ & \multicolumn{2}{|c|}{$\mathrm{M}=5 \quad$ Etot $=-6554.89506114$} \\
\hline Pt 1 & 0.05500543 & -0.03757037 & 0.00000000 \\
\hline Pt2 & 2.71130788 & 0.15502565 & 0.00000000 \\
\hline Pt 3 & 1.08058871 & 2.40998294 & 0.00000000 \\
\hline Pt 4 & 1.11358695 & 0.73642347 & -2.28933795 \\
\hline Pt 5 & 1.34585841 & -1.79449446 & -1.40123838 \\
\hline Pt 6 & 1.34585841 & -1.79449446 & 1.40123838 \\
\hline Pt7 & 1.11358695 & 0.73642347 & 2.28933795 \\
\hline Pt 8 & -1.21116731 & 1.84103365 & 1.38872370 \\
\hline Pt9 & -1.21116731 & 1.84103365 & -1.38872370 \\
\hline Pt10 & -1.23644325 & -0.81839859 & -2.13927003 \\
\hline Pt11 & -0.78494839 & -2.55648761 & 0.00000000 \\
\hline Pt12 & -1.23644325 & -0.81839859 & 2.13927003 \\
\hline Pt13 & -2.66443341 & -0.01518191 & 0.00000000 \\
\hline Pt14 & 5.20809235 & 0.62065509 & 0.00000000 \\
\hline Pt15 & 1.72512614 & 4.88797939 & 0.00000000 \\
\hline Pt16 & 2.14670577 & 1.70385189 & -4.39496479 \\
\hline Pt17 & 2.71324961 & -3.45626739 & -2.72160680 \\
\hline Pt18 & 2.71324961 & -3.45626739 & 2.7216068 \\
\hline Pt19 & 2.14670577 & 1.70385189 & 4.39496479 \\
\hline Pt20 & -2.77195722 & 3.45366463 & 2.72298659 \\
\hline Pt21 & -2.77195722 & 3.45366463 & -2.72298659 \\
\hline Pt22 & -2.14207690 & -1.66805061 & -4.37340500 \\
\hline Pt23 & -1.78430545 & -4.91349065 & 0.00000000 \\
\hline Pt & -2.14207690 & -1.66805061 & 4.37340500 \\
\hline Pt25 & -5.14336230 & -0.62865384 & 0.00000000 \\
\hline Pt26 & 3.64375021 & 2.87252000 & 0.00000000 \\
\hline Pt27 & 3.81548932 & 1.27673957 & -2.26531120 \\
\hline Pt28 & 4.15122678 & -1.49039022 & -1.44132720 \\
\hline Pt29 & 4.15122678 & -1.49039022 & 1.44132720 \\
\hline Pt 3 & 3.81548932 & 1.27673957 & 2.26531120 \\
\hline Pt31 & 1.98728652 & 3.43180516 & -2.27364320 \\
\hline Pt32 & 1.98728652 & 3.43180516 & 2.27364320 \\
\hline Pt33 & -0.60776291 & 4.37775177 & 1.39033746 \\
\hline Pt34 & -0.60776291 & 4.37775177 & -1.39033746 \\
\hline Pt35 & 2.61543845 & -0.91712573 & -3.74055880 \\
\hline Pt36 & -0.32777066 & 2.73075202 & -3.69281739 \\
\hline Pt37 & 0.00392115 & 0.02844363 & -4.56012169 \\
\hline Pt38 & 2.80973093 & -3.62955387 & 0.0000000 \\
\hline Pt39 & 0.30217694 & -2.67678673 & -3.65752452 \\
\hline Pt 40 & 0.48302998 & -4.43482977 & -1.44311040 \\
\hline Pt41 & 2.61543845 & -0.91712573 & 3.74055880 \\
\hline Pt 42 & 0.48302998 & -4.43482977 & 1.4431104 \\
\hline Pt 43 & 0.30217694 & -2.67678673 & 3.65752452 \\
\hline Pt 44 & -0.32777066 & 2.73075202 & 3.6928173 \\
\hline Pt 45 & 0.00392115 & 0.02844363 & 4.5601216 \\
\hline Pt 46 & -2.92338485 & 3.50782276 & 0.00000000 \\
\hline Pt 47 & -2.55103284 & 0.93112831 & 3.68837417 \\
\hline
\end{tabular}




\begin{tabular}{|c|c|c|c|}
\hline Pt 48 & -4.18817341 & 1.50347593 & 1.44630771 \\
\hline Pt 49 & -2.55103284 & 0.93112831 & -3.68837417 \\
\hline Pt50 & -4.18817341 & 1.50347593 & -1.44630771 \\
\hline Pt51 & -2.03368303 & -3.44379352 & -2.28520303 \\
\hline Pt52 & -3.79623890 & -1.23800261 & -2.28315168 \\
\hline Pt53 & -2.03368303 & -3.44379352 & 2.28520303 \\
\hline Pt5 4 & -3.55649418 & -2.82690838 & 0.00000000 \\
\hline Pt5 5 & -3.79623890 & -1.23800261 & 2.28315168 \\
\hline \# 73 & Cluster $=$ Pt5 $5 \quad \mathrm{Q}=0$ & \multicolumn{2}{|c|}{$\mathrm{M}=5 \quad \mathrm{Etot}=-6554.81722740$} \\
\hline Pt 1 & -3.78673028 & 0.03549592 & 3.95454730 \\
\hline Pt2 & -1.90603422 & -1.82089914 & 3.90649271 \\
\hline Pt 3 & -0.04135293 & -3.73021610 & 3.79557864 \\
\hline Pt 4 & -1.90414327 & 1.86522397 & 3.87281076 \\
\hline Pt5 & 0.01166950 & 0.01236892 & 4.08355907 \\
\hline Pt 6 & 1.90260099 & -1.88649427 & 3.90871343 \\
\hline Pt 7 & -0.00849575 & 3.72930487 & 3.82382170 \\
\hline Pt 8 & 1.92166492 & 1.88342198 & 3.91583705 \\
\hline Pt9 & 3.83421252 & -0.02576866 & 3.73998577 \\
\hline Pt10 & -3.94190718 & $-1.7685578 \varepsilon$ & 1.97808390 \\
\hline Pt11 & -1.93456073 & -3.83172180 & 1.87904160 \\
\hline Pt 1 : & -3.93187915 & 1.78593179 & 1.93467164 \\
\hline Pt13 & -1.88708475 & -0.02627104 & 1.90967162 \\
\hline Pt14 & 0.05561612 & -1.92965854 & 1.93845867 \\
\hline Pt15 & 1.86188768 & -3.90691231 & 1.89049798 \\
\hline Pt16 & -1.94826526 & 3.84131335 & 1.87204601 \\
\hline Pt17 & 0.07493282 & 1.91570717 & 1.96638135 \\
\hline Pt18 & 1.97677561 & -0.01028980 & 1.91613826 \\
\hline Pt19 & 3.92985852 & -1.89884652 & 1.86661952 \\
\hline Pt20 & 1.85672561 & 3.91261556 & 1.90336181 \\
\hline Pt21 & 3.93139880 & 1.89744010 & 1.86426448 \\
\hline Pt22 & -3.86953115 & -3.59580487 & 0.03352524 \\
\hline Pt23 & -4.12052578 & -0.0094208 & 0.00331555 \\
\hline Pt24 & -1.93347898 & -1.91199915 & -0.02121516 \\
\hline Pt25 & -0.04599831 & -4.06960126 & 0.00513599 \\
\hline Pt26 & -3.88022221 & 3.58464031 & -0.03030811 \\
\hline Pt27 & -1.92953290 & 1.89866656 & 0.03313700 \\
\hline Pt28 & 0.03186554 & -0.00028316 & -0.00669456 \\
\hline Pt29 & 1.95753088 & -1.9397630 & -0.00040855 \\
\hline Pt30 & 3.77272363 & -3.81834561 & -0.00339885 \\
\hline Pt31 & -0.01375104 & 4.0291398 & 0.01178998 \\
\hline Pt32 & 1.97199288 & 1.94193469 & 0.02678597 \\
\hline & 4.13673344 & -0.00345910 & -0.01280756 \\
\hline Pt 34 & 3.74987519 & 3.83969853 & -0.00266607 \\
\hline Pt35 & -3.74766466 & -0.0155929 & -3.93190638 \\
\hline Pt36 & -1.89544190 & -1.86629120 & -3.90879952 \\
\hline Pt37 & -0.00018611 & -3.72434596 & -3.83883034 \\
\hline Pt38 & -1.86250454 & 1.85111760 & -3.93533328 \\
\hline Pt & 0.02572953 & -0.00898010 & -4.05425186 \\
\hline Pt 40 & 1.91359573 & -1.8811039 & -3.92659109 \\
\hline Pt41 & -0.02917509 & 3.76352578 & -3.78883293 \\
\hline Pt 42 & 1.90500766 & 1.89127732 & -3.91903861 \\
\hline Pt 43 & 3.83924215 & 0.01632880 & -3.73695314 \\
\hline Pt 44 & -3.92792560 & -1.7950371 & -1.92891109 \\
\hline Pt 45 & -1.94578482 & -3.82783686 & -1.88050001 \\
\hline Pt 46 & -3.89544966 & 1.76342575 & -1.95418273 \\
\hline Pt 47 & -1.88365131 & -0.00766547 & -1.91370353 \\
\hline Pt 48 & 0.07894972 & -1.94110870 & -1.96035831 \\
\hline & 1.84291099 & $-3.9353971 \varepsilon$ & -1.88505849 \\
\hline
\end{tabular}




\begin{tabular}{|c|c|c|c|}
\hline t50 & -1.91015663 & 3.84519663 & -1.86867649 \\
\hline Pt51 & -0.00812878 & 1.93523738 & -1.94394739 \\
\hline Pt52 & 1.95929961 & 0.00803660 & -1.90959608 \\
\hline Pt53 & 3.90526676 & -1.89684843 & -1.88206703 \\
\hline Pt 54 & 1.84808944 & 3.91013421 & -1.88505535 \\
\hline Pt 55 & 3.89340678 & 1.92733753 & -1.90418048 \\
\hline 74 & Iuster $=$ Pt 75 & \multicolumn{2}{|c|}{$\mathrm{M}=5 \quad$ Etot $=-8938.89519301$} \\
\hline Pt1 & -3.72900003 & 0.24531918 & 0.2165235 \\
\hline Pt2 & 2.41094934 & -0.07023137 & 4.20634457 \\
\hline Pt3 & 4.02258414 & 4.06464776 & -1.20605177 \\
\hline Pt 4 & -0.01558795 & 0.00000520 & 5.43601065 \\
\hline Pt5 & -0.21186562 & 5.81026674 & 1.37875931 \\
\hline Pt 6 & -0.96791104 & 3.66017092 & -2.95468992 \\
\hline Pt7 & -1.39078672 & -3.64813343 & -3.01379007 \\
\hline Pt 8 & 3.19586359 & 1.86700086 & -0.17661920 \\
\hline Pt9 & 3.12145347 & -2.48975078 & -3.05183278 \\
\hline Pt10 & -3.82856369 & -2.53186660 & -2.86469808 \\
\hline Pt11 & 1.17134114 & -4.38118867 & 2.68607092 \\
\hline Pt12 & 0.50134379 & -2.26281756 & -1.37701246 \\
\hline Pt13 & 5.16029031 & -2.46441355 & 1.31679851 \\
\hline Pt14 & 3.53690571 & -4.57169299 & 1.43475276 \\
\hline Pt15 & 4.63454960 & -0.26011779 & -2.71442528 \\
\hline Pt16 & -4.04258711 & 0.19620767 & 3.09950646 \\
\hline Pt17 & 1.60094909 & 4.34183015 & 2.83640532 \\
\hline Pt18 & 5.25063108 & -2.51363454 & -1.38418844 \\
\hline Pt19 & -1.93030693 & 1.48687155 & 4.21990151 \\
\hline Pt20 & 1.84348118 & 4.69223198 & 0.10001432 \\
\hline Pt21 & -0.92903207 & 3.67794727 & 0.05444017 \\
\hline Pt22 & -5.45057323 & 1.42606041 & -1.27293249 \\
\hline Pt23 & -1.88556571 & 1.48978661 & -4.26429377 \\
\hline Pt24 & 2.38787182 & -0.13308447 & -4.19838775 \\
\hline Pt25 & 1.68463887 & 4.23853197 & -2.64262141 \\
\hline Pt26 & -1.92326543 & 1.55403801 & -1.11680091 \\
\hline Pt27 & -5.74272546 & -1.17852438 & -1.52807872 \\
\hline Pt28 & -4.30865457 & -2.78084980 & -0.07050277 \\
\hline Pt29 & -1.76324662 & 1.58043775 & 1.58357365 \\
\hline Pt 30 & 3.38453035 & 2.13260331 & -3.05178903 \\
\hline Pt31 & 0.99525441 & 2.32283672 & 1.32059852 \\
\hline Pt 32 & 1.21995261 & -4.38443928 & -2.64318865 \\
\hline Pt33 & 0.63696916 & -2.22161647 & -4.08567231 \\
\hline Pt 34 & 0.80249992 & 2.25196661 & 4.26847971 \\
\hline Pt 35 & -1.94230584 & -1.23986646 & 1.50153328 \\
\hline Pt 36 & 3.01215247 & -2.38422496 & 2.96431731 \\
\hline Pt 37 & -0.77184372 & -5.69180669 & 1.37263880 \\
\hline Pt 38 & -5.70529772 & -1.00011288 & 1.23019807 \\
\hline Pt 39 & -3.33215161 & -4.77251871 & 1.34816366 \\
\hline Pt 40 & 2.95572271 & -2.46751506 & -0.08677127 \\
\hline Pt 41 & 0.60192489 & -2.31126991 & 1.27149715 \\
\hline Pt 42 & 0.94170428 & 2.13846307 & -4.08541498 \\
\hline Pt 43 & 3.57367590 & -4.62452578 & -1.37594407 \\
\hline Pt 44 & -1.05424099 & 3.79484488 & 3.05497525 \\
\hline Pt 45 & 3.22191191 & 2.19751087 & 2.96004494 \\
\hline Pt 46 & 5.34565854 & -0.23454154 & -0.00690430 \\
\hline Pt 47 & -5.55316076 & 1.68722512 & 1.42748500 \\
\hline Pt 48 & 5.56905414 & 1.92162992 & -1.51233632 \\
\hline Pt 49 & -3.62860336 & 2.93641808 & 2.76216293 \\
\hline Pt 50 & -2.68619724 & 5.07923867 & -1.34207113 \\
\hline Pt51 & -2.75579681 & 5.11467393 & 1.3772026 \\
\hline
\end{tabular}




$\begin{array}{lrrr}\text { Pt52 } & -3.95210805 & 3.21869579 & -0.05002810 \\ \text { Pt53 } & 0.54438775 & -2.23229776 & 4.06825645 \\ \text { Pt54 } & 5.30737644 & 2.07675615 & 1.24191942 \\ \text { Pt55 } & -3.84606369 & 0.12651622 & -2.98500106 \\ \text { Pt56 } & -1.94180177 & -1.33983918 & -4.32277940 \\ \text { Pt57 } & 0.22647348 & 0.15749732 & 2.78420604 \\ \text { Pt58 } & 2.30181389 & -0.28427198 & -1.56162997 \\ \text { Pt59 } & 0.03895721 & 0.03227536 & -5.48817745 \\ \text { Pt60 } & 2.43659778 & -0.22957476 & 1.15747920 \\ \text { Pt61 } & -2.22560583 & -1.32874075 & -1.21619446 \\ \text { Pt62 } & 0.86741415 & 2.13374764 & -1.39265933 \\ \text { Pt63 } & -0.11979333 & 5.75801992 & -1.37417248 \\ \text { Pt64 } & -0.75545737 & -5.66605233 & -1.36798589 \\ \text { Pt65 } & -0.02471125 & 0.03189074 & 0.05449081 \\ \text { Pt66 } & -1.47067689 & -3.63928241 & 2.99048014 \\ \text { Pt67 } & -3.27435114 & -4.77667375 & -1.43913824 \\ \text { Pt68 } & -0.38968392 & 0.03306848 & -2.71801304 \\ \text { Pt69 } & 4.5415717 & -0.14042851 & 2.58354707 \\ \text { Pt70 } & 1.39263820 & -5.10918336 & 0.00945691 \\ \text { Pt71 } & -2.05632845 & -1.26960030 & 4.15164222 \\ \text { Pt72 } & -1.40519352 & -3.54357683 & 0.02015435 \\ \text { Pt73 } & -3.51527068 & 2.82123737 & -2.71369351 \\ \text { Pt74 } & -3.89938314 & -2.46863712 & 2.65042372 \\ \text { Pt75 } & 3.98501877 & 4.34843253 & 1.51603545\end{array}$

Table S2. Cartesian coordinates ( $\AA$ ) and binding energies (kcal/mol) of platinum clusters optimized with SC potential.

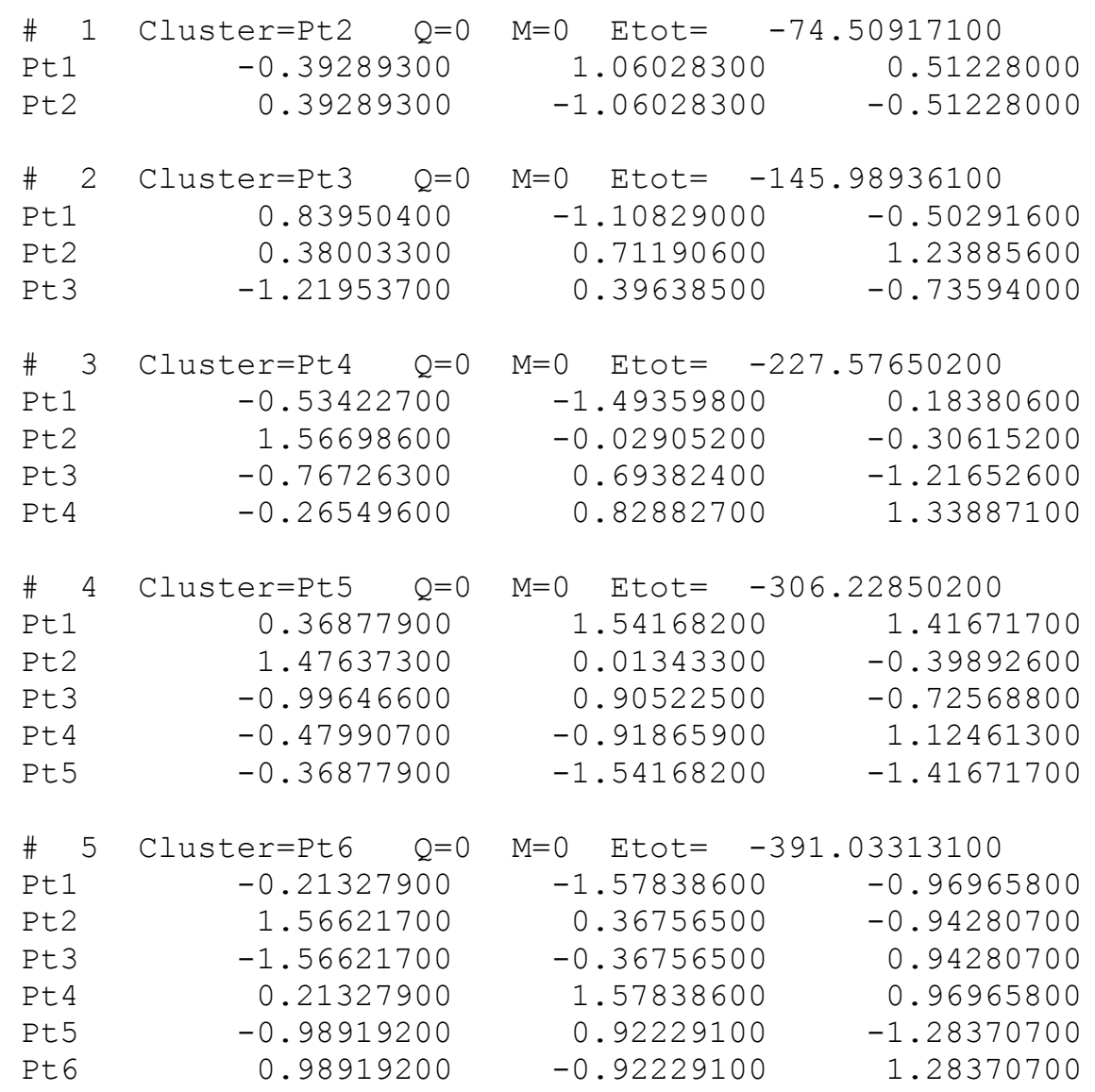




\begin{tabular}{|c|c|c|c|}
\hline 6 & luster $=$ Pt 7 & tot $=$ & -4 \\
\hline Pt1 & -1.51165800 & -1.44081000 & 0.84265600 \\
\hline Pt2 & -0.67523900 & -1.35443700 & -1.66753300 \\
\hline t3 & 1.09433700 & 0.60372200 & -1.87324800 \\
\hline$t 4$ & -1.02597600 & 0.89448700 & -0.31108600 \\
\hline$=5$ & 1.02597600 & -0.89448700 & 0.31108600 \\
\hline$=6$ & 1.35157700 & 1.72755800 & 0.50980200 \\
\hline$=7$ & -0.25901700 & 0.46396700 & 2.18832300 \\
\hline 7 & Cluster $=$ Pt 8 & Etot $=$ & -553.17646700 \\
\hline Pt1 & 0.97610800 & 0.72651700 & 1.31698500 \\
\hline t2 & 1.37824900 & -1.85486700 & 0.85078800 \\
\hline t3 & 0.43026800 & 2.31214900 & -0.72991500 \\
\hline$t 4$ & -1.46417300 & 1.70272900 & 1.01037300 \\
\hline t5 & -1.24879700 & 0.33239100 & -1.24301500 \\
\hline t 6 & 1.35977600 & -0.13588900 & -1.16086500 \\
\hline t7 & -0.34434400 & -2.16001200 & -1.13124600 \\
\hline t8 & -1.08708800 & -0.92301800 & 1.08689500 \\
\hline & ter $=$ Pt 9 & $\mathrm{M}=0 \quad \mathrm{E}$ tot $=$ & 1132 \\
\hline Pt1 & -0.09368800 & -0.83188500 & -1.73344200 \\
\hline Pt2 & -1.61731900 & 0.52743700 & 2.10384500 \\
\hline Pt3 & -0.10573100 & 2.47515400 & 1.08743100 \\
\hline t 4 & -1.51804100 & -1.54372600 & 0.41769700 \\
\hline Pt 5 & 0.63982900 & -0.05206300 & 0.85177300 \\
\hline t 6 & 0.92658100 & -2.54194700 & 32600 \\
\hline Pt 7 & -1.58588700 & 55200 & 9400 \\
\hline Pt 8 & 2.43816800 & -0.59423000 & -1.01108800 \\
\hline Pt9 & 0.91608700 & 1.59270700 & -1.21904800 \\
\hline & Cluster $=$ Pt10 & $\mathrm{M}=0 \quad \mathrm{Etot}=-7$ & 9453 \\
\hline Pt1 & 2.41903700 & 0.65372700 & -0.26628100 \\
\hline Pt2 & -0.65919600 & -2.42902600 & -0.12368100 \\
\hline Pt 3 & -1.68066900 & 1.71369300 & 0.76721100 \\
\hline Pt 4 & 1.98131600 & -1.96551600 & 0.27874600 \\
\hline Pt 5 & 0.91484200 & 2.35970800 & 1.20888500 \\
\hline$=6$ & -1.74596300 & -0.18861100 & -1.124819 \\
\hline t7 & 3200500 & 1.69 & 31600 \\
\hline Pt 8 & 0.75518500 & -0.83527500 & -1.75533200 \\
\hline Pt9 & -2.29900000 & -0.85885400 & 1.35776800 \\
\hline Pt10 & 0.18244200 & -0.14196200 & 0.86931900 \\
\hline \# 10 & Cluster=Pt11 & $=0 \quad$ Etot $=$ & 812 \\
\hline Pt1 & -1.72341300 & -1.40483300 & 347400 \\
\hline Pt2 & 1.04984700 & 2.00306700 & 126400 \\
\hline Pt 3 & 2.22998100 & 1.00052700 & 931900 \\
\hline Pt 4 & -0.54328000 & -2.40737300 & -0.90710900 \\
\hline Pt 5 & 0.41580500 & -0.33186600 & 0.35636600 \\
\hline Pt 6 & -1.56978500 & 40500 & 1.25750700 \\
\hline Pt 7 & -1.97489100 & -0.14809400 & -0.94442100 \\
\hline Pt 8 & -0.27352300 & 1.94261800 & -0.98258600 \\
\hline Pt9 & 0.02341100 & 0.03431700 & 2.92352900 \\
\hline Pt10 & 2.08629900 & -1.71813700 & -1.11539800 \\
\hline Pt11 & 0.27955000 & -0.27063100 & -2.36330500 \\
\hline \# 1 & Eer=Pt12 $Q=0$ & Etot $=-\varsigma$ & 906.6391 \\
\hline Pt 1 & -0.46244100 & 1.29316900 & 2.09400 \\
\hline t 2 & 2.07507100 & 1.36006800 & 1.1489220 \\
\hline
\end{tabular}




\begin{tabular}{|c|c|c|c|}
\hline Pt 3 & 2.51914500 & -1.03960300 & -0.22104100 \\
\hline Pt 4 & 0.23802100 & -2.49195800 & -0.06691100 \\
\hline Pt 5 & 0.01077500 & 2.72987800 & -0.15289500 \\
\hline t 6 & 1.04883900 & -0.97792400 & 2.05294600 \\
\hline t7 & -2.20728200 & 1.18274700 & -0.00047800 \\
\hline t8 & -0.82095600 & 1.17679500 & -2.32742600 \\
\hline 59 & -1.77437300 & -1.15659000 & -1.33599700 \\
\hline t10 & 0.21312000 & 0.14516700 & -0.18519600 \\
\hline-1 & -1.56922000 & -1.06887600 & 1.36361500 \\
\hline$=12$ & 0.72930200 & -1.15287200 & -2.36954200 \\
\hline 12 & Cluster=Pt13 $\mathrm{Q}=0$ & \multicolumn{2}{|c|}{$\mathrm{M}=0 \quad \mathrm{E}$ tot $=-1011.79510500$} \\
\hline t1 & 2.26264600 & -1.29936000 & 0.08110100 \\
\hline t2 & 0.49942400 & -1.60061500 & -2.00077200 \\
\hline t3 & -2.04107500 & -1.27217500 & -1.01492200 \\
\hline t 4 & 1.84796700 & 0.76793200 & -1.67624000 \\
\hline t5 & 0.00000000 & 0.00000000 & 0.00000000 \\
\hline$=6$ & 0.81187900 & -0.78473400 & 2.35362000 \\
\hline$=7$ & -0.49942400 & 1.60061500 & 2.00077200 \\
\hline$=8$ & -1.84796700 & -0.76793200 & 1.67624000 \\
\hline$E 9$ & -0.14091400 & -2.56021600 & 0.48981800 \\
\hline 10 & -2.26264600 & 1.29936000 & -0.08110100 \\
\hline 11 & -0.81187900 & 0.78473400 & -2.35362000 \\
\hline$=12$ & 2.04107500 & 1.27217500 & 1.01492200 \\
\hline & 0.14091400 & 2.56021600 & -0.48981800 \\
\hline 13 & luster $=\operatorname{Pt14} \quad \mathrm{Q}=0$ & \multicolumn{2}{|c|}{$\mathrm{M}=0 \quad$ Etot $=-1089.15633700$} \\
\hline Pt 1 & 2.11320400 & 0.41109000 & -1.60480200 \\
\hline t2 & -0.31703700 & 1.26442400 & -2.52991200 \\
\hline Pt3 & 1.23738900 & 0.36679700 & 3.63330700 \\
\hline t 4 & -1.90696800 & 1.85155800 & -0.38108100 \\
\hline t5 & -0.47287200 & 1.38435000 & 1.90317700 \\
\hline$t 6$ & -2.30793300 & -0.46858700 & 1.04123400 \\
\hline th & 0.26012200 & -1.41415700 & -2.45606000 \\
\hline Pt8 & -0.97158700 & -2.48952000 & -0.26139200 \\
\hline$=9$ & 2.06302100 & 0.47571400 & 1.13126300 \\
\hline 0 & 1.71646400 & -1.91056900 & -0 . \\
\hline & -0.09061300 & -0.02 & -0 \\
\hline & 0.11715900 & -1.35396900 & 1.97867600 \\
\hline $2+3$ & -2.22045000 & -0.52534300 & -1.70098600 \\
\hline tet14 & 0.78010000 & 2.43507200 & -0.30358800 \\
\hline & luster $=$ Pt15 $\quad \mathrm{Q}=0$ & \multicolumn{2}{|c|}{$\mathrm{M}=0 \quad \mathrm{E}$ tot $=-1177.73957900$} \\
\hline D. & -1.18081900 & -0.60313200 & -2.3255620 \\
\hline Pt 2 & 2.01300800 & 0.17998300 & 1.75549100 \\
\hline Pt3 & 2.94622200 & -0.23094300 & -0.77690200 \\
\hline Pt 4 & -0.37660700 & -1.19919200 & 2.03137900 \\
\hline Pt & -2.21628100 & -0.25907700 & 0.16043100 \\
\hline Pt & 0.37432200 & -0.19033400 & -0.25642000 \\
\hline Pt & -0.81435800 & -2.52389100 & -0.36487700 \\
\hline Pt 8 & -0.96427500 & 1.88099900 & -1.11283900 \\
\hline Pt9 & -2.41434200 & 0.39152500 & 2.72009100 \\
\hline Pt 1 & 1.64654700 & 2.10074200 & -0.20519500 \\
\hline & 1.13707100 & 0.81298800 & -2.55292200 \\
\hline & -0.32386500 & 1.55068300 & 1.5796370 \\
\hline & -2.76766100 & 2.24340300 & 0.82972000 \\
\hline & 1.21609800 & -1.88550000 & -2.07439900 \\
\hline & 1.72494000 & -2.26825400 & 0.5923680 \\
\hline
\end{tabular}




$\begin{array}{lr}\# 15 & \text { Cluster=Pt16 } Q=0 \\ \text { Pt1 } & 1.12095100 \\ \text { Pt2 } & -2.69800700 \\ \text { Pt3 } & -1.46272100 \\ \text { Pt4 } & 1.31614400 \\ \text { Pt5 } & -1.54222000 \\ \text { Pt6 } & 0.26135100 \\ \text { Pt7 } & 1.90260500 \\ \text { Pt8 } & 2.56175900 \\ \text { Pt9 } & 0.17116500 \\ \text { Pt10 } & -1.80282700 \\ \text { Pt11 } & -0.08370200 \\ \text { Pt12 } & -0.78666800 \\ \text { Pt13 } & 0.22204500 \\ \text { Pt14 } & -2.13491300 \\ \text { Pt15 } & 2.28622800 \\ \text { Pt16 } & 0.66881100\end{array}$

\# 16 Cluster $=$ Pt17 $Q=0$ $\begin{array}{ll}\text { Pt } 1 & 0.08869500\end{array}$

Pt2 $\quad-0.57295900$

Pt3 -1.64089300

Pt4 2.36957100

Pt5 0.00808700

Pt6 1.31921600

Pt7 2.12013200

Pt8 0.42096700

Pt9 0.76443400

Pt10 0.76016300

Pt11

Pt12

Pt13

Pt14

Pt15

Pt16

Pt17

\# 17 Cluster=Pt18 Q $=0$

Pt $1 \quad-3.30089400$

Pt2

Pt 3

Pt 4

Pt5

Pt 6

Pt 7

Pt 8

Pt9

Pt10

Pt11

Pt 12

Pt13

Pt14

Pt15

Pt 16

Pt 17

Pt18

\# 18 Cluster=Pt19 Q $=0$ Pt $1 \quad-0.92660000$
$\mathrm{M}=0 \quad$ Etot $=-1265.32993000$

2.56593200

2.28534900

0.04661100

$-1.82060800$

$-1.93154800$

2.42750700

$-2.94437800$

1.05928200

$-1.42682600$

2.24329500

$-1.38514700$

0.22546000

1.07738600

$-0.35860400$

0.72200300

0.65252900

$-1.15289600$

2.40456800

0.90643400

1.34300500

1.89563700

$-0.93343200$

$-1.90281500$

$-1.00524500$

$-0.15736500$

$-1.78714000$

2.08314600

$-2.43225000$

$-0.46518700$

$-0.08537400$

0.78940100

$-2.93873100$

$\mathrm{M}=0 \quad \mathrm{Etot}=-1353.18460500$

$-0.35278900$

1.73676000

2.00986900

$-1.52039900$

$-2.67042000$

$-1.40039000$

$-0.05330100$

2.01049000

$-0.40232400$

$-2.48137300$

$-2.05226700$

0.57065300

2.00969100

$-2.19607500$

1.20479400

1.84284800

$-3.20093800$

$-1.07185300$

$-0.53876000$

3.12747100

$-0.80524100$

0.01443100

0.06303600

$-3.05673800$

0.17161200

2.36903100

1.39799300

$-1.90195600$

1.17908000

$-0.50159400$

$\mathrm{M}=0 \quad \mathrm{Etot}=-1443.03616100$

$-0.71967800$

0.82303200

$-2.48957200$

2. 32398300

$-0.62291300$

$-1.32945000$

1.07918500

$-0.62847400$

1.38995400

2. 94127400

1.21658000

1.89785900

$-0.33667200$

$-1.12360400$

$-1.15848100$

0.12272500

1.88932000

0.37714200

1.40115500

$-3.01020600$

$-2.22559500$

$-2.77290700$

1.26469100

$-0.17686000$

$-1.13824100$

2. 52280900

1.40727500

1.28739800

$-2.89108100$

$-1.27578500$

3.45044800

$-0.24613500$

$-0.75893000$

$-2.81957300$

0.70015100

$-0.37082400$

$\mathrm{M}=0 \quad$ Etot $=-1545.31996300$

$-3.19778300$

0.06981300 


\begin{tabular}{|c|c|c|c|}
\hline Pt2 & -1.11074500 & -1.12444500 & -1.76382600 \\
\hline Pt 3 & 3.08456100 & -1.24257500 & -0.17542200 \\
\hline Pt 4 & 1.96869300 & 1.18231800 & 0.57929800 \\
\hline Pt5 & -1.67102500 & 3.29654700 & -1.04925600 \\
\hline t 6 & -0.17147000 & 0.63707300 & 2.27463200 \\
\hline Pt 7 & -0.85631100 & 1.39288100 & -2.90101600 \\
\hline t8 & -0.35071800 & -2.11777500 & 2.54584100 \\
\hline t9 & 1.19651900 & -2.65688000 & -1.61202000 \\
\hline t10 & 1.03173000 & 2.80718600 & -1.46441800 \\
\hline t11 & -2.97943100 & 0.85197800 & -1.21918300 \\
\hline 12 & 1.38818800 & 0.09364000 & -1.91660600 \\
\hline$=13$ & 0.54662900 & -1.07837300 & 0.34323400 \\
\hline$=14$ & -2.40354900 & 1.93198700 & 1.25684500 \\
\hline$=15$ & 2.12831500 & -0.90939000 & 2.39427800 \\
\hline 16 & 1.67102500 & -3.29654700 & 1.04925600 \\
\hline 17 & -0.54662900 & 1.07837300 & -0.34323400 \\
\hline 18 & -2.07466700 & -0.78858500 & 0.82650100 \\
\hline 19 & 0.07548400 & 3.14037200 & 1.10528200 \\
\hline 19 & luster $=\mathrm{Pt} 20 \quad \mathrm{Q}=0$ & $I=0 \quad$ Etot $=-16$ & 03810900 \\
\hline Pt1 & 0.44174300 & 1.43427100 & -2.08332900 \\
\hline t2 & -1.10176700 & -2.82444300 & -2.35759800 \\
\hline Pt3 & 0.27642100 & 0.45793300 & 3.10683300 \\
\hline Pt 4 & 2.71247400 & 0.75532400 & 1.73321400 \\
\hline t5 & -1.62641300 & -0.16639200 & -3.03744400 \\
\hline Pt 6 & -1.03944400 & -2.93535400 & 0.49170700 \\
\hline Pt7 & -1.71175700 & -0.40135800 & 1.38209900 \\
\hline t 8 & -2.89061800 & -1.33372900 & -0.89306000 \\
\hline t 9 & -0.65092900 & -2.11346900 & 3.06988700 \\
\hline Pt10 & 1.14740400 & -1.43101100 & 1.27948000 \\
\hline 1 & 1.31504700 & -2.84829100 & -1.04400700 \\
\hline 12 & 2.34618200 & 2.50337400 & 10200 \\
\hline t13 & 0.40099500 & 1.05575500 & 0.57930300 \\
\hline Pt14 & -0.23118700 & 3.43154700 & -0.26459800 \\
\hline$\Gamma$ & 1.15188000 & 2.98715100 & 2.12125900 \\
\hline & -1.49319100 & 2.26988500 & 1.88416100 \\
\hline 7 & -1.94748400 & 1.311 & -0 . \\
\hline & -0.33606600 & -0.84494100 & -0 . \\
\hline & 2.28575400 & -0.21308900 & -0.87363700 \\
\hline & 0.95095600 & -1.09456500 & -3.12994900 \\
\hline 0 & Cluster $=\mathrm{Pt} 21 \quad \mathrm{Q}=0$ & $\mathrm{M}=0 \quad \mathrm{E}$ to $=-17$ & 9921 \\
\hline Pt & -2.14379000 & 1.97356700 & 264500 \\
\hline Pt & 2.46159900 & -0.66816200 & 2100 \\
\hline Pt & -0.04308100 & 2.72558300 & 2.59512900 \\
\hline Pt 4 & 0.12801300 & -1.73845600 & 1.93741600 \\
\hline Pt5 & -0.72903700 & 1.06582700 & -1.60425500 \\
\hline Pt 6 & 0.21317600 & 3.15305800 & -0.13066000 \\
\hline Pt & 2.35103900 & 2.11239200 & 1.3267830 \\
\hline $\mathrm{Pt}$ & 1.34149100 & 0.40174800 & 3.21674800 \\
\hline Pt & 0.52394900 & -3.15669400 & -2.32423400 \\
\hline & 0.16857600 & 0.76650900 & 0.91311000 \\
\hline & 1.91760700 & 1.00665100 & -1.14739400 \\
\hline & -3.30445800 & 0.76631300 & -1.22795700 \\
\hline & -2.00233000 & -0.75011500 & 0.5229520 \\
\hline & -1.37684900 & 0.32863700 & 2.97917200 \\
\hline & -1.08007400 & -3.24077500 & -0.09921700 \\
\hline & -1.76434700 & -1.40891900 & -2.1334020 \\
\hline & -2.19247500 & 3.24220200 & -1.38424400 \\
\hline
\end{tabular}




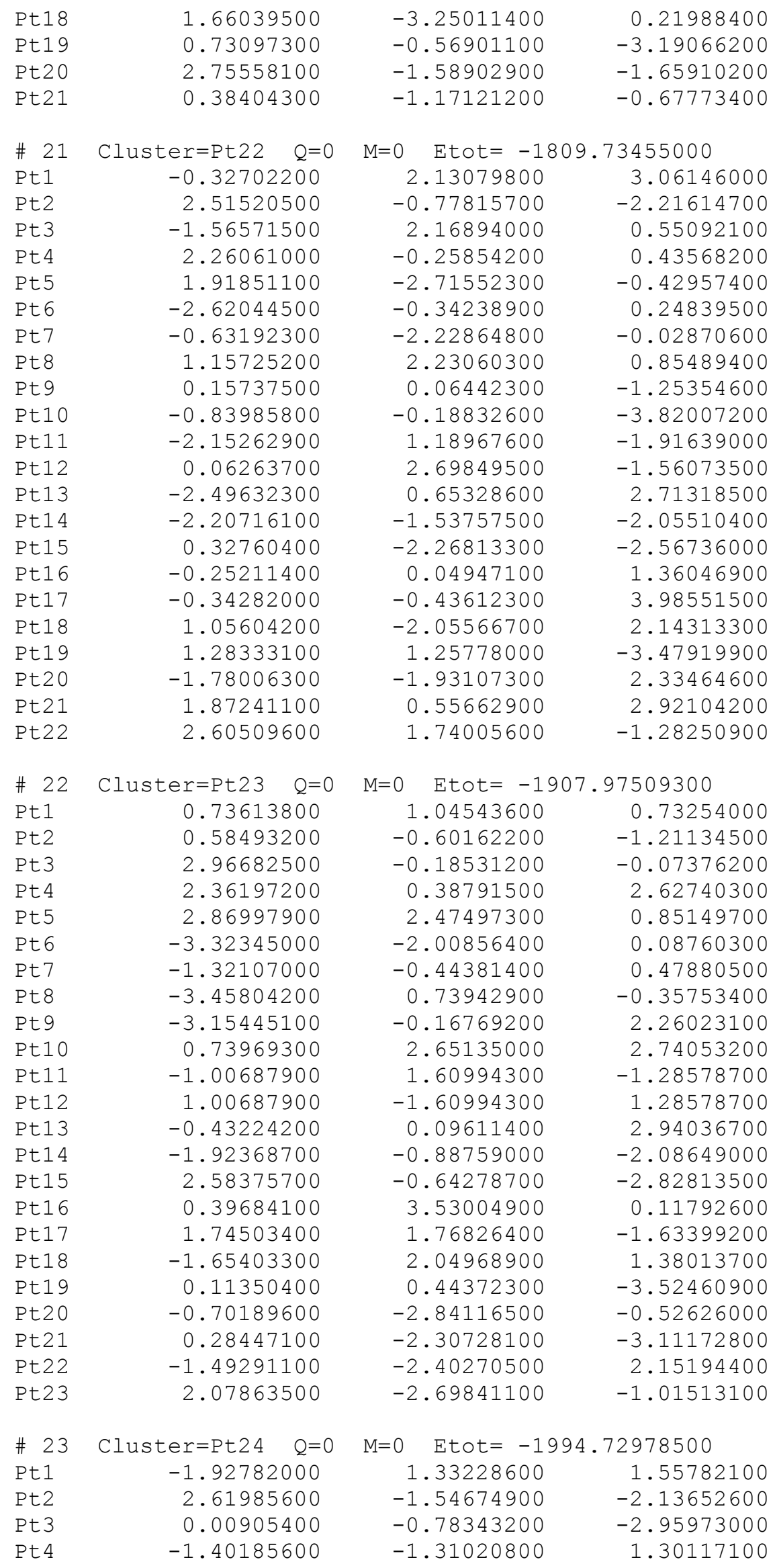




\begin{tabular}{|c|c|c|c|}
\hline Pt 5 & -1.77318500 & 3.93480800 & 1.23270200 \\
\hline Pt 6 & -0.26716100 & -3.67403000 & 0.50095400 \\
\hline Pt 7 & -2.63009500 & 2.45778800 & -0.89658500 \\
\hline t8 & 2.34313700 & 0.55638100 & 3.01357800 \\
\hline$=9$ & 0.65553400 & -1.45467100 & -0.45572100 \\
\hline$=10$ & 2.97520500 & -0.52836900 & 0.49277600 \\
\hline$=11$ & -1.05006100 & 0.42043800 & -0.81030100 \\
\hline 12 & -1.78750400 & -2.10721500 & -1.31845500 \\
\hline 13 & -0.57853900 & 1.96637100 & -2.82529200 \\
\hline 14 & 2.43229400 & -3.25229700 & 0.03925200 \\
\hline 15 & 2.55241700 & 2.23560500 & 0.81904300 \\
\hline 16 & 0.30134100 & 2.70986500 & 2.51551400 \\
\hline 17 & -0.35096800 & 0.09626400 & 3.47147700 \\
\hline 18 & -3.48192400 & -0.17297000 & -0.17285800 \\
\hline 19 & 1.17864700 & -1.85215200 & 2.13405100 \\
\hline 20 & 0.57106500 & -3.41234600 & -2.12715900 \\
\hline 21 & 0.06525200 & 2.80086900 & -0.26298200 \\
\hline 22 & -2.65106200 & 0.12692400 & -2.79951100 \\
\hline 23 & 0.60691800 & 0.56292400 & 1.11837000 \\
\hline 24 & 1.58945200 & 0.89391900 & -1.43158600 \\
\hline 24 & ter $=$ Pt 25 & Etot $=-20$ & 5401900 \\
\hline Pt1 & 1.60972200 & 2.29583300 & -2.32900700 \\
\hline$=2$ & 1.46093000 & -2.19732400 & 0.22395600 \\
\hline$=3$ & -1.51343100 & 0.16754500 & -0.11379800 \\
\hline$=4$ & -1.34761100 & 1.82526300 & 2.03648200 \\
\hline$=5$ & 1.29331700 & 2.55293000 & 2.25747700 \\
\hline$=6$ & -2.70779800 & -0.54295500 & -2.37204800 \\
\hline$=7$ & 1.25355000 & -2.03410600 & 2.89903500 \\
\hline 8 & -4.14314500 & 0.51456200 & -0.31466400 \\
\hline 9 & 3.38481000 & -0.78688200 & -1.08510600 \\
\hline 0 & 3.19826600 & -0.63530400 & 1.61897000 \\
\hline 1 & 1.84749400 & 0.42089100 & 3.73350400 \\
\hline 12 & 2.64136900 & 1.51942400 & 0.09704600 \\
\hline 13 & -0.79955000 & -2.16197800 & -1.30356100 \\
\hline 14 & 0.67336300 & 0.13335200 & 1.36392600 \\
\hline 5 & -3.01110500 & -0.29650100 & 2.02457800 \\
\hline & 0.12283200 & 25600 & 18600 \\
\hline 7 & 1.62295500 & -2.33426900 & -2.45572500 \\
\hline 18 & 2.34436000 & 0.01715900 & -3.46887900 \\
\hline 19 & -3.20031500 & -1.96446400 & -0.11065900 \\
\hline 20 & -2.52152700 & 2.61258000 & -0.32040000 \\
\hline & -0.98703500 & -2.00963600 & 1.41415300 \\
\hline & 0.85473700 & 2500 & -1.26519700 \\
\hline 3 & -0.27211700 & -0.72970100 & -3.5562720 \\
\hline 24 & -1.04210200 & 1.57702000 & -2.3920560 \\
\hline$t 25$ & -0.76196800 & -0.33166900 & 3.5444300 \\
\hline \# 25 & Cluster $=$ Pt 26 & $=0 \quad$ Etot $=-2$ & 1898 \\
\hline Pt 1 & 3.26011300 & -2.47846000 & 0.0072070 \\
\hline Pt2 & -1.10962100 & -2.90198800 & -1.5573960 \\
\hline Pt 3 & 1.10514200 & 2.90351400 & 1.5577360 \\
\hline Pt 4 & 0.30585400 & 0.40870200 & 2.19659100 \\
\hline Pt 5 & -1.12867900 & -0.99256400 & 0.3418360 \\
\hline Pt 6 & -0.32658600 & 1.48852500 & -0.2314840 \\
\hline t7 & -2.09493800 & 1.60781300 & -2.2593020 \\
\hline Pt $\varepsilon$ & 1.50344700 & -2.39978800 & -2.0146810 \\
\hline & -0.30585400 & -0.40870200 & -2.1965910 \\
\hline & 2.90707600 & 0.89666000 & 1.680233 \\
\hline
\end{tabular}




\begin{tabular}{|c|c|c|c|}
\hline t11 & 0.46177100 & 3.98844000 & -0.80639300 \\
\hline Pt12 & -2.97119600 & 1.01259800 & 0.22530500 \\
\hline t13 & -2.31110700 & -0.10621200 & 2.59341000 \\
\hline Pt1 & -2.90607100 & -0.90124100 & -1.67952000 \\
\hline t15 & 0.66678600 & -3.03910300 & 0.47261700 \\
\hline t16 & -3.72188400 & -1.50998000 & 0.79918600 \\
\hline Pt1 & 2.09841200 & -1.60475900 & 2.25825000 \\
\hline Pt18 & -1.49997400 & 2.40284200 & 2.01362900 \\
\hline+ & -0.51465600 & -2.10695900 & 2.7155350 \\
\hline t. & 4.05648300 & -0.01507200 & -0.56202200 \\
\hline$t^{2}-x-2 \cdot 2$ & 2.31211200 & 0.10163100 & -2.59269800 \\
\hline 1 & 1.45526500 & -0.49596100 & -0.11035200 \\
\hline Pt2 & 0.51017800 & 2.10848500 & -2.71519500 \\
\hline Pt2 & -2.10373800 & 3.49538000 & -0.35743100 \\
\hline 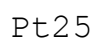 & 2.30440900 & 2.02650500 & -0.6979220 \\
\hline t2 & -1.95274500 & -3.48030800 & 0.91945300 \\
\hline 26 & uster $=$ Pt27 & $=0 \quad$ Etot $=-22$ & 581 \\
\hline Pt1 & 1.97541400 & -2.36667600 & -1.23576800 \\
\hline Pt2 & 1.91399400 & -0.70501300 & -3.38796200 \\
\hline Pt3 & 0.26292400 & -2.00864300 & 0.81866500 \\
\hline Pt 4 & 1.36789300 & 1.78645300 & -2.56895800 \\
\hline Pt5 & -1.26208400 & 1.31156900 & -2.88946800 \\
\hline Pt 6 & 0.60080400 & 3.07971400 & 1.84484500 \\
\hline Pt 7 & -0.33961200 & 2.24511100 & -0.53975800 \\
\hline Pt 8 & 2.30172600 & 2.67265400 & -0.19871700 \\
\hline Pt9 & -3.18004300 & 0.50408300 & 2.73297900 \\
\hline Pt10 & -1.46928400 & 0.09859800 & 0.70321000 \\
\hline Pt11 & 1.13852400 & 0.56948000 & 1.02101800 \\
\hline Pt12 & -2.44411000 & -0.79535700 & -1.67565800 \\
\hline Pt13 & 2.08215600 & 1.45425700 & 3.37427200 \\
\hline Pt14 & 2.88677500 & 0.16721900 & -1.02599400 \\
\hline Pt15 & -2.38999600 & -2.42038800 & 0.48977200 \\
\hline Pt16 & -1.44986800 & -1.53698900 & 2.84787400 \\
\hline Pt17 & 3.75911200 & 1.05757300 & 1.36167200 \\
\hline Pt18 & 2.89394600 & -1.46628800 & 1.13371400 \\
\hline Pt19 & -4.09996400 & -0.36150800 & 0.40390300 \\
\hline Pt20 & -0.55932000 & 0.99754000 & 3.11155200 \\
\hline Pt21 & 0.22046000 & -0.32110500 & -1.33324100 \\
\hline Pt22 & 1.01269300 & -3.19843500 & -3.57078800 \\
\hline Pt23 & -0.70914100 & -1.17866200 & -3.70763800 \\
\hline Pt24 & 1.18572200 & -1.06109100 & 3.16906800 \\
\hline Pt25 & -0.69290400 & -2.84848400 & -1.56095000 \\
\hline Pt26 & -2.96837500 & 1.72105300 & -0.84097300 \\
\hline Pt27 & -2.03744100 & 2.60333700 & 1.52332800 \\
\hline \# 27 & Cluster $=$ Pt $28 \quad Q=0$ & $\mathrm{M}=0 \quad \mathrm{E}$ tot $=-237$ & 946 \\
\hline Pt1 & 2.13529500 & 0.06870000 & -1.9090660 \\
\hline Pt 2 & 3.23958400 & -0.90782000 & 2.32151900 \\
\hline Pt3 & -1.31391700 & 1.62822700 & 3.11080200 \\
\hline Pt 4 & 0.15376000 & 0.21792600 & -3.73944600 \\
\hline Pt5 & 3.80678100 & -1.45284300 & -0.32582000 \\
\hline Pt 6 & 3.13567000 & 1.14276500 & 0.43655500 \\
\hline Pt 7 & -0.45910500 & 3.91289300 & -0.9039350 \\
\hline Pt 8 & -0.40811800 & -2.43214900 & -3.3298410 \\
\hline Pt9 & -3.49139700 & 0.47112600 & 1.9816150 \\
\hline Pt10 & 0.48292000 & 1.50249900 & -0.20586200 \\
\hline Pt 1 & 0.20529300 & 3.35258900 & 1.66511100 \\
\hline Pt12 & -2.01420500 & 2.22595700 & 0.5383970 \\
\hline
\end{tabular}




\begin{tabular}{|c|c|c|c|}
\hline t13 & -2.98398000 & -0.05342100 & -0.62832500 \\
\hline Pt14 & 2.13350500 & 3.54173500 & -0.27205100 \\
\hline Pt15 & -1.45185900 & 1.68559500 & -2.08630200 \\
\hline Pt1 & -1.88193600 & -1.01594600 & 3.54907200 \\
\hline t17 & 0.62002200 & -1.71259200 & 2.83046300 \\
\hline t18 & 1.41040500 & -0.84364200 & 0.47586700 \\
\hline Pt19 & -2.37843100 & -0.59833000 & -3.21304800 \\
\hline Pt20 & -0.46208900 & -0.71867000 & -1.34278900 \\
\hline t21 & 2.26144500 & -3.20685900 & 1.14473400 \\
\hline t2 & 1.20905500 & 0.95873100 & 2.41407600 \\
\hline t23 & 1.54336000 & -2.59983300 & -1.47913000 \\
\hline+20 & 1.11536300 & 2.47809600 & -2.58255600 \\
\hline t25 & -2.88334700 & -2.06059300 & 1.22264400 \\
\hline Pt26 & -1.01007400 & -0.19210700 & 1.21488300 \\
\hline t27 & -0.38826100 & -2.79563300 & 0.49236800 \\
\hline t28 & -2.32573900 & -2.59640200 & -1.37993700 \\
\hline 28 & I uster $=\mathrm{Pt} 29 \quad \mathrm{Q}=0$ & $=0 \quad$ Etot $=-24$ & 5238 \\
\hline Pt1 & -0.53164800 & 3.70775100 & -1.69461000 \\
\hline Pt2 & -2.61028400 & -1.14573300 & 2.53313200 \\
\hline Pt3 & -2.02218600 & 0.28176500 & -2.39804400 \\
\hline Pt 4 & 2.38495700 & 2.45954300 & 1.75005800 \\
\hline Pt5 & 0.84710400 & -1.30961200 & -0.53065500 \\
\hline Pt 6 & -3.72259900 & -1.36142500 & -1.09112100 \\
\hline Pt 7 & 0.29908100 & 2.36152000 & 3.45089900 \\
\hline Pt 8 & 0.16604900 & 1.20258200 & -1.09201600 \\
\hline Pt9 & -1.87904400 & 1.42740700 & 2.16564400 \\
\hline Pt10 & -2.30319600 & 2.10379300 & -0.43426600 \\
\hline Pt11 & 0.12452600 & -3.84358700 & -0.10682000 \\
\hline Pt12 & 2.71379500 & 0.63088600 & -0.19701200 \\
\hline Pt13 & -0.13226900 & -2.05466400 & 1.88742600 \\
\hline Pt14 & 1.15084500 & -3.16119200 & -2.50147000 \\
\hline Pt15 & -3.99970500 & 0.43528700 & 0.84537200 \\
\hline Pt16 & -1.56512500 & -0.43095600 & 0.17588400 \\
\hline Pt17 & 2.73686300 & -3.16206500 & -0.33706800 \\
\hline Pt18 & -0.25454200 & 1.91103800 & -3.63110300 \\
\hline Pt19 & 2.47469600 & -1.36485800 & 1.62046000 \\
\hline Pt20 & -1.30722700 & -2.28017300 & -1.83037800 \\
\hline Pt21 & 0.56309600 & 0.53185100 & 1.45407000 \\
\hline Pt22 & -0.13332300 & 3.07467300 & 0.88708900 \\
\hline Pt23 & -2.32982600 & -2.96418500 & 0.57320900 \\
\hline Pt24 & 2.24136700 & 1.34920000 & -2.77470300 \\
\hline Pt25 & 0.43849400 & -0.63290700 & 893300 \\
\hline Pt26 & 2.94641200 & -1.18085000 & -2.17355100 \\
\hline Pt27 & 2.17970800 & 0.45045100 & 3.55649500 \\
\hline Pt28 & 1.96090900 & 3.16765200 & -0.81478000 \\
\hline Pt29 & -0.43692900 & -0.20319300 & 3.81678900 \\
\hline \# 29 & Iuster $=\operatorname{Pt} 30 \quad Q=0$ & $I=0 \quad$ Etot $=-2$ & 04159400 \\
\hline Pt1 & -3.41887000 & -2.21592300 & -1.1708490 \\
\hline Pt2 & -3.80517200 & 0.42030200 & -0.60467900 \\
\hline Pt3 & 0.58590600 & -0.09652800 & -2.00960800 \\
\hline Pt 4 & 3.52366900 & -0.37392400 & 1.57021300 \\
\hline Pt5 & 1.56764100 & -1.11367900 & 3.2772720 \\
\hline Pt 6 & 1.96169100 & 2.04724300 & -2.73008600 \\
\hline Pt 7 & 2.48323100 & 4.01986600 & -1.04665500 \\
\hline Pt 8 & -0.18624400 & 4.20086400 & -0.5365890 \\
\hline Pt9 & -0.75189100 & 0.12387400 & 2.57892000 \\
\hline PtIu & -2.01923900 & -0.31608800 & -2.4777020 \\
\hline
\end{tabular}




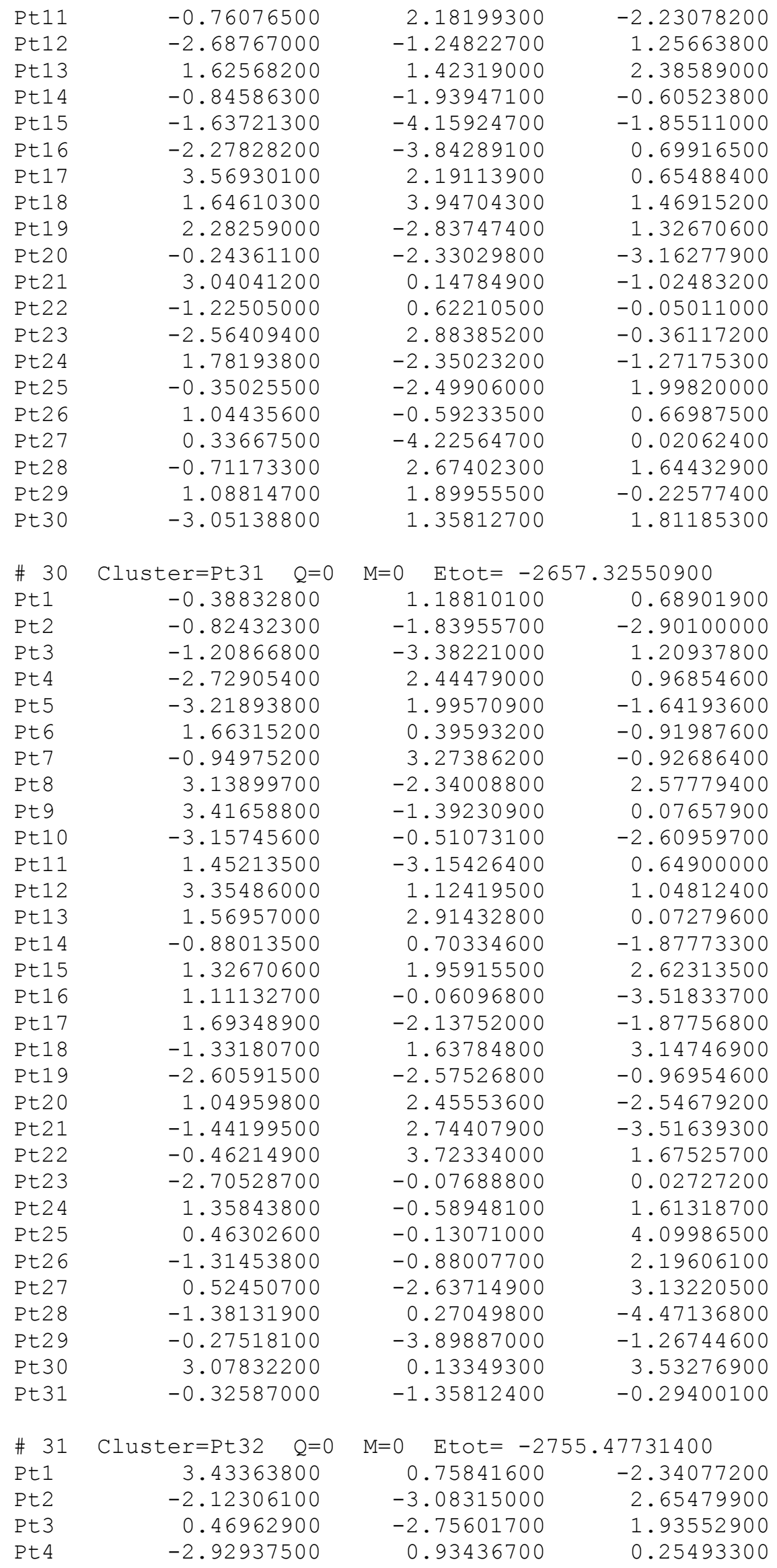




\begin{tabular}{|c|c|c|c|}
\hline Pt 5 & -0.92408100 & 0.19656800 & -1.31150000 \\
\hline Pt 6 & 1.12103500 & -0.54970100 & -2.82141400 \\
\hline Pt 7 & 1.03921900 & -1.07381600 & -0.09524400 \\
\hline Pt 8 & -0.18438100 & 1.86680800 & -3.19219500 \\
\hline Pt9 & -2.86462400 & 1.47850200 & -2.46527800 \\
\hline Pt10 & 3.37837500 & 0.20148100 & 0.32930100 \\
\hline t11 & 1.23492900 & 4.02844300 & -0.30145000 \\
\hline t12 & -3.15732800 & -1.15596700 & -1.55051600 \\
\hline t13 & 1.70907300 & -0.36355000 & 2.42559700 \\
\hline t14 & -1.50085400 & -1.41631000 & 0.65288000 \\
\hline$t 15$ & -3.71694500 & -2.81125800 & 0.50036800 \\
\hline$=16$ & -1.50075700 & -0.54990800 & -3.73027600 \\
\hline t17 & 1.32240600 & 1.45426900 & -0.90614100 \\
\hline t18 & 2.14231800 & 3.14620900 & -2.73515300 \\
\hline t19 & 3.12891000 & -1.81622500 & -1.51929500 \\
\hline t20 & -0.89243800 & -0.73028400 & 3.19885800 \\
\hline Pt21 & -2.24053400 & 1.62725100 & 2.78012000 \\
\hline 222 & -0.69201700 & -2.39314100 & -1.82023600 \\
\hline-23 & 2.01630800 & 2.22721400 & 1.59263100 \\
\hline Pt24 & -1.26750000 & -4.02151500 & 0.25522400 \\
\hline t25 & -0.42655400 & 3.47163500 & 1.77842900 \\
\hline t26 & 0.33247200 & 1.64940600 & 3.63908200 \\
\hline Pt27 & -1.11632300 & 2.77780700 & -0.74624500 \\
\hline Pt28 & 1.31493000 & -3.66060900 & -0.51760400 \\
\hline t29 & 3.62695900 & 2.76324400 & -0.52039700 \\
\hline t30 & -0.29892800 & 0.91634300 & 1.14590000 \\
\hline tet31 & 3.03170600 & -2.36502700 & 1.13551800 \\
\hline t32 & -3.46620800 & -0.75148800 & 2.29454600 \\
\hline \# 32 & Cluster $=$ Pt $33 \quad Q=0$ & $=0 \quad$ Etot $=-28$ & 4763 \\
\hline Pt1 & -3.20400000 & 1.75460600 & -2.85127000 \\
\hline Pt2 & -1.68507900 & -1.71752400 & -2.57288600 \\
\hline Pt3 & -2.67399400 & 2.26728000 & -0.26808300 \\
\hline Pt 4 & 0.48505800 & 1.94876100 & -3.72275400 \\
\hline Pt 5 & -0.39389400 & 1.27037600 & 0.77034700 \\
\hline Pt 6 & 0.91885200 & -0.81607100 & 1.79176000 \\
\hline Pt 7 & 1.30468000 & -0.22363800 & -0.78117800 \\
\hline Pt 8 & -3.40281500 & -2.24107300 & 0.78240500 \\
\hline Pt9 & 0.60108900 & 2.90133200 & 2.62694700 \\
\hline Pt10 & 3.93726400 & -0.03306900 & -0.42040900 \\
\hline Pt11 & 3.56623500 & -0.67121600 & 2.17331100 \\
\hline Pt12 & 0.71815300 & -0.71635300 & -3.36656600 \\
\hline Pt13 & 2.76325800 & 0.96520800 & -2.66695800 \\
\hline Pt14 & 2.99659300 & -1.69708900 & -2.32008500 \\
\hline Pt15 & 1.12764000 & 2.46335800 & -1.13753500 \\
\hline Pt16 & 0.56664000 & -1.45163400 & 4.36413500 \\
\hline Pt17 & -1.75687300 & -3.68879500 & -0.72104400 \\
\hline Pt18 & 2.20994800 & -2.89703800 & 2.86309200 \\
\hline Pt19 & 2.29527800 & 1.47049600 & 1.0968280 \\
\hline Pt20 & -0.95361500 & 0.77611900 & -1.79134700 \\
\hline Pt21 & -0.57291700 & 3.89960900 & 0.38039800 \\
\hline Pt22 & 0.61780700 & -2.72489400 & -1.53369000 \\
\hline Pt23 & 1.90100700 & 0.79346900 & 3.69437700 \\
\hline Pt24 & 0.22698600 & -3.34338500 & 1.08599300 \\
\hline Pt25 & -1.06136100 & -1.21978900 & 0.01262200 \\
\hline Pt26 & -3.35360300 & -0.24994000 & -1.04881000 \\
\hline Pt27 & -1.56069300 & 0.30920100 & -4.35231300 \\
\hline & 2.61790400 & -2.33174300 & 0.26875000 \\
\hline & -1.44153900 & -1.87580100 & 2.6100700 \\
\hline
\end{tabular}




\begin{tabular}{|c|c|c|c|}
\hline$t 30$ & -2.76828700 & 0.25341400 & 1.55926400 \\
\hline Pt31 & -1.18017000 & 3.41344700 & -2.20168700 \\
\hline t32 & -0.77424400 & 0.65188900 & 3.36723300 \\
\hline$t 33$ & -2.07131000 & 2.76048800 & 2.30908100 \\
\hline 33 & Cluster $=$ Pt 34 & \multicolumn{2}{|c|}{$M=0 \quad$ Etot $=-2941.92415100$} \\
\hline Pt 1 & -2.26577400 & -0.23032300 & 3.13458600 \\
\hline Pt2 & 1.39197400 & 3.01962400 & $1.374747 \mathrm{C}$ \\
\hline Pt3 & -3.61207200 & -0.97276900 & -1.8419270 \\
\hline t 4 & -0.34098500 & -1.86493000 & 2.0955450 \\
\hline t5 & -3.02809000 & 1.48700900 & -0.8839200 \\
\hline t 6 & -3.75071300 & -0.52579700 & 0.817375 \\
\hline Pt 7 & 0.12486700 & -3.70807700 & 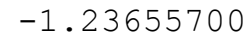 \\
\hline Pt 8 & -1.21558100 & 0.31315100 & 0.7390500 \\
\hline Pt9 & -1.06698500 & -0.13038300 & -1.89647300 \\
\hline t10 & 0.81105100 & 1.37375800 & -0.69491800 \\
\hline Pt11 & 3.31131000 & 1.43655500 & 0.3430500 \\
\hline t12 & 0.23284000 & -0.20770800 & 4. \\
\hline Pt13 & -0.96823300 & -0.55455100 & -4 \\
\hline Pt14 & 1.83206300 & 1.93480500 & 3.8079620 \\
\hline Pt15 & -1.12421000 & 2.99099800 & 0.3390380 \\
\hline Pt16 & 2.80317800 & 2.45321700 & -2.10917700 \\
\hline Pt17 & 1.26550700 & 0.33373700 & 1.7433240 \\
\hline Pt18 & 39800 & -0.211 & -1 \\
\hline Pt19 & -1.81333900 & 789900 & -0 \\
\hline Pt20 & 3.24337000 & -1.25890900 & 0.7151880 \\
\hline Pt21 & 3.72617600 & 0.37954300 & 2.79022700 \\
\hline t22 & 0.75337900 & -1.28450900 & -0.31899700 \\
\hline Pt23 & 0.93268500 & 0.96325600 & -3.36873700 \\
\hline Pt24 & -2.88427600 & -2.64 & 571200 \\
\hline Pt25 & -3.15144800 & 1.92236900 & 1 . \\
\hline Pt26 & -2.86436200 & 1.00030900 & -3.52069700 \\
\hline Pt27 & 0.87831300 & -1.72950900 & -2.97542700 \\
\hline Pt28 & 0.90767600 & 4.01270600 & -1.09333600 \\
\hline t29 & -1.68886700 & -2.55078400 & -2.87571500 \\
\hline Pt 30 & 1.58836000 & -3.41687100 & 3900 \\
\hline Pt 31 & -0.98987400 & 2.54568300 & 7000 \\
\hline Pt 32 & 2.15834200 & -1.78526600 & 3.12776200 \\
\hline Pt33 & 2.68983700 & -2.88910600 & -1.35533200 \\
\hline Pt 34 & -0.66151700 & 1.95791000 & 2.8090610 \\
\hline \# 34 & luster $=\mathrm{Pt} 35 \quad \mathrm{Q}=0$ & $=0 \quad \mathrm{Etot}=-30$ & 0414 \\
\hline Pt1 & -0.33929700 & 1.33803700 & 8180 \\
\hline Pt2 & 2.40569200 & -2.22341900 & 1.51546800 \\
\hline Pt3 & -0.91323600 & 3.99511300 & 0.09146300 \\
\hline Pt 4 & 3.35074500 & 2.10571500 & -0.01444300 \\
\hline Pt5 & -2.78055800 & -2.01913000 & -1.96299500 \\
\hline Pt 6 & -0.22163300 & -2.88555000 & -1.9792840 \\
\hline Pt 7 & 3.23769700 & 2.00788500 & -2.70375900 \\
\hline Pt 8 & 1.26093600 & 3.07069900 & 1.41719200 \\
\hline Pt9 & -3.35110900 & 0.61948100 & -2.01197600 \\
\hline Pt10 & -1.81864200 & 0.88656800 & -4.21083500 \\
\hline Pt11 & 1.17247400 & 3.02993900 & -1.31827900 \\
\hline Pt12 & 0.81764300 & -4.00962900 & 0.2400570 \\
\hline Pt13 & -2.66555800 & -1.91059100 & 2.50705900 \\
\hline Pt14 & -0.67942600 & -0.10317300 & 2.35628100 \\
\hline Pt15 & 1.72865900 & 0.35069800 & -1.2440260 \\
\hline & -2.34910400 & -0.50372100 & 0.22787600 \\
\hline & -1.37842700 & 2.46423900 & -2.07859300 \\
\hline
\end{tabular}




\begin{tabular}{|c|c|c|c|}
\hline Pt18 & 1.28596500 & -1.16356200 & -3.44876600 \\
\hline Pt19 & 2.32926800 & -2.31985100 & -1.21897000 \\
\hline Pt20 & 1.45039500 & -0.99781800 & 3.73384700 \\
\hline Pt21 & -3.23582300 & 0.72669500 & 2.45810300 \\
\hline Pt22 & -1.25778200 & -1.70722700 & -4.16268600 \\
\hline Pt23 & -1.27021900 & 2.57665300 & 2.34454900 \\
\hline Pt24 & 3.92129700 & -0.53289600 & 0.03453800 \\
\hline Pt25 & -1.74945000 & -3.14488200 & 0.27699900 \\
\hline Pt26 & -1.04261700 & -1.49714200 & 4.61197400 \\
\hline Pt27 & 1.85626500 & 0.42888100 & 1.48131500 \\
\hline Pt28 & 3.79855800 & -0.58591100 & -2.65561000 \\
\hline Pt29 & 0.24253200 & -1.35273100 & 0.19276700 \\
\hline Pt30 & -1.60319900 & 1.09536500 & 4.56384900 \\
\hline Pt31 & -0.11057200 & -2.78633100 & 2.44410300 \\
\hline Pt32 & 0.71699900 & 1.46771900 & -3.49761100 \\
\hline Pt33 & -2.89420600 & 2.14923600 & 0.17872400 \\
\hline Pt34 & 0.88013100 & 1.63946700 & 3.68489100 \\
\hline Pt35 & -0.79439700 & -0.20882700 & -1.99604000 \\
\hline \# 35 & Cluster $=$ Pt $36 \quad Q=0$ & Etot $=-313$ & 56156500 \\
\hline Pt 1 & 1.01364800 & 2.19588000 & 0.17833900 \\
\hline Pt2 & -1.02874800 & -1.61457700 & 3.03277700 \\
\hline Pt 3 & 2.22858600 & 0.20447700 & 1.68641700 \\
\hline Pt 4 & -2.91662000 & -0.99383800 & 1.12934400 \\
\hline Pt 5 & 2.75446700 & -3.56210200 & 1.81286900 \\
\hline Pt 6 & -0.92715800 & 1.15243800 & -1.33715600 \\
\hline Pt7 & -1.08701800 & -1.46286000 & -0.80879800 \\
\hline Pt 8 & -1.16685200 & 3.67819500 & -0.41934900 \\
\hline Pt 9 & -2.19896400 & -1.89688100 & -3.15838700 \\
\hline Pt10 & 1.27379100 & 4.19995400 & -1.52616700 \\
\hline Pt11 & -3.43013600 & -2.74420000 & -0.89152700 \\
\hline Pt12 & 2.94423000 & -0.71123900 & -2.62973000 \\
\hline Pt13 & -1.12591800 & -3.96312600 & -1.63832000 \\
\hline Pt14 & 0.73704800 & -4.58112200 & 0.24783700 \\
\hline Pt15 & 3.46640000 & 1.13078200 & -0.59722700 \\
\hline Pt16 & -3.10550700 & 2.56670600 & -1.96446700 \\
\hline Pt17 & 1.23076300 & -0.22605900 & -0.74658000 \\
\hline Pt18 & 3.19452000 & 3.63713100 & 0.35843900 \\
\hline Pt19 & 0.37902400 & 0.70896300 & 3.62394600 \\
\hline Pt20 & -1.59890100 & -3.39008900 & 1.00862700 \\
\hline Pt21 & -0.69277200 & 3.12310700 & -3.06992200 \\
\hline Pt22 & 0.19962000 & -0.64709400 & -3.14263200 \\
\hline Pt23 & -2.75273800 & 1.68726400 & 0.58769300 \\
\hline Pt24 & 3.29513900 & -1.67103700 & -0.03118700 \\
\hline Pt25 & 1.92278400 & 2.71807300 & 2.61264500 \\
\hline Pt26 & 0.30263600 & -3.96828400 & 2.85657000 \\
\hline Pt27 & -2.30924000 & 0.77117900 & 3.11854600 \\
\hline Pt28 & 1.27351500 & -2.71291900 & -1.62580500 \\
\hline Pt29 & -0.76071700 & 2.77038700 & 2.14690100 \\
\hline Pt30 & 0.83244400 & 4.69933900 & 1.10548600 \\
\hline Pt31 & 1.65269000 & -1.70063300 & 3.50533800 \\
\hline Pt32 & -3.32397000 & -0.08629700 & -1.43285800 \\
\hline Pt33 & -0.53722700 & 0.27242500 & 1.18592100 \\
\hline Pt3 4 & -2.03590800 & 0.77071000 & -3.69731000 \\
\hline Pt35 & 1.54347200 & 1.70356600 & -2.51804900 \\
\hline Pt36 & 0.75361600 & -2.05821800 & 1.03777700 \\
\hline \# & Cluster $=$ Pt $37 \quad Q=0$ & \multirow{2}{*}{\multicolumn{2}{|c|}{$\begin{array}{c}\mathrm{M}=0 \quad \mathrm{Etot}=-3238.06367900 \\
1.83090600 \quad 3.47016600\end{array}$}} \\
\hline & 2.36014900 & & \\
\hline
\end{tabular}




\begin{tabular}{|c|c|c|c|}
\hline Pt2 & 4.41964700 & -1.05730600 & -0.55982200 \\
\hline Pt3 & 0.45134000 & -2.06121500 & -3.67678600 \\
\hline Pt 4 & 2.17529700 & 3.71774000 & -1.55276000 \\
\hline Pt 5 & -2.75038100 & -2.37883800 & 2.09354700 \\
\hline Pt 6 & -0.37149700 & 3.13632800 & 1.19181600 \\
\hline Pt 7 & -1.81027700 & -2.87676600 & -2.46039000 \\
\hline Pt 8 & 3.42387700 & 0.38881000 & 1.47109600 \\
\hline Pt9 & -1.62101200 & 0.83509200 & 0.37335400 \\
\hline Pt10 & 2.90301200 & -3.14756100 & 0.16079500 \\
\hline Pt11 & 0.63726400 & -3.95899100 & 1.37526600 \\
\hline t12 & 0.56376400 & 2.58416200 & -3.37116500 \\
\hline 13 & -0.39227200 & -2.51518200 & 3.38984600 \\
\hline Pt14 & -2.93630700 & -0.48104000 & -2.95856500 \\
\hline Pt15 & 0.55343800 & -3.05752700 & -1.16891100 \\
\hline Pt16 & 1.76785300 & -0.75946200 & -0.35034800 \\
\hline Pt17 & -1.71709500 & -3.82790600 & 0.07163000 \\
\hline Pt18 & -2.64963600 & 2.28755300 & 2.39115000 \\
\hline Pt19 & -0.29204100 & 2.15565700 & 3.69165700 \\
\hline Pt20 & 1.90624600 & -1.70976500 & 2.19952800 \\
\hline Pt21 & -0.58426400 & -0.61882900 & -1.65533600 \\
\hline Pt22 & -1.73283900 & 1.80672400 & -2.18039800 \\
\hline Pt23 & 1.71864300 & 0.20514300 & -2.89813300 \\
\hline Pt24 & -2.74204300 & 3.23077700 & -0.11979600 \\
\hline Pt25 & -2.88404400 & -1.44646400 & -0.43721900 \\
\hline Pt26 & -3.88174100 & 0.02822800 & 1.59301400 \\
\hline Pt27 & 0.63941100 & 1.64139600 & -0.84959000 \\
\hline Pt28 & 2.80959600 & -2.19403800 & -2.37757100 \\
\hline Pt29 & -3.97448500 & 0.97488600 & -0.92707700 \\
\hline Pt30 & 3.33039300 & 1.34302500 & -1.06911100 \\
\hline Pt31 & -1.54453400 & -0.11536000 & 2.93636300 \\
\hline Pt32 & 2.28885900 & 2.80365800 & 0.96894500 \\
\hline Pt33 & 0.84215500 & -0.25744200 & 4.19344400 \\
\hline Pt34 & -0.67060400 & 0.32581700 & -4.17315300 \\
\hline Pt35 & -0.49121000 & -1.56865900 & 0.87319700 \\
\hline Pt36 & -0.47701500 & 4.04373800 & -1.33458600 \\
\hline Pt37 & 0.73235300 & 0.69271000 & 1.67590000 \\
\hline \# 37 & Cluster $=$ Pt $38 \quad Q=0$ & \multicolumn{2}{|c|}{$\mathrm{M}=0 \quad \mathrm{E}$ tot $=-3346.58730200$} \\
\hline Pt 1 & 1.69823700 & -3.64500700 & -1.42297100 \\
\hline Pt2 & 1.19254200 & -1.52310500 & 2.72845100 \\
\hline Pt 3 & -0.41187500 & 1.86016200 & -0.13325700 \\
\hline Pt 4 & -1.19254200 & 1.52310500 & -2.72845100 \\
\hline Pt 5 & -0.05187800 & -3.79048200 & 1.95562900 \\
\hline Pt 6 & -3.39466400 & -0.57119500 & 2.51891300 \\
\hline Pt 7 & -1.69823700 & 3.64500700 & 1.42297100 \\
\hline Pt 8 & -0.81707800 & -4.14310800 & -0.60164000 \\
\hline Pt9 & 2.87797500 & 2.70713900 & 1.60735500 \\
\hline Pt10 & -0.11414800 & 0.27953100 & 4.25485200 \\
\hline Pt11 & 4.16361600 & 0.92555000 & 0.0508940 \\
\hline Pt12 & 2.16498900 & -1.70849400 & -3.2540430 \\
\hline Pt13 & -2.41350000 & -0.78007500 & -3.42949400 \\
\hline Pt14 & 2.46343800 & -3.29238100 & 1.13429800 \\
\hline Pt15 & -2.96760300 & 1.37555600 & 0.69830500 \\
\hline Pt16 & 3.39466400 & 0.57119500 & -2.5189130 \\
\hline Pt17 & -1.64542000 & -0.42670200 & -0.8707010 \\
\hline Pt18 & 2.13473200 & 2.38595700 & -0.9677700 \\
\hline Pt19 & -2.13473200 & -2.38595700 & 0.9677700 \\
\hline Pt20 & -3.69914100 & 1.00151400 & -1.87303300 \\
\hline Pt21 & -2.46343800 & 3.29238100 & -1.13429800 \\
\hline
\end{tabular}




\begin{tabular}{|c|c|c|c|}
\hline Pt22 & 2.96760300 & -1.37555600 & -0.69830500 \\
\hline Pt23 & 0.81707800 & 4.14310800 & 0.60164000 \\
\hline Pt24 & -2.16498900 & 1.70849400 & 3.25404400 \\
\hline Pt25 & 0.11414800 & -0.27953100 & -4.25485100 \\
\hline Pt26 & -1.34382300 & -2.00015800 & 3.51972100 \\
\hline Pt27 & 0.87781100 & 0.07296700 & -1.69461600 \\
\hline Pt28 & 2.41350000 & 0.78007500 & 3.42949400 \\
\hline Pt29 & -4.16361600 & -0.92555000 & -0.05089400 \\
\hline Pt30 & 1.34382200 & 2.00015800 & -3.51972100 \\
\hline Pt31 & -0.35967100 & -2.23840800 & -2.45898600 \\
\hline Pt32 & -2.87797500 & -2.70713900 & -1.60735500 \\
\hline Pt33 & 3.69914100 & -1.00151400 & 1.87303300 \\
\hline Pt 34 & 0.35967100 & 2.23840800 & 2.45898600 \\
\hline Pt35 & 1.64542000 & 0.42670200 & 0.87070100 \\
\hline Pt36 & 0.41187500 & -1.86016200 & 0.13325700 \\
\hline Pt37 & 0.05187800 & 3.79048200 & -1.95562900 \\
\hline Pt38 & -0.87781100 & -0.07296700 & 1.69461600 \\
\hline \# 38 & luster $=$ Pt 39 & $1=0 \quad$ Etot $=-34$ & 03711600 \\
\hline Pt 1 & -1.23683500 & -1.97048800 & -3.99721800 \\
\hline Pt2 & 0.56812100 & 0.80607600 & -0.97127700 \\
\hline Pt3 & -1.31252200 & -3.24471300 & 0.25212800 \\
\hline Pt 4 & 2.91298000 & -1.08196500 & 2.48924500 \\
\hline Pt 5 & 2.15308700 & 3.32806700 & 2.38296500 \\
\hline Pt 6 & -3.63950200 & 1.43295100 & 0.74371800 \\
\hline Pt 7 & 1.22177600 & -3.72537000 & -0.69377600 \\
\hline Pt 8 & -0.43462800 & 0.53436100 & -3.38732000 \\
\hline Pt9 & 1.63293400 & 2.31688200 & -2.79171300 \\
\hline Pt10 & -3.07659500 & -0.01831100 & -3.45319600 \\
\hline Pt11 & 3.15416900 & 1.21519300 & -0.72312200 \\
\hline Pt12 & -0.94199900 & 2.78734800 & -1.81430700 \\
\hline Pt13 & -0.53972700 & -0.76579000 & 0.92273400 \\
\hline Pt14 & -0.32671300 & 4.27984100 & 1.72236800 \\
\hline Pt15 & 2.09694300 & -0.43728500 & -2.71293100 \\
\hline Pt16 & -2.54944300 & 3.85816600 & 0.07229000 \\
\hline Pt17 & -0.19230500 & -1.61579000 & -1.60610700 \\
\hline Pt18 & 1.30013700 & -2.94420600 & -3.32139000 \\
\hline Pt19 & -0.98109600 & 1.88684900 & 0.83939900 \\
\hline Pt20 & 2.38933300 & 1.14383900 & 3.97561700 \\
\hline Pt21 & -2.55107000 & 3.04002100 & 2.65953500 \\
\hline Pt22 & 4.03526300 & 1.33088200 & 1.82640000 \\
\hline Pt23 & 1.93882600 & -1.21149200 & -0.02403000 \\
\hline Pt24 & -2.12451900 & 0.36096900 & 2.76957900 \\
\hline Pt25 & 1.27600000 & 3.20812600 & -0.16774200 \\
\hline Pt26 & -1.99691600 & 0.29909000 & -1.07247900 \\
\hline Pt27 & -0.09145500 & 2.10605300 & 3.37766500 \\
\hline Pt28 & 0.88130500 & -2.82852200 & 1.88074900 \\
\hline Pt29 & 3.37947600 & -3.25290400 & 0.90585600 \\
\hline Pt30 & 3.47277600 & -2.53203400 & -1.70849800 \\
\hline Pt31 & 4.53225800 & -0.87603100 & 0.28555600 \\
\hline Pt32 & -3.17022300 & -1.27350000 & 0.80145600 \\
\hline Pt33 & -3.58504900 & 2.23948300 & -1.87682600 \\
\hline Pt34 & 1.45132700 & 0.95325800 & 1.48737700 \\
\hline Pt35 & 0.37946300 & -0.60008700 & 3.4366190 \\
\hline Pt36 & -4.61426400 & -0.18481700 & -1.22360900 \\
\hline Pt37 & -0.94898600 & -4.07405800 & -2.30744300 \\
\hline Pt38 & -1.63444600 & -2.31902700 & 2.7942990 \\
\hline Pt39 & -2.82788100 & -2.17106600 & -1.77257100 \\
\hline
\end{tabular}




\begin{tabular}{|c|c|c|c|}
\hline 39 & Cluster $=$ Pt $40 \quad \mathrm{Q}=0$ & Etot $=-35$ & 81687200 \\
\hline Pt 1 & -1.43579300 & -2.99491300 & 1.43485500 \\
\hline Pt2 & 2.53009000 & -4.06494200 & -0.91108400 \\
\hline Pt 3 & 0.06843100 & -2.05564500 & 3.50283700 \\
\hline Pt 4 & -1.40579900 & 2.79722200 & -1.18060700 \\
\hline Pt 5 & 1.31583500 & -3.10161200 & 1.31848300 \\
\hline Pt 6 & 4.50348300 & 0.38285900 & 1.53811000 \\
\hline Pt 7 & -1.44640600 & -1.63083400 & -0.90861200 \\
\hline Pt 8 & 0.14925900 & 4.93812600 & -0.99840300 \\
\hline t9 & 0.02035100 & -0.75322300 & 1.17180900 \\
\hline t10 & -0.17829500 & -0.97141700 & -3.32513900 \\
\hline t11 & -3.52247400 & 2.87690500 & 0.47914600 \\
\hline Pt12 & -3.65556800 & -1.59022800 & 0.63977300 \\
\hline Pt13 & -4.31466000 & 0.72479700 & 1.91104700 \\
\hline Pt14 & 2.20339500 & 0.45891400 & 0.18671800 \\
\hline Pt15 & -3.80048500 & -1.72232000 & -2.09329700 \\
\hline Pt16 & 2.41666000 & 1.89172900 & 2.45625100 \\
\hline Pt17 & -2.13771900 & 0.62724800 & 0.3703120 \\
\hline Pt18 & -0.07350700 & 1.84794400 & -3.43242500 \\
\hline Pt19 & -2.34664900 & 0.52147600 & -2.44031300 \\
\hline Pt20 & 1.65172000 & 4.10170900 & 1.04093900 \\
\hline Pt21 & -1.23606500 & 4.21368800 & 1.16307000 \\
\hline Pt22 & 0.26895800 & 3.37642400 & 3.26378400 \\
\hline Pt2 & -2.04749400 & 2.06483300 & 2.6450490 \\
\hline Pt24 & 2.31510500 & -0.84897700 & 2.56087200 \\
\hline Pt25 & 1.23425100 & -1.73478100 & -1.02198200 \\
\hline Pt26 & 0.17095400 & 0.66940600 & 3.42846700 \\
\hline Pt27 & -0.02872700 & 0.55308000 & -1.18636900 \\
\hline Pt2 & -2.90451000 & -3.85420600 & -0.68124400 \\
\hline Pt: & 0.11873600 & 1.95268700 & 1.01714900 \\
\hline Pt3 & 4.44259200 & 0.24217900 & -1.17757700 \\
\hline Pt31 & 3.56259200 & -1.87012400 & 0.33450200 \\
\hline Pt32 & 1.51326200 & 2.68403100 & -1.30406000 \\
\hline Pt3. & -0.18917700 & -4.01619600 & -0.79074800 \\
\hline $\mathrm{Pt}$ & -1.62248300 & -3.22979600 & -2.99256300 \\
\hline Pt 3 & 3.46593100 & -2.004 & -2.40060900 \\
\hline Pt 3 & 3.76213300 & 2.59443300 & 0.17106500 \\
\hline Pt37 & 1.10988400 & -3.33574800 & -3.10812000 \\
\hline Pt3 & -2.14963800 & -0.67584900 & 2.74969500 \\
\hline Pt39 & -4.49399300 & 0.58870900 & -0.79963100 \\
\hline Pt 40 & 2.16582300 & 0.34649800 & -2.63115400 \\
\hline \# 40 & I uster $=$ Pt $41 \quad Q=0$ & Etot $=-36$ & 70198900 \\
\hline Pt 1 & 0.55000200 & -1.17897600 & -1.94327800 \\
\hline Pt 2 & -2.65810800 & 1.67591000 & -0.71366800 \\
\hline Pt 3 & 1.15020000 & -3.43243200 & -0.55999000 \\
\hline Pt 4 & 0.45361100 & -1.18732400 & 0.74253700 \\
\hline Pt 5 & 1.69889300 & 2.92965400 & -0.59991800 \\
\hline Pt 6 & 3.11543900 & -2.01828100 & -1.86206600 \\
\hline Pt 7 & -1.45966300 & 0.55929800 & 1.51674700 \\
\hline Pt 8 & 2.98377300 & 3.13283000 & 1.76994700 \\
\hline Pt9 & -0.72867300 & 3.14553100 & -2.04557500 \\
\hline Pt10 & -1.28325100 & -3.22192500 & 0.73637400 \\
\hline Pt11 & -0.19690900 & 0.81570100 & 3.90202400 \\
\hline Pt12 & -1.32425000 & 0.57688700 & -2.98682600 \\
\hline Pt13 & -4.04346000 & 1.13561300 & 1.56965900 \\
\hline Pt14 & 3.86722200 & 0.48522200 & -2.68802200 \\
\hline & 0.55198700 & 3.34226900 & 3.12886900 \\
\hline & 4.91753400 & -0.57203900 & -0.42103700 \\
\hline
\end{tabular}




\begin{tabular}{|c|c|c|c|}
\hline Pt17 & 3.71926000 & 0.49088500 & 1.78124800 \\
\hline Pt18 & 2.36884700 & 0.21115400 & -0.53802100 \\
\hline Pt19 & -1.17494000 & -3.24849100 & -1.99919000 \\
\hline Pt20 & -2.82712700 & 0.08960300 & 3.75751100 \\
\hline Pt21 & -0.66685000 & -1.72258900 & -4.22531300 \\
\hline Pt22 & 4.24529100 & 2.05628100 & -0.40684100 \\
\hline Pt23 & -3.23148800 & -1.48080700 & 1.55540600 \\
\hline Pt24 & -3.07624600 & -1.53585500 & -2.94996100 \\
\hline Pt25 & -0.11431400 & 1.02272400 & -0.68540900 \\
\hline Pt26 & 3.01221500 & -2.00507600 & 0.88167800 \\
\hline Pt27 & 1.75348300 & -0.92550100 & 3.08007900 \\
\hline Pt28 & -0.70425900 & 3.15324900 & 0.75405700 \\
\hline Pt29 & -2.07914800 & 2.60756500 & 3.06629300 \\
\hline Pt30 & 2.42926800 & 1.56431800 & 3.92181600 \\
\hline Pt31 & -4.41462100 & -0.40674300 & -0.66984400 \\
\hline Pt32 & -3.56667500 & -2.98357500 & -0.70474900 \\
\hline Pt33 & -1.82605700 & -0.96324700 & -0.69399000 \\
\hline Pt34 & -0.90908000 & -1.66706200 & 2.99403700 \\
\hline Pt35 & 1.13801000 & 1.26448300 & 1.60462800 \\
\hline Pt36 & 1.03466900 & -3.37976100 & 2.15617700 \\
\hline Pt37 & -3.31709700 & 3.63391000 & 0.94210100 \\
\hline Pt38 & -3.95607800 & 0.97863200 & -2.91336700 \\
\hline Pt39 & 2.00775300 & -0.92300400 & -4.11912900 \\
\hline Pt 40 & 1.23700700 & -3.39010500 & -3.27201400 \\
\hline Pt41 & 1.32382700 & 1.37107400 & -2.86297900 \\
\hline \# 41 & er $=$ Pt 42 & ot & 95453800 \\
\hline Pt 1 & 0.33125300 & -0.27673100 & -3.84263700 \\
\hline Pt2 & -1.83959000 & -1.89318000 & -3.35777400 \\
\hline Pt 3 & -1.53455400 & 0.42300200 & -2.01152200 \\
\hline Pt 4 & 2.71438000 & 2.89736300 & -0.40901700 \\
\hline Pt 5 & 2.06832800 & 1.55263100 & -2.71937300 \\
\hline Pt 6 & -2.03698000 & -1.63965400 & 1.92367400 \\
\hline Pt 7 & -3.44486800 & -0.32721700 & -0.08944800 \\
\hline Pt 8 & -3.08315300 & 2.37842100 & -2.85356100 \\
\hline Pt9 & 4.05973400 & 1.60534300 & 1.57594700 \\
\hline Pt10 & 0.92179100 & -0.35947000 & -1.22229400 \\
\hline Pt11 & -1.21338800 & -1.92283400 & -0.73143000 \\
\hline Pt12 & -2.15469300 & 0.51699900 & -4.59922000 \\
\hline Pt13 & -2.21373200 & -3.97508500 & 0.59280000 \\
\hline Pt14 & -0.29217100 & -3.64451800 & 2.52630200 \\
\hline Pt15 & 2.33411700 & -3.58477500 & 1.80096200 \\
\hline Pt16 & -2.56694200 & 2.31331200 & -0.20392700 \\
\hline Pt17 & 0.61774500 & 4.54111500 & 0.22358400 \\
\hline Pt18 & 1.53904900 & -1.79542700 & 3.67530800 \\
\hline Pt19 & -2.77280400 & 1.02172100 & 2.30229900 \\
\hline Pt20 & -0.23260700 & 0.20784500 & 3.01025000 \\
\hline Pt21 & 0.15820500 & 2.20248700 & -0.91028100 \\
\hline Pt22 & -0.98442600 & 0.35042200 & 0.53326000 \\
\hline Pt23 & -3.63899000 & -2.66237800 & -1.43342600 \\
\hline Pt24 & 0.43238300 & -3.96616800 & -0.11145200 \\
\hline Pt25 & -0.42933300 & 2.34843300 & -3.54166700 \\
\hline Pt26 & 1.52290200 & 0.96445800 & 1.05430100 \\
\hline Pt27 & 0.65868400 & -2.68713600 & -2.5355600 \\
\hline Pt28 & 1.27620700 & 4.12762600 & -2.37543200 \\
\hline Pt29 & 0.40907400 & 2.45803600 & 4.23680000 \\
\hline Pt30 & 0.67104200 & -1.59767100 & 1.16538000 \\
\hline Pt31 & 3.25776100 & -1.00986000 & 1.69036500 \\
\hline Pt32 & 2.40165500 & 0.79902100 & 3.562827 \\
\hline
\end{tabular}




\begin{tabular}{|c|c|c|c|}
\hline Pt33 & -1.03174500 & -1.87549500 & 4.42467800 \\
\hline Pt34 & -0.60216800 & 2.67581000 & 1.73657900 \\
\hline Pt35 & -1.36144600 & 4.18776400 & -1.73041000 \\
\hline-36 & 0.65412700 & 0.01993800 & 5.47632800 \\
\hline$=3$ & 3.47319300 & 0.27636400 & -0.71595400 \\
\hline 38 & 2.59916200 & -2.35244700 & -0.60198300 \\
\hline 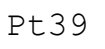 & -3.96607200 & -0.27712300 & -2.73843200 \\
\hline$-1+3>-1$ & 2.79527900 & -1.05902400 & -2.99036100 \\
\hline$=4$ & -1.50632400 & -4.24135700 & -2.01259900 \\
\hline & 2.00991500 & 3.27943900 & 2.22611700 \\
\hline 12 & luster $=\mathrm{Pt} 43 \quad \mathrm{Q}=0$ & \multicolumn{2}{|c|}{$\mathrm{M}=0 \quad \mathrm{E}$ tot $=-3821.50649400$} \\
\hline t1 & 2.40236100 & -2.10298600 & -3.6010470 \\
\hline Pt2 & 1.09943700 & -0.82067000 & 3.36348000 \\
\hline-3 & -0.26202100 & 4.34642100 & -0.01657100 \\
\hline$=4$ & -1.36360400 & -1.18599700 & -3.16194900 \\
\hline$=5$ & 1.48666100 & -4.03226600 & 0.29921100 \\
\hline 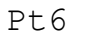 & -1.97496400 & -2.35501700 & -0.73322500 \\
\hline 7 & 0.69831400 & -2.26434200 & -1.5848560 \\
\hline 8 & -4.66007000 & -0.09550500 & 0.89065800 \\
\hline 9 & -1.29013200 & 1.63204500 & -2.72269400 \\
\hline 10 & -2.37818800 & 3.88247400 & -1.69854400 \\
\hline & -3.63454200 & -0.57737500 & 3.35778000 \\
\hline & 0.94262000 & -3.49609300 & 2.89891100 \\
\hline & -0.57076100 & -0.12238800 & -0.90616100 \\
\hline & 3.49869900 & 1.95096000 & -0.42073300 \\
\hline & 3.34088500 & -2.49794200 & -1.07593200 \\
\hline 16 & 1.77497300 & 3.13356600 & 1.37401300 \\
\hline & 0.17241000 & 3.90325100 & -2.69324200 \\
\hline & -3.25730800 & 1.86789600 & 2.20481500 \\
\hline & -3.15127200 & 0.09199800 & -1.34242200 \\
\hline & 0.84857400 & 2.06600000 & -0. \\
\hline & -0.58040100 & -4.57114600 & -1.38894900 \\
\hline $\mathrm{P}$ & 2.27448400 & 4.31219600 & -1.02451500 \\
\hline Pt2 & 1.21358600 & 1.87657700 & 3.73018400 \\
\hline & -2.06258400 & -0.34291100 & 1.22229300 \\
\hline & 2.00821600 & -0.28307900 & -0.34301000 \\
\hline & 2.82122400 & -1.9973 & 1 \\
\hline & 1.04992800 & 0.14800500 & -2.97656700 \\
\hline & -0.80484800 & 3.10398800 & 2.37804600 \\
\hline Pt29 & 3.68679300 & -0.02926900 & -2.36238000 \\
\hline & -2.81306700 & 4.25710800 & 0.95679200 \\
\hline & -1.66001800 & 2.04202100 & 0.04005100 \\
\hline & 0100 & 0.8 & 366000 \\
\hline & 4.61217200 & -0.45963100 & 0.17747100 \\
\hline & 1.97580000 & -4.47999100 & -2.33443500 \\
\hline & -4.25237600 & 2.33163000 & -0.30859500 \\
\hline & -1.22218700 & 0.65349200 & 3.59678800 \\
\hline & -1.15906200 & -4.03909300 & 1.23143900 \\
\hline & -3.46237100 & -2.55976700 & 1.5056090 \\
\hline & 2.95567900 & 0.71532300 & 1.98684100 \\
\hline & -0.00618300 & -3.42059600 & -3.82488600 \\
\hline Pt 4 & 0.19290600 & -1.78520300 & 1.00327400 \\
\hline Pt & 2.53130800 & 2.39985200 & -2.93946700 \\
\hline-4 & -1.35656900 & -2.06665200 & 3.18006700 \\
\hline 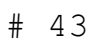 & Cluster $=$ Pt $44 \quad Q=0$ & $\mathrm{M}=0 \quad \mathrm{Etot}=-391$ & .7453 \\
\hline & -3.03713800 & 2.40171600 & 2.8583900 \\
\hline & -4.09077200 & 0.40778200 & 1.289816 \\
\hline
\end{tabular}




\begin{tabular}{|c|c|c|c|}
\hline Pt 3 & 4.17077200 & -1.50035700 & 0.43221900 \\
\hline Pt 4 & -2.27940600 & -3.77472300 & 0.09244400 \\
\hline Pt5 & 2.14535800 & 1.06935000 & -1.51011800 \\
\hline Pt 6 & -1.12232500 & 1.24681900 & 4.53904300 \\
\hline Pt 7 & -4.63791300 & 0.92286000 & -1.34205000 \\
\hline Pt 8 & -1.86616400 & 4.50101900 & -0.90365100 \\
\hline Pt9 & -0.47574100 & -3.31024400 & 2.08732200 \\
\hline Pt10 & -0.17579500 & 2.58303000 & -1.59823800 \\
\hline Pt11 & 3.30344300 & -1.17026100 & 3.02030100 \\
\hline Pt12 & 1.67739700 & 3.13618800 & 0.36232200 \\
\hline Pt13 & 0.83470000 & 1.80602900 & 2.75934100 \\
\hline Pt14 & -1.51996300 & 0.38526300 & 2.08579900 \\
\hline Pt15 & 3.02868300 & -3.47766600 & -1.08031200 \\
\hline Pt16 & 1.68179000 & 0.03955100 & -3.96311900 \\
\hline Pt17 & 3.55475400 & -1.10314000 & -2.27112100 \\
\hline Pt18 & 0.82511900 & -0.93744200 & 2.15649800 \\
\hline Pt19 & -3.66505000 & 2.90104700 & 0.24501000 \\
\hline Pt20 & 2.21120500 & -3.16144600 & 1.49333400 \\
\hline Pt21 & 1.25773500 & -0.10431200 & 4.61700600 \\
\hline Pt22 & 2.85658400 & 0.75357100 & 1.18087800 \\
\hline Pt23 & 1.66477900 & -1.30447800 & -0.36882200 \\
\hline Pt24 & 0.37651900 & -3.67768700 & -0.50497200 \\
\hline Pt25 & 0.35411500 & 0.87903900 & 0.34542900 \\
\hline Pt2 6 & -2.75781000 & 2.53180800 & -2.47777200 \\
\hline Pt27 & -0.17019900 & -0.18268900 & -2.02539300 \\
\hline Pt28 & -0.96315400 & -1.45708100 & 0.20453400 \\
\hline Pt29 & 1.18182200 & -2.39058600 & -2.79102500 \\
\hline Pt 30 & -2.69426900 & -0.15798300 & -2.92927000 \\
\hline Pt31 & 1.68264300 & 2.72501600 & -3.53422200 \\
\hline Pt32 & -1.06151900 & 2.95785300 & 1.10974600 \\
\hline Pt33 & -1.06193800 & -1.44470000 & 4.01536800 \\
\hline Pt34 & -0.67737600 & -1.21459800 & -4.42744600 \\
\hline Pt35 & 4.68647200 & 0.88912800 & -0.79639100 \\
\hline Pt36 & -2.88320900 & -1.95199000 & 2.01470600 \\
\hline Pt37 & -0.71845900 & 1.48339500 & -4.00613500 \\
\hline Pt 38 & 4.01065200 & 1.32604100 & -3.39699700 \\
\hline Pt39 & -1.49742100 & -2.53386400 & -2.19801200 \\
\hline Pt 40 & -3.41571500 & -0.07113000 & 3.89841800 \\
\hline Pt 41 & -3.49983100 & -1.44259000 & -0.64005800 \\
\hline Pt 42 & 3.54674100 & 3.27505500 & -1.58280300 \\
\hline Pt 43 & -2.11520400 & 0.89901600 & -0.49839000 \\
\hline Pt 44 & 1.33508800 & -2.75161000 & 4.03839000 \\
\hline$\# 44$ & Cluster $=$ Pt $45 \quad \mathrm{Q}=0$ & $\mathrm{M}=0 \quad \mathrm{Etot}=-4014$ & 25752800 \\
\hline Pt 1 & -3.73798100 & -0.00753000 & 2.05080100 \\
\hline Pt2 & 1.43319700 & -4.44470200 & -0.50481700 \\
\hline Pt 3 & -1.09169400 & -4.34214900 & 0.60523600 \\
\hline Pt 4 & -1.44341500 & 0.37472200 & -4.37828300 \\
\hline Pt5 & -0.42185900 & -1.12438100 & 4.76224900 \\
\hline Pt 6 & 2.14430800 & 0.58436200 & -1.38475800 \\
\hline Pt 7 & 4.61512200 & -0.32058400 & -1.35153000 \\
\hline Pt 8 & -0.45700700 & 0.09805400 & -1.92397300 \\
\hline Pt9 & 2.86226000 & -1.20078200 & -3.27302200 \\
\hline Pt10 & -0.76197300 & 2.57780500 & -2.90288000 \\
\hline Pt11 & 3.03143000 & -1.11283000 & 0.68442500 \\
\hline Pt 12 & 2.04901200 & 4.01653700 & 0.28521100 \\
\hline Pt13 & 1.89071300 & 3.08708000 & -2.35317900 \\
\hline Pt14 & -3.16186000 & -3.58918800 & -1.01862900 \\
\hline 15 & 0.54177000 & 1.44022600 & 4.6640590 \\
\hline
\end{tabular}




\begin{tabular}{|c|c|c|c|}
\hline Pt16 & -2.75768500 & 2.60143300 & 1.95091300 \\
\hline Pt17 & -1.06462000 & -3.36336500 & 3.18744000 \\
\hline Pt18 & 0.23908000 & 2.23085900 & -0.41474700 \\
\hline Pt19 & -2.24455100 & 3.22680700 & -0.69084900 \\
\hline Pt20 & -1.94312900 & 0.73892400 & 0.23009300 \\
\hline Pt21 & -0.26144200 & 1.62413300 & 2.16438500 \\
\hline Pt22 & 1.49069900 & 3.43737200 & 2.92706300 \\
\hline Pt23 & -0.66603400 & -3.71745900 & -2.09265600 \\
\hline Pt24 & 3.79777100 & 1.37705300 & -3.30351100 \\
\hline Pt25 & -2.99285900 & -1.65133200 & 4.07789500 \\
\hline Pt26 & 0.20923100 & -1.71082300 & -3.79086400 \\
\hline Pt27 & -0.04567600 & 4.70423600 & -1.33615500 \\
\hline Pt28 & -2.34211400 & -1.62758800 & -2.74187000 \\
\hline Pt29 & -1.29760900 & -1.85889000 & -0.25816500 \\
\hline Pt30 & 3.98946300 & 2.35855500 & -0.76529000 \\
\hline Pt31 & -2.95573400 & 1.02554800 & -2.22124500 \\
\hline Pt32 & 2.42096200 & 1.52791200 & 1.26614900 \\
\hline Pt33 & -3.82945600 & -0.99126800 & -0.52935400 \\
\hline Pt34 & 1.48154800 & -0.44271200 & 2.97668600 \\
\hline Pt35 & 1.87046600 & -3.75228600 & -3.10712600 \\
\hline Pt36 & -3.12407000 & -2.65940300 & 1.54120000 \\
\hline Pt37 & -2.08111100 & 0.93624300 & 4.02969800 \\
\hline Pt38 & -1.06457500 & 3.48061200 & 3.88141100 \\
\hline Pt39 & 1.18722800 & 0.89202200 & -3.89051800 \\
\hline Pt 40 & -0.56425800 & 4.15328900 & 1.28036500 \\
\hline Pt 41 & -1.21047800 & -0.90163500 & 2.26108800 \\
\hline Pt 42 & 0.57048900 & -0.19436400 & 0.52219100 \\
\hline Pt 43 & 1.19523700 & -1.94150000 & -1.28805100 \\
\hline Pt 44 & 0.84973300 & -2.65376500 & 1.42625100 \\
\hline Pt 45 & 3.65147100 & -2.88524900 & -1.25333800 \\
\hline \# 45 & Luster $=\mathrm{Pt} 46 \quad \mathrm{Q}=0$ & $=0 \quad$ Etot $=-41$ & 92021100 \\
\hline Pt 1 & -0.20393100 & -4.16139300 & -1.19783300 \\
\hline Pt2 & 3.83770800 & -0.76792600 & -1.48835400 \\
\hline Pt 3 & -2.85264100 & -3.46810200 & -0.60209200 \\
\hline Pt 4 & 1.02298300 & 3.61851700 & 2.15717800 \\
\hline Pt 5 & -4.68263400 & 1.35042900 & 0.63845400 \\
\hline Pt 6 & -1.57281600 & 4.20227000 & 2.78334100 \\
\hline Pt 7 & 0.79164300 & -3.68450200 & -3.69831800 \\
\hline Pt 8 & -1.19822200 & -4.51741300 & 1.31155400 \\
\hline Pt 9 & 0.14458900 & -1.65174000 & -2.12017300 \\
\hline Pt10 & 1.95682200 & 4.06185900 & -0.44716800 \\
\hline Pt11 & 2.32054500 & -1.40618800 & -3.692 \\
\hline Pt12 & 1.51549000 & 1.61728000 & 0.4349660 \\
\hline Pt13 & 1.65177100 & -1.05579900 & 0.03868500 \\
\hline Pt14 & 3.71102100 & 1.96807200 & -1.07172300 \\
\hline Pt15 & -2.48353800 & -0.03808000 & 1.03676200 \\
\hline Pt16 & -3.20666000 & 3.66129400 & 0.68599800 \\
\hline Pt17 & 2.11882100 & 1.24973500 & 3.0457830 \\
\hline Pt18 & 1.59854800 & 0.62465900 & -2.0733380 \\
\hline Pt19 & 0.05344500 & -0.07443300 & 1.95861200 \\
\hline Pt20 & 2.24368800 & -1.48713500 & 2.62800000 \\
\hline Pt21 & -1.81118700 & -3.09602000 & -3.13169600 \\
\hline Pt22 & -0.83748500 & -2.06666700 & 0.3591710 \\
\hline Pt23 & -2.56160600 & -0.98559300 & -1.6287360 \\
\hline Pt24 & -1.98555800 & -0.45969500 & 3.6577520 \\
\hline Pt25 & 0.59447500 & -0.47295900 & 4.5329100 \\
\hline Pt26 & -0.52872100 & 0.35044100 & -0.61928400 \\
\hline Pt27 & -0.62705800 & 4.71211100 & 0.27581200 \\
\hline
\end{tabular}




\begin{tabular}{|c|c|c|c|}
\hline te 28 & -0.22948900 & -1.38378500 & -4.71071300 \\
\hline Pt29 & -1.02957800 & 2.23831900 & 1.08359700 \\
\hline Pt30 & -0.29902800 & -2.53578300 & 2.96789900 \\
\hline Pt31 & 3.56883300 & 2.94615100 & 1.46511100 \\
\hline Pt32 & -0.99726600 & 0.70242500 & -3.18170000 \\
\hline Pt33 & 3.74395200 & 0.93779600 & -3.60321800 \\
\hline Pt34 & 1.24648500 & 0.92708100 & -4.66316800 \\
\hline Pt35 & -4.54290500 & -1.41875600 & 0.14967400 \\
\hline Pt3 & 2.10466000 & 3.03484600 & -3.00555700 \\
\hline Pt3 & -0.15326800 & 2.78503400 & -1.55115800 \\
\hline Pt3 & -0.48658800 & 1.88717500 & 3.70603700 \\
\hline Pt39 & -3.08337500 & 1.91179800 & 2.79887700 \\
\hline Pt 40 & -2.89196900 & -2.51094800 & 2.03106800 \\
\hline Pt 41 & 2.33873800 & -3.11479600 & -1.53663900 \\
\hline Pt 42 & -4.52512400 & -0.42002800 & 2.68824100 \\
\hline Pt 43 & -2.69473200 & 1.74466300 & -1.14268600 \\
\hline Pt 44 & 1.33730000 & -3.54048200 & 1.00232400 \\
\hline Pt 45 & 3.75514900 & 0.24503600 & 1.08020800 \\
\hline Pt 46 & 3.82871300 & -2.45876800 & 0.64790900 \\
\hline \# 46 & ter $=$ Pt 47 & $=0 \quad$ Etot $=-$ & 16964100 \\
\hline Pt 1 & -3.80053600 & -0.27886500 & -1.67778400 \\
\hline Pt2 & 2.24676300 & -1.75977400 & -2.83445300 \\
\hline Pt 3 & 3.97021700 & 0.18269000 & -1.91209200 \\
\hline Pt 4 & 0.05673800 & -0.53619300 & -3.95675200 \\
\hline Pt 5 & -2.42799300 & -1.63503000 & -3.60603300 \\
\hline Pt 6 & 2.79923000 & 2.67191000 & -2.43387700 \\
\hline Pt 7 & 2.02271000 & -2.89179900 & 3.92450500 \\
\hline Pt 8 & -1.08806200 & 0.51756900 & 2.83385600 \\
\hline Pt 9 & -2.63334800 & -2.71959100 & -1.10476500 \\
\hline Pt10 & 1.42591500 & 1.64370600 & 2.54100800 \\
\hline Pt11 & 0.58268200 & 4.26194000 & -1.99488500 \\
\hline Pt12 & -0.83665600 & 2.13919700 & -1.35655200 \\
\hline Pt13 & 2.67882400 & 0.17372100 & 0.45516600 \\
\hline Pt14 & 1.97958300 & -3.52151000 & 1.28098500 \\
\hline Pt15 & -2.13549300 & 1.06550000 & -3.46691700 \\
\hline Pt16 & 1.06058600 & -1.14445100 & 2.17309700 \\
\hline Pt17 & 4.24028300 & 2.22033300 & -0.11793600 \\
\hline Pt18 & -0.44382400 & -3.94236300 & 0.01986600 \\
\hline Pt19 & -2.56604600 & -1.69348600 & 3.26474500 \\
\hline Pt20 & -0.79836500 & 2.93215200 & 1.32379000 \\
\hline Pt21 & -2.33812300 & 2.76454000 & 3.48579900 \\
\hline Pt22 & 3.64487900 & -2.16649100 & -0.48572700 \\
\hline Pt23 & 2.53813700 & 0.59210900 & -4.19316500 \\
\hline Pt24 & -1.86440600 & 4.45609000 & -0.62317300 \\
\hline Pt25 & -3.52066000 & 3.14638700 & 1.12270100 \\
\hline Pt26 & 3.71637900 & -1.59457800 & 2.20585300 \\
\hline Pt27 & -0.12722900 & -1.33579000 & 4.52571800 \\
\hline Pt28 & 1.31311900 & 0.57580700 & -1.83801600 \\
\hline Pt29 & 1.90050600 & -4.05615900 & -1.38839000 \\
\hline Pt30 & 0.37916700 & 2.19379400 & -3.76530200 \\
\hline Pt31 & -2.45327600 & 0.84542900 & 0.39531100 \\
\hline Pt32 & 0.12607300 & 0.60956800 & 0.50363100 \\
\hline Pt33 & 5.30246700 & -0.22176800 & 0.4448030 \\
\hline Pt3 4 & -0.26811200 & -2.88432500 & -2.53284700 \\
\hline Pt35 & -5.09031000 & 1.09575600 & 0.27960100 \\
\hline Pt36 & -2.76918700 & -3.71381000 & 1.4364710 \\
\hline Pt37 & -1.79908600 & 3.75060100 & -3.23474100 \\
\hline Pt38 & -1.14268100 & -0.53124900 & -1.5519660 \\
\hline
\end{tabular}




\begin{tabular}{|c|c|c|c|}
\hline Pt39 & 1.59986200 & 2.73321900 & -0.01686700 \\
\hline Pt 40 & 4.07645300 & 1.11707000 & 2.46973300 \\
\hline Pt41 & -3.98107400 & -1.34092000 & 0.86161000 \\
\hline Pt 42 & -3.50508400 & 2.44108600 & -1.52163500 \\
\hline Pt 43 & -1.30969200 & -1.55041000 & 0.94395200 \\
\hline Pt 44 & -0.38617100 & -3.35531900 & 2.70935700 \\
\hline Pt 45 & 2.43651800 & -0.22087600 & 4.21370800 \\
\hline Pt 46 & -3.80954700 & 0.69541200 & 2.65816800 \\
\hline Pt 47 & 0.99787000 & -1.73082800 & -0.45955800 \\
\hline \# 47 & Cluster $=$ Pt $48 \quad Q=0$ & \multicolumn{2}{|c|}{$\mathrm{M}=0 \quad \mathrm{Etot}=-4313.89104000$} \\
\hline Pt 1 & -0.65180500 & 2.81500900 & -3.74100700 \\
\hline Pt2 & -4.78399600 & -0.90021700 & 1.52830400 \\
\hline Pt3 & -2.89529600 & 3.31406300 & 0.91102000 \\
\hline Pt 4 & 2.14983300 & 0.39634800 & -1.34238000 \\
\hline Pt5 & 2.45898000 & -0.90902100 & 0.98867600 \\
\hline Pt 6 & 3.20793800 & -0.60361400 & -3.58086100 \\
\hline Pt 7 & -0.25810700 & 3.87664600 & 0.77718900 \\
\hline Pt 8 & -3.95473100 & 1.07323200 & -0.13212900 \\
\hline Pt9 & -1.33160700 & -1.81849300 & -3.59237100 \\
\hline Pt10 & -3.01225800 & -2.33540800 & 2.99164700 \\
\hline Pt11 & -1.00491000 & -3.12457800 & -1.21856800 \\
\hline Pt12 & 0.76932300 & -0.53070700 & -4.75439500 \\
\hline Pt13 & -3.23406300 & -2.72641000 & 0.30454400 \\
\hline Pt14 & -3.43015700 & -3.02603200 & -2.37960300 \\
\hline Pt15 & 3.54637200 & -1.96432900 & -1.24494200 \\
\hline Pt16 & 4.81908900 & 0.37312900 & -1.63397400 \\
\hline Pt17 & -2.34750200 & -0.76532200 & -1.32434700 \\
\hline Pt18 & 5.11322400 & -0.90141200 & 0.73296100 \\
\hline Pt19 & 2.42499400 & 3.74966300 & 0.50511300 \\
\hline Pt20 & -3.80037100 & -0.63492300 & -3.55652700 \\
\hline Pt21 & -4.97365400 & -1.19485400 & -1.15322000 \\
\hline Pt22 & 3.46389200 & 2.69196200 & -1.76041100 \\
\hline Pt23 & 0.78965000 & 2.79790500 & -1.46707800 \\
\hline Pt24 & 0.27332100 & -0.49918800 & 2.50139200 \\
\hline Pt25 & 1.11471100 & -1.89821500 & -2.43430800 \\
\hline Pt26 & 1.09470000 & 1.46038600 & 0.87467300 \\
\hline Pt27 & -1.94299000 & -0.09286100 & 4.03178200 \\
\hline Pt28 & -2.14780500 & -0.42680400 & 1.33627400 \\
\hline Pt29 & -1.10211000 & 1.89509100 & 2.40792800 \\
\hline Pt30 & 1.64391600 & -2.87224600 & 2.65197300 \\
\hline Pt31 & -3.73265400 & 1.34472100 & 2.56968400 \\
\hline Pt32 & -2.78505100 & 1.63144400 & -2.5280550 \\
\hline Pt33 & 3.84759000 & -3.24361300 & 1.12266600 \\
\hline Pt34 & 3.78773700 & 1.43493800 & 0.63496700 \\
\hline Pt35 & -0.80107200 & -2.81249300 & 1.48990700 \\
\hline Pt36 & 0.05677500 & -0.85273200 & -0.17095500 \\
\hline Pt37 & 2.61805700 & 0.87672700 & 3.03089300 \\
\hline Pt38 & -1.70698800 & 0.56823800 & -4.7745180 \\
\hline Pt39 & 1.74208100 & -1.09532600 & 4.6688470 \\
\hline Pt 40 & -0.57364300 & -2.40831500 & 4.16518100 \\
\hline Pt41 & -0.27129300 & 0.46873200 & -2.50749800 \\
\hline Pt 42 & 3.93994000 & -1.46134300 & 3.13626800 \\
\hline Pt 43 & 1.83530300 & 1.71027100 & -3.7075240 \\
\hline Pt 44 & 1.25239700 & 3.19006000 & 2.90701400 \\
\hline Pt 45 & -1.29391900 & 1.53179800 & -0.27480100 \\
\hline Pt 46 & 1.42581600 & -3.19724900 & -0.04883000 \\
\hline Pt 47 & 0.38304200 & 1.22167500 & 4.55028000 \\
\hline Pt 48 & -1.72270000 & 3.87366600 & -1.49088000 \\
\hline
\end{tabular}




\begin{tabular}{|c|c|c|c|}
\hline \# 48 & Cluster $=$ Pt $49 \quad Q=0$ & $\mathrm{M}=0 \quad \mathrm{E}$ tot $=-44$ & 7178400 \\
\hline Pt1 & 3.76428300 & -0.47892900 & 2.13594900 \\
\hline Pt2 & 0.05491100 & 3.09074700 & 0.73292300 \\
\hline Pt 3 & -2.26257200 & -0.29176400 & -1.11304800 \\
\hline Pt 4 & -0.06653000 & -1.89006100 & -1.05595700 \\
\hline Pt 5 & -3.77498000 & 1.42674200 & -2.42089700 \\
\hline Pt 6 & 4.58761500 & 2.09199600 & 1.81472200 \\
\hline Pt 7 & 0.12536900 & -3.06505700 & 2.58990900 \\
\hline Pt 8 & 1.30610300 & 2.39505600 & -1.62810100 \\
\hline Pt9 & 1.38105500 & -3.76324700 & 0.22040400 \\
\hline Pt10 & 4.51326400 & 0.43081400 & -0.37616400 \\
\hline Pt11 & -2.60208600 & -3.00534400 & 2.69879600 \\
\hline Pt12 & -1.17617000 & -1.01270900 & 3.92298400 \\
\hline Pt13 & -1.45095600 & 2.40859200 & -1.47862200 \\
\hline Pt14 & 2.46663400 & 1.56878800 & 3.48586800 \\
\hline Pt15 & 2.11574800 & -2.84309100 & -2.28893100 \\
\hline Pt16 & -2.37854300 & -2.82102600 & -2.04526400 \\
\hline Pt17 & 2.84906300 & -3.03210600 & 2.40325100 \\
\hline Pt18 & -2.46887100 & -0.66698800 & -3.76250700 \\
\hline Pt19 & 2.04097300 & -0.68913000 & -4.00701700 \\
\hline Pt20 & -3.71684200 & 3.06595300 & -0.18398700 \\
\hline Pt21 & 2.42080300 & 3.74355900 & 1.83693100 \\
\hline Pt22 & -0.14697100 & 0.23026400 & -2.73164100 \\
\hline Pt23 & -2.15093500 & 1.34451400 & 1.04987900 \\
\hline Pt24 & -1.63290500 & 1.90723500 & -4.11641900 \\
\hline Pt25 & -4.52304300 & 0.47517800 & 0.11375700 \\
\hline Pt26 & 0.13441000 & 0.79406500 & 2.40720400 \\
\hline Pt27 & -0.21199700 & -2.28017800 & -3.70369000 \\
\hline Pt28 & 1.58343200 & -1.02625800 & 3.77336700 \\
\hline Pt29 & 3.50490700 & 1.39100100 & -2.81559000 \\
\hline Pt30 & 3.62300400 & -2.16030700 & -0.07534600 \\
\hline Pt31 & -1.38590800 & -3.74966200 & 0.37042000 \\
\hline Pt32 & 2.26489300 & 1.32283400 & 0.81046700 \\
\hline Pt33 & 2.12600900 & -0.31331000 & -1.35098400 \\
\hline Pt34 & -0.00704700 & 0.61853700 & -0.18599100 \\
\hline Pt35 & 4.30101200 & -1.21349500 & -2.55430600 \\
\hline Pt3 3 & -4.34382700 & 2.13584600 & 2.29895800 \\
\hline Pt37 & 1.40490800 & -1.22350100 & 1.10502400 \\
\hline Pt38 & -0.29061400 & -0.11783400 & -5.34953600 \\
\hline Pt39 & -4.56363000 & -1.16997400 & -2.07369200 \\
\hline Pt 40 & 0.27811400 & 1.02332800 & 5.03698400 \\
\hline Pt41 & -2.05982900 & 1.59101100 & 3.73127900 \\
\hline Pt 42 & 1.19688600 & 1.89334200 & -4.26984200 \\
\hline Pt 43 & -3.63088900 & -2.12469300 & 0.31793800 \\
\hline Pt4 4 & -3.51580100 & -0.44318700 & 2.53065300 \\
\hline Pt 45 & 3.70500100 & 3.02951500 & -0.58637700 \\
\hline Pt 46 & -2.17125000 & 3.76610400 & 2.08589800 \\
\hline Pt 47 & -1.28911600 & -1.21027500 & 1.25108600 \\
\hline Pt 48 & 0.20119300 & 3.22794200 & 3.41858400 \\
\hline Pt4 9 & -0.12827900 & -4.38083700 & -1.96932800 \\
\hline \# 49 & Cluster $=$ Pt $50 \quad Q=0$ & $=0 \quad \mathrm{Etot}=-45$ & 99462200 \\
\hline Pt 1 & 3.77176900 & 1.43538900 & -1.75447500 \\
\hline Pt 2 & -1.78848100 & -1.49013600 & -1.33560300 \\
\hline Pt 3 & 3.51391100 & -1.01327200 & 2.93980300 \\
\hline Pt 4 & 0.44016000 & -0.38580100 & -2.45270000 \\
\hline Pt 5 & -0.26477900 & -0.45405900 & 2.68310800 \\
\hline Pt 6 & -4.80614500 & 0.26866300 & 1.80623100 \\
\hline
\end{tabular}




\begin{tabular}{|c|c|c|c|}
\hline Pt 7 & -2.89106400 & 2.90693600 & -2.58479000 \\
\hline Pt 8 & -2.76613700 & -0.06766800 & 3.60446700 \\
\hline Pt9 & 1.00824900 & -2.63936700 & -3.71165300 \\
\hline Pt10 & -0.22826000 & 3.41018900 & -2.24445100 \\
\hline Pt11 & -1.38133500 & 1.20227400 & -1.22004800 \\
\hline Pt12 & -0.82870900 & -4.85889800 & -0.28496400 \\
\hline Pt13 & -1.10362000 & -2.63472500 & 1.14225900 \\
\hline Pt14 & 1.64977000 & 0.65727600 & 4.21576500 \\
\hline Pt15 & 1.46011800 & 2.97469500 & 2.73308500 \\
\hline Pt16 & -2.33135700 & -0.11290800 & 0.95412100 \\
\hline Pt17 & 5.00957800 & -0.98098000 & -1.62610200 \\
\hline Pt18 & 1.25625400 & 1.65447800 & -0.85296300 \\
\hline Pt19 & -3.15702900 & -3.54718500 & -0.39483700 \\
\hline Pt20 & -3.72943700 & -2.47061200 & -2.83607200 \\
\hline Pt21 & -1.53128200 & -2.49368200 & 3.78671800 \\
\hline Pt22 & 2.44397400 & 3.81303200 & -1.83922100 \\
\hline Pt23 & -0.99107400 & 4.15125800 & 2.38322900 \\
\hline Pt24 & -3.34641200 & 0.21957600 & -2.73996200 \\
\hline Pt25 & -1.06478000 & 1.33856700 & -3.88086100 \\
\hline Pt26 & -1.49180700 & -1.37854200 & -4.02115900 \\
\hline Pt27 & 0.68024800 & -2.81037100 & -1.05051800 \\
\hline Pt28 & -0.44314700 & 1.82180700 & 1.23598400 \\
\hline Pt29 & -3.90041000 & 1.61777300 & -0.39523000 \\
\hline Pt 30 & 4.13821800 & -2.32935400 & 0.58118500 \\
\hline Pt 31 & 0.10620800 & -0.52640800 & 0.12856900 \\
\hline Pt 32 & 1.23967500 & -2.04395300 & 4.14415000 \\
\hline Pt33 & -1.96976500 & 3.58166400 & -0.10374700 \\
\hline Pt 34 & 1.60066000 & -2.23243700 & 1.48099100 \\
\hline Pt 35 & 4.50380800 & 0.37625800 & 0.70750700 \\
\hline Pt 36 & 0.73795900 & 4.03929600 & 0.27678900 \\
\hline Pt37 & -1.31434600 & -3.72862200 & -2.65991000 \\
\hline Pt 38 & 2.52669000 & -0.72891700 & -0.74823900 \\
\hline Pt39 & 3.19960800 & 2.80341800 & 0.59485800 \\
\hline Pt 40 & 1.96572200 & 0.60287400 & 1.55086300 \\
\hline Pt 41 & -3.61793400 & -2.18385800 & 1.97497200 \\
\hline Pt 42 & -0.62777600 & -0.42590700 & 5.30348000 \\
\hline Pt 43 & 2.93878900 & -0.62939400 & -3.40817500 \\
\hline Pt 44 & -0.81989400 & 1.88749900 & 3.88971600 \\
\hline Pt 45 & 3.87203400 & 1.70330400 & 3.01354000 \\
\hline Pt 46 & -4.33772600 & -1.09832300 & -0.52288200 \\
\hline Pt 47 & 0.78983200 & -0.28233600 & -5.07248700 \\
\hline Pt 48 & 1.63603100 & 1.79441600 & -3.50328800 \\
\hline Pt 49 & 3.18209000 & -2.95777700 & -2.00708100 \\
\hline Pt 50 & -2.93864900 & 2.24485400 & 2.12002800 \\
\hline \# 50 & Cluster $=$ Pt 51 & \multicolumn{2}{|l|}{$\mathrm{M}=0 \quad \mathrm{Etot}=-46$} \\
\hline Pt 1 & 2.30390500 & -0.81180400 & 0.87650300 \\
\hline Pt2 & 0.86669000 & 0.51043200 & 5.13323800 \\
\hline Pt 3 & 1.94915700 & 2.59674500 & -3.04560500 \\
\hline Pt 4 & -3.15131200 & 1.62965800 & 2.78305800 \\
\hline Pt 5 & 2.02173200 & 0.36335000 & -1.59295000 \\
\hline Pt 6 & -3.47392200 & 2.82859200 & 0.26859600 \\
\hline Pt7 & 4.41437200 & -0.40341600 & -0.70127600 \\
\hline Pt 8 & 3.09298500 & -1.78006600 & -2.75622300 \\
\hline Pt9 & 4.03560500 & 1.10102500 & 1.60667400 \\
\hline Pt10 & -2.03846500 & -0.48165000 & 1.59038000 \\
\hline Pt11 & 0.60674400 & 3.99426000 & 1.86969300 \\
\hline Pt12 & -1.08258200 & 2.05847300 & 1.16086900 \\
\hline Pt13 & 0.61816500 & -4.12091300 & 85904900 \\
\hline
\end{tabular}




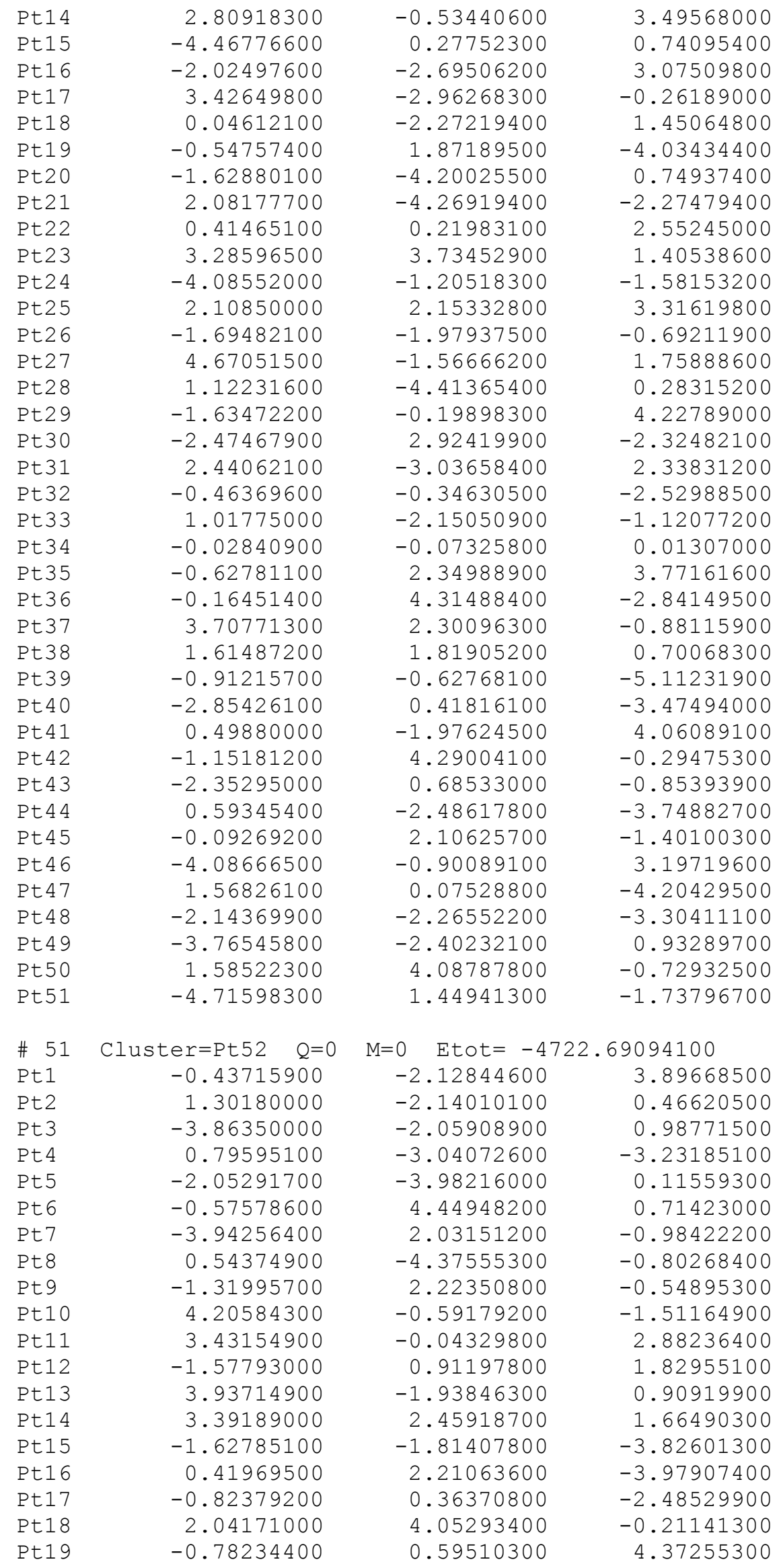




\begin{tabular}{|c|c|c|c|}
\hline Pt20 & -0.77633400 & -2.09268100 & -1.29877400 \\
\hline Pt21 & 2.93232700 & -3.08865100 & -1.41554100 \\
\hline Pt22 & -0.08639200 & 4.10762200 & -2.00401200 \\
\hline Pt23 & 5.12833900 & 0.53479300 & 0.80042400 \\
\hline Pt24 & 1.60910200 & 1.87125300 & 3.74850100 \\
\hline Pt25 & 2.16336100 & -2.52242200 & 2.97704500 \\
\hline Pt26 & -0.00957900 & 0.04629800 & -0.04692200 \\
\hline Pt27 & 1.22825300 & 1.83745600 & -1.45009500 \\
\hline Pt28 & 0.06919200 & -4.02642400 & 1.92059000 \\
\hline Pt29 & -2.49085200 & -3.58033900 & 2.80055400 \\
\hline Pt30 & 3.86371900 & 2.12988600 & -1.05565800 \\
\hline Pt31 & -2.95566200 & 3.16301700 & 1.35592800 \\
\hline Pt32 & 2.85576600 & 1.00157100 & -3.38836300 \\
\hline Pt33 & -4.20883900 & 0.68302500 & 1.46409400 \\
\hline Pt34 & 2.62898200 & -4.35599000 & 0.98999100 \\
\hline Pt35 & -1.55557400 & -4.26549400 & -2.57143800 \\
\hline Pt36 & 1.63209200 & -0.61930200 & 4.88047300 \\
\hline Pt37 & 2.47975800 & 3.66132700 & -2.88118300 \\
\hline Pt38 & -2.87341000 & -0.90667000 & 3.31766400 \\
\hline Pt39 & -3.17168300 & 1.79224000 & 3.73561800 \\
\hline Pt 40 & 0.74792600 & -0.51224800 & -4.45171400 \\
\hline Pt 41 & -2.60083600 & -0.21162400 & -0.46317600 \\
\hline Pt 42 & 1.58898300 & -0.84637800 & -1.94485000 \\
\hline Pt 43 & -1.65041100 & 0.69510200 & -4.96474600 \\
\hline Pt 44 & -0.84248400 & 3.10069100 & 3.16233400 \\
\hline Pt45 & -3.38435800 & -2.40741300 & -1.75354000 \\
\hline Pt 46 & -2.64904700 & 4.43833700 & -1.06744800 \\
\hline Pt 47 & -3.43290800 & 0.12114500 & -2.97321600 \\
\hline Pt 48 & 0.80615400 & -0.28224700 & 2.40052400 \\
\hline Pt 49 & 2.53891300 & 0.28828000 & 0.37368300 \\
\hline Pt50 & 0.77317700 & 2.21154200 & 1.23892700 \\
\hline Pt51 & -1.24374400 & -1.75445300 & 1.36640400 \\
\hline Pt52 & -2.17946600 & 2.60440700 & -3.05991500 \\
\hline \# 52 & Cluster $=$ Pt $53 \quad Q=0$ & $\mathrm{M}=0 \quad \mathrm{Etot}=-4828$ & 91339900 \\
\hline Pt1 & -0.81437200 & -0.58451300 & 5.22875100 \\
\hline Pt2 & -0.40785700 & -4.41568800 & -0.50716800 \\
\hline Pt3 & 2.36599500 & -1.09650100 & -3.57944300 \\
\hline Pt 4 & 2.14723000 & -0.36106900 & 1.59748900 \\
\hline Pt5 & 4.09471600 & -1.81846300 & 0.50684600 \\
\hline Pt 6 & -4.08267400 & 1.88079600 & -0.35324000 \\
\hline Pt7 & -0.31939900 & -1.65331200 & -4.07422600 \\
\hline Pt 8 & -2.25248400 & 3.94109000 & 0.18580400 \\
\hline Pt9 & 1.72090600 & -0.67771700 & 4.20858500 \\
\hline Pt10 & -0.63370100 & 4.86392300 & -1.81845300 \\
\hline Pt11 & -4.32915400 & -2.53921700 & 1.47543100 \\
\hline Pt12 & 4.15240000 & -0.10472800 & -1.68625800 \\
\hline Pt13 & -2.60988800 & -1.58563300 & 3.40155100 \\
\hline Pt14 & 4.35403900 & 0.95245400 & 0.88066900 \\
\hline Pt15 & 0.73528000 & 2.00606400 & 1.63737300 \\
\hline Pt16 & 1.47426600 & 4.00034100 & 3.18880900 \\
\hline Pt17 & 2.46788700 & -2.82609400 & 2.56186000 \\
\hline Pt18 & -4.13863600 & 0.16254300 & 1.86699600 \\
\hline Pt19 & 0.41374300 & 0.34627200 & -2.44439200 \\
\hline Pt20 & 1.90901600 & -1.37564000 & -0.95005800 \\
\hline Pt21 & 0.42686500 & 4.49792800 & 0.69478800 \\
\hline Pt22 & -0.71792200 & -1.92080500 & -1.43570000 \\
\hline Pt23 & 2.88763600 & 1.65960600 & 3.16863800 \\
\hline Pt24 & -0.39927800 & -0.26680800 & 2.64864300 \\
\hline
\end{tabular}




\begin{tabular}{|c|c|c|c|}
\hline Pt25 & 0.00897900 & 0.04647900 & 0.11453800 \\
\hline Pt26 & 2.95184000 & 3.35568800 & 0.96036200 \\
\hline Pt27 & -4.21935100 & 0.82754400 & -2.87380700 \\
\hline Pt28 & 0.82520100 & 0.65504400 & -5.02835400 \\
\hline Pt29 & 0.32904100 & -2.35612300 & 1.04618700 \\
\hline Pt30 & -1.45431300 & -3.91153100 & -2.99654500 \\
\hline Pt31 & -2.93536300 & -3.27230400 & -0.77300700 \\
\hline Pt32 & 4.34330100 & 2.61244400 & -1.29497900 \\
\hline Pt33 & 2.16229000 & 1.31644500 & -0.58785100 \\
\hline Pt34 & 2.62320500 & 1.65647200 & -3.20885700 \\
\hline Pt35 & -1.72305900 & 0.75329100 & -4.00952400 \\
\hline Pt36 & -2.09095700 & 0.43385400 & -1.37076900 \\
\hline Pt37 & 3.83518000 & -2.81613700 & -2.02664400 \\
\hline Pt38 & 2.27710100 & -3.86153600 & -0.01473900 \\
\hline Pt39 & -0.08491300 & -2.72787300 & 3.66712400 \\
\hline Pt 40 & 1.20897100 & -3.41438000 & -2.54814800 \\
\hline Pt41 & 0.64784800 & -4.79069600 & 1.99890400 \\
\hline Pt 42 & 0.33501500 & 1.73414700 & 4.27342500 \\
\hline Pt 43 & -2.14677300 & -1.23612300 & 0.78578500 \\
\hline Pt 44 & -1.17607600 & 3.49793400 & 2.74122300 \\
\hline Pt 45 & -1.93038200 & 1.48354700 & 1.16861200 \\
\hline Pt 46 & -2.46501400 & 2.88883500 & -2.38226800 \\
\hline Pt4 7 & -2.34280600 & 1.16061100 & 3.78117000 \\
\hline Pt 48 & -4.34031300 & -0.86797300 & -0.71074900 \\
\hline Pt 49 & -0.31352400 & 2.43644500 & -0.84825300 \\
\hline Pt50 & -2.88008400 & -1.56458800 & -2.97822900 \\
\hline Pt51 & 1.88150100 & 3.79246500 & -1.57712200 \\
\hline Pt52 & -1.86685800 & -3.71666300 & 1.76391800 \\
\hline Pt53 & 0.09569900 & 2.79985600 & -3.47469700 \\
\hline \# 53 & Luster $=$ Pt54 $Q=0$ & \multicolumn{2}{|c|}{$\mathrm{M}=0 \quad$ Etot $=-4935.08885200$} \\
\hline Pt 1 & 4.25449800 & -1.66484100 & 0.11594700 \\
\hline Pt2 & 4.36276700 & 1.10742900 & 0.38654500 \\
\hline Pt 3 & -2.23173900 & 0.12381400 & -1.23347700 \\
\hline Pt 4 & -1.46140600 & -3.87846900 & 1.98481900 \\
\hline Pt 5 & -3.94229300 & -0.13308300 & 2.20230900 \\
\hline Pt 6 & 1.18672800 & -3.55001700 & -2.62201300 \\
\hline Pt 7 & 0.59605200 & -2.47434400 & 1.03581600 \\
\hline Pt 8 & -2.04866400 & 1.03057200 & 3.89235800 \\
\hline Pt 9 & 2.20232800 & 1.57233900 & -3.55022500 \\
\hline Pt10 & 4.03313100 & 2.69349200 & -1.83346800 \\
\hline Pt11 & -0.72150600 & -2.14811000 & -1.36658200 \\
\hline Pt12 & -2.16688200 & -1.74181800 & 3.6272010 \\
\hline Pt13 & -4.00226000 & -2.86267400 & 1.90578100 \\
\hline Pt14 & -0.59387900 & -1.93848000 & -4.03722200 \\
\hline Pt15 & -1.47866200 & -4.22334700 & -2.79676400 \\
\hline Pt16 & 2.27845600 & -0.30460300 & 1.31085900 \\
\hline Pt17 & -3.76890200 & 2.59996500 & 2.43273600 \\
\hline Pt18 & -2.13736100 & 0.38031900 & -3.9004670 \\
\hline Pt19 & 0.02554000 & -0.10602400 & 0.0443840 \\
\hline Pt20 & -1.14430600 & 3.37677700 & 2.68598900 \\
\hline Pt21 & 2.91314800 & -2.67687200 & 2.33787500 \\
\hline Pt22 & 1.48192400 & 3.71098900 & -1.91171900 \\
\hline Pt23 & -0.12007900 & -0.33335600 & 2.6223940 \\
\hline Pt24 & -2.85642600 & 2.51933600 & -2.26185300 \\
\hline Pt25 & 0.16335200 & 0.15252500 & -2.5431290 \\
\hline Pt26 & 4.56507700 & -0.50940900 & 2.59722300 \\
\hline Pt27 & -1.11183600 & 4.61547600 & -1.93214100 \\
\hline Pt28 & -4.32173600 & -1.27801900 & -0.31435300 \\
\hline
\end{tabular}




\begin{tabular}{|c|c|c|c|}
\hline Pt29 & -1.86057000 & 1.24378300 & 1.22830600 \\
\hline Pt 30 & -3.04236300 & -1.96783100 & -2.69837400 \\
\hline Pt31 & 3.98313600 & -0.03807600 & -2.13136900 \\
\hline Pt 32 & -0.17283600 & -4.57324000 & -0.41634000 \\
\hline t3 & 2.01476800 & 1.29023900 & -0.89075500 \\
\hline Pt 34 & -2.78190300 & -3.59759100 & -0.45193900 \\
\hline Pt 35 & 0.63432900 & 1.76753300 & 4.10889400 \\
\hline Pt36 & 2.53078800 & -3.83061200 & -0.19814000 \\
\hline Pt37 & 1.51484900 & 4.05129900 & 2.85917100 \\
\hline Pt38 & -4.51615700 & 0.34518500 & -2.52345300 \\
\hline+20 & 3.08403000 & 1.79689900 & 2.76938100 \\
\hline 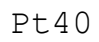 & 1.90675400 & -1.42618300 & -1.15446400 \\
\hline t 41 & -1.97415700 & -1.47270500 & 0.96764400 \\
\hline Pt 42 & -2.47723000 & 3.66352800 & 0.25317400 \\
\hline t4 & 0.44583700 & -2.70644900 & 3.68701800 \\
\hline Pt 4 & -0.54039300 & 2.24328900 & -0.93909100 \\
\hline Pt 4 & 3.81624400 & -2.76894900 & -2.36942900 \\
\hline & 0.30576400 & 0.40298800 & -5.16011200 \\
\hline-41 & 2.18185300 & -0.54731300 & 3.97817300 \\
\hline Pt 48 & -0.26702400 & -0.56733400 & 5.23944900 \\
\hline Pt 49 & -4.20569500 & 1.49552300 & -0.04627500 \\
\hline Pt5 & 0.20401800 & 4.40001000 & 0.46956800 \\
\hline Pt 5 & 2.09044700 & -1.20115300 & -3.82057800 \\
\hline Pt 52 & 0.76391500 & 1.96467300 & 1.44012000 \\
\hline Pt53 & 2.81607000 & 3.42425200 & 0.52042900 \\
\hline Pt54 & -0.40953400 & 2.54866800 & -3.59983000 \\
\hline 5 & luster $=$ Pt 55 & $=0 \quad \mathrm{E}$ & 20004000 \\
\hline Pt 1 & -2.25390800 & -3.40588100 & 1.92826700 \\
\hline Pt2 & -1.53679700 & 0.47862700 & -4.21989500 \\
\hline Pt3 & 2.96577700 & -3.26628400 & 0.96638400 \\
\hline Pt 4 & -1.35020600 & -5.05188100 & -0.08127600 \\
\hline Pt5 & 0.81059200 & 2.12885600 & 4.70766100 \\
\hline Pt 6 & 0.43995900 & -4.12362500 & 1.78899300 \\
\hline Pt 7 & 1.80202200 & -1.67139700 & -0.84583100 \\
\hline Pt 8 & 1.09683800 & 3.64499700 & 2.43090200 \\
\hline Pt9 & -1.10184200 & 1.52670000 & -1.79204100 \\
\hline Pt10 & 1.53578800 & 0.82394000 & -1.92 \\
\hline Pt11 & -2.96577700 & 3.26628400 & -0.96638400 \\
\hline Pt12 & -1.09683800 & -3.64499700 & -2.43090200 \\
\hline Pt13 & 1.10184200 & -1.52670000 & 1.79204100 \\
\hline Pt14 & -3.62571800 & 3.36289800 & 1.70183600 \\
\hline Pt15 & -1.80202200 & 1.67139700 & 0.84583100 \\
\hline Pt16 & -4.08685900 & -1.38720700 & 1.331 \\
\hline Pt17 & 2.25390800 & 3.40588100 & -1.92826700 \\
\hline Pt18 & -3.40897600 & 0.86552500 & 2.83338900 \\
\hline Pt19 & 2.21693600 & -3.07176300 & 3.60563800 \\
\hline Pt20 & -1.15506800 & 4.27140700 & 0.90512100 \\
\hline Pt21 & -1.53578800 & -0.82394000 & 1.9284080 \\
\hline Pt22 & -4.35876900 & 1.16133300 & 0.2253500 \\
\hline Pt23 & 3.64366000 & -1.01355200 & 2.46876400 \\
\hline Pt24 & -3.09004900 & -1.65779100 & 3.88001100 \\
\hline Pt25 & -3.64366000 & 1.01355200 & -2.46876400 \\
\hline Pt26 & -1.42898000 & 2.78765600 & 3.25351000 \\
\hline Pt27 & 2.92978900 & 1.62632300 & 3.02816000 \\
\hline Pt28 & -0.67106700 & -2.51084000 & -0.0403950 \\
\hline Pt29 & -2.92978900 & -1.62632300 & -3.02816000 \\
\hline Pt 30 & -0.44319900 & -2.40075800 & 3.7997730 \\
\hline Pt31 & 3.09004900 & 1.65779100 & -3.88001100 \\
\hline
\end{tabular}




\begin{tabular}{|c|c|c|c|}
\hline Pt32 & 2.46575300 & 0.53430700 & 0.62518500 \\
\hline Pt33 & 4.96116500 & 1.07504100 & 1.25789000 \\
\hline Pt34 & -2.46575300 & -0.53430700 & -0.62518500 \\
\hline Pt35 & -0.40287300 & -1.05806500 & -2.33975900 \\
\hline Pt36 & -2.21693700 & 3.07176300 & -3.60563700 \\
\hline Pt37 & -3.20370100 & -3.11007300 & -0.67977100 \\
\hline Pt38 & 0.00000000 & 0.00000000 & 0.00000000 \\
\hline Pt39 & 0.40287300 & 1.05806500 & 2.33975900 \\
\hline Pt 40 & 4.08685900 & 1.38720700 & -1.33100900 \\
\hline Pt 4 & 3.40897600 & -0.86552500 & -2.83338900 \\
\hline Pt 42 & -0.43995900 & 4.12362500 & -1.78899300 \\
\hline Pt 43 & 1.15707000 & -0.23911600 & -4.35916900 \\
\hline Pt 44 & 1.15506800 & -4.27140700 & -0.90512100 \\
\hline Pt 45 & 0.44319900 & 2.40075800 & -3.79977300 \\
\hline Pt 46 & 3.20370100 & 3.11007300 & 0.67977100 \\
\hline Pt 47 & 3.62571900 & -3.36289800 & -1.70183600 \\
\hline Pt 48 & -0.81059200 & -2.12885500 & -4.70766100 \\
\hline Pt 49 & -4.96116500 & -1.07504200 & -1.25789000 \\
\hline Pt50 & 1.53679700 & -0.47862700 & 4.21989500 \\
\hline Pt51 & 4.35876900 & -1.16133300 & -0.22535000 \\
\hline Pt52 & 1.35020600 & 5.05188100 & 0.08127600 \\
\hline Pt53 & -1.15707000 & 0.23911600 & 4.35916900 \\
\hline Pt5 4 & 0.67106700 & 2.51084000 & 0.04039500 \\
\hline Pt55 & 1.42898000 & -2.78765600 & -3.25351000 \\
\hline \# 55 & luster $=\operatorname{Pt5} 6 \quad \mathrm{Q}=0$ & \multicolumn{2}{|c|}{$\mathrm{M}=0 \quad$ Etot $=-5125.09960800$} \\
\hline Pt 1 & 3.67633900 & 2.61991400 & 0.20831800 \\
\hline Pt2 & -0.24545300 & 3.20288900 & -3.31931500 \\
\hline Pt 3 & -2.09677100 & -4.71000200 & -0.76497000 \\
\hline Pt 4 & -0.02429100 & 0.04645500 & -0.09577000 \\
\hline Pt 5 & 2.04620300 & 4.80345600 & 0.56840000 \\
\hline Pt 6 & 2.57145800 & 0.19950900 & -0.12432100 \\
\hline Pt 7 & -2.40530300 & 0.54834700 & 3.71416800 \\
\hline Pt 8 & 2.57236200 & -3.95092000 & 2.06418100 \\
\hline Pt9 & 2.12533300 & $3.5868700 \mathrm{c}$ & -1.89827100 \\
\hline Pt10 & 1.21641300 & -1.54295900 & -1.73747400 \\
\hline Pt11 & -0.11205100 & 0.70841600 & -4.56313400 \\
\hline Pt12 & -1.31745300 & 2.03555900 & -1.15784500 \\
\hline Pt13 & -0.24115200 & 4.08692500 & 1.91355900 \\
\hline Pt14 & 1.36854500 & -2.61719500 & 5.39555400 \\
\hline Pt15 & -3.77508600 & -1.23625400 & 2.07441900 \\
\hline Pt16 & -1.05376500 & -2.31852800 & -0.42622000 \\
\hline Pt17 & 0.17331400 & -3.13264400 & 3.09315900 \\
\hline Pt18 & 3.89232300 & -1.41875700 & -1.80548300 \\
\hline Pt19 & 5.19748400 & 0.35650100 & -0.15752300 \\
\hline Pt20 & -2.62607400 & $4.0485870 c$ & -2.23380900 \\
\hline Pt21 & -5.24533100 & -0.26115700 & -0.03882100 \\
\hline Pt22 & 2.56480800 & $-3.6103990 c$ & -0.68526600 \\
\hline Pt23 & 0.03660800 & -0.57637900 & 4.36778900 \\
\hline Pt24 & -3.86188800 & -0.57178300 & -2.39687200 \\
\hline Pt25 & -3.94325500 & 1.51548800 & 1.60525700 \\
\hline Pt26 & 1.13919800 & 0.50490000 & 2.18274400 \\
\hline Pt27 & 2.14162100 & 2.26319400 & -4.30913400 \\
\hline Pt28 & 3.72482000 & 1.32857100 & -2.26495100 \\
\hline Pt29 & -1.18680600 & -0.40741200 & -2.37598300 \\
\hline Pt30 & 2.31090800 & 0.93800600 & 4.50194500 \\
\hline Pt31 & -2.17059300 & -2.17479100 & 4.1287370 \\
\hline Pt32 & -1.26526300 & 1.63627300 & 1.54576100 \\
\hline Pt33 & -2.26288900 & -2.83042000 & -2.763592 \\
\hline
\end{tabular}




\begin{tabular}{|c|c|c|c|}
\hline Pt34 & -3.99524700 & 1.92188900 & -1.15345300 \\
\hline Pt35 & 3.81868600 & 0.67979300 & 2.19925700 \\
\hline Pt36 & -2.52232300 & 3.24739300 & 3.20282300 \\
\hline Pt 37 & 2.47159600 & -3.15097000 & -3.39791300 \\
\hline Pt38 & 0.05541400 & -2.03872300 & -4.10203000 \\
\hline Pt39 & 1.00494400 & 2.41131000 & 0.23424200 \\
\hline 240 & 2.21532300 & 2.92461300 & 2.57328500 \\
\hline Pt 41 & 0.19078200 & -3.98980300 & -2.11373800 \\
\hline$=42$ & -2.61221600 & 3.70164600 & 0.49349000 \\
\hline 543 & 2.51655000 & -1.50506100 & 3.28829800 \\
\hline$=44$ & -2.36325700 & -0.86584100 & -4.68428900 \\
\hline$=4$ & 3.95305200 & -1.83271200 & 0.94644700 \\
\hline$=46$ & -2.61979100 & -0.10638800 & -0.06761600 \\
\hline Pt 47 & -3.72730500 & -2.52257000 & -0.40823800 \\
\hline+4 & 0.24832200 & -4.40597300 & 0.63792700 \\
\hline$=4$ & -1.10557500 & -1.05429300 & 1.99580500 \\
\hline Pt5 & -2.53204600 & 1.61464800 & -3.50973700 \\
\hline$-\infty$ & -2.18916600 & -3.49318900 & 1.69893900 \\
\hline$=52$ & 2.34127300 & -0.45099200 & -3.91167000 \\
\hline Pt53 & -0.29388400 & 4.49281200 & -0.84521300 \\
\hline Pt 5 & 1.05257700 & 1.14803700 & -2.18949300 \\
\hline 125 & 1.27015900 & -1.94395100 & 0.96167400 \\
\hline - & -0.10218000 & 2.14806800 & 3.90596700 \\
\hline \# & Cluster $=$ Pt5 $7 \quad Q=0$ & $\mathrm{M}=0 \quad \mathrm{Etot}=-52$ & 53533600 \\
\hline Pt1 & 1.10108300 & 3.63985400 & 3.34688600 \\
\hline Pt2 & 0.30621700 & -0.88444700 & 2.36294600 \\
\hline Pt3 & -3.03042300 & 2.88801000 & 1.02147800 \\
\hline Pt 4 & -2.30908100 & -0.19748100 & -1.13913300 \\
\hline Pt 5 & 0.85177600 & 1.00955500 & 4.17899300 \\
\hline Pt 6 & 0.59377100 & 1.72569000 & 1.596870 \\
\hline Pt 7 & -0.65404700 & -1.34330500 & -4.42296900 \\
\hline Pt 8 & 3.66754100 & -2.47733900 & 1.35717100 \\
\hline Pt 9 & -1.68198800 & -0.23869200 & 4.04013000 \\
\hline Pt10 & 3.99932900 & -1.51970000 & -3.31670600 \\
\hline Pt11 & 2.03010800 & -0.83458400 & -1.7111020 \\
\hline Pt12 & -0.41859300 & -2.04092500 & -1.84530400 \\
\hline Pt13 & -1.02501900 & 2.14526000 & -0.5808470 \\
\hline Pt14 & -1.27547200 & 2.93690900 & -3.1271170 \\
\hline Pt15 & 4.90284400 & -0.08189900 & 1.91094100 \\
\hline Pt16 & 3.67475000 & 1.10407100 & -2.55910700 \\
\hline Pt17 & 0.70718200 & -4.43770800 & -1.43204000 \\
\hline Pt18 & -2.58319700 & 0.53881400 & -3.70269200 \\
\hline & -3.84017000 & 1.20 & 3.0760800 \\
\hline Pt20 & 3.04942000 & 1.80453400 & 2.6650480 \\
\hline Pt21 & -3.49546600 & 2.15741500 & -1.62534900 \\
\hline Pt22 & -1.86498700 & 0.51439500 & 1.46211700 \\
\hline Pt23 & 2.47902200 & -0.12041900 & 0.88830600 \\
\hline Pt24 & 1.55766300 & -2.77223800 & -3.50209900 \\
\hline Pt25 & 2.20088600 & 3.72307400 & 0.81697800 \\
\hline Pt26 & -3.50828100 & -1.42608700 & 2.3056520 \\
\hline Pt27 & -0.35748500 & 1.29647500 & -5.13122400 \\
\hline Pt28 & -0.13462100 & 0.56402400 & -2.61367100 \\
\hline Pt29 & 0.53611700 & -1.63065200 & 4.87574800 \\
\hline Pt 30 & -3.96221700 & -2.15181400 & -0.3479030 \\
\hline Pt31 & -3.57100700 & 3.86045500 & 3.42580600 \\
\hline Pt & -0.38073000 & -4.47170100 & 1.13530500 \\
\hline$F(3$ & -1.28785000 & -2.86418800 & 3.2230940 \\
\hline 234 & 1.84770800 & -0.11082700 & -4.2858600 \\
\hline
\end{tabular}




\begin{tabular}{|c|c|c|c|}
\hline Pt35 & 0.55317600 & 4.15196000 & -1.40346200 \\
\hline Pt36 & -2.15386900 & 4.48655600 & -1.02933000 \\
\hline Pt37 & -0.53917800 & 4.11531000 & 1.15801100 \\
\hline Pt38 & -1.19255900 & 5.03218700 & 3.55615800 \\
\hline Pt39 & 1.65462300 & 1.75522000 & -0.92190100 \\
\hline Pt 40 & -2.03211900 & -4.03538600 & -1.07044800 \\
\hline Pt 41 & 4.51647100 & -0.80925400 & -0.71155200 \\
\hline Pt 42 & 2.76164900 & -0.86922100 & 3.44502700 \\
\hline Pt 43 & -2.87322000 & -2.12175600 & -2.9179700 \\
\hline Pt 44 & 4.13179100 & 1.83566200 & 0.0956910 \\
\hline Pt 45 & 3.20780600 & -3.20578700 & -1.29499300 \\
\hline Pt 46 & -1.48401300 & -2.07278900 & 0.67111100 \\
\hline Pt 47 & -4.73047000 & -0.23766000 & -2.16659900 \\
\hline Pt 48 & 2.32394900 & -4.80199300 & 0.76142300 \\
\hline Pt 49 & 1.44910000 & -3.25982100 & 2.8616950 \\
\hline Pt50 & -0.92782200 & -3.94703700 & -3.58673900 \\
\hline Pt51 & -3.07213200 & -4.01073400 & 1.47387100 \\
\hline Pt52 & -1.35736600 & 2.38892800 & 3.29297100 \\
\hline Pt53 & -4.34933600 & 0.49613800 & 0.45799200 \\
\hline Pt5 4 & 1.19739400 & -2.46575400 & 0.3165420 \\
\hline Pt55 & 0.08452900 & -0.15785300 & -0.1234180 \\
\hline Pt5 6 & 3.24300800 & 3.69043800 & -1.72961100 \\
\hline Pt57 & 1.46380200 & 2.53254900 & -3.48089600 \\
\hline \# 57 & Cluster $=$ Pt 58 & $\Lambda=0 \quad$ Etot $=-53$ & 66890300 \\
\hline Pt 1 & -3.49425700 & 1.52340100 & 1.76052700 \\
\hline Pt2 & -2.08953900 & -0.46375200 & -4.71913000 \\
\hline Pt3 & -4.19271400 & 2.63233000 & -0.67013000 \\
\hline Pt 4 & 4.15058500 & -1.49584500 & -1.94032800 \\
\hline Pt 5 & 4.09757300 & 1.28225600 & -2.18986200 \\
\hline Pt 6 & -1.30374200 & -1.96980900 & 3.64947700 \\
\hline Pt 7 & 0.37293600 & 2.08024500 & -1.64835300 \\
\hline Pt 8 & -5.94650500 & -1.36288700 & 1.09362700 \\
\hline Pt 9 & -2.77786400 & 0.31884600 & 4.13991500 \\
\hline Pt10 & 4.69376200 & 2.84868400 & -0.01051600 \\
\hline Pt11 & -0.88696600 & -0.23165300 & -2.39060500 \\
\hline Pt12 & 1.47339300 & -1.89523900 & 3.84142400 \\
\hline Pt13 & -3.18460600 & 1.10567700 & -2.74487300 \\
\hline Pt14 & -1.38975300 & 2.53764900 & 3.24461000 \\
\hline Pt15 & -1.90275600 & 3.46234800 & -1.97765100 \\
\hline Pt16 & 1.83890200 & -0.16134500 & -2.18694900 \\
\hline Pt17 & 3.75767800 & 1.67892100 & 2.29444400 \\
\hline Pt18 & 0.24692700 & -2.08573300 & 1.46427800 \\
\hline Pt19 & -1.86578700 & -1.42943000 & -0.13870000 \\
\hline Pt20 & -1.97133300 & -3.59312100 & 1.44979200 \\
\hline Pt21 & 2.52694900 & -3.46009900 & 1.78655200 \\
\hline Pt22 & -0.75595300 & -2.60850400 & -3.62541000 \\
\hline Pt23 & 0.19188200 & -4.19420300 & 3.0369820 \\
\hline Pt24 & -1.21313800 & 0.15755000 & 2.01175200 \\
\hline Pt25 & 2.38810400 & 3.81612600 & 1.13299100 \\
\hline Pt26 & 2.02433600 & -2.53586800 & -3.41684600 \\
\hline Pt27 & -3.44006500 & -1.31654300 & 2.01561500 \\
\hline Pt28 & -2.11081900 & 3.71669600 & 0.79321300 \\
\hline Pt29 & 3.38972700 & -0.32220200 & -4.3072550 \\
\hline Pt30 & -5.30978800 & 0.18535200 & 3.19158200 \\
\hline Pt31 & 0.22993800 & 4.49647700 & -0.49817500 \\
\hline Pt32 & 0.02269000 & 4.67233600 & 2.2405760 \\
\hline Pt33 & 0.16283700 & 2.32104600 & 1.06845400 \\
\hline Pt34 & 4.81239700 & 0.11341600 & 0.24031500 \\
\hline
\end{tabular}




\begin{tabular}{|c|c|c|c|}
\hline Pt35 & -4.08778800 & -2.86636100 & -0.17622900 \\
\hline Pt36 & -0.02670600 & 0.39473500 & 4.40033000 \\
\hline Pt37 & -1.76362700 & -3.82875700 & -1.32275300 \\
\hline Pt38 & -0.84180200 & 1.89043900 & -4.0295120 \\
\hline Pt39 & 2.53905500 & -1.30619700 & 0.19335300 \\
\hline Pt 40 & -5.99750600 & 1.30987000 & 0.85355600 \\
\hline Pt41 & 2.48713100 & 1.41488800 & -0.05105900 \\
\hline Pt 42 & 2.60050300 & 3.57106900 & -1.63958700 \\
\hline Pt 43 & 0.65472800 & -0.39874700 & -4.57841200 \\
\hline Pt 44 & 0.60754800 & -4.67472900 & -2.4271080 \\
\hline Pt 45 & 1.50643000 & 0.22681800 & 2.20517300 \\
\hline Pt 46 & 0.43844500 & 4.18717800 & -3.22309800 \\
\hline Pt 47 & 2.73934600 & -3.70501200 & -0.98603800 \\
\hline Pt 48 & -4.17080200 & -0.12168300 & -0.46866000 \\
\hline Pt 49 & 0.40163400 & -4.50132500 & 0.3100220 \\
\hline Pt50 & 1.38754600 & 2.60362000 & 3.43732900 \\
\hline Pt51 & 3.81070400 & -1.09990100 & 2.5440430 \\
\hline Pt52 & 4.79821600 & -2.62528400 & 0.48116400 \\
\hline Pt53 & 1.93851900 & 1.96139100 & -3.82079700 \\
\hline Pt5 4 & -1.91806500 & 1.31020700 & -0.3847790 \\
\hline Pt55 & 2.71867300 & 0.45867600 & 4.52896100 \\
\hline Pt56 & 0.45699600 & -2.32498300 & -1.25266800 \\
\hline Pt57 & -3.13113200 & -1.69668200 & -2.49316100 \\
\hline Pt58 & 0.30692200 & -0.00235400 & -0.09141200 \\
\hline \# 58 & uster $=$ Pt59 $Q=0$ & Etot $=-54$ & 92790500 \\
\hline Pt 1 & 1.96969900 & 4.02012300 & -0.73888400 \\
\hline Pt2 & -4.57709900 & 2.04731900 & 0.30652900 \\
\hline Pt 3 & 4.47682700 & 0.15406400 & 1.88428600 \\
\hline Pt 4 & -3.48107000 & -2.72836200 & 2.83473800 \\
\hline Pt 5 & -2.36394900 & 3.68059800 & 0.49212500 \\
\hline Pt 6 & -2.52900900 & -0.38519700 & 3.90454600 \\
\hline Pt 7 & 4.56872100 & 0.32705800 & -0.89975800 \\
\hline Pt 8 & 2.99151900 & 0.37671200 & -3.20720300 \\
\hline Pt9 & -1.39972600 & -3.89081500 & -1.33234600 \\
\hline Pt10 & 1.88139000 & 3.84662900 & 2.04472500 \\
\hline Pt11 & 0.56257600 & -5.22296200 & 0.04156100 \\
\hline Pt12 & -3.33503600 & -2.44694300 & -2.64271100 \\
\hline Pt13 & 3.53489900 & -2.04688000 & -1.94946900 \\
\hline Pt14 & 0.95512200 & -1.25926600 & 2.50737400 \\
\hline Pt15 & 3.78592700 & 2.36930600 & 3.36479900 \\
\hline Pt16 & 1.41691400 & 2.67347000 & -3.11732700 \\
\hline Pt17 & 1.99965000 & 1.17504500 & 1.8454280 \\
\hline Pt18 & -2.22770400 & 0.06349800 & -3.28172700 \\
\hline Pt19 & 1.26403800 & -3.64924700 & -2.11245200 \\
\hline Pt20 & 1.07059500 & -0.98008400 & -1.91490600 \\
\hline Pt21 & -1.75669700 & 0.66989900 & -5.81928900 \\
\hline Pt22 & -1.53201000 & -1.23195700 & -1.11760500 \\
\hline Pt23 & -2.15695200 & 1.01104900 & 0.3145460 \\
\hline Pt24 & -4.09143000 & -0.34536000 & 1.5868760 \\
\hline Pt25 & -2.94727700 & 2.33568600 & -1.88737100 \\
\hline Pt26 & -2.77890100 & -1.71238700 & -5.17149100 \\
\hline Pt27 & 0.09481700 & -0.28437200 & 4.85040200 \\
\hline Pt28 & 1.16074600 & 2.19981500 & 4.1748930 \\
\hline Pt29 & -0.80176500 & 3.64021000 & 2.8099450 \\
\hline Pt30 & -1.61237000 & -1.36014700 & 1.5859610 \\
\hline Pt31 & -4.77030200 & -0.48385600 & -3.7959160 \\
\hline Pt32 & 1.13593900 & -3.93263300 & 2.40023300 \\
\hline & 0.37342400 & 0.28708400 & -4.18116900 \\
\hline
\end{tabular}




\begin{tabular}{|c|c|c|c|}
\hline Pt34 & -3.74809800 & 1.89843000 & -4.44371500 \\
\hline Pt35 & -4.02797300 & -0.18291600 & -1.20250400 \\
\hline Pt36 & -1.17992000 & 2.57563600 & -4.00846600 \\
\hline Pt37 & -1.49105200 & 1.96443400 & 4.87139500 \\
\hline$t 38$ & 2.84147800 & -3.69834900 & 0.19484400 \\
\hline Pt39 & -3.46774800 & -2.61501400 & 0.09615500 \\
\hline Pt 40 & 3.96841300 & 2.71447100 & -2.11681100 \\
\hline Pt 41 & -0.07523200 & 5.20907100 & 0.67636600 \\
\hline Pt 42 & -0.70727100 & -2.23151800 & -3.4963030 \\
\hline Pt 43 & 1.68573700 & -2.52526800 & 4.69574700 \\
\hline$=44$ & 0.39952700 & -2.60132300 & 0.19622900 \\
\hline$=4$ & 2.09345200 & 1.34643500 & -0.87725800 \\
\hline Pt 4 & 2.62278100 & -1.02991400 & 0.35114100 \\
\hline Pt 47 & 0.08155500 & 2.58761100 & 0.51247400 \\
\hline Pt 48 & -0.91395100 & -2.66801900 & 3.8162090 \\
\hline Pt 49 & -0.65110800 & 3.94927100 & -1.6888260 \\
\hline 50 & 3.41089900 & -2.33012400 & 2.5597940 \\
\hline 51 & 2.77241800 & -0.07902400 & 4.08416800 \\
\hline Pt 52 & -0.45053400 & 1.28846600 & -1.80296700 \\
\hline Pt5 & 1.93822100 & -1.97210200 & -4.1939750 \\
\hline Ptb & -1.48616800 & -4.03390600 & 1.4511940 \\
\hline Pt5 & -0.62375600 & 0.97293400 & 2.5937090 \\
\hline $\mathrm{P}$ & 3.93128000 & 2.58201400 & 0.6287490 \\
\hline 57 & -3.07989800 & 2.03391000 & 2.61975100 \\
\hline Pt 58 & 5.04153000 & -2.06563500 & 0.35896800 \\
\hline Pt59 & 0.23391300 & -0.00666700 & 0.34458700 \\
\hline \# & zer $=$ Pt 60 & $=-$ & 92656400 \\
\hline Pt 1 & -2.37600500 & -1.90050500 & -3.5210630 \\
\hline Pt2 & 3.64690300 & -1.29663700 & -1.3631950 \\
\hline Pt3 & 3.02885900 & 0.11795400 & 2.87149400 \\
\hline Pt 4 & 0.38715900 & 0.26904300 & 2.38228700 \\
\hline Pt 5 & -3.13800100 & 3.26499600 & 3.2217840 \\
\hline Pt 6 & 0.24382400 & 3.87833800 & -2.7745020 \\
\hline Pt 7 & 1.84032400 & -3.57675600 & -0.8120920 \\
\hline Pt 8 & 5.47865500 & -2.52343900 & 0.25720100 \\
\hline Pt9 & -4.21270100 & -1.74633000 & -1.41502500 \\
\hline Pt10 & 4.30411000 & -3.84060700 & -1.7806600 \\
\hline Pt11 & 2.16916100 & 0.20073900 & 0.3152650 \\
\hline Pt 12 & 2.55890900 & 0.06449500 & -3.5038130 \\
\hline Pt13 & 0.43696300 & -1.98550200 & 0.84367800 \\
\hline Pt14 & -2.70464900 & 4.10420700 & 0.63694100 \\
\hline Pt15 & 1.17097000 & 0.25516700 & 4.8942970 \\
\hline Pt16 & -4.40312100 & 1.96053200 & 1.1550650 \\
\hline Pt17 & 0.22795200 & -1.21887400 & -4.2956880 \\
\hline Pt18 & 3.82913200 & -4.60533500 & 0.7603930 \\
\hline Pt19 & 3.41001300 & 2.38084900 & 1.22733900 \\
\hline Pt20 & 5.09921000 & -1.63320600 & $2.7999020 c$ \\
\hline Pt21 & -3.79289600 & 0.45795000 & -3.0655950 \\
\hline Pt22 & -0.73356400 & -4.23705500 & -0.0228760 \\
\hline Pt23 & -3.17645800 & -3.17191500 & 0.7433750 \\
\hline Pt24 & 2.39118500 & -2.70881200 & -3.36879000 \\
\hline Pt25 & 0.75818900 & 2.46102300 & 0.76953000 \\
\hline Pt26 & -4.58955400 & -0.81569500 & 1.1955810 \\
\hline Pt27 & -1.29301000 & 2.54777300 & -1.0275150 \\
\hline Pt28 & -0.20293300 & -3.42128100 & -2.6613040 \\
\hline & -2.20343600 & 4.83049900 & -1.9708880 \\
\hline etsu & -1.94437500 & -0.94993500 & 1.5939550 \\
\hline t31 & 1.33923700 & -4.30251900 & 1.7888330 \\
\hline
\end{tabular}




\begin{tabular}{|c|c|c|c|}
\hline Pt 32 & -2.76587700 & -4.03978200 & -1.84484100 \\
\hline Pt 33 & 0.98618000 & -1.19750800 & -1.72172500 \\
\hline Pt 34 & -1.93509500 & 0.30237600 & -5.09242700 \\
\hline Pt 35 & 2.33847400 & 3.78858900 & -0.93897300 \\
\hline Pt 36 & -3.50068400 & -2.20664400 & 3.30399400 \\
\hline Pt 37 & -3.35813500 & 0.53304500 & 3.31606800 \\
\hline Pt 38 & -1.15965400 & 0.29556000 & -2.57863800 \\
\hline Pt 39 & 1.13043100 & 1.54823200 & -1.79096200 \\
\hline 40 & -1.17158900 & -0.99687500 & 4.15918200 \\
\hline 41 & -3.92654700 & 2.75334300 & -1.48006600 \\
\hline$t 42$ & 1.31495800 & -2.04519500 & 3.39432500 \\
\hline 43 & 3.47315900 & -3.68547700 & 3.29593400 \\
\hline t 44 & 2.67104100 & 2.82226000 & -3.50339000 \\
\hline Pt 45 & 0.37943300 & 1.57718800 & -4.36161800 \\
\hline 46 & -0.13403100 & 4.80770800 & -0.16359000 \\
\hline t 47 & 1.93015500 & 4.64772500 & 1.64729900 \\
\hline 48 & -0.39562000 & 0.29031400 & -0.0957 \\
\hline Pt 49 & -2.09540100 & 2.60039200 & -3.58342100 \\
\hline Pt 50 & 4.79176100 & 0.05498000 & 0.73562900 \\
\hline Pt 51 & 3.78933200 & 1.47138000 & -1.40260000 \\
\hline 52 & -1.57308200 & -1.86392600 & -0.96624700 \\
\hline Pt 53 & 3.03025000 & -2.11057000 & 1.20129500 \\
\hline Pt 54 & -5.55001200 & 0.60671900 & -0.95000500 \\
\hline Pt 55 & -1.08776700 & -3.29588700 & 2.59940000 \\
\hline Pt 56 & -0.60538700 & 4.00734200 & 2.47633400 \\
\hline Pt 57 & 1.58234400 & 2.47891700 & 3.31908600 \\
\hline Pt 58 & -1.75847000 & 1.76867100 & 1.55318600 \\
\hline Pt 59 & -0.99225900 & 1.78155500 & 4.11822300 \\
\hline Pt 60 & -2.95796100 & 0.44640700 & -0.51961700 \\
\hline$\# 60$ & Cluster $=$ Pt $61 \quad Q=0$ & Etot $=-5$ & 67799500 \\
\hline Pt 1 & 0.27875300 & 0.31894900 & 0.38943400 \\
\hline Pt 2 & -4.41186500 & -2.47199300 & -3.16267100 \\
\hline Pt3 & -0.11262500 & 2.45382300 & -1.05438600 \\
\hline Pt 4 & -0.50876900 & 4.60919200 & -2.51305300 \\
\hline Pt 5 & 1.09745800 & -3.93561200 & 3.33548700 \\
\hline Pt 6 & -1.90143300 & 1.66367000 & 0.87316500 \\
\hline Pt 7 & 1.03916900 & 0.18220000 & -2.09492500 \\
\hline Pt 8 & -0.23635000 & 2.77878900 & 4.14191400 \\
\hline Pts & 0.05879100 & -1.99822400 & -0.76456400 \\
\hline Pt 10 & 1.38673200 & 0.60777000 & 4.75821100 \\
\hline $\mathrm{P}$ & -2.67089400 & -0.99279400 & 3.53677500 \\
\hline & -3.96628400 & -2.51501000 & 1.58603300 \\
\hline & 4.50827600 & 3.11891900 & -0.88604800 \\
\hline & 4.42653500 & -0.86466100 & 1.75914800 \\
\hline & 4.03940800 & 0.65134500 & 4.01566000 \\
\hline & 0.83558800 & -2.20044300 & -3.32206200 \\
\hline Pt & -3.80358600 & -1.19554800 & -0.82440800 \\
\hline & -1.65964500 & 4.03054200 & 2.10636100 \\
\hline & -1.60572600 & 0.22821900 & -1.39659500 \\
\hline & 2.71075700 & 4.08058600 & 0.96622500 \\
\hline & -2.08712900 & -3.49146600 & -0.17265600 \\
\hline & -2.83942400 & -4.57527400 & -2.56560300 \\
\hline & 0.66402400 & 2.36532700 & -3.61788700 \\
\hline & 4.33383800 & 1.90956800 & 1.58252200 \\
\hline & 0.83966100 & 4.93129700 & 2.80069000 \\
\hline & 0.68620500 & -1.79753100 & 1.85571100 \\
\hline & 4.69229300 & -2.34770000 & -0.53816800 \\
\hline Pt28 & -3.52615900 & 0.08093200 & -3.23917500 \\
\hline
\end{tabular}




\begin{tabular}{|c|c|c|c|}
\hline Pt29 & 0.55602600 & 2.61224000 & 1.58682900 \\
\hline Pt 30 & -2.63292200 & -1.68619800 & -5.07959100 \\
\hline Pt31 & 2.02724100 & 3.91190700 & -1.73469700 \\
\hline Pt 32 & -1.45443800 & -3.26972100 & 2.51363100 \\
\hline Pt3 & -2.34157200 & 3.87169000 & -0.58592300 \\
\hline Pt 34 & -4.66709700 & -3.70986600 & -0.77740300 \\
\hline Pt 35 & -0.11228900 & -1.72081500 & 4.40999800 \\
\hline Pt 36 & 1.79392500 & 0.02939200 & -4.62094800 \\
\hline Pt 37 & 2.14845000 & 0.48222800 & 2.19148500 \\
\hline Pt 38 & -1.82729500 & -1.08397600 & 0.99386600 \\
\hline Pt39 & -0.20954800 & -4.35533700 & -1.97983100 \\
\hline$=4$ & -0.88087000 & 0.09547500 & -3.97381500 \\
\hline t41 & -0.50559200 & 0.43526600 & 2.86483300 \\
\hline Pt 42 & 0.45562300 & -4.21205700 & 0.69104400 \\
\hline Pt 4 & 2.47501300 & -1.00737800 & -0.07183900 \\
\hline Pt 44 & 0.16444000 & 4.83627800 & 0.14079700 \\
\hline Pt 4 & 2.28018800 & -3.40610900 & -1.27503700 \\
\hline t 46 & 4.66177000 & 0.38798500 & -0.73434500 \\
\hline Pt 47 & -4.10360300 & 3.02128000 & 1.36060200 \\
\hline Pt 48 & 3.30509000 & -1.16734000 & -2.61505300 \\
\hline Pt 49 & -2.05074700 & 2.38691100 & -2.91952800 \\
\hline Pt 5 & -0.02573200 & -1.65968100 & -5.77875900 \\
\hline Pt5 & -4.09550500 & 0.26294600 & 1.51079800 \\
\hline Pt 52 & -1.84125800 & -2.10677200 & -2.57234700 \\
\hline Pt53 & -1.06048200 & -3.78947900 & -4.48252300 \\
\hline Pt5 4 & 2.60244900 & -1.67039700 & 3.72471200 \\
\hline Pt 55 & -3.87531300 & 1.58096300 & -0.95344700 \\
\hline Pt 5 & -1.29817100 & 0.55409200 & 5.37075600 \\
\hline Pt5 & 2.93761300 & -3.18970000 & 1.41862600 \\
\hline Pt 58 & 3.20327700 & 1.60194700 & -2.79300500 \\
\hline Pt59 & 2.38064800 & 1.71017700 & -0.24526900 \\
\hline Pt 60 & 2.46990400 & 2.82607000 & 3.45060200 \\
\hline Pt 61 & -2.74681900 & 1.80310700 & 3.40964600 \\
\hline \# 61 & Cluster $=$ Pt $62 \quad \mathrm{Q}=0$ & $=0 \quad$ Etot $=-$ & 3684500 \\
\hline Pt 1 & 0.27512100 & 2.68188500 & -3.81633400 \\
\hline Pt2 & 3.61327600 & 2.67729200 & -0.77676100 \\
\hline Pt3 & -4.60753000 & 1.08161700 & 0.80804200 \\
\hline Pt 4 & 1.08518300 & -3.64289100 & -3.46803700 \\
\hline Pt5 & -3.06984000 & -0.34702900 & -3.50546100 \\
\hline Pt 6 & 3.02853400 & -3.02485200 & 3.11481400 \\
\hline Pt7 & 0.45159900 & 5.24268300 & 2.86461800 \\
\hline Pt 8 & -4.17373700 & -0.96294100 & -1.00812300 \\
\hline Pt9 & 2.70364500 & -1.05195200 & -1.11704100 \\
\hline Pt10 & -0.50074300 & 2.80842900 & 2.46709900 \\
\hline Pt11 & 1.07427400 & -5.44717200 & -1.39728200 \\
\hline Pt12 & 5.15211800 & -1.75462100 & -1.77626700 \\
\hline Pt13 & -3.06922500 & -3.37381500 & -0.15570500 \\
\hline Pt14 & -4.94346800 & 0.35736600 & 3.3660230 \\
\hline Pt15 & 2.97634900 & 3.00982900 & -3.43306600 \\
\hline Pt16 & -1.07065900 & -2.02511800 & 1.00984500 \\
\hline Pt17 & -2.14339900 & 0.33747800 & 0.14002600 \\
\hline Pt18 & -1.50380500 & -1.39208400 & 3.60279300 \\
\hline Pt19 & 1.88589400 & 1.67734000 & 3.28776200 \\
\hline Pt20 & 4.15100700 & -2.41304500 & 0.6884010 \\
\hline Pt21 & -0.69243900 & 0.26738900 & -4.8453300 \\
\hline Pt22 & 1.31961500 & -1.05580400 & 3.91195000 \\
\hline Pt23 & 3.63792700 & -0.38236300 & 2.54497700 \\
\hline Pt24 & -0.41405400 & 0.92779000 & 4.5845110 \\
\hline
\end{tabular}




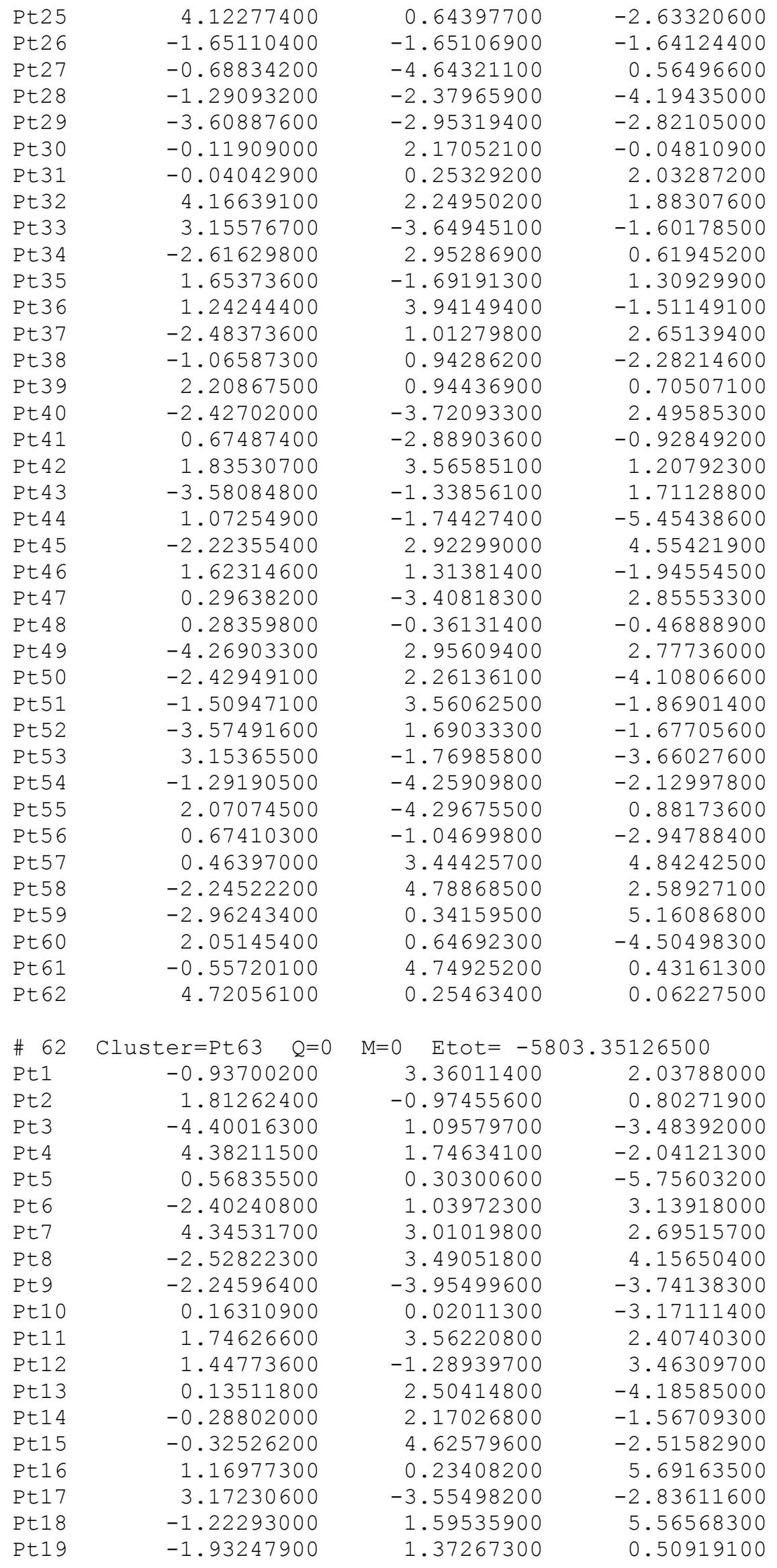




\begin{tabular}{|c|c|c|c|}
\hline Pt20 & 4.22069500 & 0.07234100 & 0.15189600 \\
\hline Pt21 & 2.76569100 & 0.91884500 & 2.35230000 \\
\hline Pt22 & -2.53525700 & -1.27226100 & 0.09285700 \\
\hline Pt23 & -0.21584200 & -2.71328400 & 0.28145300 \\
\hline Pt24 & -4.31192700 & 0.37124000 & 1.24640000 \\
\hline Pt25 & -0.62246000 & -3.08648400 & 2.91360500 \\
\hline Pt26 & -1.94055400 & 0.71406400 & -4.68041900 \\
\hline Pt27 & 1.45426700 & -1.90171900 & -1.72384500 \\
\hline Pt28 & -1.22880400 & -4.63351200 & -1.2849580 \\
\hline Pt2 & -2.01614800 & 3.88536500 & -0.44719000 \\
\hline Pt3 & 1.90508300 & -1.64349100 & -4.35051500 \\
\hline Pt 31 & -0.84743200 & -1.84730600 & -4.80744600 \\
\hline Pt 32 & -0.24264300 & -0.26228500 & -0.61958700 \\
\hline Pt 33 & -3.36684400 & -1.44553500 & -3.65753300 \\
\hline Pt3 & 3.89701600 & -1.65942000 & 2.3043790 \\
\hline Pt35 & 3.64720100 & -0.15811800 & 4.59300500 \\
\hline Pt 36 & 5.37794000 & 0.55197400 & 2.57919700 \\
\hline Pt 37 & -1.23782000 & -2.09990100 & -2.16890500 \\
\hline Pt 38 & -3.62843700 & 3.02888000 & 1.67405900 \\
\hline Pt 39 & 1.52323400 & -4.42570300 & -0.82167100 \\
\hline Pt 40 & 3.83725500 & -0.91469500 & -2.45545300 \\
\hline Pt 41 & 0.74516400 & 4.15388200 & -0.05723100 \\
\hline Pt 42 & 0.13667400 & 3.70528200 & 4.56657600 \\
\hline Pt 43 & 3.16232100 & 2.66328800 & 0.23260200 \\
\hline Pt 44 & -1.03535500 & -0.89333600 & 4.54290900 \\
\hline Pt 45 & -0.64888300 & -0.59762100 & 1.92398000 \\
\hline Pt 46 & 0.47326500 & -3.80820100 & -3.33069600 \\
\hline Pt 47 & 2.06203000 & 0.73631800 & -1.32085400 \\
\hline Pt 48 & 2.51386600 & 1.04250700 & -3.94728200 \\
\hline Pt 49 & -4.85215500 & -2.29420400 & 0.80917000 \\
\hline Pt 50 & 2.53406100 & 2.31980400 & 4.65537700 \\
\hline Pt51 & -2.30944300 & 0.41460500 & -2.04072100 \\
\hline Pt52 & 0.32385500 & 1.32346600 & 3.42985700 \\
\hline Pt53 & -2.55263900 & -3.79108900 & 1.02319900 \\
\hline Pt 54 & 3.60329300 & -2.63346100 & -0.27750300 \\
\hline Pt 55 & 2.06540800 & 3.23138100 & -2.29818700 \\
\hline Pt 56 & -4.69179800 & -0.59904000 & -1.34614200 \\
\hline Pt 57 & 1.88867000 & -3.47202400 & 1.77013800 \\
\hline Pt58 & -2.99395900 & -1.62959800 & 2.71755800 \\
\hline Pt59 & -2.40045100 & 2.90801000 & -3.03030200 \\
\hline Pt 60 & -0.18503900 & -5.18287800 & 1.19546000 \\
\hline Pt 61 & 0.74098000 & 1.63892000 & 0.84652800 \\
\hline Pt 62 & -4.07581600 & 2.09448000 & -0.92642300 \\
\hline Pt 63 & -3.59853000 & -3.16590000 & -1.4795410 \\
\hline \# 63 & Cluster $=$ Pt $64 \quad Q=0$ & $\mathrm{M}=0 \quad \mathrm{Etot}=-5907$ & 39633500 \\
\hline Pt1 & 2.48951600 & -2.37175200 & -2.97297100 \\
\hline Pt 2 & 4.93684700 & -1.97835800 & -1.83972200 \\
\hline Pt3 & -2.33510100 & -1.26747700 & -1.32687600 \\
\hline Pt 4 & 1.55292400 & 1.18825600 & -2.08183500 \\
\hline Pt5 & -4.07373400 & -0.51400200 & -3.24150900 \\
\hline Pt 6 & -1.23558700 & 3.74507500 & -2.26035300 \\
\hline Pt 7 & -3.19687400 & 4.64430900 & 1.91181900 \\
\hline Pt 8 & 4.19838900 & -0.27506000 & 1.4978190 \\
\hline Pt9 & 2.76393400 & 4.37801800 & -0.4721080 \\
\hline Pt10 & 0.93776700 & 2.70534100 & 4.3394680 \\
\hline Pt11 & -0.93137800 & -3.60721600 & -1.6074900 \\
\hline Pt12 & -3.58575900 & -3.64351900 & -1.11084900 \\
\hline Pt13 & 5.08633800 & 0.56450200 & -0.9163880 \\
\hline
\end{tabular}




\begin{tabular}{|c|c|c|c|}
\hline Pt 14 & -0.61958600 & -2.05190300 & 0.60174000 \\
\hline Pt 15 & -3.09190800 & 2.04477800 & 2.63067400 \\
\hline Pt 16 & -3.77063200 & 1.05499700 & -1.06433600 \\
\hline Pt 17 & 0.95596000 & -2.50500600 & 4.10741700 \\
\hline Pt 18 & 0.34663100 & -5.59053100 & -0.29614100 \\
\hline Pt19 & -4.98391000 & -1.34767700 & -0.84338100 \\
\hline Pt 20 & 1.86054500 & 3.56380800 & 1.94505200 \\
\hline Pt 21 & -1.45294800 & 3.86939500 & 3.81938000 \\
\hline Pt 22 & -0.17687100 & 1.96861700 & -4.01002800 \\
\hline Pt23 & 1.55517500 & -3.19081300 & -0.51899700 \\
\hline Pt24 & 0.31070400 & -1.19980400 & -1.84938300 \\
\hline Pt 25 & -4.67552300 & 0.21490400 & 1.35056000 \\
\hline Pt 26 & -3.87816600 & 3.67363800 & -1.71058600 \\
\hline Pt 27 & 1.09281700 & 0.08185600 & 5.00991900 \\
\hline Pt 28 & -2.13840000 & 2.87424200 & 0.18759000 \\
\hline Pt29 & 4.05956700 & -2.80678300 & 0.56501300 \\
\hline Pt 30 & 3.70429500 & -4.36888400 & -1.62348700 \\
\hline Pt 31 & -1.10085800 & 1.11805800 & -1.57934900 \\
\hline Pt 32 & -2.29034300 & 5.47464500 & -0.47670300 \\
\hline Pt 33 & 2.48399700 & 2.03617100 & -4.49295200 \\
\hline Pt 34 & 2.93624100 & 1.76414300 & 0.20605900 \\
\hline Pt 35 & -1.84302700 & -4.43621100 & 0.80869900 \\
\hline Pt 36 & 0.62461000 & 0.33903400 & 0.35492300 \\
\hline Pt 37 & 2.02310300 & 0.92775500 & 2.61199200 \\
\hline Pt 38 & -2.95371500 & -0.57483300 & 3.30638700 \\
\hline Pt 39 & 2.79776400 & -5.19922100 & 0.76503500 \\
\hline Pt 40 & -3.29070600 & -2.13325000 & 1.10929200 \\
\hline Pt 41 & 0.36278100 & 5.53251200 & -0.96469600 \\
\hline Pt 42 & 1.23880800 & -0.35600700 & -4.28085000 \\
\hline Pt 43 & -1.33904600 & 1.26352200 & 4.54645500 \\
\hline Pt 44 & 1.43069500 & 3.80046600 & -2.75124600 \\
\hline Pt 45 & 1.86070700 & -1.64955500 & 1.66935500 \\
\hline Pt 46 & -0.29798500 & -0.50708400 & 2.79493000 \\
\hline Pt 47 & 3.72570100 & 0.03193900 & -3.19028500 \\
\hline Pt 48 & -2.67558300 & -2.80984300 & -3.50897700 \\
\hline Pt 49 & -0.41632600 & 2.08946000 & 2.10495600 \\
\hline Pt 50 & -1.20278900 & -1.34074000 & 5.22097400 \\
\hline Pt 51 & -1.43048300 & -0.42937900 & -3.79201600 \\
\hline Pt 52 & -0.01247800 & -2.75951900 & -4.01442500 \\
\hline Pt 53 & 2.78282600 & -0.80494100 & -0.76023800 \\
\hline Pt 54 & -1.53803600 & -2.89945600 & 3.03556500 \\
\hline Pt 55 & 1.25625200 & -4.75736300 & -2.69280900 \\
\hline Pt 56 & -0.52682700 & 4.72433100 & 1.44239800 \\
\hline Pt 57 & 0.63319500 & -4.07204900 & 1.91805500 \\
\hline Pt 58 & 3.26017100 & -1.10817500 & 3.89518900 \\
\hline Pt 59 & -4.78778800 & 2.84047000 & 0.68608200 \\
\hline Pt 60 & 0.50579200 & 2.93407500 & -0.32463800 \\
\hline Pt 61 & -2.84497300 & 1.89159600 & -3.47256400 \\
\hline Pt 62 & 3.12111700 & -3.64147500 & 2.94435400 \\
\hline Pt 63 & -2.03114700 & 0.26595900 & 0.87177400 \\
\hline Pt 64 & 3.83331800 & 2.59201700 & -2.21073800 \\
\hline$\# 64$ & Cluster $=$ Pt 65 & Etot $=-60$ & 81246000 \\
\hline Pt 1 & 4.07642600 & 2.74297400 & -2.67208100 \\
\hline Pt2 & 5.66662900 & 0.85365000 & -1.57931500 \\
\hline Pt 3 & 0.17060600 & 0.18840800 & 5.30164900 \\
\hline Pt 4 & -4.35561200 & -1.44480800 & 3.28170600 \\
\hline Pt 5 & -1.39048900 & -3.16505600 & -3.72413700 \\
\hline Pt 6 & 1.18783200 & 0.74356900 & -4.16917400 \\
\hline
\end{tabular}




\begin{tabular}{|c|c|c|c|}
\hline Pt 7 & 2.75178300 & -0.04755500 & 4.53844900 \\
\hline Pt 8 & 3.25955500 & -2.44642000 & -3.83033800 \\
\hline Pt9 & -1.85666600 & 4.50709100 & 0.16155700 \\
\hline Pt10 & 1.94854700 & 1.47818600 & -1.65331200 \\
\hline Pt11 & -3.94605200 & 1.15703500 & 3.86242900 \\
\hline Pt12 & 3.52245100 & -0.42417900 & -0.58376700 \\
\hline Pt13 & -0.38520100 & 3.80248100 & 2.37678700 \\
\hline Pt14 & 3.80845000 & 1.61711600 & 2.67685500 \\
\hline Pt15 & -3.29246600 & 0.19881800 & 1.43126200 \\
\hline Pt16 & 2.98401300 & -3.62034600 & 1.50786900 \\
\hline Pt17 & -1.21556700 & -1.50811800 & 1.19684600 \\
\hline Pt18 & 1.20233000 & 1.89563600 & 3.48024900 \\
\hline Pt19 & 2.23498500 & 3.56295200 & 1.60205200 \\
\hline Pt20 & -5.16501300 & -1.67627400 & -1.33936800 \\
\hline Pt21 & 5.25848100 & -1.73922200 & -2.15803700 \\
\hline Pt22 & -5.79400000 & -0.72884000 & 1.10995200 \\
\hline Pt23 & 5.12411800 & -2.31141400 & 0.47874700 \\
\hline Pt24 & 1.12590600 & -3.79102400 & -2.85440800 \\
\hline Pt25 & -3.49052400 & -1.47037200 & -3.46947300 \\
\hline Pt26 & -0.23913300 & -2.41457400 & 4.72067100 \\
\hline Pt27 & 3.40509800 & -1.00460900 & 2.09952300 \\
\hline Pt28 & 1.53373300 & -1.15703000 & -2.24148500 \\
\hline Pt29 & 2.37128400 & 4.12058500 & -1.08856000 \\
\hline Pt 30 & -0.55938500 & 2.06716400 & -2.53098000 \\
\hline Pt 31 & 0.24068400 & 2.82558800 & -0.05959800 \\
\hline Pt 32 & -2.25265800 & -3.14162600 & 3.05624300 \\
\hline Pt33 & -1.63686300 & -4.14904800 & 0.60202900 \\
\hline Pt 34 & -3.10204400 & -3.40451800 & -1.60429100 \\
\hline Pt 35 & -2.67191100 & 3.73009200 & -2.3087450 \\
\hline Pt 36 & 1.81695400 & 0.91882300 & 1.01926800 \\
\hline Pt 37 & -2.90183700 & 2.85592500 & 2.04170800 \\
\hline Pt 38 & -1.42822100 & 2.09583600 & 4.22522900 \\
\hline Pt39 & -5.38585200 & 1.86403300 & 1.68867400 \\
\hline Pt 40 & -3.10607500 & 1.13610400 & -2.8995550 \\
\hline Pt 41 & 0.36394800 & -3.43042100 & 2.2914890 \\
\hline Pt 42 & -0.17390200 & 0.17040600 & -0.64083000 \\
\hline Pt 43 & 0.98332300 & -4.38857700 & -0.17270600 \\
\hline Pt 44 & -1.01451600 & -5.09184400 & -1.85686500 \\
\hline Pt 45 & -1.34508100 & 1.31803500 & -4.95662500 \\
\hline Pt 46 & 0.76260900 & -1.85918000 & -4.72703600 \\
\hline Pt 47 & -4.77742600 & 0.92642500 & -0.78138800 \\
\hline Pt 48 & 5.53367700 & 0.29042900 & 1.05947000 \\
\hline Pt 49 & -0.59432800 & -2.47905500 & -1.2435790 \\
\hline Pt 50 & 0.64544200 & 5.45349800 & 0.4968240 \\
\hline Pt51 & 3.13651200 & -3.08588200 & -1.17692900 \\
\hline Pt52 & -2.66831200 & -0.77289300 & -0.99902700 \\
\hline Pt53 & 2.34204400 & -2.65053600 & 3.95747100 \\
\hline Pt 54 & -3.73821800 & -2.45741600 & 0.85578600 \\
\hline Pt55 & 1.39732600 & -1.74698000 & 0.42426800 \\
\hline Pt5 6 & 0.77462900 & -0.75905300 & 2.8545090 \\
\hline Pt57 & -4.34814300 & 3.51312000 & -0.1811110 \\
\hline Pt58 & -0.15558100 & 4.68004000 & -1.97313500 \\
\hline Pt59 & -2.25349900 & 1.86232400 & -0.41085400 \\
\hline Pt 60 & 1.58122200 & 3.34128400 & -3.5663080 \\
\hline Pt 61 & -1.84439100 & -0.52471800 & $3.6516880 c$ \\
\hline Pt 62 & -0.97370200 & -0.56490200 & -3.1184500 \\
\hline Pt 63 & 3.67511600 & 0.15372100 & -3.2806290 \\
\hline Pt 64 & 3.97289300 & 2.22746000 & 0.0089930 \\
\hline 65 & -0.79593900 & 1.15768500 & 1.7918460 \\
\hline
\end{tabular}




\begin{tabular}{|c|c|c|c|}
\hline \# 65 & Cluster=Pt $66 \quad Q=0$ & $\mathrm{M}=0 \quad \mathrm{Etot}=-61$ & 96846400 \\
\hline Pt1 & -5.07522700 & -1.40666000 & -2.76449300 \\
\hline Pt2 & -1.96236700 & 2.73649200 & -2.72825700 \\
\hline Pt3 & 3.38212700 & 0.02828800 & 1.25056000 \\
\hline Pt 4 & 5.11355300 & 1.65244900 & 2.50925700 \\
\hline Pt5 & 0.55676600 & 1.64478400 & -2.74920900 \\
\hline Pt 6 & 1.37851900 & 0.19851400 & -4.87182300 \\
\hline Pt 7 & -0.78693800 & -1.44612500 & -4.59904400 \\
\hline Pt 8 & 0.25883300 & 4.26666000 & -3.02737600 \\
\hline Pt9 & -1.76701100 & -0.49946200 & 1.38579900 \\
\hline Pt10 & -0.79825700 & 1.99051600 & 1.76941000 \\
\hline Pt11 & -1.90928100 & -4.48456700 & -1.12959100 \\
\hline Pt12 & 4.30936200 & 2.31731500 & 0.00565900 \\
\hline Pt13 & -2.59782500 & -2.47092800 & -2.86124200 \\
\hline Pt14 & -4.40435400 & -3.42240500 & -1.09473000 \\
\hline Pt15 & -1.09789100 & 4.65649300 & 1.47510300 \\
\hline Pt16 & 1.65897000 & -0.75726800 & 4.61229500 \\
\hline Pt17 & -4.10424900 & 1.10341900 & -2.42449700 \\
\hline Pt18 & 5.11380600 & 0.81809100 & -2.07829300 \\
\hline Pt19 & -1.60775700 & 0.03502300 & -2.45062800 \\
\hline Pt20 & 2.30403700 & 3.35203900 & -1.49596500 \\
\hline Pt21 & 2.51785900 & -3.13281900 & -2.15776000 \\
\hline Pt22 & 3.47855800 & 3.76468600 & 2.11653000 \\
\hline Pt23 & 0.71447500 & -4.15022100 & -0.40054200 \\
\hline Pt24 & -0.96585700 & -1.12538700 & 3.90610300 \\
\hline Pt25 & -0.55418000 & 5.73507300 & -0.94608400 \\
\hline Pt26 & -2.44019300 & 1.52976800 & -0.32148700 \\
\hline Pt27 & -1.08211000 & -5.10011600 & 1.37853400 \\
\hline Pt28 & -2.74166700 & -3.00580200 & 0.99122200 \\
\hline Pt29 & 1.48937600 & -4.72910800 & 2.12127300 \\
\hline Pt 30 & -3.28243100 & 3.04351500 & 1.79400000 \\
\hline Pt 31 & -3.40517800 & -0.95096900 & -0.70986000 \\
\hline Pt 32 & 5.03981900 & -2.05982200 & 1.63309100 \\
\hline Pt33 & -3.56073800 & -1.51035600 & 3.10465400 \\
\hline Pt 34 & 0.83894400 & -0.12348100 & 2.13849400 \\
\hline Pt 35 & 0.87536000 & -1.02454500 & -2.51886900 \\
\hline Pt 36 & -4.91280300 & 2.55400000 & -0.30720000 \\
\hline Pt 37 & -4.27456900 & 0.54523800 & 1.43452800 \\
\hline Pt 38 & 2.57457800 & 1.53341700 & 3.41281100 \\
\hline Pt39 & 0.66857100 & -3.25194100 & 4.22510100 \\
\hline Pt 40 & 1.70264000 & -2.48230300 & -4.65488200 \\
\hline Pt 41 & -0.12430500 & -2.60876500 & 1.73347700 \\
\hline Pt 42 & -0.26851200 & 3.11848400 & -0.62809100 \\
\hline Pt 43 & 3.31162100 & -3.73130800 & 0.37839500 \\
\hline Pt 44 & 2.57707400 & 0.63880000 & -1.24189600 \\
\hline Pt 45 & 0.03859000 & 0.46396100 & -0.36536000 \\
\hline Pt 46 & 0.00895100 & 1.38279700 & 4.27097600 \\
\hline Pt 47 & 1.46863700 & 4.81226300 & 0.60825700 \\
\hline Pt 48 & 3.42219700 & -0.83884100 & -3.38329300 \\
\hline Pt 49 & -2.76707400 & 4.20114500 & -0.61517000 \\
\hline Pt 50 & -5.22499300 & -1.94577500 & 1.00816700 \\
\hline Pt51 & -0.09501600 & -3.50979800 & -2.91245300 \\
\hline Pt52 & 0.92886700 & 3.65111100 & 3.01037600 \\
\hline Pt53 & 4.21954400 & -0.58313900 & 3.73609300 \\
\hline Pt 54 & 2.49094500 & -2.25085300 & 2.50259100 \\
\hline Pt55 & 3.10756000 & 1.85229100 & -3.59067000 \\
\hline Pt 56 & 5.93080300 & 0.18126400 & 0.41444800 \\
\hline & -3.27484400 & -0.39220400 & -4.501240 \\
\hline
\end{tabular}




\begin{tabular}{|c|c|c|c|}
\hline Pt 58 & -2.60292300 & 1.00596200 & 3.51657200 \\
\hline Pt59 & -1.90287800 & -3.62294400 & 3.48237300 \\
\hline Pt 60 & 4.24986600 & -1.48295900 & -0.88338000 \\
\hline Pt 61 & -1.62354800 & 3.50271200 & 3.87134300 \\
\hline Pt 62 & 1.68123800 & -1.63974000 & -0.01796300 \\
\hline Pt 63 & -0.92592800 & -2.01589500 & -0.77100700 \\
\hline Pt 64 & 1.74790600 & 2.13401700 & 0.88818900 \\
\hline Pt & -5.89259700 & 0.06450700 & -0.66972000 \\
\hline Pt 66 & -1.12645200 & 1.24141700 & -4.81360700 \\
\hline$\# 66$ & Cluster $=$ Pt 67 & Etot $=-6207$. & 74618600 \\
\hline Pt 1 & 3.25642100 & 2.95910700 & -2.44177100 \\
\hline Pt2 & 1.52719900 & -0.15060900 & -5.49769100 \\
\hline Pt 3 & -0.03999900 & 4.78846600 & 1.54655300 \\
\hline Pt 4 & 0.83107500 & -4.34860000 & 2.68150800 \\
\hline Pt 5 & 5.07525500 & -1.98963700 & -0.95791900 \\
\hline Pt 6 & 3.63929400 & 1.00316300 & -4.28121800 \\
\hline Pt 7 & -1.25940500 & -5.47700900 & 1.47126700 \\
\hline Pt 8 & 1.13216700 & 1.80215700 & -3.67498600 \\
\hline Pt 9 & -1.67896900 & -3.56722500 & -2.79601600 \\
\hline Pt 10 & -1.06554400 & 3.26687500 & -4.23860600 \\
\hline Pt 1 & -0.10315000 & 2.89935100 & 3.53605100 \\
\hline Pt 1 & -2.87241400 & -3.45159200 & 2.27150900 \\
\hline Pt 1 & -2.32242800 & 4.31164000 & 2.93184400 \\
\hline Pt 1 & 3.83891800 & 2.43553300 & 0.16571400 \\
\hline Pt 1 & 4.03340200 & -2.62327700 & 1.47448000 \\
\hline Pt 1 & 1.69050000 & 1.26838500 & -1.06990300 \\
\hline Pt 1 & 3.66232400 & -0.66889400 & 3.33378200 \\
\hline Pt 1 & 1.35990300 & -1.13960900 & 4.70913600 \\
\hline Pt 19 & -0.51582700 & 2.69906600 & -1.66434500 \\
\hline Pt 2 & -0.19891500 & -4.93526000 & -0.96314600 \\
\hline Pt21 & -4.19719100 & -2.80660700 & -2.18172100 \\
\hline Pt22 & -0.09611600 & -1.85668900 & -4.1 \\
\hline Pt23 & -2.40475700 & 2.41825000 & 4.8587 \\
\hline Pt2 & 4.37501900 & 1.87612400 & 2.75549100 \\
\hline Pt 25 & 4.21921300 & 0.50064100 & -1.69355700 \\
\hline Pt 26 & -2.07120000 & -1.62343500 & -0.96724800 \\
\hline Pt 27 & -4.41847900 & 0.54202100 & 1.05061300 \\
\hline Pt 28 & 2.91686300 & -3.19564400 & 3.87661200 \\
\hline Pt 29 & -2.62044700 & -1.09301700 & -3.55459400 \\
\hline Pt 30 & 1.92726600 & -3.80290900 & 0.26068900 \\
\hline Pt 31 & 2.18887300 & 3.35123100 & 2.16209600 \\
\hline Pt 32 & -4.43615500 & -1.35445500 & 3.02000900 \\
\hline Pt 33 & -0.04823000 & 0.25740100 & 2.86836900 \\
\hline Pt 34 & 0.08168200 & -0.42740100 & 0.26355100 \\
\hline Pt 35 & -0.60459600 & -2.96464800 & 0.86011200 \\
\hline Pt 36 & 0.45348500 & -2.37789900 & -1.56079200 \\
\hline Pt 37 & 1.04741200 & 4.42111700 & -3.02163700 \\
\hline Pt 38 & -2.23932800 & 1.70053900 & 2.27513500 \\
\hline Pt 39 & 2.43666100 & -2.58519000 & -4.75581300 \\
\hline Pt 40 & -4.50849100 & 1.26904600 & 3.64709700 \\
\hline Pt 41 & -2.68885500 & 4.16773700 & -2.27456900 \\
\hline Pt 42 & 2.97818900 & -3.17260500 & -2.16178700 \\
\hline Pt 43 & 2.04357800 & -0.67627400 & -2.90673500 \\
\hline Pt 44 & -4.43452200 & 3.15786900 & 1.71537100 \\
\hline Pt 45 & 0.85884300 & -4.29293100 & -3.38638500 \\
\hline Pt 46 & -4.27049200 & -0.17731900 & -1.55821200 \\
\hline Pt 47 & 4.74751100 & -0.05663900 & 0.90535200 \\
\hline Pt 48 & -0.47761800 & 0.08401000 & -2.31864300 \\
\hline
\end{tabular}




\begin{tabular}{|c|c|c|c|}
\hline Pt 49 & -2.18846300 & -0.91174100 & 1.63569000 \\
\hline Pt50 & 1.64133500 & 3.91093300 & -0.43726900 \\
\hline Pt51 & 2.59371800 & -1.20875700 & -0.32811200 \\
\hline Pt52 & 1.51633900 & -1.80604800 & 2.08167600 \\
\hline Pt53 & 0.04459500 & 2.18178500 & 0.92147000 \\
\hline Pt5 4 & -2.32834000 & -0.22772900 & 4.25855900 \\
\hline Pt5 5 & -4.29306700 & 2.46514700 & -0.90302600 \\
\hline Pt5 6 & 2.24147900 & 0.73244700 & 1.51666400 \\
\hline Pt5 & 4.54039500 & -1.43598600 & -3.54415600 \\
\hline Pt5 & -2.73842200 & -4.16542200 & -0.35276400 \\
\hline Pt5 & -0.21974300 & 0.94884000 & 5.44847600 \\
\hline t 60 & -0.57589900 & 5.32197900 & -1.05760000 \\
\hline Pt 61 & -2.68457500 & 1.54782300 & -2.92880200 \\
\hline Pt 62 & -2.17136800 & 3.63546500 & 0.32194700 \\
\hline Pt 6 & -0.99664700 & 0.63578800 & -4.89135000 \\
\hline Pt 6 & 2.08964700 & 1.41835200 & 4.12109000 \\
\hline Pt 65 & -0.75854800 & -2.31240800 & 3.51024300 \\
\hline Pt 66 & -2.12352300 & 0.99741000 & -0.32727000 \\
\hline Pt 67 & -4.33683300 & -2.09223400 & 0.41972300 \\
\hline 67 & er $=$ Pt $68 \quad Q=0$ & Etot $=$ & 22091500 \\
\hline Pt 1 & 1.55720000 & 4.49287900 & -3.01466900 \\
\hline Pt2 & 2.61687200 & -1.96808000 & 3.91632300 \\
\hline Pt 3 & 2.95667300 & 2.70671200 & 3.75491100 \\
\hline Pt 4 & 0.37085600 & -0.45018400 & 0.10745100 \\
\hline Pt 5 & -0.84586500 & 0.59139900 & -2.04805100 \\
\hline Pt 6 & 0.18234100 & -2.45725100 & -4.16471600 \\
\hline Pt 7 & -3.59422900 & -2.10563700 & -1.85637000 \\
\hline Pt 8 & -0.77641600 & 3.24231600 & -2.35061900 \\
\hline Pt 9 & 0.29651400 & -1.73173100 & 5.32083800 \\
\hline Pt10 & -4.99624900 & -0.52280900 & -0.14037300 \\
\hline Pt11 & -3.64507200 & -2.73503200 & 0.77855600 \\
\hline Pt12 & -2.05426100 & 1.71899500 & -4.18305700 \\
\hline Pt13 & 0.29197600 & 2.93248000 & -4.79905500 \\
\hline Pt14 & -2.15418900 & -3.66365700 & -3.53562900 \\
\hline Pt15 & 0.25508400 & 2.68973900 & 3.63583800 \\
\hline Pt16 & -2.12078400 & -0.98377700 & -3.87925500 \\
\hline Pt17 & -0.78661500 & 5.82384200 & -2.76726700 \\
\hline Pt18 & -2.31786700 & -0.51227600 & -0.03248300 \\
\hline Pt19 & 1.36677900 & -3.56178000 & 2.12645900 \\
\hline Pt20 & -2.19658700 & -4.28654700 & -0.90743300 \\
\hline Pt21 & -5.02336800 & -1.17290800 & 2.49344200 \\
\hline Pt22 & 2.64011200 & -1.29363900 & 1.28687900 \\
\hline Pt23 & -3.65372800 & -3.31516000 & 3.41317800 \\
\hline Pt24 & 1.44305500 & -0.86463800 & -2.33412200 \\
\hline Pt25 & 5.03138200 & 1.8987880 & -1.17274900 \\
\hline Pt26 & 3.64901300 & -3.7191280 & 0.66479100 \\
\hline Pt27 & 2.54968200 & 1.4557870 & -5.09927900 \\
\hline Pt28 & -0.98021000 & 3.8005350 & 1.48526600 \\
\hline Pt29 & 1.50319300 & 1.8055220 & -2.65553800 \\
\hline Pt30 & -3.76457500 & 0.3952880 & 4.29085200 \\
\hline Pt31 & 1.37586300 & -2.8695570 & -0.51787700 \\
\hline Pt32 & 2.79233000 & 3.4334120 & -0.84896000 \\
\hline Pt33 & -3.74091300 & 1.0571430 & 1.6725670 \\
\hline Pt3 4 & -3.46270900 & 3.2912140 & -2.48089400 \\
\hline Pt35 & 2.39449400 & -5.2595290 & -1.11423900 \\
\hline Pt36 & 0.22021000 & 0.2350600 & -4.51544600 \\
\hline Pt37 & 3.94278800 & 0.2705020 & 3.11180700 \\
\hline Pt38 & 4.88310100 & -2.1581270 & 2.440029 \\
\hline
\end{tabular}




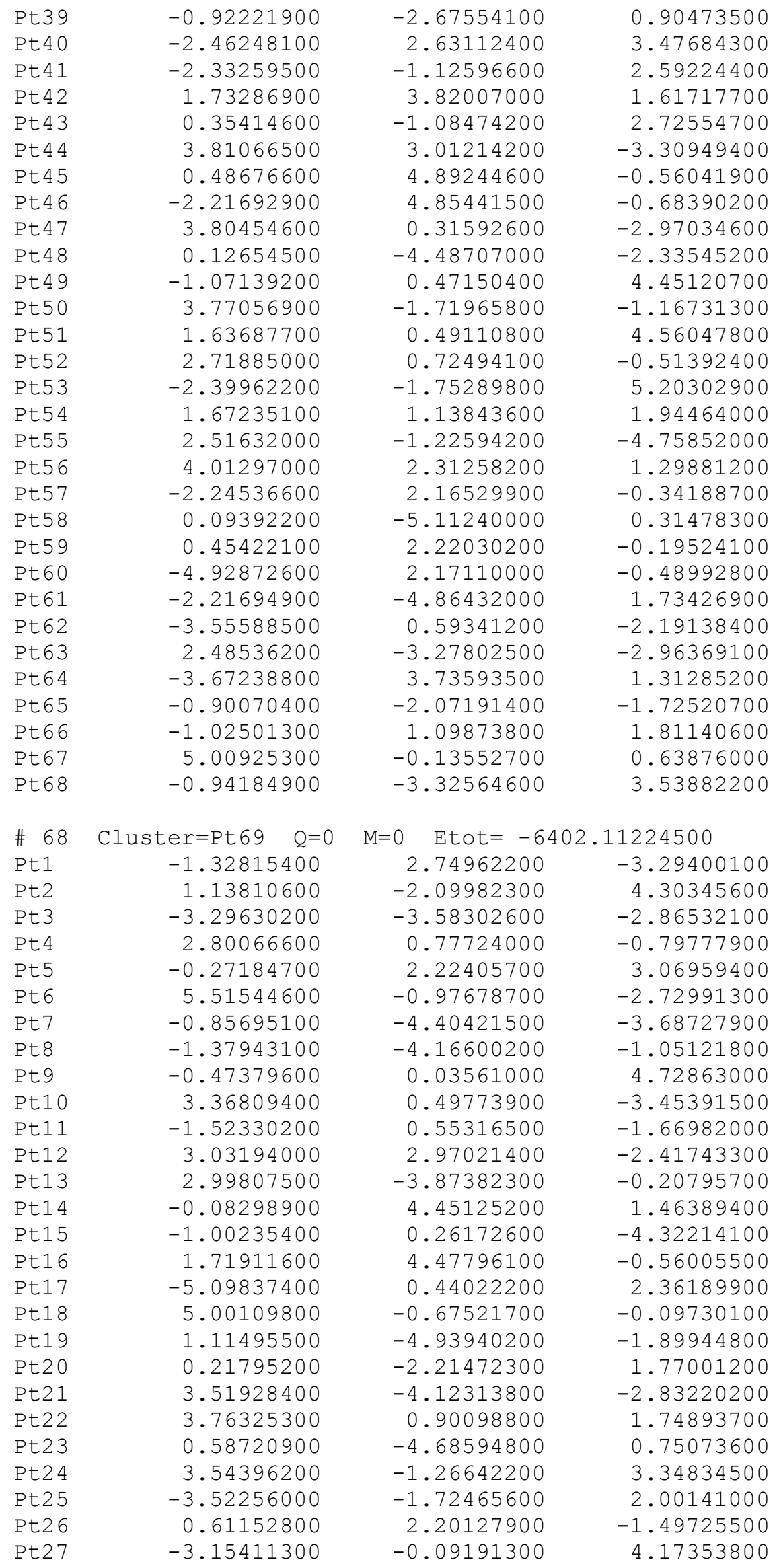




\begin{tabular}{|c|c|c|c|}
\hline Pt2 8 & 2.21249400 & 0.21794200 & 5.18909600 \\
\hline Pt29 & 2.45327400 & -3.57876400 & 2.42851900 \\
\hline Pt 30 & -3.66809800 & -1.11108400 & -1.84065100 \\
\hline Pt 31 & 3.13745500 & -1.69070900 & -1.81117300 \\
\hline Pt 32 & -2.04145000 & 2.20049700 & 5.10237000 \\
\hline Pt33 & -0.67862400 & -2.24015800 & -5.28911000 \\
\hline Pt 34 & 1.47003100 & -0.59400500 & -5.11948300 \\
\hline Pt 35 & -3.84619200 & -3.28872500 & -0.22766900 \\
\hline Pt 36 & 1.28629100 & -2.77582800 & -3.51804600 \\
\hline Pt37 & 0.60901600 & 2.34721300 & 5.6047070 \\
\hline Pt 38 & 2.61136100 & -1.40973000 & 0.82924900 \\
\hline Pt39 & -1.56077600 & -2.26665100 & 3.7967300 \\
\hline Pt 40 & 3.93699600 & 3.05985000 & 0.141254 \\
\hline Pt 41 & -0.97853200 & 4.34489100 & -1.0905690 \\
\hline Pt 42 & 0.05569200 & -4.39497200 & 3.380877 \\
\hline Pt 43 & -1.17424200 & 2.14448400 & 0.5275700 \\
\hline Pt 44 & -4.90918400 & 2.60545700 & 0.7644820 \\
\hline Pt 45 & -3.29497100 & 0.48580200 & 0.3627000 \\
\hline Pt 46 & -1.37094100 & -0.07122800 & 2.166723 \\
\hline Pt 47 & -5.44053600 & -1.13515700 & 0.1942 \\
\hline Pt 48 & 1.31098800 & 0.08806000 & 2.669 \\
\hline Pt 49 & -1.18031800 & -1.93278300 & -2.66025 \\
\hline Pt 50 & 5.20263500 & 1.49288200 & -1.69 \\
\hline Pt 51 & 3.68780200 & -1.97977800 & -4.44 \\
\hline Pt 52 & -2.96932000 & 2.08959000 & 2.56 \\
\hline Pt 53 & 0.40111800 & -0.011 & 0.13 \\
\hline Pt5 4 & -1.90444100 & -3.867 & 1.59 \\
\hline Pt 55 & -3.11433400 & -1.41667100 & -4.46405 \\
\hline Pt 56 & 0.75103900 & -2.49146800 & -0.863038 \\
\hline Pt 57 & -1.71874700 & -1.66749200 & -0.03005300 \\
\hline Pt 58 & 0.80143600 & 4.50096600 & 4.01 \\
\hline Pt 59 & -5.25294800 & 1.03114200 & -1.40357700 \\
\hline Pt 60 & 2.59713200 & 4.531 & 1.996642 \\
\hline Pt 61 & 0.82328700 & 4.38368800 & -3.10462800 \\
\hline Pt 62 & 2.43761700 & 2.37615000 & 3.59 \\
\hline Pt 63 & 0.94453200 & -0.27986400 & -2.50226100 \\
\hline Pt 64 & -2.77025000 & 4.26126800 & 0.9563 \\
\hline Pt 65 & -1.84931500 & 4.36339700 & 3.50420 \\
\hline Pt 66 & -3.46882800 & 1.07090000 & -3.4445480 \\
\hline Pt 67 & 1.15088300 & 1.91055300 & -4.12629300 \\
\hline Pt 68 & -3.13592000 & 2.70489800 & -1.26964400 \\
\hline Pt 69 & 1.50638000 & 2.27702700 & 1.0487850 \\
\hline$\# 69$ & Cluster $=\mathrm{Pt} 70 \quad \mathrm{Q}=0$ & Etot $=-6$ & 54034800 \\
\hline Pt 1 & 0.70253700 & 2.29303800 & 1.53391800 \\
\hline Pt 2 & 5.15689200 & 1.17232800 & 0.8123770 \\
\hline Pt3 & 1.91029700 & -0.88357100 & 4.71096100 \\
\hline Pt 4 & -0.59512500 & -4.33713800 & -1.11773200 \\
\hline Pt 5 & -2.78198000 & -1.16401300 & 4.93961 \\
\hline Pt 6 & 3.03382000 & -4.41786300 & $1.8361 \varepsilon$ \\
\hline Pt 7 & 0.31907100 & 0.50172600 & -2.82301800 \\
\hline Pt 8 & 5.53991200 & 1.41969600 & -1.861 \\
\hline Pt 9 & 0.16393500 & -2.32377800 & 0.5748090 \\
\hline Pt10 & 2.55133300 & 0.73579600 & 0.33604 \\
\hline Pt11 & 2.19114700 & -1.05598500 & -4.0489780 \\
\hline Pt 12 & 1.68599400 & 1.72786300 & 3.96757 \\
\hline Pt 13 & 2.03525700 & -3.89453000 & -0.618189 \\
\hline Pt & 4.63724200 & -3.40252300 & -0 \\
\hline Pt & -2.30100100 & -1.16595700 & 0 . \\
\hline
\end{tabular}




\begin{tabular}{|c|c|c|c|}
\hline Pt16 & -1.58371200 & -3.74517300 & -3.56589500 \\
\hline Pt17 & 3.71438100 & 2.98753400 & -0.64056900 \\
\hline Pt18 & -1.80824900 & 0.86114600 & -4.46118200 \\
\hline Pt19 & -3.02324000 & 1.42759300 & 4.17730100 \\
\hline Pt20 & -1.09605900 & -4.28898900 & 3.53502100 \\
\hline Pt21 & 3.29884300 & 2.75243200 & 2.02623500 \\
\hline Pt22 & 1.07627700 & 2.50820300 & -1.13234500 \\
\hline Pt23 & -1.42555100 & 2.58627400 & -0.10320900 \\
\hline Pt24 & -3.65889700 & 1.16607300 & 0.38135900 \\
\hline Pt25 & -0.34693400 & -2.30473100 & 5.20241000 \\
\hline Pt26 & -5.91227800 & -0.22678100 & 0.87247900 \\
\hline Pt27 & 1.83143700 & 4.52154400 & 0.55340200 \\
\hline Pt28 & -2.95621300 & -1.41630900 & -3.49691800 \\
\hline Pt29 & -3.06881400 & -3.17554600 & -1.38358600 \\
\hline Pt30 & -2.08559700 & -3.76593600 & 1.07693800 \\
\hline Pt31 & 1.16081300 & -2.89083000 & 3.04123700 \\
\hline Pt32 & 5.38353200 & -1.41882200 & 1.58070000 \\
\hline Pt33 & -4.30674500 & 0.92558100 & -3.38782500 \\
\hline Pt34 & -5.79878500 & 1.49019600 & -1.21083200 \\
\hline Pt35 & -1.30700400 & -1.72004100 & 2.75449400 \\
\hline Pt36 & -2.18424500 & 0.57984300 & -1.79327100 \\
\hline Pt37 & 1.03408700 & -3.30357900 & -3.07132800 \\
\hline Pt38 & -1.06340600 & 2.86553300 & -2.80252100 \\
\hline Pt39 & -2.80775300 & 4.87809600 & -0.05301800 \\
\hline Pt 40 & -0.55330900 & 0.29090400 & 4.48653000 \\
\hline Pt 41 & -0.30532700 & 4.83674700 & -1.10916800 \\
\hline Pt 42 & -5.16162700 & 1.75364200 & 2.54049600 \\
\hline Pt 43 & 3.34029300 & 1.20500700 & -4.97022200 \\
\hline Pt 44 & 3.65932700 & -2.81840300 & -2.54740900 \\
\hline Pt 45 & -0.66850200 & 4.59955600 & 1.58176000 \\
\hline Pt 46 & 4.08431200 & 3.17866700 & -3.30605900 \\
\hline Pt4 7 & 0.72157900 & 0.76867000 & -5.45624300 \\
\hline Pt 48 & -3.78931200 & -0.57047400 & 2.50622300 \\
\hline Pt 49 & -2.92500100 & 3.19471900 & 2.09056200 \\
\hline Pt50 & -4.53623200 & -2.56265700 & 0.82386100 \\
\hline Pt51 & 4.41871000 & -0.82661000 & -0.87017400 \\
\hline Pt52 & -0.82560500 & -1.75512900 & -1.87390200 \\
\hline Pt53 & -5.05112700 & 3.46374300 & 0.45183400 \\
\hline Pt5 4 & -0.05809600 & 0.25983600 & -0.16094200 \\
\hline Pt5 5 & 3.56216900 & 0.16247900 & 2.79361500 \\
\hline Pt56 & 0.40532400 & -4.87966000 & 1.35551700 \\
\hline Pt57 & -3.55902200 & 2.92462100 & -1.74800400 \\
\hline Pt58 & 2.77166600 & -1.84731800 & 1.07672000 \\
\hline Pt59 & -0.43772700 & -1.49490000 & -4.53290500 \\
\hline Pt 60 & -0.76795500 & 2.87933800 & 3.69912500 \\
\hline Pt 61 & 3.78069000 & -2.43353600 & 3.50449800 \\
\hline Pt 62 & 2.21396000 & 4.72139100 & -2.11871600 \\
\hline Pt 63 & -4.45679100 & -0.84853300 & -1.31432700 \\
\hline Pt 64 & 2.92494600 & 0.96062100 & -2.32692600 \\
\hline Pt 65 & -1.53372900 & 0.86327200 & 2.01644600 \\
\hline Pt 66 & 0.93084100 & -0.29066700 & 2.28492600 \\
\hline Pt 67 & -3.53119900 & -3.14762300 & 3.27290200 \\
\hline Pt 68 & 4.79388700 & -0.56087100 & -3.53153700 \\
\hline Pt 69 & 1.78679500 & -1.29351900 & -1.37249300 \\
\hline Pt70 & 1.45084900 & 2.76828500 & -3.80883100 \\
\hline 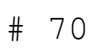 & Cluster $=$ Pt71 $\quad \mathrm{Q}=0$ & \multirow{3}{*}{\multicolumn{2}{|c|}{$\begin{aligned} & \mathrm{M}=0 \quad \text { Etot }=-6615.71032000 \\
&-2.64148700 \quad-0.75099400 \\
&-0.99084800-1.15916500\end{aligned}$}} \\
\hline Pt 1 & 0.38536300 & & \\
\hline & 2.48581400 & & \\
\hline
\end{tabular}




\begin{tabular}{|c|c|c|c|}
\hline Pt3 & 0.07502000 & 1.99098600 & -1.72889900 \\
\hline Pt 4 & 2.34229200 & 4.22800100 & 0.47005300 \\
\hline Pt 5 & -2.30648000 & 0.16797800 & -5.28954600 \\
\hline Pt 6 & -2.08791000 & 4.01498400 & 0.76698200 \\
\hline Pt 7 & -4.35756500 & -0.41645500 & -3.63087800 \\
\hline Pt 8 & 4.08709700 & -2.74974100 & 0.13717600 \\
\hline Pt 9 & 0.53506200 & 1.49599000 & 4.49311200 \\
\hline Pt10 & 0.61724500 & -1.11850400 & 3.63895300 \\
\hline Pt11 & 2.40298900 & 1.61622300 & -0.34378200 \\
\hline Pt12 & -2.38819100 & 2.73998700 & -4.48512800 \\
\hline Pt13 & -2.00306800 & 1.40227700 & -0.04970700 \\
\hline Pt14 & 4.02688900 & -0.16910100 & 0.94972200 \\
\hline Pt15 & -1.83803800 & -3.85012200 & -1.69289700 \\
\hline Pt16 & 2.06380600 & 2.97367200 & -4.78190700 \\
\hline Pt17 & 1.96191500 & -4.40874800 & 0.54507400 \\
\hline Pt18 & -4.31045600 & 2.76664400 & -0.18744100 \\
\hline Pt19 & 0.01657800 & -1.22442000 & -5.17233500 \\
\hline Pt20 & -0.61124300 & -0.46713400 & 5.94784200 \\
\hline Pt21 & 4.44440500 & 0.05048100 & -4.21123000 \\
\hline Pt22 & -0.86250800 & -1.95791700 & 1.54317400 \\
\hline Pt23 & 2.26239000 & 0.99757900 & -2.95980500 \\
\hline Pt24 & -3.15614300 & -0.54429000 & 1.41969300 \\
\hline Pt25 & 2.59097200 & -3.59961800 & -1.97810200 \\
\hline Pt26 & 4.36299900 & 2.61287500 & -3.40981900 \\
\hline Pt27 & -0.14779600 & 3.94953000 & -3.55413900 \\
\hline Pt28 & 2.36249600 & -1.61766700 & -3.80973700 \\
\hline Pt29 & 0.48086900 & -5.23765900 & -1.55972100 \\
\hline Pt30 & 1.86042800 & -1.79525700 & 1.34928800 \\
\hline Pt31 & 0.24654900 & -3.25432500 & -3.37679800 \\
\hline Pt32 & -0.52956500 & -3.03809900 & 5.14375000 \\
\hline Pt33 & -0.77838400 & -4.57265400 & 0.73425800 \\
\hline Pt34 & 2.14548500 & 0.40270700 & -5.58599800 \\
\hline Pt35 & -0.94623100 & 0.67739200 & 2.36738900 \\
\hline Pt36 & -4.34737400 & -2.45714000 & 2.89657800 \\
\hline Pt37 & 3.25585700 & 1.59207300 & 4.25741200 \\
\hline Pt38 & 4.62205200 & 0.63731800 & -1.56823000 \\
\hline Pt39 & -3.27256500 & 2.08135800 & 2.25661500 \\
\hline Pt 40 & -1.02808600 & 3.28714400 & 3.19247700 \\
\hline Pt41 & -1.91920100 & -1.23756900 & -0.87534100 \\
\hline Pt 42 & 1.71323500 & 3.41887200 & 2.99322800 \\
\hline Pt 43 & 2.16079800 & -2.88682900 & 4.94842500 \\
\hline Pt 44 & 0.28898600 & -0.01213500 & 0.06815600 \\
\hline Pt 45 & -2.07601100 & -1.85675500 & -3.5180510 \\
\hline Pt 46 & -2.11717700 & -1.27323200 & 3.8559140 \\
\hline Pt 47 & -4.42908500 & 0.11486900 & 3.70099500 \\
\hline Pt 48 & 1.77760300 & 0.81181500 & 2.16467200 \\
\hline Pt 49 & 4.67519100 & -1.92512100 & -2.38691300 \\
\hline Pt50 & -4.14594800 & -2.41153400 & -1.80696000 \\
\hline Pt51 & 3.92306700 & 2.41337000 & 1.7519830 \\
\hline Pt52 & -0.00315300 & 4.60547300 & -0.9185800 \\
\hline Pt53 & -2.03092500 & -3.85803900 & 3.04029800 \\
\hline Pt5 4 & 0.70184200 & -3.75369900 & 2.85122700 \\
\hline Pt5 5 & 3.41893200 & -3.54099600 & 2.65200100 \\
\hline Pt5 6 & -5.47120100 & 0.82919400 & 1.3128110 \\
\hline Pt57 & 3.37016400 & -0.97293500 & 3.45315500 \\
\hline Pt58 & 0.14891300 & 5.21121300 & 1.7082530 \\
\hline Pt59 & 0.15874300 & -0.64432300 & -2.5531140 \\
\hline Pt 60 & -0.06615300 & 1.36491600 & -4.37084200 \\
\hline Pt 61 & -2.19529900 & 1.31591100 & 4.6584950 \\
\hline
\end{tabular}




\begin{tabular}{|c|c|c|c|}
\hline et 62 & -3.10538800 & -3.18080100 & 0.61083000 \\
\hline Pt 63 & 4.51211600 & 3.20794800 & -0.78150200 \\
\hline Pt 64 & 0.21833100 & 2.61613700 & 0.89337200 \\
\hline Pt 65 & -2.13701900 & 0.76656600 & -2.66806000 \\
\hline Pt 66 & -4.23748400 & 0.17794500 & -0.99939000 \\
\hline Pt 67 & -2.24318800 & 3.40540300 & -1.87226600 \\
\hline Pt 68 & -4.43930600 & 2.15647900 & -2.82617100 \\
\hline Pt 69 & -5.38946000 & -1.74373900 & 0.50810400 \\
\hline Pt 70 & 2.19571500 & 3.63202200 & -2.1678520 \\
\hline Pt71 & 2.07939200 & -0.32443400 & 5.74983600 \\
\hline \# 71 & luster $=$ Pt 72 & \multicolumn{2}{|c|}{$\mathrm{M}=0 \quad$ Etot $=-6708.26541500$} \\
\hline Pt1 & 1.68202900 & -2.89738100 & 2.75235400 \\
\hline Pt2 & 5.33894000 & -1.98539500 & -0.08648900 \\
\hline Pt3 & -0.21437900 & 0.22043800 & -0.14497500 \\
\hline Pt 4 & 3.10747600 & -0.91366400 & 3.93338200 \\
\hline Pt 5 & 0.97288300 & -1.59446000 & -1.74281300 \\
\hline Pt 6 & 2.19849200 & 4.27656900 & -0.42924900 \\
\hline Pt 7 & 2.92758800 & -4.67877200 & 1.13845300 \\
\hline Pt 8 & -3.75956000 & -2.91457000 & -0.07861000 \\
\hline Pt9 & 0.16367500 & 4.69511400 & 1.33491500 \\
\hline Pt10 & -5.44183000 & 0.44414600 & 2.70220700 \\
\hline Pt11 & 1.45713200 & -0.32153000 & 1.90216800 \\
\hline Pt12 & -4.16906900 & -1.28057200 & -2.26188400 \\
\hline Pt13 & 5.11296800 & 0.56930500 & -0.94947500 \\
\hline Pt14 & 4.34566900 & -2.71689900 & 2.32745100 \\
\hline Pt15 & -1.44247200 & -4.33033600 & -0.51057900 \\
\hline Pt16 & 4.14674500 & -0.15163200 & 1.51276200 \\
\hline Pt17 & 2.66016900 & -2.13543700 & 0.31008900 \\
\hline Pt18 & 4.64520200 & 0.63850000 & -3.61414300 \\
\hline Pt19 & 0.27803800 & 2.35204000 & -4.28454300 \\
\hline Pt20 & -1.51363000 & 1.91660600 & 4.79447800 \\
\hline Pt21 & -1.67809600 & -1.77892700 & -1.36086300 \\
\hline Pt22 & -1.76285400 & 2.78855700 & -2.54875500 \\
\hline Pt23 & -0.00333200 & -2.33639900 & 0.69051600 \\
\hline Pt24 & -0.63261200 & -1.46640500 & 3.16251400 \\
\hline Pt25 & -0.44676900 & -3.58785800 & -2.95359500 \\
\hline Pt26 & -0.32615600 & 4.74374600 & -1.34775800 \\
\hline Pt27 & -2.12729200 & -1.69514100 & -4.02095600 \\
\hline Pt28 & 6.18360700 & -1.32737100 & -2.57100000 \\
\hline Pt29 & 0.75461400 & 2.27499700 & -1.60812600 \\
\hline Pt 30 & 1.71507000 & 4.28718600 & -3.09049400 \\
\hline Pt 31 & 1.97008200 & 0.47191400 & -3.19335600 \\
\hline Pt 32 & -4.82752500 & -0.44296400 & 0.22831500 \\
\hline Pt 33 & 3.92173100 & -3.93352000 & -1.27425200 \\
\hline Pt 34 & 2.96365900 & 2.48505000 & -4.66841300 \\
\hline Pt 35 & 0.81817300 & 0.52661700 & 4.40334100 \\
\hline Pt 36 & 1.00432600 & -2.04004400 & 5.22224900 \\
\hline Pt 37 & -1.92325500 & 3.56478100 & 2.64032500 \\
\hline Pt 38 & -2.35010100 & 5.11882000 & 0.43173800 \\
\hline Pt 39 & 2.23778100 & -3.38630300 & -3.32653100 \\
\hline Pt 40 & 2.67011700 & 4.22310000 & 2.23363000 \\
\hline Pt 41 & 1.23654900 & 2.23650300 & 1.05366400 \\
\hline Pt 42 & -3.18056800 & 0.80176500 & -3.7206700 \\
\hline Pt 43 & -0.84753600 & 1.09004800 & 2.32832400 \\
\hline Pt 44 & -2.09304000 & -3.49163100 & 1.98563000 \\
\hline Pt 45 & -2.94950300 & -0.05853100 & 3.6133280 \\
\hline Pt 46 & 0.24899900 & -4.86347900 & 1.5551560 \\
\hline Pt 47 & 0.59140900 & 3.08740300 & 3.5307860 \\
\hline
\end{tabular}




\begin{tabular}{|c|c|c|c|}
\hline Pt 48 & -2.70974900 & 0.69742800 & -1.05364800 \\
\hline Pt 49 & -3.34638500 & 1.54955100 & 1.41853400 \\
\hline Pt50 & 3.23310100 & -1.32534000 & -4.77981400 \\
\hline Pt51 & -4.02002400 & 2.40605400 & 3.8832460 \\
\hline Pt52 & -0.68714600 & 0.28565200 & -2.80495500 \\
\hline Pt53 & -1.26701900 & 2.68748400 & 0.14427400 \\
\hline Pt5 4 & -0.41789200 & $-4.0038170 c$ & 4.03981400 \\
\hline Pt5 5 & -3.77504200 & 3.17986400 & -0.77373100 \\
\hline Pt5 6 & 2.44741200 & $0.4279830 c$ & -0.52826500 \\
\hline Pt57 & -4.42800300 & 3.98951000 & 1.7322200 \\
\hline Pt58 & -2.69373900 & -2.60924700 & 4.46577000 \\
\hline Pt59 & -1.27176200 & -0.64593000 & 5.64714100 \\
\hline Pt 60 & -3.11219600 & $-3.7512260 c$ & -2.56270000 \\
\hline Pt 61 & 0.55172600 & -1.53542200 & -4.42265400 \\
\hline Pt 62 & 3.90600300 & 2.41373300 & 0.6555360 \\
\hline Pt 63 & 2.90499200 & 1.66955600 & 3.09923800 \\
\hline Pt 64 & 3.60936000 & -1.39797000 & -2.11761200 \\
\hline Pt 65 & -2.30627400 & -0.92229200 & 1.11807300 \\
\hline Pt 66 & 1.24239600 & $-4.1625660 c$ & -0.90192600 \\
\hline Pt 67 & -5.84466100 & 2.03418600 & 0.55488100 \\
\hline Pt 68 & -4.37569300 & -2.03260000 & 2.41883500 \\
\hline Pt 69 & 3.44808700 & 2.46818800 & -2.01600400 \\
\hline Pt70 & -1.11638400 & 0.36904100 & -5.44657800 \\
\hline Pt71 & -5.18883800 & 1.20142100 & -1.92732200 \\
\hline Pt72 & 1.55418500 & 0.52277800 & -5.84260000 \\
\hline \# 72 & uster $=$ Pt73 $\quad Q=0$ & \multicolumn{2}{|l|}{$\mathrm{M}=0 \quad$ Etot $=-68$} \\
\hline Pt 1 & -0.65713800 & -2.45764600 & 1.69768700 \\
\hline Pt2 & -0.74244400 & $3.0713170 \mathrm{C}$ & -1.16160700 \\
\hline Pt3 & 4.13885800 & $1.3364350 \mathrm{C}$ & -0.50606900 \\
\hline Pt 4 & 3.05853600 & 3.51587900 & -1.75165100 \\
\hline Pt 5 & 4.20049900 & -1.06843400 & -1.77353000 \\
\hline Pt 6 & -4.29778300 & -1.24700800 & 2.10636300 \\
\hline Pt 7 & -5.00681300 & -2.78058300 & -0.00433400 \\
\hline Pt 8 & -2.59377700 & -1.62058500 & -0.00372500 \\
\hline Pt 9 & 4.65096500 & -2.88853900 & 2.50632200 \\
\hline Pt10 & 1.03077200 & $0.3015950 \mathrm{c}$ & -4.55825600 \\
\hline Pt11 & 3.40276600 & $1.9274670 \mathrm{C}$ & 4.10357700 \\
\hline Pt12 & 0.79992200 & -0.27635400 & 2.47574400 \\
\hline Pt13 & 1.35267100 & 3.86874300 & 0.36366100 \\
\hline Pt14 & 0.77165800 & -2.42271300 & -4.24237500 \\
\hline Pt15 & 1.54208800 & -4.65664400 & 3.83028600 \\
\hline Pt16 & 4.02874400 & 3.72556100 & 0.78567700 \\
\hline Pt17 & -3.55251800 & 0.30171200 & 4.19210600 \\
\hline Pt18 & 3.21475100 & -1.26775300 & -4.28291300 \\
\hline Pt19 & -2.44736800 & 3.50136100 & 0.96082000 \\
\hline Pt20 & 2.36542100 & 4.11131900 & 2.86874800 \\
\hline Pt21 & 1.03097500 & -2.8491530 & -0.41642800 \\
\hline Pt22 & -0.26211000 & 1.90966300 & 1.23786400 \\
\hline Pt23 & 0.52680800 & -4.87305300 & 1.32308100 \\
\hline Pt24 & -1.86820000 & -0.07628200 & 2.08895800 \\
\hline Pt25 & -1.11760800 & -4.46158800 & 3.41248100 \\
\hline Pt26 & 1.29742800 & 6.24414700 & 1.59519200 \\
\hline Pt27 & -0.90566500 & -2.01209200 & -2.1178400 \\
\hline Pt28 & 2.50575100 & -0.67196900 & 0.33944100 \\
\hline Pt29 & 1.80223600 & -0.09180800 & 4.9606100 \\
\hline Pt30 & -1.94957100 & 2.30940600 & 3.3552890 \\
\hline Pt31 & -0.32553700 & 4.30071300 & 2.48640800 \\
\hline Pt32 & -5.28576100 & $1.0729750 \mathrm{C}$ & -2.6769850 \\
\hline
\end{tabular}




\begin{tabular}{|c|c|c|c|}
\hline et33 & 1.53860500 & -0.86638800 & -2.17756900 \\
\hline Pt34 & 2.20521200 & -5.23221500 & -0.74886600 \\
\hline Pt35 & -3.34875800 & -3.19794400 & -2.08175200 \\
\hline Pt36 & 3.15090500 & 1.12650400 & -3.03231500 \\
\hline Pt37 & -0.80893300 & 5.48334800 & 0.07995700 \\
\hline Pt38 & 3.71929300 & -3.07525200 & -0.01944500 \\
\hline Pt39 & 1.45802000 & 1.51073100 & -0.91634900 \\
\hline Pt 40 & 2.98664600 & -2.50255100 & 4.59064000 \\
\hline Pt 4 & 3.20087600 & -5.04135000 & 1.75289500 \\
\hline Pt 4 & -2.86062200 & 2.26270900 & -2.70322000 \\
\hline Pt 4 & 0.25700700 & -4.43254200 & -2.47326100 \\
\hline t 44 & -0.88640000 & 0.11131900 & 4.59707000 \\
\hline Pt 4 & -0.65398700 & 0.69374200 & -2.43650900 \\
\hline Pt 4 & 0.95643600 & 2.71195100 & -3.30194900 \\
\hline Pt 4 & -1.23710400 & 4.26649600 & -3.52134600 \\
\hline Pt 4 & 3.48406600 & -0.47961100 & 2.87083000 \\
\hline Pt 4 & 0.73505500 & 2.12584400 & 3.75670000 \\
\hline Pt50 & -3.08555800 & -3.65733400 & 1.71281600 \\
\hline Pt51 & 0.31993800 & -2.28687500 & 4.23775200 \\
\hline Pt52 & 1.99878400 & -2.66153500 & 2.08890300 \\
\hline Pt5 & 2.93611100 & 5.86410400 & -0.45702000 \\
\hline Pts & -4.05612700 & 1.48134900 & 1.81234400 \\
\hline Pt55 & -4.81769700 & -0.06626900 & -0.27585700 \\
\hline Pt56 & -1.68399400 & -3.55121100 & -4.16568100 \\
\hline Pt57 & -1.42158300 & -4.06583700 & -0.38218700 \\
\hline Pt5 8 & 5.06808700 & 1.54124600 & 2.01800500 \\
\hline Pt59 & -0.15969500 & -0.45674100 & -0.04293300 \\
\hline Pt 60 & -2.34921600 & -2.08922400 & 3.81825400 \\
\hline Pt61 & -2.90242500 & 4.65271700 & -1.43577500 \\
\hline Pt 62 & -4.52867000 & 2.64455600 & -0.60133300 \\
\hline Pt 63 & -3.86507600 & -1.98728300 & -4.45481300 \\
\hline Pt 64 & -1.41834300 & -0.85464800 & -4.53305400 \\
\hline Pt 65 & -1.18113800 & 1.86819600 & -4.79363000 \\
\hline Pt 66 & -3.62144100 & 0.68698600 & -4.76130200 \\
\hline Pt 67 & 2.72022600 & -3.26492900 & -2.53048300 \\
\hline Pt 68 & -5.52386400 & -1.60257700 & -2.37742200 \\
\hline Pt 69 & 5.14976800 & -0.86816800 & 0.76831300 \\
\hline Pt70 & -2.35981500 & 1.08935800 & -0.30020700 \\
\hline Pt71 & 0.85439000 & 5.09759000 & -2.00311400 \\
\hline Pt72 & -3.09079500 & -0.46170100 & -2.38248600 \\
\hline Pt73 & 2.41326100 & 1.70734800 & 1.61080000 \\
\hline \# 73 & Cluster $=$ Pt $74 \quad Q=0$ & $=0 \quad$ Etot $=-69$ & 500 \\
\hline Pt 1 & -3.19803200 & -0.48206600 & -3.3317700 \\
\hline Pt2 & 4.92712900 & 2.63774100 & 2.14382300 \\
\hline Pt 3 & -0.05590900 & 1.76648300 & 3.31234600 \\
\hline Pt 4 & -0.89632100 & 4.56663300 & -0.39957200 \\
\hline Pt 5 & 5.71171900 & 1.76707800 & -0.28975500 \\
\hline Pt 6 & -5.12015000 & -0.71962500 & -1.39328500 \\
\hline Pt 7 & -5.66030400 & -1.43262000 & 1.16579400 \\
\hline Pt 8 & -4.30152900 & -3.45275900 & 2.32498600 \\
\hline Pt9 & -1.88516400 & 1.27789600 & 1.38898800 \\
\hline Pt10 & 0.15338000 & -2.32176600 & -4.04759200 \\
\hline Pt11 & -0.49580400 & -0.79111300 & 2.5757500 \\
\hline Pt12 & 4.62536600 & -0.91335500 & -3.6880930 \\
\hline Pt13 & 4.02676700 & -2.49308300 & 0.6791700 \\
\hline Pt14 & -3.64396600 & -1.83031100 & 4.3819770 \\
\hline Pt15 & 1.86372400 & 0.43889300 & 1.95961100 \\
\hline Pt16 & -1.94670000 & 3.09050500 & 4.7020900 \\
\hline
\end{tabular}




\begin{tabular}{|c|c|c|c|}
\hline Pt17 & -5.00267700 & 0.18998700 & 3.22298600 \\
\hline Pt18 & -1.16071400 & -2.43169100 & 0.49577200 \\
\hline Pt19 & 3.57274400 & 4.66029400 & 0.98370200 \\
\hline Pt20 & 0.22092500 & -4.47704300 & 1.67298300 \\
\hline Pt21 & -1.97745400 & 1.89908000 & -3.78158100 \\
\hline Pt22 & -1.79405300 & -2.52215700 & -2.12492900 \\
\hline Pt23 & 4.35741100 & 3.78954500 & -1.45011400 \\
\hline Pt24 & 1.51827300 & -4.34849100 & -2.86665600 \\
\hline Pt25 & 0.65187400 & 1.52486300 & -4.33708900 \\
\hline Pt26 & -3.07580500 & -1.09979000 & 1.85070600 \\
\hline Pt27 & -1.21088400 & -0.28614100 & -5.20835100 \\
\hline Pt28 & -0.60075000 & -0.14742500 & -2.59490200 \\
\hline Pt29 & -3.93561800 & 1.68194600 & -1.87060200 \\
\hline Pt30 & 0.47571200 & 2.50860500 & 0.77249700 \\
\hline Pt31 & 1.74345600 & 2.28471600 & 5.25078000 \\
\hline Pt32 & 3.27034400 & 1.10735200 & -4.85068400 \\
\hline Pt33 & 3.23589300 & -1.61544600 & 3.13223900 \\
\hline Pt34 & -1.71622200 & -3.17419700 & 3.05227000 \\
\hline Pt35 & 1.26952400 & 1.62770800 & -1.68968300 \\
\hline Pt36 & 3.09224200 & 2.11557000 & 0.24005500 \\
\hline Pt37 & -2.42579900 & 0.54036300 & 3.95229500 \\
\hline Pt38 & -3.00531400 & -4.89117500 & -1.69550700 \\
\hline Pt39 & 4.50489300 & 0.06882600 & 1.42776200 \\
\hline Pt 40 & -4.36323800 & -2.87198100 & -2.85734000 \\
\hline Pt41 & 0.88375300 & -2.84160100 & 3.74644900 \\
\hline Pt 42 & -3.26744100 & 3.33058400 & 0.21959400 \\
\hline Pt43 & 2.65708200 & -0.44150100 & -0.50116200 \\
\hline Pt 44 & 3.67294900 & 0.96788500 & 3.87361500 \\
\hline Pt 45 & 1.42267000 & -2.11950800 & 1.21697000 \\
\hline Pt 46 & 2.77681700 & -2.66786700 & -4.58279700 \\
\hline Pt 47 & 2.31498100 & 3.01169800 & 2.71434400 \\
\hline Pt 48 & 0.94748400 & 5.03934600 & 1.54347100 \\
\hline Pt 49 & 3.42141800 & -2.60450300 & -1.96157100 \\
\hline Pt50 & 2.01801000 & -0.53495500 & -3.12036600 \\
\hline Pt51 & -2.72018400 & 4.05255800 & -2.32688100 \\
\hline Pt52 & 5.26186200 & -0.79534000 & -1.05474600 \\
\hline Pt53 & -3.78620500 & 2.57170700 & 2.77957000 \\
\hline Pt5 4 & -3.76174200 & -2.75590000 & -0.22681100 \\
\hline Pt5 5 & -1.44263300 & 3.84914800 & 2.15948100 \\
\hline Pt5 6 & -2.55045500 & -0.36362000 & -0.69217900 \\
\hline Pt57 & 0.38698000 & 4.30703700 & 4.09270000 \\
\hline Pt58 & 1.73677000 & 4.20170000 & -0.94330900 \\
\hline Pt59 & -1.05264200 & -1.49553200 & 5.10759900 \\
\hline Pt 60 & -1.35125000 & 2.01310500 & -1.15587700 \\
\hline Pt 61 & 0.03568700 & -0.05316800 & 0.03052700 \\
\hline Pt 62 & -2.43599300 & -2.65827600 & -4.73814500 \\
\hline Pt 63 & -2.38430500 & -4.78123300 & 0.94188300 \\
\hline Pt 64 & 1.32965600 & -0.28326400 & 4.52648600 \\
\hline Pt 65 & 1.42161500 & -0.64733200 & -5.74547500 \\
\hline Pt 66 & 2.16341400 & -4.26164700 & -0.22269900 \\
\hline Pt 67 & 3.90900200 & 1.24280500 & -2.22985800 \\
\hline Pt 68 & 0.78787600 & -2.21561900 & -1.40586400 \\
\hline Pt 69 & -0.09906600 & 3.68191400 & -2.87243400 \\
\hline Pt70 & 2.53912000 & 3.27309900 & -3.39333600 \\
\hline Pt71 & -0.58999600 & 1.06830300 & 5.86011000 \\
\hline Pt72 & -1.07825800 & -4.67749100 & -3.57612800 \\
\hline Pt73 & -4.49250800 & 0.95442100 & 0.68333100 \\
\hline Pt7 4 & -0.42344200 & -4.60880200 & -0.95756900 \\
\hline
\end{tabular}




\begin{tabular}{|c|c|c|c|}
\hline \# 74 & Cluster $=$ Pt 75 & Etot $=-7 c$ & 77508100 \\
\hline Pt1 & 3.31734800 & -1.48389000 & 3.03481800 \\
\hline Pt2 & 0.48487400 & 2.38237400 & -4.00533400 \\
\hline Pt 3 & -0.96170500 & -3.01859500 & 4.36271000 \\
\hline Pt 4 & 2.62811100 & 0.61832400 & -0.14338100 \\
\hline Pt 5 & -1.48965300 & -0.36263300 & -2.29366200 \\
\hline Pt 6 & -0.12090700 & -3.69279100 & 0.66236400 \\
\hline Pt 7 & -4.55689900 & -1.07433300 & -0.18436600 \\
\hline Pt 8 & 2.96526300 & -1.57687200 & -1.67652700 \\
\hline Pt 9 & -4.16364600 & 0.42855400 & 4.25753600 \\
\hline Pt10 & -1.31367800 & 4.22860200 & -3.07623400 \\
\hline Pt11 & -3.54435700 & -2.20053500 & 4.27099400 \\
\hline Pt12 & -3.26243300 & 2.92617700 & -1.65756600 \\
\hline Pt13 & 4.80445000 & -3.41210100 & -0.95971100 \\
\hline Pt14 & 0.38014700 & 1.52204700 & 4.46718100 \\
\hline Pt15 & 5.58922700 & -0.98676800 & -1.85284700 \\
\hline Pt16 & -3.88929600 & -3.73127200 & -0.14498600 \\
\hline Pt17 & -5.14544200 & 1.60149300 & -0.17228500 \\
\hline Pt18 & -1.58181800 & -0.35009500 & 4.39645200 \\
\hline Pt19 & -0.28735200 & 3.65041500 & 2.92325200 \\
\hline Pt20 & 4.13376800 & -1.34107800 & -4.09385900 \\
\hline Pt21 & -1.09038500 & 1.15794700 & 2.18633700 \\
\hline Pt22 & -1.60961600 & -4.09058800 & -1.62164600 \\
\hline Pt23 & 4.11463500 & 0.98010400 & 2.12744500 \\
\hline Pt24 & 1.00930700 & -1.14895000 & 4.48085400 \\
\hline Pt25 & 3.64640100 & -3.68731700 & 1.47563400 \\
\hline Pt26 & 1.68141700 & -5.55329400 & 1.40711700 \\
\hline Pt27 & -1.13096500 & -2.56676400 & -3.81437900 \\
\hline Pt28 & -2.42948000 & -3.38047100 & 2.11232200 \\
\hline Pt29 & -0.67086100 & 2.10506800 & -1.5583590 \\
\hline Pt30 & -3.03998700 & -0.70539900 & 2.08589000 \\
\hline Pt31 & 0.15036400 & 4.59246300 & -0.82373500 \\
\hline Pt32 & -2.58184100 & 0.79487500 & -0.11010000 \\
\hline Pt33 & 2.29934500 & 2.83787500 & 3.01269400 \\
\hline Pt34 & 1.95354000 & 2.71823100 & -1.69851500 \\
\hline Pt35 & 4.56542000 & 3.35963700 & -1.8750980 \\
\hline Pt36 & 0.22595800 & -5.90760300 & -0.83389500 \\
\hline Pt37 & 0.00000000 & 0.00000000 & 0.00000000 \\
\hline Pt38 & -2.95948700 & -0.72044200 & -4.55680800 \\
\hline Pt39 & 0.82059400 & 2.46814000 & 0.73807800 \\
\hline Pt 40 & 1.83423800 & -1.83511800 & 0.76010700 \\
\hline Pt41 & 0.34278200 & -2.19819000 & -1.53633000 \\
\hline Pt 42 & 5.23822000 & 1.23215600 & -0.33597700 \\
\hline Pt 43 & -0.33367800 & -0.10277000 & -4.72175200 \\
\hline Pt 44 & -4.99981600 & -2.55484400 & 2.02998200 \\
\hline Pt 45 & -2.04586900 & -5.57791900 & 0.58945300 \\
\hline Pt 46 & 3.34899100 & -3.76641000 & -3.20072300 \\
\hline Pt 47 & 1.50637100 & -1.95422600 & -3.98313400 \\
\hline Pt 48 & -4.06816600 & 0.43917300 & -2.38201600 \\
\hline Pt 49 & -4.32171100 & 4.08380100 & 0.53999200 \\
\hline Pt50 & -3.43900600 & -2.23182400 & -2.36834200 \\
\hline Pt51 & -3.68768700 & 1.96104400 & 2.08497800 \\
\hline Pt52 & 2.18618600 & -4.05029000 & -0.77949100 \\
\hline Pt53 & 3.45995500 & 3.10661200 & 0.57548400 \\
\hline Pt5 4 & -0.97716700 & 5.73329300 & 1.34933800 \\
\hline Pt55 & -2.86625300 & 4.43811000 & 2.78100500 \\
\hline Pt5 6 & 4.48145200 & -1.22998700 & 0.59768400 \\
\hline Pt57 & -1.75790800 & 3.25683100 & 0.62678700 \\
\hline & 2.95948700 & 0.72044200 & 4.55680800 \\
\hline
\end{tabular}




\begin{tabular}{|c|c|c|c|}
\hline Pt59 & 0.71881000 & -4.39999400 & -3.03206200 \\
\hline Pt 60 & 1.36546500 & -3.36635000 & 2.95917200 \\
\hline Pt 61 & 1.61391300 & 4.94127900 & 1.43146200 \\
\hline Pt 62 & -1.95537500 & -1.86468500 & -0.09648600 \\
\hline Pt 63 & 2.77861300 & 5.18825900 & -1.00373800 \\
\hline Pt 64 & -2.21785100 & 2.31417000 & 4.33541100 \\
\hline Pt 65 & 2.31062900 & 0.51947900 & -4.84367300 \\
\hline Pt 66 & -5.61910400 & 0.07424500 & 2.01652300 \\
\hline Pt 67 & -0.59041000 & -5.22361000 & 2.83046500 \\
\hline Pt 6 & 3.10996100 & 3.00532800 & -4.11611000 \\
\hline$=6$ & 3.78061700 & 0.87725400 & -2.59407400 \\
\hline$=70$ & 1.48965300 & 0.36263300 & 2.29366200 \\
\hline 271 & -2.43262600 & 5.37898300 & -0.89167400 \\
\hline$t 72$ & 1.13665600 & 0.25525200 & -2.43981800 \\
\hline$=73$ & -2.14896800 & 1.75500100 & -3.83650300 \\
\hline$=74$ & 1.32315400 & 4.83395000 & -3.24475000 \\
\hline$=75$ & -0.46391900 & -1.50161300 & 2.19995200 \\
\hline \# & luster $=$ Pt 76 & $=0 \quad$ Etot $=-711$ & 39796600 \\
\hline Pt 1 & -4.59419600 & -3.83379700 & 0.66329400 \\
\hline Pt2 & -0.61345800 & -3.59353800 & 3.10759700 \\
\hline Pt3 & 3.01846000 & -3.31020700 & -3.83618700 \\
\hline Pt 4 & 0.81771900 & -1.97026800 & -2.99768700 \\
\hline Pt 5 & 1.96682300 & -1.38441700 & -5.38423700 \\
\hline Pt 6 & -4.35770000 & -1.24633400 & 1.40297300 \\
\hline Pt 7 & 0.13826900 & 4.60903800 & 0.91766300 \\
\hline Pt 8 & -3.80758100 & 2.34913000 & -1.63052800 \\
\hline Pt9 & 4.49731300 & -0.96871200 & 0.56407800 \\
\hline Pt10 & 1.05207800 & 0.63508800 & -2.30074300 \\
\hline Pt11 & -2.78817400 & 0.84527500 & 5.3136160 \\
\hline Pt12 & -1.15566700 & 2.45856300 & 3.88987600 \\
\hline Pt13 & 3.39434500 & 1.01100400 & -0.99651600 \\
\hline Pt14 & -0.55724500 & -5.16799000 & -1.36573200 \\
\hline Pt15 & -2.73730000 & 0.37737300 & -0.05853300 \\
\hline Pt 1 & -1.92757200 & -4.22911700 & 0.82025100 \\
\hline Pt17 & -1.61383300 & -3.22341100 & -2.92711400 \\
\hline Pt18 & -4.28949400 & -2.86228500 & -3.01408000 \\
\hline Pt19 & 2.36291600 & 2.99928800 & -2.59577200 \\
\hline Pt20 & -0.10250400 & 0.50009900 & 5.44186600 \\
\hline Pt21 & 0.98941000 & -1.73894800 & 4.35248600 \\
\hline Pt22 & -0.06521900 & 0.00492000 & 0.07664400 \\
\hline Pt23 & -1.69710600 & -1.37871700 & 4.23908600 \\
\hline Pt24 & 5.59567100 & -0.32825700 & -1.83456200 \\
\hline Pt25 & -2.20599400 & 4.20223900 & -0.38663300 \\
\hline Pt26 & -0.30256900 & -2.59809400 & -0.62401100 \\
\hline Pt27 & -3.83429100 & 2.59481100 & 1.07121900 \\
\hline Pt28 & 3.20352900 & -3.34088100 & 0.85239100 \\
\hline Pt29 & 3.51525200 & 3.01407700 & 3.78336900 \\
\hline Pt30 & 3.56424400 & 2.76785900 & 1.09451000 \\
\hline Pt31 & 4.60771800 & 0.80810500 & 2.64570700 \\
\hline Pt32 & -5.40835800 & 0.96615600 & 2.54132100 \\
\hline Pt33 & -3.83340700 & 2.78844500 & 3.76452800 \\
\hline Pt34 & 0.01010900 & 2.60115000 & -3.88811500 \\
\hline Pt35 & 0.60481300 & -4.54590300 & -3.74082500 \\
\hline Pt36 & -4.06562900 & -0.25506300 & -2.34735200 \\
\hline Pt37 & 1.00414900 & -1.98636600 & 1.66631500 \\
\hline & -3.29944200 & -3.19890300 & 2.95892100 \\
\hline S & 2.22316700 & 1.21256500 & -4.66889500 \\
\hline$[40$ & -2.43357500 & 1.35949300 & -3.79291100 \\
\hline
\end{tabular}




\begin{tabular}{|c|c|c|c|}
\hline Pt 41 & -4.36313700 & -0.97702800 & 4.09040000 \\
\hline Pt 42 & 4.41962200 & -0.24688900 & -5.32271700 \\
\hline Pt 43 & -1.37310700 & -0.60669100 & -2.21179400 \\
\hline Pt 44 & 2.27628700 & 0.63493900 & 4.08523600 \\
\hline Pt 45 & -1.14236100 & 2.22279000 & 1.18019800 \\
\hline Pt 46 & 0.15408500 & 4.35353300 & -1.78317000 \\
\hline Pt 47 & 3.23150800 & -0.74720200 & -3.04760300 \\
\hline Pt 48 & -5.40149000 & 0.71050200 & -0.15738600 \\
\hline Pt 49 & 1.20537500 & 2.37672900 & -0.20918100 \\
\hline Pt50 & 0.14846000 & 4.80725500 & 3.61039200 \\
\hline Pt51 & -0.23208000 & -0.00323900 & -4.61121000 \\
\hline Pt52 & 3.34697000 & -1.58281400 & 2.94876700 \\
\hline Pt53 & -2.18497500 & 3.95470300 & -3.07828900 \\
\hline Pt5 4 & -5.64001500 & -1.89027200 & -0.88530500 \\
\hline Pt55 & -2.96885800 & -2.22028200 & -0.75536200 \\
\hline Pt5 6 & 4.69849600 & 3.34983000 & -1.28965700 \\
\hline Pt 57 & -0.07096900 & 0.23079500 & 2.76907900 \\
\hline Pt58 & 1.19468400 & 2.59712400 & 2.47830900 \\
\hline Pt59 & -1.13429400 & 1.99480100 & -1.51131200 \\
\hline Pt 60 & 0.75972700 & -4.57637200 & 0.93935000 \\
\hline Pt 61 & 2.12437900 & -1.36837800 & -0.69670300 \\
\hline Pt 62 & -2.20522400 & 4.40872600 & 2.32503700 \\
\hline Pt 63 & 2.27620700 & 0.38566700 & 1.37883000 \\
\hline Pt 64 & 2.46941100 & 4.95807300 & 2.23531400 \\
\hline Pt 65 & 2.06321400 & -3.94780200 & 3.22478300 \\
\hline Pt 66 & 1.19440500 & 2.86387600 & 5.15908900 \\
\hline Pt 67 & 4.32357500 & -2.70327200 & -1.53962000 \\
\hline Pt 68 & 4.56152200 & 1.61776700 & -3.36902900 \\
\hline Pt 69 & 2.50462100 & 4.71530500 & -0.46448700 \\
\hline Pt 70 & -2.73969600 & 0.60231300 & 2.63021500 \\
\hline Pt71 & 1.90409700 & -3.96918000 & -1.44997800 \\
\hline Pt72 & 5.74449200 & 1.40093700 & 0.25097600 \\
\hline Pt73 & -0.44961700 & -2.60677000 & -5.28800900 \\
\hline Pt74 & -2.66179300 & -1.26247100 & -4.47705200 \\
\hline Pt 75 & -3.24288200 & -4.80539100 & -1.46542700 \\
\hline Pt76 & -1.66630900 & -1.61508600 & 1.53003600 \\
\hline \# 76 & Cluster $=$ Pt77 & \multicolumn{2}{|c|}{$\mathrm{M}=0 \quad$ Etot $=-7219.12422600$} \\
\hline Pt 1 & -2.56546200 & -4.01225400 & 3.87825400 \\
\hline Pt2 & -0.73967800 & -4.35704000 & 1.91761100 \\
\hline Pt3 & 0.95249900 & -2.30561700 & 1.34768100 \\
\hline Pt 4 & -3.37214200 & -4.17578200 & 1.29549200 \\
\hline Pt 5 & -1.74162200 & -5.80686000 & -0.12969100 \\
\hline Pt 6 & -5.37011000 & 0.25813500 & -1.09599200 \\
\hline Pt7 & 1.51755400 & -5.40502500 & -2.07501100 \\
\hline Pt 8 & -2.34959200 & 2.75286900 & 4.86526700 \\
\hline Pt9 & 2.58799900 & -3.96418700 & -0.05815800 \\
\hline Pt10 & -1.89919100 & 0.23080200 & 2.09264700 \\
\hline Pt11 & -3.20755200 & 2.60634400 & 2.30964800 \\
\hline Pt12 & -0.90245900 & -1.97786900 & 3.27957900 \\
\hline Pt13 & -3.58764500 & -1.81901000 & 2.68051300 \\
\hline Pt14 & 3.98856100 & 1.92006300 & 1.60721500 \\
\hline Pt15 & -2.61315900 & 4.63006100 & 0.55483300 \\
\hline Pt16 & -1.10101400 & -5.23157600 & -2.69097100 \\
\hline Pt17 & 3.61200600 & -2.49198500 & 1.97829400 \\
\hline Pt 18 & 0.87698000 & -5.98031200 & 0.48627700 \\
\hline Pt19 & -0.75073800 & 5.70770600 & -1.10076300 \\
\hline Pt20 & -0.24747700 & 4.62301900 & -3.53675800 \\
\hline $\mathrm{P}$ & -2.72596900 & 0.08244800 & -0 \\
\hline
\end{tabular}




\begin{tabular}{|c|c|c|c|}
\hline Pt22 & 1.83794500 & 0.98035900 & -1.45630700 \\
\hline Pt23 & -1.73166600 & -2.12782200 & 0.71629100 \\
\hline Pt24 & 0.05301200 & -4.18569800 & 4.49419200 \\
\hline Pt2 5 & 3.20053600 & 3.19485100 & -0.65958500 \\
\hline Pt26 & 1.55918000 & 0.20241300 & 5.29289900 \\
\hline Pt 27 & 1.76589500 & -2.17361900 & 3.93981400 \\
\hline Pt28 & -0.86172000 & 1.15918000 & -2.09134300 \\
\hline Pt29 & 0.02455600 & 0.79302900 & -5.77171700 \\
\hline Pt 30 & 4.49997500 & 0.81237100 & -0.84441600 \\
\hline Pt 31 & 3.68350800 & 2.07128500 & -3.10116300 \\
\hline Pt32 & 0.80772500 & 4.58879100 & 3.75679600 \\
\hline Pt33 & 1.59992900 & -1.72530900 & -1.24392100 \\
\hline Pt 34 & -4.01250100 & 2.45004000 & -0.27485600 \\
\hline Pt 35 & -3.51636200 & 1.34336000 & -2.73008100 \\
\hline Pt 36 & -1.35382900 & 2.26467200 & 0.34958200 \\
\hline Pt37 & -1.07597700 & 0.37776800 & 4.68056800 \\
\hline Pt 38 & 1.89493000 & -4.52466400 & 2.53445300 \\
\hline Pt39 & 3.47318900 & -0.64388000 & -2.89964400 \\
\hline Pt 40 & -0.08850500 & -3.75125100 & -0.68007100 \\
\hline Pt 41 & -1.08305600 & -1.54759300 & -1.87503300 \\
\hline Pt 42 & 2.40871500 & -2.07400900 & -4.92440600 \\
\hline Pt 43 & 5.28368300 & -0.44755400 & 1.41007500 \\
\hline Pt 44 & 2.63906000 & -0.27292300 & 0.78891800 \\
\hline Pt 45 & 0.26080500 & 3.48074800 & -5.93312300 \\
\hline Pt 46 & -2.14759900 & 3.54910300 & -1.91761500 \\
\hline Pt 47 & -0.11241000 & -1.85669200 & 5.85326400 \\
\hline Pt 48 & 3.19370700 & -3.35770500 & -2.66457400 \\
\hline Pt 49 & -3.74069100 & -1.37682000 & -2.50798800 \\
\hline Pt 50 & -3.70789100 & 0.55129600 & 4.053938 \\
\hline Pt 51 & 1.01570200 & 2.22605900 & -3.69111100 \\
\hline Pt 52 & 1.32915600 & 2.08695600 & 0.980694 \\
\hline Pt 53 & -2.76605800 & -3.60954300 & -1.31758100 \\
\hline Pt 54 & 2.63966400 & 0.61980800 & -5.15657100 \\
\hline Pt 55 & 0.01919400 & 4.46546200 & 1.17584400 \\
\hline Pt 56 & -4.56297300 & 0.40780400 & 1.47756600 \\
\hline Pt 57 & 0.26901000 & 2.57941700 & 5.48123500 \\
\hline Pt 58 & -1.81084300 & 4.76224100 & 3.14083600 \\
\hline Pt59 & 2.36763200 & 4.44979900 & -2.92161200 \\
\hline Pt 60 & 3.44371200 & -0.12254600 & 3.36096000 \\
\hline Pt 61 & 2.83769200 & 3.31005900 & -5.32696800 \\
\hline Pt 62 & 2.65112900 & 4.28136400 & 1.7931400 \\
\hline Pt 63 & 0.79065800 & -0.46771800 & -3.49294900 \\
\hline Pt 64 & 1.86832600 & 5.53422400 & -0.48468600 \\
\hline Pt 65 & 4.26037100 & -1.90679700 & -0.62591600 \\
\hline Pt 66 & -2.07058000 & -3.00900800 & -3.90288100 \\
\hline Pt 67 & -1.87494600 & -0.28962800 & -4.1576740 \\
\hline Pt 68 & -0.52576300 & 2.40499200 & 2.9123520 \\
\hline Pt 69 & 2.14650400 & 2.25170000 & 3.5690700 \\
\hline Pt 70 & -4.39467900 & -1.96163600 & 0.0948990 \\
\hline Pt 71 & 0.56291500 & -3.18968700 & -3.2912530 \\
\hline Pt 72 & -2.73088400 & -1.68324800 & 5.2373260 \\
\hline Pt 73 & -0.21034900 & -1.90052700 & -5.5404830 \\
\hline Pt 74 & 0.78497300 & 0.05300700 & 2.7240360 \\
\hline Pt 75 & 0.52016600 & 3.34057900 & -1.2706500 \\
\hline Pt 76 & -0.04298800 & -0.09421500 & 0.1562210 \\
\hline Pt 77 & -1.63267200 & 2.42342000 & -4.3516750 \\
\hline
\end{tabular}

\# 77 Cluster=Pt78 $\quad \mathrm{Q}=0 \quad \mathrm{M}=0 \quad$ Etot $=-7298.21612600$

$\begin{array}{llll}\text { Pt } 1 & -4.19603200 & -2.61932100 & 3.35323300\end{array}$ 


\begin{tabular}{|c|c|c|c|}
\hline Pt2 & 3.74293700 & -2.66298000 & -2.68997800 \\
\hline Pt3 & -1.21562000 & 1.68370400 & 3.25224300 \\
\hline Pt 4 & 3.37956200 & -4.75471800 & -0.99338700 \\
\hline Pt 5 & -3.98243700 & 1.07953700 & 0.47030600 \\
\hline Pt 6 & -2.75490100 & -1.24712200 & 5.22651200 \\
\hline Pt 7 & -3.14969300 & -4.76958700 & 2.02031000 \\
\hline Pt 8 & -4.27982700 & 0.72405400 & -2.30438300 \\
\hline Pt9 & -1.95644200 & 1.30111200 & -1.23282300 \\
\hline Pt10 & -1.80384300 & 2.81104100 & 0.87053200 \\
\hline Pt11 & -3.37438100 & -0.08779200 & 2.86032300 \\
\hline Pt12 & -1.70325300 & -3.47959700 & 3.94752000 \\
\hline Pt13 & -1.05583500 & -3.18338400 & -0.70497600 \\
\hline Pt14 & 1.03967700 & 0.55788300 & 2.21129400 \\
\hline Pt15 & -1.78847300 & -2.12983300 & -5.26146600 \\
\hline Pt16 & -1.85775400 & -0.40211300 & -3.21044400 \\
\hline Pt17 & -2.78411100 & -2.94376600 & -2.84023700 \\
\hline Pt1 8 & 0.78149100 & 4.79147000 & 4.15545100 \\
\hline Pt19 & 1.76089800 & 5.62657600 & 1.73261400 \\
\hline Pt20 & 0.97632700 & -3.04281300 & 3.57697000 \\
\hline Pt21 & -1.05937300 & 3.21932300 & 5.41759800 \\
\hline Pt22 & 1.69938700 & -4.47393800 & -3.09420800 \\
\hline Pt23 & 0.60985700 & 3.24271100 & 2.00064500 \\
\hline Pt24 & -2.38706400 & 2.34052500 & -3.6021140 \\
\hline Pt25 & -2.23590700 & 0.54611000 & -5.63034000 \\
\hline Pt26 & 2.25718300 & -0.31116500 & -0.05252500 \\
\hline Pt27 & 0.41358100 & -1.88565000 & 1.21150000 \\
\hline Pt28 & 1.54546900 & 4.11389100 & -0.51286300 \\
\hline Pt29 & -2.25685200 & -2.31130800 & 1.55469100 \\
\hline Pt 30 & -0.17504500 & -0.69719600 & -1.1237430 \\
\hline Pt 31 & -0.09214000 & -2.42076200 & -3.1497190 \\
\hline Pt 32 & 5.00884500 & 2.25271300 & -0.65448100 \\
\hline Pt33 & -3.73570000 & -3.63899000 & -0.36101500 \\
\hline Pt 34 & -3.62634800 & 3.30725300 & -1.16953500 \\
\hline Pt35 & -3.72116000 & 2.63453500 & 2.7096530 \\
\hline Pt 36 & 2.05299400 & -2.37646600 & -4.8070800 \\
\hline Pt 37 & -0.25980700 & -2.13598800 & 5.80828900 \\
\hline Pt 38 & 4.42114600 & -0.21579300 & -1.67665800 \\
\hline Pt39 & -0.21387600 & 2.36049300 & -5.23718100 \\
\hline Pt 40 & 1.60373700 & -2.72720600 & -1.05796400 \\
\hline Pt 41 & 0.42469900 & 1.72375800 & -0.16741200 \\
\hline Pt 42 & -2.82593700 & -1.13703200 & -0.8058340 \\
\hline Pt 43 & -0.00546900 & -4.13553000 & -5.15924500 \\
\hline Pt 44 & 3.46320000 & 0.99714100 & 3.32590700 \\
\hline Pt 45 & -3.12961800 & 1.44994500 & 5.08568800 \\
\hline Pt 46 & -1.40450200 & 0.16157100 & 1.08674000 \\
\hline Pt 47 & 2.83541800 & 2.12613600 & 0.93333500 \\
\hline Pt 48 & -0.65118400 & 0.53525200 & 5.6505920 \\
\hline Pt 49 & 4.68160900 & 0.12159700 & 1.03652500 \\
\hline Pt50 & -4.13711500 & -1.07757200 & -4.32681900 \\
\hline Pt51 & 2.19622900 & -3.92244700 & 1.2971480 \\
\hline Pt52 & -1.93679800 & -5.64863100 & -0.2576920 \\
\hline Pt53 & 2.55955000 & 1.80202700 & -1.74756800 \\
\hline Pt 54 & -0.90626500 & 3.70667000 & -1.63880300 \\
\hline Pt55 & -4.79431800 & -1.48867100 & 0.9446650 \\
\hline Pt5 6 & -0.81211200 & -0.98895200 & 3.43418300 \\
\hline Pt57 & -3.41842500 & 4.85219400 & 1.0581210 \\
\hline Pt58 & 1.22192600 & 2.11999400 & 4.4228910 \\
\hline Pt59 & 0.12434100 & 1.38258400 & -2.8286270 \\
\hline & 5.25641900 & 2.57501500 & 2.0262120 \\
\hline
\end{tabular}




\begin{tabular}{|c|c|c|c|}
\hline Pt 61 & -0.47682700 & -4.39879300 & 1.68152900 \\
\hline Pt 62 & 2.86522100 & -1.48009900 & 2.33534400 \\
\hline Pt 63 & 4.67793600 & 1.91541700 & -3.34653100 \\
\hline Pt 64 & 1.61323400 & -0.58277900 & 4.59429500 \\
\hline 6 & -5.11765900 & -1.82746800 & -1.87501100 \\
\hline 666 & -1.64784400 & 4.38699200 & 3.06108400 \\
\hline$=67$ & 1.93689800 & -0.62751000 & -2.75687700 \\
\hline Pt 68 & 0.73035700 & -5.23580300 & -0.62446900 \\
\hline 69 & 3.97717200 & 4.50224700 & 0.61081600 \\
\hline 570 & 3.74421100 & 4.16185800 & -2.09081800 \\
\hline$=71$ & 4.05397400 & -2.33588300 & 0.02342600 \\
\hline$=7$ & -0.96530700 & -4.95417500 & -2.73764900 \\
\hline$=73$ & 4.05034200 & -0.53545400 & -4.36264100 \\
\hline Pt 7 & 2.26214600 & 1.48333100 & -4.47330500 \\
\hline C & 0.26285500 & -0.34157600 & -4.92932200 \\
\hline$=76$ & 3.04817100 & 3.69460000 & 3.11421100 \\
\hline 577 & 1.31028200 & 3.72481900 & -3.24081900 \\
\hline 78 & -0.69402600 & 5.21978800 & 0.63416600 \\
\hline \# 78 & Luster $=$ Pt79 $\quad \mathrm{Q}=0$ & $I=0 \quad$ Etot $=-7401$ & 99731200 \\
\hline Pt 1 & 3.95998700 & 3.59171200 & 1.47444500 \\
\hline Pt2 & 2.88060900 & 1.89502400 & -0.32314700 \\
\hline Pt 3 & 2.42026400 & -0.20409600 & 3.87638100 \\
\hline Pt 4 & 4.02235400 & -1.80354100 & 2.37085200 \\
\hline Pt 5 & -0.05883300 & -4.80913100 & 3.797408 \\
\hline Pt 6 & -0.44249800 & -2.62117700 & -5.00327900 \\
\hline Pt 7 & -0.28862300 & -0.21981900 & 3.49147300 \\
\hline Pt 8 & 5.13108600 & 3.13598000 & -1.01639700 \\
\hline Pt9 & -1.22378700 & 4.48043400 & 1.5065020 \\
\hline Pt10 & -3.13579800 & -4.41626900 & -1.7561080 \\
\hline Pt11 & -1.89497500 & 1.44081800 & 5.025380 \\
\hline Pt12 & 1.65472200 & 3.38398700 & -5.06239500 \\
\hline Pt13 & 1.44092700 & 5.12367700 & -2.97777400 \\
\hline Pt14 & 2.35097100 & 5.85196900 & 1.50735000 \\
\hline Pt15 & 0.85995800 & -2.37512400 & 4.62398100 \\
\hline Pt16 & -4.19354900 & -3.89483600 & 0.7333150 \\
\hline Pt17 & 3.20853900 & 1.12064800 & -4.90089700 \\
\hline Pt18 & 1.32196300 & 0.8321 & 1.61327800 \\
\hline Pt19 & 4.03121500 & 0.89666500 & 1.9264120 \\
\hline Pt20 & -0.82363800 & -0.66197800 & 0.87874000 \\
\hline Pt21 & 1.70664700 & -3.77881000 & -3.8609740 \\
\hline Pt22 & -2.78309800 & -2.44860200 & -3.6129970 \\
\hline Pt & -3.31102200 & -1.46787600 & 1.53802800 \\
\hline Pt24 & -3.93106300 & 2.48055100 & -1.8565140 \\
\hline Pt25 & -2.85809000 & 3.36564800 & 3.3796090 \\
\hline Pt26 & -4.41680000 & 1.20262200 & 4.10905000 \\
\hline Pt27 & -1.40510000 & -1.12263700 & -1.73892600 \\
\hline Pt28 & 1.52113600 & -4.76706900 & -1.32522900 \\
\hline Pt29 & -3.63738000 & -3.43858300 & 3.35868500 \\
\hline Pt30 & 2.99100500 & -3.53371400 & 0.57450200 \\
\hline Pt31 & 3.22966900 & -1.56255500 & -4.45215700 \\
\hline Pt32 & -0.68217200 & 3.63987800 & -3.68251100 \\
\hline Pt33 & 2.45241300 & -3.97161300 & 3.16528000 \\
\hline Pt3 4 & 3.06605300 & 2.88943500 & -2.84030100 \\
\hline Pt35 & -0.63184700 & -3.61209700 & -2.4718020 \\
\hline Pt36 & -2.43220800 & 0.93481900 & 2.35212400 \\
\hline ו & -3.62176600 & 0.02824200 & -2.94029000 \\
\hline & 5.31314300 & -1.22970200 & -2.6890530 \\
\hline & -5.84521400 & -1.69230800 & 0.72 \\
\hline
\end{tabular}




\begin{tabular}{|c|c|c|c|}
\hline et 40 & 0.50087200 & -4.30903000 & 1.22460000 \\
\hline Pt 41 & -1.14542100 & -2.63437300 & 2.68908000 \\
\hline Pt 42 & 2.90315600 & -0.79777600 & 0.12765800 \\
\hline Pt 43 & 0.38766300 & 5.51593800 & -0.42430100 \\
\hline Pt 44 & -4.98626400 & 0.77393200 & 1.51662200 \\
\hline Pt 45 & 5.16794800 & 0.48872300 & -0.57308000 \\
\hline Pt 46 & 2.90729200 & 4.61963300 & -0.78819100 \\
\hline Pt 47 & 0.78389500 & 4.80550300 & 3.39515600 \\
\hline Pt 48 & -2.99291400 & 0.48290900 & -0.28161400 \\
\hline Pt 49 & -0.32378500 & 2.43479600 & 3.07664100 \\
\hline Pt 50 & 0.73378800 & 3.05140600 & -1.46421700 \\
\hline Pt51 & 2.38341500 & 2.53742200 & 3.42378700 \\
\hline Pt52 & -3.91564200 & -1.93008300 & -1.12284700 \\
\hline Pt53 & 0.79774300 & 1.39644000 & 5.32274200 \\
\hline Pt 54 & 0.78821700 & -2.30406300 & -0.57314400 \\
\hline Pt5 5 & -1.29887700 & -0.18668900 & -4.27591900 \\
\hline Pt 56 & 5.29036500 & 1.42565400 & -3.12999300 \\
\hline Pt57 & -0.98607400 & -5.57421400 & -0.61349600 \\
\hline Pt58 & 3.05485100 & 0.16517400 & -2.37518300 \\
\hline Pt59 & 0.91073500 & -1.34575800 & -3.07728400 \\
\hline Pt 60 & -3.41772400 & 2.94052500 & 0.77339900 \\
\hline Pt61 & 0.88853000 & 1.33205900 & -3.52661700 \\
\hline Pt 62 & -5.29298000 & -1.24844000 & 3.31946900 \\
\hline Pt 63 & -0.46849100 & 1.88456400 & -5.76208300 \\
\hline Pt 64 & -2.79441500 & 2.13741000 & -4.37408600 \\
\hline Pt 65 & 3.11537200 & -2.56441600 & -1.92967600 \\
\hline Pt 66 & 0.74180700 & 0.38568000 & -1.00478900 \\
\hline Pt 67 & -1.66069200 & -3.20496500 & 5.25008800 \\
\hline Pt 68 & -5.52661400 & 0.32627400 & -1.07569800 \\
\hline Pt 69 & -2.02417300 & -5.06336600 & 1.88637200 \\
\hline Pt 70 & -1.69924600 & -3.08668200 & 0.06888100 \\
\hline Pt71 & -1.78335900 & 4.02867300 & -1.16412600 \\
\hline Pt72 & 1.06142600 & -0.39463500 & -5.60461200 \\
\hline Pt73 & 1.33444800 & -1.84801600 & 2.01844700 \\
\hline Pt 74 & 1.27248000 & 3.47330400 & 1.12585200 \\
\hline Pt75 & -0.75347500 & -0.77062100 & 6.05616500 \\
\hline Pt 76 & -2.77785800 & -0.99734900 & 4.21556200 \\
\hline Pt77 & -0.87969500 & 2.01805100 & 0.44306200 \\
\hline Pt78 & -1.42168000 & 1.53178900 & -2.17106100 \\
\hline Pt79 & 5.18017500 & -2.15808700 & -0.13546600 \\
\hline \# 79 & luster $=\operatorname{Pt} 80 \quad Q=0$ & $=0 \quad$ Etot $=-75$ & 60953500 \\
\hline Pt1 & 5.02837600 & -2.29621100 & -0.20579800 \\
\hline Pt2 & -3.46429900 & -1.80011200 & 2.59269300 \\
\hline Pt3 & -3.54050200 & -0.24540000 & -1.93041600 \\
\hline Pt 4 & 1.66654500 & -3.28425200 & 1.30334900 \\
\hline Pt5 & 4.96653500 & 1.07013500 & 1.79948100 \\
\hline Pt 6 & -0.00484700 & 5.92131300 & 1.31700500 \\
\hline Pt 7 & -0.11309400 & -2.86135900 & -0.68862200 \\
\hline Pt 8 & -5.07426400 & 1.87675800 & -1.18782200 \\
\hline Pt9 & -0.25301900 & -3.49896700 & -4.44886200 \\
\hline Pt10 & 3.44200900 & 3.26491300 & 2.59674200 \\
\hline Pt11 & 1.21305500 & 1.04554500 & -4.03338300 \\
\hline Pt12 & 3.35896900 & -4.45436000 & -0.4414450 \\
\hline Pt13 & 0.75950800 & 5.64066300 & -1.35903100 \\
\hline Pt14 & 0.82186300 & 0.31529000 & 5.34469600 \\
\hline Pt15 & 2.47092200 & -1.94615200 & -0.9378540 \\
\hline Pt16 & 0.37286400 & 0.94888300 & 2.82122400 \\
\hline Pt17 & -1.39713600 & 4.54336500 & -2.7735580 \\
\hline
\end{tabular}




\begin{tabular}{|c|c|c|c|}
\hline Pt18 & 0.68336900 & -1.46786600 & -2.92075900 \\
\hline Pt19 & 4.22457800 & -3.63191800 & 2.04132200 \\
\hline Pt20 & 0.76900600 & -5.35948200 & -0.19692700 \\
\hline Pt21 & -0.98081400 & -0.35684900 & -1.16537400 \\
\hline Pt22 & -1.76033100 & -0.08210100 & 1.46257300 \\
\hline Pt23 & -4.28744200 & 2.76348000 & 1.32761100 \\
\hline Pt24 & 1.22258500 & 3.77435000 & -3.39571000 \\
\hline Pt25 & -3.95709200 & 0.18666500 & -4.52254600 \\
\hline Pt26 & 1.81613600 & 5.37130100 & 3.32313200 \\
\hline Pt27 & 0.78756900 & -0.83708700 & 0.81654800 \\
\hline Pt28 & -4.43781800 & -2.27442500 & -3.45663300 \\
\hline Pt29 & -1.29726000 & 1.97046500 & 4.63883000 \\
\hline Pt30 & -4.34423400 & 0.02742700 & 0.72261700 \\
\hline Pt31 & 1.62790600 & 3.17251600 & -0.81033500 \\
\hline Pt32 & -4.34027400 & -3.83581700 & 1.04061700 \\
\hline Pt33 & -3.37182000 & 3.59299300 & 3.79931000 \\
\hline Pt34 & 0.24444300 & -1.04128300 & -5.51692300 \\
\hline Pt35 & 4.20899100 & 2.98532900 & -0.08067700 \\
\hline Pt36 & 1.25153700 & -1.51294100 & 3.37612000 \\
\hline Pt37 & 1.34029900 & 2.87419900 & 4.39687300 \\
\hline Pt38 & -1.35179100 & -0.76455200 & 4.03424500 \\
\hline Pt39 & -0.78730200 & 4.52519600 & 3.61193900 \\
\hline Pt 40 & -0.48019300 & 2.10512400 & -2.19330900 \\
\hline Pt41 & -1.81123100 & -4.64889900 & 0.42520700 \\
\hline Pt 42 & -3.50172900 & 4.15814100 & -0.97019300 \\
\hline Pt 43 & -2.66991600 & -2.08439200 & -0.04734600 \\
\hline Pt 44 & -2.55393800 & 1.72565400 & -0.41767200 \\
\hline Pt 45 & -0.43528800 & -3.30917800 & 4.46072800 \\
\hline Pt 46 & 3.32503200 & 4.82333600 & -1.94369500 \\
\hline Pt 47 & 4.20247400 & 0.25289900 & -0.71222100 \\
\hline Pt 48 & 0.00460400 & -5.07503000 & 2.43395500 \\
\hline Pt 49 & 1.56171800 & -4.02900700 & -2.46633100 \\
\hline Pt50 & -3.60509100 & -4.11548500 & -1.57651300 \\
\hline Pt51 & 2.32306000 & -2.60969700 & -4.66629100 \\
\hline Pt52 & -5.21703100 & -2.04540500 & -0.83771800 \\
\hline Pt53 & 5.82614400 & -1.44625100 & 2.25539500 \\
\hline Pt5 4 & -0.88577200 & -2.56214200 & 1.94124900 \\
\hline Pt5 5 & -1.70902800 & 2.55863700 & 2.05185200 \\
\hline Pt5 6 & -2.55097400 & -4.31205400 & 3.03216600 \\
\hline Pt57 & 3.31221200 & -1.12085900 & 1.53683400 \\
\hline Pt58 & -2.82639400 & -4.33450500 & -4.17301400 \\
\hline Pt59 & -1.40948200 & 0.14567600 & -3.75637700 \\
\hline Pt 60 & 2.94345700 & 0.70138600 & 3.6226830 \\
\hline Pt 61 & -3.47364900 & 0.90414700 & 3.24813200 \\
\hline Pt 62 & -0.93912700 & 3.92349700 & -0.20081000 \\
\hline Pt 63 & -1.82697000 & 6.33602500 & -0.71992000 \\
\hline Pt 64 & 4.11724500 & -3.08488600 & -2.67763800 \\
\hline Pt 65 & -3.04518800 & 2.28552300 & -3.00996300 \\
\hline Pt 66 & -1.89219300 & -2.33324800 & -2.65299500 \\
\hline Pt 67 & 3.36305200 & 2.15702800 & -2.60767200 \\
\hline Pt 68 & 3.82525300 & -1.85065100 & 4.05826300 \\
\hline Pt 69 & 2.55801300 & -5.76280700 & 1.80443200 \\
\hline Pt70 & -0.92682300 & 2.63600000 & -4.74045700 \\
\hline Pt71 & -0.05853100 & 1.54717100 & 0.3186100 \\
\hline Pt72 & -1.04037500 & -4.87904200 & -2.20503900 \\
\hline Pt73 & -2.63984200 & 5.01573000 & 1.5562830 \\
\hline Pt74 & 2.14318700 & -4.00683900 & 3.8312960 \\
\hline Pt75 & -2.34899000 & -1.89057100 & -5.24651100 \\
\hline Pt76 & 3.29004000 & -0.55972900 & -3.1882270 \\
\hline
\end{tabular}




\begin{tabular}{|c|c|c|c|}
\hline Pt77 & 1.61392000 & 0.53318100 & -1.42002200 \\
\hline Pt78 & 0.87573900 & 3.44306700 & 1.81369000 \\
\hline Pt79 & 2.43754100 & 1.33860400 & 1.04835600 \\
\hline Pt 80 & 2.61134000 & 5.15020200 & 0.6965510 \\
\hline \# 80 & Cluster $=$ Pt $81 \quad Q=0$ & $\mathrm{M}=0 \quad \mathrm{Etot}=-76$ & 34552900 \\
\hline Pt 1 & -1.61892300 & 0.25874800 & -1.54587900 \\
\hline Pt2 & -3.54027500 & -5.24018600 & 1.93230500 \\
\hline Pt3 & -0.45437500 & -1.52405100 & -0.02091400 \\
\hline Pt 4 & 3.45914200 & 0.18481000 & 3.0988790 \\
\hline Pt 5 & -0.28812900 & 4.74984900 & -1.48021400 \\
\hline Pt 6 & 2.40677400 & 4.77638800 & -0.92997900 \\
\hline Pt 7 & -4.47971900 & -2.65866700 & 1.6641520 \\
\hline Pt 8 & -0.06342500 & 2.20953500 & -2.48772900 \\
\hline Pt9 & 0.59419100 & 5.28936500 & 1.06810800 \\
\hline Pt10 & -2.38364700 & -2.33838400 & -1.56007400 \\
\hline Pt11 & 3.18678900 & 2.27321900 & -4.54521800 \\
\hline Pt12 & 2.70554500 & -2.49089900 & 3.09878600 \\
\hline Pt13 & 5.54850200 & 0.67395300 & -0.12216900 \\
\hline Pt14 & -4.32951400 & -3.17377100 & -3.1103640 \\
\hline Pt15 & 2.15580500 & 0.30975000 & -6.11779800 \\
\hline Pt16 & 1.86178300 & -5.10342700 & 2.9967790 \\
\hline Pt17 & -1.98924100 & -3.36625900 & 0.9521640 \\
\hline Pt18 & 2.30536300 & -4.63449800 & -1.76423500 \\
\hline Pt19 & 1.96992000 & -2.21333800 & -0.69185900 \\
\hline Pt20 & 0.51050500 & 2.23197800 & -5.1086390 \\
\hline Pt21 & -4.88079600 & -1.60823400 & -0.90036600 \\
\hline Pt22 & 0.83930900 & 2.81518800 & 0.03863200 \\
\hline Pt23 & 3.03812900 & 1.24125300 & 0.57316600 \\
\hline Pt24 & -3.61457600 & 1.90375300 & 4.32348700 \\
\hline Pt25 & 4.01329000 & -1.36066000 & 0.88029300 \\
\hline Pt26 & 2.55292100 & -2.31542200 & -3.33057000 \\
\hline Pt27 & -1.10198900 & 0.23251800 & -4.19198700 \\
\hline Pt28 & 1.07173000 & 0.33375700 & -0.95890800 \\
\hline Pt29 & 5.11254200 & 1.66360600 & -2.64591600 \\
\hline Pt30 & -4.27474200 & 3.59845900 & 0.18079800 \\
\hline Pt31 & -1.20093100 & 1.04230200 & 3.56124100 \\
\hline Pt32 & -2.13439500 & -4.77116600 & -2.64099100 \\
\hline Pt33 & -3.17950900 & 1.65443400 & 1.68675700 \\
\hline Pt34 & -1.21854900 & 5.72456500 & 3.04055600 \\
\hline Pt35 & 4.17671200 & 4.19762400 & -2.90439700 \\
\hline Pt36 & -1.68028700 & 1.28522100 & 6.13968600 \\
\hline Pt37 & -1.16503200 & 4.10161500 & -3.9844400 \\
\hline Pt38 & -3.42893200 & -0.62287200 & 3.2902480 \\
\hline Pt39 & 1.48471600 & -0.70890200 & 1.53970200 \\
\hline Pt 40 & -2.86537800 & -0.79018300 & 0.65064300 \\
\hline Pt41 & 0.10788800 & -6.27263400 & -2.09029300 \\
\hline Pt 42 & 3.19682100 & -4.04011000 & 0.82285500 \\
\hline Pt4 3 & -0.95514200 & -1.39609600 & 2.5357130 \\
\hline Pt 44 & 3.19057800 & 2.69241600 & 4.0776730 \\
\hline Pt 45 & -2.10398400 & 5.20708100 & 0.52187500 \\
\hline Pt 46 & 1.00898000 & -5.75088800 & 0.45854100 \\
\hline Pt 47 & 0.99494000 & 4.23740300 & 3.58109100 \\
\hline Pt 48 & 1.60232300 & 0.30403800 & -3.5427650 \\
\hline Pt 49 & 4.42760700 & -2.88306000 & -1.3843390 \\
\hline Pt50 & 4.61536800 & 3.21653700 & -0.4062800 \\
\hline Pt51 & 1.51436600 & 4.17924300 & -3.4655920 \\
\hline Pt52 & -3.38706600 & 4.12393700 & 2.72533700 \\
\hline Pt53 & -5.29377300 & -0.02221200 & 1.34447100 \\
\hline
\end{tabular}




\begin{tabular}{|c|c|c|c|}
\hline Pt5 4 & -1.89365200 & -2.45786900 & -4.20115700 \\
\hline Pt5 5 & 1.01791900 & -0.51450100 & 4.18811700 \\
\hline Pt5 6 & -0.96311600 & 3.26781400 & 1.99958900 \\
\hline Pt57 & 1.24411000 & 1.75068900 & 2.54207400 \\
\hline Pt58 & 2.83053800 & 3.77783100 & 1.59723700 \\
\hline Pt59 & 3.57899800 & -0.31447900 & -1.66836900 \\
\hline Pt 60 & -0.72126200 & 0.84177300 & 0.97504000 \\
\hline Pt 61 & -1.85831600 & 2.71604500 & -0.51886100 \\
\hline Pt 62 & 0.60765100 & -1.68735900 & -5.16933900 \\
\hline Pt 63 & -1.47113800 & -1.20892600 & 5.12959400 \\
\hline Pt 64 & 0.70256600 & -3.30025100 & 1.48269700 \\
\hline Pt 65 & 2.61007100 & 2.25635700 & -1.92378200 \\
\hline Pt 66 & -0.17135100 & -3.88074100 & -1.05161300 \\
\hline Pt 67 & 0.36205600 & -4.03452600 & -3.68252200 \\
\hline Pt 68 & -3.98947300 & -4.24620100 & -0.59572800 \\
\hline Pt 69 & 0.05391700 & -1.62677000 & -2.57719500 \\
\hline Pt70 & 0.75646000 & 2.01764000 & 5.16695500 \\
\hline Pt71 & -1.73604800 & -5.82300500 & -0.07990800 \\
\hline Pt72 & 0.20729200 & -3.19847400 & 4.12353700 \\
\hline Pt73 & -4.09655100 & 1.08138900 & -0.88412500 \\
\hline Pt74 & -2.77003700 & 2.12656800 & -3.06716100 \\
\hline Pt75 & -3.60441300 & -0.52405800 & -3.14015900 \\
\hline Pt76 & 4.09936200 & -0.27769700 & -4.30117300 \\
\hline Pt77 & -2.53954400 & -3.26914700 & 3.58229500 \\
\hline Pt78 & -2.96262200 & 4.61120400 & -2.00589000 \\
\hline Pt79 & -0.84607900 & -5.22586000 & 2.50380500 \\
\hline Pt 80 & 4.98861400 & 2.18432100 & 2.09914300 \\
\hline Pt 81 & -1.45616000 & 3.53161000 & 4.62487600 \\
\hline \# 81 & Cluster $=$ Pt $82 \quad \mathrm{Q}=0$ & \multicolumn{2}{|c|}{$\mathrm{M}=0 \quad$ Etot $=-7706.51235200$} \\
\hline Pt 1 & 5.16336000 & -2.16648100 & 0.23348700 \\
\hline Pt2 & -5.83345700 & 2.85281900 & 0.92244900 \\
\hline Pt3 & -4.93044600 & 1.63032400 & 3.16126200 \\
\hline Pt 4 & -2.56138600 & 2.34688100 & 4.23699300 \\
\hline Pt 5 & 2.20444500 & -2.76810900 & 3.85293300 \\
\hline Pt 6 & 2.14688200 & 2.40435700 & 4.00532800 \\
\hline Pt 7 & 1.21814700 & 3.62714900 & 1.73254900 \\
\hline Pt 8 & 2.09866700 & -4.15179900 & 1.51219100 \\
\hline Pt9 & 2.65635700 & -4.26520600 & -3.16149100 \\
\hline Pt10 & 3.55230700 & 3.00184200 & 0.43744100 \\
\hline Pt11 & 4.47991700 & -3.40565000 & 2.54039500 \\
\hline Pt12 & 2.69472700 & 5.55852300 & 0.56175500 \\
\hline Pt13 & -1.27509900 & 1.59142100 & -1.68525400 \\
\hline Pt14 & 4.38081200 & -4.75635900 & 0.19993800 \\
\hline Pt15 & -2.76690300 & -0.36763500 & -0.48560800 \\
\hline Pt16 & -0.26092800 & 1.70594600 & 2.92601000 \\
\hline Pt17 & 2.00330200 & -5.48762300 & -0.85570800 \\
\hline Pt18 & -3.47366500 & 3.59430900 & 1.99939100 \\
\hline Pt & 2.05412700 & 1.06127800 & 1.63736700 \\
\hline Pt20 & -2.08336400 & 4.19363100 & -1.57675200 \\
\hline Pt21 & 0.21288000 & 3.54938100 & -2.87241000 \\
\hline Pt22 & 1.13647000 & 2.30619000 & -0.62330300 \\
\hline Pt23 & 0.54751500 & -0.87471700 & 2.82021700 \\
\hline Pt24 & -2.68083700 & -5.52803700 & -0.6128960 \\
\hline Pt25 & 1.74066900 & -3.00461400 & -5.38240700 \\
\hline Pt26 & -3.67938800 & 0.85842900 & -2.74708900 \\
\hline Pt27 & -1.84007100 & -1.58237700 & 1.7688260 \\
\hline Pt28 & 3.59431000 & 4.33706400 & 2.78684700 \\
\hline Pt29 & -2.60248000 & -4.19284100 & 1.73616800 \\
\hline
\end{tabular}




\begin{tabular}{|c|c|c|c|}
\hline Pt 30 & 2.95000700 & -0.18246400 & 3.90610500 \\
\hline Pt31 & 3.45644000 & 1.65240100 & -1.91549900 \\
\hline Pt32 & 5.01795200 & -3.53111800 & -2.11155500 \\
\hline Pt33 & -2.18608000 & 2.81050100 & -3.91701300 \\
\hline Pt34 & -1.93908300 & -2.93954400 & -0.58454300 \\
\hline Pt35 & -2.05178500 & -4.30498900 & -2.94375500 \\
\hline Pt36 & -0.57217300 & -2.36712900 & -4.14436100 \\
\hline Pt37 & -4.46666100 & 3.43594900 & -2.59181400 \\
\hline Pt38 & -0.16334300 & 3.04325300 & 5.26049100 \\
\hline Pt39 & 4.26299700 & -0.93402600 & -2.01515900 \\
\hline Pt 40 & 0.12044500 & 2.14906000 & -5.18403800 \\
\hline Pt 41 & 2.61661500 & 4.23397900 & -1.80211200 \\
\hline Pt 42 & -0.46972200 & -1.00165500 & -1.78645500 \\
\hline Pt 43 & 1.83561900 & -1.65557100 & -3.05416000 \\
\hline Pt 44 & -0.09492100 & -2.12038400 & 5.12063300 \\
\hline Pt 45 & -0.29493300 & -4.86124200 & 0.45815200 \\
\hline$=46$ & 0.27211200 & -4.93774500 & -4.20142000 \\
\hline Pt 47 & -4.32660400 & -3.64464100 & -1.60565400 \\
\hline Pt 48 & 0.45391300 & -2.23810600 & 0.47561900 \\
\hline Pt 49 & -3.55051200 & 2.21375900 & -0.36736400 \\
\hline Pt 50 & 2.84768000 & -1.52078800 & 1.52334400 \\
\hline Pt51 & 5.21323900 & -0.81711200 & $2.5952850 c$ \\
\hline Pt 52 & -1.17346300 & 2.95323700 & 0.67706000 \\
\hline Pt53 & -0.39247700 & -6.17112100 & -1.90201500 \\
\hline Pt 54 & -2.87656500 & -1.72661500 & -2.85403900 \\
\hline Pt 55 & -4.13995700 & -0.95000300 & 3.06847400 \\
\hline Pt 56 & 0.31137300 & 4.90341000 & -0.52221200 \\
\hline Pt57 & -4.25258800 & -2.31725200 & 0.73439000 \\
\hline Pt 58 & -2.65324300 & 0.99688400 & $1.8725370 \mathrm{C}$ \\
\hline $\mathrm{P}$ & -1.08961000 & 4.30092300 & 3.04922700 \\
\hline Pt 60 & -5.15519000 & -1.07605500 & -1.52429500 \\
\hline Pt 61 & 0.65079700 & 0.46993600 & 5.17811800 \\
\hline Pt 62 & 2.74967100 & -2.87263800 & -0.81679100 \\
\hline Pt 63 & -2.48463600 & -2.82867500 & 4.0683050 \\
\hline Pt 64 & -1.38485300 & 0.21748300 & -4.04739800 \\
\hline $\mathrm{P}$ & -4.36840600 & 4.77898200 & -0.25898000 \\
\hline Pt 66 & 4.11249000 & -2.30162700 & -4.33795800 \\
\hline Pt 67 & -1.98671300 & 5.52565800 & 0.79243600 \\
\hline Pt 68 & -5.93473700 & 1.51273600 & -1.40933700 \\
\hline Pt 69 & 1.02394600 & 0.93514900 & -2.9546840 \\
\hline Pt 70 & 3.31579500 & 0.27963200 & -4.2604460 \\
\hline Pt 71 & 0.34896700 & -3.58418600 & -1.87395800 \\
\hline Pt72 & -1.76220200 & -0.21922300 & 4.14930700 \\
\hline Pt73 & -0.19540500 & -3.50670800 & 2.8040040 \\
\hline Pt74 & 1.31560400 & 4.97174000 & 4.0705900 \\
\hline Pt75 & 4.37144900 & 0.42163500 & 0.3376760 \\
\hline Pt76 & 1.95394400 & -0.28330100 & -0.71917600 \\
\hline Pt77 & -0.35505600 & 0.36034500 & 0.56375400 \\
\hline Pt78 & 4.43372900 & 1.76601900 & $2.6982550 c$ \\
\hline Pt79 & -5.06707300 & 0.26620200 & 0.8286100 \\
\hline Pt 80 & 2.50063800 & 2.85452900 & -4.1359030 \\
\hline Pt 81 & 0.41563200 & 6.19693000 & 1.84770600 \\
\hline Pt 82 & 0.94007100 & -0.42451200 & $-5.3066130 c$ \\
\hline \# 82 & Cluster $=$ Pt $83 \quad \mathrm{Q}=0$ & \multicolumn{2}{|c|}{$\mathrm{M}=0 \quad$ Etot $=-7803.33200700$} \\
\hline Pt 1 & 4.10496300 & -1.02671200 & 4.1334080 \\
\hline Pt2 & 2.34221100 & 2.09998300 & 1.5570330 \\
\hline Pt3 & 4.81642900 & 3.17034700 & 1.1555740 \\
\hline Pt 4 & -2.99118100 & 3.16521300 & -1.14895 \\
\hline
\end{tabular}




\begin{tabular}{|c|c|c|c|}
\hline Pt 5 & -2.08848500 & 3.53614800 & -3.67249600 \\
\hline Pt 6 & -2.06532600 & 5.06276100 & 0.53017900 \\
\hline Pt 7 & 4.58838400 & 0.66096800 & 2.08969300 \\
\hline Pt 8 & -1.68076600 & -6.03046900 & 0.64849000 \\
\hline Pt9 & 2.09184500 & -1.92746800 & -1.70187400 \\
\hline Pt10 & -4.54659100 & -3.09314800 & -2.76324800 \\
\hline Pt11 & -4.29732900 & 1.84933200 & 3.14858800 \\
\hline Pt12 & 4.09668800 & -3.44311200 & -2.64065900 \\
\hline Pt13 & 0.13079000 & 2.07037200 & -3.13894400 \\
\hline Pt14 & 4.16516200 & 3.79385000 & -1.40744800 \\
\hline Pt15 & -0.37704500 & -3.01228900 & -1.30858500 \\
\hline Pt16 & -1.95146600 & -3.64442600 & -3.42682000 \\
\hline Pt17 & 2.98561200 & -1.52046900 & -4.21401300 \\
\hline Pt18 & -1.44274100 & 4.41288200 & 3.08576300 \\
\hline Pt19 & -5.44683000 & -3.52683800 & -0.24042100 \\
\hline Pt20 & 1.65929300 & -2.10072200 & 4.53837200 \\
\hline Pt21 & -5.21786000 & -1.00011100 & -1.14352600 \\
\hline Pt22 & 3.22041100 & -3.89192800 & -0.11428600 \\
\hline Pt23 & 0.77456700 & 2.94544500 & 3.64326100 \\
\hline Pt24 & -4.01759000 & 4.36354100 & 2.23497600 \\
\hline Pt25 & -4.94592600 & 2.45204600 & 0.56159500 \\
\hline Pt26 & -2.32577500 & 2.48461400 & 1.40792400 \\
\hline Pt27 & -1.72156700 & -1.11589100 & -4.36420200 \\
\hline Pt28 & -1.44724600 & 1.43307700 & -5.24564200 \\
\hline Pt29 & 2.99364500 & 1.45865800 & 4.13500100 \\
\hline Pt30 & -0.80664100 & -5.58386600 & -1.87886900 \\
\hline Pt31 & -5.21505900 & -0.08434700 & 1.45295000 \\
\hline Pt32 & 1.65140000 & -4.52845900 & -2.26985300 \\
\hline Pt33 & -0.13424600 & -0.47589800 & -2.25417500 \\
\hline Pt3 4 & 1.69152100 & 2.73152200 & -1.02368500 \\
\hline Pt35 & -2.86668400 & -4.12693300 & -0.91995700 \\
\hline Pt36 & -0.79854000 & -3.16714200 & 4.90731200 \\
\hline Pt37 & 4.58343800 & -0.84375600 & -2.12859800 \\
\hline Pt38 & 0.37095600 & 4.59163500 & -4.05548000 \\
\hline Pt39 & 6.11458800 & -0.13260300 & -0.00642500 \\
\hline Pt 40 & 2.09847900 & -0.44804100 & 2.45094700 \\
\hline Pt41 & -1.01247600 & -0.90122200 & 0.27734400 \\
\hline Pt 42 & 1.92891100 & 5.25657700 & -1.92981800 \\
\hline Pt 43 & 3.23456900 & 1.03042000 & -5.10149000 \\
\hline Pt 44 & -4.93327000 & 1.52998300 & -2.05112400 \\
\hline Pt 45 & 1.02718500 & 5.46646500 & 2.69302800 \\
\hline Pt 46 & -1.28025300 & -3.44776500 & 1.18344000 \\
\hline Pt 47 & -2.35271200 & 1.02248400 & -2.7509060 \\
\hline Pt 48 & 2.33646700 & 0.60862100 & -2.62350500 \\
\hline Pt 49 & 4.80967400 & 1.68940400 & -2.99688700 \\
\hline Pt50 & 3.89683500 & 1.26904500 & -0.50476800 \\
\hline Pt51 & -2.59208400 & -1.53911700 & -1.82901900 \\
\hline Pt52 & -3.45988300 & -2.00072900 & 0.67072800 \\
\hline Pt53 & 0.39396300 & 6.09801000 & 0.14254500 \\
\hline Pt5 4 & -3.76336500 & -4.58927300 & 1.5911450 \\
\hline Pt55 & 2.60987700 & 3.16723500 & -3.54506900 \\
\hline Pt5 6 & 0.13681100 & 3.56191000 & 1.03671200 \\
\hline Pt57 & -4.54034100 & -0.69117200 & 4.03068000 \\
\hline Pt58 & -1.94107500 & -0.68228700 & 4.8820860 \\
\hline Pt59 & -1.67665000 & -5.11161400 & 3.23052800 \\
\hline Pt 60 & -3.21213900 & 0.56133900 & -0.24097000 \\
\hline Pt 61 & -0.37104300 & -1.53640400 & 2.8389790 \\
\hline Pt 62 & 5.63879900 & -2.76089100 & -0.51348100 \\
\hline Pt 63 & 0.52509800 & -2.60990700 & -3.8365210 \\
\hline
\end{tabular}




\begin{tabular}{|c|c|c|c|}
\hline Pt64 & -2.58737900 & -0.06792200 & 2.31881100 \\
\hline Pt 65 & 1.42508100 & 0.18585400 & -0.11519600 \\
\hline Pt 66 & 0.76642500 & -0.05796500 & -4.76925500 \\
\hline Pt 67 & -5.44423100 & -2.61340000 & 2.34500200 \\
\hline$t 68$ & 1.18943500 & -2.36503100 & 0.79543200 \\
\hline Pt 69 & 0.77237000 & -4.05769600 & 2.85790600 \\
\hline Pt70 & 3.22359300 & -2.97303800 & 2.46271600 \\
\hline Pt71 & 0.76881200 & -4.98020900 & 0.26965800 \\
\hline$t 72$ & -0.12303000 & 1.02407000 & 1.96656500 \\
\hline Pt73 & 5.64143600 & -1.84608400 & 2.05073600 \\
\hline$t 74$ & 3.24427200 & 3.99464300 & 3.2053460 \\
\hline Pt75 & -0.53537100 & 4.20552300 & -1.54585300 \\
\hline Pt76 & -0.77533600 & 1.64855800 & -0.63743200 \\
\hline Pt77 & -1.71601200 & 1.87638900 & 4.00819300 \\
\hline$=78$ & 3.64163900 & -1.24765400 & 0.39384600 \\
\hline$t 79$ & 1.01588600 & 2.49279600 & -5.63114400 \\
\hline t80 & 0.53353700 & 0.37824000 & 4.5390400 \\
\hline Pt81 & 2.61889100 & 4.66262200 & 0.64790000 \\
\hline Pt82 & -2.86008900 & -2.64686700 & 3.24626300 \\
\hline Pt83 & -4.29831600 & -0.56170900 & -3.67509500 \\
\hline \# 83 & er $=$ Pt 84 & $=0 \quad$ Etot $=-7 \mathrm{~s}$ & 01971900 \\
\hline Pt1 & 0.49607600 & -0.65220500 & -1.20838700 \\
\hline Pt2 & -0.76752600 & 1.04634900 & 2.9868610 \\
\hline Pt3 & -3.34120800 & 0.47945200 & -3.73950800 \\
\hline Pt 4 & 0.39088000 & 3.09468500 & 1.60682400 \\
\hline Pt5 & -3.96115500 & -2.24688800 & -1.0514480 \\
\hline Pt 6 & 3.07802400 & -0.37943300 & -4.0922040 \\
\hline Pt 7 & 3.20982400 & 3.18678000 & 4.16288000 \\
\hline Pt 8 & -0.89974200 & 1.11869200 & 0.26581100 \\
\hline Pt9 & 4.87377300 & -2.40329400 & 2.55593000 \\
\hline Pt10 & 2.05099000 & -2.52954900 & 0.01007400 \\
\hline Pt11 & 3.44982300 & -4.30537900 & -1.50054400 \\
\hline Pt12 & -1.66639700 & -1.49568400 & 0.2189090 \\
\hline Pt13 & 1.52447000 & 1.46292100 & -5.28974700 \\
\hline Pt14 & -4.45308000 & 2.10772500 & 3.15924500 \\
\hline Pt15 & 1.65549000 & 1.39788500 & -2.58079600 \\
\hline Pt16 & -2.41369200 & -4.11204800 & $0.1683940 c$ \\
\hline Pt17 & 2.79843800 & 3.42245600 & -3.93590800 \\
\hline Pt18 & 0.01784000 & -3.44126500 & 4.1507490 \\
\hline Pt19 & -4.05264100 & -2.13174600 & -3.75067800 \\
\hline Pt20 & 1.65852100 & 5.04703000 & 2.94424000 \\
\hline Pt21 & 2.06933100 & 174800 & 5.5178520 \\
\hline Pt22 & -2.24484400 & -4.17143500 & 2.8854920 \\
\hline Pt23 & -4.73918300 & 2.25207500 & -2.2167250 \\
\hline Pt24 & 1.90326500 & -2.43735500 & -2.6893320 \\
\hline Pt25 & 4.35984900 & 1.56869300 & -2.73670800 \\
\hline Pt26 & -2.52275500 & -4.01106800 & -2.5506270 \\
\hline Pt27 & -2.60605300 & 3.09225200 & -3.6672490 \\
\hline Pt28 & 1.31840900 & -5.14894000 & -0.0595840 \\
\hline Pt29 & 1.42823200 & -5.19661000 & 2.6490850 \\
\hline Pt30 & 0.64051600 & -0.74019500 & 1.49562900 \\
\hline Pt31 & -2.30382700 & 2.90025300 & 1.75400500 \\
\hline Pt32 & 3.11516600 & 3.30140900 & 1.4695730 \\
\hline Pt33 & -2.43969700 & 2.97791400 & -0.9427260 \\
\hline Pt34 & -3.05466800 & 0.30136200 & 1.6999800 \\
\hline Pt & 6.01776700 & -0.37570400 & 1.1972610 \\
\hline Pt36 & 5.87547900 & -0.30309400 & -1.5095160 \\
\hline Pt37 & 3.58551600 & -4.38175500 & 1.2281720 \\
\hline
\end{tabular}




\begin{tabular}{|c|c|c|c|}
\hline Pt 38 & -5.18184300 & -0.49848700 & 3.12918000 \\
\hline Pt39 & -3.82330800 & -2.32364600 & 1.65716500 \\
\hline Pt 40 & 4.76146900 & -2.35763400 & -0.15267200 \\
\hline Pt 41 & -1.29704900 & 5.04262900 & -2.30978800 \\
\hline Pt 42 & -1.37620900 & -1.63856900 & 5.61100700 \\
\hline Pt 43 & -0.83495100 & -5.93123600 & 1.38253400 \\
\hline Pt 44 & -1.05372100 & 1.21770400 & -2.46047700 \\
\hline Pt 45 & -1.03957300 & 4.87507200 & 3.06695500 \\
\hline Pt 46 & -3.64289400 & -2.36875300 & 4.34815400 \\
\hline Pt 47 & -0.39737000 & -3.19695100 & -3.99824100 \\
\hline Pt 48 & 1.53576200 & 5.15165900 & 0.24596200 \\
\hline Pt 49 & -4.62888400 & 2.17164200 & 0.47088900 \\
\hline Pt 50 & 2.98020000 & 3.38050200 & -1.23911800 \\
\hline Pt 51 & 4.63664200 & 1.40742000 & 2.69944100 \\
\hline Pt 52 & 2.18905600 & -2.60890800 & 2.73710400 \\
\hline Pt53 & -3.19280400 & 0.37866900 & -1.00888600 \\
\hline Pt 54 & 0.36407100 & -0.57927400 & -3.90393700 \\
\hline Pt 55 & 1.16493100 & -5.01993800 & -2.77423900 \\
\hline Pt 56 & 4.52478200 & 1.51300700 & -0.01 \\
\hline Pt 57 & 1.92955300 & 1.23429200 & 2.82 \\
\hline Pt 58 & -5.34684200 & -0.42810100 & 0.4 \\
\hline Pt 59 & 4.57843800 & -2.25259200 & -2.86 \\
\hline Pt 60 & 1.39539800 & 5.20251700 & -2.45 \\
\hline Pt 61 & -1.91766400 & -1.32763400 & -5.1 \\
\hline Pt 62 & -0.62783500 & 0.96379300 & 5.6 \\
\hline Pt 63 & 0.25542800 & 3.17416700 & -1.10 \\
\hline Pt 64 & 0.788 & -0.83226500 & 4.21 \\
\hline Pt & 0.51252100 & 3.03307600 & 4.31 \\
\hline Pt 66 & -1.79274200 & -1.39761300 & -2.487567 \\
\hline Pt 67 & 1.79821000 & -2.39635500 & -5.34560400 \\
\hline Pt 68 & -0.96488600 & -5.84861100 & $-1.32457^{\prime}$ \\
\hline Pt 69 & 0.09842700 & 3.26798000 & -3.83250400 \\
\hline Pt 70 & -1.18001700 & 1.28301600 & -5.17 \\
\hline Pt 71 & 3.33830800 & -0.55301900 & 1.34076400 \\
\hline Pt 72 & -1.15349100 & 4.98916000 & 0.37692700 \\
\hline Pt 73 & -5.45568100 & -0.35168600 & -2.26885200 \\
\hline Pt 74 & -0.24537500 & -3.26283400 & -1.26706500 \\
\hline Pt 75 & -0.10411600 & -3.34380900 & 1.43 \\
\hline Pt 76 & -3.97239000 & 4.83080900 & -2.07239200 \\
\hline Pt 77 & 0.27584300 & -0.55899500 & -6.55918000 \\
\hline Pt 78 & -3.83078100 & 4.74524000 & 0.59947700 \\
\hline Pt 79 & 3.20017300 & -0.46951100 & -1.36022100 \\
\hline Pt 80 & 1.81360300 & 1.32556900 & 0.12482200 \\
\hline Pt 81 & -2.93053900 & 0.24177100 & 4.42 \\
\hline Pt 82 & 3.49706700 & -0.62063900 & 4.05668300 \\
\hline Pt 83 & -2.18596000 & 2.84483100 & 4.45170900 \\
\hline Pt 84 & -1.51287100 & -1.55649800 & 2.9216980 \\
\hline \# 84 & Cluster $=$ Pt $85 \quad Q=0$ & Etot $=-80$ & 14628600 \\
\hline Pt 1 & -2.75998400 & -3.46339700 & -3.7721120 \\
\hline Pt2 & 2.84361200 & 1.29047000 & -3.4487180 \\
\hline Pt 3 & 1.82814600 & 3.27167600 & 0.8005340 \\
\hline Pt 4 & 3.38637900 & 1.62494600 & -0.7316250 \\
\hline Pt 5 & 0.78808200 & -2.02447700 & -3.3451100 \\
\hline Pt 6 & -0.44219500 & 5.07025500 & -1.6666840 \\
\hline Pt 7 & 5.24637200 & 1.63620800 & 1.2359370 \\
\hline Pt 8 & -1.71451000 & 0.65304700 & -0.8497340 \\
\hline Pt 9 & -3.20159400 & -1.09464700 & 2.1188900 \\
\hline Pt 10 & 0.76610000 & -2.43056200 & 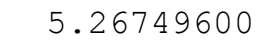 \\
\hline
\end{tabular}




\begin{tabular}{|c|c|c|c|}
\hline Pt11 & 4.24548000 & -2.68328600 & -1.75670900 \\
\hline Pt12 & -1.49097500 & 0.22964100 & 3.78864300 \\
\hline Pt13 & 3.66877200 & 3.26949400 & 2.76702100 \\
\hline Pt14 & -2.44849900 & 1.48298100 & 1.56543200 \\
\hline Pt15 & 0.78814200 & -5.61813600 & -1.95791600 \\
\hline Pt16 & 1.56533300 & -2.76076200 & -5.73925300 \\
\hline Pt17 & 0.33449400 & 0.58362800 & -2.74829200 \\
\hline Pt18 & -3.44515800 & 2.69180900 & -0.71310200 \\
\hline Pt19 & 1.09737400 & 4.13907800 & 3.26346700 \\
\hline Pt20 & 1.44184000 & -5.22038800 & 0.74019400 \\
\hline Pt21 & 1.23840600 & 2.93275300 & -1.84245900 \\
\hline Pt22 & 2.73147400 & 0.90637200 & 1.83498200 \\
\hline Pt23 & -3.15748800 & 2.93374000 & -4.47826600 \\
\hline Pt24 & 3.64373500 & -1.50305600 & 2.86060000 \\
\hline Pt25 & 1.82826000 & -1.42820500 & 0.90592000 \\
\hline Pt26 & 0.29057000 & 0.19655000 & 5.80441900 \\
\hline Pt27 & 1.54999100 & 4.92450800 & -3.55289900 \\
\hline Pt28 & -4.91659500 & -2.46914200 & 0.44495100 \\
\hline Pt29 & -1.35814100 & 2.62592700 & -2.56954800 \\
\hline Pt30 & -4.25866700 & 0.12924200 & -0.13356300 \\
\hline Pt31 & 0.15529100 & 5.37813300 & 0.98303600 \\
\hline Pt32 & 2.52970500 & -4.04916600 & -3.50383300 \\
\hline Pt33 & 1.70957700 & -3.26727800 & -1.08052200 \\
\hline Pt3 4 & 2.76858700 & 5.61010600 & 1.71058700 \\
\hline Pt35 & -1.03927000 & -2.44748200 & 3.26945500 \\
\hline Pt36 & 1.06500000 & -0.61828700 & 3.33784600 \\
\hline Pt37 & -1.54268800 & 3.88494400 & 2.53735600 \\
\hline Pt38 & -0.72614500 & -0.56552700 & 1.38630000 \\
\hline Pt39 & 0.90026300 & 0.95644100 & -0.10377000 \\
\hline Pt 40 & -1.81748300 & -1.60458100 & 5.69958400 \\
\hline Pt41 & 4.34034700 & 3.99571300 & 0.18798100 \\
\hline Pt 42 & -3.57824500 & -1.00400400 & -4.76501200 \\
\hline Pt4 3 & -1.04775100 & 4.64382400 & -4.28296200 \\
\hline Pt 44 & -4.13115300 & 3.53051000 & 1.75951300 \\
\hline Pt 45 & -3.43810400 & -4.21055500 & -1.14925400 \\
\hline Pt 46 & 4.52235500 & 0.88389800 & 3.78664000 \\
\hline Pt 47 & -1.02139400 & -1.89576600 & -5.31567900 \\
\hline Pt 48 & 0.16853900 & 1.78878100 & 2.33499100 \\
\hline Pt 49 & -1.85713800 & -5.84580300 & -2.70060700 \\
\hline Pt50 & 2.46859900 & -0.69701200 & -1.63756300 \\
\hline Pt51 & -2.43757400 & -1.89336700 & -0.29959000 \\
\hline Pt52 & -4.95402500 & 0.97092000 & 2.36397800 \\
\hline Pt53 & -1.77558000 & -1.15025000 & -2.8609480 \\
\hline Pt5 4 & 3.30448000 & -1.41257900 & -4.06741000 \\
\hline Pt5 5 & -4.31027400 & -1.75813500 & -2.19681200 \\
\hline Pt5 6 & 1.56467800 & -3.32083300 & 2.80192000 \\
\hline Pt57 & -0.78846300 & 3.01604200 & 0.06095100 \\
\hline Pt58 & -2.26659400 & 1.01960700 & 6.22815700 \\
\hline Pt59 & -0.15109600 & -4.34969300 & -4.2715460 \\
\hline Pt 60 & -5.66486400 & -1.60543500 & 2.8922230 \\
\hline Pt 61 & 0.63437900 & 2.55450500 & -4.51500700 \\
\hline Pt 62 & -0.91830100 & -3.55845000 & -1.83101900 \\
\hline Pt 63 & 1.99208300 & 1.76593600 & 4.33177300 \\
\hline Pt 64 & 4.37291600 & -0.77271000 & 0.2989530 \\
\hline Pt 65 & -3.02241200 & 4.71594300 & -2.42237200 \\
\hline Pt 66 & -0.57939900 & -5.05473900 & 2.63757000 \\
\hline Pt 67 & 2.17638300 & 5.30591600 & -0.92266000 \\
\hline Pt 68 & 3.41403400 & -5.25855800 & -1.19287200 \\
\hline Pt 69 & -3.99668500 & -0.29023500 & 4.5926650 \\
\hline
\end{tabular}




\begin{tabular}{|c|c|c|c|}
\hline Pt70 & -2.79504900 & -3.80681900 & 1.56596300 \\
\hline Pt71 & 2.84609700 & -0.63789200 & 5.29966900 \\
\hline Pt72 & 3.58722500 & -3.42668000 & 0.85042200 \\
\hline Pt73 & 3.74638700 & 3.67138900 & -2.46303500 \\
\hline Pt74 & -1.23864900 & -5.52028800 & -0.02540000 \\
\hline Pt75 & -3.56745800 & -2.91796200 & 4.03783400 \\
\hline Pt76 & 0.01440600 & -1.30295600 & -0.97924800 \\
\hline Pt77 & -0.27513000 & -3.17664500 & 0.80973900 \\
\hline Pt78 & -3.21980100 & 2.29414400 & 4.02201800 \\
\hline Pt79 & -1.49810000 & 0.80391300 & -4.69986500 \\
\hline Pt 80 & 1.12017000 & -0.09787300 & -5.20082400 \\
\hline Pt 81 & 5.84756800 & 2.33059100 & -1.33879300 \\
\hline Pt 82 & -0.59402800 & 2.59653400 & 4.78193600 \\
\hline Pt 83 & 4.95235500 & -0.03434500 & -2.28478700 \\
\hline Pt 84 & -3.56712900 & 0.87597400 & -2.77315700 \\
\hline Pt 85 & -2.47019900 & 5.06187500 & 0.22299300 \\
\hline \# 85 & Cluster $=$ Pt $86 \quad Q=0$ & $\Lambda=0 \quad$ Etot $=-81$ & 95321400 \\
\hline Pt 1 & -2.53081900 & 2.37744400 & -1.22799500 \\
\hline Pt2 & 1.89419300 & 3.27724600 & -1.14900900 \\
\hline Pt3 & 0.57281700 & -1.64130700 & -2.40739500 \\
\hline Pt 4 & -0.40203800 & 2.77013700 & 1.93566700 \\
\hline Pt 5 & 1.61418700 & 4.65066700 & -3.44363900 \\
\hline Pt 6 & 1.57211300 & 4.51707000 & 1.34923500 \\
\hline Pt 7 & -3.40831900 & -0.18764400 & -1.05965400 \\
\hline Pt 8 & -3.80083000 & -3.10329400 & 2.70373800 \\
\hline Pt 9 & -2.08951700 & -2.15378800 & -2.41483800 \\
\hline Pt10 & -1.45945500 & 0.89054100 & 3.63030300 \\
\hline Pt11 & 0.72392800 & 4.05222400 & 3.98617500 \\
\hline Pt12 & 3.74339300 & 1.23255300 & -0.90556800 \\
\hline Pt13 & -1.22357000 & 0.40892700 & -2.63228500 \\
\hline Pt14 & -0.09455300 & 1.60703600 & -0.49939200 \\
\hline Pt15 & 4.56125400 & 1.12289600 & 1.75656800 \\
\hline Pt16 & 0.34740200 & -1.16403100 & 3.85589000 \\
\hline Pt17 & -0.55215400 & -2.86749800 & -4.52833500 \\
\hline Pt18 & 3.76375100 & -3.70640200 & -2.73736800 \\
\hline Pt19 & 4.95627100 & -1.36064100 & 2.91436400 \\
\hline Pt20 & -0.92982800 & 5.34492500 & 682100 \\
\hline Pt21 & 1.08871600 & -4.26311700 & -2.81694800 \\
\hline Pt22 & 1.70198900 & -0.40988700 & -0.27614500 \\
\hline Pt23 & 1.15385000 & -4.90722800 & 1.07361100 \\
\hline Pt24 & -2.45556000 & -5.26871200 & -0.29247700 \\
\hline Pt25 & 3.11147500 & -3.18345700 & 1.98984100 \\
\hline Pt26 & -4.74071600 & 1.79526700 & 0.34054100 \\
\hline Pt27 & -5.43383200 & -1.87510400 & -0.66432900 \\
\hline Pt28 & 4.02301300 & 3.56136400 & 0.52794700 \\
\hline Pt29 & -1.63350100 & -2.88161800 & 4.43149700 \\
\hline Pt30 & -0.35408800 & 2.13423400 & 5.72228300 \\
\hline Pt31 & 3.21302500 & -1.10382200 & -2.31996200 \\
\hline Pt 32 & -2.88928600 & 3.62504600 & 1.25709600 \\
\hline Pt33 & -5.82668600 & 0.58190700 & -1.82314400 \\
\hline Pt34 & -2.77878800 & 3.77248400 & -3.53807100 \\
\hline Pt35 & 1.48844700 & 0.03531900 & 5.95510400 \\
\hline Pt36 & -2.02253300 & 3.49906300 & 3.92656700 \\
\hline Pt37 & 3.70636000 & 0.65272700 & 4.3670810 \\
\hline Pt38 & 2.52844000 & -0.55991500 & 2.2960010 \\
\hline Pt39 & 2.13299800 & -2.33887100 & -4.48541200 \\
\hline Pt 40 & -4.94864500 & 3.15059300 & -1.99419600 \\
\hline Pt41 & 0.03634700 & -6.09819100 & -1.08988800 \\
\hline
\end{tabular}




\begin{tabular}{|c|c|c|c|}
\hline Pt 42 & 3.23783900 & 3.14957400 & 3.16231400 \\
\hline Pt 43 & -2.29145400 & 1.01020100 & 1.05199900 \\
\hline Pt 44 & -1.25931000 & -0.51801200 & 5.89540100 \\
\hline Pt 45 & 5.67971900 & -1.95212300 & -1.83957600 \\
\hline Pt 46 & -0.88666700 & 5.56793500 & -2.62122400 \\
\hline Pt 47 & 0.12231300 & 0.23053600 & 1.68493000 \\
\hline Pt 48 & -2.35748800 & -0.80450600 & -4.75313500 \\
\hline Pt 49 & -3.22011100 & -3.37330600 & -4.51646800 \\
\hline Pt50 & -3.10455800 & 4.97844300 & -1.11586600 \\
\hline Pt5: & -1.34363600 & -4.07630500 & 1.90783000 \\
\hline Pt52 & 2.80498900 & -1.99660200 & 4.53062000 \\
\hline Pt53 & 2.19316500 & -2.98929400 & -0.64190000 \\
\hline Pt5 4 & 1.23519200 & 1.43276600 & 3.68515400 \\
\hline Pt55 & 0.34122400 & -0.27101900 & -4.75182100 \\
\hline Pt 5 & 1.47214600 & 0.94501400 & -2.56943800 \\
\hline Pt57 & -4.09322400 & -3.86681200 & -1.99507000 \\
\hline Pt58 & 5.20943300 & 0.50342100 & -3.03045000 \\
\hline Pt59 & 3.45315200 & 2.59727500 & -3.26643000 \\
\hline Pt 60 & -1.60466100 & -4.76626500 & -2.82826300 \\
\hline Pt 61 & 1.29988600 & 5.86625400 & -1.02417000 \\
\hline Pt 62 & 0.57711100 & -2.56789800 & 6.05851500 \\
\hline Pt 63 & 2.70372500 & -5.56199900 & -1.04158900 \\
\hline Pt 64 & -3.95668000 & 1.70054400 & 3.01261900 \\
\hline Pt 65 & 4.20146100 & -1.22053700 & 0.28481400 \\
\hline Pt 66 & -0.33483000 & 2.96253200 & -2.74767600 \\
\hline Pt 67 & -3.47807100 & -0.78391500 & 4.20274300 \\
\hline Pt 68 & -3.04904100 & 1.55895400 & 5.60584700 \\
\hline Pt 69 & -0.97412400 & -0.95295100 & -0.33416400 \\
\hline Pt70 & -4.34494400 & -0.66227200 & 1.53537800 \\
\hline Pt71 & 1.23293500 & 2.32765400 & -4.86603100 \\
\hline Pt72 & -0.47399500 & -3.52989800 & -0.69351500 \\
\hline Pt73 & -1.82807100 & -1.42373600 & 2.20415200 \\
\hline Pt7 4 & 4.65989400 & -3.84864500 & -0.16299400 \\
\hline Pt75 & -4.55133400 & -1.40597900 & -3.19679500 \\
\hline Pt76 & 2.07126800 & 1.88141000 & 1.12927400 \\
\hline Pt77 & 4.78205100 & -1.79837800 & -4.38678300 \\
\hline Pt78 & 0.63229000 & -2.31507300 & 1.41435400 \\
\hline Pt79 & -2.98993000 & -2.66730700 & 0.14199000 \\
\hline Pt 80 & -3.69130000 & 1.18999300 & -3.41400000 \\
\hline Pt 81 & 0.88061000 & -3.79621400 & 3.61239300 \\
\hline Pt 82 & -0.57373700 & 4.35292400 & -5.02465700 \\
\hline Pt 83 & 3.02735600 & 0.26098800 & -4.65941100 \\
\hline Pt 84 & -0.65633100 & 4.21020000 & -0.30565900 \\
\hline Pt 85 & -1.46790700 & 1.79128600 & -4.92468000 \\
\hline Pt 86 & 2.35439600 & 2.64599600 & 5.72115800 \\
\hline \# 86 & Cluster $=$ Pt $87 \quad Q=0$ & $\mathrm{M}=0 \quad \mathrm{Etot}=-82$ & 10240900 \\
\hline Pt 1 & 2.04202900 & 0.66463700 & 2.76331100 \\
\hline Pt2 & -1.12035800 & -2.35691100 & 4.56313300 \\
\hline Pt 3 & 0.03602900 & 2.21414300 & 3.63408200 \\
\hline Pt 4 & -3.67549600 & -4.81874900 & -0.47464300 \\
\hline Pt 5 & 4.52626600 & 2.96294700 & 0.88136200 \\
\hline Pt 6 & -2.74516000 & 0.12092400 & 0.78209600 \\
\hline Pt 7 & 4.46199800 & 1.57380800 & 3.3353430 \\
\hline Pt 8 & -1.51714300 & 1.34162000 & 5.6525590 \\
\hline Pt9 & -3.77376200 & 1.59343900 & 4.15501700 \\
\hline Pt10 & 5.61182100 & -3.59268000 & -1.2279430 \\
\hline Pt11 & -1.66248500 & 2.26151000 & -0.53283700 \\
\hline Pt12 & 3.27082600 & -4.72897200 & -0.2895920 \\
\hline
\end{tabular}




\begin{tabular}{|c|c|c|c|}
\hline Pt13 & 2.32673900 & 1.93270000 & 5.06201900 \\
\hline Pt14 & 0.06713400 & 3.54523000 & 1.18051200 \\
\hline Pt15 & -0.14551600 & -4.11038900 & 2.65836800 \\
\hline Pt16 & -2.14749900 & -2.27539400 & 1.99752400 \\
\hline Pt17 & 0.33106800 & 6.17784900 & 1.04614200 \\
\hline Pt18 & -4.61689400 & -0.90333100 & -2.41435000 \\
\hline Pt19 & 1.72172200 & 1.67290800 & -3.44070300 \\
\hline Pt20 & -2.11249300 & -0.08097300 & -1.87117700 \\
\hline Pt21 & -5.25327900 & -0.70028800 & 0.25580300 \\
\hline Pt22 & 0.35151700 & -1.46892900 & 2.51986900 \\
\hline Pt23 & -2.71199000 & -4.87851000 & 2.09614300 \\
\hline Pt24 & -1.58860300 & -3.79518100 & -4.65368600 \\
\hline Pt25 & 2.70370700 & -0.89297100 & -3.94782500 \\
\hline Pt26 & 3.65752200 & -2.07189200 & -0.36135300 \\
\hline Pt27 & -4.27683200 & -0.75473700 & 2.82825000 \\
\hline Pt28 & 5.52343200 & 1.22584800 & -1.11515400 \\
\hline Pt29 & -0.04207000 & -0.47868600 & -3.63945100 \\
\hline Pt30 & 0.59946900 & -0.21705900 & 4.88818500 \\
\hline Pt31 & 1.60840400 & -2.80603800 & 4.42516900 \\
\hline Pt32 & 3.45747600 & 3.01318600 & -1.71754600 \\
\hline Pt33 & 5.06504600 & -1.18157000 & -2.46871200 \\
\hline Pt3 4 & -1.46906400 & 0.85759200 & -5.52040300 \\
\hline Pt35 & -3.71159900 & 3.80665500 & 0.27720100 \\
\hline Pt36 & 3.04806300 & -1.89988300 & 2.35420100 \\
\hline Pt37 & 2.11000500 & 2.00782600 & 0.38414600 \\
\hline Pt38 & -3.20111500 & -0.80572700 & 5.33605700 \\
\hline Pt39 & 0.94990900 & -3.01554700 & -4.12690000 \\
\hline Pt 40 & -4.80117400 & 1.65242600 & 1.60051400 \\
\hline Pt41 & -1.66419300 & -5.21345600 & -2.30303600 \\
\hline Pt 42 & 2.60948200 & -4.53540200 & 2.42390200 \\
\hline Pt 43 & 0.39869900 & 0.71418400 & -1.31225600 \\
\hline Pt 44 & 3.32205500 & -3.34953100 & -2.71744400 \\
\hline Pt 45 & 0.95896600 & 5.97698400 & -1.59634400 \\
\hline Pt 46 & 4.39870800 & 1.25708000 & -3.63674100 \\
\hline Pt 47 & -2.39479800 & 3.36198400 & -5.00713300 \\
\hline Pt 48 & -0.66785600 & -1.39453100 & -0.01690100 \\
\hline Pt 49 & 2.42667400 & 4.65602300 & 0.25518400 \\
\hline Pt50 & -3.52962600 & 1.24633800 & -3.73486200 \\
\hline Pt51 & -3.64044600 & -3.43550600 & -2.88321200 \\
\hline Pt52 & -4.67688900 & -3.10448600 & 1.46930100 \\
\hline Pt53 & -3.07645800 & 3.60356300 & -2.38799400 \\
\hline Pt5 4 & -1.39341200 & 4.94615100 & -0.67372800 \\
\hline Pt5 5 & -2.59389100 & -1.29193500 & -4.2388780 \\
\hline Pt5 6 & 3.35839000 & -0.60816600 & 4.67569900 \\
\hline Pt57 & -1.70061700 & 0.05016100 & 3.32748500 \\
\hline Pt58 & 0.85834300 & -5.74267300 & 0.66311100 \\
\hline Pt59 & -0.99733500 & 2.03032000 & -3.14694800 \\
\hline Pt 60 & -2.03076000 & 3.74163700 & 4.41896800 \\
\hline Pt61 & 3.06809800 & 0.33180900 & -1.55093100 \\
\hline Pt 62 & -1.11714800 & -2.60425200 & -2.33374500 \\
\hline Pt 63 & -0.72978300 & 4.69944500 & -3.32454600 \\
\hline Pt 64 & -1.16297300 & -4.03050000 & 0.06282200 \\
\hline Pt 65 & 6.13528100 & -1.25052600 & 0.11168200 \\
\hline Pt 66 & 4.16567700 & 0.30978200 & 0.9968350 \\
\hline Pt 67 & -3.13981600 & -2.20563900 & -0.54485700 \\
\hline Pt 68 & -1.99757000 & 5.10519600 & 1.9977930 \\
\hline Pt 69 & 1.95687300 & 4.35242800 & -3.5742950 \\
\hline Pt70 & 1.37355000 & -1.79341400 & -1.78857000 \\
\hline Pt71 & -0.22679500 & 0.91522100 & 1.3031580 \\
\hline
\end{tabular}




\begin{tabular}{|c|c|c|c|}
\hline Pt72 & -3.65093300 & -3.1373730 & 4.00248000 \\
\hline Pt73 & -0.51087700 & -1.6643790 & -5.96260800 \\
\hline Pt74 & 0.28005600 & 3.0018800 & -5.27874800 \\
\hline Pt75 & -5.63207600 & -3.0385260 & -1.07538800 \\
\hline Pt76 & 0.27786300 & 4.8905770 & 3.47248400 \\
\hline Pt77 & 2.40598700 & 3.3546580 & 2.69412900 \\
\hline Pt78 & 0.69465100 & 3.3438980 & -1.45433000 \\
\hline Pt79 & 1.31130500 & -3.1415930 & 0.58273300 \\
\hline Pt 80 & 0.90771000 & -4.4438870 & -1.76654700 \\
\hline Pt 81 & 5.48579800 & -1.0195160 & 2.85716100 \\
\hline Pt 82 & 1.75347000 & -0.5749960 & 0.50771400 \\
\hline Pt83 & -2.24951600 & 2.4309670 & 2.10520300 \\
\hline Pt 84 & -4.28566800 & 3.9665010 & 2.92840300 \\
\hline Pt 85 & 1.21006600 & 0.4693190 & -5.79388600 \\
\hline Pt 86 & -4.21620000 & 1.4685870 & -1.08076700 \\
\hline Pt 87 & 5.03028800 & -3.4352380 & 1.45372100 \\
\hline \# 87 & Cluster $=\operatorname{Pt} 88 \quad \mathrm{Q}=0$ & $=0 \quad$ Etot $=-\varepsilon$ & 53177400 \\
\hline Pt 1 & 5.54080900 & 0.7025680 & 2.45998200 \\
\hline Pt2 & -2.99527400 & 3.5359810 & 3.92080000 \\
\hline Pt3 & 2.64757900 & -0.0513570 & -5.53567200 \\
\hline Pt 4 & 1.81466200 & 5.2550900 & -0.00277700 \\
\hline Pt5 & 3.70680500 & -0.5197150 & 4.02799000 \\
\hline Pt 6 & 5.26496300 & 3.0856970 & -2.30263800 \\
\hline Pt 7 & -0.43098100 & 2.6444830 & 3.90639400 \\
\hline Pt 8 & -4.64314900 & 3.2133040 & 1.79192400 \\
\hline Pt 9 & -1.14422700 & 4.7783910 & 2.37327500 \\
\hline Pt10 & 1.38806500 & 0.1351560 & -0.54098900 \\
\hline Pt11 & -0.73392700 & 6.1493860 & 0.05207300 \\
\hline Pt12 & 5.81380300 & -2.2263030 & -1.83913000 \\
\hline Pt13 & -3.24628000 & -1.0708080 & 4.79953100 \\
\hline Pt14 & 4.92418200 & 1.6905950 & 0.02669300 \\
\hline Pt15 & 2.08687100 & 2.3398560 & -4.33757100 \\
\hline Pt16 & -1.77289200 & -4.7124020 & 1.38392700 \\
\hline Pt17 & 3.95269200 & 2.9187810 & 2.24644300 \\
\hline Pt18 & 0.14634400 & -6.1129590 & 0.10348600 \\
\hline Pt19 & 3.87798400 & -0.9945060 & -3.32845300 \\
\hline Pt20 & 3.25307900 & 1.3476340 & -2.09287400 \\
\hline Pt21 & -2.29338700 & 1.3904300 & 5.42451300 \\
\hline Pt22 & -0.71769200 & -2.0042010 & 4.76793800 \\
\hline Pt23 & 0.18451200 & 4.9136010 & -2.18279700 \\
\hline Pt24 & -2.43667800 & 1.7792230 & -5.40863000 \\
\hline Pt25 & -5.68955100 & 0.5276810 & -1.56387600 \\
\hline Pt26 & -4.47142800 & 4.0623020 & -1.85675700 \\
\hline Pt27 & -2.54424000 & -2.7792610 & -2.87080100 \\
\hline Pt28 & -2.37219000 & 5.7725740 & -2.08208500 \\
\hline Pt29 & 1.16205200 & -0.7771290 & 3.16397900 \\
\hline Pt30 & 1.41728800 & 3.8791140 & 2.31630600 \\
\hline Pt31 & -3.96580800 & 1.0764090 & 3.30932300 \\
\hline Pt 32 & 4.64276700 & -1.7891260 & 1.79775100 \\
\hline Pt33 & 0.91922000 & -4.1955140 & 4.87572500 \\
\hline Pt34 & -1.83537600 & -4.9117290 & -1.32987700 \\
\hline Pt35 & 0.48232000 & 1.3943530 & 1.69005800 \\
\hline Pt36 & -3.72679600 & 1.9529980 & -0.40088500 \\
\hline Pt37 & -4.89012400 & -1.3966000 & 2.6425240 \\
\hline Pt38 & 0.27153100 & -5.8578250 & 2.7985970 \\
\hline Pt39 & 0.10324300 & 0.8626830 & -5.49127500 \\
\hline Pt 40 & -1.38550100 & 0.1558860 & 3.2338340 \\
\hline Pt41 & -3.87947500 & 1.7425550 & -3.13592900 \\
\hline
\end{tabular}




\begin{tabular}{|c|c|c|c|}
\hline Pt 42 & 1.28294500 & -0.07450400 & -3.24317500 \\
\hline Pt 43 & -2.43580600 & -2.53975500 & -0.13448300 \\
\hline Pt 44 & 2.78959500 & -3.00859200 & 3.34660900 \\
\hline Pt 45 & 2.32470600 & 2.59920600 & 0.09773900 \\
\hline Pt 46 & -3.24034100 & -0.60135200 & -4.34894000 \\
\hline Pt 47 & -2.82037600 & 4.46076800 & 0.26059200 \\
\hline Pt 48 & 2.73799100 & 4.01097400 & -2.24944000 \\
\hline Pt 49 & -1.16033000 & 1.03737500 & -0.46839100 \\
\hline Pt50 & -0.68674900 & -1.52096200 & -4.4313150 \\
\hline Pt51 & -0.46565300 & -1.07889800 & 1.0086340 \\
\hline Pt52 & 1.98587300 & -2.22182900 & -1.74385900 \\
\hline Pt53 & 1.86581800 & -2.43724500 & -4.44613600 \\
\hline Pt5 4 & 3.94574200 & -0.76376600 & -0.61912100 \\
\hline Pt55 & 4.61590100 & 1.41985900 & -4.3841710 \\
\hline Pt5 6 & 3.83610500 & -3.67260700 & -3.0069810 \\
\hline Pt57 & 0.02567700 & -3.70590900 & -2.90388300 \\
\hline Pt58 & 3.99188000 & -3.45965000 & -0.28963800 \\
\hline Pt59 & 2.12936200 & 1.69326300 & 3.842609 \\
\hline Pt 60 & 0.11970100 & -3.45235400 & -0.17882700 \\
\hline Pt 61 & 0.25801500 & 0.46102200 & 5.3836000 \\
\hline Pt 62 & -5.01883700 & -1.62622200 & -0.0677280 \\
\hline Pt 63 & 1.84856000 & -1.74416900 & 5.56247200 \\
\hline Pt 64 & -0.46401800 & 3.24912800 & -4.26262500 \\
\hline Pt 65 & -4.14403900 & -3.52286700 & 4.11151700 \\
\hline Pt 66 & -3.01745900 & 4.11647000 & -4.15145500 \\
\hline Pt 67 & 0.68941300 & 2.26740100 & -2.02955800 \\
\hline Pt 68 & 3.02762900 & 0.45187000 & 1.59704000 \\
\hline Pt 69 & -1.88459300 & 3.15840000 & -1.93999400 \\
\hline Pt70 & 6.46266000 & -0.56099000 & 0.24174700 \\
\hline Pt71 & 0.20668900 & -3.20865300 & 2.50341500 \\
\hline Pt72 & 1.99566100 & -4.91141800 & -1.45962800 \\
\hline Pt73 & 4.35272500 & 4.32495400 & -0.0672870 \\
\hline Pt7 4 & 2.08806200 & -1.99699800 & 0.94708600 \\
\hline Pt75 & -4.38287900 & -3.97231500 & -1.27646200 \\
\hline Pt76 & 2.14047500 & -4.67457800 & 1.26489000 \\
\hline Pt77 & -1.28376700 & 0.84007400 & -3.17866000 \\
\hline Pt78 & -4.29793300 & -3.76990900 & 1.42689200 \\
\hline Pt79 & -2.08270800 & 2.29456300 & 1.73936200 \\
\hline Pt 80 & -5.58503000 & 0.75396800 & 1.13812400 \\
\hline Pt 81 & -1.65039200 & -4.45782100 & 4.08218900 \\
\hline Pt 82 & -0.57867400 & -1.32872200 & -1.71253700 \\
\hline Pt 83 & -3.03396300 & -0.17649600 & 1.08729600 \\
\hline Pt 84 & -5.08279000 & -1.82169300 & -2.7738390 \\
\hline Pt 85 & 5.86452500 & 0.45278000 & -2.19102700 \\
\hline Pt 86 & -0.24060500 & 3.51738000 & 0.16674600 \\
\hline Pt 87 & -3.13290300 & -0.40079600 & -1.62860300 \\
\hline Pt8 8 & -2.32165900 & -2.30568100 & 2.56915900 \\
\hline \# 88 & Cluster $=\operatorname{Pt} 89 \quad \mathrm{Q}=0$ & Etot $=-8424$ & 22308200 \\
\hline Pt1 & -1.60824200 & 2.37076000 & 3.9154830 \\
\hline Pt2 & 5.28450700 & -0.53829200 & -1.10675600 \\
\hline Pt 3 & 0.31559400 & 4.80738100 & 0.57222800 \\
\hline Pt 4 & 0.33745200 & -0.01771100 & 6.27223900 \\
\hline Pt 5 & 0.68946900 & -5.83526300 & -0.1112820 \\
\hline Pt 6 & -5.44190700 & -0.47756000 & 1.5286270 \\
\hline Pt 7 & 0.44338900 & 3.15534700 & -3.65857100 \\
\hline Pt 8 & -4.88164100 & 3.72498100 & -0.0942520 \\
\hline Pt9 & -2.01741000 & -5.62067900 & -0.30261500 \\
\hline Pt10 & -1.23601500 & 4.89442600 & 2.8558910 \\
\hline
\end{tabular}




\begin{tabular}{|c|c|c|c|}
\hline Pt11 & 0.10292000 & 2.66159000 & -1.03618300 \\
\hline Pt12 & -1.95244200 & -0.21283300 & 4.83694400 \\
\hline Pt13 & 4.04591800 & -1.00928100 & 3.47168300 \\
\hline Pt14 & 3.35420300 & -0.33565000 & -5.13594900 \\
\hline Pt15 & 2.95631000 & 0.22890500 & -0.00008400 \\
\hline Pt16 & -2.34693800 & 3.44235200 & -3.84983300 \\
\hline Pt17 & 4.61607700 & 3.12109300 & 1.86779500 \\
\hline Pt18 & 0.71268700 & 2.54729100 & 5.36254900 \\
\hline Pt19 & -4.75709000 & -3.13693100 & 1.08121000 \\
\hline Pt20 & -0.48264100 & -3.56516300 & -1.02193400 \\
\hline Pt21 & -1.77280100 & 0.28590900 & 2.22345700 \\
\hline Pt22 & 0.69550500 & -3.64243300 & 1.44329800 \\
\hline Pt23 & 0.66319400 & -2.11391300 & -3.01317500 \\
\hline Pt24 & 5.06063800 & 1.46158200 & 3.97730800 \\
\hline Pt25 & -3.66924800 & 5.15066300 & -2.12205300 \\
\hline Pt26 & -2.14502500 & -3.97097700 & 3.92221400 \\
\hline Pt27 & 1.83948100 & -5.88568100 & 2.35379100 \\
\hline Pt28 & 1.87886300 & -2.22868700 & -0.54313600 \\
\hline Pt29 & -0.48272400 & -5.70679800 & -2.57965200 \\
\hline Pt30 & -2.58978800 & 2.93782100 & -1.22538100 \\
\hline Pt31 & 2.42173600 & 1.89721400 & -2.12684000 \\
\hline Pt32 & 5.29084700 & -1.51437700 & -3.64401700 \\
\hline Pt33 & 5.20716800 & 0.46149200 & 1.45365600 \\
\hline Pt3 4 & -1.39581700 & 2.81253800 & 1.24661900 \\
\hline Pt35 & -0.98437200 & 1.64138000 & -5.47504700 \\
\hline Pt36 & 1.67467700 & -1.76222500 & 4.65232500 \\
\hline Pt37 & -3.39639100 & -1.70790000 & 3.01422600 \\
\hline Pt38 & -5.99566500 & 2.20415500 & 1.92081200 \\
\hline Pt39 & -3.82338500 & -1.66467000 & -3.41336000 \\
\hline Pt 40 & -1.27079000 & 1.19629300 & -2.89252700 \\
\hline Pt41 & -2.05832500 & -3.44580300 & 1.28333600 \\
\hline Pt 42 & 4.10157800 & 4.74971800 & -0.26908600 \\
\hline Pt4 3 & 2.83016300 & 2.33031400 & -4.72370300 \\
\hline Pt 44 & 0.66508300 & -4.25305100 & -4.57431900 \\
\hline Pt 45 & -0.86135700 & -5.67483900 & 2.18988100 \\
\hline Pt 46 & -0.60832000 & -1.94048300 & 3.13881300 \\
\hline Pt 47 & 1.01870700 & 0.45139000 & -4.06395100 \\
\hline Pt 48 & 3.00370600 & -2.90418200 & -4.13298000 \\
\hline Pt 49 & 0.46108700 & 0.49877000 & 3.67781300 \\
\hline Pt50 & 4.17453500 & -2.03350400 & 0.92013400 \\
\hline Pt51 & -3.67194500 & 0.46948700 & -1.77080600 \\
\hline Pt52 & -5.98663200 & 1.20456100 & -0.65118600 \\
\hline Pt53 & -5.36299800 & -1.49762800 & -1.0837320 \\
\hline Pt5 4 & -2.47446600 & 5.09093700 & 0.3721370 \\
\hline Pt5 5 & 4.74791300 & 2.13016100 & -0.68836600 \\
\hline Pt5 6 & 2.34530800 & 2.88521700 & 0.40340000 \\
\hline Pt57 & 2.96375900 & -3.47762000 & 2.92625600 \\
\hline Pt58 & -4.80266900 & 2.70410200 & -2.70868600 \\
\hline Pt59 & 3.07302100 & 3.29923900 & 4.1728740 \\
\hline Pt 60 & 0.56320400 & -4.20301600 & 4.06884600 \\
\hline Pt 61 & -1.53435800 & 0.74741800 & -0.33819700 \\
\hline Pt 62 & 0.61746300 & 0.99730200 & 1.08216200 \\
\hline Pt 63 & -1.35212500 & -0.94011100 & -4.56354000 \\
\hline Pt 64 & 2.82823500 & 1.22219400 & 2.5182290 \\
\hline Pt 65 & -3.65748900 & 3.59258700 & 2.42800300 \\
\hline Pt 66 & 2.98065700 & -0.74819000 & -2.52785500 \\
\hline Pt 67 & -4.03016700 & 1.00844000 & 3.4323790 \\
\hline Pt 68 & -0.93003000 & 4.93489700 & -1.95506000 \\
\hline Pt 69 & -0.44737400 & -1.43152700 & 0.5454660 \\
\hline
\end{tabular}




\begin{tabular}{|c|c|c|c|}
\hline Pt70 & 2.62592800 & 4.97049400 & 2.03770600 \\
\hline Pt71 & -1.64768900 & -1.33144100 & -1.91851900 \\
\hline Pt72 & -1.70035700 & -3.47158200 & -3.50859800 \\
\hline Pt73 & -3.45105200 & 0.92393400 & -4.40632500 \\
\hline Pt7 4 & 4.17813800 & 3.76001500 & -2.80367900 \\
\hline Pt75 & 2.72308000 & 0.72352900 & 5.15746000 \\
\hline Pt76 & -0.73379500 & -2.46116000 & 5.73231200 \\
\hline Pt77 & -3.14522900 & -1.17776400 & 0.36488600 \\
\hline Pt78 & 1.82689000 & 4.57385400 & -1.73080600 \\
\hline Pt79 & 4.77043900 & 1.12562900 & -3.24251700 \\
\hline Pt 80 & -5.84049100 & 0.19818900 & -3.21639600 \\
\hline Pt 81 & 1.87966500 & -4.41213800 & -2.13401100 \\
\hline Pt 82 & -3.26806900 & -3.36673800 & -1.23399900 \\
\hline Pt 83 & 1.80186000 & -1.23325600 & 2.00612800 \\
\hline Pt 84 & 0.81346600 & 3.10457700 & 2.73076700 \\
\hline Pt 85 & 4.20071500 & -3.01035800 & -1.65300600 \\
\hline Pt 86 & 1.04453700 & -1.74259100 & -5.62368800 \\
\hline Pt 87 & 0.69356500 & 0.01352100 & -1.43726600 \\
\hline Pt 88 & -3.74928400 & 1.46926900 & 0.78960000 \\
\hline Pt89 & 3.04121600 & -4.47891300 & 0.361979 \\
\hline \# 89 & $e r=\operatorname{Pt} 90$ & \multicolumn{2}{|c|}{$\mathrm{M}=0 \quad$ Etot $=-8525.76475000$} \\
\hline Pt 1 & 2.74457200 & -0.98073100 & 0.27319900 \\
\hline Pt2 & 4.25338100 & -3.15718800 & -0.03572000 \\
\hline Pt 3 & 4.30857700 & 3.00993900 & 3.18935700 \\
\hline Pt 4 & -0.46580800 & 1.08836600 & 2.56011000 \\
\hline Pt 5 & 6.25003700 & 1.70339400 & 1.7260200 \\
\hline Pt 6 & 2.85956800 & 1.13213600 & -1.46290800 \\
\hline Pt 7 & 4.59628200 & 0.22401400 & 3.37903200 \\
\hline Pt 8 & 2.57319300 & 1.52738600 & 4.82398000 \\
\hline Pt 9 & -3.84500300 & 0.42981700 & -0.64747700 \\
\hline Pt10 & -2.07532100 & -5.87600800 & 2.26804400 \\
\hline Pt11 & 1.73315200 & 2.67967000 & 2.55160200 \\
\hline Pt12 & 2.39172300 & -4.65788200 & 3.01269800 \\
\hline Pt13 & 1.68370700 & -2.13554900 & -5.55167200 \\
\hline Pt14 & 2.01972000 & -0.04688400 & 2.74180500 \\
\hline Pt15 & -2.53832000 & -2.47983900 & -4.11559800 \\
\hline Pt16 & 2.25245800 & 3.40869800 & -0.04831300 \\
\hline Pt17 & -2.20766700 & 1.01122200 & 4.57679300 \\
\hline Pt18 & -1.16272300 & 1.70073600 & -5.90814100 \\
\hline Pt19 & 0.16131500 & 4.83542800 & 2.87150600 \\
\hline Pt20 & 0.69111400 & 5.57918500 & 0.21906000 \\
\hline Pt21 & 4.44727800 & -1.04158600 & -1.80774 \\
\hline Pt22 & 2.32265600 & -0.48106900 & -3.53312800 \\
\hline Pt23 & 3.95954900 & 3.47361800 & -2.11682800 \\
\hline Pt24 & 2.27215700 & 4.26513700 & 4.56679500 \\
\hline Pt25 & -3.88861900 & 3.96744200 & -2.03722600 \\
\hline Pt26 & 0.94328800 & -2.41749200 & 3.37269800 \\
\hline Pt27 & 4.51633900 & 1.13256000 & -3.5127040 \\
\hline Pt28 & -1.59528600 & -1.26946800 & 3.1224000 \\
\hline Pt29 & -6.42943600 & 0.08904500 & -1.25924500 \\
\hline Pt30 & -2.87115400 & 0.24830400 & -4.35651000 \\
\hline Pt31 & -0.20531200 & -4.78744400 & 3.90572900 \\
\hline Pt32 & -2.44624600 & -1.57350800 & 0.5215540 \\
\hline Pt33 & -5.65562600 & 0.34887900 & 1.33814200 \\
\hline Pt34 & -2.73959700 & -3.65868200 & 3.7011730 \\
\hline Pt35 & 5.45278200 & 1.42641000 & -0.9090490 \\
\hline Pt36 & 3.58870000 & -2.24689900 & 2.49074200 \\
\hline Pt37 & -2.90178300 & 2.84669400 & 0.21862400 \\
\hline
\end{tabular}




\begin{tabular}{|c|c|c|c|}
\hline Pt 38 & 0.06248300 & -2.07403300 & -3.43634900 \\
\hline Pt39 & -3.09575900 & -3.24976000 & -1.52959300 \\
\hline Pt 40 & -5.52490600 & 2.50336700 & -0.40158900 \\
\hline Pt 41 & 2.81411000 & 5.05789900 & 1.98034600 \\
\hline Pt 42 & -4.80919300 & 1.53714200 & -2.89953400 \\
\hline Pt 43 & -3.57878000 & -3.94048800 & 1.09348300 \\
\hline Pt 44 & -4.24712600 & -1.68335100 & 2.53231300 \\
\hline Pt 45 & -1.92788500 & -0.84580800 & -2.07362900 \\
\hline Pt 46 & 4.83886000 & 3.75437500 & 0.53449200 \\
\hline Pt 47 & 0.29914800 & -0.12578900 & 4.83683300 \\
\hline Pt 4 & 2.01787700 & 2.28704500 & -3.76800600 \\
\hline Pt 49 & 0.02619500 & 2.67487200 & 4.63910000 \\
\hline Pt50 & 3.07682400 & -5.53958500 & 0.50082500 \\
\hline Pt51 & 1.62696200 & -3.34852700 & 0.82304900 \\
\hline Pt52 & -1.07262900 & -4.48644200 & -2.92707700 \\
\hline Pt 53 & -3.12294100 & 2.97080800 & -4.50117700 \\
\hline Pt 54 & 1.21088100 & 1.11643000 & 0.55491300 \\
\hline Pt55 & 5.32850800 & -0.74105200 & 0.85652100 \\
\hline Pt 56 & 3.31395900 & 5.72766800 & -0.64900000 \\
\hline Pt57 & -3.04188700 & 0.70996700 & 1.95292500 \\
\hline Pt 5 & 0.14959400 & -1.17718400 & 1.13320900 \\
\hline Pt5s & -0.96155700 & -3.51163000 & 1.69052500 \\
\hline Pt 60 & 1.41329900 & 4.62215500 & -2.30545800 \\
\hline Pt 61 & -2.19850300 & 1.89395000 & -2.26948900 \\
\hline Pt 62 & -1.94805200 & 5.28214600 & 1.09203300 \\
\hline Pt 63 & -0.23918800 & 0.63428800 & -3.66692400 \\
\hline Pt 64 & -4.73613500 & 2.77765800 & 2.20587500 \\
\hline Pt 65 & 3.59097100 & -4.81780100 & -2.07325700 \\
\hline Pt 66 & 0.37276700 & 2.26559800 & -1.63822800 \\
\hline Pt 67 & -2.12160700 & 3.19273100 & 2.87664100 \\
\hline Pt 68 & 0.66153800 & -0.45849300 & -1.42984400 \\
\hline Pt 69 & -0.33753400 & 3.19620900 & 0.82881200 \\
\hline Pt 70 & -0.88132500 & -2.54629700 & 5.34831600 \\
\hline Pt71 & -0.85588800 & -1.01424100 & -5.7296420 \\
\hline Pt 72 & -0.51685100 & 3.38487200 & -3.88830400 \\
\hline $\mathrm{P}$ & -1.28373700 & 0.77481500 & -0.03915200 \\
\hline Pt 74 & -1.26260800 & 4.39863600 & -1.42395100 \\
\hline Pt 75 & -0.52878400 & -3.70490500 & -5.47471600 \\
\hline Pt 76 & 2.82617600 & -1.24017100 & 4.94912200 \\
\hline Pt 77 & 0.49747000 & -5.74817900 & 1.3908200 \\
\hline Pt78 & 1.34669500 & 0.58150500 & -5.80630000 \\
\hline $\mathrm{P}$ & -4.52108600 & -1.20449500 & -2.69991000 \\
\hline 0 & -3.36018800 & -1.38417100 & 5.0935020 \\
\hline Pt 81 & -4.80526700 & 0.60907700 & 3.9214140 \\
\hline Pt 82 & -4.54523000 & 4.88287000 & 0.4613150 \\
\hline Pt 83 & 2.14019400 & -2.62802800 & -1.7464500 \\
\hline Pt 84 & 3.71553000 & 1.40288800 & 1.13551500 \\
\hline Pt 85 & 3.76355700 & -2.73203600 & $-3.8554090 c$ \\
\hline Pt 86 & -0.47132000 & -2.83708100 & -0.88967100 \\
\hline Pt 87 & -1.59221400 & -5.21586100 & -0.3257650 \\
\hline Pt 88 & 1.53437100 & -4.29252800 & -3.8004380 \\
\hline Pt 89 & 1.00877300 & -5.07281300 & -1.22094600 \\
\hline Pt90 & -5.04221100 & -1.93607600 & $-0.0908900 c$ \\
\hline \# 90 & Cluster $=$ Pt $91 \quad \mathrm{Q}=0$ & $\mathrm{M}=0 \quad$ Etot $=-86$ & 25364200 \\
\hline Pt 1 & 3.78436500 & 0.19614600 & -4.3477470 \\
\hline Pt2 & -0.98347200 & -5.66240100 & 0.4824820 \\
\hline Pt3 & -2.58814700 & -1.28479400 & 0.0219080 \\
\hline$\tau 4$ & -0.11558800 & 4.61826600 & 2.9302460 \\
\hline
\end{tabular}




\begin{tabular}{|c|c|c|c|}
\hline Pt 5 & -1.58076400 & 2.81791100 & 4.41459500 \\
\hline Pt 6 & -2.35995500 & -2.25051200 & -2.50551300 \\
\hline Pt 7 & 2.47410100 & -2.26795800 & -4.51994100 \\
\hline Pt 8 & 0.14092200 & -3.85350400 & 4.74963300 \\
\hline Pt9 & 1.09211300 & -4.66443100 & -4.57944700 \\
\hline Pt10 & 1.97334400 & 5.62754200 & -2.05362600 \\
\hline Pt11 & -2.93454600 & 0.41309700 & 4.37960300 \\
\hline Pt12 & -0.89680200 & -0.45264300 & -3.98781300 \\
\hline Pt13 & -0.66497800 & 6.10223700 & -1.55167800 \\
\hline Pt14 & 5.83747000 & -0.00192100 & -1.02638700 \\
\hline Pt15 & -0.53147500 & -5.92811200 & 3.13436200 \\
\hline Pt16 & -0.96383700 & 3.60203200 & -0.73519300 \\
\hline Pt17 & 1.83987200 & 0.37172600 & 2.26697000 \\
\hline Pt18 & 5.46070100 & 2.68324800 & -0.63975900 \\
\hline Pt19 & -0.73824800 & -3.22252300 & -0.51712000 \\
\hline Pt20 & 0.81035800 & -1.72631100 & 6.30112600 \\
\hline Pt21 & -4.63507500 & -1.00251500 & -3.18451500 \\
\hline Pt22 & 1.62252200 & -0.32169400 & -2.90878200 \\
\hline Pt23 & -4.87428200 & 0.00467200 & -0.56953700 \\
\hline Pt24 & -3.14152900 & 0.83270100 & -4.69760600 \\
\hline Pt25 & 0.06369300 & 4.12710100 & -3.25656900 \\
\hline Pt26 & 3.51768500 & 1.16625200 & -1.78192600 \\
\hline Pt27 & -2.88702300 & -3.75602000 & 1.05668500 \\
\hline Pt28 & -1.31416700 & -0.10715900 & -6.55067200 \\
\hline Pt29 & -0.81685100 & 0.84648300 & 2.75914500 \\
\hline Pt 30 & 1.71431600 & -2.04480900 & -0.78796100 \\
\hline Pt 31 & -1.59588900 & -4.24447400 & -4.11545500 \\
\hline Pt 32 & -1.71945600 & -1.87081600 & 5.30307800 \\
\hline Pt33 & 1.94916000 & -4.77973500 & 2.91280400 \\
\hline Pt 34 & -0.10161000 & -2.40835800 & -5.62928700 \\
\hline Pt 35 & 1.10048800 & 2.37250400 & 3.96386500 \\
\hline Pt36 & 1.96156500 & 2.20175000 & -3.85535000 \\
\hline Pt 37 & 1.35615000 & 0.64591300 & -0.36109000 \\
\hline Pt 38 & -0.06664100 & -1.10236100 & 1.08032000 \\
\hline Pt 39 & -0.48739200 & -0.80460400 & -1.45699500 \\
\hline Pt 40 & 0.30274500 & -2.72434400 & -3.00550100 \\
\hline Pt 41 & 3.75468900 & 1.86633200 & 3.41762200 \\
\hline Pt 42 & -0.63136300 & 2.04356400 & -4.97297100 \\
\hline Pt 43 & -2.39707000 & -4.00889000 & 3.73244100 \\
\hline Pt 44 & -4.22599100 & 4.40861300 & 1.17576700 \\
\hline Pt 45 & 3.94198900 & -1.55751400 & -2.22909300 \\
\hline Pt 46 & 2.29231400 & 0.06942000 & 4.89913200 \\
\hline Pt 47 & 2.16697600 & -2.33188900 & 1.83124700 \\
\hline Pt 48 & 5.85094100 & -1.73967700 & 1.12972100 \\
\hline Pt 49 & -3.34323900 & 0.67831000 & 1.67534500 \\
\hline Pt 50 & 3.88330000 & 3.70820100 & -2.63011200 \\
\hline Pt51 & 3.96370200 & -3.27423000 & -0.05878400 \\
\hline Pt 52 & -5.41878400 & 0.26093100 & 3.26433600 \\
\hline Pt53 & 5.68485400 & 1.71028900 & -3.16331200 \\
\hline Pt 54 & -4.89253800 & 1.73027000 & -2.73071200 \\
\hline Pt 55 & -2.67818800 & 0.44975100 & -2.09809600 \\
\hline Pt 56 & 4.39809200 & -3.52488500 & 2.60158200 \\
\hline Pt 57 & 2.56961800 & -4.02569200 & -2.34630400 \\
\hline Pt58 & -4.24983100 & -3.71439700 & -3.5765930 \\
\hline Pt59 & 1.25047400 & 0.05023700 & -5.52224900 \\
\hline Pt 60 & 4.12345800 & -0.84166100 & 3.04935300 \\
\hline Pt 61 & 0.94750500 & 5.18322100 & 0.4467530 \\
\hline Pt 62 & 2.62191900 & -2.64113300 & 4.48324500 \\
\hline Pt 63 & 3.61122300 & -0.55725100 & 0.3681010 \\
\hline
\end{tabular}




\begin{tabular}{|c|c|c|c|}
\hline Pt 64 & -4.70645600 & -1.73428600 & 1.57600200 \\
\hline Pt 65 & 3.24868500 & 2.12673600 & 0.76555200 \\
\hline Pt 66 & 5.54331300 & 0.94857000 & 1.52362200 \\
\hline Pt 67 & -4.11528100 & 2.66860500 & 3.34725500 \\
\hline Pt 68 & 1.51767500 & -4.53642400 & 0.22694500 \\
\hline Pt 69 & -4.54388700 & -2.76400400 & -1.00924800 \\
\hline Pt70 & -2.44707300 & 2.98713800 & -3.02850700 \\
\hline Pt71 & -2.76552500 & 5.03357100 & 3.37537500 \\
\hline Pt72 & 0.63780200 & 2.63390800 & 1.28545700 \\
\hline Pt73 & 0.37194400 & -1.40810000 & 3.67993800 \\
\hline Pt74 & -1.25532200 & 1.11396800 & 0.11201600 \\
\hline Pt75 & -5.58501500 & 2.01682600 & 1.15271800 \\
\hline Pt76 & 1.66670800 & 3.13529900 & -1.22784600 \\
\hline Pt77 & -3.50128400 & 2.43756500 & -0.49955500 \\
\hline Pt78 & -1.72443900 & 5.60638500 & 0.92512700 \\
\hline Pt79 & -0.38010300 & 0.54610400 & 5.39802200 \\
\hline Pt 80 & -1.99774700 & 3.06026900 & 1.73983500 \\
\hline Pt 81 & -2.82101500 & -1.92474800 & -5.11908900 \\
\hline Pt 82 & -2.15839100 & -1.56484300 & 2.64730700 \\
\hline Pt83 & -0.30335000 & -3.52889700 & 2.08910900 \\
\hline Pt 84 & 2.54830900 & 4.14443100 & 2.43129000 \\
\hline Pt 85 & 3.59173100 & 4.65538000 & -0.07761000 \\
\hline Pt 86 & -0.21891100 & 1.62643800 & -2.37833100 \\
\hline Pt 87 & -4.23035100 & -1.99533400 & 4.23125000 \\
\hline Pt 88 & 0.06111500 & -5.23294700 & -2.07538000 \\
\hline Pt 89 & 5.16217300 & 3.62104200 & 1.89236500 \\
\hline Pt90 & -3.16738500 & 4.98752100 & -1.29654800 \\
\hline Pt91 & -2.65581400 & -4.74966900 & -1.56484400 \\
\hline \# 91 & Cluster $=$ Pt92 $\quad \mathrm{Q}=0$ & $I=0 \quad$ Etot $=-872$ & 68669000 \\
\hline Pt 1 & 3.03478900 & -4.08944700 & -1.73269500 \\
\hline Pt2 & 3.48454700 & -1.58844400 & -2.97455100 \\
\hline Pt3 & 0.42921800 & 3.51486400 & 1.21751800 \\
\hline Pt 4 & 1.70427900 & -3.96007700 & 5.07610400 \\
\hline Pt5 & 0.80073200 & 1.50967900 & 5.54127000 \\
\hline Pt 6 & 0.17530300 & 4.81772100 & 3.55102400 \\
\hline Pt 7 & -2.11332000 & -3.81295400 & 2.12330700 \\
\hline Pt 8 & 1.00483600 & 0.27743300 & 3.20670800 \\
\hline Pt9 & -3.20679400 & -1.81270200 & 3.69546500 \\
\hline Pt10 & 0.06375600 & 5.17695800 & -0.93549800 \\
\hline Pt11 & -0.73992900 & 2.27815700 & 3.39096400 \\
\hline Pt12 & 0.24167600 & -4.53349300 & -1.70358900 \\
\hline Pt13 & 0.38597100 & -4.89345400 & 2.82824600 \\
\hline Pt14 & -1.34810200 & -0.68998700 & -5.03851700 \\
\hline Pt15 & -0.52657700 & 1.04277000 & 1.07271900 \\
\hline Pt16 & 4.56042500 & -4.83394800 & 0.43483400 \\
\hline Pt17 & -4.53298400 & 2.65800700 & 2.64639300 \\
\hline Pt18 & -3.22351000 & 2.49100800 & -2.45269800 \\
\hline Pt19 & 2.18093500 & 1.49959300 & 1.0597560 \\
\hline Pt20 & 2.77238300 & 5.55417300 & -0.96460700 \\
\hline Pt21 & -1.34218800 & 5.55576300 & 1.39584100 \\
\hline Pt22 & -0.89624500 & -3.38133800 & -5.21811400 \\
\hline Pt23 & 3.15959500 & -4.45411700 & 2.78298600 \\
\hline Pt24 & 0.63356200 & 1.07724300 & -5.7493040 \\
\hline Pt25 & 2.66050800 & 2.73699800 & -4.82914500 \\
\hline Pt26 & 5.06842900 & 1.68432900 & -4.1524950 \\
\hline Pt27 & 2.00467900 & 2.79262500 & 3.4198960 \\
\hline Pt28 & -1.23081500 & -0.14572400 & 4.68796700 \\
\hline Pt29 & -2.13109400 & -3.50279600 & $-0.651917 d$ \\
\hline
\end{tabular}




\begin{tabular}{|c|c|c|c|}
\hline Pt30 & 2.98090800 & 1.13972400 & -2.65543800 \\
\hline Pt31 & -3.01808100 & 1.91761600 & 4.80259100 \\
\hline Pt 32 & 1.43096900 & -2.42330400 & 2.97270400 \\
\hline Pt33 & 3.16349300 & 3.97254300 & 1.19879000 \\
\hline Pt34 & 5.06579900 & -2.40740900 & -0.79743900 \\
\hline Pt35 & 4.45685900 & 1.81993200 & 2.40000100 \\
\hline Pt36 & 1.32052800 & -2.07580800 & -1.44068300 \\
\hline Pt37 & -2.83980600 & 0.88130500 & -0.28300300 \\
\hline Pt38 & -0.76870800 & -2.92572200 & 4.44803100 \\
\hline Pt39 & -4.61143500 & 2.89431900 & -0.10511700 \\
\hline Pt 40 & -2.51881500 & 4.32167200 & 3.58731500 \\
\hline Pt41 & -5.59634400 & -1.76076000 & -0.44385000 \\
\hline Pt 42 & -1.75885000 & 2.02153700 & -4.74060100 \\
\hline Pt 43 & -2.64846700 & 4.67700900 & -0.87445300 \\
\hline Pt 44 & -1.04497300 & -1.11140900 & -0.43379600 \\
\hline Pt 45 & 4.36504500 & 2.07044300 & -0.41572600 \\
\hline Pt 46 & 3.30299800 & -0.42571700 & -0.52783300 \\
\hline Pt 47 & 3.26024800 & 0.56854100 & 4.59797700 \\
\hline Pt 48 & -2.74927500 & 0.62828100 & 2.46324900 \\
\hline Pt 49 & 1.50189500 & 4.68558300 & -3.22582800 \\
\hline Pt50 & 1.83886500 & -5.33798400 & 0.46727800 \\
\hline Pt51 & -2.26748100 & 3.03666400 & 1.25100100 \\
\hline Pt52 & -5.14377700 & 0.68218200 & -1.65216400 \\
\hline Pt53 & 0.96995500 & -0.50966000 & -3.58054200 \\
\hline Pt5 4 & -1.42075200 & 0.44712900 & -2.58898200 \\
\hline Pt55 & -4.98301400 & 0.22804300 & 3.81876600 \\
\hline Pt5 6 & 2.85468400 & -2.85890600 & 0.65915100 \\
\hline Pt57 & 1.42990200 & -3.23135400 & -3.83922100 \\
\hline Pt58 & 0.14558700 & -3.29315400 & 0.70963600 \\
\hline Pt59 & 5.15739100 & -2.63979700 & 1.99999900 \\
\hline Pt 60 & -0.93455100 & -5.70124900 & 0.49834200 \\
\hline Pt 61 & -1.22465300 & 4.24137700 & -3.18816700 \\
\hline Pt 62 & -3.24707600 & -5.86748700 & -0.89344000 \\
\hline Pt 63 & 1.35817800 & 5.98686500 & 1.33349400 \\
\hline Pt 64 & 0.53331600 & 2.17972800 & -3.30032800 \\
\hline Pt 65 & -3.31465700 & -1.31485100 & -1.79221000 \\
\hline Pt 66 & -5.51176500 & -1.96916600 & 2.28215800 \\
\hline Pt 67 & 3.96141400 & 3.65931700 & -2.60145100 \\
\hline Pt 68 & 5.60901400 & -0.15009200 & 0.78120400 \\
\hline Pt 69 & 5.44195400 & 0.08964300 & -2.00077000 \\
\hline Pt70 & -0.98073300 & -1.37171000 & 2.29889400 \\
\hline Pt71 & 3.09237600 & 0.04805100 & -5.12697200 \\
\hline Pt72 & 1.83494000 & 3.08476700 & -1.0667970 \\
\hline Pt73 & 1.04671000 & -1.61682100 & -5.97960700 \\
\hline Pt74 & -5.12428600 & 0.46538100 & 1.09782600 \\
\hline Pt75 & 1.26414000 & -1.24424700 & 5.38030200 \\
\hline Pt76 & 3.38698900 & -0.64692300 & 2.20609100 \\
\hline Pt77 & -5.60380400 & -1.53321200 & -3.15979300 \\
\hline Pt78 & -3.27485300 & -1.55574800 & 0.93585900 \\
\hline Pt79 & -4.01975400 & 5.04927200 & 1.4436350 \\
\hline Pt 80 & -0.96134600 & -2.26359200 & -2.82361700 \\
\hline Pt 81 & -3.71500600 & 0.24503300 & -3.97393400 \\
\hline Pt 82 & -2.10930500 & -4.67591300 & -3.08830300 \\
\hline Pt83 & -3.27388100 & -2.47134500 & -4.2178790 \\
\hline Pt 84 & 0.21697700 & 3.75299500 & -5.44110300 \\
\hline Pt 85 & 0.85993300 & 0.61855100 & -1.1803760 \\
\hline Pt 86 & -4.43365100 & -3.96475200 & 0.6887850 \\
\hline Pt 87 & -0.89018900 & 2.65187100 & -1.04710600 \\
\hline Pt 88 & -0.98411900 & 3.53617600 & 5.6829670 \\
\hline
\end{tabular}




\begin{tabular}{|c|c|c|c|}
\hline Pt89 & 3.70326900 & -2.19489100 & 4.36296400 \\
\hline Pt90 & 5.62829400 & -0.39644000 & 3.53818400 \\
\hline Pt91 & -4.45528600 & -3.72528700 & -2.04028800 \\
\hline Pt92 & 1.17200400 & -0.90568100 & 0.8917520 \\
\hline \# 92 & Cluster $=$ Pt $93 \quad \mathrm{Q}=0$ & $\mathrm{M}=0 \quad \mathrm{Etot}=-880$ & 34859700 \\
\hline Pt1 & 5.69647200 & -1.48020500 & -0.01989300 \\
\hline Pt2 & -0.97822900 & -2.32813800 & -2.53518700 \\
\hline Pt3 & 3.21462900 & 5.53915200 & -3.9570860 \\
\hline Pt 4 & -4.38954100 & -3.98671100 & 0.1586510 \\
\hline Pt 5 & -4.02151200 & 3.41335000 & 0.84186600 \\
\hline Pt 6 & -1.53503900 & 0.52520200 & 1.12054500 \\
\hline Pt 7 & -4.04770000 & 0.75712700 & 0.2076310 \\
\hline Pt 8 & -1.46692800 & 3.12513700 & 1.76821500 \\
\hline Pt9 & -0.19718400 & 5.37149600 & -2.17205900 \\
\hline Pt10 & 0.83172500 & 4.44505100 & -4.51898800 \\
\hline Pt11 & 2.69582000 & 0.28865500 & 3.1479130 \\
\hline Pt12 & -1.54581900 & 3.29980600 & -5.04065900 \\
\hline Pt13 & -1.73993600 & -3.84859700 & -0.46803500 \\
\hline Pt14 & -3.39637500 & 4.13400700 & 3.3610570 \\
\hline Pt15 & -3.83071800 & -3.22848700 & 2.69923000 \\
\hline Pt16 & 6.22465800 & 1.02694500 & -0.91217800 \\
\hline Pt17 & 1.77405600 & -0.75005200 & -4.6796200 \\
\hline Pt18 & 3.10521000 & 2.92497300 & -4.61767300 \\
\hline Pt19 & 5.00895200 & 3.17119100 & 0.21918900 \\
\hline Pt20 & 1.49961700 & -2.98014100 & 1.4529980 \\
\hline Pt21 & 0.28796900 & -0.85037000 & 2.58191100 \\
\hline Pt22 & -2.09098700 & 2.37755500 & -0.77771900 \\
\hline Pt23 & 2.74557800 & -5.13402000 & 0.34161200 \\
\hline Pt24 & -4.72786600 & 0.01519400 & -2.34631600 \\
\hline Pt25 & -1.79209500 & -0.21774700 & 5.75010800 \\
\hline Pt26 & -0.45769200 & 0.14788100 & -3.45553000 \\
\hline Pt27 & -4.31251500 & -0.00657700 & 4.79380600 \\
\hline Pt28 & 0.21545300 & 0.90424000 & -0.86685500 \\
\hline Pt29 & -0.36944600 & 2.03810000 & 6.22424100 \\
\hline Pt30 & 4.42481300 & 2.41727500 & -2.32922900 \\
\hline Pt31 & -0.30123900 & 2.73955000 & -2.76294600 \\
\hline Pt32 & -5.93092600 & 1.75163900 & 1.86791100 \\
\hline Pt33 & 5.46171400 & -3.51524300 & -1.79590900 \\
\hline Pt34 & -3.44435600 & 1.50881500 & 2.74275500 \\
\hline Pt35 & 3.82922500 & -0.09869300 & -1.43753900 \\
\hline Pt36 & 3.23658900 & 2.81791500 & 2.24788600 \\
\hline Pt37 & 0.11230400 & -5.2872 & 1.0016790 \\
\hline Pt38 & -0.33538300 & -1.58708300 & 0.0132440 \\
\hline Pt39 & -1.40610100 & 5.72061900 & 2.37282200 \\
\hline Pt 40 & 3.31694000 & -4.37389200 & 2.85067000 \\
\hline Pt41 & 2.07013200 & -0.46636500 & 0.57966900 \\
\hline Pt 42 & -2.59127400 & -3.62716100 & -4.21494800 \\
\hline Pt4 3 & 6.01145000 & -1.00952700 & -2.6784960 \\
\hline Pt 44 & 1.44031700 & 2.43849100 & 4.2529270 \\
\hline Pt 45 & 5.11114900 & -3.98430600 & 0.84550400 \\
\hline Pt 46 & -2.36987100 & -0.96726100 & 3.18309600 \\
\hline Pt4 7 & -2.01732300 & 4.99807000 & -0.15160200 \\
\hline Pt 48 & 1.97509400 & 1.26072900 & -2.8506110 \\
\hline Pt 49 & 0.68024700 & -4.51820300 & 3.5262380 \\
\hline Pt50 & -2.19197700 & -0.22257500 & -1.4527340 \\
\hline Pt51 & -2.75264600 & 1.62590100 & -3.3382110 \\
\hline Pt52 & 2.19454900 & 6.47073000 & -1.63032800 \\
\hline Pt53 & 3.27570500 & -2.59802600 & -0.55819700 \\
\hline
\end{tabular}




\begin{tabular}{|c|c|c|c|}
\hline Pt5 4 & 4.17359800 & 0.39276900 & -4.11143700 \\
\hline Pt5 5 & 2.10466800 & 3.85848900 & -2.20890000 \\
\hline Pt5 6 & -1.97501700 & -4.61778100 & 4.12207800 \\
\hline Pt57 & -2.88229900 & 2.26001200 & 5.26404000 \\
\hline Pt58 & -3.21967100 & -2.46984200 & 5.21394600 \\
\hline Pt59 & 2.10848800 & -2.24729200 & 4.01207700 \\
\hline Pt 60 & -4.94057500 & -0.76035800 & 2.28407700 \\
\hline Pt 61 & -0.88119500 & 3.89836100 & 4.32475400 \\
\hline Pt 62 & 0.92905600 & 4.31464000 & 2.34023100 \\
\hline Pt 63 & -2.55230600 & -5.38346000 & 1.58606800 \\
\hline Pt 64 & -2.85901300 & -0.95832300 & -4.05603300 \\
\hline Pt 65 & 0.87898800 & -3.71165900 & -1.08067700 \\
\hline Pt 66 & 2.72026300 & 4.68475200 & 0.33926000 \\
\hline Pt 67 & 3.63134600 & -2.15917100 & -3.25346100 \\
\hline Pt 68 & -3.63466400 & -2.46459900 & -1.94401200 \\
\hline Pt 6 & 0.87823900 & -0.10097200 & 5.13828900 \\
\hline Pt70 & 0.31156900 & 3.53216300 & -0.21892800 \\
\hline Pt71 & -2.98710400 & -1.71786400 & 0.63327800 \\
\hline Pt72 & -0.54971300 & -2.37671800 & 4.66148100 \\
\hline Pt73 & 2.62310300 & 2.04279300 & -0.30221800 \\
\hline Pt74 & -0.75657700 & -5.00020500 & -2.81013900 \\
\hline Pt 7 & 4.49047400 & 5.02012200 & -1.66668500 \\
\hline Pt76 & -6.54596600 & 1.00536800 & -0.62504000 \\
\hline Pt77 & -1.56551000 & -6.51205600 & -0.70060800 \\
\hline Pt78 & 4.48404800 & 0.66241800 & 1.12991900 \\
\hline Pt79 & -0.61539800 & -1.95553800 & -5.17070200 \\
\hline Pt 80 & -2.59680600 & 4.23193000 & -2.68774800 \\
\hline Pt 8 & -5.52833600 & -1.50176100 & -0.24134500 \\
\hline Pt 82 & 0.84797300 & 1.67690900 & 1.69830900 \\
\hline Pt83 & 1.06128500 & -6.35225100 & -1.36376300 \\
\hline Pt 84 & 3.92080200 & -1.85776400 & 2.01147900 \\
\hline Pt 85 & -1.14823000 & -3.11080000 & 2.08225200 \\
\hline Pt 8 & 0.40191800 & 6.13888900 & 0.38286800 \\
\hline Pt 87 & 1.42884000 & -1.23169300 & -2.01245800 \\
\hline Pt 88 & -4.62852700 & 2.65112700 & -1.67009300 \\
\hline Pt 8 & -3.39779800 & -5.13983900 & -2.11676200 \\
\hline Pts & 3.06376300 & -4.68150100 & -2.30868000 \\
\hline Pts & -0.93271500 & 1.28112100 & 3.68324400 \\
\hline Pt & 1.22157900 & -3.32746000 & -3.76006700 \\
\hline Pt93 & 0.71773700 & 1.79885600 & -5.18272100 \\
\hline & luster $=\operatorname{Pt} 94 \quad \mathrm{Q}=0$ & $I=0 \quad$ Etot $=-89$ & 56110200 \\
\hline Pt 1 & -1.02812300 & 5.98414300 & -1.1953610 \\
\hline Pt2 & 2.11146800 & -1.91169300 & -2.71480400 \\
\hline Pt3 & 2.06215900 & -2.77727500 & 5.07685300 \\
\hline Pt 4 & -5.70551500 & 0.96991600 & -0.68935600 \\
\hline Pt 5 & 0.89014900 & 4.38152200 & -0.19057000 \\
\hline Pt 6 & -2.75726300 & 5.35661700 & 0.81066600 \\
\hline Pt 7 & -2.18808200 & -2.21307700 & -4.6503240 \\
\hline Pt 8 & 1.29142800 & 2.42490200 & 4.7321520 \\
\hline Pt 9 & -2.45008900 & 3.16349700 & -3.42237000 \\
\hline Pt10 & 4.04270000 & 0.90293100 & -4.53940100 \\
\hline Pt11 & -2.63792600 & -3.39928000 & 2.75527700 \\
\hline Pt12 & 1.90404100 & 2.42971200 & -1.7215510 \\
\hline Pt13 & 4.66529200 & -3.16824300 & 1.0921520 \\
\hline Pt 1 & -4.93834200 & 0.16039100 & -3.1903990 \\
\hline Pt15 & 1.51893800 & 0.58717000 & -3.72360100 \\
\hline Pt16 & 5.45516600 & -1.30848400 & -0.83378800 \\
\hline Pt17 & -1.29758200 & -0.31800800 & -0.4501160 \\
\hline
\end{tabular}




\begin{tabular}{|c|c|c|c|}
\hline Pt18 & 4.59440600 & -0.49225800 & 1.72922600 \\
\hline Pt19 & -0.50287500 & -5.52854300 & -3.85152000 \\
\hline Pt20 & -0.89953900 & -5.48848400 & 2.48095700 \\
\hline Pt21 & 1.15477900 & -0.01485300 & -1.23016900 \\
\hline Pt22 & -3.44931800 & 3.46898300 & 2.69303700 \\
\hline Pt23 & -2.38639200 & 1.56615000 & 1.12051600 \\
\hline Pt24 & -1.32987100 & -3.52603800 & -2.34556900 \\
\hline Pt25 & 0.08838100 & 4.10281200 & -2.84862400 \\
\hline Pt26 & 3.28704300 & 4.08699800 & 3.89995400 \\
\hline Pt27 & -2.37685200 & 0.48373400 & -4.09219600 \\
\hline Pt28 & -0.50125700 & -3.65295600 & 4.47155800 \\
\hline Pt29 & -0.39717500 & -4.93998900 & -0.11755900 \\
\hline Pt30 & 2.44326400 & 5.50448200 & 1.69937600 \\
\hline Pt31 & 1.89587000 & 1.18760600 & -6.24556300 \\
\hline Pt32 & -4.28024900 & 3.18845600 & 0.07817900 \\
\hline Pt33 & 2.15952600 & -4.12603500 & 0.50761700 \\
\hline Pt34 & -3.08388300 & -4.66574400 & -3.99655000 \\
\hline Pt35 & 3.56935800 & 3.61568700 & 0.05977400 \\
\hline Pt36 & -4.31294100 & -1.21773700 & 2.95885000 \\
\hline Pt37 & 2.48299100 & -2.17039000 & 2.46103600 \\
\hline Pt38 & -6.33924700 & -0.91937700 & 1.17026300 \\
\hline Pt39 & 1.34509800 & -4.45321500 & -2.19323100 \\
\hline Pt 40 & 1.73338700 & 2.96533900 & $2.0733700 \mathrm{C}$ \\
\hline Pt 41 & -2.21395900 & -1.50300200 & 4.7148470 \\
\hline Pt42 & -4.69814100 & -3.08501800 & 0.96179600 \\
\hline Pt43 & -3.14369800 & 1.26853200 & -1.51670200 \\
\hline Pt 44 & -2.22205100 & -5.98389800 & -1.78573200 \\
\hline Pt 45 & -3.53957200 & 5.03057100 & -1.7918440 \\
\hline Pt 46 & -0.72938100 & 0.70255600 & 5.4623870 \\
\hline Pt47 & 4.65041800 & -1.64126100 & -3.5093160 \\
\hline Pt 48 & -1.93163000 & 5.61667900 & 3.39067000 \\
\hline Pt 49 & -4.99533300 & 2.85011000 & -2.54389100 \\
\hline Pt50 & -3.80669600 & -0.61682700 & 0.36145000 \\
\hline Pt51 & -2.97856900 & -5.22204300 & 0.7175200 \\
\hline Pt52 & -0.88768000 & 3.71359500 & 1.8259310 \\
\hline Pt53 & -4.93026900 & 1.27318800 & 1.94613100 \\
\hline Pt54 & -0.09125300 & -1.77014100 & 6.39489600 \\
\hline Pt55 & 5.32358500 & 1.41670700 & -0.22048200 \\
\hline Pt56 & -3.94253000 & -3.90153600 & -1.53313100 \\
\hline Pt57 & 2.29404000 & 3.09255300 & -4.27757900 \\
\hline Pt58 & -4.74769500 & -2.53148300 & -3.78365500 \\
\hline Pt59 & -3.02061600 & -1.41007900 & -2.13435200 \\
\hline Pt 60 & 0.41176500 & -2.45395500 & -0.67763200 \\
\hline Pt61 & 3.90760200 & 1.62545500 & 4.8945790 \\
\hline Pt 62 & 3.92628400 & -3.54416900 & -1.59007700 \\
\hline Pt63 & -0.48784500 & -1.08142300 & -2.94578700 \\
\hline Pt 64 & -0.15506600 & -0.51338900 & -5.52507300 \\
\hline Pt 65 & -2.86706800 & 0.99385600 & 3.74886800 \\
\hline Pt 66 & -5.58620500 & -1.72854200 & -1.31087300 \\
\hline Pt 67 & 3.61349700 & 0.28517300 & -1.9726320 \\
\hline Pt68 & -1.34825700 & 3.18436200 & 4.4526840 \\
\hline Pt 69 & 1.66542400 & -4.66576600 & 3.13660100 \\
\hline Pt70 & 2.51162900 & -1.33651000 & -5.29504900 \\
\hline Pt71 & -0.18886600 & 6.25118700 & 1.4093830 \\
\hline Pt72 & 1.91741600 & -0.07393900 & 5.68271900 \\
\hline Pt73 & 6.10052100 & 0.58754900 & -2.7336860 \\
\hline Pt74 & 2.78407700 & 1.08012900 & 0.4937390 \\
\hline Pt75 & -0.06190100 & -3.04905400 & 1.8745750 \\
\hline & 4.08623600 & -1.08313900 & 4.3481420 \\
\hline
\end{tabular}




\begin{tabular}{|c|c|c|c|}
\hline Pt77 & -1.68326400 & 3.43900900 & -0.77517300 \\
\hline Pt78 & 4.39463200 & 2.20918200 & 2.29198100 \\
\hline Pt79 & 4.18761000 & -3.74476600 & 3.69054700 \\
\hline Pt 80 & 0.74646500 & -0.58182100 & 1.26668400 \\
\hline Pt 81 & -2.13858800 & -2.77497100 & 0.14019800 \\
\hline Pt 82 & 2.34730500 & $0.5158950 c$ & 3.07327500 \\
\hline Pt83 & 2.65244900 & 4.90948500 & -2.20184900 \\
\hline Pt 84 & -1.77591800 & -0.90830300 & 2.13035400 \\
\hline Pt 85 & 0.67577100 & 4.88255000 & 3.67286900 \\
\hline Pt 86 & 0.44106400 & -3.06909100 & -4.48845100 \\
\hline Pt 87 & 2.91106100 & -1.58049600 & -0.09835100 \\
\hline Pt 88 & 0.32436100 & -1.17347000 & 3.81948500 \\
\hline Pt 89 & -0.28687600 & $2.2057050 c$ & -4.90319600 \\
\hline Pt90 & 0.15338500 & 1.84749700 & 0.27010200 \\
\hline Pt91 & -0.61621900 & 1.55816800 & -2.34380800 \\
\hline Pt92 & -0.30024200 & 1.28663000 & 2.86998100 \\
\hline Pt93 & 3.08243800 & -3.82404600 & -4.22235100 \\
\hline Pt94 & 4.43342900 & 2.78644300 & -2.48991400 \\
\hline \# 94 & Luster $=$ Pt95 $\quad \mathrm{Q}=0$ & Etot $=$ & 03361400 \\
\hline Pt1 & 3.86013200 & 2.45132800 & 3.01535600 \\
\hline Pt2 & 0.13008500 & $-3.3750940 c$ & -5.11365900 \\
\hline Pt 3 & 2.56812800 & 4.46253800 & 1.57743100 \\
\hline Pt 4 & -0.38658700 & $-2.1953550 c$ & -2.79833000 \\
\hline Pt 5 & -4.17858600 & -4.14089600 & 0.27128700 \\
\hline Pt 6 & 1.25684000 & -5.42567400 & -3.71907600 \\
\hline Pt 7 & 1.47246600 & -0.28137900 & -3.32672400 \\
\hline Pt 8 & -2.11663200 & -3.74141000 & 2.02321000 \\
\hline Pt9 & -4.64369300 & $4.8934770 c$ & 0.76951900 \\
\hline Pt10 & 1.49953100 & $4.9365150 c$ & -3.05869800 \\
\hline Pt11 & 3.18410000 & -1.57400000 & 4.98367400 \\
\hline Pt12 & 5.40676100 & 2.24544800 & -3.37483100 \\
\hline Pt13 & 0.59391800 & 6.30686200 & 1.38949100 \\
\hline Pt14 & -1.76575300 & -3.50699000 & -0.74498000 \\
\hline Pt15 & -2.92913900 & $4.5995820 c$ & -1.31818300 \\
\hline Pt16 & 0.55030100 & -0.81240000 & 5.62280100 \\
\hline Pt17 & 2.47685700 & 1.14597500 & 5.06176400 \\
\hline Pt18 & -0.80389200 & 1.20847700 & 1.02665600 \\
\hline Pt19 & -0.58790300 & -0.71421800 & -5.08784900 \\
\hline Pt20 & 2.35475700 & 5.96006700 & -0.67042900 \\
\hline Pt21 & -1.18155100 & -2.68434900 & 4.47306000 \\
\hline Pt22 & -3.83100300 & -3.96238600 & -2.44414100 \\
\hline Pt23 & 3.03830800 & 3.06788100 & -4.41814800 \\
\hline Pt24 & 2.13813300 & -4.96458100 & 0.96136100 \\
\hline Pt25 & 1.06591500 & -0.57788800 & 1.15455600 \\
\hline Pt26 & -5.33971700 & -2.06933800 & -1.09054600 \\
\hline Pt27 & 1.98637100 & -1.43366800 & -5.65490400 \\
\hline Pt28 & 1.66410200 & -1.73802500 & -1.10405000 \\
\hline Pt29 & 4.14843700 & 2.64751600 & 0.21240200 \\
\hline Pt30 & 4.97712300 & -1.94811300 & 2.84939800 \\
\hline Pt31 & -2.61203900 & -5.98361900 & -1.06922900 \\
\hline Pt32 & -4.81740600 & 2.65992400 & -0.77462100 \\
\hline Pt33 & 2.57002800 & -6.14360400 & -1.44064700 \\
\hline Pt34 & -4.92962700 & 0.37630900 & -2.3063970 \\
\hline Pt35 & -5.28788400 & 0.2225670 & 0.42450800 \\
\hline Pt36 & 3.92435000 & 4.14661300 & -2.06133700 \\
\hline Pt37 & 0.76981700 & 2.35395000 & -3.1883060 \\
\hline Pt38 & 1.21171600 & 3.22995100 & 3.64983800 \\
\hline Pt39 & -0.09289500 & 3.75941500 & 1.22441200 \\
\hline
\end{tabular}




\begin{tabular}{|c|c|c|c|}
\hline Pt 40 & 5.61129700 & 0.74333000 & -1.13441700 \\
\hline Pt41 & -5.62906600 & -2.20973500 & 1.62764000 \\
\hline Pt 42 & 1.83134400 & 1.90963100 & 1.38261600 \\
\hline Pt 43 & 1.65789900 & 3.41567900 & -0.82282900 \\
\hline Pt 44 & 1.15601400 & -2.02223800 & 3.30462000 \\
\hline Pt 45 & 3.33457700 & 0.06725900 & 0.03739700 \\
\hline Pt 46 & 2.22810300 & -2.94131600 & -3.42337700 \\
\hline Pt4 7 & -1.29712100 & -4.72061500 & -3.13708800 \\
\hline Pt 48 & 0.47183400 & 0.63248200 & 3.38254100 \\
\hline Pt 49 & -0.78811500 & 5.08190600 & 3.45510500 \\
\hline Pt50 & -2.45179600 & -2.64608700 & -4.48769300 \\
\hline Pt51 & -2.87143500 & 0.80815900 & -0.63508100 \\
\hline Pt52 & -1.22148200 & -1.20002700 & 2.26496900 \\
\hline Pt53 & 1.25915500 & -3.48472200 & 5.47538200 \\
\hline Pt5 4 & 0.77887700 & -4.20716800 & -1.38756000 \\
\hline Pt55 & -2.04026100 & 5.63636700 & 1.08413700 \\
\hline Pt56 & -1.10707900 & 0.46080700 & -2.70294200 \\
\hline Pt57 & -0.55050700 & -5.56060400 & 0.65724200 \\
\hline Pt58 & 1.31322900 & 1.21877400 & -5.56850900 \\
\hline Pt59 & -3.26682300 & -1.62810600 & 0.59493300 \\
\hline Pt 60 & -2.12661400 & 3.74461800 & 5.44304500 \\
\hline Pt61 & 5.04507600 & 0.36439200 & 4.37449600 \\
\hline Pt 62 & 3.73858900 & 0.42627700 & -4.57266300 \\
\hline Pt 63 & -1.26901700 & 1.95626000 & -4.93023300 \\
\hline Pt 64 & 3.04370500 & -0.10501900 & 2.75308100 \\
\hline Pt 65 & -1.15765900 & 4.24845500 & -3.39211400 \\
\hline Pt 66 & 3.54462200 & -3.64145300 & -1.10732200 \\
\hline Pt 67 & -3.08024000 & 2.33637900 & -2.88821400 \\
\hline Pt 68 & 0.26523100 & -3.04514000 & 0.94309700 \\
\hline Pt 69 & -0.28845500 & 5.33999900 & -1.01175900 \\
\hline Pt70 & 5.39997300 & 0.55946300 & 1.64342500 \\
\hline Pt71 & 5.26679700 & -1.76997400 & 0.07155800 \\
\hline Pt72 & -0.08083700 & -6.70535400 & -1.73335400 \\
\hline Pt73 & -4.97944700 & -1.90155600 & -3.78923300 \\
\hline Pt74 & 3.97820300 & -1.11285100 & -2.33076600 \\
\hline Pt75 & -2.93499100 & -1.45461700 & -2.12365100 \\
\hline Pt76 & -3.15276400 & 0.02323300 & -4.38831600 \\
\hline Pt77 & -2.71591600 & 3.04200300 & 0.89239500 \\
\hline Pt78 & 3.05114900 & -3.90626200 & 3.40873300 \\
\hline Pt79 & 3.18148200 & 1.54880300 & -2.16922800 \\
\hline Pt 80 & -5.11870200 & 2.49383600 & 1.96655100 \\
\hline Pt 81 & -3.86950100 & 1.93628000 & 4.33882200 \\
\hline Pt 82 & -3.57759900 & -1.80786700 & 3.35063100 \\
\hline Pt83 & -0.16487600 & 1.89160700 & 5.62237200 \\
\hline Pt 84 & -1.90874400 & 0.04635900 & 4.55300900 \\
\hline Pt 85 & -3.16054000 & 0.62987400 & 2.10531200 \\
\hline Pt 86 & 4.78150900 & -4.22987800 & 1.27119800 \\
\hline Pt 87 & 0.62830200 & 3.84941000 & -5.40166200 \\
\hline Pt 88 & 0.34987000 & -4.58520600 & 3.11261200 \\
\hline Pt 89 & -0.90715800 & -1.02323200 & -0.46838600 \\
\hline Pt90 & 2.91297600 & -2.39322100 & 1.20848800 \\
\hline Pt91 & -3.40710700 & 4.34756700 & 3.12653200 \\
\hline Pt92 & -1.44910300 & 2.46839100 & 3.25069400 \\
\hline Pt93 & -0.99733900 & 2.73837100 & -1.17452000 \\
\hline Pt9 4 & 0.92155200 & 0.87378400 & -0.99169100 \\
\hline Pt95 & -5.52493900 & 0.06108400 & 3.14433800 \\
\hline & luster $=\operatorname{Pt96} \quad \mathrm{Q}=0$ & \multirow{2}{*}{\multicolumn{2}{|c|}{$\begin{array}{c}\mathrm{M}=0 \quad \mathrm{Etot}=-9138.84518900 \\
1.79861500 \quad 4.36769600\end{array}$}} \\
\hline & 3.23244400 & & \\
\hline
\end{tabular}




\begin{tabular}{|c|c|c|c|}
\hline Pt2 & 5.75254600 & 0.42253100 & -1.20705200 \\
\hline Pt3 & 1.27906400 & -1.35323900 & -6.48349500 \\
\hline Pt 4 & -6.74446600 & -0.42210300 & -1.06910100 \\
\hline Pt 5 & 1.85386200 & -4.12696300 & 0.12440600 \\
\hline Pt 6 & -2.40076800 & -5.41493000 & 1.61636500 \\
\hline Pt 7 & -1.37511300 & -2.95002500 & 1.91748800 \\
\hline Pt 8 & -1.06635500 & 0.50841500 & 5.83314700 \\
\hline Pt9 & -3.14053500 & -1.27289200 & -5.03012500 \\
\hline Pt10 & -0.65180400 & -2.68815000 & -2.44754300 \\
\hline Pt11 & 2.34372500 & -3.33444100 & 4.75109200 \\
\hline Pt12 & 3.87412300 & 4.72402000 & -1.77950300 \\
\hline Pt13 & -4.03775000 & 3.78220100 & -3.14378000 \\
\hline Pt14 & -3.88479900 & 2.77705600 & 4.62225000 \\
\hline Pt15 & 3.44977900 & 4.61233000 & 0.91323800 \\
\hline Pt16 & 3.07909000 & 0.10364400 & -1.65064500 \\
\hline Pt17 & -4.08456500 & -0.11617100 & -0.60574300 \\
\hline Pt18 & 4.40013200 & 2.21693200 & -2.73088300 \\
\hline Pt19 & -1.39826500 & 0.19758500 & -0.13935900 \\
\hline Pt20 & 2.59351300 & 2.62921200 & -0.71985200 \\
\hline Pt21 & 0.23311700 & -1.95230100 & -0.00416000 \\
\hline Pt22 & 0.34829400 & 4.13466000 & 3.13785500 \\
\hline Pt23 & 6.62661800 & 1.16957300 & 1.23653000 \\
\hline Pt24 & 4.82717600 & 2.80523000 & 2.42058700 \\
\hline Pt25 & -4.81367800 & 0.84081600 & 2.98027000 \\
\hline Pt26 & 3.99239300 & -2.33157300 & 2.85516800 \\
\hline Pt27 & 3.38009300 & -0.84877800 & 5.08382000 \\
\hline Pt28 & -0.95479200 & 1.58042500 & -3.61924200 \\
\hline Pt29 & -5.69009600 & -1.11646100 & 1.31891700 \\
\hline Pt30 & 5.59192500 & -1.31158200 & 0.91305000 \\
\hline Pt31 & 3.77542600 & -2.80044800 & -3.98884400 \\
\hline Pt32 & 2.17339300 & -0.63158400 & -4.08633900 \\
\hline Pt33 & 5.03037900 & 0.15969000 & 3.16749300 \\
\hline Pt34 & 2.35479000 & -0.16236700 & 2.70291700 \\
\hline Pt35 & -0.30529000 & -3.67326700 & 4.30628300 \\
\hline Pt36 & 1.70970900 & 1.89760300 & -3.15979200 \\
\hline Pt37 & -1.92826000 & -1.48649100 & 4.18675100 \\
\hline Pt38 & 1.39765800 & 3.40587300 & 5.53312500 \\
\hline Pt39 & -4.06807100 & -3.29108000 & 1.46526800 \\
\hline Pt 40 & -3.33493600 & -3.02515500 & -2.93498400 \\
\hline Pt41 & -2.29841700 & -0.53648700 & -2.57791100 \\
\hline Pt 42 & -5.86058800 & 1.53297300 & 0.57765200 \\
\hline Pt 43 & -0.07555800 & 2.32904600 & -1.16965400 \\
\hline Pt 44 & 1.29877300 & -2.63173000 & 2.37855700 \\
\hline Pt 45 & 0.83126100 & 1.18533000 & -5.63902700 \\
\hline Pt 46 & -1.50140400 & -3.42859500 & -4.89875000 \\
\hline Pt4 7 & -5.12407700 & -2.58720100 & -0.93475500 \\
\hline Pt 48 & 3.62772200 & -4.51636700 & -1.88671800 \\
\hline Pt 49 & -1.80450100 & 0.84234800 & -6.07677200 \\
\hline Pt50 & -1.41046000 & 4.13543800 & -2.6833060 \\
\hline Pt51 & -5.41113900 & 1.68983200 & -2.1105670 \\
\hline Pt52 & -2.73717700 & 1.99465400 & -1.63904500 \\
\hline Pt53 & -1.24718900 & 3.10144800 & 5.09177800 \\
\hline Pt5 4 & -3.01263500 & -0.80129800 & 1.77739000 \\
\hline Pt5 5 & -4.58171300 & -1.79654100 & 3.7109330 \\
\hline Pt5 6 & -3.63343400 & 1.26062700 & -4.11614400 \\
\hline Pt57 & -3.70806200 & 0.17778000 & 5.3551170 \\
\hline Pt58 & 3.46815400 & 1.46999800 & -5.1675860 \\
\hline Pt59 & 4.50497400 & -3.78751100 & 0.58060800 \\
\hline & -2.45011900 & -2.26969200 & -0.46905800 \\
\hline
\end{tabular}




\begin{tabular}{|c|c|c|c|}
\hline Pt 61 & 4.82844400 & -0.32427600 & -3.65600100 \\
\hline Pt 62 & -1.87067500 & 4.02056200 & 0.00021900 \\
\hline Pt 63 & 0.79630400 & 4.33803600 & 0.46009700 \\
\hline Pt 64 & -2.11960200 & 1.15812700 & 3.41670500 \\
\hline Pt 65 & 1.58647600 & 0.80803500 & 6.26807600 \\
\hline Pt 66 & -1.66925200 & -5.14691200 & -2.80009700 \\
\hline Pt 67 & -0.32919700 & -0.47849600 & 2.23944200 \\
\hline Pt 68 & 5.26784900 & 2.96104400 & -0.26914500 \\
\hline Pt 69 & 2.15317500 & 2.47223200 & 1.9537010 \\
\hline Pt 70 & -0.79781100 & -4.44261500 & -0.33283300 \\
\hline Pt71 & -4.96627100 & -0.85342200 & -3.05534200 \\
\hline Pt72 & -0.49655700 & -0.94941100 & -4.54672900 \\
\hline Pt73 & -3.45405600 & -4.73494300 & -0.79180100 \\
\hline Pt 74 & -4.93006300 & 3.46825400 & 2.22748000 \\
\hline Pt75 & 3.01166700 & 3.97799400 & -4.2335120 \\
\hline Pt 76 & 1.14147500 & -3.14355200 & -4.44964600 \\
\hline Pt77 & 4.71396300 & -2.06702500 & -1.54707900 \\
\hline Pt78 & 0.37169500 & 3.68265600 & -4.69787100 \\
\hline Pt79 & 1.23514100 & 4.45036700 & -2.22711400 \\
\hline Pt 80 & 3.96760400 & 0.84234800 & 0.7827260 \\
\hline Pt 81 & 0.98402300 & -4.85333600 & -2.35619800 \\
\hline Pt 82 & -0.51506400 & 2.17129200 & 1.48808300 \\
\hline Pt 83 & -2.29860100 & 3.81957700 & 2.68144000 \\
\hline Pt 84 & 2.91662500 & -1.63084200 & 0.45635200 \\
\hline Pt 85 & 2.89979200 & -4.78395800 & 2.53036300 \\
\hline Pt 86 & -2.26698100 & 3.34963100 & -5.14373100 \\
\hline Pt 87 & -4.51321200 & 3.66442600 & -0.45985400 \\
\hline Pt 88 & 2.01862900 & -2.37026500 & -1.98706900 \\
\hline Pt 89 & 0.24880200 & -5.11933000 & 2.09121100 \\
\hline Pt90 & 0.72192900 & -1.17101600 & 4.64373400 \\
\hline Pt91 & 0.54842800 & 1.47572600 & 3.87676500 \\
\hline Pt92 & 2.98850800 & 4.41087100 & 3.59291200 \\
\hline Pt93 & 1.28162800 & 0.51659500 & 0.32274600 \\
\hline Pt94 & -3.18132300 & 1.83722000 & 1.03385200 \\
\hline Pt95 & -2.95570700 & -3.96527800 & 3.83728900 \\
\hline Pt96 & 0.38809600 & -0.21077600 & -2.10518400 \\
\hline \# 96 & Cluster $=$ Pt $97 \quad Q=0$ & \multicolumn{2}{|c|}{$\mathrm{M}=0 \quad$ Etot $=-9234.24548800$} \\
\hline Pt1 & -1.41845400 & -5.68043800 & 2.52179900 \\
\hline Pt2 & 3.69002600 & -1.71533600 & 5.76679500 \\
\hline Pt 3 & 0.65967800 & 4.00798500 & -0.56598600 \\
\hline Pt 4 & -1.73906700 & -4.28014600 & -1.05519300 \\
\hline Pt 5 & 0.22840300 & 3.39282800 & 2.09710300 \\
\hline Pt 6 & -0.12341600 & -1.94547500 & -5.59489100 \\
\hline Pt 7 & 2.49401800 & -3.74219500 & 4.39783500 \\
\hline Pt 8 & -1.50775600 & 3.08121400 & 4.16042000 \\
\hline Pt 9 & 2.68491300 & -1.28470700 & 3.32500400 \\
\hline Pt10 & -1.28953400 & -3.74628700 & -3.79289100 \\
\hline Pt11 & 2.65787800 & 2.80643600 & 0.97744100 \\
\hline Pt12 & -2.93132200 & -3.64195700 & 3.53575300 \\
\hline Pt13 & 0.31224600 & -5.34874600 & 0.46089400 \\
\hline Pt14 & 0.91224000 & 1.99978500 & -2.34328000 \\
\hline Pt15 & -3.69749600 & 2.33324200 & -1.49782500 \\
\hline Pt16 & -3.54090600 & 2.58033300 & 2.3655660 \\
\hline Pt 17 & 4.89820300 & 0.04125000 & 4.07426600 \\
\hline Pt18 & -3.71185400 & -3.07434400 & -2.57086400 \\
\hline Pt19 & -1.72278300 & -1.61946300 & 4.9945200 \\
\hline Pt20 & 0.94054500 & -2.36723800 & -3.17145300 \\
\hline Pt21 & -1.24242400 & 1.12679900 & 2.3388340 \\
\hline
\end{tabular}




\begin{tabular}{|c|c|c|c|}
\hline Pt22 & -5.50106900 & 2.01267200 & 0.51528500 \\
\hline Pt23 & 3.72010500 & -4.73989900 & 2.18198300 \\
\hline Pt24 & -5.45475400 & -3.40116000 & -0.51672800 \\
\hline Pt25 & 1.21575400 & 3.02226500 & 4.59368300 \\
\hline Pt26 & -2.54600300 & 3.92941100 & -4.69293600 \\
\hline Pt27 & 2.66316000 & -1.99897700 & -5.17896100 \\
\hline Pt28 & -1.46156500 & 0.94519800 & -1.49963800 \\
\hline Pt29 & 4.68394400 & -1.44983800 & -3.29597100 \\
\hline Pt30 & 2.91803000 & -1.82894200 & -1.33306900 \\
\hline Pt31 & -2.97576300 & 0.76083100 & 4.36247800 \\
\hline Pt32 & 2.61778300 & 2.45877600 & -4.33035400 \\
\hline Pt33 & -2.60161400 & 1.40180700 & -3.86574600 \\
\hline Pt3 4 & 1.27397700 & -5.72897500 & 2.96231200 \\
\hline Pt35 & 2.46442200 & 0.64982700 & 5.21814900 \\
\hline Pt36 & -3.22515800 & 0.59469600 & 0.52084600 \\
\hline Pt37 & -0.22845600 & -3.71505300 & 4.01873200 \\
\hline Pt38 & 6.05493900 & 1.79374900 & 2.31796100 \\
\hline Pt39 & -2.60590400 & -1.32815600 & -4.40933900 \\
\hline Pt 40 & 4.09855700 & 0.34970900 & -5.32444800 \\
\hline Pt41 & -1.65273100 & 5.44831900 & -0.52860900 \\
\hline Pt 42 & -5.53906500 & -0.70828400 & 0.01709900 \\
\hline Pt43 & 1.70747100 & -0.86794900 & 0.90655700 \\
\hline Pt 44 & 4.93381100 & -2.69411700 & 3.56159600 \\
\hline Pt 45 & -2.03233400 & 4.87467100 & 2.12737900 \\
\hline Pt 46 & -4.95446700 & -2.49918800 & 2.01290700 \\
\hline Pt 47 & -2.66826300 & -1.18031800 & 2.49773400 \\
\hline Pt 48 & 3.97020900 & -2.29220700 & 1.08534900 \\
\hline Pt 49 & 2.40913700 & 4.77571400 & 2.82789600 \\
\hline Pt50 & -4.03124900 & 4.33689400 & 0.30514800 \\
\hline Pt51 & 3.67444600 & 2.43102300 & 3.49753300 \\
\hline Pt52 & -4.92862200 & 0.13095800 & -4.33181800 \\
\hline Pt53 & 2.40154100 & -0.04666600 & -3.37269900 \\
\hline Pt5 4 & 0.74481000 & -4.89615000 & -2.26013900 \\
\hline Pt5 5 & -0.18311600 & 2.52119300 & -4.74101900 \\
\hline Pt5 6 & 4.82362600 & 4.14119800 & 1.70262400 \\
\hline Pt57 & -4.88573200 & 2.83094000 & -3.82880100 \\
\hline Pt58 & 1.05849800 & 4.48860600 & -3.22805600 \\
\hline Pt59 & -4.39583300 & -1.55951100 & 4.50484500 \\
\hline Pt 60 & -0.26428700 & 0.71454200 & 4.84856400 \\
\hline Pt61 & -3.71761700 & -0.35568300 & -2.01145400 \\
\hline Pt 62 & -6.01897300 & 1.07195600 & -2.01654400 \\
\hline Pt 63 & -1.45647600 & -1.76780800 & -2.02109100 \\
\hline Pt 64 & 3.88614800 & 0.46209900 & 1.59397200 \\
\hline Pt 65 & 6.15056900 & -0.91419700 & 1.85358000 \\
\hline Pt 66 & 3.13166300 & 3.41329800 & -1.71992000 \\
\hline Pt 67 & -4.97455600 & 0.22785100 & 2.53793000 \\
\hline Pt 68 & -5.96626900 & 3.75080800 & -1.51718800 \\
\hline Pt 69 & 2.89819000 & 0.86944600 & -0.85652000 \\
\hline Pt70 & -0.95571400 & -0.80896200 & 0.49955300 \\
\hline Pt71 & -1.40271800 & 0.47049500 & -6.13280800 \\
\hline Pt72 & -1.33441700 & 3.46962800 & -2.37308200 \\
\hline Pt73 & -0.02438100 & 5.34614200 & 3.9052770 \\
\hline Pt74 & -0.32228500 & -0.00053500 & -3.78040500 \\
\hline Pt75 & 0.38934800 & 5.92878900 & 1.28429200 \\
\hline Pt76 & 1.49396200 & -3.30527600 & 1.91724600 \\
\hline Pt77 & 5.09886000 & 2.21164600 & -0.18528300 \\
\hline Pt78 & 1.36793300 & 0.42327500 & -5.79982800 \\
\hline Pt79 & 2.76213900 & -4.35538400 & -0.3585310 \\
\hline & 5.1981 & -0.50871 & 8 \\
\hline
\end{tabular}




\begin{tabular}{|c|c|c|c|}
\hline Pt 81 & 0.48409300 & 1.47149700 & 0.29457000 \\
\hline Pt 82 & -5.99389900 & -1.62125600 & -2.50808300 \\
\hline Pt 83 & 1.16832400 & -4.30129900 & -4.92113900 \\
\hline Pt 84 & 1.46226000 & 1.06846000 & 2.76036500 \\
\hline Pt 85 & 5.14435300 & -3.22514700 & -1.19430300 \\
\hline Pt 86 & -3.63517600 & 4.85642600 & -2.36793700 \\
\hline Pt 87 & 0.72190400 & -0.44681200 & -1.44775500 \\
\hline t 88 & -3.48452700 & -4.59223300 & 1.02688200 \\
\hline Pt89 & 0.51836000 & -2.88597900 & -0.53232000 \\
\hline Pt 90 & 4.66237900 & 1.30352200 & -2.79712600 \\
\hline Pt9 & 0.99388100 & -1.67134400 & 5.41818900 \\
\hline t9 & 2.85827100 & 5.34931300 & 0.20735300 \\
\hline t93 & -1.76009600 & 2.90692500 & 0.31150100 \\
\hline Pt94 & 3.19238600 & -3.81697400 & -3.09174800 \\
\hline ty & -3.19717900 & -2.12675600 & -0.01713800 \\
\hline Pt9 & 0.00130300 & -1.23974700 & 2.96174100 \\
\hline$P+Q$ & -1.19157800 & -3.25861800 & 1.49325200 \\
\hline \# 97 & Iuster $=$ Pt98 $\quad Q=0$ & $I=0 \quad$ Etot $=-934$ & 18357900 \\
\hline Pt1 & -3.50818800 & 2.40021000 & 0.01785100 \\
\hline Pt2 & -3.21433200 & 4.03087300 & -2.17436300 \\
\hline Pt 3 & 0.73304200 & -4.06840100 & 3.73046300 \\
\hline Pt 4 & -1.50074000 & 2.14970300 & 1.76528200 \\
\hline Pt5 & -0.91361900 & -3.30394400 & 5.80201300 \\
\hline Pt 6 & 5.88246800 & -2.36918000 & -0.66831300 \\
\hline Pt 7 & -0.48301600 & 1.05733600 & 4.08191700 \\
\hline Pt 8 & 0.29689200 & 5.97594700 & -0.11185200 \\
\hline Pt9 & 0.01771300 & -1.26103100 & -0.59188600 \\
\hline Pt10 & 2.11260800 & -0.10479100 & 0.64126100 \\
\hline Pt11 & -5.55574700 & 2.65392400 & -1.74073400 \\
\hline Pt12 & 0.46039900 & 2.98437100 & -2.44646100 \\
\hline Pt13 & 5.76798600 & 1.87338200 & 2.46824000 \\
\hline Pt14 & 2.69215300 & -2.41957900 & -4.19423900 \\
\hline Pt15 & -5.05285900 & -0.18342400 & 3.75525300 \\
\hline Pt16 & -1.80089200 & 3.39155500 & 4.07711900 \\
\hline Pt17 & 0.37473700 & 0.29140000 & -2.76466000 \\
\hline Pt18 & -3.99261400 & 2.18285700 & 2.77069700 \\
\hline Pt19 & 2.41458200 & -1.25324600 & -1.78281100 \\
\hline Pt20 & 2.88077300 & 3.06513700 & -3.65213500 \\
\hline Pt21 & -6.69296800 & 0.32733500 & -0.70630400 \\
\hline Pt22 & -6.16227300 & -2.09619900 & 0.48577800 \\
\hline Pt23 & -1.16358800 & -4.61217100 & -3.51326900 \\
\hline Pt24 & -1.65726500 & 0.53814100 & -4.55967900 \\
\hline Pt25 & 1.00258900 & 3.25 & 4.44316000 \\
\hline Pt26 & 6.65107300 & -0.30300100 & 1.03598100 \\
\hline Pt27 & -3.02138600 & -1.77102400 & 4.83624300 \\
\hline Pt28 & 1.80094000 & -0.36507400 & 3.32738900 \\
\hline Pt29 & 2.81261400 & 0.32699600 & -3.97606200 \\
\hline Pt 30 & 4.92921500 & 1.51146700 & -2.67303400 \\
\hline Pt 31 & 1.93441000 & 5.20855100 & -2.16878200 \\
\hline Pt 32 & -0.06607800 & 4.41752000 & 2.10429300 \\
\hline Pt33 & 0.63520600 & -0.86384200 & -5.17752800 \\
\hline Pt 34 & -1.64988200 & -2.28395100 & 1.32288400 \\
\hline Pt 35 & -4.14303600 & -2.35964900 & 2.32206100 \\
\hline Pt 36 & -0.82974700 & -0.58624600 & 6.14287800 \\
\hline Pt 37 & -4.81682500 & 4.70006400 & -0.07559600 \\
\hline Pt38 & -6.23043500 & 0.57538100 & -3.36725300 \\
\hline Pt3 & -2.46154300 & 6.03198300 & -0.45571000 \\
\hline Pt 40 & 1.31874300 & -3.56682500 & -0.72692300 \\
\hline
\end{tabular}




\begin{tabular}{|c|c|c|c|}
\hline Pt 41 & 2.64303300 & -5.84551400 & -0.88588900 \\
\hline Pt 42 & 3.33406700 & 1.83676400 & 3.72731600 \\
\hline Pt 43 & 0.53263300 & -3.57754900 & -5.37370800 \\
\hline Pt 44 & -1.16867200 & 3.73193900 & -0.37351600 \\
\hline Pt 45 & 4.77958900 & -4.66657000 & 0.34008500 \\
\hline Pt 46 & 1.50392200 & -1.98478800 & 5.42709700 \\
\hline Pt4 7 & 3.59337400 & 2.06475100 & 0.94507700 \\
\hline Pt 48 & 3.88264100 & -0.60551300 & 4.92193100 \\
\hline Pt 49 & 3.77272500 & -3.58249700 & -1.94812900 \\
\hline Pt50 & -4.19899500 & 0.33627000 & -1.64314800 \\
\hline Pt51 & 6.91074700 & -0.04537200 & -1.65145400 \\
\hline Pt52 & -1.98265600 & -1.04553300 & -2.35694300 \\
\hline Pt53 & 3.43908800 & -2.38970800 & 0.50456100 \\
\hline Pt5 4 & 1.03686000 & -2.41086600 & 1.67368300 \\
\hline Pt5 5 & 0.27512500 & -2.41076600 & -2.97934900 \\
\hline Pt5 6 & 5.08961600 & 4.23211100 & 1.21785400 \\
\hline Pt57 & 3.17270200 & -2.70522500 & 3.27725200 \\
\hline Pt58 & 3.04426600 & 5.77190400 & 0.26316500 \\
\hline Pt59 & 1.56605900 & 3.60275800 & -0.01645200 \\
\hline Pt 60 & -3.91784000 & 1.92937800 & -3.82816300 \\
\hline Pt61 & -0.57090600 & -1.70710000 & 3.75492100 \\
\hline Pt 62 & -1.55204200 & 3.27453200 & -4.23091300 \\
\hline Pt 63 & 2.36498700 & -4.71901500 & 1.56384400 \\
\hline Pt 64 & 4.02340900 & 3.68898100 & -1.22935700 \\
\hline Pt 65 & -0.37799000 & -4.66941600 & 1.20566500 \\
\hline Pt 66 & -4.69256600 & 0.08284400 & 1.06927300 \\
\hline Pt 67 & 2.50872600 & 1.47137700 & -1.51377600 \\
\hline Pt 68 & 6.03753200 & 2.10628900 & -0.22704000 \\
\hline Pt 69 & 0.10985700 & 1.41297400 & -0.33019200 \\
\hline Pt70 & -1.41322400 & -3.43777400 & -1.08362700 \\
\hline Pt71 & 5.61041700 & -2.65230900 & 2.02289800 \\
\hline Pt72 & -0.82283700 & 5.33879600 & -2.52878600 \\
\hline Pt73 & -3.46296200 & -3.24070300 & -2.89286800 \\
\hline Pt74 & -3.65975400 & -2.05437800 & -0.42231000 \\
\hline Pt75 & -2.20669100 & 0.09924000 & 0.07730500 \\
\hline Pt7 6 & -3.11356400 & -4.46022400 & 0.84852800 \\
\hline Pt77 & 4.83453100 & -1.23198800 & -2.94396200 \\
\hline Pt78 & -2.03812500 & -3.93749800 & 3.36864200 \\
\hline Pt79 & -2.61910700 & -0.15628300 & 2.75027800 \\
\hline Pt 80 & -1.88962600 & 1.67914400 & -2.08805300 \\
\hline Pt 81 & -2.95162800 & 1.01335000 & 5.10046900 \\
\hline Pt 82 & 0.81250100 & 4.57623500 & -4.56726500 \\
\hline Pt83 & -0.10681500 & -5.78512500 & -1.27489000 \\
\hline Pt 84 & 0.72924900 & 1.87568700 & -4.90666700 \\
\hline Pt 85 & 1.59215800 & -4.74234500 & -3.15346100 \\
\hline Pt 86 & 4.49228600 & -0.07428200 & -0.50835700 \\
\hline Pt 87 & -2.85789800 & -5.58566500 & -1.60412600 \\
\hline Pt 88 & -2.81361900 & 4.46702800 & 1.72913000 \\
\hline Pt 89 & -5.70831800 & -1.82166300 & -2.18168200 \\
\hline Pt90 & -6.00850000 & 2.42967100 & 0.93062500 \\
\hline Pt91 & 4.24879000 & -0.33952100 & 2.23671500 \\
\hline Pt92 & -1.74003900 & -2.21021500 & -4.77293500 \\
\hline Pt93 & -4.01161200 & -0.81412000 & -4.09897200 \\
\hline Pt9 4 & -5.11844400 & -4.19901400 & -0.9522680 \\
\hline Pt95 & 2.67823000 & 4.20760800 & 2.44618100 \\
\hline Pt96 & -0.23395300 & -0.12238400 & 1.74755300 \\
\hline Pt97 & 1.19096500 & 2.02255400 & 2.1167290 \\
\hline Pt98 & 1.62718800 & 0.79705900 & 5.69831000 \\
\hline
\end{tabular}




\begin{tabular}{|c|c|c|c|}
\hline \# 98 & Cluster $=$ Pt 99 & Etot $=-9$ & 14592500 \\
\hline Pt1 & 3.72719300 & -4.10906800 & 2.07162600 \\
\hline Pt2 & 3.41219100 & -1.99161700 & -1.13858200 \\
\hline Pt 3 & -2.76914300 & -0.78026600 & 1.62985500 \\
\hline Pt 4 & -1.06439600 & -0.26680700 & 3.75360500 \\
\hline Pt 5 & 1.58563100 & 0.11114300 & 4.57763800 \\
\hline Pt 6 & -0.49548900 & 1.42656300 & 5.76823200 \\
\hline Pt 7 & 2.46114600 & 5.15752400 & 3.51302100 \\
\hline Pt 8 & 1.86647900 & 3.45511800 & 1.57558500 \\
\hline Pt 9 & 3.63749400 & -4.62244100 & -0.68535800 \\
\hline Pt10 & 0.40393400 & 1.69457500 & -6.27279200 \\
\hline Pt11 & -5.17923900 & -2.58902700 & -0.75326100 \\
\hline Pt12 & 1.42329400 & 5.28300600 & -3.32029300 \\
\hline Pt13 & 0.94109000 & -4.99556000 & -1.42389600 \\
\hline Pt14 & -3.85792800 & -4.95308400 & -1.24242300 \\
\hline Pt15 & -1.47407500 & -6.25637800 & -1.10306000 \\
\hline Pt16 & -1.14745000 & 5.22540400 & -2.33954800 \\
\hline Pt17 & -3.01153800 & -3.31902200 & 0.71601000 \\
\hline Pt18 & 2.17507300 & 3.52024000 & -5.27973300 \\
\hline Pt19 & -3.45345200 & -1.55893100 & 4.12374200 \\
\hline Pt20 & 5.49912700 & -2.76888400 & 0.33667400 \\
\hline Pt21 & -2.10301700 & -3.16440900 & -5.28644500 \\
\hline Pt22 & -1.32857000 & -5.42361500 & 3.36915600 \\
\hline Pt23 & -5.09104000 & -2.08424100 & 1.95760700 \\
\hline Pt24 & 2.17701700 & 0.84405300 & 2.04236000 \\
\hline Pt25 & -0.44018400 & 0.48962100 & 1.29319900 \\
\hline Pt26 & 0.32828000 & -2.32140600 & 4.98914800 \\
\hline Pt27 & 4.03288500 & 4.96798300 & 1.25971800 \\
\hline Pt28 & -5.31838000 & -4.63258100 & 1.03565900 \\
\hline Pt29 & 2.96885500 & -2.30432900 & 4.0748860 \\
\hline Pt30 & -3.68723200 & -2.89692000 & -3.06170200 \\
\hline Pt31 & -0.52625700 & -0.00300500 & -1.35236700 \\
\hline Pt32 & -2.76950600 & 4.40974500 & 2.07606800 \\
\hline Pt33 & 1.08706400 & -4.16010600 & 3.06277000 \\
\hline Pt34 & 2.73373700 & -3.64997600 & -3.15167900 \\
\hline Pt35 & -2.15867300 & 1.62433500 & -5.3246170 \\
\hline Pt36 & -0.38984800 & 3.49408400 & -4.35904100 \\
\hline Pt37 & -2.89981400 & 3.94743000 & -0.64069800 \\
\hline Pt38 & 1.24705800 & 1.77742000 & -0.37154300 \\
\hline Pt39 & -2.33493300 & -5.22107400 & -3.50529400 \\
\hline Pt 40 & -0.15606800 & 4.83207300 & 2.8264030 \\
\hline Pt41 & -2.94500300 & -5.93686800 & 1.20131000 \\
\hline Pt 42 & -2.94586300 & 3.40040300 & -3.34443200 \\
\hline Pt 43 & -4.42141800 & 3.04257100 & 3.75073300 \\
\hline Pt 44 & 0.95154700 & -1.53115900 & 2.52451500 \\
\hline Pt 45 & 2.53917900 & 0.87760100 & -4.81359300 \\
\hline Pt 46 & -4.66280600 & 2.07346400 & -1.63684800 \\
\hline Pt 47 & 2.03438100 & 0.02770400 & -2.34170600 \\
\hline Pt 48 & 4.23147900 & 0.14289600 & 3.57811300 \\
\hline Pt 49 & 5.35068800 & -3.24258600 & -2.37417200 \\
\hline Pt50 & 3.88738100 & 4.13521900 & -3.21249700 \\
\hline Pt51 & 1.84336400 & -5.88428800 & 1.03572900 \\
\hline Pt52 & 5.90290700 & 1.98393700 & -0.56147700 \\
\hline Pt53 & -4.68361500 & 1.54193600 & -4.30817200 \\
\hline Pt5 4 & -2.85675100 & -1.28167300 & -1.06286600 \\
\hline Pt55 & 0.66958100 & 6.97331300 & -1.32027400 \\
\hline Pt5 6 & 1.50048100 & -0.75551200 & 0.09186400 \\
\hline Pt57 & 1.29349800 & -1.56082300 & -4.40136800 \\
\hline & 0.94909300 & 4.35749100 & -0.84228000 \\
\hline
\end{tabular}




\begin{tabular}{|c|c|c|c|}
\hline Pt59 & 3.69308700 & 0.59552600 & -0.23104700 \\
\hline Pt 60 & 0.77737400 & 3.83188800 & 5.20528500 \\
\hline Pt 61 & 0.04132100 & -3.96375800 & -3.83082400 \\
\hline Pt 62 & -0.59649300 & -4.67090400 & 0.8891680 \\
\hline Pt 63 & -1.72957600 & -1.02709600 & 6.19163200 \\
\hline Pt 64 & -4.93717800 & -0.00139900 & 0.16089200 \\
\hline Pt 65 & 4.41851500 & 2.29692800 & 1.75202800 \\
\hline Pt 66 & 0.18100700 & 2.14703900 & 3.26360700 \\
\hline Pt 67 & -2.60083100 & 0.76935500 & -2.8203780 \\
\hline Pt 68 & -0.87355600 & -0.73723000 & -5.8520500 \\
\hline Pt 69 & -1.29492100 & -1.58616900 & -3.33180500 \\
\hline Pt70 & 0.80891100 & -2.34750800 & -1.85955100 \\
\hline Pt71 & 1.72325700 & 2.63541600 & -2.82648600 \\
\hline Pt72 & -2.42732400 & 1.76238800 & 2.51253800 \\
\hline Pt73 & 5.69477100 & -0.63501800 & -1.4947830 \\
\hline Pt7 4 & -4.96590900 & -0.51485900 & -2.54965500 \\
\hline Pt75 & -0.04951700 & 0.82767700 & -3.82180200 \\
\hline Pt76 & 5.52684400 & -2.23439800 & 3.04013000 \\
\hline Pt77 & -1.05925100 & 5.73019100 & 0.37132000 \\
\hline Pt78 & -2.56197700 & 1.28999000 & -0.1568520 \\
\hline Pt79 & -4.78955200 & 0.49448300 & 2.87084500 \\
\hline Pt 80 & 3.47543200 & 3.22131900 & -0.71291000 \\
\hline Pt 81 & 3.99636200 & -1.20275100 & -3.64845300 \\
\hline Pt 82 & -4.57469600 & 2.57774300 & 1.07129800 \\
\hline Pt 83 & -1.83874100 & 3.45539600 & 4.50878500 \\
\hline Pt 84 & 3.13245200 & 5.84248500 & -1.18983400 \\
\hline Pt 85 & -3.71404200 & -4.12958400 & 3.1800460 \\
\hline Pt 86 & 1.56718000 & 6.10650600 & 1.1021760 \\
\hline Pt 87 & -3.09481600 & 1.02086700 & 5.00298100 \\
\hline Pt 88 & -0.85215800 & 2.60363900 & -1.85156300 \\
\hline Pt 89 & -3.41474700 & -0.81019400 & -4.83042100 \\
\hline Pt90 & 1.68217100 & -3.30204000 & 0.56013800 \\
\hline Pt91 & 2.82613800 & 2.51995300 & 4.00637900 \\
\hline Pt92 & 3.50004800 & -1.48878800 & 1.56177200 \\
\hline Pt93 & 5.78447000 & -0.12164400 & 1.26220100 \\
\hline Pt9 4 & -0.72313700 & -2.04122100 & 0.40362000 \\
\hline Pt95 & -1.51135600 & -3.63392700 & -1.55384400 \\
\hline Pt96 & 4.27254100 & 1.46147300 & -2.73463800 \\
\hline Pt97 & -1.36813400 & -2.81422500 & 2.84822800 \\
\hline Pt98 & -2.04886000 & -3.58379000 & 5.28746000 \\
\hline Pt99 & -0.76455000 & 3.10504600 & 0.84115800 \\
\hline \# 99 & luster $=\operatorname{Pt} 100 \quad \mathrm{Q}=0$ & $=0 \quad$ Etot $=-95$ & .7367 \\
\hline Pt 1 & 0.94380000 & -3.85434800 & -4.3913500 \\
\hline Pt2 & 5.46995900 & 0.20341000 & 2.08037700 \\
\hline Pt3 & 3.65797200 & -3.26074200 & -0.57082800 \\
\hline Pt 4 & 2.63955500 & -1.76725600 & 5.28768200 \\
\hline Pt 5 & -0.87790200 & 6.05151400 & 0.52267500 \\
\hline Pt 6 & -0.50333900 & -1.39665200 & -1.8743480 \\
\hline Pt 7 & 1.97430600 & -1.13634500 & -0.7266390 \\
\hline Pt 8 & 2.32644400 & 2.70224000 & -0.33902700 \\
\hline Pt 9 & -1.93982500 & 1.97046500 & -4.88375100 \\
\hline Pt10 & 3.09906800 & 3.01083400 & -4.11144100 \\
\hline Pt11 & 3.15158400 & 4.87977100 & 2.6484250 \\
\hline Pt12 & -5.95320500 & -0.05539600 & 2.3054460 \\
\hline Pt13 & -4.82984100 & 4.04122500 & -1.0487790 \\
\hline Pt14 & 0.31453000 & 0.98719700 & -0.90290100 \\
\hline Pt15 & -0.55556400 & 1.61297300 & 6.13603200 \\
\hline Pt16 & -5.06353900 & -0.90326200 & -2.799014 \\
\hline
\end{tabular}




\begin{tabular}{|c|c|c|c|}
\hline Pt17 & 1.18980200 & -1.44280300 & 3.05484500 \\
\hline Pt18 & 4.57948100 & 2.65539700 & -1.86383900 \\
\hline Pt19 & 0.29108200 & 5.59330800 & -1.89697200 \\
\hline Pt20 & -5.36176600 & -2.60789300 & 1.58126100 \\
\hline Pt21 & -3.88663500 & -4.53018300 & -2.46408000 \\
\hline Pt22 & -1.97930300 & -2.68620100 & 4.88211100 \\
\hline Pt23 & 0.03013300 & -0.95606100 & 5.47404200 \\
\hline Pt24 & -3.44421600 & 5.27462600 & 0.93644300 \\
\hline Pt25 & 2.88257600 & 4.76609500 & -2.03276900 \\
\hline Pt26 & -4.48377800 & 1.15684700 & -4.45856500 \\
\hline Pt27 & -5.41616600 & 2.00291500 & 0.63229400 \\
\hline Pt28 & -4.26247700 & 1.49641600 & -1.78539600 \\
\hline Pt29 & 5.09498700 & -3.58655400 & 1.69644100 \\
\hline Pt30 & -1.41347400 & -0.62470400 & 3.19305700 \\
\hline Pt31 & 0.02612700 & 3.66605600 & 4.48622000 \\
\hline Pt32 & -0.82582900 & -3.16376600 & 2.50033800 \\
\hline Pt33 & -1.43301400 & 3.98832700 & 2.18897800 \\
\hline Pt34 & -4.56378100 & 1.19186100 & 4.27932200 \\
\hline Pt35 & -5.60788900 & -2.94249800 & -1.10210900 \\
\hline Pt36 & 2.54249900 & 0.93821700 & -2.42418500 \\
\hline Pt37 & 0.56400000 & 5.69573700 & 2.79315300 \\
\hline Pt38 & 1.75984800 & 0.62834600 & 1.35681000 \\
\hline Pt39 & 2.30505700 & 0.61725300 & -5.11609200 \\
\hline Pt 40 & -2.57338300 & 4.43135000 & 4.55029600 \\
\hline Pt41 & 2.87119600 & -3.57428000 & 3.23688700 \\
\hline Pt42 & 3.42141400 & -1.49175200 & 1.52736000 \\
\hline Pt43 & -3.64811000 & -4.25921300 & 0.21697400 \\
\hline Pt 44 & -1.99951100 & 1.92246000 & 3.88046600 \\
\hline Pt45 & -1.67693100 & 2.28163700 & -2.19349500 \\
\hline Pt 46 & 2.81460400 & -5.62992100 & -1.56289800 \\
\hline Pt 47 & -4.03619600 & 3.23925900 & 2.60444600 \\
\hline Pt 48 & 1.40507000 & -3.20969700 & 0.97203700 \\
\hline Pt 49 & 4.32762100 & 4.38868400 & 0.24733000 \\
\hline Pt50 & 0.36826500 & -5.88693900 & -2.69610600 \\
\hline Pt51 & 3.38393300 & -3.59798300 & -3.26101600 \\
\hline Pt52 & -0.18888800 & 0.33816300 & -6.21859900 \\
\hline Pt53 & 5.68374100 & -1.53780500 & 0.02537400 \\
\hline Pt54 & -1.07225400 & -3.46997000 & -0.17552400 \\
\hline Pt55 & -3.37052700 & -3.92584700 & 2.89557100 \\
\hline Pt56 & -3.67370000 & 3.54804800 & -3.45909000 \\
\hline Pt57 & -3.06054900 & -2.17276200 & -1.47525400 \\
\hline Pt58 & 3.93888600 & -1.52995100 & -4.91986600 \\
\hline Pt59 & 1.73608800 & 5.26512000 & 0.38027300 \\
\hline Pt 60 & -2.76235600 & -0.43022400 & -5.82103700 \\
\hline Pt 61 & 0.88252000 & 3.06614500 & -2.60057900 \\
\hline Pt 62 & -2.24527600 & 0.20755900 & -0.49846800 \\
\hline Pt 63 & 4.22044700 & -1.20954100 & -2.2503070 \\
\hline Pt 64 & -3.34670200 & -2.48656300 & -4.15776300 \\
\hline Pt 65 & 0.61388600 & -3.50723500 & 4.74125500 \\
\hline Pt 66 & 0.59644400 & -5.61362000 & -0.0143870 \\
\hline Pt 67 & -1.10183700 & 4.34748400 & -3.87845300 \\
\hline Pt 68 & -3.40558200 & 0.68406500 & 1.8955810 \\
\hline Pt 69 & 6.21926700 & 0.51340400 & -1.6605170 \\
\hline Pt70 & 0.82846900 & -5.26945900 & 2.66828400 \\
\hline Pt71 & 2.60076400 & 2.84519100 & 4.34594500 \\
\hline Pt72 & -3.99299500 & -1.37874000 & 3.6104240 \\
\hline Pt73 & -4.82467900 & -0.55379200 & -0.1067300 \\
\hline Pt 74 & 0.63358800 & 2.75180500 & -5.2535160 \\
\hline & 1.32 & 81200 & -2.8770770 \\
\hline
\end{tabular}




\begin{tabular}{|c|c|c|c|}
\hline Pt76 & -1.63701100 & -5.52848700 & 1.52620900 \\
\hline Pt77 & -2.49372900 & -0.10571600 & -3.16770700 \\
\hline Pt78 & 4.88080900 & -1.84053200 & 3.75652900 \\
\hline Pt79 & 6.00492000 & 2.25881300 & 0.40007300 \\
\hline Pt 80 & -0.25499500 & -1.08971600 & 0.79763100 \\
\hline Pt81 & 3.07237300 & -5.30350700 & 1.11997600 \\
\hline Pt 82 & 3.22400900 & 0.29182100 & 3.62163200 \\
\hline Pt83 & -0.27402600 & 3.53057000 & -0.20919200 \\
\hline Pt 84 & -2.83946800 & 2.74853900 & 0.20523500 \\
\hline Pt 85 & 4.76205200 & 0.85831900 & -3.92519500 \\
\hline Pt 86 & 0.60159400 & 1.10506700 & 3.75180900 \\
\hline Pt 87 & 2.02822000 & 0.78315100 & 6.00265000 \\
\hline Pt 88 & -1.87856700 & -5.82366400 & -1.17342800 \\
\hline Pt89 & 1.49254700 & -1.78696800 & -6.05307400 \\
\hline Pt90 & -2.28703900 & 4.84244100 & -1.48334500 \\
\hline Pt91 & -2.81357400 & -1.86340600 & 1.20037700 \\
\hline Pt92 & -2.56909000 & -0.11581300 & 5.57235000 \\
\hline Pt93 & 3.98823400 & 0.57529400 & -0.16509300 \\
\hline Pt94 & -0.75822200 & -1.73261100 & -4.55655600 \\
\hline Pt95 & 1.72617800 & -1.45072800 & -3.39739300 \\
\hline Pt96 & 0.06517500 & 0.67794400 & -3.57174600 \\
\hline Pt97 & -0.84089200 & 1.44801200 & 1.48687900 \\
\hline Pt98 & 1.16770800 & 3.16954100 & 2.06146300 \\
\hline Pt99 & 1.15928900 & -3.51802700 & -1.70473300 \\
\hline Pt100 & 3.78621100 & 2.34202800 & 1.94296600 \\
\hline \#100 & Cluster $=$ Pt101 $\mathrm{Q}=0$ & $I=0 \quad$ Etot $=-9$ & 68595000 \\
\hline Pt1 & -1.76321500 & -1.75454800 & -2.59969200 \\
\hline Pt2 & -5.95349600 & 0.27748600 & -2.40030400 \\
\hline Pt3 & 0.47038800 & 2.38768000 & 2.65018600 \\
\hline Pt 4 & 0.69610900 & -5.71942100 & 2.84363000 \\
\hline Pt5 & 5.04873000 & 3.20817600 & 2.34531600 \\
\hline Pt 6 & 0.54444100 & -5.82407200 & 0.14372000 \\
\hline Pt 7 & -5.19431100 & -2.22811700 & 3.05568200 \\
\hline Pt 8 & 4.91898300 & 3.14848800 & -0.35710400 \\
\hline Pt9 & -3.97022400 & 2.17130800 & 4.22160000 \\
\hline Pt10 & -1.80397800 & 1.18908800 & 5.54603800 \\
\hline Pt11 & -5.36446400 & -2.30925000 & $0.3560650 c$ \\
\hline Pt12 & -3.99583800 & 4.29281100 & -2.6244280 \\
\hline Pt13 & -4.27663700 & 2.01880200 & -1.18706300 \\
\hline Pt14 & 0.17711300 & 2.22523200 & -2.82183300 \\
\hline Pt15 & -1.60870400 & 5.55973600 & -2.7899780 \\
\hline Pt16 & -0.36564100 & -0.20253200 & -6.82224800 \\
\hline Pt17 & 4.63952300 & -3.62653100 & 2.57015000 \\
\hline Pt18 & 2.41340600 & 2.60402100 & -4.32832300 \\
\hline Pt19 & -1.32758300 & -1.51510300 & 5.6007850 \\
\hline Pt20 & 1.09202100 & -2.05724800 & 0.0025460 \\
\hline Pt21 & 4.53393600 & 0.76590000 & -1.62207300 \\
\hline Pt22 & -2.38968300 & 0.86466000 & -5.38224700 \\
\hline Pt23 & -3.95373400 & -0.77521400 & -3.8867550 \\
\hline Pt24 & 4.37078800 & 0.68238100 & -4.3277850 \\
\hline Pt25 & -1.47880000 & 5.66057900 & -0.0887890 \\
\hline Pt26 & 3.62778000 & -1.54694100 & 3.9731620 \\
\hline Pt27 & -1.61551600 & -1.67222500 & 0.13622700 \\
\hline Pt28 & 0.94610100 & -2.13982400 & -2.73346100 \\
\hline Pt29 & 0.80249400 & -2.22572700 & -5.4615880 \\
\hline Pt 30 & -0.07340500 & -0.04066000 & -1.3696150 \\
\hline Pt3 & -3.85986200 & 4.39687700 & 0.0763400 \\
\hline & 5.53506 & 0 & $2.96 / 5$ \\
\hline
\end{tabular}




\begin{tabular}{|c|c|c|c|}
\hline Pt33 & -5.66397400 & 0.43785600 & 3.00170900 \\
\hline Pt34 & -4.13179400 & 2.09903200 & 1.51546900 \\
\hline Pt35 & 2.85355800 & 2.84782600 & 3.88418900 \\
\hline Pt 36 & -2.12615000 & -5.44430300 & 0.27557700 \\
\hline Pt37 & 4.35000100 & -3.78690100 & -2.83186300 \\
\hline Pt38 & -1.69520500 & 3.37163000 & 1.35861500 \\
\hline Pt39 & 2.26862700 & -4.09729700 & 1.35638700 \\
\hline Pt 40 & -1.98129100 & -5.33868300 & 2.97582300 \\
\hline Pt41 & 2.81421500 & 4.93077800 & -3.00835400 \\
\hline Pt 42 & 2.29042200 & 0.40077600 & -0.13465400 \\
\hline Pt 43 & 4.75920700 & 3.04780700 & -3.05669700 \\
\hline Pt 44 & 2.43856400 & 0.48226300 & 2.60126700 \\
\hline Pt 45 & 4.51889800 & -3.71470700 & -0.13191300 \\
\hline Pt 46 & -3.78177400 & -0.68940700 & -1.14470200 \\
\hline Pt 47 & 0.36564000 & 0.20253200 & 6.82224800 \\
\hline Pt 48 & -1.56004700 & 3.47546000 & 4.10210500 \\
\hline Pt 49 & -0.74748100 & -3.87922900 & -3.96644600 \\
\hline Pt50 & 3.18762800 & -1.79074600 & -4.23935000 \\
\hline Pt51 & -1.31918200 & 5.72010600 & 2.61203400 \\
\hline Pt52 & -1.46994000 & -1.59210000 & 2.87232600 \\
\hline Pt53 & 3.10373800 & 5.09114800 & 2.39365800 \\
\hline Pt5 4 & -5.48383400 & -2.38848700 & -2.34633000 \\
\hline Pt55 & -4.40520800 & 1.93036500 & -3.89448200 \\
\hline Pt5 6 & -3.45376900 & -3.47035000 & -3.78514400 \\
\hline Pt57 & 1.97009500 & -4.24164600 & -4.05294000 \\
\hline Pt58 & 0.86561800 & 4.73783600 & 3.88622800 \\
\hline Pt59 & 2.14529000 & 0.31981500 & -2.87075200 \\
\hline Pt 60 & -3.01878500 & -3.22940700 & 4.33093800 \\
\hline Pt 61 & -0.45084800 & -3.69179200 & 1.50161500 \\
\hline Pt 62 & 2.54286800 & 2.66727400 & -1.58332300 \\
\hline Pt 63 & 0.03167100 & 2.14974600 & -5.55017100 \\
\hline Pt 64 & -1.91328800 & -1.83953100 & -5.32750000 \\
\hline Pt 65 & -3.32144900 & -3.40319200 & -1.07729300 \\
\hline Pt 66 & -3.51358200 & -0.53140900 & 4.32575800 \\
\hline Pt 67 & 3.31192300 & -1.69816100 & -1.49494400 \\
\hline Pt 68 & 1.38819900 & -1.90129900 & 5.46669700 \\
\hline Pt 69 & -2.09046500 & 1.02375600 & 0.08164700 \\
\hline Pt70 & -5.83293100 & 0.34994200 & 0.30222900 \\
\hline Pt71 & -2.00019900 & 3.23165500 & -4.11040700 \\
\hline Pt72 & 3.45799600 & -1.61724900 & 1.23053300 \\
\hline Pt73 & 5.82458600 & -1.19586600 & 2.43447700 \\
\hline Pt74 & -3.63570200 & -0.60849500 & 1.58077500 \\
\hline Pt75 & -1.94520100 & 1.10565300 & 2.81771000 \\
\hline Pt76 & 0.40658700 & -5.87979100 & -2.55838300 \\
\hline Pt77 & -3.70631500 & 4.45318100 & 2.77758500 \\
\hline Pt78 & 0.22016900 & 0.12195400 & 4.10799700 \\
\hline Pt79 & 2.68894100 & 2.74818500 & 1.14215300 \\
\hline Pt 80 & 0.57825800 & 4.59544200 & -1.52375500 \\
\hline Pt 81 & 0.32353800 & 2.30494100 & -0.0857670 \\
\hline Pt 82 & -0.22016900 & -0.12195400 & -4.10799700 \\
\hline Pt83 & -1.84127800 & 3.29071800 & -1.36686100 \\
\hline Pt 84 & 0.61737600 & 2.47417400 & 5.37811400 \\
\hline Pt 85 & 0.43063400 & 4.49689400 & -4.22985300 \\
\hline Pt 86 & -2.23847600 & 0.94320500 & -2.6543090 \\
\hline Pt 87 & 0.72310100 & 4.67567300 & 1.17877600 \\
\hline Pt 88 & 2.59024900 & 0.56421000 & 5.3290790 \\
\hline Pt 89 & 2.00454400 & 0.23978200 & -5.5992060 \\
\hline Pt90 & 4.67877900 & 0.84613000 & 1.08045800 \\
\hline Pt91 & 1.23937600 & -1.97737600 & 2.73855800 \\
\hline
\end{tabular}




\begin{tabular}{|c|c|c|c|}
\hline Pt92 & -0.59692100 & -3.77270300 & -1.22386100 \\
\hline Pt93 & 2.12378400 & -4.17752800 & -1.34614500 \\
\hline Pt 94 & -2.27081400 & -5.49905300 & -2.42619000 \\
\hline Pt95 & 4.80577200 & 0.92332300 & 3.78829600 \\
\hline t96 & -3.17660600 & -3.32296200 & 1.62523800 \\
\hline Pt97 & 2.97893800 & 5.02667000 & -0.30888400 \\
\hline Pt 98 & -0.30732900 & -3.63542300 & 4.24606700 \\
\hline Pt99 & 2.40507900 & -4.00070400 & 4.06314200 \\
\hline Pt 100 & 0.07340500 & 0.04066000 & 1.36961500 \\
\hline+101 & 5.70094700 & -1.29022500 & -0.26724100 \\
\hline \#101 & Cluster $=$ Pt102 $\mathrm{Q}=0$ & \multicolumn{2}{|c|}{$\mathrm{M}=0 \quad$ Etot $=-9766.54815400$} \\
\hline Pt 1 & -0.57381100 & -3.78844600 & -1.25579400 \\
\hline Pt2 & 2.28352700 & 3.23246600 & -0.04656900 \\
\hline Pt 3 & -5.38954500 & -2.42810500 & 0.26958800 \\
\hline Pt 4 & 5.14324900 & -0.28960000 & -2.65386800 \\
\hline Pt 5 & -0.19534500 & 6.16803300 & -0.07195900 \\
\hline Pt 6 & 5.66325500 & -1.45136900 & 1.88031500 \\
\hline Pt7 & 4.25912800 & 1.45463900 & -4.50939900 \\
\hline Pt 8 & -6.24080600 & -0.69061500 & -1.62376400 \\
\hline Pt9 & -1.75874000 & 3.16828300 & 1.76845000 \\
\hline Pt10 & 2.46910600 & 5.87764700 & 0.37471800 \\
\hline Pt11 & 2.43281200 & 0.96301700 & 5.5894540 \\
\hline Pt12 & -2.32312300 & -3.60771700 & 3.67044600 \\
\hline Pt13 & 2.29706100 & -1.72819900 & 5.20098600 \\
\hline Pt14 & 3.03047700 & 3.76279800 & -3.79105200 \\
\hline Pt15 & 0.68343200 & 4.44791000 & 1.82860700 \\
\hline Pt16 & -4.49005700 & -4.15794000 & 2.14758900 \\
\hline Pt17 & 0.37046100 & -0.89932400 & 0.97306300 \\
\hline Pt18 & 4.08020300 & -0.25826500 & 3.76818800 \\
\hline Pt19 & 6.47971200 & 1.72278000 & -1.42413200 \\
\hline Pt20 & 0.51898300 & 1.77121300 & 1.3875020 \\
\hline Pt21 & -2.67945700 & 0.26912600 & -2.44600400 \\
\hline Pt22 & 4.26750600 & -3.49319500 & 0.70709300 \\
\hline Pt23 & 0.37229400 & 4.07701500 & -4.28576300 \\
\hline Pt24 & -0.75537600 & 4.09556200 & 4.11605900 \\
\hline Pt25 & -2.73011900 & -4.40933000 & -2.78648600 \\
\hline Pt26 & -5.06405000 & 3.63047000 & -0.14987400 \\
\hline Pt27 & -3.60157500 & -2.65605000 & -4.65606900 \\
\hline Pt28 & 1.60947000 & 1.77099500 & -5.01868300 \\
\hline Pt29 & 2.46991500 & 0.01334200 & -3.09270800 \\
\hline Pt 30 & -1.61830900 & 5.81546400 & 2.21227700 \\
\hline Pt31 & 1.40796000 & 0.01668700 & 3.2965280 \\
\hline Pt 32 & -4.83340500 & -0.35302000 & -3.9193040 \\
\hline Pt33 & 2.14511500 & -4.39532900 & 4.77618900 \\
\hline Pt34 & 3.16584400 & 1.48505500 & 1.85406300 \\
\hline Pt 35 & -2.35101700 & -0.29670000 & -5.08948200 \\
\hline Pt 36 & 0.31077700 & -5.56833200 & 0.64438800 \\
\hline Pt37 & -6.35459400 & 1.27464000 & 0.2358840 \\
\hline Pt38 & -0.36479500 & 3.52759500 & -0.52223100 \\
\hline & 6.00250600 & -1.99808700 & -0.75392300 \\
\hline Pt 40 & 2.48370000 & -4.95166300 & 2.15074100 \\
\hline Pt 41 & -0.17850700 & 0.31019200 & -3.56674200 \\
\hline Pt 42 & -3.56581000 & 2.01741100 & -4.3522100 \\
\hline Pt 43 & -1.90751600 & 0.50319200 & 1.3481390 \\
\hline Pt 44 & -0.51792900 & 0.86011600 & -0.94065100 \\
\hline Pt 45 & -2.43360300 & -1.57908100 & 5.5164710 \\
\hline Pt 46 & -1.02085700 & -1.25293900 & 3.25927100 \\
\hline & -0.02357500 & -4.99791900 & 3.27825200 \\
\hline
\end{tabular}




\begin{tabular}{|c|c|c|c|}
\hline Pt 48 & 0.70524400 & -1.44572400 & -1.65656700 \\
\hline Pt 49 & 4.76266600 & 0.27535000 & 0.00043300 \\
\hline Pt 50 & -1.79229100 & -1.48687100 & -0.53498400 \\
\hline Pt51 & 0.17736200 & -0.26904200 & -6.16312600 \\
\hline Pt 52 & -4.98526900 & 1.66461300 & -2.06418300 \\
\hline Pt 53 & 1.26128900 & -2.65609700 & 2.88410800 \\
\hline Pt 54 & 3.35223200 & -1.73407000 & -1.19207700 \\
\hline Pt55 & 2.11755100 & 0.56300000 & -0.46540200 \\
\hline-56 & 1.55902900 & 2.71380700 & 3.71476400 \\
\hline-57 & 3.73173400 & -2.27772800 & -3.82646400 \\
\hline$=58$ & 0.62034900 & -6.08079400 & -1.99294200 \\
\hline$=59$ & -2.27596700 & 4.36053900 & -4.74743000 \\
\hline$=60$ & 4.07256600 & 4.67677700 & -1.46040900 \\
\hline Pt 61 & 3.91406200 & -2.93738900 & 3.34369300 \\
\hline$=62$ & -0.26539500 & -4.36695700 & -3.89171100 \\
\hline$=63$ & -3.97082100 & -2.10253800 & -2.03400500 \\
\hline 64 & 5.81594400 & 1.22405100 & 2.29506700 \\
\hline$=65$ & -5.50303800 & -0.46793900 & 2.12442000 \\
\hline Pt 66 & -4.20240900 & 1.89724900 & 1.75084700 \\
\hline Pt 67 & -1.24242400 & 5.28168400 & -2.42355300 \\
\hline$=68$ & 2.84047300 & -0.53627200 & -5.69453800 \\
\hline$=69$ & -3.15019900 & 2.84591200 & 4.07888200 \\
\hline Pt70 & 0.00727100 & -0.32643900 & 5.60459900 \\
\hline$=71$ & -0.13911300 & -3.00245000 & 5.16649000 \\
\hline Pt72 & -4.07228300 & -0.09037600 & -0.15366700 \\
\hline Pt73 & 3.01473700 & -1.19448300 & 1.44678300 \\
\hline Pt74 & -0.90557300 & -3.24401800 & 1.37518600 \\
\hline Pt75 & 0.13229500 & 2.34842500 & 5.98331900 \\
\hline Pt 76 & -3.68134800 & 4.01287900 & -2.46397200 \\
\hline Pt77 & -3.09484500 & -3.83739200 & -0.14703200 \\
\hline Pt78 & -2.65452300 & 4.94519300 & -0.12927200 \\
\hline Pt79 & 1.05781300 & -2.01805900 & -4.28990200 \\
\hline Pt 80 & 3.35881900 & 4.16291500 & 2.26807900 \\
\hline Pt 81 & 1.42216700 & 5.00592600 & -1.95758800 \\
\hline Pt 82 & 2.80552000 & -5.47405800 & -0.50171400 \\
\hline Pt 83 & 1.23761700 & 2.32507000 & -2.38137900 \\
\hline Pt 84 & -2.26873900 & 1.09529200 & 5.94626600 \\
\hline Pt 85 & -4.01954300 & 4.56272600 & 2.17479300 \\
\hline Pt 86 & -0.87530300 & 1.41865400 & 3.67149300 \\
\hline Pt 87 & -1.13025600 & -2.61375600 & -5.76398400 \\
\hline Pt 88 & -2.79474100 & 2.26020500 & -0.56191900 \\
\hline Pt 89 & -3.18877700 & -1.84014400 & 1.74952700 \\
\hline Pt 90 & 4.58984200 & -4.00086300 & -1.92615700 \\
\hline Pt91 & -1.40208400 & 2.61866300 & -2.85316400 \\
\hline Pt92 & 3.88042300 & 2.02261500 & -1.90004000 \\
\hline Pt93 & 4.95427100 & 2.97425800 & 0.43460100 \\
\hline Pt9 4 & -1.45724800 & -2.03881700 & -3.15883800 \\
\hline Pt95 & -1.05578500 & 2.05822100 & -5.47379800 \\
\hline Pt96 & 1.93341000 & -3.75216200 & -2.40374500 \\
\hline Pt97 & 1.59424900 & -3.20071700 & 0.25201800 \\
\hline Pt98 & -1.85077000 & -6.12348400 & -0.88459600 \\
\hline Pt99 & -3.32643300 & 0.16239500 & 3.63782000 \\
\hline Pt100 & 4.21847400 & 2.41305600 & 4.14309900 \\
\hline Pt101 & -4.60384500 & -2.19268500 & 4.0072370 \\
\hline Pt102 & -2.20295800 & -5.56257100 & 1.74704100 \\
\hline & Cluster=Pt103 $\mathrm{Q}=0$ & \multirow{3}{*}{\multicolumn{2}{|c|}{ 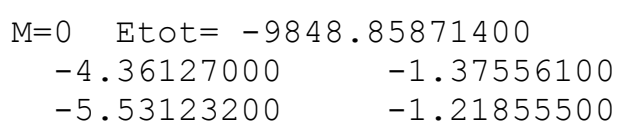 }} \\
\hline$L \perp$ & 3.14888200 & & \\
\hline & -1.15710000 & & \\
\hline
\end{tabular}




\begin{tabular}{|c|c|c|c|}
\hline Pt3 & 0.93082700 & 0.87631100 & 1.74644600 \\
\hline Pt 4 & 6.26760400 & 0.79044300 & -2.17763600 \\
\hline Pt 5 & 5.36549000 & 1.53375100 & 0.26914100 \\
\hline Pt 6 & -1.50602500 & 3.90105500 & -4.11944800 \\
\hline Pt 7 & -3.76031100 & 3.90818700 & -2.53775800 \\
\hline Pt 8 & 2.61319800 & -2.30002000 & 0.31453600 \\
\hline Pt9 & 3.73062400 & 1.68684100 & -1.92337300 \\
\hline Pt10 & 4.20050400 & -2.46534100 & 2.52225700 \\
\hline Pt11 & 0.78554200 & -3.84417500 & 1.55834700 \\
\hline Pt12 & 2.78652000 & 2.42127900 & 0.51096200 \\
\hline Pt13 & 6.93518500 & -1.59470500 & -1.18611400 \\
\hline Pt14 & -3.39416700 & -5.46332400 & 0.35740200 \\
\hline Pt15 & -1.64547900 & -3.44560200 & 0.45981700 \\
\hline Pt16 & -1.32026700 & 3.53061400 & -1.45181800 \\
\hline Pt17 & -2.10466100 & -3.14175200 & 5.14314900 \\
\hline Pt18 & 1.28934700 & -5.88723400 & -0.10714700 \\
\hline Pt19 & 2.32915700 & -4.01748700 & 3.75034500 \\
\hline Pt20 & 5.03694700 & -0.17174600 & 3.70542000 \\
\hline Pt21 & 2.49110700 & 0.69123700 & 3.94584200 \\
\hline Pt22 & -2.40052300 & 2.62812100 & 0.85990200 \\
\hline Pt23 & -1.00730400 & -2.28107900 & 2.85606000 \\
\hline Pt24 & 1.72870200 & 0.54099500 & -3.36469900 \\
\hline Pt25 & -0.99333600 & 1.79775200 & -5.77087300 \\
\hline Pt26 & 0.23875600 & 3.33819400 & 0.74644000 \\
\hline Pt27 & 5.15112000 & -3.17916100 & 0.06240300 \\
\hline Pt28 & -0.81112900 & 1.45561800 & -3.11736400 \\
\hline Pt29 & 0.39528500 & -3.05948000 & -3.81247400 \\
\hline Pt30 & -4.12615400 & -3.06148000 & -0.61944600 \\
\hline Pt31 & 2.11350800 & 4.88828600 & -0.48197100 \\
\hline Pt32 & -3.05911800 & 1.46086600 & -1.53740600 \\
\hline Pt33 & -2.58845400 & 3.97572400 & 4.39524800 \\
\hline Pt34 & -3.58745100 & -5.10124100 & -2.32124100 \\
\hline Pt35 & 1.79909500 & 3.13455400 & 2.94670800 \\
\hline Pt36 & 4.29985000 & -0.36514700 & -3.64111900 \\
\hline Pt37 & 0.03633800 & 4.66920700 & 4.29234000 \\
\hline Pt 3 & 4.65319100 & 3.94543800 & -0.71627100 \\
\hline Pt39 & -0.11360900 & -0.99880300 & -2.11653000 \\
\hline Pt 40 & -1.37182500 & -5.10390500 & -3.87931300 \\
\hline Pt41 & -4.16863200 & 4.14637300 & 2.18852600 \\
\hline Pt 42 & -2.80551400 & -0.70771300 & 4.17706200 \\
\hline Pt 43 & -5.92935300 & 2.08573600 & 2.07701200 \\
\hline Pt 4 & -2.77826700 & -4.34460400 & 2.76211200 \\
\hline Pt 45 & -5.26093700 & -0.33540300 & 3.07407100 \\
\hline Pt 46 & 1.20943000 & 2.62138500 & -1.69563500 \\
\hline Pt 47 & -4.29594600 & -2.69009600 & -3.32023400 \\
\hline Pt 48 & 0.07939900 & -1.38799200 & 0.55374100 \\
\hline Pt 49 & 4.42165900 & -0.73730200 & -0.93088700 \\
\hline Pt50 & -4.54752000 & -2.76171900 & 4.03601000 \\
\hline Pt51 & -4.86532100 & 3.01734400 & -0.2235710 \\
\hline Pt52 & -1.70764600 & 0.16687700 & 1.85822300 \\
\hline Pt53 & -4.97788000 & -0.25729200 & -4.29469500 \\
\hline Pt5 4 & 3.59333300 & 2.05630800 & -4.61138400 \\
\hline Pt5 5 & -5.49092400 & 1.80473100 & -2.60711900 \\
\hline Pt5 6 & 2.95007600 & -3.93476000 & -4.0369650 \\
\hline Pt57 & -1.90738500 & 1.55789900 & 5.4053190 \\
\hline Pt58 & 1.89401600 & 0.15767200 & -0.68757200 \\
\hline & 1.08896600 & -5.47868200 & -2.7954140 \\
\hline Pt 60 & 1.63566600 & -1.57878600 & 2.75365500 \\
\hline & -0.30969900 & -4.72123600 & 3.8448810 \\
\hline
\end{tabular}




\begin{tabular}{|c|c|c|c|}
\hline$t 62$ & -3.09685400 & 5.07642700 & -0.13278800 \\
\hline Pt 63 & 3.47926700 & -0.01840800 & 1.50882300 \\
\hline Pt 64 & -3.49136600 & 1.72277300 & 3.19335400 \\
\hline Pt 65 & -3.26803500 & 1.83824100 & -4.22489000 \\
\hline Pt 66 & 1.12314800 & 5.56780600 & 1.97599400 \\
\hline Pt 67 & -0.84154200 & 2.43187700 & 3.06441600 \\
\hline Pt 68 & 1.56414600 & 0.91015100 & -6.01860100 \\
\hline Pt 69 & -1.85332300 & -3.05819300 & -2.20336900 \\
\hline Pt 70 & -2.08720200 & -2.69283700 & -4.8815750 \\
\hline Pt 71 & -2.35955200 & -0.99689300 & -0.53476700 \\
\hline Pt72 & -4.13939000 & 0.55794800 & 0.77396900 \\
\hline Pt73 & 3.06978400 & 4.13852600 & -2.93145100 \\
\hline Pt 74 & -2.76185900 & -0.25890000 & -5.85549200 \\
\hline Pt 75 & -0.14926600 & -0.01195900 & 4.06048600 \\
\hline Pt 76 & 0.55194200 & -2.46108400 & 5.0858830 \\
\hline Pt77 & 5.57547400 & 3.17806000 & -3.1445120 \\
\hline Pt78 & -5.90131100 & -1.50244300 & 0.72148000 \\
\hline Pt79 & -3.45781200 & -1.90566600 & 1.76306100 \\
\hline Pt 80 & -1.22024400 & -0.87785400 & 6.36543700 \\
\hline Pt 81 & 2.42716700 & -1.90057700 & -2.36678900 \\
\hline Pt 82 & -2.55193100 & -0.61190600 & -3.20082600 \\
\hline Pt 83 & 3.30749100 & -4.73830600 & 1.31570500 \\
\hline Pt 84 & 1.04770400 & 2.99886600 & -4.38452300 \\
\hline Pt 85 & -2.00797700 & 5.96504900 & -2.4422960 \\
\hline Pt 86 & 0.59286700 & -3.44962900 & -1.11618100 \\
\hline Pt 87 & 4.97492000 & -2.77990200 & -2.63093400 \\
\hline Pt 88 & 0.70545500 & 2.26061600 & 5.30252800 \\
\hline Pt 89 & 3.32938400 & 2.92920200 & 5.1100650 \\
\hline Pt 90 & 6.02580400 & -0.88955800 & 1.26421100 \\
\hline Pt91 & -4.81483600 & -0.63202500 & -1.61906900 \\
\hline Pt92 & -0.62654900 & 1.07182800 & -0.4497200 \\
\hline Pt93 & -0.29835800 & -0.63690800 & -4.80153600 \\
\hline Pt 94 & 4.38739600 & 2.24788100 & 2.70643100 \\
\hline Pt95 & 2.27906700 & -1.51318500 & -5.0463800 \\
\hline Pt96 & -0.94819700 & -5.88553900 & 1.46476900 \\
\hline Pt97 & 3.18698700 & -1.72567800 & 4.94975700 \\
\hline Pt98 & 0.52837000 & 5.06722500 & -2.69364900 \\
\hline Pt99 & 1.40036800 & -0.16278800 & 6.26145200 \\
\hline Pt100 & -1.53400200 & 4.90410200 & 2.0880920 \\
\hline Pt101 & -6.57864900 & 0.92048700 & -0.28130600 \\
\hline Pt102 & -0.44579000 & 5.78716600 & -0.23891100 \\
\hline Pt103 & 3.66992100 & 4.65698200 & $1.7298770 \mathrm{C}$ \\
\hline \#103 & Cluster $=$ Pt104 $\mathrm{Q}=0$ & Etot $=-99$ & 04106000 \\
\hline Pt 1 & 0.23381100 & 3.59778600 & 1.3851070 \\
\hline Pt2 & 0.95989400 & 3.11317300 & -1.1894950 \\
\hline Pt3 & 1.17027000 & -4.69441100 & 4.83575000 \\
\hline Pt 4 & -0.53751600 & -1.85868100 & -1.5845870 \\
\hline Pt 5 & -5.69739900 & 0.21786400 & 1.7293150 \\
\hline Pt 6 & 2.85545300 & 3.57313300 & 0.6728120 \\
\hline Pt7 & -5.58317800 & -1.14463400 & -3.3352460 \\
\hline Pt 8 & 2.35698900 & 5.42324300 & -1.2762890 \\
\hline Pt9 & 2.75836300 & 3.51318600 & -3.19200100 \\
\hline Pt10 & -3.67454600 & 1.64888100 & 0.6171430 \\
\hline Pt11 & -0.41660600 & 0.78872500 & -1.0681200 \\
\hline Pt 12 & -2.41620600 & 3.59905100 & 2.1116790 \\
\hline Pt13 & -1.36488700 & -0.03387900 & 5.9894150 \\
\hline Pt14 & 6.07834600 & -1.55732000 & 0.6889080 \\
\hline & -2.92975700 & 5.39734300 & 0.1495570 \\
\hline
\end{tabular}




\begin{tabular}{|c|c|c|c|}
\hline Pt16 & 1.63146300 & 5.91555200 & 1.30501600 \\
\hline Pt17 & -1.59277200 & 1.79767100 & -5.52409300 \\
\hline Pt18 & 4.76483800 & 4.07925300 & 2.51700900 \\
\hline Pt19 & -2.93990000 & 1.16780800 & -1.94385200 \\
\hline Pt20 & 2.55522800 & -2.37812200 & 4.73024500 \\
\hline Pt21 & -1.15003600 & 1.27512700 & 1.49407000 \\
\hline Pt22 & -3.23337400 & -4.14476100 & -2.98831000 \\
\hline Pt23 & 2.01904800 & -0.56285300 & 2.76311000 \\
\hline Pt24 & 5.21209100 & 2.18250300 & 0.63313400 \\
\hline Pt25 & -5.07718500 & -2.97004000 & -1.40171400 \\
\hline Pt26 & 1.47652300 & 1.26407500 & 0.77996800 \\
\hline Pt27 & -1.36137500 & -4.32676300 & 3.96357500 \\
\hline Pt28 & 5.70979800 & 0.36941800 & 2.57297900 \\
\hline Pt29 & 3.66504200 & -0.27540800 & -3.12122200 \\
\hline Pt30 & -2.64060900 & -2.00815000 & 4.56730400 \\
\hline Pt31 & -1.67559100 & 3.10787400 & -0.47427100 \\
\hline Pt32 & 0.11282800 & -1.04879400 & 0.90765000 \\
\hline Pt33 & 0.70504600 & 0.35108400 & -5.58975900 \\
\hline Pt3 4 & -1.89515600 & 1.79063500 & 4.05615800 \\
\hline Pt35 & -0.28562500 & 5.45255900 & -0.57265400 \\
\hline Pt36 & 0.75909000 & 1.77468800 & 3.34537300 \\
\hline Pt37 & 0.44481900 & 4.94651000 & -3.14548000 \\
\hline Pt38 & 4.34122900 & -1.98049200 & 2.71501300 \\
\hline Pt39 & -1.46274000 & 4.44402300 & -4.99024600 \\
\hline Pt 40 & -1.19405600 & -2.70375400 & -4.07947600 \\
\hline Pt41 & -3.47070700 & 2.99413300 & -3.90813300 \\
\hline Pt 42 & 1.22313300 & -1.47150800 & -3.59730100 \\
\hline Pt43 & 5.58217000 & 0.26343600 & -1.25081700 \\
\hline Pt 44 & 2.67175100 & 2.30500600 & 5.18721300 \\
\hline Pt 45 & 1.35049700 & 1.17990500 & -3.07821400 \\
\hline Pt 46 & -2.69220700 & -5.94005400 & -1.03619700 \\
\hline Pt4 7 & 2.40579400 & -2.48273500 & 0.85999800 \\
\hline Pt 48 & 1.12739300 & -4.13031900 & -4.14340500 \\
\hline Pt 49 & 2.93742300 & -4.30822500 & 2.82227200 \\
\hline Pt50 & 4.66951000 & 3.97996400 & -1.30921800 \\
\hline Pt51 & 2.15878000 & 4.12781600 & 3.26050200 \\
\hline Pt52 & 4.18609100 & -2.08382400 & -1.17674400 \\
\hline Pt53 & 4.02040000 & -4.73129700 & -1.71161700 \\
\hline Pt5 4 & -0.62029400 & -0.55476700 & 3.46394100 \\
\hline Pt5 5 & -0.00939000 & -3.69274100 & 0.38590000 \\
\hline Pt56 & -0.14709600 & -6.31288100 & -0.17166600 \\
\hline Pt57 & -2.02712600 & -5.16475500 & 1.46824400 \\
\hline Pt58 & 1.63467400 & -5.92561700 & -2.18199600 \\
\hline Pt59 & 0.64436000 & -2.87972800 & 2.88098200 \\
\hline Pt 60 & 3.50690500 & -2.93956700 & -3.67359800 \\
\hline Pt61 & -0.67248400 & -4.51398500 & -2.13304600 \\
\hline Pt 62 & -5.15592600 & -1.59921900 & 3.66130200 \\
\hline Pt 63 & -1.06581000 & -0.02620400 & -3.55650200 \\
\hline Pt 64 & -6.19831300 & 2.01861300 & -0.22873000 \\
\hline Pt 65 & -3.61225800 & 0.33678900 & -4.44722800 \\
\hline Pt 66 & 2.28988600 & -5.14944600 & 0.32462300 \\
\hline Pt 67 & -4.45151700 & 2.13086900 & 3.17460900 \\
\hline Pt 68 & -0.94173000 & 2.62514400 & -3.03806700 \\
\hline Pt 69 & 5.03623100 & 2.05956800 & -3.20010800 \\
\hline Pt70 & -4.54085200 & -4.76225400 & 0.55455800 \\
\hline Pt71 & 0.04236500 & 2.28829900 & 5.88285900 \\
\hline Pt72 & -4.22544900 & 3.49238000 & -1.34707700 \\
\hline Pt73 & -4.94913100 & 3.93619500 & 1.2210180 \\
\hline & 4.24265 & 5.85359 & 596 \\
\hline
\end{tabular}




\begin{tabular}{|c|c|c|c|}
\hline Pt75 & 5.26945200 & 2.28987000 & 4.42829600 \\
\hline Pt76 & 2.97831200 & -1.11450400 & -5.60053200 \\
\hline Pt77 & 0.82992300 & 3.00631100 & -5.04259900 \\
\hline Pt78 & -0.09439000 & -2.37661000 & 5.43114100 \\
\hline Pt 79 & 3.78771300 & -0.15489600 & 0.73924800 \\
\hline Pt 80 & -2.53833300 & -3.31121300 & -0.49820800 \\
\hline Pt 81 & 3.37709700 & 1.76265200 & 2.61951100 \\
\hline Pt 82 & 0.51656000 & -5.52322500 & 2.34761500 \\
\hline Pt 83 & -3.06440200 & -1.48537700 & -2.46141700 \\
\hline Pt 84 & -3.89542600 & -3.92476100 & 3.05376500 \\
\hline Pt 85 & -3.90704300 & 0.31940200 & 5.10993300 \\
\hline Pt 86 & 1.29724000 & -0.04111900 & 5.33435900 \\
\hline Pt 87 & -5.46164600 & 1.51114800 & -2.79113300 \\
\hline Pt 88 & 1.87654300 & -0.65315400 & -1.11029600 \\
\hline Pt 89 & -0.46585200 & 4.11672500 & 3.95349400 \\
\hline Pt 90 & -0.99975000 & 5.90518600 & 1.99384200 \\
\hline Pt 91 & -3.14851100 & -0.17683900 & 2.58022700 \\
\hline Pt92 & -2.41064100 & -0.66528300 & 0.02723300 \\
\hline Pt93 & -4.95754800 & -0.30597400 & -0.84099800 \\
\hline Pt94 & 3.93106700 & -0.03993800 & 4.59793300 \\
\hline Pt95 & -4.43597500 & -2.11620500 & 1.10543100 \\
\hline Pt96 & -1.88347900 & -2.49872900 & 1.99855000 \\
\hline Pt97 & 3.26243300 & 1.66820200 & -1.22101700 \\
\hline Pt98 & -1.71331700 & -0.87697200 & -6.01656300 \\
\hline Pt99 & 1.74916800 & -3.29722800 & -1.63421700 \\
\hline Pt100 & 3.12434500 & 1.54017800 & -5.05998700 \\
\hline Pt101 & 0.59228600 & -2.30778500 & -6.07202800 \\
\hline 02 & 4.68921500 & -3.89104300 & 0.77987100 \\
\hline & -3.73453300 & -2.32243500 & -4.92600100 \\
\hline Pt104 & -2.20189000 & 4.93792700 & -2.43372900 \\
\hline \#104 & Cluster $=$ Pt105 $Q=0$ & \multicolumn{2}{|c|}{$\mathrm{M}=0 \quad$ Etot $=-10068.31810800$} \\
\hline Pt 1 & 2.82013000 & -0.66355000 & 3.6752810 \\
\hline Pt2 & -1.80631300 & 5.27632300 & -2.22229300 \\
\hline Pt 3 & -1.34508000 & -1.82189700 & -3.53141800 \\
\hline Pt 4 & -4.08474900 & -2.02914100 & 5.00042800 \\
\hline Pt5 & -1.98782800 & -0.75329200 & -1.14022200 \\
\hline Pt 6 & -5.76538600 & 98200 & 2.54777300 \\
\hline Pt 7 & -1.59601500 & -1.00282100 & 5.33594900 \\
\hline Pt 8 & 3.81289800 & -3.17015700 & 3.54833100 \\
\hline Pt9 & -5.42719900 & -2.14790900 & -0.25346300 \\
\hline Pt10 & -4.22756600 & 2.45607000 & 3.53523000 \\
\hline Pt11 & 0.62134000 & -5.77233600 & 1.21740400 \\
\hline Pt12 & 0100 & -2.26288100 & 1.60323200 \\
\hline Pt13 & -1.72819100 & -0.35897200 & -5.78704800 \\
\hline Pt14 & 2.78067400 & 5.46393500 & -1.00252300 \\
\hline Pt15 & -3.34406600 & 4.41395400 & 1.86084600 \\
\hline Pt16 & 1.83293200 & 1.85798300 & 3.82431900 \\
\hline Pt17 & 3.78885900 & -0.11723600 & 1.1851090 \\
\hline Pt18 & 2.52270600 & 3.36147000 & 5.8983520 \\
\hline Pt19 & -2.34874900 & 1.88079800 & 1.68322000 \\
\hline Pt20 & -4.51991900 & -0.23075700 & -1.97261300 \\
\hline Pt21 & 3.48822500 & 0.87235900 & 5.73394600 \\
\hline Pt22 & -0.03249100 & 2.47660200 & 5.72013400 \\
\hline Pt23 & -0.35633200 & -3.23277100 & 1.35803800 \\
\hline Pt24 & -3.97618400 & 4.33713300 & -0.81017000 \\
\hline Pt25 & -5.05732600 & 0.45726500 & 5.16415000 \\
\hline Pt2 & 5.36805100 & -0.63667000 & -3.73781200 \\
\hline Pt27 & 1.47073700 & 3.76074100 & -5.20425500 \\
\hline
\end{tabular}




\begin{tabular}{|c|c|c|c|}
\hline Pt28 & 4.80417100 & -2.66608400 & 1.04357700 \\
\hline Pt29 & 5.72520200 & -2.09949300 & -1.47948000 \\
\hline Pt 30 & -0.99596400 & -3.30571800 & -1.29759300 \\
\hline Pt31 & -2.53910600 & -5.26587100 & -2.27996100 \\
\hline Pt32 & 1.14822200 & 3.37981900 & -0.57486300 \\
\hline Pt33 & 2.14109800 & 0.82866000 & -0.74029400 \\
\hline Pt 34 & 4.42583400 & 4.52108200 & 0.94475500 \\
\hline Pt 35 & -3.85905600 & -1.27603000 & -4.40111300 \\
\hline Pt 36 & -0.70034700 & 0.91431300 & 3.61385300 \\
\hline Pt 37 & 3.78226500 & 2.95013300 & -1.16846400 \\
\hline Pt 38 & -4.42329900 & -4.65576800 & -0.41813300 \\
\hline Pt39 & -3.34507200 & 3.27727100 & -3.22909800 \\
\hline Pt 40 & -6.38487000 & 0.37908100 & -0.10236900 \\
\hline Pt 41 & 1.27744700 & -4.17449000 & 3.32400100 \\
\hline Pt 42 & 5.43113800 & -1.03037400 & 3.12205200 \\
\hline Pt43 & 0.81033300 & 4.86936800 & -2.82660500 \\
\hline Pt 44 & 3.40716900 & 4.40106400 & -3.42035600 \\
\hline Pt4 5 & 6.38404900 & -0.51505300 & 0.62866600 \\
\hline Pt 46 & -0.61989200 & -3.51084900 & 5.14745100 \\
\hline Pt 47 & -2.86956700 & -3.79282100 & -4.53570700 \\
\hline Pt 48 & 3.79339900 & -2.79368700 & -3.31062900 \\
\hline Pt49 & -3.80406200 & -4.56873800 & 2.22753100 \\
\hline Pt50 & 1.79082800 & 2.30269100 & -2.97120000 \\
\hline Pt51 & 5.42701400 & 2.01076200 & 0.79787600 \\
\hline Pt52 & 2.80146800 & 2.42185700 & 1.34414100 \\
\hline Pt53 & 1.52453400 & -3.85948800 & -0.47299000 \\
\hline Pt5 4 & 4.12897300 & -4.26382000 & -1.05277400 \\
\hline Pt55 & -3.87496400 & -0.13614300 & 0.6974620 \\
\hline Pt5 6 & -0.73261700 & -2.87404100 & -5.9256470 \\
\hline Pt57 & -3.53922100 & -2.75022200 & -2.1311120 \\
\hline Pt58 & -4.89231600 & 2.40800600 & 0.87540200 \\
\hline Pt59 & 3.48533300 & 3.99029200 & 3.44379800 \\
\hline Pt 60 & 2.50709300 & -6.38635600 & -0.62183900 \\
\hline Pt 61 & 2.78088300 & -0.24108000 & -3.1317770 \\
\hline Pt 62 & -0.46596200 & 1.25575700 & -0.1565850 \\
\hline Pt 63 & 1.17638700 & 0.29007400 & 1.74897300 \\
\hline Pt 64 & 0.18564700 & 2.85182400 & 1.92870200 \\
\hline Pt 65 & -4.81002700 & -2.06868400 & 2.38617200 \\
\hline Pt 66 & -2.33533500 & 0.72179800 & -3.37086000 \\
\hline Pt 67 & 1.79842200 & -3.40451600 & -5.10034800 \\
\hline Pt 68 & -2.98173400 & 1.79747300 & -0.9748900 \\
\hline Pt 69 & -1.69483500 & 3.46093100 & 3.80774400 \\
\hline Pt70 & 1.91683500 & -2.53893100 & 5.39315800 \\
\hline Pt71 & -1.34386800 & -0.67553400 & 1.50486100 \\
\hline Pt72 & 4.76289300 & 0.43064000 & -1.32697000 \\
\hline Pt73 & 0.86530100 & 4.40734500 & $4.0285250 c$ \\
\hline Pt74 & 0.93542200 & -0.03292900 & $5.5811430 c$ \\
\hline Pt75 & 3.43237000 & -1.27827200 & -5.52222700 \\
\hline Pt76 & -1.27625100 & -5.12000700 & $3.0553960 c$ \\
\hline Pt77 & -0.79658900 & 5.38999100 & 2.1075950 \\
\hline Pt78 & -0.01116400 & -5.85006800 & -1.45378700 \\
\hline Pt79 & -2.23426100 & -2.60008400 & 3.18571300 \\
\hline Pt 80 & 4.39961600 & 1.88818800 & -3.60117800 \\
\hline Pt81 & 3.13523300 & -1.72289600 & -0.8974450 \\
\hline Pt82 & 2.46884800 & 1.24907600 & -5.3805230 \\
\hline Pt83 & -4.83048100 & 1.24490000 & -4.21825500 \\
\hline Pt84 & 2.16318800 & -4.92094200 & -2.8678930 \\
\hline Pt85 & -1.14902700 & 4.16720600 & -4.6210140 \\
\hline & -1.91412700 & -5.24012500 & 0.3928420 \\
\hline
\end{tabular}




\begin{tabular}{|c|c|c|c|}
\hline Pt 87 & -0.16492000 & 1.64433400 & -4.80165800 \\
\hline Pt 88 & 1.17264000 & -2.37637300 & -2.70657800 \\
\hline Pt 89 & -3.20993000 & -0.07413300 & 3.33586700 \\
\hline Pt 90 & -1.46039400 & 3.80816700 & 0.01380500 \\
\hline Pt91 & -2.57612100 & 1.50203800 & 5.47375800 \\
\hline Pt92 & -2.44461200 & 6.33438600 & 0.18235200 \\
\hline Pt93 & 0.81676600 & -0.87753700 & -4.96125700 \\
\hline tet94 & 3.16826600 & -4.79651000 & 1.46409900 \\
\hline Pt95 & -0.81601500 & 2.73207100 & -2.38227900 \\
\hline Pt96 & 0.16320400 & 5.92309300 & -0.40922900 \\
\hline Pt97 & 1.82797500 & 4.98281100 & 1.52633000 \\
\hline 促 & 4.48179400 & 1.49137400 & 3.28617700 \\
\hline Pt99 & -0.36229900 & -4.38589600 & -3.71314900 \\
\hline Pt100 & -2.88612300 & -2.67468100 & 0.53857000 \\
\hline t101 & -5.48279100 & 2.29788000 & -1.80292200 \\
\hline & 0.17814100 & 0.17846000 & -2.54507800 \\
\hline & -2.69391700 & 2.16510100 & -5.60764300 \\
\hline & 0.28601200 & -1.61947500 & 3.45314700 \\
\hline 5 & 0.53146600 & -1.30582300 & -0.32164300 \\
\hline 105 & Luster $=$ Pt10 $\quad \mathrm{Q}=0$ & $I=0 \quad E$ tot $=-101$ & 39741400 \\
\hline Pt 1 & -0.15037800 & 6.30599200 & -1.29773400 \\
\hline Pt2 & 2.52886000 & -5.64258300 & 0.88147400 \\
\hline Pt 3 & 1.57985800 & 4.90169400 & -2.83320600 \\
\hline Pt 4 & 1.11419000 & -0.66795600 & -0.60198200 \\
\hline Pt 5 & -0.50200000 & 3.87343400 & -4.23602900 \\
\hline Pt 6 & 0.14241000 & -2.57822100 & -4.78434400 \\
\hline Pt 7 & -3.33758500 & 0.29460100 & -1.69601600 \\
\hline Pt 8 & 0.79173500 & -4.23484300 & 2.41582200 \\
\hline Pt9 & -0.70031100 & 0.95729400 & -1.74905000 \\
\hline Pt10 & 0.41815000 & 4.48273400 & 3.57813200 \\
\hline Pt11 & 1.04836600 & -0.48141200 & -3.30101000 \\
\hline Pt12 & -0.79518300 & -3.83168100 & 0.20714800 \\
\hline Pt13 & -2.23174600 & 5.27169000 & -2.71541000 \\
\hline Pt14 & -0.64917300 & 0.78032800 & 0.94050300 \\
\hline Pt15 & -5.03028600 & 1.54662600 & 2.53810900 \\
\hline Pt16 & -1.59329000 & -1.13546400 & -3.22415400 \\
\hline Pt & 3.57804700 & -4.60641200 & -1.4 \\
\hline Pt18 & -1.49818400 & -1.31807000 & -0.51654400 \\
\hline Pt & -4.22557000 & 3.84757300 & 1.29310200 \\
\hline Pt20 & 1.68455700 & 4.70176600 & -0.11181700 \\
\hline Pt21 & -0.43031500 & 3.64481100 & -1.51478800 \\
\hline Pt22 & -0.89547600 & 0.72245000 & 5.60342000 \\
\hline Pt & 1.83156200 & -3.16671500 & 0.12422500 \\
\hline Pt24 & -3.26990700 & -5.47920400 & -3.39478200 \\
\hline Pt25 & -2.53899900 & 2.73534500 & -5.62355900 \\
\hline Pt26 & 3.38437600 & -1.17018200 & 4.91726800 \\
\hline Pt27 & -3.56747100 & -3.47551000 & 3.97235200 \\
\hline Pt28 & 2.16010500 & 3.02087700 & 2.0297030 \\
\hline Pt29 & -1.69862900 & -5.93676200 & -1.2361590 \\
\hline Pt 30 & 0.94643900 & -5.29554200 & -1.33321000 \\
\hline Pt 31 & -0.94078300 & -2.81167200 & 3.93394900 \\
\hline Pt32 & 5.14021500 & 1.83639900 & -3.14627600 \\
\hline Pt33 & 0.07875000 & -1.72444600 & 1.66386100 \\
\hline Pt 34 & 2.96075400 & 4.03467200 & 4.38487500 \\
\hline Pt 35 & 4.88545400 & -0.85950100 & -3.4259870 \\
\hline & 5.10935500 & -2.58071600 & 3.37837300 \\
\hline & 3.31123800 & 3.46049900 & -4.3323320 \\
\hline & 2.78237400 & -1.90714700 & -4.8464490 \\
\hline
\end{tabular}




\begin{tabular}{|c|c|c|c|}
\hline Pt39 & -0.06150900 & 6.10864400 & 1.41170100 \\
\hline Pt 40 & 3.41686400 & 3.27864000 & -1.63012500 \\
\hline Pt41 & -5.88218200 & -0.57196500 & 1.06836700 \\
\hline Pt 42 & 0.92891000 & 2.77698400 & 5.67234900 \\
\hline Pt 43 & -0.65242200 & -4.86539300 & -3.51430800 \\
\hline Pt 44 & -0.37262400 & 3.45465700 & 1.18618300 \\
\hline Pt 45 & 4.22335500 & 4.26664100 & 0.77870300 \\
\hline Pt 46 & -5.11250300 & 1.73533800 & -0.18125400 \\
\hline Pt 47 & 3.63587400 & -1.09628600 & 0.23748100 \\
\hline Pt 48 & -2.47094300 & 2.40849100 & -0.22120900 \\
\hline Pt 49 & 0.24745300 & -2.77130700 & -2.06682500 \\
\hline Pt50 & 4.37524100 & -3.60627600 & 0.98580800 \\
\hline Pt51 & -0.76902800 & 1.14659800 & -4.44716100 \\
\hline Pt52 & -2.50009000 & 2.56681900 & -2.91708100 \\
\hline Pt53 & -1.85335100 & -4.91986400 & 2.45378400 \\
\hline Pt5 4 & -2.54196900 & -2.39726000 & 1.73784600 \\
\hline Pt5 5 & 6.13708900 & -1.49981400 & 1.11018600 \\
\hline Pt5 6 & 3.06798600 & 0.78519100 & -4.60761400 \\
\hline Pt57 & 1.87834900 & 0.34647300 & 1.77678000 \\
\hline Pt58 & -2.67382500 & -1.36905400 & 5.43995500 \\
\hline Pt59 & 0.85535700 & -0.71310400 & 4.04044800 \\
\hline Pt 60 & -4.15455500 & 3.65032100 & 3.99089200 \\
\hline Pt61 & 4.59781200 & -3.53730500 & -3.67644800 \\
\hline Pt 62 & -1.62705100 & 3.22777700 & 4.84980700 \\
\hline Pt 63 & -4.24330400 & -1.81630200 & -3.18239300 \\
\hline Pt 64 & 5.68867100 & 0.15433000 & -1.03943800 \\
\hline Pt 65 & -4.31635300 & -0.97370100 & 3.27823900 \\
\hline Pt 66 & 0.94419300 & -0.25765800 & -5.98992000 \\
\hline Pt 67 & 2.67970200 & 1.35306400 & 4.16505200 \\
\hline Pt 68 & 3.13751900 & 0.57206100 & -1.88889200 \\
\hline Pt 69 & 4.41427800 & -0.07136300 & 2.64560000 \\
\hline Pt70 & 5.38790900 & -2.53275900 & -1.29492900 \\
\hline Pt71 & -3.44072000 & -4.53733900 & 0.28321600 \\
\hline Pt72 & -5.15658800 & -3.09766300 & 1.79509600 \\
\hline Pt73 & -2.12182600 & 4.89532900 & 2.71307900 \\
\hline Pt74 & 0.12138400 & 1.78950700 & 3.31540600 \\
\hline Pt75 & -5.95387000 & -0.39079800 & -1.65410700 \\
\hline Pt76 & -2.37193800 & -3.40911800 & -1.96925700 \\
\hline Pt77 & -3.44389600 & 0.48126600 & -4.41379500 \\
\hline Pt78 & -2.41105100 & 2.22215200 & 2.47312100 \\
\hline Pt79 & 1.22044500 & 2.43169800 & -5.73268200 \\
\hline Pt 80 & -2.19255000 & 5.10603900 & 0.00116200 \\
\hline Pt 81 & -4.98567300 & -4.08837000 & -1.90058500 \\
\hline Pt 82 & -3.43003700 & 1.14946400 & 4.73124500 \\
\hline Pt 83 & 1.61185600 & -3.20709200 & 4.80324600 \\
\hline Pt 84 & -5.14907500 & 1.90988000 & -2.89406100 \\
\hline Pt 85 & -2.49603700 & -3.22620500 & -4.68296000 \\
\hline Pt 86 & -0.10311000 & -6.32087500 & 0.93577400 \\
\hline Pt 87 & 6.42630300 & 1.18861300 & 1.3484860 \\
\hline Pt 88 & -4.26955600 & 4.02331300 & -1.42491100 \\
\hline Pt 89 & 5.93588000 & 2.83172600 & -0.74995300 \\
\hline Pt90 & 4.71182300 & 2.62844400 & 2.87183300 \\
\hline Pt91 & 1.38457200 & 2.01254000 & -0.34836600 \\
\hline Pt92 & -3.27221700 & 0.11885900 & 1.0168100 \\
\hline Pt93 & 1.64384100 & 0.26558100 & 6.41474300 \\
\hline Pt9 & 1.98143200 & -4.21088000 & -3.6048930 \\
\hline Pt & -0.13280900 & -1.77739400 & 6.3002380 \\
\hline Pt & 3.90954100 & 1.58405600 & 0.49702500 \\
\hline & -4.25122400 & 4.14772700 & -4.13078600 \\
\hline
\end{tabular}




\begin{tabular}{|c|c|c|c|}
\hline Pt98 & 1.31483000 & 2.21026000 & -3.04513600 \\
\hline Pt99 & 2.86451600 & -2.11032600 & -2.14692500 \\
\hline Pt 100 & -6.68294200 & -2.67880400 & -0.40267600 \\
\hline t101 & 3.33153300 & -4.62277400 & 3.26464700 \\
\hline$t 102$ & 2.60484200 & -2.14994700 & 2.50757400 \\
\hline Pt103 & -1.69184900 & -0.92314300 & -5.91102200 \\
\hline Pt104 & 2.47161100 & 5.67711200 & 2.28572900 \\
\hline Pt105 & -1.67839100 & -0.28358700 & 3.19888400 \\
\hline$=106$ & -4.10541400 & -1.98661600 & -0.45004600 \\
\hline \#106 & Cluster $=$ Pt11 $6 \quad \mathrm{Q}=0$ & $\mathrm{M}=0 \quad \mathrm{E}$ to $\mathrm{t}=-11202$ & 04784300 \\
\hline Pt1 & 4.42169300 & -0.69072000 & -3.94286200 \\
\hline Pt2 & 2.34860800 & -0.94334000 & -2.30875700 \\
\hline Pt3 & -4.57216000 & -1.23747600 & -4.73187200 \\
\hline Pt 4 & -1.07775700 & -4.84357400 & -2.8082290 \\
\hline Pt5 & 4.69131200 & 3.07744200 & -2.76560000 \\
\hline Pt 6 & 2.25049200 & 0.41590600 & 0.0981390 \\
\hline Pt7 & 0.39531200 & -3.29426500 & -4.63675100 \\
\hline Pt 8 & 0.03312600 & -3.37866400 & 0.56545700 \\
\hline Pt9 & -4.76524200 & 3.65868800 & -0.7388960 \\
\hline Pt10 & 0.21317800 & -5.54114600 & 4.5638800 \\
\hline 11 & -1.83379200 & 0.13073200 & -1.3665440 \\
\hline Pt12 & 4.84165300 & 2.07860000 & 3.53337500 \\
\hline Pt13 & 1.07764000 & -3.27619200 & 5.8367910 \\
\hline Pt14 & -6.27054100 & 2.67567700 & -2.78448000 \\
\hline Pt15 & 4.70704600 & 1.72002600 & -5.14431500 \\
\hline Pt16 & 0.94882100 & -3.27271600 & -2.0086000 \\
\hline Pt17 & 2.88274300 & 5.13180100 & -2.3667140 \\
\hline Pt18 & 2.64698000 & 1.41312100 & -3.52997600 \\
\hline Pt19 & 0.03599600 & 1.23081600 & -4.466312 \\
\hline Pt20 & 0.87522600 & 3.47616600 & -3.1173910 \\
\hline Pt21 & -0.28872800 & -5.64493600 & 1.9041940 \\
\hline Pt22 & 2.57825600 & 2.77901700 & -1.1152170 \\
\hline Pt23 & -3.73178100 & -1.36928400 & 2.6012980 \\
\hline Pt24 & 2.23153500 & 2.75873300 & 4.13521400 \\
\hline Pt25 & 4.66036500 & -2.12798900 & -1.57397300 \\
\hline Pt26 & -3.83994900 & 3.78319400 & -3.3450190 \\
\hline Pt27 & 0.21668200 & 1.14445600 & 3.2646490 \\
\hline Pt28 & -2.04400700 & -4.95252400 & -0.1388190 \\
\hline Pt29 & 1.16374400 & 5.80772200 & -4.37588000 \\
\hline Pt30 & -5.97359900 & -2.77970200 & -2.85799100 \\
\hline Pt31 & 6.34952200 & 2.26854400 & -0.70720800 \\
\hline Pt32 & -1.64938200 & -2.62768300 & -1.4529380 \\
\hline Pt33 & 4.48674800 & -0.75575900 & 0.8729630 \\
\hline Pt34 & -1.60772400 & 2.48674100 & -2.6163690 \\
\hline Pt35 & -0.21833500 & -1.12257000 & -3.2292990 \\
\hline Pt36 & -4.15255200 & -5.00217600 & 1.6003630 \\
\hline Pt37 & 4.22283400 & 1.98197500 & 0.89851400 \\
\hline Pt38 & -4.68606000 & 1.49801300 & -4.6647250 \\
\hline & 0.65415600 & -5.61660700 & -0.7305660 \\
\hline Pt 40 & 1.92032500 & -0.98028400 & 7.0415660 \\
\hline Pt41 & 3.19760600 & -4.54505800 & -1.27502300 \\
\hline Pt 42 & 6.22597800 & 3.54821400 & 1.69695000 \\
\hline Pt 43 & -4.11353500 & -3.63865300 & 3.9751610 \\
\hline Pt 44 & 0.24684100 & 5.77026600 & -1.8117940 \\
\hline Pt 45 & 3.61508800 & -2.20717100 & 5.2936180 \\
\hline & -4.66246400 & 2.27737700 & 1.6629280 \\
\hline Pt4 & -2.28715000 & 4.77563000 & -1.2855560 \\
\hline t 48 & 0.75691700 & 1.19389400 & 5.8856760 \\
\hline
\end{tabular}




\begin{tabular}{|c|c|c|c|}
\hline Pt 49 & -1.68096700 & -1.24040700 & 0.93971600 \\
\hline Pt50 & 0.32624800 & -1.12530900 & -0.69064800 \\
\hline Pt51 & 6.64194900 & -0.45448000 & -0.71033300 \\
\hline Pt52 & 1.83890800 & -0.86864300 & -4.95869000 \\
\hline Pt53 & 1.97357600 & 5.07312400 & 0.24240100 \\
\hline Pt54 & 0.84326000 & -1.06416000 & 1.84494000 \\
\hline Pt55 & -5.80294800 & -1.51954200 & 4.27189000 \\
\hline Pt5 6 & -3.18903300 & 4.59467200 & 1.32396700 \\
\hline Pt57 & -1.19766000 & -1.19232800 & 3.57183600 \\
\hline Pt58 & 4.58608700 & 4.37152000 & -0.32332300 \\
\hline$=59$ & -6.03589100 & -0.10349300 & 1.95044300 \\
\hline Pt 60 & -6.06608900 & -1.45462400 & -0.46284500 \\
\hline Pt 61 & -4.44999700 & 0.83033500 & 4.01840300 \\
\hline Pt 62 & 3.39288300 & 0.55484700 & 5.33173600 \\
\hline Pt 63 & 2.09920800 & 1.57315400 & -6.15996100 \\
\hline Pt 64 & 5.27851200 & -3.40713300 & 3.47958300 \\
\hline Pt 65 & -2.37240900 & 0.96367900 & 2.33616400 \\
\hline Pt 66 & 2.83168900 & 0.50522700 & 2.70930400 \\
\hline Pt 67 & -6.15565300 & -0.04764000 & -2.84051700 \\
\hline Pt 68 & -2.52916100 & 2.37005200 & -0.02935900 \\
\hline Pt 69 & -2.30462200 & -2.61430100 & -4.07171600 \\
\hline Pt70 & -0.30639500 & 1.05683200 & 0.64862100 \\
\hline Pt71 & -6.19322700 & 1.30811800 & -0.42389000 \\
\hline Pt72 & 5.10947800 & -0.67431900 & 3.54487300 \\
\hline Pt73 & -1.58319900 & 5.46083800 & 3.35153900 \\
\hline Pt74 & 0.54311100 & -3.31898200 & 3.18646100 \\
\hline Pt75 & 3.05639700 & -2.26014100 & 2.64846100 \\
\hline Pt76 & 6.71759100 & -1.89357200 & 1.6932510 \\
\hline Pt77 & -2.36817400 & -5.72138200 & 3.6381710 \\
\hline Pt78 & 1.66007600 & 2.66255400 & 1.4729610 \\
\hline Pt79 & -0.94394700 & 3.25590300 & 1.99828100 \\
\hline Pt 80 & 6.39899700 & 0.96483000 & -3.11459100 \\
\hline Pt 81 & 0.59598400 & 1.15439000 & -1.88666700 \\
\hline Pt 82 & 6.50911900 & 0.83664500 & 1.7012510 \\
\hline Pt83 & -5.84136100 & -2.86744400 & 1.9024010 \\
\hline Pts & 0.31087200 & 3.60320800 & -5.73438000 \\
\hline Pt 85 & 2.50402000 & -2.33768300 & 0.03653200 \\
\hline Pt 86 & 2.26059200 & -4.65196700 & 1.40487100 \\
\hline Pt 87 & 2.94695700 & 3.78726600 & -4.78904300 \\
\hline Pt8 8 & 4.32867000 & 0.66250500 & -1.51256200 \\
\hline Pt 89 & -3.88929500 & -1.34059500 & -2.13080500 \\
\hline Pt90 & -0.03694800 & 3.42001400 & -0.56284800 \\
\hline Pt91 & -3.03995300 & 0.30578000 & -6.49953300 \\
\hline Pt92 & -1.33845500 & 4.83706700 & -3.8804950 \\
\hline Pt93 & -2.02258500 & -3.49812500 & 2.26636600 \\
\hline Pt94 & 3.03763100 & -3.10977200 & -3.68917800 \\
\hline Pt95 & 3.65972300 & 4.24693900 & 2.28241700 \\
\hline Pt96 & -0.55519000 & 1.35260400 & -7.03142700 \\
\hline Pt97 & -1.86976400 & 1.03086600 & 5.01314800 \\
\hline Pt98 & -0.42095900 & 3.35915300 & 4.6446440 \\
\hline Pt99 & 2.77814900 & -4.51825700 & 4.0858110 \\
\hline Pt 100 & 1.37554700 & -1.01267800 & 4.43285800 \\
\hline Pt101 & -4.04099600 & 1.40497500 & -2.06501200 \\
\hline Pt102 & -0.67036600 & -1.15698900 & 6.2015240 \\
\hline Pt103 & -0.67664300 & 5.65313500 & 0.78615900 \\
\hline Pt104 & -3.94136800 & 0.03231600 & 0.2803890 \\
\hline Pt105 & 4.73784900 & -3.52725200 & 0.8281930 \\
\hline Pt106 & -2.21556800 & 2.59518700 & -5.2425050 \\
\hline Pt107 & -1.53896200 & -3.47529000 & 4.9680540 \\
\hline
\end{tabular}




\begin{tabular}{|c|c|c|c|}
\hline Pt108 & -3.73395900 & -2.73426000 & 0.21334300 \\
\hline Pt109 & -3.03101200 & 3.17691300 & 3.70864200 \\
\hline Pt110 & 1.03412000 & 4.88954300 & 2.83845000 \\
\hline Pt111 & -3.26909900 & -1.33843900 & 5.26958100 \\
\hline t112 & -0.80027900 & -1.05533100 & -5.81553600 \\
\hline Pt113 & 1.57576000 & -5.43518000 & -3.33579200 \\
\hline t114 & -5.81731500 & -4.18187100 & -0.49953900 \\
\hline Pt115 & -3.71147900 & -4.11720500 & -2.20185600 \\
\hline Pt116 & -2.43369600 & 0.19034100 & -3.93764300 \\
\hline \#107 & Cluster $=\operatorname{Pt} 121 \quad \mathrm{Q}=0$ & \multicolumn{2}{|c|}{$\mathrm{M}=0 \quad$ Etot $=-11716.17135400$} \\
\hline Pt 1 & -0.19359000 & -2.57313100 & 4.28878700 \\
\hline Pt2 & 1.48178800 & 2.53476700 & -4.74367300 \\
\hline Pt 3 & 3.40566300 & -4.42112900 & -4.62276300 \\
\hline Pt 4 & 4.17141300 & 2.76123600 & -4.50712700 \\
\hline Pt5 & 3.56025200 & 4.02068700 & 2.23260700 \\
\hline Pt 6 & -0.01687900 & -1.21408300 & 0.78180700 \\
\hline Pt 7 & -5.40351500 & 1.12118600 & -3.10605600 \\
\hline Pt 8 & 1.71486700 & -6.81459000 & 1.95328800 \\
\hline Pt9 & 1.41307200 & -3.04741900 & 2.16247000 \\
\hline Pt10 & -3.45636400 & 2.27245200 & 5.99938000 \\
\hline Pt11 & -3.00624000 & 2.82748900 & -0.59402300 \\
\hline Pt12 & -1.87712700 & 3.53250600 & 1.76716100 \\
\hline Pt13 & -3.85421800 & -3.38129100 & -1.01842100 \\
\hline Pt14 & -4.04984600 & -3.51424000 & 1.71294700 \\
\hline Pt15 & 1.29205500 & 0.85480100 & -0.37445400 \\
\hline Pt16 & -4.62812700 & 1.58147000 & 3.65725800 \\
\hline Pt17 & 5.13949700 & 1.80410600 & 2.20289400 \\
\hline Pt18 & -0.31117700 & 1.31715200 & 1.74215000 \\
\hline Pt19 & -2.41820500 & -5.22779800 & 0.36836800 \\
\hline Pt20 & -0.76370700 & 4.27146700 & 4.15513900 \\
\hline Pt21 & 4.02149000 & 1.09571500 & -0.15481700 \\
\hline Pt22 & -2.58926900 & -5.34963600 & 3.09915100 \\
\hline Pt23 & -4.52992400 & 5.03475300 & -0.58312700 \\
\hline Pt24 & 0.84797900 & 5.51237900 & -0.13336700 \\
\hline Pt25 & 2.43802200 & 3.29092200 & -0.14373400 \\
\hline Pt26 & -3.41768300 & 5.73346800 & 1.79870800 \\
\hline Pt27 & -5.47341100 & -1.65510200 & 0.31649800 \\
\hline Pt28 & 1.95515000 & 6.19141300 & 17200 \\
\hline Pt29 & -1.93568800 & -4.87162400 & -5.06196000 \\
\hline Pt30 & 1.17834100 & 4.00916500 & -2.42736500 \\
\hline Pt31 & 1.20102000 & -4.40055700 & 5.69099200 \\
\hline Pt32 & -1.53167000 & 3.77892500 & -2.65122500 \\
\hline Pt33 & 3.05247300 & -4.76616000 & 0.82091200 \\
\hline Pt3 4 & 3.85606700 & -0.28110000 & 3.3870930 \\
\hline Pt35 & 3.52278300 & 2.27502200 & 4.33769600 \\
\hline Pt36 & 4.36832300 & -2.68765500 & -0.33903200 \\
\hline Pt37 & 2.53991600 & -2.35818400 & 4.54923800 \\
\hline Pt38 & -2.05837600 & 0.41958500 & 7.38938400 \\
\hline Pt39 & -5.76171000 & 0.87417400 & 1.28459200 \\
\hline Pt 40 & 1.63499900 & -2.90018000 & -0.5570050 \\
\hline Pt 41 & 1.65957500 & -0.11158900 & -4.00724000 \\
\hline Pt 42 & 1.92658500 & -6.68109700 & -0.75165200 \\
\hline Pt43 & -3.44836900 & 4.02700400 & 3.91932200 \\
\hline Pt 44 & -3.78005000 & -0.57515900 & -4.48292600 \\
\hline Pt 45 & 5.44289600 & -0.74288500 & 1.22807100 \\
\hline Pt 46 & 2.23959400 & 0.18589000 & 5.51681900 \\
\hline Pt 47 & -5.25599100 & -1.50167400 & -2.3939910 \\
\hline & -1.20511 & 3067 & -4.96510 \\
\hline
\end{tabular}




\begin{tabular}{|c|c|c|c|}
\hline Pt 49 & -4.61000700 & 3.32394900 & 1.55415600 \\
\hline Pt 50 & 0.03059800 & 1.57483800 & -2.65716500 \\
\hline Pt51 & -1.90574200 & 1.79899800 & 3.86550900 \\
\hline Pt52 & 4.13179200 & -2.82525600 & 2.39274400 \\
\hline Pt53 & -3.22224500 & -0.27733700 & 5.06249400 \\
\hline Pt5 4 & -0.53748600 & -6.72549900 & -3.67351800 \\
\hline Pt5 5 & -0.78894500 & 2.52573600 & 6.23380400 \\
\hline Pt5 6 & 2.93412300 & -0.82165700 & -1.71573600 \\
\hline Pt57 & 1.92514400 & 4.48451900 & 4.36605300 \\
\hline Pt58 & 2.13039800 & -6.50049900 & -3.45336700 \\
\hline Pt59 & -2.54427700 & -1.28562300 & -2.16778600 \\
\hline Pt 60 & -1.05253100 & -0.34141300 & -4.23091900 \\
\hline Pt61 & -1.63237400 & -0.74607000 & 2.91184300 \\
\hline Pt 62 & -0.73398400 & 5.97636400 & 2.04418000 \\
\hline Pt 63 & 0.10169300 & -5.12964200 & 3.32701000 \\
\hline Pt 64 & -0.96147400 & -7.04038600 & 1.73185800 \\
\hline Pt 65 & -2.27432300 & 0.36200900 & -6.51804900 \\
\hline Pt 66 & 3.25991900 & -4.60755700 & -1.90498700 \\
\hline Pt 67 & -2.93049600 & -2.82107500 & 4.09475400 \\
\hline Pt 68 & -0.07301400 & 4.77406200 & -4.71328400 \\
\hline Pt 69 & 2.72831700 & -0.97710200 & 1.00615100 \\
\hline Pt70 & 2.79136100 & -4.89566700 & 3.54566100 \\
\hline Pt71 & -4.36589300 & -0.97745900 & 2.70349000 \\
\hline Pt72 & -1.47519200 & -4.62695700 & 5.46862500 \\
\hline Pt73 & 3.54010200 & 5.72710700 & 0.08500100 \\
\hline Pt74 & -0.38316600 & 6.23114400 & -2.45725000 \\
\hline Pt 75 & 2.75328500 & 1.80982600 & -2.43695100 \\
\hline Pt76 & 1.12667500 & -0.51209500 & 3.14052300 \\
\hline Pt77 & 4.54191200 & -2.51280700 & -3.07541300 \\
\hline Pt78 & 5.64909300 & -0.58995500 & -1.50388700 \\
\hline Pt79 & -0.74774300 & -6.90673100 & -0.97263000 \\
\hline Pt 80 & 5.15208600 & 3.53592000 & 0.07696700 \\
\hline Pt 81 & 2.30191600 & 6.44200200 & -2.22005200 \\
\hline Pt 82 & 0.58018100 & -2.04206500 & -5.62025000 \\
\hline Pt 83 & -2.10919600 & -2.26437500 & -5.82176900 \\
\hline Pt8 4 & -3.05134600 & 1.09657000 & 1.52062100 \\
\hline Pt 85 & 3.26498400 & -1.80999900 & -5.37989700 \\
\hline Pt 86 & -5.71734300 & 2.59553600 & -0.82328400 \\
\hline Pt 87 & 5.46846300 & 2.04959300 & -2.22434800 \\
\hline Pt 88 & 1.82128700 & -2.72922100 & -3.27735700 \\
\hline Pt89 & 0.31447100 & -4.95998400 & 0.59316700 \\
\hline Pt90 & 6.72244700 & 1.33477200 & 0.06210400 \\
\hline Pt91 & -1.76837900 & -2.11182100 & 6.45144300 \\
\hline Pt92 & 0.73772400 & -4.64649600 & -4.87113200 \\
\hline Pt93 & -4.26198200 & 3.57055800 & -2.89200300 \\
\hline Pt9 4 & 0.60925900 & 0.64575600 & 7.61134800 \\
\hline Pt95 & -1.44632400 & 0.62229000 & -0.60074800 \\
\hline Pt96 & -2.69119500 & 1.34742100 & -2.88564800 \\
\hline Pt97 & 2.61288100 & 4.98109900 & -4.48200100 \\
\hline Pt98 & 2.42677700 & 1.56183600 & 1.97468200 \\
\hline Pt99 & 0.41577200 & 0.57485900 & -6.31909800 \\
\hline Pt100 & 0.80834400 & 2.02955000 & 4.09075900 \\
\hline Pt101 & 0.83483800 & 3.76299400 & 1.99186800 \\
\hline Pt102 & 0.19345900 & -1.05360400 & -1.92854800 \\
\hline Pt103 & 4.39121000 & 0.11687700 & -3.81175300 \\
\hline Pt104 & -1.32916600 & -3.27952300 & 1.93538300 \\
\hline Pt105 & -2.76164400 & -1.44217100 & 0.55177900 \\
\hline Pt106 & -4.17620200 & 0.39957700 & -0.83220100 \\
\hline & 0.52575100 & -4.80291900 & \\
\hline
\end{tabular}




\begin{tabular}{|c|c|c|c|}
\hline Pt108 & -3.06839600 & 5.98435300 & -2.66284600 \\
\hline Pt109 & -0.28422100 & 3.05384500 & -0.36718300 \\
\hline Pt110 & -2.75744600 & 4.52352200 & -4.92468600 \\
\hline Pt111 & -3.89973400 & 2.07414500 & -5.16710600 \\
\hline Pt112 & -2.20242300 & -5.06841900 & -2.35565000 \\
\hline Pt113 & 3.09919800 & 0.81672100 & -6.07659000 \\
\hline Pt114 & -1.83879600 & 5.28373800 & -0.35573600 \\
\hline Pt115 & -3.61049400 & -3.19932900 & -3.74496600 \\
\hline t116 & -0.48910300 & -0.03517200 & 5.25589100 \\
\hline Pt117 & 3.90643600 & 4.26682300 & -2.21872600 \\
\hline t118 & -1.12414400 & -3.13371400 & -0.78500300 \\
\hline t119 & 1.88419200 & 2.72668600 & 6.44436800 \\
\hline Pt120 & 0.90576700 & -1.88533100 & 6.67374600 \\
\hline Pt121 & -0.89299600 & -2.95884500 & -3.50117100 \\
\hline \#108 & Cluster $=$ Pt122 $Q=0$ & Etot $=-11807$ & 96954200 \\
\hline Pt 1 & 3.38876200 & 5.11703600 & 1.59408700 \\
\hline Pt2 & -0.91818200 & 4.10844200 & 4.94055800 \\
\hline Pt 3 & 3.62239100 & 3.27254300 & 3.63851400 \\
\hline Pt 4 & 0.99930500 & 6.46878300 & 1.22876700 \\
\hline Pt 5 & -4.20211100 & 1.92621500 & 1.46358800 \\
\hline Pt 6 & -2.43782000 & 3.60630300 & 2.77837100 \\
\hline Pt 7 & -0.94488000 & -1.30868400 & 6.13216300 \\
\hline Pt 8 & 1.25483000 & 4.66626100 & 3.31136700 \\
\hline Pt 9 & -0.31392600 & 4.13248200 & 1.13915700 \\
\hline Pt10 & -2.06244900 & 2.08826000 & -1.70888500 \\
\hline Pt11 & -5.05450500 & 3.74456100 & 3.25441000 \\
\hline Pt12 & -4.71883200 & 2.27150800 & -1.28989700 \\
\hline Pt13 & 2.04412900 & 0.00143000 & -6.94283000 \\
\hline Pt14 & 5.77798300 & -2.21348200 & -0.36106100 \\
\hline Pt15 & -4.21511300 & -0.74619400 & 2.04328300 \\
\hline Pt16 & -5.08295800 & -1.65150600 & 4.43927100 \\
\hline Pt17 & 2.72994700 & -5.92529300 & -0.29068700 \\
\hline Pt18 & -4.07910200 & 6.06370200 & -0.85450400 \\
\hline Pt19 & 7.02485900 & -0.22003800 & -1.75872400 \\
\hline Pt20 & 3.10590400 & 6.88753000 & -0.45846600 \\
\hline Pt21 & 4.43970900 & -4.19781200 & 1.04034400 \\
\hline Pt22 & -2.00867000 & -6.65173300 & 0.32296400 \\
\hline Pt23 & 1.04598200 & -3.53604700 & -4.00803000 \\
\hline Pt24 & 2.06301100 & 2.79429800 & 1.50683200 \\
\hline Pt25 & 5.79596500 & 2.17755000 & -1.33541600 \\
\hline Pt26 & -3.69939900 & 5.75384900 & 1.83537800 \\
\hline Pt27 & -1.14668800 & 5.96320800 & 2.89508000 \\
\hline Pt28 & 0.58877900 & 6.75827800 & -1.50002300 \\
\hline Pt29 & 2.03091700 & -2.68354100 & -6.34451900 \\
\hline Pt30 & -0.08113500 & 2.29532300 & 3.17259900 \\
\hline Pt31 & 2.36993900 & -5.66078600 & -3.00717000 \\
\hline Pt 32 & 0.05010700 & 0.96156600 & -2.98023100 \\
\hline Pt33 & -0.09215100 & -0.40127500 & 3.7636620 \\
\hline Pt 34 & -6.78294900 & 2.05456600 & 1.9768930 \\
\hline Pt 35 & 4.12190600 & -3.97972700 & -1.69890200 \\
\hline Pt36 & -2.46549200 & 0.94224000 & 3.39222200 \\
\hline Pt37 & 4.13429100 & 3.20893400 & -3.29403200 \\
\hline Pt 38 & -1.11844500 & -4.06221700 & -2.37376900 \\
\hline Pt39 & -5.49369800 & 4.11258800 & 0.57703400 \\
\hline Pt 40 & 0.72445300 & 0.48854500 & 1.3835770 \\
\hline Pt 41 & -4.74509100 & -2.19813000 & -0.30234100 \\
\hline Pt 42 & 1.76810800 & -0.04040900 & -1.0437480 \\
\hline & 2.04249 & -1.56623300 & \\
\hline
\end{tabular}




\begin{tabular}{|c|c|c|c|}
\hline Pt 44 & -1.16532900 & 3.10977400 & -4.05097400 \\
\hline Pt 45 & -1.39204000 & -2.24737000 & -4.51151100 \\
\hline Pt 46 & -0.34736900 & -2.92129500 & 2.68443300 \\
\hline Pt 47 & 2.39230800 & 4.17878500 & -5.16373800 \\
\hline Pt 48 & -3.74676500 & -4.08385000 & 3.99436800 \\
\hline Pt 49 & 3.12322200 & -2.10704000 & -0.00095400 \\
\hline Pt 50 & 1.21892900 & -2.51063100 & 4.88512300 \\
\hline Pt51 & 2.71832500 & 0.87317300 & -3.34125700 \\
\hline Pt52 & -5.11065100 & 1.06007300 & 3.90420700 \\
\hline Pt53 & 1.45034500 & 0.05193600 & 5.91302300 \\
\hline Pt5 4 & -2.86169000 & 3.91372800 & 0.05993400 \\
\hline Pt 55 & -3.37715900 & 0.13887000 & -0.33215500 \\
\hline Pt 56 & 1.42052300 & -3.82068900 & -1.32864700 \\
\hline Pt57 & -4.12472300 & -5.48436100 & 1.66072500 \\
\hline Pt58 & -1.94737600 & 6.55635100 & -2.51785200 \\
\hline Pt59 & 2.26182200 & 0.94464700 & 3.49751000 \\
\hline Pt 60 & -1.61257800 & -5.31225200 & 2.72393700 \\
\hline Pt 61 & 0.20489100 & -6.23825600 & -1.34191800 \\
\hline Pt 62 & -2.89342500 & -3.15299000 & 1.61214300 \\
\hline Pt 63 & 3.34760500 & -3.64977400 & 3.52517900 \\
\hline Pt 64 & 3.70847000 & -3.67607700 & -4.40884300 \\
\hline Pt 65 & -6.79505400 & -0.63155800 & 2.55850900 \\
\hline Pt 66 & -0.32351900 & -1.42397500 & -6.84387200 \\
\hline Pt 67 & -2.29408700 & -6.43107200 & -2.39996500 \\
\hline Pt 68 & 3.14030100 & 2.25005700 & -0.96776200 \\
\hline Pt 69 & -3.59283400 & 1.66862000 & -3.84786500 \\
\hline Pt70 & 1.75697700 & -4.03342900 & 1.36518900 \\
\hline Pt71 & -3.31827600 & 2.74327900 & 5.16911000 \\
\hline Pt72 & 1.79389400 & 4.57204700 & -0.52983500 \\
\hline Pt73 & -1.39924600 & 0.47812500 & -5.18210500 \\
\hline Pt 74 & 0.48441100 & 2.28334400 & -0.61432800 \\
\hline Pt75 & -1.61720400 & -0.83283200 & 1.58642800 \\
\hline Pt 76 & 5.40805000 & -1.95432800 & -3.09512600 \\
\hline Pt77 & -0.15560700 & -5.92561600 & -4.05484800 \\
\hline Pt78 & 2.74914000 & -1.85067900 & -2.71994000 \\
\hline Pt79 & -3.60465600 & -2.79195300 & -2.87265800 \\
\hline Pt 80 & -1.60747200 & 1.80115200 & 1.01529700 \\
\hline Pt 81 & 0.98223700 & 1.87336200 & -5.30127800 \\
\hline Pt 82 & -0.93656700 & 1.41696100 & 5.60614000 \\
\hline Pt 83 & 0.53155200 & -6.42090500 & 1.38008500 \\
\hline Pt 84 & 3.60131100 & -1.12116100 & 4.60555200 \\
\hline Pt 85 & -3.35352600 & -4.55550200 & -0.76098200 \\
\hline Pt 86 & -0.37769200 & -4.09442600 & -6.15820800 \\
\hline Pt 87 & -3.30577300 & 4.21667400 & -2.67745000 \\
\hline Pt 88 & 1.46187000 & 2.76939200 & 5.31735400 \\
\hline Pt 89 & -5.98335000 & 0.24649000 & 0.12643800 \\
\hline Pt 90 & -2.45647400 & -1.73019900 & 3.92103900 \\
\hline Pt91 & 4.74782800 & -1.68298200 & 2.15091500 \\
\hline Pt 92 & 0.94249200 & -5.01895100 & 3.74701300 \\
\hline Pt93 & 3.80354000 & 1.40363200 & 5.61967800 \\
\hline Pt 94 & 2.76521700 & 5.57181100 & -2.82480900 \\
\hline Pt95 & -1.19861900 & -3.84871200 & 5.04810300 \\
\hline Pt 96 & -2.52685200 & -4.69097300 & -4.53660000 \\
\hline Pt 97 & 0.23904800 & 5.44775900 & -3.8786200 \\
\hline Pt 98 & 4.94418400 & 0.86998900 & 3.19933000 \\
\hline Pt99 & 0.08652500 & -1.71736600 & -2.34909000 \\
\hline Pt100 & 3.67672200 & 1.77829900 & -5.6306290 \\
\hline Pt101 & -0.74085000 & 4.40528100 & -1.59487500 \\
\hline Pt102 & 1.04957400 & -0.84758000 & -4.6440610 \\
\hline
\end{tabular}




\begin{tabular}{|c|c|c|c|}
\hline Pt103 & -0.77954500 & -4.31330400 & 0.32162100 \\
\hline Pt104 & -2.29464900 & -0.42060300 & -2.78683400 \\
\hline Pt105 & -0.80864600 & 0.04818300 & -0.70399200 \\
\hline Pt 106 & -2.09031300 & -2.21214900 & -0.73883900 \\
\hline Pt107 & 0.46375800 & -1.97443800 & 0.32213400 \\
\hline Pt108 & -1.55909000 & 6.32616000 & 0.18510500 \\
\hline Pt109 & 4.47829900 & 4.56875600 & -0.89661600 \\
\hline Pt 110 & 3.04812800 & -6.11654800 & 2.40560400 \\
\hline Pt111 & 3.76226100 & -0.96355600 & -5.06710200 \\
\hline Pt112 & 1.41691100 & 3.23817300 & -2.90152700 \\
\hline Pt113 & -3.33312800 & 0.04485500 & 5.76557700 \\
\hline Pt114 & -4.94818300 & -0.34102800 & -2.42857900 \\
\hline Pt115 & 4.38935000 & -0.13027000 & -1.39136300 \\
\hline Pt116 & 5.39019600 & 0.78326500 & -3.70776000 \\
\hline Pt117 & -5.52483800 & -3.09866000 & 2.15717000 \\
\hline Pt118 & 4.76680300 & 2.74999000 & 1.16988200 \\
\hline Pt119 & -3.31928200 & -2.63943500 & 6.26982100 \\
\hline Pt120 & 6.02765600 & 0.32408000 & 0.71870000 \\
\hline Pt121 & 3.37537000 & 0.41009200 & 1.07049900 \\
\hline Pt122 & -3.76578900 & -0.92128500 & -4.90064500 \\
\hline \#109 & uster $=\mathrm{Pt12} 2 \mathrm{Q}=0$ & $=0 \quad \mathrm{Etot}=-119$ & 44944600 \\
\hline Pt1 & -1.03335500 & -0.00166400 & 0.2309930 \\
\hline Pt2 & -5.09614900 & 1.36208600 & 1.60874000 \\
\hline Pt 3 & 0.49669400 & -5.81504900 & -2.00490400 \\
\hline Pt 4 & 1.99903400 & 0.23233400 & -3.14066200 \\
\hline Pt 5 & 4.18344200 & -0.16018100 & 1.02733900 \\
\hline Pt 6 & 1.21550700 & 4.19894700 & 2.36497400 \\
\hline Pt 7 & -5.02818700 & 0.54188200 & -5.34229100 \\
\hline Pt 8 & 3.53301900 & 4.45322300 & -4.44690000 \\
\hline Pt9 & -1.28477500 & -3.22567900 & -5.29631400 \\
\hline Pt10 & 3.29979000 & -0.53717200 & 5.64057000 \\
\hline Pt11 & 3.18242600 & 5.14887500 & -1.83362600 \\
\hline Pt 12 & 5.31478000 & -2.50115100 & 1.75538600 \\
\hline Pt13 & -3.01651700 & -3.26329700 & 5.91460600 \\
\hline Pt14 & -1.61937100 & -5.56208000 & -3.78722100 \\
\hline Pt15 & -3.77448900 & -4.05657000 & -4.58331200 \\
\hline Pt16 & 3.09323900 & -1.34050900 & -1.17152000 \\
\hline Pt17 & 2.57893400 & -5.93533900 & -0.1697 \\
\hline Pt18 & 2.09426800 & 4.05189900 & 4.91995900 \\
\hline Pt19 & -2.99496700 & -0.47787500 & 6.23134800 \\
\hline Pt20 & 0.08522300 & 2.07575900 & 1.28215800 \\
\hline Pt21 & -2.03069300 & 2.30001500 & -0.45249300 \\
\hline Pt22 & 3.14113000 & 1.41139000 & -0.93746300 \\
\hline Pt23 & -4.65684900 & 2.45648800 & -0.9654660 \\
\hline Pt24 & -1.15701000 & 4.11599700 & -4.6718820 \\
\hline Pt25 & -2.00507900 & -6.63465000 & -1.27046800 \\
\hline Pt26 & -4.37554200 & 0.30996100 & -2.74905100 \\
\hline Pt27 & -3.01007800 & 4.66283100 & -1.19261500 \\
\hline Pt28 & 1.93858100 & -4.89427900 & 4.15905900 \\
\hline Pt29 & -1.58739400 & 1.03827100 & 4.5125540 \\
\hline Pt30 & 2.44973900 & -0.30825400 & 3.1230180 \\
\hline Pt31 & -3.60360600 & -3.72280300 & 0.72936500 \\
\hline Pt32 & -0.47642000 & 3.81970900 & -2.04461800 \\
\hline Pt33 & 0.70316600 & 6.04938700 & -0.99579800 \\
\hline Pt3 4 & 1.64045000 & 3.62108000 & -0.2584050 \\
\hline Pt35 & 1.30674200 & 0.46712300 & -5.7595170 \\
\hline Pt 36 & 3.49586300 & -1.02987900 & -4.9726040 \\
\hline & 00 & 6.34833900 & 3.46154 \\
\hline
\end{tabular}




\begin{tabular}{|c|c|c|c|}
\hline Pt38 & 1.23019500 & -2.30272100 & -5.93434700 \\
\hline Pt39 & -3.48036900 & -1.70084900 & -5.98864700 \\
\hline Pt 40 & 0.47340100 & 1.45951700 & -1.30674500 \\
\hline Pt41 & 2.74298900 & 2.04825300 & 1.71458900 \\
\hline Pt 42 & -2.46526000 & 1.24021000 & 2.00503500 \\
\hline Pt 43 & -0.42253800 & 3.21629600 & 5.70901200 \\
\hline Pt 44 & 4.21470400 & -3.72336000 & -0.47487100 \\
\hline Pt 45 & -2.80725100 & -5.34529200 & 4.13243200 \\
\hline Pt 46 & -3.47497900 & 3.66845100 & 1.35801300 \\
\hline Pt 47 & -0.14583000 & 5.54165600 & 4.24025200 \\
\hline Pt 48 & 1.56335300 & -0.07975900 & 0.61788100 \\
\hline Pt 49 & -1.41512900 & 6.17421700 & -2.78901400 \\
\hline Pt50 & -5.35191700 & -1.85777200 & -4.01972800 \\
\hline Pt51 & -1.30256000 & 3.37172700 & 3.11491200 \\
\hline Pt52 & 3.54394900 & 1.73325000 & -4.73759200 \\
\hline Pt53 & -2.21185200 & 5.82391000 & 2.46544300 \\
\hline Pt5 4 & -3.16584100 & -4.28773500 & -1.95388800 \\
\hline Pt55 & -3.40192600 & 2.80671600 & -5.60526500 \\
\hline Pt5 6 & -0.25829300 & -1.02939400 & -4.09415600 \\
\hline Pt57 & 1.51513300 & -3.57994600 & -0.87087400 \\
\hline Pt58 & 3.64390800 & 1.85242200 & 4.27659000 \\
\hline Pt59 & -2.10759600 & -2.11905800 & -0.82834900 \\
\hline Pt 60 & -2.91935200 & 2.32009600 & 6.38949000 \\
\hline Pt 61 & 0.00969800 & -2.26411900 & 0.91303900 \\
\hline Pt 62 & -2.63305300 & 4.66460500 & 4.98380400 \\
\hline Pt 63 & -3.59800800 & 0.07996900 & -0.20403800 \\
\hline Pt 64 & -0.96906700 & -0.80897600 & -6.66189000 \\
\hline Pt 65 & -5.14486500 & -1.43727200 & 1.37064600 \\
\hline Pt 66 & -2.69404800 & 2.55905700 & -3.01027000 \\
\hline Pt 67 & 2.78291800 & 5.78611000 & 0.82720500 \\
\hline Pt 68 & 3.00803300 & -4.87220200 & -2.68594800 \\
\hline Pt 69 & -2.43866500 & 0.44635800 & -4.74902300 \\
\hline Pt70 & 4.70402700 & 0.15494500 & -2.78423600 \\
\hline Pt71 & 5.09750300 & -0.39113300 & 3.55570600 \\
\hline Pt72 & 6.81282900 & -0.24106400 & 1.44031100 \\
\hline Pt73 & 0.05465300 & -6.81998700 & 0.53943700 \\
\hline Pt74 & -1.63556600 & -1.72977200 & 4.27712400 \\
\hline Pt75 & 0.65312900 & -0.45090700 & 5.16784600 \\
\hline Pt76 & 4.59526600 & -2.61371300 & -2.98510000 \\
\hline Pt77 & 1.32643800 & 3.22769000 & -5.46396900 \\
\hline Pt78 & -5.27271200 & 2.69352400 & -3.63262600 \\
\hline Pt79 & -0.19711500 & -0.22300200 & 2.66224500 \\
\hline Pt 80 & 0.87666000 & -2.54461400 & 3.42309300 \\
\hline Pt 81 & -1.02095600 & -4.45097900 & -0.16775400 \\
\hline Pt 82 & -6.17673200 & 0.16540000 & -0.68085400 \\
\hline Pt83 & -0.55016400 & -4.11745300 & 5.08525400 \\
\hline Pt 84 & 5.39283000 & 1.98376500 & 2.13684100 \\
\hline Pt 85 & -2.41895200 & -6.07662100 & 1.45326800 \\
\hline Pt 86 & -3.85571700 & 2.52463700 & 3.8568470 \\
\hline Pt 87 & -0.77893100 & -1.97595800 & 6.81580600 \\
\hline Pt 88 & 0.28863100 & 6.62499000 & 1.68297300 \\
\hline Pt 89 & 3.39012400 & -3.75786100 & -5.14527700 \\
\hline Pt90 & 5.79398800 & 1.34148100 & -0.53491800 \\
\hline Pt91 & 2.66554600 & -2.40184500 & 1.3360950 \\
\hline Pt92 & 0.90149000 & -4.68614100 & -4.49393200 \\
\hline Pt93 & -2.51251100 & -1.47501000 & 1.7740980 \\
\hline Pt94 & -0.60207800 & -3.40090500 & -2.6587530 \\
\hline Pts & 4.69156500 & 2.91985200 & -2.51445300 \\
\hline Pt96 & 3.73335600 & -4.74766300 & 2.05504100 \\
\hline
\end{tabular}




\begin{tabular}{|c|c|c|c|}
\hline Pt97 & 1.91078300 & -2.48773500 & -3.33182400 \\
\hline Pt98 & -1.42740400 & -3.80218800 & 2.50474900 \\
\hline Pt99 & -2.77198600 & -1.91947100 & -3.39118200 \\
\hline Pt 100 & 2.10922200 & -6.91862500 & 2.33315200 \\
\hline Pt101 & 0.95397900 & 1.89832700 & 3.80097800 \\
\hline Pt102 & -4.73607200 & -2.09586400 & -1.35265700 \\
\hline Pt103 & -3.61862000 & 4.90008500 & -3.82152200 \\
\hline t104 & -0.92096500 & 1.95510000 & -6.42679700 \\
\hline t105 & -0.86609100 & 4.44797900 & 0.58912900 \\
\hline Pt106 & 4.34218800 & 3.60223300 & 0.14819200 \\
\hline t107 & 1.06277600 & -4.57740300 & 1.61851900 \\
\hline$=108$ & 3.88908100 & 4.20070500 & 2.81612700 \\
\hline 109 & -3.95194400 & -3.00483600 & 3.38654900 \\
\hline Pt110 & -3.96189300 & -0.24443600 & 3.68456500 \\
\hline Pt111 & 1.79959300 & 1.68989600 & 6.32466000 \\
\hline Pt 112 & 1.72155200 & -2.79459400 & 5.94324000 \\
\hline Pt113 & 2.00536300 & 2.94703200 & -2.86958100 \\
\hline Pt114 & -1.75094600 & 0.21983800 & -2.22645400 \\
\hline Pt115 & 0.42653700 & -1.23341300 & -1.53578600 \\
\hline Pt116 & 5.74573500 & -1.43125100 & -0.77074700 \\
\hline & 1.07603400 & 5.34361600 & -3.64087100 \\
\hline 18 & 3.56516100 & -2.67261800 & 3.89172100 \\
\hline & -0.34963600 & -6.16960600 & 3.24417400 \\
\hline Pt 120 & -1.77094700 & 6.81915200 & -0.12618200 \\
\hline Pt121 & -3.15884700 & 0.67617700 & -7.30373400 \\
\hline Pt122 & -0.21054800 & 1.71414700 & -3.86081000 \\
\hline & -0.73023000 & 0.82256700 & 7.05382900 \\
\hline \#110 & Cluster $=$ Pt124 $\mathrm{Q}=0$ & $=0 \quad$ Etot $=-12$ & 5549 \\
\hline Pt 1 & -1.13006200 & -6.21269500 & 4.88340500 \\
\hline Pt2 & -0.63547200 & 2.26866000 & 1.10721300 \\
\hline Pt 3 & -2.44749900 & 2.97212300 & -0.76738900 \\
\hline Pt 4 & -1.72650800 & -0.20484500 & 1.21967600 \\
\hline Pt 5 & 0.36048000 & -0.05866300 & -6.26359600 \\
\hline Pt 6 & -1.62334900 & 6.62116200 & 0.04628000 \\
\hline Pt 7 & 4.20682200 & -1.24932000 & 4.38003100 \\
\hline Pt 8 & -5.13351000 & -0.15828600 & 4.27212700 \\
\hline Pt9 & -0.61772800 & -1.54298200 & 4.55820500 \\
\hline Pt10 & 3.52245200 & -2.92032100 & -4.88948700 \\
\hline Pt11 & 1.78197300 & -0.12490800 & 5.10191800 \\
\hline Pt12 & 0.99922000 & -2.35792300 & 6.49866300 \\
\hline Pt13 & 0.88343500 & -2.71143700 & -5.65882700 \\
\hline Pt14 & -4.75441200 & 4.10997300 & 2.08079600 \\
\hline Pt15 & -3.59283900 & 6.57 & 1.94365200 \\
\hline Pt16 & 1.53098400 & -4.15482600 & 1.59944900 \\
\hline Pt17 & 1.24977800 & 2.62331500 & 4.47092300 \\
\hline Pt18 & -2.51905600 & 0.21832100 & 3.71091200 \\
\hline Pt19 & 2.19331800 & -1.98331100 & 0.23080900 \\
\hline Pt20 & -1.30173000 & -3.72388900 & 5.99799700 \\
\hline Pt21 & -4.23407100 & 3.66274200 & -2.65259500 \\
\hline Pt22 & -4.87929000 & -1.54605900 & -1.95227400 \\
\hline Pt23 & -4.30153300 & -4.17548800 & -1.31839000 \\
\hline Pt2 4 & -4.10258400 & 2.39466100 & 4.17568700 \\
\hline Pt25 & 3.60880500 & -4.19567000 & -0.25094700 \\
\hline Pt26 & 3.98927600 & 0.08870300 & -2.4359850 \\
\hline Pt27 & -0.14007400 & 4.42606100 & -0.36925000 \\
\hline Pt28 & 6.11992300 & -3.79168300 & -1.13911400 \\
\hline Pt29 & 0.95025200 & -3.98376500 & -1.06625000 \\
\hline PEsu & -0.44517400 & 2.93414800 & -2.66812700 \\
\hline
\end{tabular}




\begin{tabular}{|c|c|c|c|}
\hline Pt31 & 0.08630600 & 5.07947700 & -4.17355800 \\
\hline Pt32 & -0.31035100 & 0.37665500 & -3.71067700 \\
\hline Pt33 & -2.42153100 & -2.34973600 & 2.63314200 \\
\hline Pt 34 & 0.86394300 & -6.34791700 & 3.02058800 \\
\hline Pt35 & -3.26241500 & -1.96231500 & 5.17591600 \\
\hline Pt36 & -1.76137100 & -6.11769700 & 2.23560100 \\
\hline Pt37 & 1.61748800 & -4.50246200 & -3.66065900 \\
\hline Pt38 & 3.05139600 & 6.40768700 & -1.10130600 \\
\hline Pt39 & -1.68001200 & -3.73756000 & -1.83096500 \\
\hline Pt 40 & 2.86143300 & -6.39776700 & 1.12343300 \\
\hline Pt41 & -3.25153300 & 1.93753200 & 1.64879900 \\
\hline Pt 42 & 0.21638800 & -2.25509700 & -3.10564400 \\
\hline Pt 43 & 2.66760800 & 1.40736200 & -5.88281800 \\
\hline Pt 44 & -3.73783400 & -4.38937200 & 1.38024700 \\
\hline Pt 45 & -4.29249600 & -0.57672400 & 1.75870100 \\
\hline Pt 46 & 0.79245800 & 0.15422700 & 0.68853900 \\
\hline Pt4 7 & -1.03328100 & -4.24842500 & -4.40216600 \\
\hline Pt 48 & -3.32068300 & 0.63611000 & 6.19274200 \\
\hline Pt 49 & 6.34582200 & 3.03912900 & -2.15424900 \\
\hline Pt50 & 4.55048300 & 2.24258700 & -4.04942800 \\
\hline Pt51 & 5.41381100 & -2.10253200 & -3.10813000 \\
\hline Pt52 & 3.83651500 & 2.62402200 & -1.42820600 \\
\hline Pt53 & -0.43410900 & -1.77493600 & -0.56180900 \\
\hline Pt5 4 & -5.34560700 & 1.11951900 & -2.52860300 \\
\hline Pt5 5 & 4.65547400 & -0.32898100 & -5.03440800 \\
\hline Pt5 6 & 0.09511500 & 0.59315400 & 3.15182100 \\
\hline Pt57 & 0.21887600 & 2.53642900 & -5.28152600 \\
\hline Pt58 & -4.01376400 & -0.49272800 & -4.35764500 \\
\hline Pt59 & 1.89996100 & -2.68819500 & 3.97679900 \\
\hline Pt 60 & -3.09077500 & -4.51075400 & 4.06135500 \\
\hline Pt 61 & 3.16969900 & 4.12554000 & 3.12887300 \\
\hline Pt 62 & 4.23264200 & -4.66229700 & -2.86761500 \\
\hline Pt 63 & 7.21237300 & -1.28402100 & -1.28655800 \\
\hline Pt 64 & -5.85451300 & 1.57878800 & 2.21055800 \\
\hline Pt 65 & -2.28146700 & 3.17518000 & 6.04301700 \\
\hline Pt 66 & -4.48018700 & 2.13891600 & -4.90375900 \\
\hline Pt 67 & 4.38457800 & 4.78001000 & -2.96884400 \\
\hline Pt 68 & -0.67892000 & 1.02386800 & 5.66386900 \\
\hline Pt 69 & 0.18060600 & -1.92778700 & 2.07171400 \\
\hline Pt70 & -2.08277400 & 1.04909200 & -5.61616200 \\
\hline Pt71 & -2.22980300 & -1.15209800 & -2.46279900 \\
\hline Pt72 & 5.10912200 & -0.17444400 & 1.95429100 \\
\hline Pt73 & -0.47960700 & -4.05611300 & 3.4597100 \\
\hline Pt74 & 1.01857200 & 6.52112000 & 0.81353600 \\
\hline Pt75 & 2.53151300 & 3.96955800 & -4.84119700 \\
\hline Pt76 & 3.19068300 & -5.00600300 & 3.48764200 \\
\hline Pt77 & 0.26619100 & -6.23062300 & 0.32424400 \\
\hline Pt78 & 1.32023800 & 2.23207600 & -0.74783700 \\
\hline Pt79 & -2.96491600 & 4.90896400 & 4.0123530 \\
\hline Pt 80 & -2.92473300 & -5.72043800 & -3.1032260 \\
\hline Pt 81 & 2.78725400 & -1.15185000 & -6.83458000 \\
\hline Pt 82 & -1.10187300 & -3.94606700 & 0.82047300 \\
\hline Pt83 & 5.84616700 & 0.92429000 & -0.53605500 \\
\hline Pt 84 & -1.44361800 & -1.17832700 & 7.0433910 \\
\hline Pt 85 & 3.74723100 & 1.45789500 & 3.80715700 \\
\hline Pt 86 & 2.32753100 & -6.18811500 & -1.56726100 \\
\hline Pt 87 & 1.84143300 & 4.32906300 & -2.24230200 \\
\hline Pt 88 & -5.01978900 & -2.73816600 & 3.20123800 \\
\hline Pt 89 & -0.31030800 & -5.98874400 & -2.35492900 \\
\hline
\end{tabular}




\begin{tabular}{|c|c|c|c|}
\hline et 90 & 4.74523800 & -1.64637300 & -0.39690800 \\
\hline Pt91 & 3.97464700 & -2.77559700 & 2.08409100 \\
\hline Pt92 & 2.11246200 & -0.71365300 & -4.29303200 \\
\hline Pt93 & -2.36126100 & -5.95255800 & -0.45041800 \\
\hline Pt94 & 1.98788100 & 1.83007700 & -3.31914400 \\
\hline Pt95 & 2.86841200 & -2.46374900 & -2.30508900 \\
\hline Pt96 & -1.94520800 & 5.17419200 & -2.28584600 \\
\hline Pt97 & -1.44018100 & 2.72010500 & 3.57904700 \\
\hline Pt98 & -6.87128200 & -0.94804300 & 2.3175070 \\
\hline Pt99 & 1.17810500 & -4.86735700 & 5.37144900 \\
\hline Pt100 & -2.71281400 & 1.46588800 & -3.02555100 \\
\hline t101 & -1.58360000 & -1.61887100 & -5.06434900 \\
\hline Pt102 & -2.11420100 & 4.40351500 & 1.51533300 \\
\hline Pt 103 & -5.62932200 & -2.57038200 & 0.50467200 \\
\hline t104 & -6.14938800 & 0.08096400 & -0.10160300 \\
\hline Pt105 & 2.50712300 & -0.53051200 & 2.51828900 \\
\hline Pt106 & -3.55192700 & 0.44701300 & -0.64742500 \\
\hline Pt107 & 0.53894600 & 4.32562500 & 2.36007900 \\
\hline Pt108 & -0.99858900 & 6.48167300 & 2.74840500 \\
\hline Pt109 & -2.21988600 & 3.62418600 & -4.56536500 \\
\hline Pt110 & 0.39199100 & 6.58367100 & -1.86884300 \\
\hline Pt111 & -0.38219400 & 4.79937000 & 4.84957800 \\
\hline Pt112 & 2.54464300 & 4.28951000 & 0.45530600 \\
\hline Pt113 & -5.10318600 & 2.64412300 & -0.23186300 \\
\hline Pt114 & 1.45126700 & -0.27648400 & -1.77548700 \\
\hline Pt 115 & -0.94923800 & 0.80329400 & -1.15476000 \\
\hline Pt116 & -3.49104700 & -3.13462000 & -3.75227100 \\
\hline Pt117 & 2.38440700 & 6.46536100 & -3.73753400 \\
\hline Pt118 & 3.29711000 & 0.52938700 & 0.12254800 \\
\hline Pt119 & 5.08496400 & 4.70165900 & -0.34135200 \\
\hline Pt 120 & -3.03347100 & -2.18268400 & -0.04596000 \\
\hline Pt121 & 6.49360400 & 0.50375600 & -3.17202200 \\
\hline Pt122 & 1.98787500 & 2.10943400 & 1.91752900 \\
\hline Pt123 & -3.93609500 & 5.15515500 & -0.35121300 \\
\hline Pt124 & 4.58174900 & 2.56288300 & 1.31186700 \\
\hline & luster $=\operatorname{Pt1} 46 \mathrm{Q}=0$ & $=0 \quad \mathrm{E}$ to $\mathrm{t}=-143$ & 2194 \\
\hline Pt 1 & 2.11072600 & -0.27189100 & -1.50472900 \\
\hline Pt2 & -2.22468700 & -5.95012200 & 0.15913800 \\
\hline Pt3 & -2.62999200 & -1.65022200 & 3.20294300 \\
\hline Pt 4 & -0.50488800 & -1.91534300 & 1.61563400 \\
\hline Pt 5 & 6.35430500 & -1.88570000 & -2.05351100 \\
\hline Pt 6 & -1.59898200 & 0.75398900 & 4.12716700 \\
\hline Pt 7 & 4.62772300 & -5.00250400 & 1.20463600 \\
\hline Pt 8 & -6.24176900 & 0.95094000 & 4.62878300 \\
\hline Pt 9 & 3.02173900 & -6.15928500 & -0.75069500 \\
\hline Pt10 & -1.95256600 & 2.93669200 & -5.32633400 \\
\hline Pt11 & 3.60608500 & -4.55207700 & 3.7638760 \\
\hline Pt12 & 6.42642700 & 2.62794100 & -0.36967600 \\
\hline Pt13 & -0.44355500 & -3.25613200 & -6.04741600 \\
\hline Pt14 & -4.55739900 & 2.19054500 & -4.66926100 \\
\hline Pt15 & -4.12286200 & 1.62270600 & 0.51746200 \\
\hline Pt16 & 4.74174400 & -4.07021200 & -1.42119200 \\
\hline Pt 17 & -0.33461100 & 1.19908400 & -6.7883330 \\
\hline Pt18 & -1.35360100 & -1.20419100 & -7.61625200 \\
\hline Pt19 & 1.71410100 & 5.95144700 & -4.86836000 \\
\hline Pt20 & 0.05879400 & 2.88207700 & 3.4798460 \\
\hline Ptz & 3.05297000 & -0.43018400 & 6.21645300 \\
\hline Pt22 & -3.76313500 & -5.34200100 & -2.0850660 \\
\hline
\end{tabular}




\begin{tabular}{|c|c|c|c|}
\hline Pt23 & 1.22545200 & -1.11517600 & -6.69969300 \\
\hline Pt24 & 3.83644400 & 5.40145100 & 2.10896200 \\
\hline Pt 25 & -1.13981500 & 1.17372400 & 6.72422600 \\
\hline Pt26 & 3.11941900 & 2.32337700 & 5.75820500 \\
\hline Pt27 & 6.42065700 & 0.86790400 & -2.51151300 \\
\hline Pt28 & -2.02070600 & 0.33328300 & 1.52950800 \\
\hline Pt29 & -4.18952400 & -1.11320000 & 0.97226300 \\
\hline Pt 30 & -6.35163400 & 0.17195900 & -0.07534900 \\
\hline Pt31 & 2.21425900 & 4.40737800 & 4.14057300 \\
\hline Pt 32 & 4.20984800 & -0.58148900 & -3.04896100 \\
\hline Pt33 & 6.29695000 & -2.86239800 & 0.55233000 \\
\hline Pt 34 & 0.04757200 & 0.03257600 & 0.01325700 \\
\hline Pt 35 & 3.81406600 & -1.01037900 & -5.68542400 \\
\hline Pt 36 & -6.33488200 & -0.81037400 & 2.53394500 \\
\hline Pt37 & 1.43875300 & 1.26256500 & 7.64164300 \\
\hline Pt38 & -6.26769800 & 1.94299200 & 2.07632300 \\
\hline Pt39 & 3.29643900 & 3.71584200 & -4.84520100 \\
\hline Pt 40 & -1.18104500 & -6.32941400 & -2.39832600 \\
\hline Pt41 & -0.39819600 & 2.41481400 & 0.89633400 \\
\hline Pt 42 & -2.13061400 & -4.34816500 & -4.11490600 \\
\hline Pt43 & 3.30533400 & 5.48930000 & -2.68852000 \\
\hline Pt 44 & -3.22062400 & -5.43018900 & 2.71089300 \\
\hline Pt 45 & 4.91887500 & 3.21494700 & -2.63458700 \\
\hline Pt 46 & -0.06480600 & -4.39717900 & 0.74803500 \\
\hline Pt 47 & -3.21087600 & -3.65668000 & 4.86716900 \\
\hline Pt 48 & 4.76800800 & 3.35911700 & 3.78472400 \\
\hline Pt 49 & 2.12858200 & 1.45761500 & 0.59318000 \\
\hline Pt50 & -2.96748700 & 0.48797200 & -6.19082200 \\
\hline Pt51 & 4.21064800 & -1.56398000 & -0.4935190 \\
\hline Pt52 & 2.03841300 & -2.87834800 & 5.3507520 \\
\hline Pt53 & -1.62445200 & -3.86003400 & -1.48253600 \\
\hline Pt5 4 & -4.78467700 & -4.89161500 & 0.47393500 \\
\hline Pt55 & 4.26149500 & 2.91819700 & 1.18756800 \\
\hline Pt5 6 & -0.04897100 & -1.54536000 & 4.2152370 \\
\hline Pt57 & -4.13235700 & -0.13970600 & -1.6250320 \\
\hline Pt58 & 2.55181700 & -3.69093700 & 0.15481300 \\
\hline Pt59 & -1.93709500 & 5.83744700 & -1.80067300 \\
\hline Pt 60 & -2.08933700 & 3.24492300 & 5.11621900 \\
\hline Pt 61 & 4.76656100 & 0.62119000 & 4.28559100 \\
\hline Pt 62 & -1.62888600 & -5.89199600 & 4.89088000 \\
\hline Pt 63 & -4.54173500 & 5.06067600 & -1.1804390 \\
\hline Pt 64 & 2.05481100 & -1.22407500 & 1.03570900 \\
\hline Pt 65 & -2.93641700 & 6.21844600 & 0.77485300 \\
\hline Pt 66 & -0.62941100 & -6.42106900 & 2.39668200 \\
\hline Pt 67 & -0.39736000 & 5.34439100 & 4.40789800 \\
\hline Pt68 & 2.56759400 & -0.83957800 & 3.62239200 \\
\hline Pt 69 & -0.61357100 & -3.55132300 & 5.88582000 \\
\hline Pt70 & -2.02976900 & -1.38995000 & -0.56564300 \\
\hline Pt71 & -4.12249800 & 0.64065600 & $3.0725130 c$ \\
\hline Pt72 & -4.14130300 & -2.83589100 & -1.1540730 \\
\hline Pt73 & 0.50421200 & 0.43640000 & 2.5196480 \\
\hline Pt74 & 6.30575500 & -1.08874400 & 2.70885100 \\
\hline Pt75 & -4.65561800 & 4.12843300 & 1.44490900 \\
\hline Pt76 & -1.44929500 & 3.30300900 & -2.6736550 \\
\hline Pt77 & 1.68636900 & -0.69448100 & -4.10275600 \\
\hline Pt78 & -4.68120800 & -0.56451100 & -4.25982700 \\
\hline Pt79 & 4.64348900 & -2.13340800 & 4.6943110 \\
\hline Pt 80 & 4.09704800 & -2.50235600 & 2.0759400 \\
\hline & -6.21036400 & 2.91931600 & -0.5285230 \\
\hline
\end{tabular}




\begin{tabular}{|c|c|c|c|}
\hline Pt 82 & 0.71420000 & 6.48106000 & -2.37397300 \\
\hline Pt 83 & 0.52828000 & 3.31504300 & 6.07300400 \\
\hline Pt 84 & 6.32804300 & -0.89306000 & -4.60620300 \\
\hline Pt 85 & 0.59468500 & 1.97706500 & -1.59086800 \\
\hline Pt 86 & -1.96479900 & 1.28532600 & -1.01055700 \\
\hline Pt 87 & 2.72790200 & 3.47204300 & -1.03465200 \\
\hline Pt 88 & -0.41442800 & -0.37490500 & -2.49427800 \\
\hline Pt89 & -2.54705500 & -1.83734300 & -3.14218100 \\
\hline Pt90 & 1.26505700 & 6.38883200 & 2.42244600 \\
\hline Pt91 & -4.65660300 & 3.10459600 & 4.03841200 \\
\hline Pt92 & -1.30764600 & 7.28369600 & 2.70122500 \\
\hline Pt93 & -1.06471600 & -3.89538200 & 3.24625200 \\
\hline Pt94 & 2.17475700 & -3.18594500 & -5.09182600 \\
\hline Pt 95 & 0.15132500 & 4.45778400 & -0.72416300 \\
\hline Pt96 & -0.30424300 & 6.93007800 & 0.17726100 \\
\hline Pt 97 & 0.98205300 & -5.26001400 & 4.35879200 \\
\hline Pt98 & 0.48173100 & -5.28470400 & -4.38294500 \\
\hline Pt99 & 6.43810500 & -0.11577200 & 0.09831100 \\
\hline Pt100 & -2.63955300 & -3.41248900 & 1.06033000 \\
\hline Pt101 & 0.38864200 & -6.86991600 & -0.15357800 \\
\hline Pt102 & -4.69159200 & -3.30647500 & -3.75524100 \\
\hline Pt103 & 1.15149300 & 3.95539800 & -3.22273200 \\
\hline Pt104 & -0.85147400 & 4.83848100 & 1.79477300 \\
\hline Pt105 & -3.72799200 & 1.06873500 & 5.70886300 \\
\hline Pt106 & 0.96897900 & 0.84736900 & 5.06953200 \\
\hline Pt107 & -2.46462600 & 3.75065600 & -0.13021400 \\
\hline Pt108 & 4.27696000 & 1.17410800 & -0.94663700 \\
\hline Pt109 & 2.28474600 & 1.31644600 & -5.84101500 \\
\hline Pt110 & -6.07163300 & 3.84816300 & -3.10104900 \\
\hline Pt111 & 2.02253000 & -5.77820200 & 1.82435100 \\
\hline Pt112 & -4.76535400 & -1.35070500 & 4.77884600 \\
\hline Pt113 & 6.36870500 & 1.64499400 & 2.25284100 \\
\hline Pt114 & 0.69912200 & 3.61045500 & -5.86241100 \\
\hline Pt115 & 2.63545800 & 1.89782500 & 3.16538500 \\
\hline Pt116 & 0.93787600 & -4.77829900 & -1.77031500 \\
\hline Pt117 & -6.22002000 & 1.14560500 & -2.68463300 \\
\hline Pt118 & 2.60899700 & -2.71722400 & -2.44306900 \\
\hline Pt119 & -2.99395200 & 5.23868500 & 3.38887200 \\
\hline Pt120 & 6.15786900 & -3.79094900 & 3.12540400 \\
\hline Pt121 & -6.28695300 & -1.59806700 & -2.22846500 \\
\hline Pt122 & 0.48784700 & -2.35302700 & -0.87120200 \\
\hline Pt123 & 2.30839400 & 6.01040700 & -0.13633200 \\
\hline Pt124 & -2.19887300 & -1.25766700 & 5.86427600 \\
\hline Pt125 & 1.53668900 & -3.24338100 & 2.69774400 \\
\hline Pt126 & -3.03477400 & -2.26534900 & -5.73294500 \\
\hline Pt127 & 1.39216700 & -7.22394000 & -2.67696800 \\
\hline Pt128 & -0.89638800 & 5.31902400 & -4.33549200 \\
\hline Pt129 & 4.85151100 & 1.40898600 & -4.75684500 \\
\hline Pt130 & -0.88223600 & -0.78793500 & -5.04446100 \\
\hline Pt131 & 0.13604800 & 1.60532800 & -4.19084300 \\
\hline Pt132 & 4.74261100 & -3.04609400 & -4.01509300 \\
\hline Pt133 & 4.21982700 & 0.20118700 & 1.64912800 \\
\hline Pt134 & -4.83342100 & -3.15653200 & 2.65572200 \\
\hline Pt135 & 3.07927800 & -5.17945300 & -3.3649000 \\
\hline Pt136 & 4.86140400 & 4.94955900 & -0.45859800 \\
\hline Pt137 & 0.02769700 & -2.82196300 & -3.45466300 \\
\hline & -3.52002300 & 4.61021700 & -3.7398620 \\
\hline & -6.34448300 & -2.57773700 & 0.38530900 \\
\hline Pt140 & -4.00971900 & 2.56116400 & -2.0514290 \\
\hline
\end{tabular}




\begin{tabular}{|c|c|c|c|}
\hline Pt 141 & 1.71339800 & 3.91932500 & 1.50671700 \\
\hline Pt142 & 0.42013700 & -1.14037100 & 6.81286000 \\
\hline Pt143 & 2.71731200 & 1.70997000 & -3.17983800 \\
\hline t144 & -2.48032000 & 0.89853100 & -3.59716400 \\
\hline t145 & -6.27036800 & -4.29383700 & -1.74738800 \\
\hline t14 & -2.52179400 & 2.77713700 & 2.46714800 \\
\hline \#112 & luster $=\operatorname{Pt1} 47 \mathrm{Q}=0$ & $\mathrm{M}=0 \quad \mathrm{E}$ tot $=-14$ & 71318700 \\
\hline Pt 1 & -0.35433200 & -1.62043300 & -7.67602700 \\
\hline Pt2 & -1.99453600 & 1.97856000 & 6.31390500 \\
\hline Pt 3 & 1.80755600 & -4.84826200 & 4.58097800 \\
\hline Pt 4 & 2.44961700 & 5.92675800 & -2.57510700 \\
\hline Pt 5 & -0.05153800 & 2.49570900 & 0.65648300 \\
\hline Pt 6 & 1.19293800 & 4.22355400 & -0.94667200 \\
\hline Pt 7 & 0.23489000 & 1.07419800 & 5.08850200 \\
\hline Pt 8 & -5.99301900 & 3.35166000 & 0.77997100 \\
\hline Pt9 & 0.40372500 & -2.74895000 & 5.7632410 \\
\hline Pt10 & 5.79577300 & 3.76098000 & 0.14938200 \\
\hline Pt11 & 6.07544000 & 0.49293800 & 3.25642500 \\
\hline Pt12 & -2.51427300 & -2.13511000 & -6.0727260 \\
\hline Pt13 & -3.00550800 & 3.10845100 & -2.89937600 \\
\hline Pt14 & -5.82342000 & -1.85846200 & 1.88906400 \\
\hline Pt15 & 1.21831300 & 1.63521700 & -1.5823940 \\
\hline Pt16 & 4.37900600 & -1.73398900 & -2.21799800 \\
\hline Pt17 & -4.51291200 & 4.33373400 & 2.93438900 \\
\hline Pt18 & 2.20813300 & 3.58623600 & 1.55587800 \\
\hline Pt19 & -2.44961700 & -5.92675800 & 2.57510700 \\
\hline Pt20 & 2.21124400 & 1.01187600 & 0.86527300 \\
\hline Pt21 & 4.66212700 & 2.62653900 & 4.37311500 \\
\hline Pt22 & -3.96967800 & -0.00342000 & -5.01760000 \\
\hline Pt23 & 2.45725600 & 3.29812400 & -3.19158200 \\
\hline Pt24 & 1.99453600 & -1.97856000 & -6.31390500 \\
\hline Pt25 & 4.53382200 & -4.68911200 & 4.37372100 \\
\hline Pt26 & 1.01423700 & 0.58835900 & -6.81053800 \\
\hline Pt27 & 3.51236500 & 5.34733400 & -0.06304900 \\
\hline Pt28 & 6.72782700 & 3.07868600 & 2.63263800 \\
\hline Pt29 & -3.70678200 & -4.97523400 & 4.81451600 \\
\hline Pt30 & -1.19293800 & -4.22355400 & 0.94667200 \\
\hline Pt31 & -2.38282300 & -2.81108400 & 5.84621400 \\
\hline Pt32 & -1.57624400 & 4.12740500 & -0.79854700 \\
\hline Pt33 & 2.18996500 & -4.04264300 & -4.44924200 \\
\hline Pt 34 & -6.60575000 & 2.61573000 & 3.34585900 \\
\hline Pt35 & 1.63900100 & -6.71229600 & 0.1304540 \\
\hline Pt 36 & 2.16917600 & 6.16772400 & 2.23877700 \\
\hline Pt37 & -3.46543500 & -0.79289000 & 2.74222200 \\
\hline Pt 38 & 2.17112100 & -0.85971600 & -1.09968800 \\
\hline Pt39 & -3.50645800 & -2.70644900 & 0.73320100 \\
\hline Pt 40 & 4.73640600 & 2.47452000 & -4.38194900 \\
\hline Pt 41 & 2.33963200 & -6.01117300 & -2.4799560 \\
\hline Pt 42 & -1.21831300 & -1.63521700 & 1.5823940 \\
\hline Pt 43 & 6.77607100 & 1.19406100 & 0.64601500 \\
\hline Pt 44 & -6.72782700 & -3.07868600 & -2.63263900 \\
\hline Pt 45 & 5.99301900 & -3.35166000 & -0.77997100 \\
\hline Pt 46 & -2.36668900 & -4.20085500 & -4.2055040 \\
\hline Pt 47 & -1.77236000 & 0.49160500 & -6.66148100 \\
\hline Pt 48 & 5.82342000 & 1.85846200 & -1.8890640 \\
\hline Pt 49 & 2.51427300 & 2.13511000 & 6.0727260 \\
\hline Pt50 & 6.73493200 & -0.72494500 & -1.36872600 \\
\hline Pt51 & 0.01395000 & 5.67460900 & 3.94422200 \\
\hline
\end{tabular}




\begin{tabular}{|c|c|c|c|}
\hline Pt 52 & 0.33622300 & -5.89768600 & 2.45742200 \\
\hline Pt53 & -1.80755600 & 4.84826200 & -4.58097800 \\
\hline Pt 54 & -2.10073400 & 1.42352500 & 3.70380800 \\
\hline Pt55 & -2.37989700 & -1.57909700 & -3.46413600 \\
\hline Pt 56 & -3.40314300 & -0.25257200 & 5.41197700 \\
\hline Pt 57 & 4.45993200 & 2.04088700 & 1.74519800 \\
\hline Pt58 & 0.10394900 & -5.03367900 & -1.32408300 \\
\hline Pt59 & 1.14759600 & 6.80905000 & -0.27951100 \\
\hline Pt 60 & -5.71335200 & 0.08361900 & 3.88701400 \\
\hline Pt61 & -0.01395000 & -5.67460900 & -3.94422200 \\
\hline Pt 62 & -2.27249700 & 3.43066300 & 1.79555000 \\
\hline Pt 63 & -2.18996500 & 4.04264300 & 4.44924200 \\
\hline Pt 64 & 0.11645900 & 0.53259000 & 2.52289100 \\
\hline Pt 65 & -0.10394900 & 5.03367900 & 1.32408300 \\
\hline Pt 66 & -6.10605500 & 1.44425800 & -1.25095000 \\
\hline Pt 67 & 1.64262100 & -1.03120200 & 4.04921200 \\
\hline Pt 68 & 2.37989700 & 1.57909700 & 3.46413600 \\
\hline Pt 69 & 2.10073400 & -1.42352500 & -3.70380800 \\
\hline Pt 70 & 3.46543500 & 0.79289000 & -2.74222200 \\
\hline Pt71 & -2.33963200 & 6.01117300 & 2.47995600 \\
\hline Pt72 & 3.40314300 & 0.25257200 & -5.41197700 \\
\hline Pt73 & 4.51291200 & -4.33373400 & -2.93438900 \\
\hline Pt 74 & -4.37900600 & 1.73398900 & 2.21799800 \\
\hline Pt 75 & 3.18083900 & -2.61790800 & 5.54861100 \\
\hline Pt 76 & -0.11645900 & -0.53259000 & -2.52289100 \\
\hline Pt 77 & -0.33622300 & 5.89768600 & -2.45742200 \\
\hline Pt78 & -2.16917600 & -6.16772400 & -2.23877700 \\
\hline Pt 79 & 6.10605500 & -1.44425800 & 1.25095000 \\
\hline Pt 80 & 1.12656100 & 1.12735100 & -4.19733700 \\
\hline Pt 81 & -4.73640600 & -2.47452000 & 4.38194900 \\
\hline Pt 82 & 3.78437800 & -0.54116900 & 2.35442500 \\
\hline Pt 83 & -0.96951000 & -4.94468500 & 4.72952500 \\
\hline Pt 84 & 3.96967800 & 0.00342000 & 5.01760000 \\
\hline Pt 85 & 0.96951000 & 4.94468500 & -4.72952500 \\
\hline Pt 86 & -6.77607100 & -1.19406100 & -0.64601500 \\
\hline Pt 87 & 5.29478400 & -4.05038500 & 1.82151100 \\
\hline Pt 88 & -3.11404500 & 5.73358200 & -2.27750300 \\
\hline Pt 89 & 3.50645800 & 2.70644900 & -0.73320100 \\
\hline Pt 90 & -4.48928300 & -4.64629900 & -2.45285600 \\
\hline Pt91 & -6.07544000 & -0.49293800 & -3.25642500 \\
\hline Pt92 & -4.45993200 & -2.04088600 & -1.74519800 \\
\hline Pt93 & 1.49013800 & -1.54117700 & 1.43751700 \\
\hline Pt 94 & -0.18620300 & -3.66175600 & -5.85791200 \\
\hline Pt95 & -2.17112100 & 0.85971600 & 1.09968800 \\
\hline Pt 96 & 0.05153800 & -2.49570900 & -0.65648300 \\
\hline Pt97 & -1.63900100 & 6.71229600 & -0.13045500 \\
\hline Pt98 & 0.27792000 & -3.24761800 & 3.08762600 \\
\hline Pt99 & 3.74335500 & -2.45472800 & 0.34540400 \\
\hline Pt100 & -5.33606500 & 2.12479300 & -3.84316600 \\
\hline Pt101 & 0.35433200 & 1.62043300 & 7.67602700 \\
\hline Pt102 & -0.40372500 & 2.74895000 & -5.76324100 \\
\hline Pt103 & -3.51236500 & -5.34733400 & 0.06304900 \\
\hline Pt104 & 2.27249800 & -3.43066400 & -1.79555000 \\
\hline Pt105 & 1.57624400 & -4.12740500 & 0.7985470 \\
\hline Pt106 & 5.33606500 & -2.12479300 & 3.84316600 \\
\hline Pt107 & -2.20813300 & -3.58623600 & -1.55587800 \\
\hline Pt108 & -4.48063100 & -0.15557200 & 0.2396710 \\
\hline Pt109 & -1.12656100 & -1.12735100 & 4.19733700 \\
\hline Pt110 & 2.36668900 & 4.20085500 & 4.2055040 \\
\hline
\end{tabular}




\begin{tabular}{|c|c|c|c|}
\hline t111 & -4.66212700 & -2.62653900 & -4.37311500 \\
\hline Pt112 & 4.34006800 & -2.31397300 & -4.85464700 \\
\hline t113 & -4.77768700 & -4.40011200 & 2.36029300 \\
\hline Pt114 & -1.49013800 & 1.54117700 & -1.43751700 \\
\hline Pt115 & 3.11404500 & -5.73358200 & 2.27750300 \\
\hline Pt116 & 4.77768700 & 4.40011200 & -2.36029300 \\
\hline Pt117 & 4.48063100 & 0.15557200 & -0.23967100 \\
\hline Pt118 & 6.60575000 & -2.61573000 & -3.34585900 \\
\hline Pt119 & 0.06637600 & 3.09620300 & 3.2506650 \\
\hline Pt120 & -5.79577200 & -3.76098000 & -0.14938200 \\
\hline Pt121 & 2.38282300 & 2.81108400 & -5.84621400 \\
\hline t122 & 1.77236000 & -0.49160500 & 6.66148100 \\
\hline Pt123 & -2.45725600 & -3.29812400 & 3.19158200 \\
\hline Pt124 & 3.86045600 & -5.09134200 & -0.33132700 \\
\hline et125 & -4.53382200 & 4.68911200 & -4.37372100 \\
\hline Pt126 & -1.01423700 & -0.58835900 & 6.81053800 \\
\hline Pt127 & -6.73493200 & 0.72494500 & 1.36872600 \\
\hline Pt128 & 5.71335200 & -0.08361900 & -3.88701400 \\
\hline Pt129 & -1.14759600 & -6.80905000 & 0.27951100 \\
\hline Pt130 & 0.15680700 & -7.59332700 & -1.99738500 \\
\hline Pt131 & -0.15680700 & 7.59332700 & 1.9973850 \\
\hline Pt132 & -3.78437800 & 0.54116900 & -2.35442500 \\
\hline Pt133 & -3.86045600 & 5.09134200 & 0.33132700 \\
\hline Pt134 & -1.64262100 & 1.03120300 & -4.04921200 \\
\hline Pt135 & -5.29478400 & 4.05038500 & -1.82151100 \\
\hline Pt136 & -0.23489000 & -1.07419800 & -5.08850200 \\
\hline Pt137 & -0.27792000 & 3.24761800 & -3.08762600 \\
\hline Pt138 & 3.00550800 & -3.10845100 & 2.89937600 \\
\hline Pt139 & -0.06637600 & -3.09620300 & -3.25066500 \\
\hline Pt140 & 4.48928300 & 4.64629900 & 2.45285600 \\
\hline Pt141 & -4.34006800 & 2.31397300 & 4.85464700 \\
\hline Pt142 & -3.74335500 & 2.45472800 & -0.34540400 \\
\hline Pt143 & 0.00000000 & 0.00000000 & 0.00000000 \\
\hline Pt144 & -2.21124400 & -1.01187600 & -0.86527300 \\
\hline Pt145 & 3.70678200 & 4.97523400 & -4.81451600 \\
\hline Pt146 & -3.18083900 & 2.61790800 & -5.54861100 \\
\hline Pt 147 & 0.18620300 & 3.66175600 & 5.8579120 \\
\hline \#113 & luster $=\operatorname{Pt1} 48 \quad \mathrm{Q}=0$ & $=0 \quad E$ tot $=-1$ & 7426 \\
\hline Pt1 & -4.90288700 & 2.99608500 & -3.56985300 \\
\hline Pt2 & -3.07301700 & -4.42681800 & -1.55641900 \\
\hline Pt3 & -5.56777300 & 0.58257800 & 4.02792600 \\
\hline Pt 4 & -6.29857700 & 1.99735800 & 1.73761600 \\
\hline Pt5 & -6.95346900 & 0.15005300 & -0.24737000 \\
\hline Pt 6 & 2.91076300 & 7.24058700 & 3.62022700 \\
\hline Pt 7 & -6.25918800 & 0.64463400 & -2.88669300 \\
\hline Pt 8 & -0.58547300 & 3.65332700 & -1.75089100 \\
\hline Pt9 & -4.10630300 & -2.43377800 & 0.0055800 \\
\hline Pt10 & 1.04323400 & 0.35392500 & -4.29640100 \\
\hline Pt11 & 4.10726300 & -3.86947300 & -3.46238900 \\
\hline Pt12 & -4.85652400 & -4.24556900 & 1.89305900 \\
\hline Pt13 & 1.49757500 & -0.57794600 & -6.74434900 \\
\hline Pt14 & 0.70749700 & -7.23184600 & -1.09618400 \\
\hline Pt15 & 3.57990000 & 7.72103600 & 0.9897726 \\
\hline Pt16 & -1.01401100 & -1.81764600 & -6.7300530 \\
\hline Pt17 & -3.69419500 & 0.60163000 & -3.67452400 \\
\hline Pt18 & 3.11133000 & 1.25625900 & -2.6911410 \\
\hline Pt19 & -4.94884300 & 4.33258900 & 1.06453100 \\
\hline Pt20 & 1.92529800 & 4.79462800 & -5.519261 \\
\hline
\end{tabular}




\begin{tabular}{|c|c|c|c|}
\hline Pt21 & -3.55010700 & 6.58651100 & 0.37802300 \\
\hline Pt22 & 0.81189200 & -2.34152700 & -4.83966100 \\
\hline Pt23 & 2.97025700 & 3.47854200 & 1.49794900 \\
\hline Pt24 & 2.22271700 & -5.16202800 & -0.31295100 \\
\hline Pt25 & 5.55127500 & 3.52511500 & 0.70844400 \\
\hline Pt26 & 0.96901600 & 5.68917300 & -0.94524500 \\
\hline Pt27 & 4.88258600 & -0.54321600 & -1.63103400 \\
\hline Pt28 & 4.51790600 & 5.52738800 & 2.27788200 \\
\hline Pt29 & -4.27168200 & 2.96423500 & 3.39359500 \\
\hline Pt30 & 2.88060400 & -1.44693100 & -3.26279200 \\
\hline Pt31 & 0.58172400 & 1.25871900 & -1.83601800 \\
\hline Pt32 & -3.76116500 & -1.22125900 & 5.13878200 \\
\hline Pt33 & 2.68959200 & -6.15893800 & -2.75791300 \\
\hline Pt34 & 0.30949500 & 7.60230100 & 3.00945300 \\
\hline Pt35 & -3.49255000 & 1.51126600 & 5.62541800 \\
\hline Pt36 & -4.59920200 & -6.44710400 & -2.33330400 \\
\hline Pt37 & -3.80381900 & -6.26221000 & 0.28125000 \\
\hline Pt38 & 3.70855500 & 5.33718000 & -0.33173300 \\
\hline Pt39 & 4.77506600 & -5.24579600 & -1.13575200 \\
\hline Pt 40 & 1.25154500 & 3.02530900 & -3.66534200 \\
\hline Pt41 & 1.06225900 & -2.78285200 & -0.16822500 \\
\hline Pt 42 & -0.91001500 & 6.29997700 & 0.99369100 \\
\hline Pt 43 & -1.14711700 & -3.41740700 & -3.22457100 \\
\hline Pt 44 & 6.80648000 & -1.08790800 & 0.18057700 \\
\hline Pt 45 & -2.38710100 & 4.19613200 & 0.23842800 \\
\hline Pt 46 & 0.11577400 & 5.49057100 & -3.58747700 \\
\hline Pt 47 & 2.90042900 & -3.29429200 & 5.51550900 \\
\hline Pt 48 & -1.63501500 & -0.29084200 & 6.67988200 \\
\hline Pt 49 & 7.40254200 & -0.58658100 & -2.44702100 \\
\hline Pt50 & 1.52613400 & -3.77247300 & -2.59158700 \\
\hline Pt51 & -3.76066700 & 1.94002400 & 0.91781800 \\
\hline Pt52 & -0.71559300 & -4.38963100 & -5.66129000 \\
\hline Pt53 & 3.28353900 & 1.00638300 & 2.58253500 \\
\hline Pt5 4 & 5.41900000 & -1.49417400 & -4.09728300 \\
\hline Pt55 & -4.25621100 & 4.82880900 & -1.59933600 \\
\hline Pt5 6 & -4.40588700 & 0.10975600 & -1.05562600 \\
\hline Pt57 & 1.72347200 & 2.13293600 & -6.16889100 \\
\hline Pt58 & 6.13478000 & -2.92595600 & -1.80567200 \\
\hline Pt59 & -1.86358700 & -2.99272000 & 6.12636500 \\
\hline Pt 60 & -2.53012900 & -0.42542500 & 0.76358100 \\
\hline Pt61 & -1.96682600 & -6.88160100 & -1.72807500 \\
\hline Pt 62 & 2.88717400 & 2.02230300 & 5.07953500 \\
\hline Pt 63 & 1.98272500 & -4.74626300 & -5.02467500 \\
\hline Pt 64 & 0.98583500 & 8.09113200 & 0.3625370 \\
\hline Pt 65 & 2.14518000 & 3.29024400 & -1.10322800 \\
\hline Pt 66 & 3.59219200 & -2.86930100 & -0.98316800 \\
\hline Pt 67 & 2.58822800 & 4.54110600 & 3.95661500 \\
\hline Pt 68 & -6.02718900 & -1.92804500 & -1.85646600 \\
\hline Pt 69 & -3.04425300 & 2.38840000 & -5.5709470 \\
\hline Pt70 & -1.55955600 & -2.42665200 & -0.78655800 \\
\hline Pt71 & -3.01806600 & -2.92603900 & -5.14148300 \\
\hline Pt72 & -1.22588800 & 1.83109600 & -3.71316900 \\
\hline Pt73 & -2.68136300 & -5.45601600 & -4.01974100 \\
\hline Pt74 & 1.30634700 & -0.03084000 & 4.21755400 \\
\hline Pt75 & 3.35116000 & -2.39668800 & -5.7011150 \\
\hline Pt76 & -3.06808000 & 2.43491500 & -1.7317840 \\
\hline Pt77 & 1.42649400 & 1.49389100 & 0.73210800 \\
\hline Pt78 & 1.01466300 & 2.51495800 & 3.1660140 \\
\hline & 2.38367500 & -0.50205600 & -0.8221410 \\
\hline
\end{tabular}




\begin{tabular}{|c|c|c|c|}
\hline Pt 80 & -2.43016000 & 4.28445700 & -3.63839100 \\
\hline Pt 81 & 6.51970400 & 1.47379400 & -0.88379900 \\
\hline Pt 82 & -5.65705300 & -4.46479700 & -0.77016400 \\
\hline Pt83 & 0.64662200 & -1.79914100 & 6.17788900 \\
\hline Pt 84 & -7.55018800 & -0.34773800 & 2.37971900 \\
\hline Pt 85 & -1.22692300 & 1.83741200 & 0.10039200 \\
\hline Pt 86 & -0.07197300 & -0.46551100 & -0.02648100 \\
\hline Pt 87 & -0.50619900 & 0.48551900 & 2.34383500 \\
\hline Pt 88 & 3.97788000 & 1.50006900 & -0.06776900 \\
\hline Pt89 & 3.54857900 & -1.52459700 & 3.61169400 \\
\hline Pt90 & -5.25853000 & -1.42048100 & -4.48452300 \\
\hline Pt91 & 0.58325000 & 3.54695300 & 5.59209300 \\
\hline Pt92 & -3.27146300 & -0.34241100 & -6.14530100 \\
\hline Pt93 & 1.71579400 & -0.98741600 & 1.77078200 \\
\hline Pt94 & -1.40572000 & -3.93535700 & 3.65814100 \\
\hline Pt 95 & 3.13029400 & -0.56041000 & 6.07986400 \\
\hline Pt96 & -2.12922400 & 3.87383900 & 4.95664400 \\
\hline Pt97 & -0.78608700 & 0.91555000 & -6.23511900 \\
\hline Pt 98 & 5.65032800 & 1.22676700 & -3.52240100 \\
\hline Pt99 & 0.36390300 & -1.38986600 & -2.40467500 \\
\hline Pt100 & -0.94742700 & 1.46791800 & 4.77309200 \\
\hline Pt101 & -3.95006800 & -3.92724000 & 4.50785000 \\
\hline Pt102 & 1.80588300 & 5.85435900 & 1.64406200 \\
\hline Pt103 & -5.66951000 & 2.52533800 & -0.92976000 \\
\hline Pt104 & 0.00529700 & -5.87618700 & -3.42246400 \\
\hline Pt105 & -5.79358400 & -2.14286000 & 3.46479800 \\
\hline Pt106 & 6.11332000 & -1.58133700 & 2.82183700 \\
\hline Pt107 & -4.96150700 & -3.96542700 & -3.42879200 \\
\hline Pt108 & -0.25336500 & -6.35335600 & 3.54512700 \\
\hline Pt109 & 5.89409700 & 0.98830100 & 1.79482700 \\
\hline Pt110 & -0.19291900 & 4.94436000 & 3.31069000 \\
\hline Pt111 & -1.18581700 & -1.24367700 & 4.24043200 \\
\hline Pt112 & -1.44681200 & -0.87159500 & -4.28275200 \\
\hline Pt113 & 2.90330100 & -3.36127500 & 1.65945900 \\
\hline Pt114 & 3.80093400 & 3.04611600 & -4.55106300 \\
\hline Pt115 & 1.56398200 & -6.96772900 & 1.55642800 \\
\hline Pt116 & 2.26989900 & -5.18802600 & 3.57619700 \\
\hline Pt117 & -0.72968100 & -2.17648600 & 1.79408000 \\
\hline Pt118 & 5.12557200 & 0.48797500 & 4.42736800 \\
\hline Pt119 & -0.55407000 & 3.62496200 & -5.57674500 \\
\hline Pt120 & -0.43637600 & -4.83600300 & -0.94523200 \\
\hline Pt121 & -1.75454200 & 6.06762100 & -1.62097200 \\
\hline Pt122 & 1.00265300 & 6.25054600 & 5.2702830 \\
\hline Pt123 & 2.78316400 & 7.52440000 & -1.60846500 \\
\hline Pt124 & 4.25968200 & -1.04086500 & 0.99276100 \\
\hline Pt125 & 2.81012700 & 5.13413300 & -2.95045200 \\
\hline Pt126 & 0.26010400 & 3.86318700 & 0.88040300 \\
\hline Pt127 & 4.72887600 & 3.32965200 & -1.95166000 \\
\hline Pt128 & 4.85501600 & 3.02928000 & 3.3731910 \\
\hline Pt129 & -3.02794500 & 0.53658400 & 3.1977670 \\
\hline Pt130 & 5.50807700 & -3.45626700 & 0.86044000 \\
\hline Pt131 & -2.95211100 & -5.99644500 & 2.90726100 \\
\hline Pt132 & 4.07827100 & -5.74429500 & 1.53203300 \\
\hline Pt133 & 3.61696400 & 0.33064200 & -5.1964010 \\
\hline Pt134 & 1.07714200 & -2.74115500 & 3.65873800 \\
\hline Pt135 & -1.70438600 & 2.85157600 & 2.5262830 \\
\hline Pt136 & -6.65127500 & -2.40940500 & 0.8184490 \\
\hline Pt137 & 4.74269600 & -3.92167400 & 3.49793400 \\
\hline Pt138 & -1.12868300 & -6.69090900 & 0.92362400 \\
\hline
\end{tabular}




$\begin{array}{lrrr}\text { Pt139 } & -5.02975800 & -0.38519100 & 1.56722000 \\ \text { Pt140 } & -2.26330000 & -4.21930800 & 1.05358100 \\ \text { Pt141 } & 0.87996100 & 0.94268000 & 6.65718200 \\ \text { Pt142 } & -3.41566600 & -1.93560300 & -2.63719900 \\ \text { Pt143 } & -3.25286600 & -2.17204600 & 2.63732900 \\ \text { Pt144 } & 0.41813700 & -4.50230700 & 5.53877700 \\ \text { Pt145 } & -2.86645100 & 5.26817500 & 2.67952000 \\ \text { Pt146 } & -1.86103600 & 0.06160800 & -1.83092600 \\ \text { Pt147 } & 0.41107500 & -4.57385100 & 1.68312500 \\ \text { Pt148 } & 0.17024700 & 7.85725700 & -2.22714800\end{array}$

Table S3. Cartesian coordinates ( $\AA$ ) and binding energies (kcal/mol) of platinum clusters optimized with SCG5 potential.

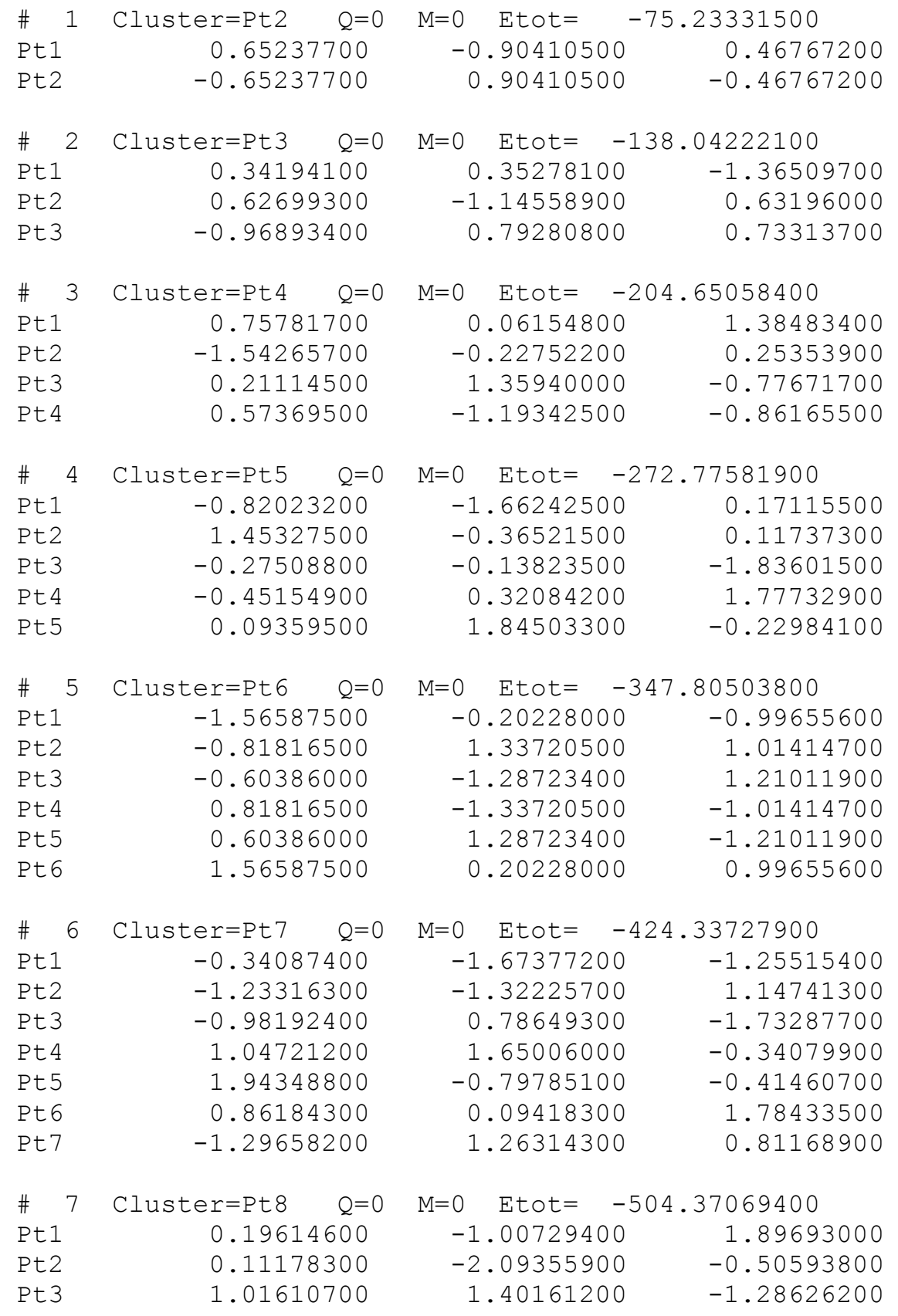




$\begin{array}{lr}\text { Pt4 } & -1.32403600 \\ \text { Pt5 } & -0.96314800 \\ \text { Pt6 } & 2.09103800 \\ \text { Pt7 } & -1.96348900 \\ \text { Pt8 } & 0.83560000\end{array}$

\# 8 Cluster=Pt9 $\mathrm{Q}=0$

$\begin{array}{lr}\text { Pt1 } & -1.38057600 \\ \text { Pt2 } & 0.58745400 \\ \text { Pt3 } & 1.04758400 \\ \text { Pt } 4 & -1.55457800 \\ \text { Pt5 } & 1.08804200 \\ \text { Pt6 } & 2.28843500 \\ \text { Pt7 } & -2.05516700 \\ \text { Pt8 } & -0.35418600 \\ \text { Pt9 } & 0.33299100\end{array}$

\# 9 Cluster=Pt10 $Q=0$

Pt 2

Pt3

Pt 4

Pt5

Pt 6

Pt 7

Pt 8

Pt 9

Pt10

\# 10 Cluster=Pt11 Q $=0$

Pt $1 \quad-1.98258100$

Pt2 $\quad-0.74591600$

Pt3 0.12983200

Pt4 -1.72437500

Pt5

Pt 6

Pt 7

Pt 8

Pt9

Pt 10

Pt11

\# 11 Cluster=Pt12 Q $=0$

Pt1 $\quad-2.11853900$

Pt2 0.14803600

Pt3 $\quad-0.15067800$

Pt $4 \quad-1.06816900$

Pt5 2.11853900

Pt $6 \quad-0.14803600$

Pt7 1.06816900

Pt8 -1.55149900

Pt9 -2.12017200

Pt10 0.15067800

Pt11 $\quad 1.55149900$

Pt12 2.12017200

\# 12 Cluster $=$ Pt13 Q $=0$

Pt $1 \quad 1.58346700$

Pt2 -1.11514200
1.69924000
$-0.10473100$
$-0.17975000$
$-1.92132600$
0.12912600
-0.51219600
-0.77221900
0.44704100
1.46416600
1.34515800

$$
\begin{array}{cr}
M=0 \quad \text { Etot }=-666.29637600 \\
-0.03707500 & -1.65087500 \\
1.46625800 & -0.45704100 \\
-0.64500600 & 1.08570200 \\
-1.92479600 & 0.06938900 \\
1.40998200 & 1.65191300 \\
2.01812900 & -0.72597500 \\
0.50277500 & 0.93147800 \\
-1.04483700 & 2.28952700 \\
-1.95833500 & -0.92534900 \\
0.21290300 & -2.26876900
\end{array}
$$
$\mathrm{M}=0 \quad$ Etot $=-748.28764200$
$\begin{array}{ll}0.47730700 & 0.70540800\end{array}$
$2.59808400 \quad-0.23755800$
$0.23218400 \quad 2.23222400$
$0.84301700 \quad-1.91799800$
$1.84919100 \quad 0.77157600$
$-1.91054400 \quad 1.60363700$
$-1.82221700 \quad 1.14146400$
$-1.55240100 \quad-1.40522100$
$-1.57689500 \quad-0.94214600$
$1.05191300-1.66333400$
$-0.18963900 \quad-0.28805100$

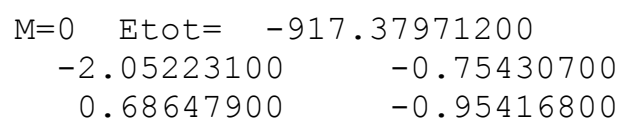

0.68613300

$-2.38982700$

$-0.41673700$

$-1.70821600$

$-0.68613300$

2.38982700

1.70821600

1.48431100

$-1.04842000$

0.41673700

$\begin{array}{lr}1.29526700 & -1.48431100 \\ 0.83290600 & 1.04842000\end{array}$

$\begin{array}{rr}1.29526700 & -1.48431100 \\ 0.83290600 & 1.04842000\end{array}$ 


\begin{tabular}{|c|c|c|c|}
\hline Pt 3 & 1.60471400 & 1.86114500 & 1.29509500 \\
\hline Pt 4 & 1.42016200 & -0.72877100 & 1.49993800 \\
\hline t5 & -2.88183000 & 0.03963500 & 0.86749700 \\
\hline t 6 & 1.04316900 & 1.18400000 & -2.35756300 \\
\hline t7 & -1.02645100 & 1.82563400 & 1.45858200 \\
\hline t 8 & -0.36006500 & -2.56935300 & 0.93105700 \\
\hline t9 & -0.96253500 & -0.57759900 & 2.54089400 \\
\hline t10 & 2.68278200 & 0.32118400 & -0.54553400 \\
\hline t11 & -0.10582500 & -1.17216500 & -2.51267500 \\
\hline 12 & 0.17273700 & 2.94130500 & -0.61478100 \\
\hline 13 & -2.05518300 & -1.75926200 & -0.8540370 \\
\hline 13 & luster $=\operatorname{Pt14} \quad \mathrm{Q}=0$ & \multicolumn{2}{|c|}{$\mathrm{M}=0 \quad$ Etot $=-1007.89192900$} \\
\hline Pt1 & 0.57788800 & 1.83021100 & 1.89367100 \\
\hline t2 & 1.67013400 & -1.73985500 & 0.95406700 \\
\hline t3 & -0.78270400 & -1.61684500 & -2.01067300 \\
\hline t 4 & 2.71339700 & 0.70600200 & 0.83044300 \\
\hline & -0.04521600 & 0.04710400 & -0.02583000 \\
\hline t 6 & 1.83369100 & -1.52272800 & -1 \\
\hline t 7 & -0.02044500 & 2.72890000 & -0.56320600 \\
\hline Pt 8 & 0.20010400 & -0.59597100 & 2.85576200 \\
\hline $1 F$ & -0.69469100 & 1.02070000 & -2.49801700 \\
\hline 0 & 1.84117800 & 1.11352900 & -1.62472600 \\
\hline & -2.62099300 & 0.02280800 & -0.9 \\
\hline & -1.94674700 & 1.731 & 0 . \\
\hline 13 & -2.04599400 & -0.90016200 & 1.50772400 \\
\hline & -0.67960200 & -2.82470100 & 0.33136700 \\
\hline 14 & Cluster=Pt15 $\quad \mathrm{Q}=0$ & \multicolumn{2}{|c|}{$\mathrm{M}=0 \quad$ Etot $=-1100.58339200$} \\
\hline Pt1 & -2.34906500 & 0.07703300 & -1.27645600 \\
\hline te 2 & 0.81015100 & -1.97595000 & -1.6 \\
\hline t3 & 0.98940800 & 1.80085200 & -1.71213000 \\
\hline Pt 4 & 1.66158200 & 2.43144200 & 0.77186700 \\
\hline Pt & -0.81015200 & 1.97595100 & 1.61017400 \\
\hline & 1.42597300 & -2.53269500 & 0.90587600 \\
\hline 7 & 0.36112900 & 0.06444700 & 4800 \\
\hline 8 & -1.42597200 & 2.53269400 & 87500 \\
\hline & 2.34906600 & -0.07703400 & 1.27645600 \\
\hline & 0.00000000 & 0.00000200 & 0.00000000 \\
\hline & -2.72642600 & 0.16569800 & 1.34450500 \\
\hline & -1.66158200 & -2.43144400 & -0.77186700 \\
\hline & -0.36112900 & -0.0644 & -3 \\
\hline & -0.98940700 & -1.8008 & 1. \\
\hline & 2.72642600 & -0.16569800 & -1.34450 \\
\hline$\|$ & Cluster=Pt16 $Q=0$ & \multicolumn{2}{|c|}{$\mathrm{M}=0 \quad$ Etot $=-1189.15590500$} \\
\hline Pt & 2.34461600 & -1.96164000 & 0.58199500 \\
\hline + & 1.60539800 & 2.80179600 & 0.30278100 \\
\hline Pt & 0.85175800 & 2.02445300 & 824400 \\
\hline Pt & 0.51664400 & -2.87906600 & -1.08707900 \\
\hline Pt & -1.28970100 & -2.40236900 & 0.78944000 \\
\hline Pt 6 & -0.33710100 & 0.06149100 & 0.42494200 \\
\hline Pt & -2.77804200 & -0.28666100 & 1.35875200 \\
\hline Pt & 3.03826900 & 0.57984900 & 0.4127730 \\
\hline & 1.47925900 & -0.45173400 & -1.44522700 \\
\hline & 0.54733400 & -1.44952600 & 2.42265100 \\
\hline & -2.44882100 & 0.54973300 & -1.15531600 \\
\hline & -1.31725500 & 1.71710200 & 2.22762200 \\
\hline & -1.87411800 & -1.97105900 & -1.7668190 \\
\hline
\end{tabular}




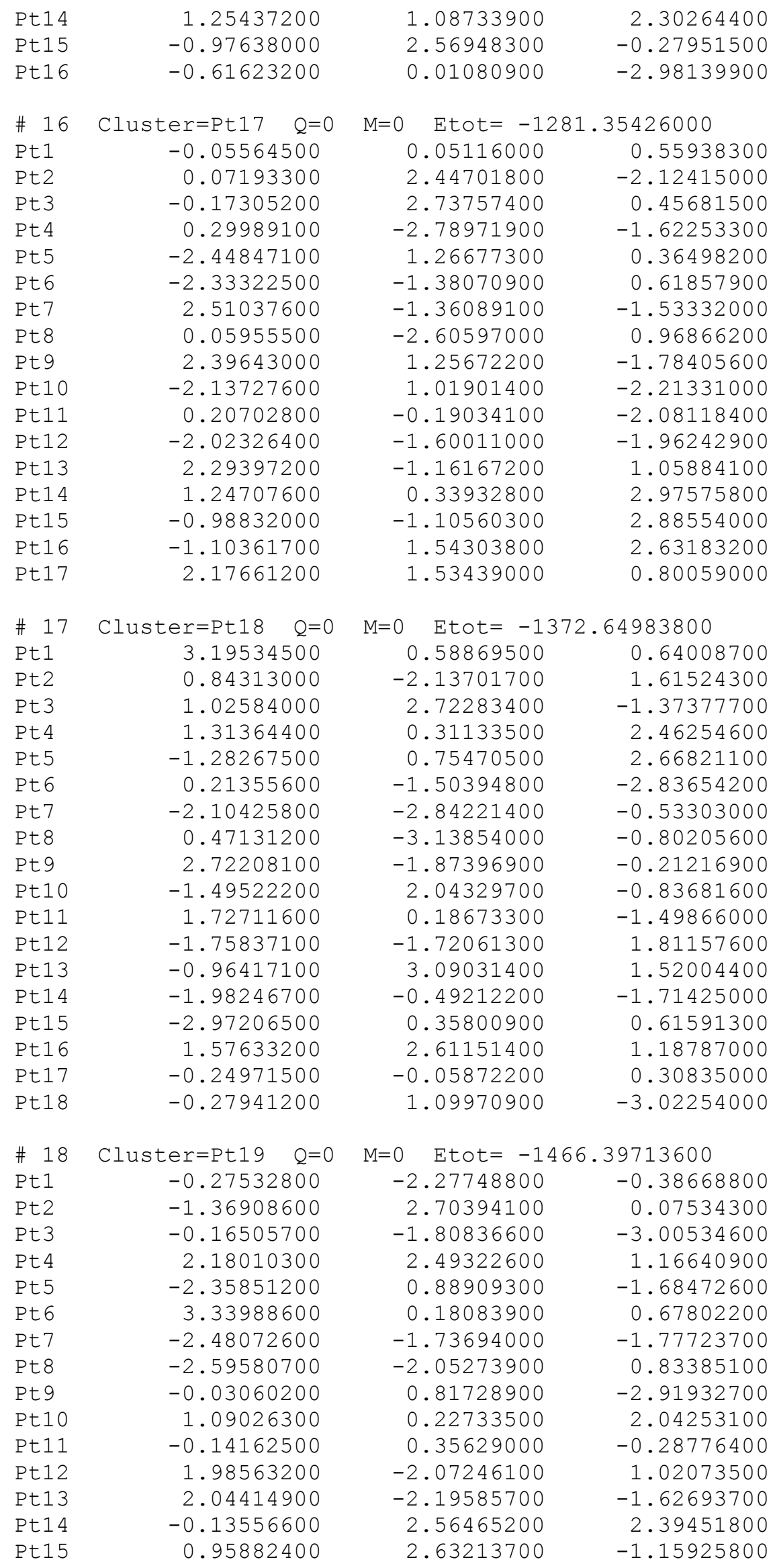




\begin{tabular}{|c|c|c|c|}
\hline 16 & -2.64364800 & 0.55318400 & 0.91573100 \\
\hline Pt17 & 2.26403900 & 0.40180800 & -1.68704700 \\
\hline t18 & -1.30006900 & 0.32395700 & 3.13881000 \\
\hline+19 & -0.36687100 & -1.99989900 & 2.26837800 \\
\hline 19 & Cluster $=$ Pt20 & \multicolumn{2}{|c|}{$M=0 \quad$ Etot $=-1559.01052600$} \\
\hline Pt 1 & -1.42665600 & -0.79563000 & -2.84010300 \\
\hline Pt2 & 1.33058900 & 2.81884900 & 0.0032340 \\
\hline t3 & -0.24979900 & 1.55591500 & -3.0032390 \\
\hline t 4 & 0.14571700 & 0.39569800 & 0.4599590 \\
\hline$t 5$ & -1.73985500 & 1.83698100 & 1.8206580 \\
\hline t 6 & -0.38732500 & -0.08705700 & 3.10745300 \\
\hline t7 & -3.45317900 & -0.00 & 1.1 \\
\hline Pt 8 & 2.71672200 & 0.90555700 & 1.27013900 \\
\hline t9 & 2.23989900 & 1.81260100 & -2.22229200 \\
\hline t10 & 0.78035500 & 2.20745400 & 2.51095200 \\
\hline t11 & -0.09949900 & -3.12585500 & 0.90646300 \\
\hline t12 & -1.17950000 & 2.39410200 & -0 . \\
\hline t13 & 2.15248000 & -2.40 & -0 \\
\hline t14 & 3.45948000 & -0.17558400 & -0 \\
\hline t15 & -0.11342500 & -2.85905600 & -1.73623800 \\
\hline t16 & -3.03841900 & 0.66416900 & -1.35262900 \\
\hline t17 & 1.01594800 & -0.48337100 & -1.87523400 \\
\hline t18 & -1.69 & -1 & -0 . \\
\hline t19 & -2.02764000 & -1.93 & 2.2 \\
\hline t20 & 1.57235700 & -1.3 & 1.9 \\
\hline 20 & ter $=$ Pt 21 & \multicolumn{2}{|l|}{$\mathrm{M}=0 \quad \mathrm{Etot}=-16$} \\
\hline t1 & -0.54752600 & 0.90307800 & 1.08228400 \\
\hline Pt2 & 3.03507200 & 0.26266400 & 500 \\
\hline Pt3 & 0.71725200 & 3.17077200 & 1.42691200 \\
\hline Pt 4 & 1.23827100 & 1.93961200 & -2.05964300 \\
\hline Pt5 & -0.94008500 & -0.86019300 & -0.81423700 \\
\hline t 6 & 1.76855500 & -2.02629400 & 1.51785500 \\
\hline$t 7$ & -0.28820000 & -1.07028100 & 2.88252900 \\
\hline Pt 8 & 3898400 & -0.41326600 & 6439600 \\
\hline Pt9 & -3.29775500 & 50000 & -1 \\
\hline 10 & 1.48761000 & -0.04288500 & -0.26804700 \\
\hline t11 & -0.00354900 & -3.19134700 & -1.55551100 \\
\hline $\mathrm{Pt}$ & -0.26456200 & -1.23927700 & -3.31640300 \\
\hline 3 & -3.03674300 & -1.78147000 & 0.45703800 \\
\hline Pt14 & -2.64000400 & 0.00057600 & 2.3737 \\
\hline Pt & 0.97826500 & 00 & 3. \\
\hline Pt & -0.68492900 & -2.85228300 & 0.96586200 \\
\hline Pt17 & 2.44999700 & -2.36533700 & -1.00350500 \\
\hline Pt & -0.81848400 & 2.89562500 & -0.69496800 \\
\hline Pt19 & -1.21521300 & 1.11362200 & -2.61163500 \\
\hline Pt20 & -2.90101600 & 1.95264700 & 0.61286300 \\
\hline Pt21 & 2.77405900 & 2.21473600 & 0.0622 \\
\hline \# 21 & Cluster=Pt22 $\mathrm{Q}=0$ & \multicolumn{2}{|c|}{$\mathrm{M}=0 \quad \mathrm{E}$ tot $=-1753.65312900$} \\
\hline Pt 1 & 0.42439100 & -2.57193300 & 2.37948400 \\
\hline Pt2 & -2.70677900 & -0.36045600 & -1.29134400 \\
\hline Pt 3 & 1.18468700 & 1.16507100 & 3.3290520 \\
\hline Pt 4 & -2.17300500 & 2.15319900 & -0.66338700 \\
\hline Pt5 & -2.11823000 & -1.97038000 & 2.02178100 \\
\hline Pt 6 & 0.60610200 & -3.37757600 & -0.1303940 \\
\hline Pt7 & -0.97908900 & -0.22895600 & -3.28349600 \\
\hline 20 & -0.09350200 & -0.90410100 & -0.85580800 \\
\hline
\end{tabular}




\begin{tabular}{|c|c|c|c|}
\hline Pt9 & -0.70316700 & 3.49090800 & 1.07822800 \\
\hline Pt10 & 1.59926500 & -0.83896400 & -2.92076600 \\
\hline Pt11 & 2.31408400 & -0.78906000 & 1.95466100 \\
\hline t12 & -1.37606700 & 1.77091500 & 2.96879800 \\
\hline$t 13$ & 2.50470900 & -1.59343200 & -0.55817700 \\
\hline t14 & -1.97225200 & -2.76756900 & -0.49312400 \\
\hline t15 & 1.85758800 & 2.88506500 & 1.43848200 \\
\hline 16 & -0.28902300 & -2.79465700 & -2.64538100 \\
\hline t17 & -0.26484900 & -0.16064000 & 1.61245000 \\
\hline t18 & 0.41002600 & 1.56440200 & -0.28366900 \\
\hline t19 & 2.08832600 & 1.68123600 & -2.29548100 \\
\hline 20 & -0.45429400 & 2.28278900 & -2.65318400 \\
\hline 21 & 2.98751200 & 0.93228300 & 0.06260900 \\
\hline t22 & -2.84643300 & 0.43185600 & 1.22866500 \\
\hline 22 & luster $=P$ t2 $3 \quad Q=0$ & \multicolumn{2}{|c|}{$\mathrm{M}=0 \quad$ Etot $=-1850.61811900$} \\
\hline Pt 1 & 1.16207500 & 0.69604600 & 3.1229660 \\
\hline t2 & -1.39649500 & -0.04226300 & 3.10416300 \\
\hline t3 & 1.08066500 & -3.76168900 & 0.65577200 \\
\hline Pt 4 & -0.77535000 & 2.61349100 & 2.88386300 \\
\hline et 5 & 1.86122700 & -1.70545300 & 5500 \\
\hline t 6 & -0.69734400 & -2.44376200 & 2.26485200 \\
\hline Pt 7 & 1.57134600 & -0.58119800 & -3.28608800 \\
\hline Pt 8 & 2.98869000 & -0.63541500 & 0.15390300 \\
\hline t9 & -2.89290200 & 0.27518400 & 0.95660800 \\
\hline t10 & -0.45684300 & -3.22663600 & -1.55672800 \\
\hline t11 & -2.21182800 & 2.80152100 & 0.55 \\
\hline$t 12$ & 0.51617700 & 3.04317800 & -2.01938800 \\
\hline t13 & 2.12663600 & -2.48114000 & -1.53774300 \\
\hline Pt14 & 2.28831400 & 1.77029100 & 0.99468500 \\
\hline 15 & 0.38033200 & -1.32021400 & 0 . \\
\hline t16 & -0.14352800 & 0.539 & -1 \\
\hline t17 & 0.37165100 & 3.54701700 & 0.56906600 \\
\hline Pt18 & 2.42353500 & 1.26380700 & -1.59727800 \\
\hline t19 & -2.03059000 & 2.30827500 & -2.03810400 \\
\hline t20 & -0.29548900 & 1.00114500 & 0.89746100 \\
\hline t21 & -0.97542000 & -1.31 & $-3 \cdot 3$ \\
\hline t22 & -2.19252600 & -2.13 & 0.1 \\
\hline t23 & -2.70233400 & -0.21532800 & -1.6349480 \\
\hline \# 23 & Cluster $=$ Pt $24 \quad Q=0$ & \multicolumn{2}{|c|}{$\mathrm{M}=0 \quad \mathrm{Etot}=-1947.32906100$} \\
\hline Pt 1 & -0.09552300 & -1.15097600 & -0.92741200 \\
\hline Pt2 & -0.45132100 & 1.28531600 & 3.30120100 \\
\hline Pt 3 & -2.45994800 & -1.786 & 1. \\
\hline Pt 4 & 0.84298400 & 3.43390000 & 0.50136300 \\
\hline Pt5 & 1.01304600 & -2.77725900 & 0.90509400 \\
\hline Pt 6 & -1.55668100 & 2.86801200 & 1.45082900 \\
\hline Pt 7 & -2.26263600 & 1.90127300 & -0.90789000 \\
\hline Pt 8 & -2.51303000 & -1.69921800 & -1.88440700 \\
\hline Pt 9 & 3.33342200 & 2.33679200 & 0.00745900 \\
\hline Pt10 & -0.26253400 & -3.16332400 & -2.54888500 \\
\hline Pt11 & -1.20987700 & 0.40560800 & -2.81608000 \\
\hline Pt12 & 2.41167200 & 1.20524900 & -2.34197900 \\
\hline Pt13 & 1.94834300 & 1.85120400 & 2.35173500 \\
\hline Pt14 & 0.13812900 & 2.46742000 & -1.85779100 \\
\hline Pt15 & 2.36105200 & -0.71544700 & 1.86338300 \\
\hline 16 & 1.06428400 & -0.85561800 & -3.29982900 \\
\hline Pt17 & 2.16964300 & -2.43831400 & -1.4494580 \\
\hline 18 & -3.56530700 & -0.20423800 & 0.02290800 \\
\hline
\end{tabular}




\begin{tabular}{|c|c|c|c|}
\hline t19 & -3.07088800 & 0.82653200 & 2.54142600 \\
\hline Pt20 & -0.03971300 & -1.28159400 & 2.81328400 \\
\hline Pt21 & -1.11734300 & 0.30073400 & 0.92469900 \\
\hline Pt22 & 1.21286600 & 0.85024200 & 0.00271400 \\
\hline t23 & -1.40767100 & -3.28191400 & -0.03403600 \\
\hline t24 & 3.51703100 & -0.37744700 & -0.49160700 \\
\hline 24 & luster $=$ Pt 25 & \multicolumn{2}{|c|}{$\mathrm{M}=0 \quad$ Etot $=-2044.78930600$} \\
\hline Pt1 & -2.23952800 & -1.82905100 & -2.2805920 \\
\hline Pt2 & 1.61849100 & -2.04969800 & 2.7799580 \\
\hline Pt3 & 2.88154900 & -1.99754800 & 0.3993890 \\
\hline t 4 & 3.47326000 & 0.37616700 & 1.53098200 \\
\hline Pt5 & 0.98740700 & -3.47932400 & -0.69378900 \\
\hline Pt 6 & 2.94440400 & -0.91975500 & -2.01160400 \\
\hline Pt 7 & -2.57230500 & 0.79158500 & -2.28247200 \\
\hline Pt 8 & -1.14750600 & 0.84442100 & -0.06397900 \\
\hline Pt9 & -0.25629800 & -3.52237400 & 1.68387200 \\
\hline t10 & 3.53447300 & 1.43560100 & -0.86878200 \\
\hline Pt11 & -1.89308900 & 3.12054900 & -1.10785000 \\
\hline Pt12 & 0.99546600 & -0.25150700 & 0.9897990 \\
\hline Pt13 & -2.22614700 & 1.06001000 & 2.3065150 \\
\hline Pt1 & -3.74785800 & 0.69468400 & 0.14112600 \\
\hline Pt15 & 1.07870600 & 0.77501600 & -1.38764800 \\
\hline Pt16 & -3.41338100 & -1.90782400 & 141800 \\
\hline Pt17 & 0.40399900 & -1.57547400 & -2.46006600 \\
\hline Pt18 & 1.46030000 & 1.92656600 & 2.35695300 \\
\hline Pt19 & -1.97469300 & -1.60253600 & 2.34911400 \\
\hline Pt20 & -0.28253100 & 0.73051800 & -3.59840700 \\
\hline Pt21 & -0.83451300 & -1.72729500 & -0.09931400 \\
\hline Pt22 & -0.05226400 & -0.05172700 & 3.375 \\
\hline Pt23 & -0.71358300 & 3.03830300 & 1.28797400 \\
\hline Pt24 & 1.59825200 & 3.07054200 & -0.05685700 \\
\hline Pt25 & 0.37739000 & 3.05015200 & -2.42123600 \\
\hline \# 25 & Cluster=Pt26 $\quad \mathrm{Q}=0$ & \multicolumn{2}{|c|}{$\mathrm{M}=0 \quad$ Etot $=-2145.77859100$} \\
\hline Pt1 & 0.38072900 & 3.06821600 & -1.68211400 \\
\hline Pt2 & -0.61634300 & -3.72147000 & 0.66396400 \\
\hline Pt3 & 3.24935500 & 0.68642500 & 1.24979700 \\
\hline Pt 4 & -1.37830700 & 3.29623000 & 0.35492900 \\
\hline Pt 5 & 0.45964000 & -1.36135100 & 0.61335500 \\
\hline Pt 6 & -1.29434100 & -0.27397100 & -0.97898900 \\
\hline Pt 7 & -1.22818200 & 0.51961900 & 3.53950900 \\
\hline Pt 8 & -1.35590800 & 78700 & -3 \\
\hline Pt9 & 1.35219600 & -3.15810100 & -1.04345400 \\
\hline Pt10 & 0.71734400 & 3.18716800 & 1.99936100 \\
\hline Pt11 & -3.76249600 & 0.62363100 & -0.70084600 \\
\hline Pt12 & 1.47491400 & -1.82485300 & 2.96666500 \\
\hline Pt13 & -0.54697000 & -2.18878600 & -2.70156500 \\
\hline Pt & 1.09161000 & 1.21436900 & -3.42771300 \\
\hline Pt15 & -1.16547400 & -1.96264300 & 2.61125500 \\
\hline Pt16 & 2.98683500 & -1.96260100 & 0.77533700 \\
\hline Pt17 & -2.42907300 & -2.65276100 & -0.92987700 \\
\hline Pt18 & -2.89346800 & 1.46868000 & 1.68670000 \\
\hline Pt19 & 3.69196900 & -0.25053600 & -1.1589110 \\
\hline Pt20 & -3.15941100 & -1.16223600 & 1.12196700 \\
\hline Pt21 & -0.39382700 & 1.01319500 & 1.12208900 \\
\hline Pt22 & 2.39341000 & 2.84142300 & -0.0105440 \\
\hline Pt23 & 1.88554500 & -1.21373300 & -2.76987600 \\
\hline Pt24 & 1.22348800 & 0.64878800 & -0.8893536 \\
\hline
\end{tabular}




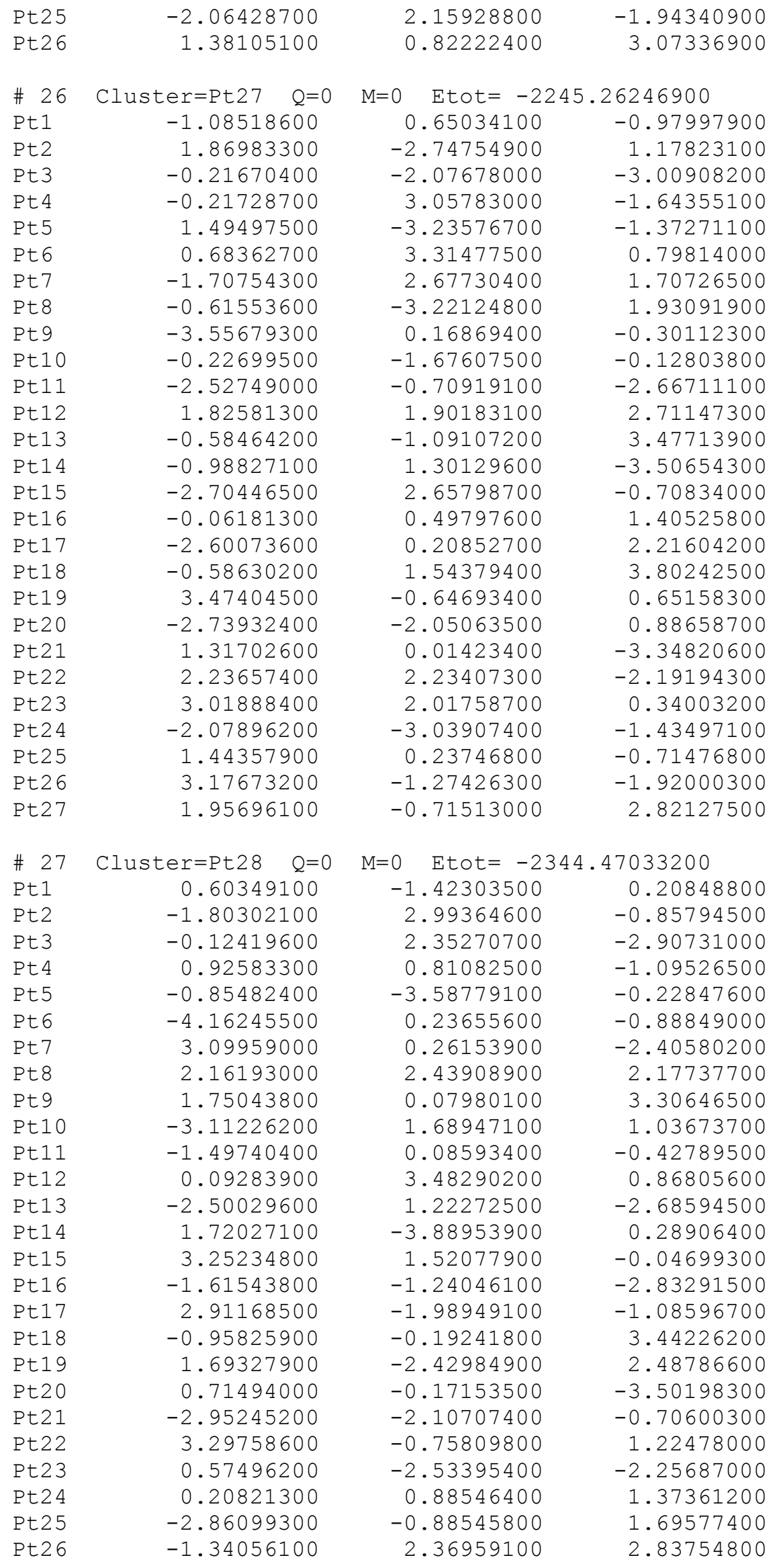




\begin{tabular}{|c|c|c|c|}
\hline $\begin{array}{l}\text { Pt27 } \\
\text { Pt28 }\end{array}$ & $\begin{array}{r}-0.91137300 \\
1.68612800\end{array}$ & $\begin{array}{r}-2.53910600 \\
3.31678000\end{array}$ & $\begin{array}{r}2.21272000 \\
-1.23288700\end{array}$ \\
\hline 28 & ter $=\operatorname{Pt} 29$ & \multicolumn{2}{|c|}{$\mathrm{M}=0 \quad$ Etot $=-2443.05521300$} \\
\hline Pt 1 & 1.34719500 & -0.28060300 & -1.0579420 \\
\hline Pt2 & -2.64573600 & -2.54775500 & 1.50696100 \\
\hline Pt 3 & -3.64242100 & -1.17710300 & -1.60226000 \\
\hline Pt 4 & -0.22340300 & 3.01216500 & -2.19667200 \\
\hline Pt 5 & -3.34098700 & 1.48490500 & -1.29194700 \\
\hline Pt 6 & 0.21099600 & -2.12724300 & 3.55673400 \\
\hline Pt 7 & -1.51383900 & -1.55039300 & -3.21571700 \\
\hline Pt 8 & 1.11781400 & -1.58040000 & -3.34545000 \\
\hline Pt 9 & -1.86708500 & 3.37818100 & -0.20270400 \\
\hline Pt10 & 0.33729700 & -0.63445400 & 1.32139000 \\
\hline Pt11 & 3.20068100 & -2.09397400 & -0.55688600 \\
\hline Pt12 & -1.67607800 & -2.87398000 & -0.94436400 \\
\hline Pt13 & 2.70816800 & -0.01195000 & 2.59100300 \\
\hline Pt14 & -2.16054800 & 1.96093300 & 1.97493000 \\
\hline Pt15 & -1.62377700 & 1.05618100 & -3.20865100 \\
\hline Pt16 & 3.12050400 & 1.42079700 & -2.06 \\
\hline Pt17 & 0.91961700 & -3.1 & -1 \\
\hline Pt18 & -1.24886300 & -0.24 & -0 \\
\hline Pt19 & 0.21578200 & 2.86460800 & 2.58585900 \\
\hline Pt20 & 0.47001300 & 0.54522400 & 3.81026000 \\
\hline Pt21 & 3.71263000 & 0.34784300 & 0.20927500 \\
\hline Pt22 & $2.29^{\circ}$ & -2.4 & 000 \\
\hline Pt23 & -1.79302200 & -0.46664600 & 2.9 \\
\hline Pt24 & 1.91316500 & 3.42645700 & 4800 \\
\hline Pt25 & 0.14460900 & 1.71716000 & 0.20823900 \\
\hline Pt26 & 2.51529600 & 2.33688200 & 1.46992700 \\
\hline Pt27 & 0.97 & 1.0 & -3. \\
\hline Pt28 & -0.11169000 & -3.2 & 1.1 \\
\hline Pt29 & -3.35740900 & -0.08 & 0.82587900 \\
\hline \# 29 & Luster $=P$ t $30 \quad Q=0$ & $=0 \quad$ Etot $=-25$ & 455 \\
\hline Pt 1 & -0.86875300 & -2.86218600 & 3.01627300 \\
\hline Pt2 & 3.43324400 & 0.281 & 5400 \\
\hline Pt 3 & -0.51 & -0.3 & 3. \\
\hline Pt 4 & -3.20251800 & -0.79261700 & -2.67588900 \\
\hline Pt5 & -0.55336400 & 2.18139900 & 3.17121700 \\
\hline Pt 6 & -3.55099700 & -0.07762900 & -0.09473300 \\
\hline Pt 7 & 2.06082900 & 1.49813200 & -2.47652200 \\
\hline Pt 8 & -1.12185100 & -0.1 & -1.14942500 \\
\hline Pt 9 & -2.87 & 1.7 & -1 \\
\hline Pt10 & -2.59093400 & -0.92900700 & 2.24690600 \\
\hline Pt11 & -2.96889300 & -2.66519200 & 0.24821800 \\
\hline Pt12 & -1.90997400 & 3.38443300 & -0.13762000 \\
\hline Pt13 & 3.86895400 & -0.35 & -1.71673000 \\
\hline Pt14 & -0.13620700 & -1.0 & 1.25460700 \\
\hline Pt 15 & 1.92486500 & 800 & 29200 \\
\hline Pt16 & -0.14525600 & 1.48803000 & 0.60057500 \\
\hline Pt17 & 1.81077300 & -1.05261600 & -3.27634400 \\
\hline Pt18 & -0.43469800 & -3.50035200 & 0.42796300 \\
\hline Pt19 & 0.20231800 & 4.01366200 & 1.37634600 \\
\hline Pt20 & 1.40331400 & 866400 & -0.7057570 \\
\hline Pt21 & -0.74710500 & -0.78520300 & -3.73451800 \\
\hline Pt22 & 1.62110000 & -2.88531300 & 2.04164900 \\
\hline Pt23 & -2.66125700 & 1.64611800 & 1.68960400 \\
\hline Pt24 & -1.39476100 & -2.64897400 & -1.91367600 \\
\hline
\end{tabular}




\begin{tabular}{|c|c|c|c|}
\hline$t 25$ & 1.95949900 & -0.43361200 & 2.93583000 \\
\hline Pt26 & 0.55245000 & 3.29689800 & -1.20409300 \\
\hline Pt27 & 2.96889400 & 2.66519200 & -0.24821800 \\
\hline Pt28 & 3.16738300 & -2.25565000 & 0.03249300 \\
\hline t29 & -0.49795800 & 1.74564600 & -3.05802400 \\
\hline t30 & 1.19971500 & -2.95815200 & -1.56741000 \\
\hline 30 & luster $=$ Pt 31 & Etot $=-2$ & 47933700 \\
\hline Pt1 & 0.00475800 & 1.93511100 & -0.7988690 \\
\hline Pt2 & 1.82499600 & 0.17545700 & -0.0621800 \\
\hline Pt3 & 0.01360900 & -0.51640300 & -1.8003590 \\
\hline Pt 4 & -3.29518400 & -0.93143600 & 1.77419600 \\
\hline Pt 5 & -3.65627600 & 1.61113400 & 1.11381100 \\
\hline Pt 6 & 1.38258400 & -2.67167200 & -2.41334100 \\
\hline Pt7 & 1.61791300 & 2.41550700 & 2.85804600 \\
\hline Pt 8 & -3.25551500 & -1.95407600 & -0.71972200 \\
\hline Pt9 & 3.51202800 & 0.46928100 & -1.99066800 \\
\hline Pt10 & 1.39087700 & -3.62225300 & 0.06466600 \\
\hline Pt11 & -2.50036400 & 2.57126300 & -1.12367600 \\
\hline Pt12 & -1.68816500 & -0.35713200 & -3.75474400 \\
\hline Pt13 & -0.02310600 & -1.51517700 & 0.6038590 \\
\hline Pt14 & 1.70461500 & 3.54390700 & 0.43141400 \\
\hline Pt15 & -1.31276100 & -2.67682400 & -2.45887400 \\
\hline Pt16 & 1.75952300 & -0.40366500 & -3 \\
\hline Pt17 & 1.33247800 & -2.56752400 & 5546900 \\
\hline Pt18 & 3.29539500 & -1.88433200 & -0.65739500 \\
\hline Pt19 & 2.12954900 & 2.72161800 & -2.08315000 \\
\hline Pt20 & -0.04674500 & 0.87178700 & 1.61340100 \\
\hline Pt21 & -1.37288400 & -2.59407200 & 2.53878300 \\
\hline Pt22 & -3.55016800 & 0.33557500 & -2.08788000 \\
\hline Pt23 & -1.86021200 & 0.18070000 & -0.12291500 \\
\hline Pt24 & 1.28887200 & -0.12851600 & 3.61892200 \\
\hline Pt25 & -0.94489900 & 3.86538200 & 0.66909500 \\
\hline Pt26 & -1.76638700 & 2.43297000 & 2.73456700 \\
\hline Pt27 & 0.02674100 & 1.63112300 & -3.38593900 \\
\hline Pt28 & -1.34458800 & -3.6 & 2051400 \\
\hline Pt29 & 3.25092100 & -0.85844300 & 1.86249600 \\
\hline Pt 30 & 3.51424000 & 1.71003100 & 1.16045500 \\
\hline Pt31 & -1.43184300 & -0.12569300 & 3.57442700 \\
\hline \# 31 & Cluster $=$ Pt $32 \quad \mathrm{Q}=0$ & $\mathrm{M}=0 \quad \mathrm{E}$ tot $=-2$ & 5406 \\
\hline Pt1 & -3.54755800 & -1.61644500 & 655200 \\
\hline Pt2 & 1.39891500 & -1.70573000 & -3.60359900 \\
\hline Pt3 & -3.78297000 & -0.36684500 & -1.54070800 \\
\hline Pt 4 & -0.71942100 & -0.19453500 & -3.96155900 \\
\hline Pt5 & -1.24495900 & 0.20110800 & -1.43714400 \\
\hline Pt 6 & 2.71135100 & 0.30370400 & -2.47592800 \\
\hline Pt 7 & 0.85940400 & -0.56144000 & 3.81120100 \\
\hline Pt 8 & -0.12592400 & -3.58945800 & 1.63675800 \\
\hline Pt9 & 1.88757600 & -3.43255500 & -0.09591100 \\
\hline Pt10 & -1.00235400 & 1.21999000 & 1.00141300 \\
\hline Pt11 & 0.94885300 & 1.47461200 & -0.76220000 \\
\hline Pt12 & -0.00753800 & 2.89290500 & 2.76734200 \\
\hline Pt13 & -3.10587400 & 1.92897300 & -0.4544110 \\
\hline Pt14 & -1.75526200 & 3.72235900 & 0.95682000 \\
\hline Pt15 & -1.96470700 & -3.48420600 & -0.37575100 \\
\hline Pt16 & 3.48475300 & 2.05451000 & -0.67431500 \\
\hline Pt17 & -1.42041500 & 0.78879800 & 3.56578200 \\
\hline Pt18 & 0.06080700 & 3.92175300 & -0.93032800 \\
\hline
\end{tabular}




\begin{tabular}{|c|c|c|c|}
\hline t19 & 0.99173100 & -1.15987100 & -1.08031800 \\
\hline Pt20 & -1.61421800 & 2.59372900 & -2.51792100 \\
\hline t21 & -1.40532200 & -1.84612700 & 3.18596800 \\
\hline t22 & 2.30400400 & 1.56405800 & 2.89590000 \\
\hline t23 & 3.59684400 & -1.56844200 & -0.83403600 \\
\hline 24 & -0.97659500 & -1.33449000 & 0.66004500 \\
\hline t25 & 0.10222700 & -3.42876900 & -2.06403400 \\
\hline t 2 & 2.14700200 & -2.35117700 & 2.32289800 \\
\hline t2 & 1.23688300 & -0.13853000 & 1.27325900 \\
\hline t2 $2 \cdot r \cdot r \cdot r$ & 0.82064400 & 1.89725500 & -3.3763920 \\
\hline t2 & -2.08863200 & -2.04720600 & -2.63673300 \\
\hline t3 & 1.85987500 & 3.34178200 & 0.93763200 \\
\hline t3 & -3.45447500 & 0.76275000 & 1.89148800 \\
\hline t3 & 3.80535300 & 0.15753700 & 1.13823000 \\
\hline 32 & cluster $=$ Pt $33 \quad \mathrm{Q}=0$ & $I=0 \quad$ Etot $=-28$ & 20344400 \\
\hline Pt 1 & -1.61454500 & 0.72200200 & -0.55408700 \\
\hline Pt2 & 0.89007900 & 1.34292900 & -0.91616300 \\
\hline Pt 3 & 1.04014800 & 2.08786100 & -3.44681300 \\
\hline Pt 4 & -3.80813300 & 0.87429100 & 0.90663300 \\
\hline Pt 5 & 3.06252900 & 2.52980600 & -0.06418500 \\
\hline Pt 6 & 4.00658200 & 0.34200700 & 1.07327700 \\
\hline Pt 7 & 3.13452900 & 1.04567100 & -2.27366300 \\
\hline Pt 8 & 1.99283300 & -2.15426000 & 2.70725700 \\
\hline Pt9 & -0.13111900 & 0.54141900 & 7200 \\
\hline Pt10 & 3.68648900 & -1.22613300 & -0.99731500 \\
\hline Pt11 & -1.16566100 & -3.37156500 & -0.86897100 \\
\hline Pt 1 & 0.07594000 & -1.07273300 & -1.50948100 \\
\hline Pt13 & -2.90759300 & -0.99394000 & 2.57802700 \\
\hline Pt & -0.97984900 & 3.49414700 & 1.61677100 \\
\hline Pt & 1.14239400 & -3.42383900 & -2.07216300 \\
\hline Pt & 0.06524900 & -3.71185400 & 1.46946900 \\
\hline Pt 1 & -1.99545100 & -1.87107400 & -2.90838700 \\
\hline Pt18 & -0.17671900 & -0.19760000 & -3.96394300 \\
\hline Pt & -2.23467200 & 1.55708000 & 2.94448400 \\
\hline Pt20 & 1.62678500 & -0.68659900 & 0.56 \\
\hline Pt & 0.76958800 & 3.90215900 & -0.3497 \\
\hline Pt22 & -3.70868000 & -0.68809100 & -1.24713200 \\
\hline Pt23 & 1.60810900 & 2.97510700 & 2.12230600 \\
\hline Pt24 & -0.06534600 & 1.04253500 & 1.53098200 \\
\hline Pt & 2.12938000 & -1.25696800 & -3.15816600 \\
\hline Pt26 & 2.17463100 & 0.49571500 & 2.91660300 \\
\hline Pt & -3.21974700 & -2.58 & 0. \\
\hline Pt28 & -0.56977800 & -1.96157400 & 3.40744700 \\
\hline Pt29 & -2.83825800 & 3.03726000 & -0.20232000 \\
\hline Pt30 & 2.37249900 & -3.17668800 & 0.25104200 \\
\hline Pt31 & -1.00897000 & 2.87585000 & -2.04763200 \\
\hline Pt32 & -2.44032800 & 0.85992200 & -3.04085400 \\
\hline Pt33 & -0.91291400 & -1.34813400 & 0.8855380 \\
\hline \# 33 & Cluster $=$ Pt $34 \quad Q=0$ & $\mathrm{M}=0 \quad \mathrm{Etot}=-29$ & 62296300 \\
\hline Pt 1 & -1.81443100 & 0.46117600 & -0.24601500 \\
\hline Pt2 & -3.10755000 & 2.67289700 & 0.31074300 \\
\hline Pt3 & -0.45463400 & -1.71764400 & 0.2839100 \\
\hline Pt 4 & 0.54031800 & -3.22123800 & 2.2316420 \\
\hline Pt 5 & 1.78539800 & 2.77176200 & -2.33315200 \\
\hline Pt 6 & -0.15876500 & 0.35565000 & 1.8151570 \\
\hline Pt 7 & -3.28613300 & -2.17603800 & -1.38930400 \\
\hline Pt 8 & 0.43338800 & 1.86023700 & -0.226715 \\
\hline
\end{tabular}




\begin{tabular}{|c|c|c|c|}
\hline Pt9 & -1.40748300 & -0.76998200 & -3.86863100 \\
\hline Pt10 & -0.85198500 & 3.72922600 & 1.18571200 \\
\hline Pt11 & -0.15423400 & 1.53781600 & -3.71261500 \\
\hline Pt12 & -1.47009200 & -0.69639100 & 3.86247100 \\
\hline Pt13 & 3.90787400 & -0.20359100 & -1.39739900 \\
\hline Pt14 & 1.71573200 & 1.51862600 & 3.23893000 \\
\hline Pt15 & -0.79392000 & 2.35283500 & 3.41624000 \\
\hline Pt16 & 1.79373500 & -0.33108500 & 0.19329700 \\
\hline Pt17 & -3.74461600 & -0.97092500 & 0.89224700 \\
\hline Pt18 & 2.22100700 & 0.36193300 & -3.33667700 \\
\hline Pt19 & 3.11179500 & 1.99944800 & -0.21349000 \\
\hline Pt20 & 0.72913200 & -3.99404900 & -0.31882200 \\
\hline Pt21 & -1.91751500 & -3.98102300 & -0.06426100 \\
\hline Pt22 & 1.12223300 & -1.05506500 & 3.64699500 \\
\hline Pt23 & -3.32747400 & 0.23788800 & -2.37687800 \\
\hline Pt24 & -0.17666500 & 4.18756500 & -1.31609400 \\
\hline Pt25 & 2.85960200 & -2.53486000 & -0.76115200 \\
\hline Pt26 & 2.86809000 & -2.03228100 & 1.86771800 \\
\hline Pt27 & -2.07773700 & -2.58893800 & 2.16736400 \\
\hline Pt28 & 1.59489900 & -2.16514900 & -3.05322100 \\
\hline Pt29 & 1.76136800 & 3.46666600 & 1.45233200 \\
\hline Pt 30 & 3.68690100 & 0.47044300 & 1.85639200 \\
\hline Pt 31 & 0.16288200 & -0.27677700 & -1.81533200 \\
\hline Pt 32 & -2.00720600 & 2.47882600 & -2.08874100 \\
\hline Pt33 & -0.80979900 & -2.73313600 & -2.17725800 \\
\hline Pt 34 & -2.73411700 & 0.98517800 & 2.2746070 \\
\hline \# 34 & cluster $=$ Pt $35 \quad \mathrm{Q}=0$ & \multicolumn{2}{|c|}{$\mathrm{M}=0 \quad$ Etot $=-3059.52799500$} \\
\hline Pt1 & 1.33801600 & -0.82064600 & -0.85368300 \\
\hline Pt2 & 0.51389100 & 4.00036000 & 0.52956400 \\
\hline Pt3 & -1.67305000 & 3.43792200 & 1.87448000 \\
\hline Pt 4 & -0.19149300 & 1.82672200 & 3.31084700 \\
\hline Pt5 & -1.89279300 & -0.61004300 & -3.91839600 \\
\hline Pt 6 & 0.72263000 & -0.44892100 & -4.03617100 \\
\hline Pt 7 & 0.45928000 & 1.64558300 & -0.67280500 \\
\hline Pt 8 & -2.51162200 & 0.58372100 & 3.38978800 \\
\hline Pt9 & 0.78881300 & -0.43164300 & 4.11401100 \\
\hline Pt10 & 2.78831000 & 2.85151700 & -0.29683200 \\
\hline Pt11 & -3.07266300 & 2.53457200 & -0.15772100 \\
\hline Pt12 & -1.94402400 & -2.64616100 & -2.24109300 \\
\hline Pt13 & -0.63977200 & 1.62257100 & -3.10465100 \\
\hline Pt14 & 3.20651600 & -2.25496400 & -2.42581900 \\
\hline Pt15 & 2.86204100 & 0.32374600 & -2.72364600 \\
\hline Pt16 & -0.55024100 & -1.56123000 & 0.85098800 \\
\hline Pt17 & -1.10084400 & 3.65632300 & -1.50837000 \\
\hline Pt18 & -1.35078800 & 0.93882600 & 1.03171100 \\
\hline Pt19 & 1.75996600 & 2.68595500 & -2.72058800 \\
\hline Pt20 & 3.62183400 & 0.61095100 & 2.37111900 \\
\hline Pt21 & 1.13290500 & 0.29827900 & 1.53997000 \\
\hline Pt22 & -3.90202600 & 0.36971400 & 1.17289700 \\
\hline Pt23 & -3.07544500 & -2.17693300 & 1.35220600 \\
\hline Pt24 & 2.03667000 & 2.69826000 & 2.26115000 \\
\hline Pt25 & -1.19649300 & -0.28797700 & -1.30876400 \\
\hline Pt26 & 0.63721500 & -2.70906400 & -2.6603050 \\
\hline Pt27 & -3.18358400 & 1.10859100 & -2.35058100 \\
\hline Pt28 & 0.48443600 & -3.43153300 & 2.39444300 \\
\hline Pt29 & -1.56194500 & -3.87165700 & 0.03638000 \\
\hline Pt 30 & 3.33412200 & -2.22890900 & 0.19449800 \\
\hline Pt31 & -1.35101000 & -1.77215500 & 3.343821 \\
\hline
\end{tabular}




\begin{tabular}{|c|c|c|c|}
\hline$t 32$ & 3.72187500 & 0.39019900 & -0.23320700 \\
\hline Pt 33 & -3.71665900 & -1.11291200 & -0.98905400 \\
\hline t34 & 2.47846300 & -1.74701900 & 2.61072100 \\
\hline t35 & 1.02747100 & -3.47204700 & -0.17690900 \\
\hline 35 & Cluster $=$ Pt $36 \quad \mathrm{Q}=0$ & \multicolumn{2}{|c|}{$\mathrm{M}=0 \quad \mathrm{E}$ tot $=-3159.55694400$} \\
\hline Pt 1 & 0.53657000 & -3.67125900 & 1.38692900 \\
\hline Pt2 & 0.34910200 & 3.46181500 & 2.3258160 \\
\hline Pt3 & 0.31170200 & 1.46833100 & 4.0902760 \\
\hline Pt 4 & -0.63626600 & 1.83305500 & 0.5228170 \\
\hline Pt5 & -1.96179400 & -2.78088300 & 1.3556100 \\
\hline Pt 6 & -2.07723700 & 0.50690500 & 3.51370700 \\
\hline Pt 7 & 2.44996900 & 0.44595500 & 3.01581700 \\
\hline Pt 8 & 0.84410700 & -1.49895500 & -0.0679250 \\
\hline Pt9 & -3.30271700 & 1.57888800 & 0.3564620 \\
\hline Pt10 & 1.81198200 & 0.84133200 & 0.43803500 \\
\hline Pt11 & 2.89556500 & -2.19432900 & -1.53622800 \\
\hline Pt12 & -1.58449500 & 2.00728300 & -2.9156030 \\
\hline Pt13 & -3.78790500 & -1.97753600 & -0.58231200 \\
\hline Pt14 & 0.93315000 & 2.89826900 & -2.61123 \\
\hline Pt15 & 1.46756700 & -1.90350600 & -3.7316960 \\
\hline Pt16 & 3.63104400 & 0.36020900 & -1.40226900 \\
\hline Pt17 & -1.61765100 & -0.53358400 & -0.05044200 \\
\hline Pt18 & 4.15135800 & -0.19673400 & \\
\hline Pt19 & -1.95137700 & -3.847 & -1 \\
\hline Pt20 & 0.17901000 & 0.56659800 & -1.60071000 \\
\hline Pt21 & -2.64248100 & -1.41045300 & -3.71249400 \\
\hline Pt22 & -3.36404200 & 0.32617000 & -1.91503500 \\
\hline Pt23 & 2.04375100 & 0.65205900 & -3.45239800 \\
\hline Pt24 & -1.07802600 & -1.92435200 & 7998300 \\
\hline Pt25 & 0.00636400 & -0.17650000 & 2.0391740 \\
\hline Pt26 & 2.84618700 & 2.79556100 & 1.86601800 \\
\hline Pt27 & -2.22618500 & 2.86787200 & 2.36725100 \\
\hline Pt28 & 2.98086100 & -2.53988000 & 1.09610300 \\
\hline Pt29 & -2.06308300 & 3.55943900 & -0.86747700 \\
\hline Pt 30 & 0.45901200 & 4.07877900 & 661100 \\
\hline Pt 31 & 1.51294000 & -1.99543400 & 98100 \\
\hline Pt 32 & -0.44252200 & -0.04109400 & -4.14823300 \\
\hline Pt 33 & -0.64240000 & -1.88177200 & -2.17400500 \\
\hline Pt 34 & 2.84604000 & 2.90165600 & -0.77691100 \\
\hline Pt 35 & 0.72673900 & -3.92481500 & -1.22712900 \\
\hline Pt 36 & -3.60484100 & -0.65171600 & 1.71654500 \\
\hline \# 36 & uster $=\operatorname{Pt} 37 \quad \mathrm{Q}=0$ & $=0 \quad \mathrm{Etot}=-3$ & 96554600 \\
\hline Pt1 & 1.32002300 & -0.74852100 & 0.6200280 \\
\hline Pt2 & 1.38362500 & 1.47872800 & -3.77730400 \\
\hline Pt3 & 0.10762700 & -0.81261100 & 4.16170600 \\
\hline Pt 4 & 2.25347200 & -3.15975300 & 0.25137000 \\
\hline Pt 5 & -0.26557700 & 3.06935400 & -2.39513900 \\
\hline Pt 6 & 4.16701300 & 1.49481700 & -0.06041600 \\
\hline Pt 7 & 1.35895200 & 1.43128100 & 3.54300900 \\
\hline Pt 8 & 0.31284600 & 1.65903400 & -0.19391500 \\
\hline Pt9 & 1.46291800 & -2.76398600 & -2.25112600 \\
\hline Pt10 & -0.53301300 & 0.46959100 & 1.9532780 \\
\hline Pt11 & -2.96295400 & 1.91442100 & 1.80177100 \\
\hline Pt12 & 3.21581700 & 0.17230500 & 2.15595000 \\
\hline Pt13 & -2.01251400 & 3.09068200 & -0.3879810 \\
\hline Pt14 & -3.04605200 & -1.96748600 & 2.32560400 \\
\hline Pt15 & 2.27108400 & 2.60901100 & 1.37809500 \\
\hline
\end{tabular}




\begin{tabular}{|c|c|c|c|}
\hline Pt16 & -3.18110100 & -3.11857700 & -0.07835500 \\
\hline Pt17 & -0.06690900 & -4.26744700 & 0.70496700 \\
\hline Pt18 & -2.02726600 & 0.45135500 & -0.18350900 \\
\hline Pt19 & 2.18073400 & 0.45762800 & -1.49674200 \\
\hline Pt20 & 1.93523600 & -2.14573300 & 2.75619200 \\
\hline Pt21 & 3.36810700 & 2.57660500 & -2.35495800 \\
\hline Pt22 & -4.35240700 & -0.11603000 & 0.94068300 \\
\hline Pt23 & 3.88921200 & -1.12443900 & -0.10958200 \\
\hline Pt24 & -0.05159000 & 3.93182700 & 1.16987500 \\
\hline Pt25 & -3.36689200 & 1.58163800 & -2.09973900 \\
\hline Pt26 & -0.94355100 & 2.69455400 & 3.29618300 \\
\hline Pt27 & -2.36654700 & 0.13639700 & 3.76613100 \\
\hline Pt28 & 1.66887600 & 3.92774400 & -0.85220700 \\
\hline Pt29 & -1.21293300 & 0.88224100 & -3.44514700 \\
\hline Pt30 & -1.03804700 & -1.79607200 & 0.65800200 \\
\hline Pt31 & 0.52795600 & -1.07115900 & -4.11559000 \\
\hline Pt32 & -0.99266600 & -3.30732400 & -1.54677000 \\
\hline Pt33 & -1.99164700 & -1.63038800 & -3.28042800 \\
\hline Pt34 & -3.81066300 & -0.98305500 & -1.49406500 \\
\hline Pt35 & -0.63124100 & -3.01561600 & 2.9354880 \\
\hline Pt36 & 3.58108500 & -1.28739900 & -2.7312620 \\
\hline Pt37 & -0.15101300 & -0.71361700 & -1.56409700 \\
\hline \# 37 & Cluster $=\mathrm{Pt} 38$ & $=0 \quad$ Etot $=-33$ & 46566000 \\
\hline Pt 1 & -3.88881000 & 0.49121900 & 1.33330900 \\
\hline Pt2 & -2.19688100 & -3.05118600 & -2.61175200 \\
\hline Pt3 & -0.41052100 & 1.94046100 & 1.0554560 \\
\hline Pt 4 & 3.37682800 & 2.10754900 & 0.00799600 \\
\hline Pt 5 & -0.67916400 & -0.98813700 & -2.31051100 \\
\hline Pt 6 & -0.64180000 & -3.07244400 & 2.26961500 \\
\hline Pt 7 & -0.44629900 & -0.41473200 & 2.14677200 \\
\hline Pt 8 & 0.81599700 & 0.88744600 & 4.09763700 \\
\hline Pt 9 & 2.08412600 & 2.92230800 & -2.15729100 \\
\hline Pt10 & -1.92707500 & 0.49189800 & -4.04893400 \\
\hline Pt11 & -2.48222000 & 2.22493100 & 2.70128400 \\
\hline Pt12 & 0.01147700 & 3.82635900 & -0.76334200 \\
\hline Pt13 & 0.39272500 & -3.20277200 & -3.24881500 \\
\hline Pt14 & 2.87140800 & 1.55199900 & 2.60262300 \\
\hline Pt15 & 1.91696700 & -3.19338200 & 1.40456300 \\
\hline Pt16 & -1.66964700 & -3.70692400 & -0.10159200 \\
\hline Pt17 & 0.24586800 & 1.24406800 & -1.34879400 \\
\hline Pt18 & -0.10152500 & 3.23931100 & 30098100 \\
\hline Pt19 & -3.44372400 & 1.76714300 & -0.9778530 \\
\hline Pt20 & -1.57865500 & 2.67465200 & -2.59611600 \\
\hline Pt21 & 0.84084700 & -4.19902500 & -0.85455800 \\
\hline Pt22 & 0.14213200 & -1.81285200 & 0.03930900 \\
\hline Pt23 & -3.53431400 & -1.89371500 & 0.21038900 \\
\hline Pt24 & 0.53333500 & 1.68153800 & -3.94749700 \\
\hline Pt25 & 1.59751600 & 0.28050900 & 0.6756100 \\
\hline Pt26 & 3.69701900 & 0.86236800 & -2.4246990 \\
\hline Pt27 & 3.18483500 & -2.91449900 & -0.95183300 \\
\hline Pt28 & 1.15741900 & -1.75698500 & 3.71496400 \\
\hline Pt29 & -1.75155800 & 0.25728400 & 4.30727600 \\
\hline Pt30 & 1.87030000 & -0.82024300 & -1.6820270 \\
\hline Pt31 & 1.62307900 & 3.59573700 & 1.34091700 \\
\hline Pt32 & 3.22503600 & -1.06402700 & 2.25387700 \\
\hline Pt33 & -2.75919800 & -1.56034400 & 2.7027530 \\
\hline Pt34 & -2.30762300 & 3.65057300 & 0.47750100 \\
\hline & 4.03210600 & -0.51250800 & -0.18159900 \\
\hline
\end{tabular}




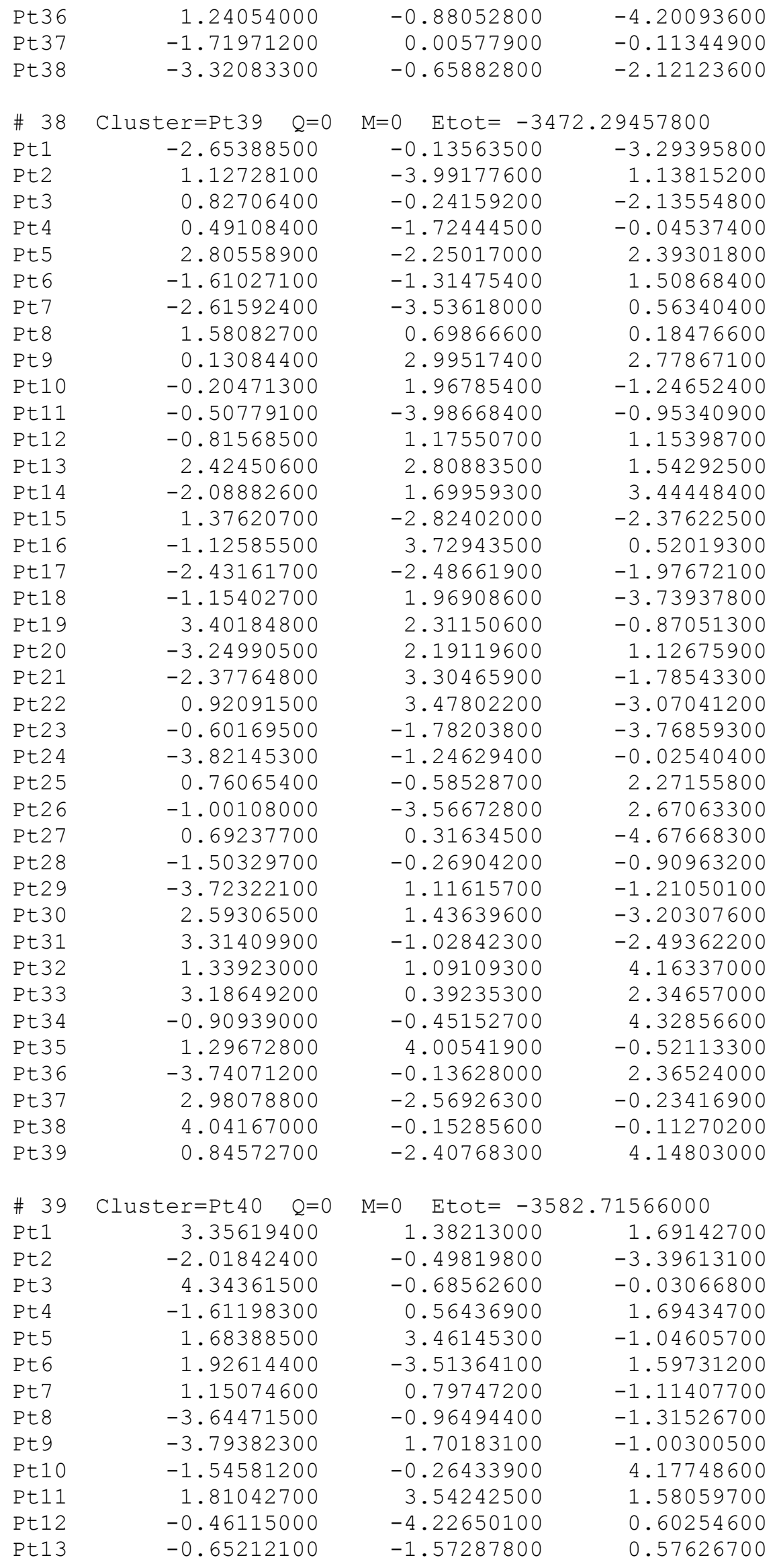




\begin{tabular}{|c|c|c|c|}
\hline Pt14 & 0.96722000 & 0.30093300 & 1.46910000 \\
\hline Pt15 & 3.62511300 & 1.68569600 & -0.90732500 \\
\hline Pt16 & -0.85222300 & 4.01772000 & -1.54980900 \\
\hline Pt17 & 2.76959900 & 0.67890200 & -3.20121200 \\
\hline Pt18 & 3.05459800 & -1.22256500 & 2.22481600 \\
\hline Pt19 & 1.47991900 & 1.80750400 & 3.58500200 \\
\hline Pt20 & 1.01345900 & -0.79101800 & 3.85939900 \\
\hline Pt21 & -0.25361000 & 2.35879500 & 0.39665700 \\
\hline Pt22 & -2.00388700 & 2.08996000 & -2.91340200 \\
\hline Pt23 & -2.01106800 & -2.98519000 & -2.51461500 \\
\hline Pt24 & -2.61911700 & 3.58223800 & 0.40823700 \\
\hline Pt25 & -1.09971400 & 2.29914100 & 3.65796000 \\
\hline Pt26 & 3.38941300 & -1.71532400 & -2.31752200 \\
\hline Pt27 & 0.35779000 & -4.04065900 & -1.88036800 \\
\hline Pt28 & 0.00751000 & -1.40732200 & -1.94828600 \\
\hline Pt29 & 2.79723600 & -3.84918800 & -0.86007000 \\
\hline Pt30 & 1.86733000 & -1.51742500 & -0.14134500 \\
\hline Pt31 & 0.60549500 & 2.29799900 & -3.2089100 \\
\hline Pt32 & -1.46151700 & 0.45338300 & $-0.916197 C$ \\
\hline Pt33 & -4.13771900 & 0.06142300 & 1.09271000 \\
\hline Pt34 & -3.48570800 & 2.20096500 & 2.48862700 \\
\hline Pt35 & 0.45013200 & -0.14670400 & -4.24532200 \\
\hline Pt36 & -2.68226500 & -1.77071500 & 2.32583000 \\
\hline Pt37 & -0.65719700 & 4.34037300 & 2.03707000 \\
\hline Pt38 & -0.29377800 & -2.83381200 & 2.86710800 \\
\hline Pt39 & -2.75509600 & -3.05078200 & 0.00969400 \\
\hline Pt 40 & 1.38510300 & -2.56787800 & -3.83260400 \\
\hline 4 & Cluster $=\operatorname{Pt} 41 \quad \mathrm{Q}=0$ & $=0 \quad \mathrm{Etot}=-36$ & 56807800 \\
\hline Pt 1 & -1.38671800 & 0.83791400 & -3.6395290 \\
\hline Pt2 & 3.90471800 & 2.05634000 & -1.93364000 \\
\hline Pt3 & -0.98262900 & -1.30889600 & -0.66055700 \\
\hline Pt 4 & 1.56574000 & -0.93770600 & 0.0643590 \\
\hline Pt5 & -4.33926000 & 0.33301200 & -0.10764300 \\
\hline Pt 6 & 1.88363500 & -2.34072800 & -3.1594670 \\
\hline Pt 7 & -3.10949100 & 2.58380400 & 0.50873000 \\
\hline Pt 8 & -0.96765700 & 3.97972300 & -0.06797500 \\
\hline Pt9 & -0.24714600 & -1.42245700 & -4.4091020 \\
\hline Pt10 & 1.46926300 & -1.82788900 & 3.7896380 \\
\hline Pt11 & -1.00454000 & 3.30116100 & -2.6182430 \\
\hline Pt12 & -0.86997600 & 1.41233900 & -0.7688870 \\
\hline Pt13 & -3.06280900 & -0.72520200 & -2.20332600 \\
\hline Pt14 & -0.24890100 & -1.55892200 & 1.82576700 \\
\hline Pt15 & -2.02585900 & 0.248 & 1.2038830 \\
\hline Pt16 & -1.14076100 & -3.81626500 & 0.7118880 \\
\hline Pt17 & -3.28149000 & 0.18633300 & 3.49493600 \\
\hline Pt18 & 0.65994300 & -0.13686500 & -2.27157200 \\
\hline Pt19 & 3.10944600 & -0.00317200 & -3.35393800 \\
\hline Pt20 & 3.95377100 & 0.48004500 & 0.2012950 \\
\hline Pt21 & 3.72110700 & -1.63699300 & -1.3674820 \\
\hline Pt22 & -3.18295700 & -2.83424700 & -0.57845200 \\
\hline Pt23 & -2.06137400 & 2.40804000 & 2.87086600 \\
\hline Pt24 & 0.48526700 & -3.50861900 & -1.29890600 \\
\hline Pt25 & -3.20644000 & 1.89458300 & -2.02583300 \\
\hline Pt26 & 0.05836900 & 1.49813800 & 4.1040580 \\
\hline Pt 27 & -1.63227700 & -2.89618000 & -2.7494870 \\
\hline Pt28 & 1.64330000 & 4.15624800 & -0.09962900 \\
\hline PEZ & 1.62944000 & 3.30840400 & -2.6344410 \\
\hline t 30 & 1.70038100 & 1.57934000 & -0.6482570 \\
\hline
\end{tabular}




\begin{tabular}{|c|c|c|c|}
\hline$t 31$ & -2.10858100 & -2.98972300 & 3.02600300 \\
\hline Pt32 & 0.46432700 & 0.93685700 & 1.53736700 \\
\hline t33 & 0.94199800 & -3.90957000 & 2.29172100 \\
\hline t3 & 0.29586400 & 3.45750100 & 2.18840000 \\
\hline t35 & 2.39620800 & 0.59608800 & 3.37112200 \\
\hline 26 & 3.36666800 & -1.38817800 & 1.95981800 \\
\hline-3 & -0.99653500 & -0.92087000 & 4.27684200 \\
\hline c. & 2.77922000 & 2.45807500 & 1.58832100 \\
\hline & -3.85728800 & -1.59912200 & 1.63248700 \\
\hline & 1.12099200 & 1.39521100 & -4.36326700 \\
\hline+2 & 2.56303700 & -3.34608400 & 0.31213200 \\
\hline 41 & Iuster $=$ Pt $42 \quad \mathrm{Q}=0$ & $=0 \quad E$ tot $=-37$ & 04703100 \\
\hline Pt1 & 2.15212300 & 1.13796200 & -3.55495800 \\
\hline Pt2 & 0.92091800 & 3.97014500 & 1.24160100 \\
\hline Pt3 & 2.73213600 & 2.26826200 & 2.26704300 \\
\hline Pt 4 & -1.32711500 & 0.79050000 & -2.02774100 \\
\hline t 5 & -0.25886200 & -0.96187700 & 1.72942100 \\
\hline Pt 6 & -0.94231500 & 0.13202800 & 4.13355000 \\
\hline Pt7 & 3.55520700 & 0.68942500 & -1.34953200 \\
\hline t 8 & -1.11493700 & 3.72260000 & 2.89159200 \\
\hline Pt9 & 1.34429600 & 1.46336500 & 4.38009200 \\
\hline Pt10 & -1.27746200 & -0.47475600 & -4.32583300 \\
\hline Pt11 & -2.78520600 & -1.06323300 & 2.47408600 \\
\hline Pt & 1.27463200 & -2.24319400 & 0.08217200 \\
\hline Pt. & -0.26660200 & 1.96712100 & -4.11928100 \\
\hline PtI & -1.10515300 & -1.42824000 & -0.68749000 \\
\hline 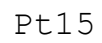 & -1.83189500 & 0.77828300 & 0.52375700 \\
\hline Pt16 & -1.11544400 & -3.22067600 & 2.78409800 \\
\hline Pt 17 & -2.17304300 & 3.19848600 & -2.77624500 \\
\hline Pt & 2.81904900 & 2.96589900 & -0.31466200 \\
\hline Pt & 1.26874500 & 3.37787700 & -2.43026900 \\
\hline Pt2 & 2.90009700 & -0.37152500 & 3.12788800 \\
\hline Pt21 & -3.66082800 & 1.80957500 & -1.11450900 \\
\hline $\mathrm{P}$ & 3.87841400 & -2.59350600 & 0.06575800 \\
\hline Pt23 & -0.76518400 & 4.25149800 & -0.82288200 \\
\hline Pt24 & -0.70877600 & -4.03918000 & -0.80725600 \\
\hline Pt25 & 0.09222800 & 1.57418600 & 2.11848800 \\
\hline Pt26 & 1.88803400 & 0.21396900 & 0.77375500 \\
\hline Pt27 & -2.75792900 & 1.61762500 & 2.86510700 \\
\hline Pt & -3.59640800 & 0.13743300 & -3.17071900 \\
\hline Pt29 & -2.67545400 & -3.08224800 & 0.68192000 \\
\hline Pt & 0.84829500 & -1.88968300 & -3.67 \\
\hline Pt 31 & 0.33972300 & 1.93802200 & -0.44068000 \\
\hline Pt & 2.60688100 & -2.90980900 & 2.34184000 \\
\hline Pt33 & 0.82313100 & -1.83881700 & 3.95328100 \\
\hline Pt 34 & -4.29976000 & -0.15986400 & 0.48606800 \\
\hline Pt 35 & 1.05092200 & -0.27570200 & -1.6373020 \\
\hline Pt 36 & 3.19797500 & -1.72305100 & -2.34336500 \\
\hline & -3.60887100 & -1.83019100 & -1.43981400 \\
\hline Pt & 0.72188700 & -4.46269400 & 1.35633000 \\
\hline Pt39 & -2.65569400 & 3.31018900 & 0.77465000 \\
\hline Pt 40 & 4.47224000 & -0.19141600 & 0.94179200 \\
\hline Pt 41 & -1.57029500 & -2.72433100 & -2.9347920 \\
\hline Pt 42 & 1.61029800 & -3.83045800 & -2.0215160 \\
\hline \# 42 & Cluster $=$ Pt $43 \quad \mathrm{Q}=0$ & \multirow{3}{*}{\multicolumn{2}{|c|}{$\begin{array}{l}\mathrm{M}=0 \quad \text { Etot }=-3895.65773300 \\
0.87105600\end{array}$}} \\
\hline Pt1 & 4.08728100 & & \\
\hline & -3.32153200 & & \\
\hline
\end{tabular}




\begin{tabular}{|c|c|c|c|}
\hline Pt 3 & 1.98212100 & 1.47365700 & 3.54222900 \\
\hline Pt 4 & -0.52004800 & -1.36463800 & 2.14017200 \\
\hline Pt5 & -3.04669700 & -3.10998200 & -0.10276400 \\
\hline Pt 6 & -1.87814100 & 3.75624000 & -1.50919600 \\
\hline Pt 7 & 0.49480600 & -3.44859500 & 3.39105500 \\
\hline t 8 & 3.31352200 & -1.73353100 & 1.54910000 \\
\hline Pt9 & -2.91188900 & 2.72380100 & 0.69719300 \\
\hline t10 & -3.16528300 & 1.08666700 & 2.79009600 \\
\hline t11 & 0.94158600 & 2.40398800 & 0.21745100 \\
\hline t12 & 3.46580200 & 3.08395300 & 0.24218800 \\
\hline t13 & 0.65440900 & -1.89894000 & -0.10375300 \\
\hline 114 & -2.05851000 & -0.47359100 & -4.27437000 \\
\hline t15 & 1.45485300 & 0.12679100 & 1.37977000 \\
\hline t16 & 0.07650900 & 4.85723400 & -0.19574600 \\
\hline t17 & -4.07053100 & -0.82975700 & 1.15103900 \\
\hline 18 & -0.89465600 & 1.30658400 & -1.23992700 \\
\hline t19 & -1.62421600 & -3.49814500 & -2.40996600 \\
\hline$=20$ & 1.77003900 & -1.15954400 & 3.66197700 \\
\hline$=21$ & 1.53243500 & 0.27808200 & -1.19779900 \\
\hline Pt22 & -1.76607000 & -0.85413500 & -0.10889900 \\
\hline t23 & -0.65641500 & 1.79501900 & 3.87 \\
\hline $\operatorname{tet} 24$ & -2.65425000 & 1.81713000 & -3.13315800 \\
\hline Pt25 & 1.82300500 & -3.89218400 & 1.20082500 \\
\hline Pt26 & 1.88301300 & 3.81179600 & -1.80066100 \\
\hline t27 & 3.33048000 & -1.67650400 & -1.09795600 \\
\hline t28 & -0.53835900 & -4.22914300 & -0.12552300 \\
\hline t29 & -1.81990100 & -3.65618500 & 2.15364200 \\
\hline Pt 30 & -0.96035700 & 1.09244400 & 1.38590100 \\
\hline t31 & -3.69252000 & 0.78385500 & -0.92689800 \\
\hline Pt 32 & 3.95354300 & 0.85687500 & 1.61856800 \\
\hline Pt 33 & 0.38709000 & 0.44787100 & -4.50916900 \\
\hline t34 & -0.75897500 & 3.65243700 & 1.99281600 \\
\hline t35 & -0.06997600 & 2.72922500 & -3.27683800 \\
\hline Pt36 & -0.64154700 & -0.68470200 & 4.67659700 \\
\hline Pt 37 & 1.83582200 & -3.83505400 & -1.44423000 \\
\hline $\mathrm{P}$ & 2.45808900 & 1.65870400 & -3.18926500 \\
\hline Pt39 & -0.41684500 & -1.02777600 & -2.33137100 \\
\hline Pt 40 & 1.88378400 & 3.74757600 & 2.271 \\
\hline t41 & 2.31267300 & -0.99541700 & -3.43439000 \\
\hline Pt 42 & 0.64388800 & -3.01431800 & -3.63748300 \\
\hline Pt 43 & -2.81803300 & -1.48680600 & 3.38072600 \\
\hline \# 43 & luster $=\operatorname{Pt} 44 \quad \mathrm{Q}=0$ & $=0 \quad$ Etot $=-40$ & 13369500 \\
\hline Pt1 & 1.82033200 & 4.01729400 & 1.0584300 \\
\hline Pt2 & 0.32192900 & -0.97510300 & -4.52893400 \\
\hline Pt3 & 1.86155100 & -0.76488900 & 0.09472100 \\
\hline Pt 4 & -1.59775900 & 3.99929500 & 1.47185900 \\
\hline Pt 5 & -1.61570900 & -3.51070100 & 2.14961800 \\
\hline Pt 6 & 0.91236600 & 1.50644800 & 0.96588300 \\
\hline Pt 7 & -1.30623900 & 3.49332300 & -2.78957100 \\
\hline Pt 8 & -4.13562200 & -0.35200200 & -0.90563600 \\
\hline Pt9 & -4.00198200 & 1.01222700 & 1.37688800 \\
\hline Pt10 & 4.00923000 & 0.63970600 & 0.82748800 \\
\hline Pt11 & -2.13144700 & 2.00582500 & 3.12526400 \\
\hline Pt12 & -3.01091300 & -0.43585900 & 3.4933910 \\
\hline Pt13 & 2.85970700 & 2.15755600 & 2.6618160 \\
\hline & -1.14042800 & 2.22649200 & -0.46364800 \\
\hline & 3.15874400 & 1.00759900 & -2.87959600 \\
\hline & -0.09601200 & 4.61291900 & -0.6464120 \\
\hline
\end{tabular}




\begin{tabular}{|c|c|c|c|}
\hline Pt17 & -0.11848400 & -4.09930500 & -1.21368300 \\
\hline Pt18 & 2.95414600 & 2.47256500 & -0.71237600 \\
\hline Pt19 & 4.08920500 & -0.93470800 & -1.30013900 \\
\hline Pt20 & -2.92424200 & -2.04048500 & -2.54847900 \\
\hline Pt21 & 0.13867600 & 1.60068600 & -4.01228700 \\
\hline Pt22 & 1.45344400 & -2.90258300 & 3.49226100 \\
\hline Pt23 & -0.49713500 & -1.93340400 & 0.35994500 \\
\hline Pt24 & -0.80431300 & -3.17927600 & -3.58966000 \\
\hline Pt25 & 0.63297300 & -0.66282000 & 2.36639800 \\
\hline Pt26 & -1.49424600 & -0.19665900 & -1.33196000 \\
\hline Pt27 & 2.37493800 & 0.10750700 & 4.19135000 \\
\hline Pt28 & -3.27722300 & -1.65279900 & 1.21566500 \\
\hline Pt29 & 0.86336600 & 0.97344000 & -1.57862200 \\
\hline Pt30 & -3.15992600 & 1.71989900 & -2.19500500 \\
\hline Pt31 & 0.58314400 & -1.61353000 & -1.98708200 \\
\hline Pt32 & -1.47162200 & 0.31012600 & 1.23748800 \\
\hline Pt33 & 1.36568500 & 3.44843900 & -2.54270900 \\
\hline Pt34 & -2.52743200 & -3.54237300 & -0.41266600 \\
\hline Pt35 & -3.56642500 & 3.17306100 & -0.05099400 \\
\hline Pt36 & -2.02641100 & 0.08837500 & -3.90654700 \\
\hline Pt37 & 0.32706900 & 3.00009400 & 3.04246800 \\
\hline Pt38 & 3.11862700 & -3.04670700 & 0.17428400 \\
\hline Pt39 & 3.26975700 & -1.44409600 & 2.25732600 \\
\hline Pt 40 & 2.71002100 & -1.47781700 & -3.52839400 \\
\hline Pt41 & 0.87451600 & -4.02400000 & 1.20944100 \\
\hline Pt 42 & -0.15272000 & 0.76658300 & 4.45272400 \\
\hline Pt 43 & 2.25657300 & -3.69581200 & -2.20589400 \\
\hline Pt 44 & -0.89970700 & -1.85453100 & 4.1055850 \\
\hline \# 44 & luster $=\operatorname{Pt} 45 \quad \mathrm{Q}=0$ & $=0 \quad \mathrm{Etot}=-41$ & 69338100 \\
\hline Pt 1 & -0.45023700 & -0.54235600 & -2.01244200 \\
\hline Pt2 & -0.68594700 & 1.88023300 & 0.78094700 \\
\hline Pt3 & -1.65000100 & 0.53308800 & -4.09722100 \\
\hline Pt 4 & -1.85555700 & -3.66172100 & 1.59535900 \\
\hline Pt 5 & -1.77207700 & -2.03539100 & 3.68446900 \\
\hline Pt 6 & 3.10491600 & -0.63271200 & -3.02868300 \\
\hline Pt 7 & 4.09673000 & 0.67908900 & 1.87092600 \\
\hline Pt 8 & 1.51115600 & -1.84345000 & 1.64031900 \\
\hline Pt9 & -2.73526700 & 3.32531200 & 1.53428500 \\
\hline Pt10 & 0.79495600 & -0.46241900 & -4.33161400 \\
\hline Pt11 & 1.97752200 & 1.76109400 & -3.53045400 \\
\hline Pt12 & -4.28289000 & -0.46934900 & -1.47691700 \\
\hline Pt13 & -0.07393300 & -2.78219700 & -3.38639400 \\
\hline Pt14 & 0.82300700 & 2.92820700 & 2.69626600 \\
\hline Pt15 & -1.47666900 & 1.80459500 & -1.71331100 \\
\hline Pt16 & 3.55286100 & -2.67697800 & 0.06981700 \\
\hline Pt17 & 3.96862500 & -1.86551400 & 2.53273900 \\
\hline Pt18 & 1.57787700 & 0.73734300 & 1.27042100 \\
\hline Pt19 & -0.38442000 & 4.46504600 & 0.87975700 \\
\hline Pt20 & -0.39309900 & 2.88978000 & -3.83284000 \\
\hline Pt21 & -3.43340100 & 3.37780100 & -1.01672900 \\
\hline Pt22 & -1.00691300 & 4.38037300 & -1.71646100 \\
\hline Pt23 & -4.25895000 & 1.37489300 & 0.56278900 \\
\hline Pt24 & 2.18481000 & 0.96197400 & 3.8271310 \\
\hline Pt25 & 1.52762700 & 4.03118900 & -0.90129400 \\
\hline Pt26 & 4.26186900 & -0.15987400 & -0.64309600 \\
\hline Pt27 & -0.49317700 & -2.06895900 & 0.0417690 \\
\hline Pt28 & 1.01161300 & 1.48485500 & -1.13903700 \\
\hline Pt29 & -1.56046300 & 1.75812300 & 3.3027220 \\
\hline
\end{tabular}




\begin{tabular}{|c|c|c|c|}
\hline $\operatorname{ct} 30$ & 1.71575900 & -4.37997900 & 1.03342700 \\
\hline Pt 31 & -3.24790300 & -0.19615500 & 2.43602800 \\
\hline Pt 32 & -3.75825300 & 1.62849500 & -2.97799100 \\
\hline Pt33 & 0.32258600 & -3.60936400 & 3.16484000 \\
\hline Pt 34 & 1.76011500 & -0.93344000 & -0.75991500 \\
\hline Pt 35 & -0.63491500 & -0.39206100 & 2.01465800 \\
\hline Pt36 & -0.14787700 & -0.07696700 & 4.56789000 \\
\hline Pt 37 & -2.37605600 & -3.57070300 & -1.05800100 \\
\hline Pt3 & 3.52770100 & 2.27318200 & -1.37945600 \\
\hline Pt3 & -2.54216400 & -1.77766200 & -2.99666200 \\
\hline Pt 40 & 0.23483900 & -4.25368900 & -1.15201000 \\
\hline Pt 41 & 2.31184000 & -3.03790700 & -2.24392100 \\
\hline Pt 42 & 2.96346400 & 2.99687100 & 1.11035100 \\
\hline Pt 43 & 1.92586400 & -1.67930700 & 4.20743400 \\
\hline Pt 44 & -3.78227800 & -2.07433900 & 0.62267100 \\
\hline Pt 45 & -2.15328800 & -0.08904600 & -0.05256400 \\
\hline$\# 45$ & Cluster=Pt $46 \quad \mathrm{Q}=0$ & $=0 \quad \mathrm{Etot}=-42$ & 3200 \\
\hline Pt1 & 3.45017000 & -3.42047100 & -1.29062300 \\
\hline Pt2 & 3.01645700 & -0.63251700 & 3.04550600 \\
\hline Pt3 & -1.39570400 & -1.31264400 & 0.95066800 \\
\hline Pt 4 & -0.92410400 & -0.38160400 & 4.23519800 \\
\hline Pt5 & 5.07837800 & -0.66523300 & -0.11067100 \\
\hline Pt 6 & 0.74632100 & 0.55711600 & -1.96096800 \\
\hline Pt 7 & 0.70689600 & -3.14409000 & -3.27033300 \\
\hline Pt 8 & -3.82092700 & -1.35870100 & -1.91781800 \\
\hline Pt 9 & -2.85457600 & -1.79532100 & 3.06182000 \\
\hline Pt10 & -4.84661200 & -0.04299600 & 0.11671700 \\
\hline Pt11 & -1.93773000 & -0.63529600 & -3.89066700 \\
\hline Pt12 & 4.15633800 & 1.19015400 & 1.47853400 \\
\hline Pt13 & 2.98650800 & -1.77820300 & -3.36265600 \\
\hline Pt14 & 1.17418600 & -1.25475800 & 1.26529100 \\
\hline Pt15 & 1.49758000 & -3.85842700 & 1.69486300 \\
\hline Pt16 & 0.91192300 & -2.19056100 & 3.72535900 \\
\hline Pt17 & 2.34987300 & 2.89428600 & 2.33446100 \\
\hline Pt18 & -0.95003000 & 4.32903200 & 0.92735300 \\
\hline Pt19 & 1.34500800 & 1.12025500 & 4.06916500 \\
\hline Pt20 & -2.90262400 & 1.68927100 & -2.87336200 \\
\hline Pt21 & 3.63781300 & 2.48418900 & -0.90157200 \\
\hline Pt22 & -0.52470500 & 1.65257800 & -4.07060000 \\
\hline Pt23 & -0.23691100 & 0.79469200 & 1.98583200 \\
\hline Pt24 & 2.56149300 & -0.98604600 & -0.89603700 \\
\hline Pt25 & -0.11961600 & 3.18322800 & 3.14293000 \\
\hline Pt26 & -2.57151600 & 1.01170600 & 0.89312500 \\
\hline Pt27 & 4.25650100 & 0.33955300 & -2.39444300 \\
\hline Pt28 & -3.72847500 & -2.43809500 & 0.50996600 \\
\hline Pt29 & 0.11542200 & -1.84406400 & -1.06317900 \\
\hline Pt 30 & -2.28351500 & 1.74806000 & 3.45808200 \\
\hline Pt 31 & 0.41494700 & 3.43133000 & -2.37021400 \\
\hline Pt 32 & -4.16349000 & 2.40568500 & -0.65794400 \\
\hline Pt 33 & -0.50941500 & 2.06434300 & -0.27884800 \\
\hline Pt 34 & 1.78660300 & 1.13278800 & 0.38962600 \\
\hline Pt 35 & -3.31318400 & 3.41312000 & 1.66273600 \\
\hline Pt 36 & -1.67634400 & -0.01774600 & -1.3144120 \\
\hline Pt 37 & -4.35492400 & 0.31075300 & 2.6946660 \\
\hline Pt 38 & 1.51118400 & 3.79576800 & 0.00095600 \\
\hline Pt 39 & -1.52308700 & -3.76586300 & -0.1420580 \\
\hline Pt 40 & 1.00205100 & -4.29581900 & -0.84243500 \\
\hline Pt 41 & 2.46838800 & 2.04067100 & -3.241358 \\
\hline
\end{tabular}




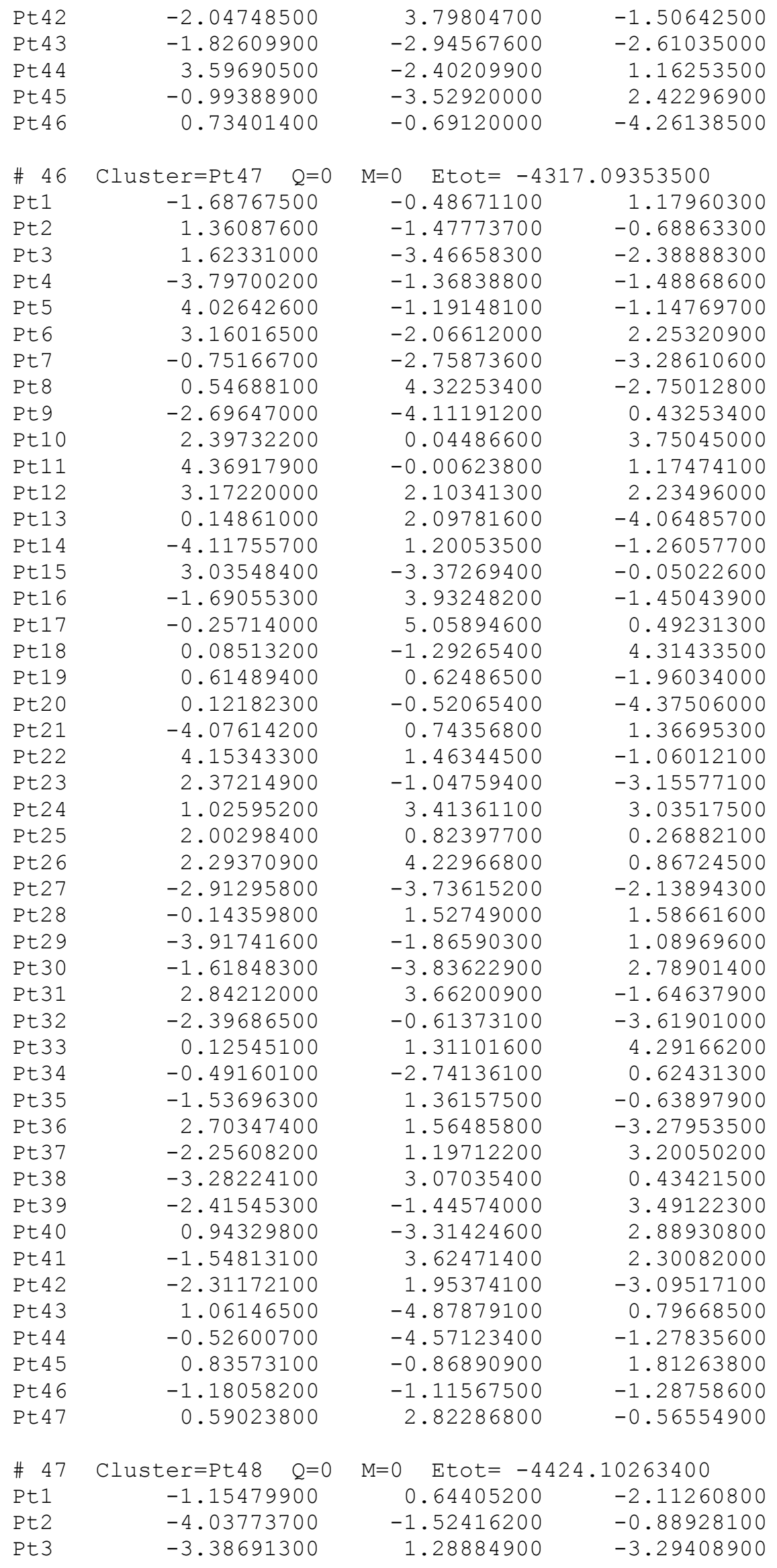




\begin{tabular}{|c|c|c|c|}
\hline Pt 4 & -4.67258000 & 1.05001700 & -1.02361700 \\
\hline Pt 5 & -0.77773600 & 1.05483500 & 2.22076600 \\
\hline Pt 6 & -1.58829500 & 3.55487800 & 2.17621200 \\
\hline Pt 7 & 0.93216700 & -1.09965100 & -2.13803300 \\
\hline Pt 8 & 2.67533900 & 1.67754500 & -3.86574600 \\
\hline Pt9 & -0.51019300 & -4.01754500 & 1.98697900 \\
\hline Pt10 & -1.42538000 & -1.83205400 & -0.99984400 \\
\hline Pt11 & 4.08966100 & 1.01799800 & -1.71783300 \\
\hline t12 & 0.08290300 & -0.02405900 & 0.04441400 \\
\hline t13 & 1.39100400 & 1.45771200 & -1.60920300 \\
\hline t14 & 0.07217000 & 2.18701100 & -3.86093600 \\
\hline t15 & 1.60178000 & 1.77956800 & 1.09421100 \\
\hline Pt16 & -2.05054900 & 0.79963900 & 4.48011300 \\
\hline Pt17 & 0.10214000 & -2.23446000 & 3.96350300 \\
\hline t18 & 0.70924000 & 3.98168100 & -1.88120600 \\
\hline t19 & -1.23657400 & -1.50252800 & 1.69193600 \\
\hline Pt20 & -2.50392600 & -1.72728900 & 3.95757700 \\
\hline t21 & -0.53253100 & -2.93941400 & -3.31240000 \\
\hline Pt22 & 0.56832500 & 0.36385600 & 4.50080200 \\
\hline Pt23 & -3.22573000 & $3.0016180 \mathrm{C}$ & 0.04659200 \\
\hline Pt24 & -0.65942200 & 2.43706400 & -0.11704500 \\
\hline Pt25 & 0.83626400 & -2.48708900 & 0.21192100 \\
\hline Pt26 & -3.90950200 & -1.07341600 & 1.80516600 \\
\hline Pt27 & -3.44163600 & 1.53426800 & 2.34440100 \\
\hline Pt28 & -1.90125600 & 3.24765900 & -2.29241100 \\
\hline Pt29 & 2.08318400 & 4.27416400 & 1.62921700 \\
\hline Pt 30 & 0.72432700 & 2.86313700 & 3.40981600 \\
\hline Pt31 & 2.22196200 & -0.84938300 & -4.38828200 \\
\hline Pt32 & 1.78464900 & -3.59124300 & -2.06568400 \\
\hline Pt33 & 3.45221800 & -2.95000000 & 0.07186500 \\
\hline Pt34 & -2.83006600 & -3.36621700 & 0.74501800 \\
\hline Pt35 & -0.39569600 & -0.42067300 & -4.40017200 \\
\hline Pt36 & -1.82782400 & -4.36106800 & -1.49375900 \\
\hline Pt37 & 2.11479900 & -3.19844600 & 2.43373100 \\
\hline Pt38 & 3.62347600 & -1.58031800 & -2.25513200 \\
\hline Pt39 & 2.88783300 & 1.11011500 & 3.32468600 \\
\hline Pt 40 & -2.45291600 & 0.40290700 & 0.17985000 \\
\hline Pt41 & -0.15944200 & 4.93777300 & 0.42912200 \\
\hline Pt 42 & 2.61393600 & -0.44231700 & -0.12897700 \\
\hline Pt 43 & 3.87792800 & -1.12537600 & 2.07752000 \\
\hline Pt 44 & -2.71326400 & -1.27812100 & -3.22828400 \\
\hline Pt 45 & 1.28506800 & -0.68917500 & 2.21778700 \\
\hline Pt 46 & 4.22525300 & 1.35856200 & 0.96282000 \\
\hline Pt4 7 & 3.01917000 & 3.28943900 & -0.64284700 \\
\hline Pt 48 & 0.41916800 & -5.00034700 & -0.28863400 \\
\hline \# 48 & Cluster $=$ Pt $49 \quad Q=0$ & \multicolumn{2}{|c|}{$\mathrm{M}=0 \quad$ Etot $=-4536.48541000$} \\
\hline Pt 1 & 1.48919400 & -1.2411400 & 1.69514800 \\
\hline Pt2 & 4.34325700 & 0.9956940 & 0.52591300 \\
\hline Pt 3 & -1.19485400 & -3.8279920 & -2.01263000 \\
\hline Pt 4 & -2.25556900 & 3.7025800 & -1.15550200 \\
\hline Pt 5 & -3.68415900 & -1.4168550 & -3.05807200 \\
\hline Pt 6 & 2.25645200 & -0.9141160 & -4.35948700 \\
\hline Pt 7 & 4.18619400 & -0.8439760 & -2.58762500 \\
\hline Pt 8 & 3.14429700 & -2.9198550 & -1.31138200 \\
\hline Pt9 & -0.50300400 & 0.4384940 & 2.48724000 \\
\hline Pt10 & -1.48919400 & 1.24114000 & -1.69514800 \\
\hline Pt11 & 3.41146200 & -1.1589520 & 3.45703200 \\
\hline & -1.76450900 & -1.3594020 & -1.2923580 \\
\hline
\end{tabular}




\begin{tabular}{|c|c|c|c|}
\hline Pt13 & -2.45127600 & 0.36768100 & 0.69836300 \\
\hline Pt14 & -1.11438600 & -1.93014900 & 1.29008700 \\
\hline Pt15 & -1.64306700 & -1.61346200 & 3.85083700 \\
\hline Pt16 & 2.45127600 & -0.36768100 & -0.69836300 \\
\hline Pt17 & 0.65459600 & -2.41287500 & -0.61740800 \\
\hline Pt18 & 0.00000000 & 0.00000000 & 0.00000000 \\
\hline Pt19 & -0.92931500 & 0.80405300 & -4.31529200 \\
\hline Pt20 & -2.25645200 & 0.91411600 & 4.35948700 \\
\hline Pt21 & 1.15547800 & -2.99214200 & -3.13748800 \\
\hline Pt22 & -0.37472700 & 4.97466500 & 0.21119300 \\
\hline Pt23 & 1.37742900 & 4.49653000 & -1.67816000 \\
\hline Pt24 & -0.65459600 & 2.41287500 & 0.61740800 \\
\hline Pt25 & -3.41146200 & 1.15895300 & -3.45703200 \\
\hline Pt26 & -4.18619300 & 0.84397600 & 2.58762500 \\
\hline Pt27 & -1.21036100 & -1.85061000 & -3.90411900 \\
\hline Pt28 & 1.11438600 & 1.93014900 & -1.29008700 \\
\hline Pt29 & 0.44977200 & -3.20980800 & 3.10269500 \\
\hline Pt30 & 1.21036100 & 1.85061000 & 3.90411900 \\
\hline Pt31 & 0.37472800 & -4.97466500 & -0.2111930 \\
\hline Pt32 & -3.00065100 & -3.33522000 & -0.06543700 \\
\hline Pt33 & -3.14429600 & 2.91985500 & 1.31138200 \\
\hline Pt34 & -1.37742900 & -4.49653000 & 1.67816000 \\
\hline Pt35 & 1.19485400 & 3.82799200 & 2.01263000 \\
\hline Pt36 & -1.15547800 & 2.99214200 & 3.13748800 \\
\hline Pt37 & 0.50300400 & -0.43849400 & -2.4872400 \\
\hline Pt38 & -0.44977200 & 3.20980800 & -3.10269500 \\
\hline Pt39 & 2.25556900 & -3.70258000 & 1.15550200 \\
\hline Pt 40 & -4.06221200 & 1.65896900 & -0.93708600 \\
\hline Pt 41 & 0.92931500 & -0.80405300 & 4.3152920 \\
\hline Pt 42 & -3.63188500 & -1.68574900 & 2.0247310 \\
\hline Pt 43 & 3.68415900 & 1.41685500 & 3.0580720 \\
\hline Pt 44 & 4.06221200 & -1.65896900 & 0.93708600 \\
\hline Pt 45 & -4.34325700 & -0.99569400 & -0.5259130 \\
\hline Pt 46 & 3.00065100 & 3.33522000 & 0.06543700 \\
\hline Pt 47 & 3.63188500 & 1.68574900 & -2.02473100 \\
\hline Pt 48 & 1.64306700 & 1.61346200 & -3.8508370 \\
\hline Pt 49 & 1.76450900 & 1.35940200 & 1.29235800 \\
\hline \# 49 & Cluster $=$ Pt50 $\quad Q=0$ & $=0 \quad$ Etot $=-46$ & 51982800 \\
\hline Pt 1 & 2.41255800 & 2.74343100 & 2.4894080 \\
\hline Pt2 & -0.28058900 & -2.52438900 & 4.2892230 \\
\hline Pt3 & -3.86719600 & 1.17912700 & -1.95510800 \\
\hline Pt 4 & -0.77081600 & 0.63694300 & 2.28699200 \\
\hline Pt 5 & -4.58712500 & 1.80945000 & 0.4985200 \\
\hline Pt 6 & -1.77538800 & 4.01445300 & 0.7645210 \\
\hline Pt 7 & 1.29068500 & 0.94837000 & 4.15777000 \\
\hline Pt 8 & -1.07976000 & -0.09221500 & 4.80459000 \\
\hline Pt 9 & 0.10838800 & 4.28752800 & -2.54048400 \\
\hline Pt10 & -0.48077300 & -4.32359300 & 1.2106890 \\
\hline Pt11 & -1.86523600 & 1.57318600 & -3.7913820 \\
\hline Pt12 & 1.84804900 & 0.24308700 & 1.70561800 \\
\hline Pt13 & -2.29227000 & -0.97239100 & -4.38897600 \\
\hline Pt14 & -0.09431200 & 1.78228500 & -1.84873200 \\
\hline Pt15 & -1.76145500 & -0.37803800 & -1.90522800 \\
\hline Pt16 & 2.26548400 & 0.91702900 & -0.8128770 \\
\hline Pt 17 & 0.80223400 & -0.73372300 & -2.4248050 \\
\hline Pt18 & -2.30359300 & -2.94905100 & -2.62553700 \\
\hline$F(1)$ & 3.90466100 & -1.56273400 & 1.5746340 \\
\hline$t<0$ & -2.27861100 & 3.39098100 & -1.8023370 \\
\hline
\end{tabular}




\begin{tabular}{|c|c|c|c|}
\hline et21 & 0.31675900 & -3.24326300 & -3.21677500 \\
\hline Pt22 & -2.81490100 & $-3.5355550 c$ & -0.00470000 \\
\hline Pt23 & 4.31744800 & 0.95174800 & 2.24012700 \\
\hline Pt24 & -2.01996300 & 1.51711700 & 0.04021000 \\
\hline Pt25 & 0.22369800 & 4.21655700 & 2.51955000 \\
\hline Pt26 & 2.09967800 & -1.55890900 & 3.61944700 \\
\hline Pt27 & -3.11133900 & -0.35231300 & 3.14625100 \\
\hline Pt28 & -2.29151800 & -2.86045200 & 2.59150400 \\
\hline Pt29 & 2.06172500 & -3.58501400 & 1.79598500 \\
\hline Pt 30 & 2.82573500 & -2.39709600 & -2.67694700 \\
\hline Pt3 & -0.87965600 & 2.49972100 & 4.14187500 \\
\hline Pt 32 & 4.71655900 & 1.63235600 & -0.26078200 \\
\hline Pt 33 & -2.90027500 & 2.25025100 & 2.46349000 \\
\hline Pt 34 & 0.26069800 & -1.25589100 & -4.93024700 \\
\hline Pt3 & 0.09844100 & -1.82456500 & 1.80679900 \\
\hline Pt3 & 0.34407100 & -4.93627600 & -1.21134700 \\
\hline Pt 37 & -2.25465800 & -1.02787400 & 0.70293800 \\
\hline Pt38 & 0.00655700 & -0.06713800 & -0.06658900 \\
\hline Pt39 & -0.48697200 & -2.49421400 & -0.77676800 \\
\hline Pt 40 & 1.96682300 & $-1.7247070 c$ & -0.18034300 \\
\hline Pt 41 & 2.29801600 & 2.79392000 & -2.65749200 \\
\hline Pt 42 & -4.80603500 & -0.71622100 & 1.16943500 \\
\hline Pt 43 & 2.81028200 & 3.43480000 & -0.08893900 \\
\hline Pt 44 & 4.30324000 & -0.85662200 & -1.02116200 \\
\hline Pt 45 & 0.47873400 & 2.35365800 & 0.67918400 \\
\hline Pt 46 & 3.13488800 & 0.25923700 & -3.24485900 \\
\hline Pt 47 & 0.78219400 & 1.23737400 & -4.27170300 \\
\hline Pt 48 & -4.07885500 & -1.44083600 & -1.25753200 \\
\hline Pt 49 & 2.79221300 & -4.14647400 & -0.68808200 \\
\hline Pt 50 & 0.61147400 & 4.88693800 & -0.04902600 \\
\hline \# 50 & luster $=$ Pt51 $\quad \mathrm{Q}=0$ & \multicolumn{2}{|c|}{$\mathrm{M}=0 \quad$ Etot $=-4753.18655200$} \\
\hline Pt1 & 2.47883000 & 2.6792740 & 3.43826300 \\
\hline Pt2 & 0.66401200 & -2.0054820 & 3.94630100 \\
\hline Pt3 & -4.20945800 & 0.4858210 & 2.61568900 \\
\hline Pt 4 & 1.74628900 & 0.8739980 & 1.67535100 \\
\hline Pt5 & 1.04885800 & -3.3448670 & -3.53682700 \\
\hline Pt 6 & -3.08736400 & 1.1806580 & -3.04228100 \\
\hline Pt 7 & 1.80709600 & -4.0923370 & 0.10123200 \\
\hline Pt 8 & 4.30910200 & 0.4971140 & 1.28457600 \\
\hline Pt9 & 0.47117600 & -2.2059900 & -1.23388700 \\
\hline Pt10 & -1.82654500 & -4.6493380 & 0.43834500 \\
\hline Pt11 & 0.57021000 & 0.1122260 & -2.50415100 \\
\hline Pt12 & -1.48319100 & 4.6566600 & 0.95553800 \\
\hline Pt13 & -2.19870900 & 4.2481470 & -1.52999000 \\
\hline Pt14 & 1.27607900 & -1.7800790 & 1.34590000 \\
\hline Pt15 & -0.47994800 & 2.2324850 & 1.18175200 \\
\hline Pt16 & 4.77343300 & 0.5550430 & -1.34535700 \\
\hline Pt17 & -2.39620100 & -0.0973140 & 4.41256100 \\
\hline Pt18 & 3.01142300 & -0.8636000 & 3.20606400 \\
\hline Pt19 & -1.21503300 & 1.8353010 & -1.32810800 \\
\hline Pt20 & -1.83160400 & -0.7969280 & -1.62182900 \\
\hline Pt21 & 3.01552000 & -2.7240840 & -1.89175700 \\
\hline Pt22 & -2.45092300 & 0.4151320 & 0.6569010 \\
\hline Pt23 & 1.99878600 & 2.1071190 & -3.42726500 \\
\hline Pt24 & -1.15710600 & 2.1292030 & 3.76173600 \\
\hline Pt25 & 1.13705800 & -1.0416950 & -4.77953100 \\
\hline Pt26 & -3.76723100 & 2.3192190 & -0.67250200 \\
\hline Pt27 & 0.03358700 & -3.9430730 & 2.152276 \\
\hline
\end{tabular}




\begin{tabular}{|c|c|c|c|}
\hline te 28 & -0.66401200 & 2.00548200 & -3.94630100 \\
\hline Pt29 & 3.45785300 & -3.10914000 & 1.89695900 \\
\hline Pt30 & 0.00000000 & 0.00000000 & 0.00000000 \\
\hline Pt31 & 3.72594700 & 2.93350400 & -1.62991700 \\
\hline Pt32 & 1.05499100 & 0.65529600 & 4.31727000 \\
\hline Pt33 & 0.35830900 & 4.08707800 & 2.84097300 \\
\hline Pt34 & -1.34739400 & -2.07876000 & 0.69532900 \\
\hline Pt35 & 0.31410400 & 3.87183700 & -2.23685800 \\
\hline Pt 3 & -1.54465700 & -2.99438800 & -3.01882300 \\
\hline Pt3 & -0.63125600 & -0.16744700 & 2.48636000 \\
\hline Pt38 & 3.11724100 & -0.35608600 & -3.19046700 \\
\hline Pt39 & -3.01552000 & 2.72408400 & 1.89175700 \\
\hline Pt 40 & 1.43270900 & 2.00169100 & -0.74885000 \\
\hline Pt41 & -4.37823200 & -0.96344000 & -2.24669100 \\
\hline Pt 42 & -1.92549300 & -2.41618100 & 3.24774700 \\
\hline Pt 43 & 1.08508400 & 4.38241200 & 0.28752900 \\
\hline Pt 44 & -4.98902600 & 0.09610200 & 0.06406100 \\
\hline Pt 45 & -1.40003400 & -0.59205700 & -4.21695300 \\
\hline Pt 46 & -3.26649800 & -2.95484000 & -0.95200200 \\
\hline Pt4 7 & -0.14271600 & -4.73822300 & -1.55967700 \\
\hline Pt 48 & 2.45969500 & -0.44162600 & -0.60476600 \\
\hline Pt 49 & 3.26649700 & 2.95484000 & 0.95200200 \\
\hline Pt50 & -3.84953000 & -1.88513700 & 1.44671900 \\
\hline Pt51 & 4.64379000 & -1.79761300 & -0.03440000 \\
\hline \# 51 & I uster $=\operatorname{Pt} 52 \quad \mathrm{Q}=0$ & Etot $=-48$ & 23215700 \\
\hline Pt 1 & -1.70324300 & 2.70435100 & -3.12897700 \\
\hline Pt2 & -2.43314600 & 0.47119300 & -4.33006900 \\
\hline Pt 3 & 3.84405500 & 2.68553400 & -1.75413500 \\
\hline Pt 4 & -1.33128000 & -4.66072200 & -1.33665300 \\
\hline Pt 5 & 4.80108600 & 0.21517700 & -1.33977400 \\
\hline Pt 6 & 1.71855100 & 4.08227400 & -2.33398200 \\
\hline Pt 7 & 3.02054700 & 0.67536900 & -3.28614300 \\
\hline Pt 8 & -4.20638200 & 1.20745900 & 1.10768200 \\
\hline Pt9 & -4.22155400 & -1.13533900 & 2.40441500 \\
\hline Pt10 & -0.81415400 & -2.08127900 & -1.27012100 \\
\hline Pt11 & 4.24456000 & -2.33572000 & -1.34514000 \\
\hline Pt12 & 4.27682900 & 0.42683200 & 1.23757600 \\
\hline Pt13 & 1.12284700 & 4.36573900 & 0.24104500 \\
\hline Pt14 & -2.57358600 & -3.13118200 & 1.89140100 \\
\hline Pt15 & 1.11332900 & -3.90444100 & -1.94485100 \\
\hline Pt16 & 1.85477300 & -4.06856900 & 0.62058200 \\
\hline Pt17 & 1.79545200 & -1.74393200 & -0.58551800 \\
\hline Pt18 & -2.37669400 & -0.81429000 & 0.54952600 \\
\hline Pt19 & 2.45804700 & -1.93978900 & -3.23447600 \\
\hline Pt20 & -0.79661400 & 4.65690800 & -1.60322600 \\
\hline Pt21 & 0.88739600 & -0.28535200 & 4.41697500 \\
\hline Pt22 & 3.71224600 & -2.20817800 & 1.2193070 \\
\hline Pt23 & 0.00000000 & 0.00000000 & 0.00000000 \\
\hline Pt24 & -1.72659300 & 1.82771000 & 0.53491100 \\
\hline Pt25 & 3.31841600 & 2.90984500 & 0.81513800 \\
\hline Pt26 & -0.78360200 & 0.13735200 & 2.44435700 \\
\hline Pt27 & 0.85972600 & 2.00892200 & 1.35384000 \\
\hline Pt28 & -3.26164800 & -2.99997900 & -0.7108520 \\
\hline Pt29 & 0.17461100 & 2.22937100 & -1.2700940 \\
\hline Pt30 & -2.66107800 & 4.26228100 & 0.18139500 \\
\hline Pt31 & -0.12051900 & -2.22397200 & 1.2857460 \\
\hline Pt32 & -2.57521400 & 2.12582300 & 3.01923200 \\
\hline Pt33 & 2.37925600 & 2.99678300 & 3.261857 \\
\hline
\end{tabular}




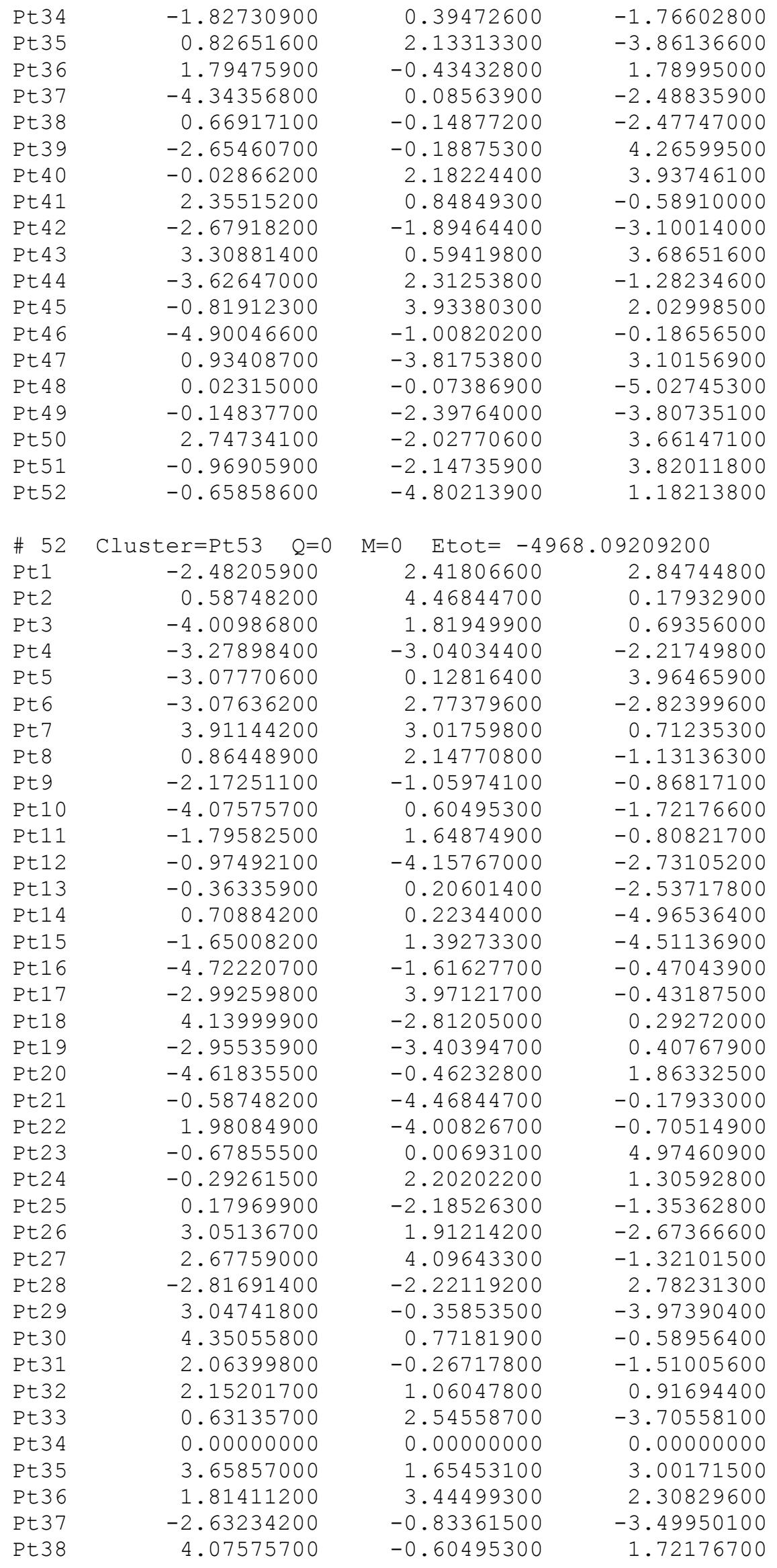




\begin{tabular}{|c|c|c|c|}
\hline Pt39 & -0.32232900 & -2.31720600 & 3.82652400 \\
\hline Pt 40 & 2.36014000 & -3.94847000 & 1.93702000 \\
\hline Pt 41 & 1.81632000 & -1.64948600 & 0.75944300 \\
\hline Pt 42 & -0.15941600 & -4.47019800 & 2.38479400 \\
\hline Pt43 & 4.36164200 & -1.49025500 & -1.94168700 \\
\hline Pt 44 & 2.17693000 & -2.64658800 & -2.97766800 \\
\hline Pt 45 & -0.83960200 & 3.96722200 & -2.01082600 \\
\hline Pt 46 & -0.24697900 & -2.01300300 & -4.00030500 \\
\hline Pt 47 & 0.37791900 & -0.09526800 & 2.54162200 \\
\hline t 48 & -0.75157300 & -2.16446800 & 1.17906300 \\
\hline t 4 & -2.07855800 & 0.15643200 & 1.50561200 \\
\hline$=50$ & 1.90679500 & 0.55984900 & 4.58973100 \\
\hline 551 & 2.29165200 & -1.75770300 & 3.39550600 \\
\hline Pt52 & -1.54325400 & 4.53143500 & 1.66727200 \\
\hline Pt53 & 0.00862700 & 2.32219200 & 3.90093700 \\
\hline$\# 5$ & uster $=$ Pt 54 & $=0 \quad$ Etot $=-507]$ & 00381800 \\
\hline Pt 1 & -0.40034200 & 4.84878200 & -1.3584990 \\
\hline Pt2 & 0.25369400 & -2.56620100 & 0.0914740 \\
\hline Pt3 & 2.41365900 & -1.03680200 & 0.10803300 \\
\hline Pt 4 & -0.00048700 & 2.24371300 & -1.33980000 \\
\hline Pt 5 & 1.93290700 & -2.39374600 & -2.14253700 \\
\hline Pt 6 & 3.88825200 & -3.15536900 & -0.54305300 \\
\hline Pt 7 & -3.47119300 & 2.85827200 & -2.15865300 \\
\hline Pt 8 & 1.46131600 & 0.20762700 & -2.11410900 \\
\hline Pt9 & -1.09583000 & 3.51479100 & 3.44605300 \\
\hline Pt10 & -0.79370400 & 0.91847000 & 3.67131200 \\
\hline Pt11 & -3.49718000 & 0.56193800 & -3.50662700 \\
\hline Pt12 & -0.63074600 & -1.40507400 & -2.07603100 \\
\hline Pt13 & 0.02825400 & 2.27320800 & 1.35021400 \\
\hline Pt14 & -2.11420700 & 0.77837600 & 1.34431000 \\
\hline Pt15 & -0.60461800 & -4.09085600 & -2.45459400 \\
\hline Pt16 & -1.16971200 & 3.40653300 & -3.4606080 \\
\hline Pt17 & 4.82329000 & -0.68128800 & -0.90585700 \\
\hline Pt18 & -3.43527400 & 2.94336700 & 2.2026040 \\
\hline Pt19 & 2.25977500 & -3.44135200 & 1.5313870 \\
\hline Pt20 & 3.62082800 & -0.69476500 & -3.29185500 \\
\hline Pt21 & 0.01627100 & 0.01916300 & 0.0 \\
\hline Pt22 & 1.42262000 & -0.01715600 & -4.7576750 \\
\hline Pt23 & 4.80442000 & 1.83060700 & -0.06474600 \\
\hline Pt24 & 2.14287000 & 3.90063500 & 1.30896300 \\
\hline Pt25 & -2.90059600 & -2.76402500 & -2.2622560 \\
\hline Pt26 & -0.25692200 & -3.91129000 & 2.36752000 \\
\hline Pt27 & -0.63181900 & -1.2 & 2.18686900 \\
\hline Pt28 & -1.99054700 & -1.42870800 & -4.37003200 \\
\hline Pt29 & 1.45551200 & 0.14355600 & 4.82231800 \\
\hline Pt30 & 2.20156100 & 1.59391000 & -0.00456100 \\
\hline Pt31 & 0.46360000 & -2.48448800 & -4.29226300 \\
\hline Pt32 & -0.80945900 & 0.80218600 & -3.5926800 \\
\hline Pt33 & 4.50596300 & -0.67059800 & 1.7526430 \\
\hline Pt34 & -2.18476500 & -1.55392800 & 0.05442100 \\
\hline Pt35 & -4.33586500 & 1.57269700 & 0.05071300 \\
\hline Pt36 & 1.60226900 & -4.64762500 & -0.84362500 \\
\hline Pt37 & 0.42629300 & -2.35898600 & 4.38676800 \\
\hline Pt 38 & 3.62245700 & 1.76982000 & 2.31336500 \\
\hline Pt39 & 1.34151400 & 2.47037700 & -3.6738170 \\
\hline Pt 40 & -0.91922900 & -4.91403200 & 0.0304620 \\
\hline Pt 41 & -2.00216900 & -1.31892300 & 4.46066800 \\
\hline & 2.77399000 & -1.70976100 & 3.4051140 \\
\hline
\end{tabular}




\begin{tabular}{|c|c|c|c|}
\hline et 43 & -3.32920800 & -3.89873100 & 0.07609200 \\
\hline Pt 44 & -0.35463800 & 4.87952100 & 1.28600900 \\
\hline Pt 45 & -3.48087700 & 0.67493800 & 3.58497600 \\
\hline Pt 46 & 3.55757900 & 1.83757200 & -2.37665800 \\
\hline Pt 47 & -2.12228900 & 0.72477300 & -1.27853800 \\
\hline Pt 48 & 1.42782200 & 2.57010700 & 3.62513100 \\
\hline Pt 49 & -2.05635500 & 3.26212000 & 0.00648000 \\
\hline Pt 50 & -4.34969100 & -0.74862400 & -1.31329300 \\
\hline Pt 5 & -4.29718600 & -0.75688600 & 1.46721500 \\
\hline t5 & -2.78365900 & -2.79052200 & 2.39496500 \\
\hline t5 & 1.47691500 & 0.23646100 & 2.19215300 \\
\hline t54 & 2.09493600 & 3.88877100 & -1.37104600 \\
\hline \# 54 & luster $=\operatorname{Pt} 55 \quad \mathrm{Q}=0$ & \multicolumn{2}{|c|}{$\mathrm{M}=0 \quad$ Etot $=-5180.77779300$} \\
\hline Pt1 & 3.19628000 & -2.76767000 & 1.86874800 \\
\hline Pt2 & 2.16533700 & -0.33492900 & 1.41106900 \\
\hline Pt3 & 0.52091100 & -4.45318200 & -1.18764700 \\
\hline Pt 4 & -2.34403700 & -3.24740700 & 2.91841100 \\
\hline Pt5 & 1.42726200 & 1.98909000 & 3.18776300 \\
\hline Pt 6 & 4.70533600 & -1.38553700 & -1.13013100 \\
\hline Pt7 & 3.73241000 & 1.09240200 & -0.29447500 \\
\hline Pt 8 & -0.95659900 & 2.38388900 & 4.33494000 \\
\hline Pt9 & 1.76204100 & 4.66247200 & 0.70555700 \\
\hline Pt10 & -1.06812800 & -4.39168000 & 0.91550900 \\
\hline Pt11 & -0.40329300 & 5.07157800 & -0.74628200 \\
\hline Pt12 & -3.44283700 & 2.98166000 & -0.87734100 \\
\hline Pt13 & 0.81850200 & -2.46485700 & 0.63087900 \\
\hline Pt14 & 0.08677200 & 4.19569700 & 2.67124400 \\
\hline Pt15 & 1.81288800 & -3.01655500 & -3.04336800 \\
\hline Pt16 & 3.67938600 & 0.67760200 & 3.36715100 \\
\hline Pt17 & -4.25388600 & -2.02364000 & 1.54303000 \\
\hline Pt18 & -1.13548500 & 0.39969000 & -2.37067300 \\
\hline Pt19 & -1.94441700 & 2.82940200 & -3.16008100 \\
\hline Pt20 & -2.52155500 & 0.56580400 & -0.16072000 \\
\hline Pt21 & 2.83362700 & 3.49032900 & -1.38126900 \\
\hline Pt22 & -0.86923200 & 1.08973600 & -4.89790300 \\
\hline Pt23 & -0.27243800 & -1.96726500 & -1.70856200 \\
\hline Pt24 & 4.78981900 & -0.67920300 & 1.39019800 \\
\hline Pt25 & -1.86215900 & -1.07741700 & 4.49753400 \\
\hline Pt26 & 0.54649200 & 3.72823000 & -2.78845100 \\
\hline Pt27 & -0.16757100 & -0.75247700 & 2.47309400 \\
\hline Pt28 & -2.88772500 & -1.62213400 & -1.77131600 \\
\hline Pt29 & 3.00769400 & -3.45254200 & -0.67055000 \\
\hline Pt 30 & 2.19007500 & -1.44509700 & 3.90838800 \\
\hline Pt31 & 1.29429600 & 1.35600100 & -1.90863200 \\
\hline Pt 32 & -1.72749400 & -1.82374000 & 0.66050200 \\
\hline Pt 33 & -0.55498500 & -2.58669600 & -4.31826400 \\
\hline Pt 34 & 3.60340400 & 2.85632300 & 1.82334700 \\
\hline Pt 35 & -1.80935700 & -3.97812200 & -2.44919900 \\
\hline Pt 36 & -2.61016200 & -0.82238100 & -4.25031200 \\
\hline Pt 37 & -3.16536500 & 2.56832600 & 2.94554300 \\
\hline Pt 38 & -3.33329100 & -3.75042600 & -0.29719800 \\
\hline Pt 39 & -4.94265300 & -0.52011400 & -0.59333100 \\
\hline Pt 40 & 0.94749200 & -0.52043400 & -3.7666180 \\
\hline Pt 41 & 2.12524600 & -0.99692300 & -1.17890000 \\
\hline Pt 42 & 1.21566500 & -4.48644800 & 2.23349400 \\
\hline Pt 43 & -2.84271000 & 0.02729500 & 2.3874890 \\
\hline Pt 44 & 1.60101600 & 1.95557800 & -4.47072700 \\
\hline Pt 45 & 0.03303900 & -2.93363300 & 3.9689040 \\
\hline
\end{tabular}




\begin{tabular}{|c|c|c|c|}
\hline Pt 46 & -0.80551100 & 2.49078800 & -0.76466900 \\
\hline Pt 47 & -4.64879800 & 1.53777100 & 1.04186500 \\
\hline Pt 48 & 1.43042200 & 2.08924000 & 0.57229700 \\
\hline Pt 49 & 0.42332900 & 0.23592200 & 4.89351400 \\
\hline Pt 50 & 0.01182500 & 0.01015300 & -0.03179900 \\
\hline Pt51 & 3.51871300 & -1.00998300 & -3.46920100 \\
\hline Pt52 & -3.78578200 & 0.91884000 & -2.56292500 \\
\hline Pt53 & -1.99826400 & 4.03399400 & 1.06717500 \\
\hline Pt5 4 & -0.89654400 & 1.69870000 & 1.75381500 \\
\hline Pt 55 & 3.77100100 & 1.57397700 & -2.92091500 \\
\hline \# 55 & Cluster $=$ Pt5 $6 \quad Q=0$ & \multicolumn{2}{|c|}{$\mathrm{M}=0 \quad$ Etot $=-5289.79590400$} \\
\hline Pt1 & 1.00949900 & -2.31132000 & 2.78186300 \\
\hline Pt2 & -3.50635300 & -2.74294400 & 1.37443700 \\
\hline Pt3 & -1.63994300 & -1.48958700 & -1.65390500 \\
\hline Pt 4 & 1.90891800 & 3.72763700 & -1.93985800 \\
\hline Pt5 & -0.12163600 & 3.46269300 & -3.65936100 \\
\hline Pt 6 & -1.64217000 & -2.70986300 & 3.35592200 \\
\hline Pt 7 & -4.49487600 & -0.50248200 & 2.33621300 \\
\hline Pt 8 & 3.23322900 & 2.16849100 & 2.38954400 \\
\hline Pt9 & -0.13247900 & 1.76552900 & 5.2380940 \\
\hline Pt10 & 0.51749500 & 1.97450000 & 2.74719000 \\
\hline Pt11 & -2.12703000 & 2.05220200 & 3.48285100 \\
\hline Pt12 & 3.47295900 & -1.10196400 & -2.98184600 \\
\hline Pt13 & -0.96389400 & -2.35361000 & 0.79710600 \\
\hline Pt14 & 3.44484100 & -1.36260700 & 3.30671400 \\
\hline Pt15 & -3.49919500 & -0.28806800 & -3.18348600 \\
\hline Pt16 & -3.88926500 & 1.90625900 & 1.47239100 \\
\hline Pt17 & -2.00068600 & -2.34084100 & -4.16457700 \\
\hline Pt18 & -1.32252400 & 1.90632700 & 0.90964400 \\
\hline Pt19 & 3.20110700 & 1.57827800 & -2.99487600 \\
\hline Pt20 & -0.23194700 & -3.72942900 & -1.36693200 \\
\hline Pt21 & 2.51618200 & -3.46261200 & -2.03496400 \\
\hline Pt22 & 4.16298700 & -2.01564000 & -0.44127700 \\
\hline Pt23 & -0.77141100 & -0.27127500 & 2.35881500 \\
\hline Pt24 & 0.70268600 & 1.35697500 & -2.18375700 \\
\hline Pt25 & -0.76111800 & -0.05960400 & -3.90488100 \\
\hline Pt26 & 0.02692800 & -0.90124400 & 4.79737900 \\
\hline Pt27 & 0.90361100 & -1.31988600 & -2.25501100 \\
\hline Pt28 & 1.23369500 & 1.46337000 & -4.77929500 \\
\hline Pt29 & 1.55799100 & -2.15900400 & 0.13955100 \\
\hline Pt 30 & -4.23021900 & -1.78735500 & -1.03816400 \\
\hline Pt 31 & 3.25609600 & -3.42056500 & 1.6825240 \\
\hline Pt 32 & -0.02667400 & -0.10177400 & -0.14160100 \\
\hline Pt 33 & 0.48991000 & -3.42352100 & -3.85557800 \\
\hline Pt 34 & 2.59015900 & 0.12036700 & -0.79401200 \\
\hline Pt 35 & 1.26138500 & 2.14076800 & 0.24227400 \\
\hline Pt 36 & 3.45995800 & 3.55601400 & 0.17497600 \\
\hline Pt 37 & -1.82954600 & 1.12288700 & -1.58113700 \\
\hline Pt 38 & -2.64346000 & -0.32374000 & 0.51105300 \\
\hline Pt 39 & 4.76876900 & 1.54752200 & -0.88221200 \\
\hline Pt 40 & 1.51105800 & -1.16285700 & -4.80978500 \\
\hline Pt 41 & -0.69131800 & 3.52533800 & -1.09275800 \\
\hline Pt 42 & -3.29556500 & 3.26997500 & -1.8920440 \\
\hline Pt 43 & 1.81544300 & -0.02559800 & 1.7070780 \\
\hline Pt 44 & -2.63888000 & -0.42319400 & 4.18382400 \\
\hline Pt 45 & -0.16807700 & -4.56808900 & 2.1213870 \\
\hline Pt 46 & -4.43140600 & 0.99149000 & -0.9836570 \\
\hline Pt 47 & -2.36271600 & 2.00857800 & -4.03829500 \\
\hline
\end{tabular}




\begin{tabular}{|c|c|c|c|}
\hline Pt 48 & -2.08209600 & -4.70043800 & 0.27545000 \\
\hline Pt 49 & 1.70592900 & 4.23796000 & 3.11476600 \\
\hline Pt50 & -2.81236100 & -3.80225400 & -2.09834500 \\
\hline Pt51 & 1.62485600 & -4.82387500 & 0.12127500 \\
\hline Pt52 & -2.72238500 & 4.11913300 & 0.52853600 \\
\hline Pt53 & -0.90185100 & 4.18454900 & 2.43775600 \\
\hline Pt5 4 & 4.40140800 & 0.04781700 & 1.24638200 \\
\hline Pt5 5 & 0.98157600 & 4.74918400 & 0.55639200 \\
\hline$P+56$ & 2.18240500 & 0.70139600 & 4.36022800 \\
\hline 30 & Cluster=Pt57 $\mathrm{Q}=0$ & $\mathrm{M}=0 \quad \mathrm{Etot}=-5397$ & 84324900 \\
\hline Pt 1 & -2.33440100 & 0.84257000 & -1.13829600 \\
\hline Pt2 & -3.29513100 & -1.76234700 & -1.54159300 \\
\hline Pt3 & 4.58417500 & -0.47113100 & -1.35964000 \\
\hline Pt 4 & 0.25451700 & -4.49097500 & -1.40571400 \\
\hline Pt5 & -2.26593000 & -0.19124500 & -4.57189200 \\
\hline Pt 6 & 0.94716800 & 1.64567600 & 1.52911700 \\
\hline Pt 7 & 2.13540900 & -0.28812800 & 2.94673300 \\
\hline Pt 8 & -0.96995200 & 1.67244000 & -3.25338200 \\
\hline Pt9 & 0.35625000 & -2.98190100 & -3.58915900 \\
\hline Pt10 & -1.80259300 & 3.89887300 & 2.77174900 \\
\hline Pt11 & -0.14365800 & 2.32824700 & -0.82013200 \\
\hline Pt12 & -4.06972100 & 1.14081200 & 2.21867500 \\
\hline Pt13 & 0.51925300 & -3.48933900 & 3.67513600 \\
\hline Pt14 & -4.29876800 & 0.27066900 & -2.85571900 \\
\hline Pt15 & 1.09779900 & 4.38385400 & 0.44440600 \\
\hline Pt16 & 4.97900100 & 1.83105500 & -0.11886900 \\
\hline Pt17 & 2.52096600 & -1.47228900 & -3.56555300 \\
\hline Pt18 & -4.81890400 & 0.26941200 & -0.21813400 \\
\hline Pt19 & 3.64532600 & -2.95537900 & -1.70781900 \\
\hline Pt20 & 0.85494200 & -2.06043000 & 1.42417400 \\
\hline Pt21 & 0.79953900 & 3.80442100 & 2.99340100 \\
\hline Pt22 & 0.40888600 & -0.25788700 & -4.60928700 \\
\hline Pt23 & -1.67577000 & -2.60188300 & 2.56296000 \\
\hline Pt24 & 2.42825500 & 2.22030100 & -0.50118300 \\
\hline Pt25 & 4.46830100 & 0.31908900 & 1.96396400 \\
\hline Pt26 & -2.57632300 & -0.69488600 & 0.94867700 \\
\hline Pt27 & 3.00171500 & 2.22987100 & 3.08257600 \\
\hline Pt28 & -2.27694600 & -2.72806500 & -3.79014800 \\
\hline Pt29 & 2.31620200 & -3.98659500 & 0.36243400 \\
\hline Pt30 & -4.68043700 & -2.31976100 & 0.59290200 \\
\hline Pt31 & -3.34675600 & 2.75699200 & -2.63616400 \\
\hline Pt32 & -1.57449600 & 4.2878 & 0.18521700 \\
\hline Pt33 & 4.34133100 & -2.06422900 & 0.73574900 \\
\hline Pt34 & -0.18433600 & -0.13733000 & 0.00516100 \\
\hline Pt35 & 1.51222200 & 4.04897200 & -2.18288500 \\
\hline Pt36 & 3.60603900 & 0.90794800 & -3.34597000 \\
\hline Pt37 & -3.79476400 & 2.77961500 & 0.00465100 \\
\hline Pt38 & 1.24344300 & -2.03629100 & -1.23766800 \\
\hline Pt39 & -4.04955100 & -1.42361500 & 3.0078570 \\
\hline Pt 40 & -1.12877000 & 4.19205800 & -2.43068200 \\
\hline Pt41 & 0.59979300 & -1.07996100 & 4.90608200 \\
\hline Pt 42 & -0.14899100 & -4.57089300 & 1.29463100 \\
\hline Pt43 & 2.43591500 & -0.25322100 & 0.2601490 \\
\hline Pt 44 & 4.05630100 & 3.24612800 & -2.23793000 \\
\hline Pt 45 & 2.94437400 & -2.83716900 & 2.8349450 \\
\hline Pt 46 & -1.14060000 & -2.55578600 & -0.19971300 \\
\hline Pt 47 & -0.55103600 & -0.19082600 & 2.61282000 \\
\hline Pt 48 & -2.05552300 & 1.54726400 & 3.92581200 \\
\hline
\end{tabular}




\begin{tabular}{|c|c|c|c|}
\hline Pt 49 & -2.83090700 & -4.29608000 & 0.94710500 \\
\hline Pt50 & -2.03875300 & -1.00654600 & 4.65942500 \\
\hline Pt51 & -1.66017200 & 1.78216700 & 1.25284300 \\
\hline Pt52 & 0.59999600 & 1.53788900 & 4.33579700 \\
\hline Pt53 & 1.30744400 & 0.46788800 & -2.08516100 \\
\hline Pt5 4 & -2.43376100 & -4.30602900 & -1.67293600 \\
\hline Pt55 & 1.42710900 & 2.19432700 & -4.13956600 \\
\hline Pt5 6 & -0.89567200 & -0.96218000 & -2.37074700 \\
\hline Pt57 & 3.65095000 & 3.86603000 & 1.10079400 \\
\hline \# 57 & Cluster $=\operatorname{Pt} 58 \quad \mathrm{Q}=0$ & \multicolumn{2}{|c|}{$\mathrm{M}=0 \quad$ Etot $=-5506.66803200$} \\
\hline Pt 1 & -1.81461300 & -4.31559700 & 2.37933600 \\
\hline Pt2 & -0.99635800 & 0.14645400 & -2.23415500 \\
\hline Pt3 & -2.79972600 & -4.07475800 & -0.06242100 \\
\hline Pt 4 & 1.86636800 & 1.63256300 & 4.27754900 \\
\hline Pt5 & 0.96779300 & -3.03885400 & -3.98603200 \\
\hline Pt 6 & -0.92840100 & -1.23267100 & -4.51463400 \\
\hline Pt7 & -0.30417000 & 4.25389400 & -1.98887100 \\
\hline Pt 8 & -1.00029900 & 4.34269400 & 1.98502200 \\
\hline Pt9 & 0.81848600 & 0.54087900 & -5.28030800 \\
\hline Pt10 & -4.22379200 & 2.37187400 & -2.12837600 \\
\hline Pt11 & -3.45106700 & -2.27287400 & 3.08349700 \\
\hline Pt12 & 2.19152300 & -1.07845900 & 1.61488600 \\
\hline Pt13 & -0.45749000 & -4.68544800 & -1.18334600 \\
\hline Pt14 & -0.59782700 & 2.54398300 & 4.01228000 \\
\hline Pt15 & 2.64233800 & -1.00933200 & -4.09884300 \\
\hline Pt16 & -3.69395100 & -2.54693400 & -2.09617300 \\
\hline Pt17 & -1.44990500 & 0.17484300 & 4.94479300 \\
\hline Pt18 & 3.76713000 & -1.76891100 & -1.72398400 \\
\hline Pt19 & 1.80512900 & -3.49943800 & 2.66119800 \\
\hline Pt20 & 2.08258500 & -3.92871800 & -1.72204600 \\
\hline Pt21 & -4.77633200 & 0.55673600 & 1.14320700 \\
\hline Pt22 & 0.01391900 & -0.18764100 & 2.78595000 \\
\hline Pt23 & 0.14259600 & -0.23387700 & 0.15425100 \\
\hline Pt24 & 3.80471200 & 2.98732000 & 1.62406600 \\
\hline Pt25 & -0.36552000 & -2.62106600 & 3.83984600 \\
\hline Pt26 & 2.86014000 & 3.03812300 & -3.38135000 \\
\hline Pt27 & -1.39965200 & -1.91664600 & 1.47117700 \\
\hline Pt28 & 0.39442300 & -5.21065800 & 1.24999000 \\
\hline Pt29 & 3.26028600 & -1.70528400 & 3.91555100 \\
\hline Pt30 & -0.96654000 & 1.79194100 & 1.36198700 \\
\hline Pt31 & -1.53220800 & -3.54052100 & -3.33984400 \\
\hline Pt32 & -2.44938500 & -0.02179800 & -0.01721 \\
\hline Pt33 & 4.07313400 & 0.74074300 & -2.76804900 \\
\hline Pt34 & 4.65578400 & -0.78131500 & 0.67130600 \\
\hline Pt35 & 0.77867400 & -2.80658200 & 0.30078500 \\
\hline Pt36 & -2.25885800 & 4.77527900 & -0.30077600 \\
\hline Pt37 & 1.12250800 & -1.44701200 & -1.92053700 \\
\hline Pt38 & 0.29745100 & 2.92739100 & -4.16949700 \\
\hline Pt39 & -4.31418400 & -2.00167100 & 0.55566700 \\
\hline Pt 40 & 0.72638200 & 2.25361900 & -0.63182200 \\
\hline Pt41 & 2.57063000 & 0.32425600 & -0.57362700 \\
\hline Pt 42 & 4.80965400 & 1.66030900 & -0.41268700 \\
\hline Pt43 & -2.97217600 & 2.76348500 & 2.8212670 \\
\hline Pt 44 & -4.03504900 & 2.97295700 & 0.42864300 \\
\hline Pt 45 & 3.87973800 & 0.61962200 & 2.85074200 \\
\hline Pt 46 & 1.08923100 & -0.76376700 & 5.08022400 \\
\hline Pt 47 & 3.01974500 & 3.59649500 & -0.84243300 \\
\hline Pt 48 & 0.96769600 & 4.73752100 & 0.28860700 \\
\hline
\end{tabular}




\begin{tabular}{|c|c|c|c|}
\hline Pt 49 & -2.11847100 & 2.03473600 & -3.67636600 \\
\hline Pt 50 & -4.66432300 & -0.13894900 & -1.42291500 \\
\hline Pt51 & -1.29776600 & -2.15298900 & -1.12310000 \\
\hline Pt52 & -3.21583300 & -0.37955300 & -3.60252600 \\
\hline Pt53 & 1.66296700 & 1.46842400 & 1.63070000 \\
\hline Pt 54 & -1.85174000 & 2.30360800 & -1.03849600 \\
\hline Pt55 & 3.41970500 & -3.18896400 & 0.51700300 \\
\hline Pt 56 & 1.38289900 & 0.99247700 & -2.79198400 \\
\hline Pt5 & -2.65582100 & 0.17021800 & 2.63154400 \\
\hline+5 & 1.51783000 & 3.82784300 & 2.75134400 \\
\hline 58 & Cluster $=$ Pt59 $\quad \mathrm{Q}=0$ & \multicolumn{2}{|c|}{$\mathrm{M}=0 \quad$ Etot $=-5617.08242500$} \\
\hline Pt1 & -2.32858800 & 0.95485600 & 4.07527800 \\
\hline Pt2 & 4.04495000 & 1.81722500 & 2.04788300 \\
\hline Pt3 & -0.15718300 & -1.40536200 & -4.87773700 \\
\hline Pt 4 & 0.11896400 & 4.22089700 & 2.33250500 \\
\hline Pt 5 & -4.08156400 & 2.54911200 & -0.54107700 \\
\hline Pt 6 & 1.49383500 & $-1.8257240 c$ & -0.21637900 \\
\hline Pt 7 & 2.71630900 & 0.40268800 & 0.22201600 \\
\hline Pt 8 & -3.50476000 & -0.32121700 & -3.33226800 \\
\hline Pt9 & -1.28499800 & -2.69044600 & 4.00384400 \\
\hline Pt10 & -2.50708200 & 4.16848600 & 2.44502800 \\
\hline Pt11 & -1.93661200 & -4.48890700 & 2.16169900 \\
\hline Pt12 & -3.73650000 & -2.32711300 & -1.54806000 \\
\hline Pt13 & -2.98688200 & 2.28137300 & -2.92733100 \\
\hline Pt14 & 2.43687300 & 0.91160100 & 4.04731600 \\
\hline Pt15 & -3.64140600 & -2.72645800 & 1.12359500 \\
\hline Pt16 & 1.33379200 & 2.41505300 & -0.71730300 \\
\hline Pt17 & -3.91073400 & $1.9753370 \mathrm{C}$ & 2.09574000 \\
\hline Pt18 & 2.76581500 & 4.53410500 & -0.28594300 \\
\hline Pt19 & 0.09341000 & 4.7328590 & -0.23972900 \\
\hline Pt20 & 4.73755200 & -0.1496880 & -1.35589200 \\
\hline Pt21 & 1.70877000 & -3.0336720 & -3.97247600 \\
\hline Pt22 & -1.33128100 & 0.2016400 & -2.00605300 \\
\hline Pt23 & 0.01548000 & 0.3410290 & 0.21222900 \\
\hline Pt24 & 1.87207300 & -4.5542660 & 2.17826600 \\
\hline Pt25 & 3.72421700 & -2.6219970 & -1.45827800 \\
\hline Pt26 & -1.30287500 & -0.6581790 & 2.21788300 \\
\hline Pt27 & 3.55735500 & -1.4223990 & 3.37887800 \\
\hline Pt28 & -3.48735500 & -1.3797350 & 3.45161300 \\
\hline Pt29 & -2.23630000 & -2.6031910 & -3.74333200 \\
\hline Pt 30 & -2.56872500 & 4.6815540 & -0.12670500 \\
\hline Pt 31 & 4.29422900 & 2.3857910 & -0.55290900 \\
\hline Pt 32 & 1.33579600 & -2.6940490 & 3.99331900 \\
\hline Pt33 & 3.43213000 & 1.6547810 & -2.96752500 \\
\hline Pt 34 & -1.51162000 & -1.7916130 & -0.19943200 \\
\hline Pt 35 & -4.87242200 & 0.0516190 & -1.12114500 \\
\hline Pt 36 & -2.67882500 & 0.4640580 & 0.26738000 \\
\hline Pt 37 & -2.29576100 & -4.3167780 & -0.55365100 \\
\hline Pt 38 & 0.00018800 & -2.8349030 & 1.63776500 \\
\hline Pt 39 & -1.36730100 & 4.3623310 & -2.53969200 \\
\hline Pt 40 & 1.34696800 & -0.6818340 & 2.19936200 \\
\hline Pt 41 & 1.35843900 & 1.9218880 & 1.85312400 \\
\hline Pt 42 & -0.06346500 & -2.0545390 & -2.3066640 \\
\hline Pt 43 & 0.06363300 & 2.2215040 & -3.04961500 \\
\hline Pt 44 & -1.28671500 & 2.4620330 & -0.69639100 \\
\hline Pt 45 & 3.55228100 & -2.8733100 & 1.1268720 \\
\hline Pt 46 & 3.25832100 & -0.9824050 & -3.45603800 \\
\hline & 2.04485300 & 3.9049210 & -2.8080990 \\
\hline
\end{tabular}




\begin{tabular}{|c|c|c|c|}
\hline Pt 48 & -1.48229900 & 0.85097400 & -4.62179500 \\
\hline Pt 49 & 1.35111500 & 0.11842600 & -2.02306700 \\
\hline Pt 50 & -4.86824900 & -0.40655900 & 1.46111600 \\
\hline Pt51 & -1.23831700 & 1.95237200 & 1.87711000 \\
\hline Pt52 & -0.81341600 & -4.54666700 & -2.70363000 \\
\hline Pt53 & 0.03967100 & $-0.3657410 c$ & 4.51216800 \\
\hline Pt5 4 & 4.90433800 & -0.59076300 & 1.28123500 \\
\hline Pt 55 & 1.52190100 & -4.08724100 & -1.51599300 \\
\hline Pt56 & 0.07956000 & 2.26732500 & 4.18481400 \\
\hline t57 & 1.48996600 & 0.66259400 & -4.60893500 \\
\hline t58 & 0.04310700 & $-5.1227020 c$ & 0.37094700 \\
\hline Pt59 & 2.74534200 & 4.08902900 & 2.31416000 \\
\hline \# 59 & luster $=$ Pt $60 \quad \mathrm{Q}=0$ & \multicolumn{2}{|c|}{$\mathrm{M}=0 \quad$ Etot $=-5725.86267100$} \\
\hline Pt 1 & -3.83909000 & -1.87090300 & -2.31363700 \\
\hline Pt2 & -2.33677600 & -0.0618440 & 1.54200400 \\
\hline Pt3 & 0.30636300 & 4.9677000 & -0.18737900 \\
\hline Pt 4 & -2.44245700 & -4.0122120 & -1.69571200 \\
\hline Pt5 & 1.66887400 & -2.1950350 & 0.77137800 \\
\hline Pt 6 & 4.55268300 & -1.0627560 & -1.11503300 \\
\hline Pt7 & -2.91791100 & -2.3921480 & 0.29442700 \\
\hline Pt 8 & -4.12614500 & -1.8399370 & 2.57354100 \\
\hline Pt9 & 2.59603700 & 3.3968780 & -2.53708700 \\
\hline Pt10 & -2.28962200 & 4.7048780 & 0.02992900 \\
\hline Pt11 & 3.42168400 & -0.3556820 & -3.51795300 \\
\hline Pt12 & -1.68461200 & 2.1353560 & 4.54031700 \\
\hline Pt13 & -0.85314900 & 1.8035290 & -2.08085000 \\
\hline Pt14 & -2.06843400 & -2.8267010 & 3.93277700 \\
\hline Pt15 & 0.92796500 & 2.6385480 & 4.38297800 \\
\hline Pt16 & -2.20962200 & 3.6105630 & 2.42189400 \\
\hline Pt17 & -1.75524200 & -4.3980280 & 1.77688100 \\
\hline Pt18 & 2.77254900 & 3.9877220 & 0.05341600 \\
\hline Pt19 & -4.15049600 & 1.8907400 & 1.72703600 \\
\hline Pt20 & 3.72686900 & -3.4931070 & -1.76418200 \\
\hline Pt21 & 2.57357000 & 0.2628870 & 0.27208400 \\
\hline Pt22 & 0.90122300 & -4.1567250 & 2.40434000 \\
\hline Pt23 & -0.36684200 & -5.0258390 & -0.38424900 \\
\hline Pt24 & 1.24010300 & 0.1050180 & 5.11727500 \\
\hline Pt25 & -0.28137400 & -0.6463400 & -2.66684400 \\
\hline Pt26 & 4.46141300 & 2.1053630 & 0.77596200 \\
\hline Pt27 & -0.51836200 & 2.4504980 & -4.61727200 \\
\hline Pt28 & 1.94174400 & -1.5192490 & -1.66341900 \\
\hline Pt29 & -1.08195300 & -0.3524330 & 3.89440400 \\
\hline Pt 30 & -0.41396100 & 1.6419780 & 2.23039700 \\
\hline Pt 31 & 1.19547900 & -4.0376010 & -2.31695500 \\
\hline Pt 32 & -4.26533100 & 2.9541860 & -0.67740100 \\
\hline Pt 33 & -2.51565000 & 3.8283370 & -2.44871000 \\
\hline Pt 34 & -4.79211800 & -0.3808070 & 0.47299600 \\
\hline Pt 35 & -0.00082000 & -0.0641660 & 0.16110600 \\
\hline Pt 36 & 1.60940000 & 1.0290900 & -1.98992300 \\
\hline Pt 37 & 2.21115800 & -4.6867860 & 0.14953300 \\
\hline Pt 38 & -2.88848000 & 1.5564260 & -3.75935000 \\
\hline Pt 39 & -3.60065000 & 0.4375400 & 3.85435000 \\
\hline Pt 40 & 3.48812200 & 0.2481570 & 3.6540730 \\
\hline Pt 41 & 0.39270300 & 4.2135040 & 2.32923000 \\
\hline Pt 42 & -0.68021200 & -2.0286040 & 1.80085100 \\
\hline Pt 43 & 2.23763000 & 2.1762170 & 2.1401770 \\
\hline Pt 44 & 4.23753500 & 1.4801540 & -1.75187100 \\
\hline Pt 45 & 1.49100800 & -2.0664680 & -4.209160 \\
\hline
\end{tabular}




\begin{tabular}{|c|c|c|c|}
\hline Pt 46 & -1.94208100 & 2.11165900 & 0.19810200 \\
\hline Pt 47 & 4.18496900 & -2.93777700 & 0.85618300 \\
\hline Pt 48 & -2.25491200 & -0.20272600 & -1.05859300 \\
\hline Pt 49 & 0.06938800 & -0.05554500 & -5.19654200 \\
\hline Pt50 & 0.09602400 & 4.20101800 & -2.69085900 \\
\hline Pt51 & 2.90952000 & -2.37715800 & 3.12333700 \\
\hline Pt52 & -4.54499300 & 0.66887600 & -1.92422800 \\
\hline Pt53 & 0.56045500 & -2.38815300 & 4.34538500 \\
\hline Pt5 4 & 4.81550000 & -0.43372500 & 1.47109600 \\
\hline Pt5 5 & -2.31440900 & -0.95430700 & -4.29509000 \\
\hline Pt5 6 & -0.90725600 & -3.07868400 & -3.59377200 \\
\hline Pt57 & 1.23583700 & -0.33077800 & 2.48682100 \\
\hline Pt58 & 1.99297900 & 1.64896300 & -4.50787200 \\
\hline Pt59 & -0.46185000 & -2.42682200 & -0.81463500 \\
\hline Pt 60 & 0.68602800 & 2.40326400 & -0.0056990 \\
\hline 60 & Cluster $=$ Pt $61 \quad \mathrm{Q}=0$ & $I=0 \quad$ Etot $=-58$ & 96769900 \\
\hline Pt 1 & -1.00214800 & 4.54410000 & -1.36491600 \\
\hline Pt2 & -1.36200600 & -0.36969900 & 4.89452400 \\
\hline Pt 3 & 0.43011800 & 0.95218600 & -2.48062500 \\
\hline Pt 4 & 4.71538700 & -0.14195900 & 1.43998200 \\
\hline Pt5 & -0.12531500 & -4.12988400 & -2.52343100 \\
\hline Pt 6 & 2.94740700 & -2.88683700 & -2.57712700 \\
\hline Pt 7 & -0.49807100 & 1.07369200 & -4.93872700 \\
\hline Pt 8 & 0.56475600 & 4.98645300 & 0.77438700 \\
\hline Pt 9 & -1.69414000 & -0.37617400 & 2.30244100 \\
\hline Pt10 & -1.99941000 & 4.42319500 & 1.25102300 \\
\hline Pt11 & 2.02509900 & 1.77154000 & -4.41621000 \\
\hline Pt12 & 1.96965700 & -4.63527000 & -0.85911200 \\
\hline Pt13 & -4.53118700 & -1.79451200 & 0.23715300 \\
\hline Pt14 & 0.56820900 & -1.64579400 & -2.92602200 \\
\hline Pt15 & -4.32386900 & -0.43179400 & -2.11120500 \\
\hline Pt16 & 0.42220000 & -1.34341700 & -5.44565000 \\
\hline Pt17 & 2.82275700 & 2.03200600 & -1.90440900 \\
\hline Pt18 & 2.26553600 & -0.54302800 & -1.34806100 \\
\hline Pt19 & 2.76781200 & -0.76221600 & -4.14931700 \\
\hline Pt20 & -1.14527500 & 1.93055100 & 1.3103830 \\
\hline Pt21 & -2.97359600 & 0.35371300 & 0.15275500 \\
\hline Pt22 & 0.45698200 & 1.56187900 & 4.66436700 \\
\hline Pt23 & -3.10777700 & -2.06650200 & 3.70547600 \\
\hline Pt24 & -1.64384400 & 1.95392700 & -1.33099800 \\
\hline Pt25 & 0.02968100 & 0.04865100 & -0.04614500 \\
\hline Pt26 & 2.61208200 & 4.46819400 & -0.85604500 \\
\hline Pt27 & 1.07194200 & 3.79930500 & -2.92150000 \\
\hline Pt28 & 0.80975000 & 0.29279900 & 2.39799500 \\
\hline Pt29 & -1.38623600 & 3.19721900 & -3.67427800 \\
\hline Pt30 & 4.92172400 & 1.80302900 & -0.32268600 \\
\hline Pt31 & 1.23522300 & 2.88338400 & 2.27823900 \\
\hline Pt32 & 4.60087400 & 0.16880600 & -2.4146880 \\
\hline Pt33 & 2.24965600 & -0.37829300 & 4.4634440 \\
\hline Pt34 & 0.67920400 & 2.63514100 & -0.43299900 \\
\hline Pt35 & -3.85527300 & 2.49365200 & 1.51411200 \\
\hline Pt36 & 3.48145700 & 1.52605800 & 3.13276300 \\
\hline Pt37 & -1.74303700 & -4.49009800 & -0.4775030 \\
\hline Pt38 & 2.38230500 & -1.40955500 & 1.0796910 \\
\hline Pt39 & -2.83606400 & 1.53776800 & 3.7578950 \\
\hline Pt 40 & -1.67387100 & -2.44686400 & -3.9599540 \\
\hline Pt41 & 3.66339900 & 3.46661700 & 1.36052900 \\
\hline Pt 42 & -3.24333600 & -3.66697700 & 1.58872800 \\
\hline
\end{tabular}




\begin{tabular}{|c|c|c|c|}
\hline Pt 43 & -1.01588100 & 3.41530500 & 3.52346900 \\
\hline Pt 44 & 0.60744600 & -2.47574200 & -0.50333200 \\
\hline Pt 45 & 0.26907900 & -4.70676900 & 1.18865000 \\
\hline Pt 46 & -3.51650200 & 3.76953300 & -0.84743100 \\
\hline Pt4 7 & 2.50423900 & 1.14794500 & 0.63518700 \\
\hline Pt 48 & 1.96438600 & -3.78642700 & 2.99918900 \\
\hline Pt 49 & 0.11624100 & -2.18341100 & 2.01108900 \\
\hline Pt50 & 3.63961400 & -3.70575800 & 0.95458800 \\
\hline Pt5 & -3.68900500 & 2.08260000 & -2.97270700 \\
\hline Pt5 & -3.21738600 & -2.82606000 & -1.87871100 \\
\hline Pt53 & -5.13853300 & 1.63738400 & -0.66799600 \\
\hline t54 & 0.43872900 & -2.28606000 & 4.60896600 \\
\hline Pt5 5 & -1.27548300 & -3.95663400 & 3.31331400 \\
\hline Pt5 6 & -1.63118900 & -0.59668000 & -1.97693400 \\
\hline Pt5 & 3.86640700 & -1.96847900 & 3.14899000 \\
\hline Pt58 & -2.76806100 & -0.04385900 & -4.27171600 \\
\hline Pt59 & 4.46865800 & -1.80784100 & -0.65419400 \\
\hline Pt 60 & -4.37803300 & -0.14523900 & 2.34202600 \\
\hline Pt 61 & -1.79348900 & -1.94879900 & 0.22327500 \\
\hline \# 61 & Iuster $=$ Pt $62 \quad \mathrm{Q}=0$ & Etot $=-5$ & 02111000 \\
\hline Pt 1 & -1.93703400 & 0.24604500 & 1.67056100 \\
\hline Pt2 & -3.53892100 & 0.88269800 & -3.46079700 \\
\hline Pt 3 & -1.86889800 & 4.32077400 & 2.81682800 \\
\hline Pt 4 & 4.32194000 & 1.28542200 & 0.10281400 \\
\hline Pt 5 & -0.61893900 & -2.01633700 & 1.57253700 \\
\hline Pt 6 & 2.11596500 & 1.02133100 & 4.60902400 \\
\hline Pt 7 & 1.21609400 & -3.61396400 & 0.57632700 \\
\hline Pt 8 & -2.69060300 & -1.61483400 & 0.04489600 \\
\hline Pt9 & -4.38766700 & 0.18423600 & -1.01718700 \\
\hline Pt10 & 3.45473700 & 1.45259000 & -3.49810000 \\
\hline Pt11 & -2.72415700 & -4.15299200 & -0.55761000 \\
\hline Pt12 & -0.08816800 & 1.79261200 & -2.36989200 \\
\hline Pt13 & 1.90933500 & 1.29882100 & 1.33961200 \\
\hline Pt1 & 2.11190500 & 0.91361300 & -1.28663800 \\
\hline Pt15 & -0.23339100 & -5.04674000 & -1.03224700 \\
\hline Pt16 & 1.22969700 & 2.47906700 & -4.54165800 \\
\hline Pt17 & -2.31460800 & -3.09547600 & 3.27506600 \\
\hline Pt1 & -0.72407600 & -0.70733700 & -5.06889200 \\
\hline Pt19 & -1.76233600 & -0.13232000 & -1.82188800 \\
\hline Pt2 & 1.06007800 & 4.23552900 & -2.58511900 \\
\hline Pt21 & 0.25550300 & -0.01331400 & 3.04487700 \\
\hline Pt22 & -1.10397800 & -4.67472100 & 1.50127500 \\
\hline Pt23 & 0.47599300 & -2.93527900 & -4.20845400 \\
\hline Pt24 & -1.00574100 & -0.46853600 & 5.28819000 \\
\hline Pt25 & -1.62922700 & -3.81243700 & -2.95353800 \\
\hline Pt26 & -4.19958000 & -3.20342400 & 1.46876700 \\
\hline Pt27 & 2.53381700 & -1.52361800 & 4.06151000 \\
\hline Pt28 & -0.43048700 & 2.02519500 & 4.63529000 \\
\hline Pt29 & -1.37790100 & 1.82804400 & -4.64653100 \\
\hline Pt30 & 1.90152800 & -4.11240700 & -2.28909900 \\
\hline Pt31 & 0.82847900 & 3.00529600 & -0.25123300 \\
\hline Pt32 & -0.46564200 & -2.33904300 & -1.09430400 \\
\hline Pt33 & -4.41020700 & -2.35873600 & -1.78897700 \\
\hline Pt3 4 & -2.79570600 & -1.62296800 & -3.73437900 \\
\hline Pt35 & 4.39760000 & -1.36478200 & 0.62557100 \\
\hline Pt36 & -2.85922300 & 1.97790400 & 3.44205300 \\
\hline Pt37 & 1.12240500 & -4.65844300 & 2.94818900 \\
\hline Pt38 & -4.58302700 & -0.60131800 & 1.5665820 \\
\hline
\end{tabular}




\begin{tabular}{|c|c|c|c|}
\hline Pt39 & 2.89011800 & -1.99407700 & -3.54082500 \\
\hline Pt 40 & -2.86234000 & 4.19571300 & 0.40994300 \\
\hline Pt41 & -3.16287900 & -0.64585500 & 3.80818600 \\
\hline Pt 42 & 3.35468000 & 3.21102600 & -1.56540000 \\
\hline Pt 43 & 3.78471700 & 2.96806800 & 2.06643300 \\
\hline Pt 44 & 1.91896300 & -1.25291200 & 1.51684900 \\
\hline Pt 45 & 0.57623800 & -0.66898000 & -2.80108300 \\
\hline Pt 46 & 1.86809100 & -0.02446200 & -4.99815600 \\
\hline Pt 47 & 3.33799900 & -3.33441600 & 2.2584660 \\
\hline Pt 48 & 0.10300800 & -0.03954200 & 0.01051200 \\
\hline Pt 49 & -0.70085400 & 5.10664800 & -0.75895300 \\
\hline Pt50 & -3.67158400 & 2.78180000 & -1.66723100 \\
\hline Pt51 & -1.59262600 & 1.95631800 & -0.26226100 \\
\hline Pt52 & 0.41642000 & 4.83835900 & 1.64356100 \\
\hline Pt 5 & -1.61830800 & 3.87129000 & -2.93794600 \\
\hline Pt 5 & 4.32070000 & -0.49224700 & -1.89908800 \\
\hline Pt55 & 2.72420200 & 4.73428300 & 0.43547900 \\
\hline Pt56 & -3.96303000 & 1.88374700 & 1.00401300 \\
\hline Pt57 & 0.11197100 & -2.50576800 & 4.09409500 \\
\hline Pt58 & 3.68953700 & -3.76539900 & -0.37779500 \\
\hline Pt5 & 1.60376600 & 3.36113500 & 3.49328400 \\
\hline Pt & 2.11554800 & -1.69024500 & -1.01061400 \\
\hline Pt 61 & 3.94068500 & 0.30648100 & 2.69528700 \\
\hline Pt 62 & -0.37058400 & 2.31888600 & 1.99982000 \\
\hline \# 62 & I uster $=$ Pt $63 \quad \mathrm{Q}=0$ & $=0 \quad$ Etot $=-60$ & 45438300 \\
\hline Pt 1 & 1.29719400 & -4.85615900 & 1.92386400 \\
\hline Pt2 & -2.66481700 & 4.26541900 & 1.84187300 \\
\hline Pt 3 & -0.25371500 & 5.17647200 & 1.15034400 \\
\hline Pt 4 & -4.03855900 & 2.11248200 & 2.34294900 \\
\hline Pt 5 & 0.09647700 & 0.10905200 & 4.64656000 \\
\hline Pt 6 & 4.79641800 & -1.44201800 & -0.87936900 \\
\hline Pt 7 & -2.52553500 & 1.29141100 & -1.14211600 \\
\hline Pt 8 & -2.74749200 & 0.49519000 & -4.12721200 \\
\hline Pt9 & -3.89740100 & -2.51267400 & -2.49241300 \\
\hline Pt10 & 2.35056600 & -2.92708700 & 3.40742200 \\
\hline Pt11 & 2.58855200 & -0.36091200 & 0.23144700 \\
\hline Pt12 & 0.04105400 & 1.46160800 & 2.33014500 \\
\hline Pt13 & -1.00897900 & -0.48650100 & -2.35439200 \\
\hline Pt14 & -4.57318400 & 0.04001900 & -2.25650500 \\
\hline Pt15 & -1.08656800 & 2.73952900 & 0.40879900 \\
\hline Pt16 & 1.35640900 & -1.41417400 & -1.78809100 \\
\hline Pt17 & -2.74125500 & -1.18466000 & -0.48849200 \\
\hline Pt18 & 3.12901800 & -4.42643600 & -0.10157600 \\
\hline Pt19 & -0.09106300 & 1.88520100 & -1.82345100 \\
\hline Pt20 & 3.31513300 & -3.06082900 & -2.33913100 \\
\hline Pt21 & 1.76581300 & 4.63622000 & -0.52372800 \\
\hline Pt22 & 4.81773900 & -0.34110200 & 2.74353800 \\
\hline Pt23 & -0.53181800 & 3.77901600 & 3.41998800 \\
\hline Pt24 & -4.89115600 & 1.82336800 & -0.14232800 \\
\hline Pt25 & 1.08155600 & -0.87089800 & 2.36590500 \\
\hline Pt26 & -0.72435800 & -2.69123700 & -0.95197400 \\
\hline Pt27 & -2.01142300 & -2.02379500 & -4.26415500 \\
\hline Pt28 & 2.28781300 & 0.97826600 & -2.0049190 \\
\hline Pt29 & -2.19118000 & 0.52868900 & 1.3983400 \\
\hline Pt30 & 3.91564000 & -2.39314100 & 1.37718300 \\
\hline Pt31 & 3.76409700 & 2.91206800 & -0.97731300 \\
\hline Pt32 & -2.19876200 & -0.91938200 & 3.75507200 \\
\hline Pt33 & -1.85200000 & -4.18192600 & -2.761333 \\
\hline
\end{tabular}




\begin{tabular}{|c|c|c|c|}
\hline Pt34 & 0.56521300 & -1.50400300 & -4.32992300 \\
\hline Pt35 & -0.02699000 & -0.03507400 & 0.04350500 \\
\hline Pt36 & -1.12879300 & -1.89583200 & 1.49335800 \\
\hline Pt37 & 1.34059400 & -2.63835000 & 0.54272400 \\
\hline Pt38 & -5.30100300 & -0.81697400 & 0.11971900 \\
\hline Pt39 & 0.80317900 & -3.76985300 & -2.96207000 \\
\hline Pt 40 & -2.01634400 & 1.68210800 & 4.02066200 \\
\hline Pt 41 & -0.60819500 & 3.54665200 & -3.74271900 \\
\hline Pt 42 & -3.23400100 & 3.83336700 & -0.7179140 \\
\hline Pt 43 & 1.90811100 & 3.99158800 & 2.1258640 \\
\hline Pt 44 & -0.17430800 & -2.55471200 & 4.04239000 \\
\hline Pt 45 & -4.46474300 & -0.47237000 & 2.54181200 \\
\hline Pt 46 & 0.59493500 & -4.99009700 & -0.62781500 \\
\hline Pt 47 & 1.47432400 & 2.10628800 & 0.23952600 \\
\hline Pt 48 & 1.58608400 & 2.42109300 & 4.31252400 \\
\hline Pt 49 & -3.10592400 & 2.89751900 & -3.16266200 \\
\hline Pt50 & -2.00668500 & -4.81221900 & -0.12177300 \\
\hline Pt51 & -0.18344200 & 1.02012700 & -4.38155200 \\
\hline Pt52 & 2.36949300 & 1.53674300 & -4.57982700 \\
\hline Pt53 & -4.18833800 & -3.30524600 & 0.01483800 \\
\hline Pt5 4 & 2.73245800 & -0.46780100 & 4.40036000 \\
\hline Pt5 5 & 4.84282500 & 0.76253100 & -2.32763400 \\
\hline Pt56 & -0.78821300 & 4.69169100 & -1.38841800 \\
\hline Pt57 & 2.74600500 & 1.19608700 & 2.32428900 \\
\hline Pt58 & 4.94243100 & 0.89536300 & 0.37931600 \\
\hline Pt59 & -1.22549400 & -4.35135200 & 2.37282500 \\
\hline Pt 60 & 3.07960600 & -0.85920000 & -3.76310800 \\
\hline Pt 61 & 4.34282300 & 3.14289200 & 1.63285100 \\
\hline Pt 62 & -3.41254900 & -2.89132900 & 2.47266500 \\
\hline Pt 63 & 1.96272500 & 3.49928300 & -2.89874300 \\
\hline \# 63 & luster $=\operatorname{Pt} 64 \quad \mathrm{Q}=0$ & $\mathrm{M}=0 \quad \mathrm{Etot}=-61$ & 84502800 \\
\hline Pt 1 & 1.01027300 & 1.45289900 & -2.69441600 \\
\hline Pt2 & 2.06148300 & 3.01759900 & 1.21741700 \\
\hline Pt 3 & 3.04063500 & -4.06667400 & -0.10176500 \\
\hline Pt 4 & 3.61196700 & 3.50218800 & -0.83999800 \\
\hline Pt 5 & 2.04513400 & -1.12128500 & 4.94827200 \\
\hline Pt 6 & -1.82413900 & -0.26050900 & 1.74106500 \\
\hline Pt 7 & 0.69638800 & -2.92482300 & -0.57869100 \\
\hline Pt 8 & 1.64447900 & -0.94472300 & -1.96683400 \\
\hline Pt9 & -0.31645400 & 1.92064200 & 2.00172500 \\
\hline Pt10 & -2.25521100 & 1.89316900 & 0.31194200 \\
\hline Pt11 & 0.58515900 & 3.19951600 & -4.5799070 \\
\hline Pt12 & -0.09629300 & 1.99314200 & 5.1648720 \\
\hline Pt13 & -1.68178100 & -2.43565100 & 0.31460400 \\
\hline Pt14 & -1.17111100 & 4.61185400 & -1.59093900 \\
\hline Pt15 & 3.89920400 & -2.96404900 & 2.13703300 \\
\hline Pt16 & -3.53693000 & 3.10171700 & -1.69113400 \\
\hline Pt17 & 1.73077900 & -3.29627900 & 3.5171800 \\
\hline Pt18 & -2.85516200 & 4.44976900 & 0.4829010 \\
\hline Pt19 & -4.20042800 & 0.74350300 & -2.70733800 \\
\hline Pt20 & -1.87072600 & 3.19869200 & -3.71652900 \\
\hline Pt21 & 2.43392500 & 1.05610800 & -0.51392900 \\
\hline Pt22 & 5.17577900 & -1.06479600 & 0.8234690 \\
\hline Pt23 & 4.33997200 & -2.18418500 & -1.4664610 \\
\hline Pt24 & -2.21229300 & 2.70749400 & 3.7319920 \\
\hline Pt25 & 4.13883100 & 2.01535500 & 2.45067100 \\
\hline Pt26 & -2.92518300 & -0.42086100 & -0.64343200 \\
\hline Pt27 & -2.28447600 & 0.68032000 & -4.498572 \\
\hline
\end{tabular}




\begin{tabular}{|c|c|c|c|}
\hline Pt28 & 1.17755700 & 3.66353900 & 3.56926100 \\
\hline Pt29 & -2.66274800 & 0.16783900 & 4.25859400 \\
\hline Pt30 & 0.26602300 & 0.61574500 & -5.09247000 \\
\hline Pt31 & 1.80977700 & 0.43753500 & 1.95276500 \\
\hline Pt32 & 0.41447100 & -1.81788700 & 1.77750400 \\
\hline Pt33 & 0.70351700 & 5.05892800 & 0.25146000 \\
\hline Pt34 & 0.97198000 & -4.68083400 & 1.44267600 \\
\hline Pt35 & 2.69336300 & -0.01753900 & -4.23602000 \\
\hline Pt36 & -3.10639000 & -2.17105500 & 3.06575700 \\
\hline Pt37 & -4.88513800 & 1.29942600 & -0.17643200 \\
\hline Pt38 & 1.68788500 & -4.63579900 & -2.29382500 \\
\hline Pt39 & -3.04466900 & -3.96269600 & -1.42927400 \\
\hline Pt 40 & -2.26960200 & -1.92213400 & -4.12127900 \\
\hline Pt 41 & 3.34425600 & 2.46747500 & -3.28455300 \\
\hline Pt 42 & 2.39788300 & 1.43155100 & 4.37302900 \\
\hline Pt4 3 & -5.23214200 & -1.37213900 & 0.11783600 \\
\hline Pt 44 & -0.89789200 & -5.02372000 & -0.37071000 \\
\hline Pt 45 & 1.40698700 & 4.13756000 & -2.2294510 \\
\hline Pt 46 & 4.44267000 & 0.29620100 & -2.2719280 \\
\hline Pt 47 & 2.81543700 & -2.64764300 & -3.57401000 \\
\hline Pt 48 & -1.59316300 & -4.29940400 & 2.15719000 \\
\hline Pt 49 & 2.60517500 & -1.44128000 & 0.38668300 \\
\hline Pt50 & -3.98515100 & -3.51392800 & 0.99667600 \\
\hline Pt51 & -0.94278500 & 4.51051000 & 2.25855900 \\
\hline Pt52 & -0.89611900 & -1.35532000 & -1.96718600 \\
\hline Pt53 & 0.06135100 & 0.04542900 & -0.01434400 \\
\hline Pt5 4 & -4.14009200 & 2.63437400 & 1.98793900 \\
\hline Pt5 5 & -4.26149400 & -1.86185300 & -2.37988800 \\
\hline Pt 56 & -4.41628000 & -0.00107600 & 2.2472040 \\
\hline Pt57 & 0.38689800 & -1.84136500 & -4.22053000 \\
\hline Pt58 & 0.12822700 & 2.57466400 & -0.51907700 \\
\hline Pt59 & -0.75177200 & -2.69542700 & 4.10688800 \\
\hline Pt 60 & 4.98486500 & 1.41304700 & 0.03982600 \\
\hline Pt 61 & -0.77861300 & -3.83263000 & -2.86507600 \\
\hline Pt 62 & 3.91432400 & -0.52565700 & 3.1402810 \\
\hline Pt63 & -0.07846300 & -0.15785900 & 3.7213460 \\
\hline Pt 64 & -1.45395200 & 1.16329300 & -2.05861800 \\
\hline \# 64 & Cluster $=$ Pt $65 \quad Q=0$ & Etot $=-62$ & 65232200 \\
\hline Pt 1 & 4.75854700 & -1.74615500 & -0.57169100 \\
\hline Pt2 & 2.25147000 & 0.21105700 & -4.57215200 \\
\hline Pt3 & -4.54826700 & 0.62763000 & 2.66990400 \\
\hline Pt 4 & -4.71983100 & -1.61514900 & 1.31843800 \\
\hline Pt 5 & -1.56997100 & -4.71754100 & -1.1106890 \\
\hline Pt 6 & -1.04491500 & 0.03792400 & -2.55117000 \\
\hline Pt 7 & -2.58452700 & 0.72913900 & -0.39114200 \\
\hline Pt 8 & -0.62061800 & -2.21199200 & -1.34250100 \\
\hline Pt9 & -3.05937800 & 2.80151300 & 2.99164200 \\
\hline Pt10 & -0.52871800 & 5.05357100 & 0.9910640 \\
\hline Pt11 & 3.97166500 & 3.06529300 & 0.1010830 \\
\hline Pt12 & -4.02854100 & 2.09413500 & -2.13422000 \\
\hline Pt13 & -3.12576600 & -1.46159600 & -1.81321600 \\
\hline Pt14 & 2.87954700 & -3.15477600 & -3.10254400 \\
\hline Pt15 & 4.05959600 & -0.80111800 & -2.93712300 \\
\hline Pt16 & -1.36785100 & -1.23557200 & -4.8381990 \\
\hline Pt 17 & 1.99308500 & 3.92935200 & -1.4474080 \\
\hline Pt18 & -3.00469600 & -2.80513500 & 2.9650470 \\
\hline Pt19 & 2.84775800 & 2.59566100 & -3.57607900 \\
\hline$t<0$ & 0.42019100 & 1.37187200 & 2.6157310 \\
\hline
\end{tabular}




\begin{tabular}{|c|c|c|c|}
\hline et21 & -2.98949900 & 4.52643100 & -1.79915600 \\
\hline Pt22 & -3.84289700 & -3.48633300 & -0.33938100 \\
\hline Pt23 & 2.89898300 & 0.17082400 & -0.76412000 \\
\hline Pt24 & -4.62228200 & 2.14748200 & 0.49377300 \\
\hline Pt25 & -0.49767000 & -3.38393300 & 3.59528900 \\
\hline Pt26 & 1.83171600 & -2.18238600 & 3.92720500 \\
\hline Pt27 & -0.30468900 & -0.97778900 & 4.83138400 \\
\hline Pt28 & -0.60350900 & 3.76485500 & 3.28223900 \\
\hline Pt2 & 3.18643800 & -3.84404500 & -0.62492500 \\
\hline Pt3 & 3.99147200 & 1.93843900 & 2.52674700 \\
\hline Pt3 & 3.43658700 & -0.12095100 & 4.10738700 \\
\hline Pt3 & -0.38127100 & 4.98427900 & -1.63591000 \\
\hline Pt33 & -2.88434200 & -0.61481100 & 4.35663600 \\
\hline Pt 3 & -3.42606100 & 0.12915000 & -3.86962500 \\
\hline Pt3 & 0.42558200 & -4.07032500 & -2.85555600 \\
\hline Pt3 & 1.58685800 & -1.90980300 & 0.08803500 \\
\hline Pt 3 & 4.70399900 & 1.60992300 & -2.01305700 \\
\hline Pt3 & 5.16008300 & 0.67461500 & 0.50755200 \\
\hline Pt39 & -2.43281900 & -4.88590300 & 1.41570500 \\
\hline Pt 4 & 2.03290600 & 4.56211100 & 1.15521000 \\
\hline Pt 4 & 0.05640600 & -1.18358300 & 2.19840600 \\
\hline Pt 4 & -1.32751900 & 1.54048600 & 4.60476300 \\
\hline Pt 43 & 1.97762600 & 3.29338500 & 3.48982400 \\
\hline Pt 4 & -0.16350100 & 1.10754100 & -4.93662600 \\
\hline Pt 4 & -5.11606800 & -0.08454200 & -0.82199600 \\
\hline Pt 46 & -0.31128000 & -3.44101800 & 0.92019800 \\
\hline Pt 47 & 2.36281900 & -0.04876100 & 1.73815900 \\
\hline Pt 48 & -1.97664000 & 0.51266300 & 2.15290300 \\
\hline Pt 49 & 1.39350900 & -1.06723600 & -2.42800200 \\
\hline Pt50 & 1.04355700 & 1.48139100 & -2.05021800 \\
\hline Pt5 & -1.32105400 & 2.52750400 & -1.77437400 \\
\hline Pt52 & -2.13794500 & -1.59691700 & 0.70937600 \\
\hline Pt53 & -3.03277900 & 4.20965400 & 0.78692700 \\
\hline Pt5 4 & 1.32338100 & 1.15321200 & 5.05155400 \\
\hline Pt5 & -1.00579100 & 2.48835700 & 0.76542500 \\
\hline Pt5 6 & 1.04731400 & -2.21415700 & -4.75921100 \\
\hline Pt57 & 1.04338700 & -5.20063700 & 2.20556400 \\
\hline Pt 5 & 0.28595100 & 3.43810000 & -3.71588500 \\
\hline Pt 5 & 2.91081200 & -3.39829900 & 1.91928300 \\
\hline Pt 60 & -2.07112200 & -3.30500600 & -3.35651400 \\
\hline Pt & 4.61601100 & -1.43591500 & 2.05467600 \\
\hline Pt 62 & 0.97446500 & -5.26199000 & -0.51432400 \\
\hline Pt & -2.26436600 & 2.51081700 & -4.20913700 \\
\hline Pt 6 & -0.08657000 & 0.08620600 & -0.07479500 \\
\hline Pt 6 & 1.53103100 & 2.08879900 & 0.39381700 \\
\hline \# 65 & Cluster $=$ Pt $66 \quad Q=0$ & $1=0 \quad$ Etot $=-638$ & 75305500 \\
\hline Pt 1 & -3.11191700 & -3.45453900 & -1.9818120 \\
\hline Pt2 & 5.09257500 & -1.26993300 & -0.48527500 \\
\hline Pt 3 & 1.37975200 & -2.65893500 & -0.10370200 \\
\hline Pt 4 & -3.45900800 & -4.14560600 & 1.41800400 \\
\hline Pt 5 & 3.79819700 & -3.51099900 & -0.77781800 \\
\hline Pt 6 & -0.31257200 & -0.14383500 & 3.20621700 \\
\hline Pt 7 & -0.74093900 & -2.64837200 & -4.8738050 \\
\hline Pt 8 & 1.26739700 & -0.96527400 & -2.0591830 \\
\hline Pt9 & -1.50713200 & -4.70910500 & -0.28208600 \\
\hline Pt10 & 4.18078800 & 0.91020800 & -3.2708300 \\
\hline Pt 1 & 1.64215700 & -2.90322400 & -3.79875600 \\
\hline Pt12 & 2.13158000 & -0.28624300 & -4.4623550 \\
\hline
\end{tabular}




\begin{tabular}{|c|c|c|c|}
\hline Pt13 & -2.72626900 & -2.28023900 & 3.13118400 \\
\hline Pt14 & 2.93287700 & -3.83215800 & 1.73252200 \\
\hline Pt15 & 0.11301000 & 0.13258000 & 0.02009400 \\
\hline Pt16 & -4.06212200 & 1.32757400 & -2.75295400 \\
\hline Pt17 & -0.61554500 & 1.76416300 & 4.99101000 \\
\hline Pt18 & -0.74411100 & -2.11811100 & 4.90700800 \\
\hline Pt19 & -0.66968600 & -4.22881800 & -2.77388100 \\
\hline Pt20 & -3.43607900 & 3.63137000 & -1.72301100 \\
\hline Pt21 & 2.22870700 & 1.37943700 & -1.61365700 \\
\hline Pt22 & -1.02260800 & -0.61209100 & -3.24202600 \\
\hline Pt23 & 3.74442100 & -1.67871800 & -2.73988200 \\
\hline Pt24 & -2.23796700 & 3.52983300 & 3.94816900 \\
\hline Pt25 & -4.06401400 & 3.05012700 & 0.83085900 \\
\hline Pt26 & -0.75665100 & -1.90670600 & 1.36752900 \\
\hline Pt27 & -3.74438000 & 1.59813600 & 3.02799000 \\
\hline Pt28 & 3.34870500 & 3.60277400 & -0.77411900 \\
\hline Pt29 & 3.04667400 & 1.00446900 & 4.26821400 \\
\hline Pt30 & -4.50831000 & -1.18090700 & -1.92780800 \\
\hline Pt31 & 1.04610900 & -0.23918600 & 5.47091900 \\
\hline Pt32 & 1.16629800 & -3.55946400 & 3.78208300 \\
\hline Pt33 & -2.68906200 & 0.57510300 & -4.88223800 \\
\hline Pt34 & -4.55929900 & -0.65694800 & 1.94335800 \\
\hline Pt35 & 1.64233300 & -1.54961600 & 2.20830300 \\
\hline Pt36 & 1.65334600 & 1.05021500 & 2.01663100 \\
\hline Pt37 & 2.99011400 & 3.36432300 & 1.78721300 \\
\hline Pt38 & -4.87868400 & 0.81366900 & -0.24274500 \\
\hline Pt39 & 4.68127800 & 1.32590000 & -0.66173100 \\
\hline Pt 40 & -2.30761300 & 0.19960000 & -1.16742600 \\
\hline Pt 41 & 2.75412400 & 3.25268500 & -3.34985600 \\
\hline Pt 42 & -2.52238100 & -0.09290500 & 4.65310100 \\
\hline Pt 43 & 0.72637800 & 2.00737800 & -4.50790700 \\
\hline Pt 44 & 0.88965400 & -5.12688300 & 0.66678900 \\
\hline Pt 45 & -1.37314000 & 5.01403800 & -0.96957500 \\
\hline Pt 46 & -2.10621100 & 0.36651100 & 1.40419500 \\
\hline Pt 47 & 0.66063000 & 4.68425300 & 1.93370000 \\
\hline Pt 48 & 0.90768000 & 2.66105300 & 0.14658400 \\
\hline Pt 49 & 0.23693500 & 4.09361600 & -2.87947400 \\
\hline Pt 50 & -0.21348000 & -0.14312000 & -5.67219600 \\
\hline Pt51 & -1.66775700 & 2.48665800 & -0.09560200 \\
\hline Pt52 & -0.60992300 & 2.31710700 & 2.29889700 \\
\hline Pt53 & 1.41430600 & 3.06112400 & 3.90743600 \\
\hline Pt54 & 3.02386700 & -1.63957600 & 4.40384800 \\
\hline Pt55 & -1.95907900 & 4.54662100 & 1.55593800 \\
\hline Pt5 6 & -3.11054700 & -1.88548100 & 0.18737700 \\
\hline Pt 57 & -0.28491000 & 1.60605100 & -2.10196900 \\
\hline Pt58 & 1.21229900 & 5.14129100 & -0.64678800 \\
\hline Pt59 & 1.71784000 & -4.67095700 & -1.82766700 \\
\hline Pt 60 & -0.90743500 & -2.14936500 & -1.18501100 \\
\hline Pt 61 & 4.31415600 & -1.56205800 & 2.05225800 \\
\hline Pt 62 & 4.28782100 & 1.08266800 & 1.91973100 \\
\hline Pt 63 & -3.17452600 & -1.90665600 & -4.10534800 \\
\hline Pt 64 & 2.71245900 & -0.39443600 & 0.15361700 \\
\hline Pt 65 & -1.91948400 & 2.81387000 & -3.76600000 \\
\hline Pt 66 & -0.94162900 & -4.28394200 & 2.36371800 \\
\hline \# 66 & Cluster $=$ Pt $67 \quad Q=0$ & \multirow{4}{*}{\multicolumn{2}{|c|}{ 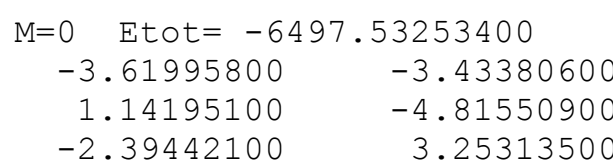 }} \\
\hline Pt 1 & 0.59497600 & & \\
\hline Pt2 & -0.24874900 & & \\
\hline & 2.87908500 & & \\
\hline
\end{tabular}




\begin{tabular}{|c|c|c|c|}
\hline Pt 4 & -0.08119800 & 3.68034400 & -4.15294600 \\
\hline Pt5 & 2.25910800 & 1.07963800 & -0.52710400 \\
\hline Pt 6 & 3.68081700 & 3.27795900 & -0.00221900 \\
\hline Pt 7 & 4.56424600 & -1.46996000 & 1.41877400 \\
\hline Pt 8 & -4.57983000 & -1.15376700 & 1.77249400 \\
\hline Pt9 & -4.33330400 & -0.68736800 & -2.76445000 \\
\hline Pt10 & -4.22228200 & 1.08632800 & 3.08865400 \\
\hline Pt11 & 2.51683300 & 3.43245000 & -3.62292100 \\
\hline Pt12 & 3.85168700 & -3.60114100 & -1.58745600 \\
\hline Pt13 & -1.64655200 & -0.89286500 & -2.29074000 \\
\hline Pt14 & 4.39734200 & 0.81900300 & 2.70060700 \\
\hline Pt15 & 1.84043400 & 0.99850500 & 2.00906500 \\
\hline Pt16 & 2.98291900 & -2.60421800 & -3.88626500 \\
\hline Pt17 & 1.47158800 & -4.60275600 & -1.10286700 \\
\hline Pt18 & 2.17774700 & 4.89985400 & -1.42600500 \\
\hline Pt19 & -1.51671800 & 1.55398900 & -1.34231300 \\
\hline Pt20 & 0.72582100 & 2.73497000 & -1.82570400 \\
\hline Pt21 & 0.65552500 & -3.70576400 & 3.65146800 \\
\hline Pt22 & -0.56563600 & 1.31497700 & 2.96591500 \\
\hline Pt23 & -1.99587100 & -3.49860500 & 3.12595000 \\
\hline Pt24 & 2.35732300 & 0.93829800 & -4.39383100 \\
\hline Pt25 & 3.24351500 & 3.17582900 & 2.58592200 \\
\hline Pt26 & -3.24786600 & -0.16612500 & -0.36876600 \\
\hline Pt27 & -0.12924100 & -2.74897300 & 1.2981440 \\
\hline Pt28 & -0.81608200 & -2.06454200 & 5.05308100 \\
\hline Pt29 & 5.24706800 & -1.39154800 & -1.12385900 \\
\hline Pt 30 & -4.95565100 & 1.58910800 & 0.58317600 \\
\hline Pt31 & -4.89267800 & -2.18674500 & -0.67103200 \\
\hline Pt 32 & -1.07234300 & 4.78871700 & 0.57051900 \\
\hline Pt33 & -3.20865700 & -3.17884400 & -2.57752000 \\
\hline Pt 34 & 4.12355000 & 1.90904000 & -2.23260300 \\
\hline Pt 35 & -2.34583000 & 2.42234500 & -3.68165500 \\
\hline Pt36 & -4.24129900 & 1.80036400 & -1.93278800 \\
\hline Pt 37 & 3.14663500 & -3.70748600 & 0.9378690 \\
\hline Pt 38 & -1.79164500 & -0.81014900 & 1.78674900 \\
\hline Pt39 & -2.59321200 & -0.02038800 & -4.61243400 \\
\hline Pt 40 & 0.87876900 & -1.25324800 & -4.70728800 \\
\hline Pt 41 & 0.97774600 & -5.11474400 & 1.42215900 \\
\hline Pt 42 & -1.14739800 & -4.57975900 & -1.68318600 \\
\hline Pt 43 & -2.61917300 & 3.17491900 & 3.3422150 \\
\hline Pt 44 & -2.27957700 & 1.12529500 & 4.94333300 \\
\hline Pt 45 & -3.56286200 & 3.94133800 & 0.97725700 \\
\hline Pt 46 & -0.18811800 & 3.99635100 & 2.97274800 \\
\hline Pt 47 & -2.33123000 & 1.66066600 & 1.1616330 \\
\hline Pt 48 & -2.11124700 & -2.50488900 & -0.29708400 \\
\hline Pt 49 & 0.17851000 & 0.32847000 & 5.36058300 \\
\hline Pt 50 & 1.58084600 & 4.58600700 & 1.11205800 \\
\hline Pt51 & -3.09631700 & -1.21591500 & 3.99444200 \\
\hline Pt52 & 4.39810800 & -0.39131700 & -3.44509700 \\
\hline Pt53 & -0.40744900 & 5.12759700 & -1.9531190 \\
\hline Pt5 4 & 2.67006700 & -1.23291700 & -1.65276000 \\
\hline Pt55 & 4.84817300 & 0.89621100 & 0.11190600 \\
\hline Pt 56 & -3.88618300 & -3.65940300 & 1.27567000 \\
\hline Pt 57 & 0.11744800 & 2.44958100 & 0.6714420 \\
\hline Pt58 & 0.66442000 & 0.21370100 & -2.47704400 \\
\hline Pt59 & 1.41835300 & 2.42844100 & 4.3319550 \\
\hline Pt 60 & -1.49216100 & -2.40054500 & -4.4511720 \\
\hline Pt 61 & 0.33656700 & -2.23518100 & -1.2193680 \\
\hline Pt 62 & 2.62140000 & -0.06541200 & 4.4321600 \\
\hline
\end{tabular}




\begin{tabular}{|c|c|c|c|}
\hline t63 & 2.01059300 & -1.33847200 & 0.84244200 \\
\hline Pt 64 & -1.61596300 & -4.92190500 & 0.86821700 \\
\hline Pt 65 & -2.68327800 & 3.90946400 & -1.48232500 \\
\hline Pt 66 & 0.55834100 & -1.01921900 & 3.05478700 \\
\hline$=6$ & -0.06996100 & -0.04316300 & 0.06670400 \\
\hline \# 67 & Cluster $=$ Pt 68 & \multicolumn{2}{|c|}{$\mathrm{M}=0 \quad$ Etot $=-6606.54103000$} \\
\hline Pt 1 & -2.43323100 & 0.34416800 & 2.08643500 \\
\hline Pt2 & 5.02366300 & 0.56780400 & -0.19901500 \\
\hline Pt3 & -2.47901200 & -1.80937300 & -4.18801200 \\
\hline Pt 4 & 3.10389200 & 2.62511100 & 3.9090400 \\
\hline Pt 5 & -0.64555800 & 4.69185500 & -1.81046700 \\
\hline Pt 6 & 3.20988300 & -3.93727000 & 1.15246900 \\
\hline Pt 7 & 0.81930000 & -2.70685900 & 1.53821100 \\
\hline Pt 8 & -0.09063900 & 1.48355300 & 2.10833400 \\
\hline Pt9 & -3.71655100 & 3.02021700 & -3.18638700 \\
\hline Pt10 & 3.98130000 & 2.62389500 & -1.66599200 \\
\hline Pt11 & 0.16458300 & 5.01301500 & 0.69790100 \\
\hline Pt12 & -4.68308900 & 2.26882400 & 0.04324000 \\
\hline Pt13 & 0.44631500 & -0.65762200 & 5.42452400 \\
\hline Pt14 & -3.82297100 & 2.59370500 & 2.49400000 \\
\hline Pt15 & -1.48403500 & -4.33336100 & 1.59045300 \\
\hline Pt16 & -2.46274200 & -0.53755900 & 4.59203500 \\
\hline Pt 1 & 0.67785900 & 1.83814300 & 4.60566400 \\
\hline Pt18 & 2.51016600 & -2.74196400 & -4.34667600 \\
\hline Pt19 & -4.94277000 & -0.32622200 & 0.36322100 \\
\hline Pt20 & -0.17095100 & 0.85782100 & -5.02771500 \\
\hline Pt21 & 2.45295700 & -4.33599800 & -1.34182000 \\
\hline Pt2 & -4.70344500 & -1.82432200 & -2.79494900 \\
\hline Pt23 & -2.41775000 & -2.27193800 & -1.54903200 \\
\hline Pt24 & -4.64451000 & -0.63024200 & 2.95402700 \\
\hline Pt25 & 1.95378000 & 4.47916000 & -1.29997600 \\
\hline Pt26 & -1.56552100 & 2.14950300 & -1.98301800 \\
\hline Pt27 & -2.69026300 & -4.74898300 & -0.73702100 \\
\hline Pt 2 & 3.83417500 & 4.11525500 & 0.50296300 \\
\hline Pt29 & 0.02174800 & $0.034^{\circ}$ & -0.07394200 \\
\hline Pt30 & -2.01419300 & 2.36383100 & 0.56037600 \\
\hline Pt31 & 2.44852000 & 0.74254500 & -0.66056100 \\
\hline Pt32 & 0.47554200 & -3.93969700 & -3.09967500 \\
\hline Pt33 & 0.09932400 & -1.74758500 & -4.61156600 \\
\hline Pt 3 & 0.69149300 & -3.06626000 & 4.22152100 \\
\hline Pt35 & -0.34870300 & 3.88088400 & 3.16799800 \\
\hline Pt36 & 3.00665100 & -2.40147700 & 3.2608790 \\
\hline Pt37 & 0.98137100 & -5.27499700 & 1.80792400 \\
\hline Pt38 & -0.33159600 & 3.33114700 & -4.04540800 \\
\hline Pt39 & -0.87389900 & -0.42236900 & -2.51861800 \\
\hline Pt 40 & -0.39185300 & -0.96232400 & 3.00061100 \\
\hline Pt41 & -1.89235200 & -3.03737400 & 3.9039490 \\
\hline Pt 42 & -2.53602000 & 0.12427400 & -0.55851900 \\
\hline Pt 43 & -4.51929000 & 0.70731400 & -2.13114300 \\
\hline Pt 44 & 4.24071000 & 0.18608200 & -2.70796300 \\
\hline Pt 45 & 0.12307100 & -2.37340000 & -1.04864600 \\
\hline Pt4 6 & 4.06476900 & -2.41152900 & -2.25615900 \\
\hline Pt 47 & -2.65370200 & 0.79653900 & -4.0865810 \\
\hline Pt 48 & 5.01067400 & -2.06711100 & 0.22657200 \\
\hline Pt 49 & 2.47521300 & -1.82904900 & -0.25012000 \\
\hline Pt50 & 2.45458400 & 0.74350100 & -4.55973000 \\
\hline Pt51 & 1.85131700 & -0.31270500 & 1.77374800 \\
\hline & 1.77250200 & -0.94911100 & -2.59082100 \\
\hline
\end{tabular}




\begin{tabular}{|c|c|c|c|}
\hline Pt 53 & -3.67791900 & -2.87097400 & 2.01538700 \\
\hline Pt54 & 4.86669100 & 2.11888600 & 1.94344400 \\
\hline Pt55 & 0.85467600 & 1.46557700 & -2.57308200 \\
\hline Pt5 6 & -2.12901500 & -4.24561700 & -3.26717500 \\
\hline Pt57 & 2.07960200 & 4.62570200 & 2.46321400 \\
\hline Pt58 & 2.34378400 & 3.29218700 & -3.63673400 \\
\hline$=59$ & 2.70168900 & 0.04508800 & 4.24104900 \\
\hline Pt 60 & -1.56586900 & -1.80977900 & 0.87490300 \\
\hline FLO & 0.42881000 & 2.59634300 & -0.28777000 \\
\hline$=62$ & -3.09429100 & 4.21291100 & -0.93215700 \\
\hline$=6$ & -4.67528600 & -2.86687700 & -0.37904000 \\
\hline 64 & -0.02861700 & -4.95414700 & -0.61953000 \\
\hline 65 & -2.30987600 & 4.70805100 & 1.61055400 \\
\hline Pt 6 & 4.45476100 & -0.45932700 & 2.24486100 \\
\hline Pt 6 & 2.29579900 & 2.21566000 & 1.44992600 \\
\hline-68 & -1.92565700 & 2.00012300 & 4.19558400 \\
\hline \# 68 & Cluster $=$ Pt $69 \quad \mathrm{Q}=0$ & $=0 \quad \mathrm{Etot}=-67$ & 97965000 \\
\hline Pt1 & -4.03010800 & 2.51284900 & -2.2363350 \\
\hline Pt2 & 3.15902300 & -0.42250000 & -1.84072500 \\
\hline Pt3 & 0.90366200 & 0.32357700 & 5.30557400 \\
\hline Pt 4 & 1.79381500 & -4.40097700 & 1.15908400 \\
\hline Pt5 & 0.54766200 & -5.53183300 & -0.89975900 \\
\hline Pt 6 & 3.24013500 & 0.68148900 & 4.11898100 \\
\hline Pt 7 & -1.24711600 & -1.13995200 & -2.31435100 \\
\hline Pt 8 & 1.14267700 & -1.93346900 & -1.44371400 \\
\hline Pt9 & -2.16265900 & -1.50091400 & 0.18529500 \\
\hline Pt10 & -3.27872900 & -2.31178200 & -3.51099900 \\
\hline Pt11 & -0.45801700 & 1.26824800 & 2.06292900 \\
\hline Pt12 & -2.40410400 & 0.77310200 & -1.05873100 \\
\hline 7 & 3.57224400 & -1.72493900 & 3.1070260 \\
\hline Pt14 & 3.74760600 & -1.65835800 & -4.06157100 \\
\hline Pt15 & 2.80803200 & 3.99484700 & 2.26049400 \\
\hline Pt16 & -0.33585100 & 2.31796600 & -1.53056600 \\
\hline Pt17 & -5.37049300 & 1.39809900 & 0.96288200 \\
\hline Pt18 & 2.04491500 & 1.59540900 & -0.6907210 \\
\hline Pt19 & -0.38433100 & -1.93913800 & 4.88653400 \\
\hline Pt2 & 0.37081200 & 5.00813100 & -1.31874700 \\
\hline Pt21 & 0.70316100 & 3.66608600 & -3.55798500 \\
\hline Pt22 & 4.79925100 & -2.44690500 & -1.72830000 \\
\hline Pt23 & -3.03122600 & 4.12998200 & 2.41340000 \\
\hline Pt24 & 3.90473400 & -2.86553900 & 0.73569900 \\
\hline Pt2 2 & 0.25166500 & -2.27415700 & 1.01709000 \\
\hline Pt26 & -1.20993600 & 2.89507400 & 3.93422700 \\
\hline Pt27 & 5.59049600 & 0.19334600 & -1.24241800 \\
\hline Pt28 & -1.90548800 & 2.76314500 & 0.51286100 \\
\hline Pt29 & 1.63788900 & -3.22815000 & -3.66319400 \\
\hline Pt30 & -2.94416600 & 0.62361100 & 1.47784200 \\
\hline Pt31 & 4.61119200 & 1.98496700 & 2.17170300 \\
\hline Pt 32 & -1.75073800 & 2.65475800 & -3.71039500 \\
\hline Pt33 & -3.27177900 & 0.30190100 & -3.49479300 \\
\hline Pt 34 & -4.83695500 & -1.19598400 & 1.21955900 \\
\hline Pt35 & 1.15452100 & -0.70322000 & 2.90214600 \\
\hline Pt36 & -1.17224600 & 5.28635200 & 0.81478500 \\
\hline Pt37 & -1.38486200 & -1.05810400 & 2.62557000 \\
\hline Pt38 & -1.93479400 & -5.34140500 & 0.30142800 \\
\hline & 2.34883500 & -0.74275000 & 0.59095400 \\
\hline & -2.49481300 & -3.14497400 & 3.67566400 \\
\hline & -0.04530700 & 0.05388000 & -0.1886810 \\
\hline
\end{tabular}




\begin{tabular}{|c|c|c|c|}
\hline Pt 42 & 2.69344000 & -4.03189100 & -1.34765200 \\
\hline Pt 43 & 2.74086000 & 4.40454700 & -0.35143800 \\
\hline Pt 44 & -0.81404700 & -2.40963100 & -4.56771400 \\
\hline Pt 45 & 1.38582900 & -0.91557000 & -4.98325600 \\
\hline Pt 46 & -0.51340300 & -4.69817500 & -3.24102900 \\
\hline Pt 47 & -1.70757700 & 0.44110800 & 4.83161000 \\
\hline Pt 48 & -4.34075500 & 3.65724100 & 0.13757300 \\
\hline Pt 49 & 1.46492200 & -3.24226000 & 3.53098500 \\
\hline Pt50 & 0.36076600 & 4.75101900 & 2.87586100 \\
\hline Pt51 & 0.62372700 & 3.27642300 & 0.71808400 \\
\hline Pt52 & 2.08317800 & 1.49041800 & 1.89743900 \\
\hline Pt53 & -0.90767000 & -3.37419500 & -1.02942300 \\
\hline Pt5 4 & 4.89095600 & -0.43346400 & 1.20662100 \\
\hline Pt 5 & 0.83578800 & 0.33759600 & -2.69754400 \\
\hline Pt5 & 4.52186400 & 2.44278300 & -0.41152700 \\
\hline Pt5 & 1.42759800 & 2.68064200 & 4.21492800 \\
\hline Pt5 & -0.60181700 & -4.38456300 & 2.34216900 \\
\hline Pt59 & -3.51426900 & -3.47943900 & 1.28174600 \\
\hline Pt 6 & 2.00336800 & 1.65854600 & -4.61637600 \\
\hline Pt 6 & 3.12589800 & 3.04924000 & -2.61002700 \\
\hline Pt 6 & -2.20928900 & 4.35996900 & -1.59277600 \\
\hline Pt 6 & -0.92816300 & 0.33186200 & -4.64025900 \\
\hline Pt 64 & 4.39161800 & 0.94840200 & -3.66264700 \\
\hline Pt 6 & -3.81370800 & -0.77804900 & 3.63787500 \\
\hline Pt 6 & -3.67421300 & 1.88345200 & 3.64331800 \\
\hline Pt 6 & -4.93420500 & 0.12906400 & -1.35096300 \\
\hline Pt 6 & -2.97311100 & -4.52182600 & -2.09592800 \\
\hline Pt 6 & -4.29619900 & -2.43501900 & -1.0693880 \\
\hline \# 69 & Cluster $=$ Pt $70 \quad Q=0$ & $I=0 \quad$ Etot $=-68$ & 70986400 \\
\hline Pt 1 & 3.26659200 & -4.15916600 & -0.78037300 \\
\hline Pt2 & 2.71756500 & 0.51281700 & -4.17918400 \\
\hline Pt3 & -1.80010200 & 0.53830200 & -1.83964500 \\
\hline Pt 4 & -4.06043100 & -0.49846500 & -2.91963100 \\
\hline Pt5 & -2.10384000 & -0.75196600 & -4.66317300 \\
\hline Pt 6 & 2.74537100 & -3.42054200 & -3.28796700 \\
\hline Pt 7 & 4.19431900 & -2.02772400 & 2.23230000 \\
\hline Pt 8 & -5.32530400 & -1.80150500 & 1.19184200 \\
\hline Pt9 & -3.86347600 & -0.85951600 & 3.19611400 \\
\hline Pt10 & -3.36350600 & 1.80960600 & 3.34260100 \\
\hline Pt11 & -1.74937400 & -1.12278900 & 1.59258100 \\
\hline Pt12 & -1.34841600 & -4.93481700 & 1.35232400 \\
\hline Pt & -4.83205400 & 0.8 & 1.38873800 \\
\hline Pt14 & 2.39674500 & -3.97434500 & 1.71030700 \\
\hline Pt15 & 3.00018900 & -0.13820800 & 3.73237700 \\
\hline Pt16 & 4.58813500 & -1.96349500 & -0.35193100 \\
\hline Pt17 & -1.27377500 & 3.38261400 & 3.91113000 \\
\hline Pt18 & 0.80713100 & -5.02969000 & -0.23864700 \\
\hline Pt & -2.17978900 & 1.36640800 & 0.6830220 \\
\hline Pt20 & -3.18607500 & -3.23336300 & 0.4964930 \\
\hline Pt21 & -4.89330400 & -2.59463100 & -1.42653200 \\
\hline Pt22 & 5.11622500 & 0.48366200 & 2.28641900 \\
\hline Pt23 & -4.14392100 & 2.94499300 & -0.17666000 \\
\hline Pt24 & -1.35261500 & -1.96636400 & -1.0193860 \\
\hline Pt25 & -0.98190800 & 2.87758500 & -1.0602430 \\
\hline Pt26 & -0.00163100 & 0.01486800 & 0.0165100 \\
\hline Pt27 & 4.13126700 & -1.25902400 & -2.8107980 \\
\hline Pt28 & 0.60530800 & -1.17417700 & 2.91466900 \\
\hline Pt29 & 0.20208100 & -3.97267700 & 3.306140 \\
\hline
\end{tabular}




\begin{tabular}{|c|c|c|c|}
\hline Pt 30 & 2.17647800 & 2.32636300 & 4.12373100 \\
\hline Pt31 & 5.94531800 & 0.27677700 & -0.27101600 \\
\hline Pt 32 & -3.49200500 & -0.65410100 & -0.29859800 \\
\hline Pt33 & -1.77702400 & -4.57118300 & -1.32550800 \\
\hline Pt34 & 2.02952200 & -0.10938200 & -1.66885400 \\
\hline Pt35 & -2.80760500 & 4.48983000 & -1.88509600 \\
\hline Pt36 & -0.01396600 & 1.52583900 & 5.31270600 \\
\hline Pt37 & 4.27727500 & 3.35578900 & -0.38388600 \\
\hline Pt38 & 0.12074000 & -2.77753200 & 0.95930000 \\
\hline Pt39 & -0.88858700 & 0.92291000 & 2.91394900 \\
\hline$=40$ & -0.70033700 & -3.03457600 & -4.58791000 \\
\hline$=4$ & -0.62580600 & 3.96473400 & -3.45150000 \\
\hline Pt 42 & -0.03724500 & -1.01254300 & -3.02145600 \\
\hline Pt 43 & 0.97011800 & 4.49306300 & 3.20232200 \\
\hline Pt 44 & -0.22027900 & 2.97257100 & 1.44503900 \\
\hline Pt 45 & 0.86383400 & 4.87023200 & -1.44676300 \\
\hline$=46$ & -0.95802400 & -2.17102600 & 4.84390100 \\
\hline Pt 47 & -3.74363300 & 2.13992300 & -2.68499000 \\
\hline Pt 48 & -2.81914300 & -2.86237900 & -3.06128700 \\
\hline Pt 49 & 1.39624900 & 2.18424100 & -0.50724200 \\
\hline Pt 50 & 2.43555500 & -2.74292100 & 4.08014200 \\
\hline Pt51 & 1.54150300 & 2.86505100 & -4.74365300 \\
\hline Pt 52 & 0.38144400 & 1.54043100 & -2.83147100 \\
\hline Pt 53 & -2.57513800 & -3.18124700 & 3.05866000 \\
\hline Pt 54 & 2.18374900 & -1.26973700 & 0.70370300 \\
\hline Pt 55 & -5.52770100 & 0.87652300 & -1.17080100 \\
\hline Pt56 & 3.49773300 & 0.90631200 & 0.2529050 \\
\hline Pt 57 & 4.34773900 & 1.32108100 & -2.2429610 \\
\hline Pt5 & -1.13409600 & 5.18025700 & 0.2553180 \\
\hline $\mathrm{P}$ & 3.80140600 & 2.77933800 & 2.13654300 \\
\hline Pt 60 & 2.09691800 & 4.35782600 & 0.84814500 \\
\hline Pt 61 & 1.49867900 & 1.05201800 & 1.90037000 \\
\hline Pt 62 & 0.39597700 & -4.61920300 & -2.82663900 \\
\hline Pt 63 & 1.59125600 & -1.76136100 & -4.9665940 \\
\hline Pt 64 & 1.18793700 & -0.80586600 & 5.4945070 \\
\hline $\mathrm{P}$ & 2.74197400 & 3.40070000 & -2.50021300 \\
\hline Pt 66 & -2.24457900 & 0.08503500 & 5.03471100 \\
\hline Pt 67 & -2.78604400 & 3.92104300 & 1.83387300 \\
\hline Pt 68 & 1.19964000 & -2.55690800 & -1.3737750 \\
\hline Pt 69 & -1.87174600 & 1.88451100 & -4.4703110 \\
\hline Pt 70 & 0.23050800 & 0.50095200 & -5.2787000 \\
\hline \# 70 & Cluster $=$ Pt7 1 & \multicolumn{2}{|c|}{$\mathrm{M}=0 \quad$ Etot $=-6935.63899100$} \\
\hline Pt1 & -3.20498600 & -3.97743700 & -2.2683740 \\
\hline Pt2 & 3.03433500 & 4.11605600 & 1.8649120 \\
\hline Pt3 & -2.63986500 & -1.94620900 & -4.5566800 \\
\hline Pt 4 & -0.20869400 & -2.91170400 & $-4.742917 C$ \\
\hline Pt 5 & 0.65813200 & 5.03104200 & 2.56036800 \\
\hline Pt 6 & -1.61528800 & 3.02933000 & 0.19491700 \\
\hline Pt 7 & 1.79072800 & 3.20819600 & 4.1230080 \\
\hline Pt 8 & -0.23291400 & 2.57361700 & 2.3662220 \\
\hline Pt9 & 0.79693400 & -0.46408200 & -4.89950900 \\
\hline Pt10 & -2.29244700 & 0.87605300 & -1.09663200 \\
\hline Pt11 & 3.53504500 & 1.12538400 & 4.2040160 \\
\hline Pt12 & -0.26743400 & 5.34186900 & $0.0853770 \mathrm{c}-\mathrm{r}-\mathrm{r}-\mathrm{s}$ \\
\hline Pt13 & -3.41020200 & 0.04204200 & 3.8894460 \\
\hline Pt & -0.05785500 & 0.02465200 & 0.0564660 \\
\hline & 4.75384800 & -0.52172500 & -1.6217190 \\
\hline & -1.88324300 & -1.58758100 & 2.4919350 \\
\hline
\end{tabular}




\begin{tabular}{|c|c|c|c|}
\hline Pt17 & 2.65753900 & 3.88161300 & -3.17291400 \\
\hline Pt18 & 2.15447200 & -4.56743300 & 2.13775200 \\
\hline Pt19 & 0.94771200 & -5.59108800 & 0.02606600 \\
\hline Pt20 & -2.06916500 & 0.86803700 & 1.56921400 \\
\hline Pt21 & -2.18632700 & 4.71232700 & -1.73374500 \\
\hline Pt22 & -4.84472200 & 0.56849700 & 1.71217700 \\
\hline Pt23 & 0.34255400 & 4.96745300 & -2.53860800 \\
\hline Pt24 & -0.58676700 & 2.56107300 & -2.14298700 \\
\hline Pt25 & 3.63650900 & -1.34412300 & 3.24768000 \\
\hline Pt26 & -0.25071500 & 0.02299800 & -2.53007800 \\
\hline Pt27 & -0.80749300 & -3.34304800 & 4.32092700 \\
\hline Pt28 & 1.57137500 & -3.04414500 & -0.08726100 \\
\hline Pt29 & 2.14009200 & 1.60381100 & 2.07279500 \\
\hline Pt 30 & -4.85561500 & -2.01810900 & -1.53041300 \\
\hline Pt31 & -3.91971000 & 0.03292900 & -3.25204100 \\
\hline Pt 32 & -0.17892700 & 1.63643100 & 4.86114700 \\
\hline Pt 33 & 3.78639600 & -2.96109200 & -1.54802400 \\
\hline Pt 34 & -1.56832300 & -4.86196800 & -0.33681100 \\
\hline Pt 35 & 2.16549200 & -0.82395300 & 1.15457700 \\
\hline Pt 36 & -2.91317400 & -3.97377300 & 2.83241100 \\
\hline Pt 37 & 1.52095400 & -0.45684000 & 4.84493800 \\
\hline Pt 38 & -0.99630400 & -2.37756700 & 0.12901900 \\
\hline Pt 39 & 3.26786300 & -0.00036100 & -3.82591000 \\
\hline Pt 40 & -4.05756100 & 2.99215200 & -0.96410900 \\
\hline Pt 41 & 0.16624100 & 0.01445000 & 2.66068400 \\
\hline Pt 42 & -5.02460800 & 0.55380500 & -0.89044500 \\
\hline Pt 43 & 3.94498000 & -2.94989700 & 1.09210000 \\
\hline Pt 44 & 1.74394700 & 2.01175400 & -4.89165900 \\
\hline Pt 45 & 4.91631500 & -0.50073800 & 1.01810900 \\
\hline Pt 46 & -3.88274700 & 2.99405200 & 1.63889800 \\
\hline Pt 47 & 0.90525800 & 2.41709700 & -0.01583200 \\
\hline Pt 48 & 4.51991400 & 3.41598400 & -0.24604600 \\
\hline Pt 49 & -2.22569100 & -1.57136500 & -2.01814200 \\
\hline Pt 50 & -0.78826300 & -4.80675100 & -2.91043000 \\
\hline Pt51 & 4.43602600 & 1.99392900 & -2.44399800 \\
\hline Pt52 & 2.30136000 & -2.43733400 & -3.75228700 \\
\hline Pt53 & 2.00729400 & -0.83975100 & -1.44513700 \\
\hline Pt 54 & 1.81503200 & -4.61473500 & -2.24434400 \\
\hline Pt5 5 & -2.43226900 & 2.50783600 & 3.81495200 \\
\hline Pt 56 & -2.94490500 & 2.49083800 & -3.32629600 \\
\hline Pt 57 & -4.57622400 & -1.97567200 & 2.39105900 \\
\hline Pt58 & -3.85142400 & -3.69492500 & 0.35838700 \\
\hline Pt59 & 3.22755600 & 1.13180700 & -0.21342000 \\
\hline Pt 60 & 1.89114200 & -3.01961000 & 4.32924000 \\
\hline Pt61 & -0.57013500 & -4.97238100 & 2.13948200 \\
\hline Pt 62 & 0.16368100 & -2.50186500 & -2.18686900 \\
\hline Pt 63 & 0.50332800 & -2.52318200 & 2.19747600 \\
\hline Pt 64 & 4.74154000 & 1.95569000 & 1.94068200 \\
\hline Pt 65 & 1.78738600 & 1.59220200 & -2.31055400 \\
\hline Pt 66 & 2.18041700 & 4.59023400 & -0.62237100 \\
\hline Pt 67 & -0.68425500 & 2.98473200 & -4.70564800 \\
\hline Pt 68 & -3.24716800 & -1.08534900 & 0.31922500 \\
\hline Pt 69 & -1.93075000 & 4.69471900 & 2.18954100 \\
\hline Pt 70 & -1.65475900 & 0.51434200 & -4.6981530 \\
\hline Pt71 & -1.15046500 & -0.81323900 & 4.93515400 \\
\hline H & Cluster $=$ Pt72 $\quad \mathrm{Q}=0$ & \multirow{3}{*}{\multicolumn{2}{|c|}{$\begin{array}{c}\mathrm{M}=0 \quad \mathrm{Etot}=-7047.18621100 \\
0.82994500\end{array}$}} \\
\hline Pt1 & 3.40799200 & & \\
\hline & 2.28855100 & & \\
\hline
\end{tabular}




\begin{tabular}{|c|c|c|c|}
\hline Pt 3 & 1.14405300 & 0.62996700 & 2.18328400 \\
\hline Pt 4 & -2.43503900 & -1.71655700 & 1.73624300 \\
\hline Pt 5 & -2.29272100 & 2.59968900 & 0.53063600 \\
\hline Pt 6 & 1.48017800 & -1.28573400 & -4.63026500 \\
\hline Pt 7 & -2.10011600 & -4.28574600 & -2.67738300 \\
\hline Pt 8 & -0.86700300 & 0.07643100 & -5.07921300 \\
\hline Pt9 & -2.20661500 & 5.15543900 & -0.16304600 \\
\hline Pt10 & 4.57152300 & -0.99152500 & 1.86586100 \\
\hline Pt11 & -4.71034300 & 1.06325300 & -1.88105700 \\
\hline Pt12 & 0.09984500 & 5.43497000 & 1.20787700 \\
\hline Pt13 & 3.75360600 & 1.62932300 & -3.88625200 \\
\hline Pt14 & 4.21542100 & 3.40968600 & -0.75009200 \\
\hline Pt15 & 1.36853700 & -3.82982400 & -3.81873500 \\
\hline Pt16 & -2.12622600 & 4.50264200 & 2.40892500 \\
\hline Pt17 & 2.19064000 & 2.47230000 & 0.72461700 \\
\hline Pt18 & -3.26652900 & 0.47976900 & -3.98267100 \\
\hline Pt19 & 4.75799600 & 0.86127900 & -1.44522400 \\
\hline Pt20 & 2.33110800 & -4.03736700 & 3.16715300 \\
\hline Pt21 & 0.02519100 & 2.64656800 & -0.75027700 \\
\hline Pt22 & 1.06047700 & 5.03586900 & -1.22180100 \\
\hline Pt23 & -0.09125600 & 0.14928100 & -0.05157000 \\
\hline Pt24 & -0.14573400 & -2.34002000 & 0.65006900 \\
\hline Pt25 & -1.49475500 & 0.70627600 & 2.12573300 \\
\hline Pt26 & -4.83751100 & 1.89315800 & 0.63750400 \\
\hline Pt27 & -2.32578500 & -2.40292100 & -0.76959700 \\
\hline Pt28 & 3.69105200 & -0.93154400 & -3.21621300 \\
\hline Pt29 & -2.17325400 & 1.88963800 & -1.97113300 \\
\hline Pt30 & 4.66799500 & -1.67804600 & -0.74491200 \\
\hline Pt31 & 2.04620500 & -1.77836900 & 1.91394900 \\
\hline Pt32 & -0.95091200 & -2.45749200 & -4.35159300 \\
\hline Pt33 & -4.59843000 & -3.19596100 & 1.99032500 \\
\hline Pt3 4 & -4.87919000 & -0.64962600 & 1.32797800 \\
\hline Pt35 & 1.13443500 & 3.80720900 & 3.96813900 \\
\hline Pt36 & 1.08486600 & 1.26900400 & 4.72957000 \\
\hline Pt37 & -1.35580800 & 0.08032200 & 4.94571100 \\
\hline Pt38 & 2.78652400 & 4.03603400 & -2.92188900 \\
\hline Pt39 & 2.15239700 & -2.50319200 & -0.55919600 \\
\hline Pt 40 & -1.31706600 & -0.54260600 & -2.30711000 \\
\hline Pt41 & -1.79200800 & -4.92824600 & -0.04466700 \\
\hline Pt42 & 2.17003700 & -5.07246900 & -0.13893000 \\
\hline Pt 43 & -4.78777500 & -1.45890400 & -1.17147900 \\
\hline Pt 44 & -1.13232700 & 4.38132200 & -2.46867300 \\
\hline Pt 45 & -2.72051700 & 0.09660100 & -0.14122400 \\
\hline Pt4 6 & -3.57848800 & 2.34940100 & 2.88355000 \\
\hline Pt 47 & 2.61908500 & -0.01594800 & 0.10371100 \\
\hline Pt 48 & -2.22111400 & -4.28584000 & 2.48690200 \\
\hline Pt 49 & -2.36496300 & -2.35371000 & 4.35457600 \\
\hline Pt50 & -4.35083800 & -4.01735500 & -0.47422300 \\
\hline Pt51 & 0.26075800 & -4.70512100 & 1.62216000 \\
\hline Pt52 & 0.61619100 & 3.67721400 & -4.37942500 \\
\hline Pt53 & -0.02067000 & -2.75741700 & -1.89611200 \\
\hline Pt5 4 & -1.89023200 & 2.70552300 & -4.44049300 \\
\hline Pt55 & -0.07086700 & 2.89912800 & 1.84457000 \\
\hline Pt5 6 & 1.53969600 & 1.29136500 & -5.27077500 \\
\hline Pt57 & 0.21295200 & -5.33642200 & -1.83994400 \\
\hline Pt58 & 4.64181400 & 1.54972800 & 1.16973000 \\
\hline Pt59 & -0.22223800 & -1.36854300 & 3.05398500 \\
\hline Pt 60 & -0.09391500 & -3.73357700 & 4.1370970 \\
\hline & 55 & -1.30753200 & 3 \\
\hline
\end{tabular}




\begin{tabular}{|c|c|c|c|}
\hline Pt 62 & -1.25695300 & 2.59828900 & 4.19100800 \\
\hline Pt 63 & 3.45680700 & 3.37405500 & 2.82787200 \\
\hline Pt 64 & 3.27302700 & -1.72041400 & 4.21332900 \\
\hline Pt 65 & 3.60067500 & -3.46638300 & -2.49744100 \\
\hline t 66 & -4.02737200 & 3.59576600 & -1.28672700 \\
\hline 67 & 3.94921100 & -3.52167000 & 1.11113600 \\
\hline$=68$ & -3.65734700 & -0.15632900 & 3.60027500 \\
\hline Pt 69 & -3.36817700 & -2.03897400 & -3.30186900 \\
\hline Pt70 & 0.13172400 & 1.47625900 & -3.08031500 \\
\hline 571 & 1.33084000 & -0.58014100 & -2.09897000 \\
\hline$t 72$ & 2.71352900 & 5.00798400 & 0.82426800 \\
\hline 72 & Cluster $=$ Pt73 $\quad \mathrm{Q}=0$ & \multicolumn{2}{|c|}{$\mathrm{M}=0 \quad$ Etot $=-7159.87964700$} \\
\hline Pt1 & 0.40623500 & -3.21230600 & -2.13311200 \\
\hline Pt2 & 1.10307800 & -2.92415700 & 0.37237800 \\
\hline Pt 3 & -0.03195000 & -0.94502700 & -0.90963500 \\
\hline Pt 4 & -3.61454600 & -1.14671100 & -4.47714700 \\
\hline Pt 5 & 3.17784000 & 4.30554200 & -0.10340200 \\
\hline Pt 6 & -3.60131700 & 2.62099700 & -1.0587120 \\
\hline Pt 7 & 2.94522400 & -3.66275200 & 2.21739300 \\
\hline Pt 8 & 1.49208600 & -5.56846100 & -2.27399900 \\
\hline Pt9 & 2.24289600 & -5.30330200 & 0.23037100 \\
\hline Pt10 & -3.95099600 & 0.85112700 & 3.43189700 \\
\hline 11 & 2.93181700 & 1.75845400 & 0.48702600 \\
\hline$=12$ & -0.69147200 & 2.48236000 & 5.001829 \\
\hline 13 & -4.98115200 & -1.74154200 & 0.51754500 \\
\hline Pt14 & 3.70777200 & -3.15247300 & -0.34720700 \\
\hline Pt15 & 0.71528300 & 3.12470300 & -0.0494700 \\
\hline Pt16 & -5.41565700 & 0.85821500 & -1.7245400 \\
\hline Pt17 & 1.18720600 & -2.83026900 & -4.68083000 \\
\hline 18 & 2.20051200 & 3.03617900 & -3.47888000 \\
\hline Pt19 & -1.81987800 & -1.81343000 & -2.57389000 \\
\hline Pt20 & 1.38859300 & -0.19758700 & -5.16525200 \\
\hline Pt21 & -0.31950700 & 5.60549400 & 0.08436100 \\
\hline Pt22 & 3.96388100 & 0.65248200 & 4.7187990 \\
\hline Pt23 & -2.84006400 & 3.03649800 & -3.55133700 \\
\hline $\mathrm{P}$ & 0.23671600 & 2.17685400 & -5.02514800 \\
\hline Pt25 & -1.45980900 & -2.03660400 & 4.20662000 \\
\hline Pt26 & 4.36057100 & -1.30845400 & -2.1855440 \\
\hline Pt27 & -2.14859400 & 1.00209700 & -5.09019700 \\
\hline Pt28 & -1.06418000 & -5.40764000 & -1.57583200 \\
\hline Pt29 & -2.61361300 & 3.08367600 & 3.25196300 \\
\hline Pt & 3.60589700 & -1.87293900 & 4.08635200 \\
\hline Pt31 & -3.26116000 & -3.98496200 & -2.2679170 \\
\hline Pt32 & -2.15615500 & 4.75774000 & -1.61653400 \\
\hline Pt33 & -0.32691000 & 3.05831600 & -2.60518800 \\
\hline Pt3 4 & 2.52291100 & -0.75560600 & -0.21958800 \\
\hline Pt35 & -2.15290400 & -3.78096800 & 2.24582700 \\
\hline Pt36 & 4.38695900 & -1.44311500 & 1.57423800 \\
\hline Pt37 & -0.77071300 & -1.46822700 & 1.57859500 \\
\hline Pt38 & -1.11525400 & 5.14344000 & 2.59344700 \\
\hline Pt39 & 2.19439800 & 1.28809300 & 2.92566900 \\
\hline Pt 40 & -2.11013100 & 0.28123600 & 5.24192400 \\
\hline Pt41 & -1.49560000 & -3.13860700 & -0.29521100 \\
\hline Pt 42 & 1.79275200 & -1.10831300 & -2.73543100 \\
\hline Pt4 3 & 1.51917700 & 1.24079600 & -1.64083300 \\
\hline 44 & 1.78152700 & -1.21665600 & 2.29173600 \\
\hline & -4.48447000 & -1.62484500 & -2.0407620 \\
\hline & 5830500 & -1 & \\
\hline
\end{tabular}




\begin{tabular}{|c|c|c|c|}
\hline Pt 47 & 4.80545500 & 1.07784400 & 2.24212800 \\
\hline Pt 48 & -4.45120500 & 0.87604800 & 0.79404800 \\
\hline Pt 49 & -1.01991300 & -1.43132100 & -5.09451900 \\
\hline Pt50 & 1.71329600 & 3.50987200 & 4.28845500 \\
\hline 51 & -3.86102800 & -4.12537300 & 0.28857000 \\
\hline 52 & 3.41764200 & 0.68967500 & -3.66130200 \\
\hline$=53$ & -2.59819200 & -0.76060500 & -0.22870000 \\
\hline Pt5 4 & 1.43061400 & 0.87118700 & 5.48203200 \\
\hline Pt55 & 1.08547600 & -1.70457200 & 4.87447100 \\
\hline Pt56 & -0.16163300 & 0.21868400 & 3.47863100 \\
\hline Pt57 & 1.34523000 & 4.96673200 & -1.88911900 \\
\hline$=58$ & -2.95070500 & 0.56514500 & -2.55082400 \\
\hline$=59$ & 0.61792500 & 0.69056000 & 0.92888600 \\
\hline Pt 60 & 2.99797400 & -3.47117500 & -2.87130300 \\
\hline Pt61 & 5.02762400 & 0.37036700 & -0.29842600 \\
\hline$=62$ & 4.09630900 & 2.39772700 & -1.74132500 \\
\hline Pt 63 & 1.46219800 & 4.81558700 & 1.92746600 \\
\hline$=64$ & -4.35492900 & 3.52782700 & 1.27630100 \\
\hline$=65$ & 0.41427800 & -3.52803600 & 2.992179 \\
\hline$=66$ & -0.29486000 & -5.17856300 & 0.96944900 \\
\hline Pt 67 & -1.24401800 & -3.93301500 & -4.07789800 \\
\hline t 68 & -0.03791700 & 2.67136000 & 2.46734500 \\
\hline Pt 69 & -1.92540600 & 0.93264500 & 1.67463700 \\
\hline Pt70 & 3.68801300 & 3.46369200 & 2.39404600 \\
\hline Pt71 & -0.38521500 & 0.35500000 & -3.162820 \\
\hline Pt72 & -1.17560100 & 1.38909400 & -0.82022000 \\
\hline Pt 73 & -1.81040400 & 3.43502300 & 0.67513100 \\
\hline 7 & Cluster $=$ Pt7 $4 \quad \mathrm{Q}=0$ & $=0 \quad$ Etot $=-72$ & 21094000 \\
\hline Pt1 & 0.55362800 & -0.06837300 & 5.6148160 \\
\hline Pt2 & -1.69667000 & -1.41794400 & 5.20732400 \\
\hline Pt 3 & 1.69166500 & 0.52873300 & 3.24006400 \\
\hline Pt 4 & -0.25223800 & -4.29949600 & -2.9458740 \\
\hline Pt 5 & -1.62750300 & 5.05472900 & -1.48761900 \\
\hline Pt 6 & 1.53223600 & 0.87143600 & -5.4961610 \\
\hline Pt 7 & -3.47940300 & -3.76501300 & 2.7145850 \\
\hline Pt 8 & 4.49943300 & 1.10075800 & -3.01393400 \\
\hline Pt9 & 1.31375400 & -3.55975800 & 4.9231140 \\
\hline Pt10 & -1.04459100 & 2.73046200 & -2.4816440 \\
\hline Pt11 & -2.15049300 & -0.96908100 & -2.4073460 \\
\hline Pt12 & -0.02754000 & 2.97316200 & -4.9434580 \\
\hline Pt13 & -0.09815000 & -1.24462500 & -5.68866400 \\
\hline Pt14 & -1.84364000 & -0.33615500 & 2.78787500 \\
\hline Pt15 & -4.04329200 & 37600 & 3.584509 \\
\hline Pt16 & 1.18563800 & -2.75829400 & 1.1717250 \\
\hline Pt17 & 2.04918300 & 4.99430200 & -0.78416300 \\
\hline Pt18 & -3.89049000 & -2.73441200 & 0.31271400 \\
\hline Pt19 & -4.63709600 & 2.85422700 & -0.03816500 \\
\hline Pt20 & 2.30723500 & -3.44950800 & -2.2721010 \\
\hline Pt21 & 0.89042400 & 2.45242500 & 4.8352490 \\
\hline Pt22 & -1.36462300 & -2.65126400 & 1.6958180 \\
\hline Pt23 & 1.86374300 & -2.83997900 & -4.81574300 \\
\hline Pt24 & -1.90297600 & -2.78881400 & -4.37871600 \\
\hline Pt25 & 3.43638200 & -0.77013800 & -4.56822500 \\
\hline Pt26 & -2.62758600 & -0.38405200 & -5.1648050 \\
\hline Pt27 & -0.44298700 & 1.84234600 & 2.54824100 \\
\hline Pt28 & 3.91932300 & 3.28571500 & -1.58405500 \\
\hline 29 & -4.22143500 & 0.54703700 & -3.2978570 \\
\hline 0 & -2.79270300 & 1.32085900 & -1.229208 \\
\hline
\end{tabular}




\begin{tabular}{|c|c|c|c|}
\hline Pt 31 & -1.17943800 & -3.78803600 & 4.08601100 \\
\hline Pt 32 & -1.98839600 & -4.76775100 & 0.25584200 \\
\hline Pt33 & 1.66603300 & -1.26915100 & -0.91802300 \\
\hline Pt 34 & 2.71330100 & -1.32283700 & 4.79630000 \\
\hline Pt35 & 2.17742600 & 4.26138100 & 1.75747500 \\
\hline Pt 36 & -2.21921800 & -0.69631300 & 0.22945200 \\
\hline Pt 37 & 3.09922400 & -4.27504100 & 0.10748800 \\
\hline t38 & 3.24733000 & 0.82521200 & -0.74649300 \\
\hline Pt39 & 0.60582400 & -4.82598000 & -0.53254900 \\
\hline Pt 4 & -3.59314000 & 3.52762100 & -2.43891300 \\
\hline Pt & -0.12998600 & 5.07612000 & 0.70826800 \\
\hline t4 & 1.34309800 & 2.54173400 & -1.45599600 \\
\hline Pt 43 & -2.70437500 & -3.62629200 & -1.99279900 \\
\hline Pt 44 & 1.81614100 & 0.41492900 & -2.88624300 \\
\hline Pt 4 & 4.35976100 & 0.20652600 & 3.27111400 \\
\hline Pt 4 & 4.05840900 & -1.46255200 & -2.08458500 \\
\hline Pt 4 & 0.20833400 & -0.33196100 & 1.13710500 \\
\hline Pt 48 & -2.65971600 & 4.30846900 & 0.88460300 \\
\hline Pt 49 & 4.17122800 & 2.68297600 & 0.98374700 \\
\hline Pt50 & -2.48971400 & 3.37839500 & 3.35733900 \\
\hline Pt & -0.03646600 & -5.04073600 & 2.03859700 \\
\hline Pt 5 & -3.98018100 & -1.45280300 & 3.82665000 \\
\hline Pt53 & 1.63652100 & 1.82285500 & 0.99932600 \\
\hline Pt5 4 & 4.14447500 & -2.42395500 & 2.88677200 \\
\hline Pt 55 & -4.58380200 & -0.44898900 & 1.45447100 \\
\hline Pt56 & 5.30392600 & 0.31456000 & 0.74480300 \\
\hline Pt 57 & -1.62183200 & 1.30779400 & 4.89685200 \\
\hline Pt 5 & 0.34041800 & -1.65917400 & 3.42614000 \\
\hline Pt & -2.79185500 & 1.63357300 & 1.34793100 \\
\hline Pt 60 & -5.31495900 & 0.44778900 & -0.88859300 \\
\hline Pt 61 & 0.63390000 & 4.67525000 & -2.97906300 \\
\hline Pt 6 & 3.24914900 & 2.59461700 & 3.52534000 \\
\hline Pt 63 & -0.01182600 & 4.34811800 & 3.25067100 \\
\hline Pt 64 & -0.73841400 & 2.60593700 & 0.10064000 \\
\hline Pt 65 & -0.57556200 & 0.60981400 & -3.87356000 \\
\hline Pt 66 & -2.56996600 & 2.25078600 & -4.59757800 \\
\hline Pt 67 & 0.30025200 & -1.72268500 & -3.07127800 \\
\hline Pt 6 & 2.46321400 & -4.42547400 & 2.71182000 \\
\hline Pt 6 & -0.27872000 & 0.43732000 & -1.24957500 \\
\hline Pt & -4.62037000 & -1.81141000 & -2.08707900 \\
\hline Pt71 & -0.63065100 & -2.46741700 & -0.78009100 \\
\hline Pt72 & 2.84076800 & -0.73643700 & 1.30625600 \\
\hline Pt73 & 2.47413900 & 2.83573200 & -3.85938500 \\
\hline Pt 74 & 4.76649100 & -2.26717400 & 0.31249500 \\
\hline \# 74 & Cluster $=$ Pt75 $Q=0$ & \multicolumn{2}{|c|}{$\mathrm{M}=0 \quad$ Etot $=-7381.26288400$} \\
\hline Pt1 & 0.85929200 & 0.43521100 & -5.26518300 \\
\hline Pt2 & -5.39042900 & 0.95013900 & -1.82265600 \\
\hline Pt3 & 3.15261200 & -2.74758300 & 3.15982900 \\
\hline Pt 4 & -0.12528900 & -5.31898500 & 0.1826540 \\
\hline Pt5 & -0.75193100 & 1.18190400 & 0.42435900 \\
\hline Pt 6 & 3.91811300 & -3.37331400 & -3.35066000 \\
\hline Pt7 & 0.65483800 & -0.80249500 & -0.30417200 \\
\hline Pt 8 & -3.74858600 & -0.93244400 & -2.64060400 \\
\hline Pt9 & -2.11624100 & -4.37522500 & 1.72985000 \\
\hline Pt10 & 5.03496100 & 0.32227400 & 2.39038900 \\
\hline Pt11 & 1.47711200 & -0.77620900 & 2.14806900 \\
\hline Pt12 & -2.71199800 & -2.91648600 & 3.94048800 \\
\hline Pt13 & -1.06028100 & 4.96719100 & -1.81414000 \\
\hline
\end{tabular}




\begin{tabular}{|c|c|c|c|}
\hline Pt14 & -1.45478400 & -0.05276800 & -1.74923100 \\
\hline Pt15 & 1.72822000 & 1.65867900 & 1.02377400 \\
\hline Pt16 & -2.74667300 & -3.00401300 & -3.93371100 \\
\hline Pt17 & 1.72476800 & -4.70729700 & 2.10637800 \\
\hline Pt18 & -1.89583400 & 2.47553800 & -1.54317600 \\
\hline Pt19 & 0.63414400 & -3.20755500 & -4.61574200 \\
\hline Pt20 & 5.42382100 & 1.39119200 & 0.02098600 \\
\hline Pt21 & 3.12676400 & -0.23975500 & 4.09461900 \\
\hline Pt22 & -1.87656100 & 5.41288700 & 0.69471500 \\
\hline Pt23 & 4.99881800 & -2.12657100 & 1.37623400 \\
\hline Pt24 & 4.54370400 & -3.55707800 & -0.80421000 \\
\hline Pt25 & -3.38199100 & 0.66951200 & -0.22196300 \\
\hline Pt26 & 1.61889100 & 1.72785200 & 4.9517110 \\
\hline Pt27 & -0.27721200 & -3.87348000 & 3.64105800 \\
\hline Pt28 & -2.25449600 & 4.33410700 & 3.08082700 \\
\hline Pt29 & 0.12175900 & 3.71376000 & 4.12045000 \\
\hline Pt30 & -2.49298900 & 0.67146900 & 2.28011500 \\
\hline Pt31 & -3.94484400 & 2.71188700 & -3.14390500 \\
\hline Pt32 & 2.80159300 & -1.57619000 & -1.7821280 \\
\hline Pt33 & 4.10669500 & 2.54907800 & -1.92809300 \\
\hline Pt34 & -4.36572800 & 2.45658200 & 2.89737300 \\
\hline Pt35 & 0.51521100 & -1.14479000 & -2.93769500 \\
\hline Pt36 & 1.12434900 & -1.90403800 & 4.64926900 \\
\hline Pt37 & -0.44327500 & 0.09113700 & 5.35279800 \\
\hline Pt38 & 2.04684200 & 4.49244800 & 2.36760300 \\
\hline Pt39 & -3.70859300 & 4.34767000 & -1.04039300 \\
\hline Pt 40 & 2.46381500 & -5.13469600 & -0.42236900 \\
\hline Pt 41 & -2.66138200 & 2.90998400 & 0.91856000 \\
\hline Pt 42 & -5.48943100 & -0.76448600 & 0.31093400 \\
\hline Pt43 & 2.62477000 & 4.63745600 & -1.37292300 \\
\hline Pt 44 & 0.94440400 & 4.08129500 & -3.35880700 \\
\hline Pt4 5 & 3.35936900 & -0.20668600 & 0.41423600 \\
\hline Pt 46 & -4.29098300 & -2.81969600 & -0.90370700 \\
\hline Pt 47 & 5.37608800 & -1.00238100 & -1.15137500 \\
\hline Pt 48 & 2.59515800 & -1.42937700 & -4.51499400 \\
\hline Pt 49 & -1.50985800 & 3.33404600 & -4.09031800 \\
\hline Pt50 & -5.19534100 & 2.56667200 & 0.35155300 \\
\hline Pt51 & 2.09012800 & 0.94150300 & -1.55056700 \\
\hline Pt52 & -3.88914100 & -2.41209100 & 1.6533650 \\
\hline Pt53 & 1.81765000 & -4.90335600 & -2.97739800 \\
\hline Pt5 4 & -2.96235400 & -0.41466700 & 4.66868900 \\
\hline Pt55 & -1.83067400 & -1.20788100 & 0.61488200 \\
\hline Pts & 4.18208000 & 0.19964800 & -3.18845100 \\
\hline Pt57 & -1.11843100 & -1.29392100 & -5.02738300 \\
\hline Pt58 & 2.49537400 & -2.65274700 & 0.5987160 \\
\hline Pt59 & 0.08794500 & 1.53282500 & -3.05550500 \\
\hline Pt 60 & 0.72890900 & 5.49741300 & 0.21251700 \\
\hline Pt 61 & 3.33477400 & 2.25120100 & 3.02577100 \\
\hline Pt 62 & -1.74564100 & -2.56328600 & -1.57602000 \\
\hline Pt 63 & -2.04659700 & 2.09548100 & 4.49390300 \\
\hline Pt 64 & 3.72263000 & 3.33885100 & 0.63914800 \\
\hline Pt 65 & -0.18127100 & 3.45310600 & 1.54738700 \\
\hline Pt 66 & 0.60625700 & 3.00606300 & -0.91546600 \\
\hline Pt 67 & 2.50171800 & 2.05993000 & -4.00540900 \\
\hline Pt 68 & -2.56734100 & -4.88282000 & -0.79778400 \\
\hline Pt69 & -0.00226800 & 1.23249700 & 2.94017400 \\
\hline Pt70 & -4.88231500 & -0.19232400 & 2.81501000 \\
\hline Pt71 & -0.01234100 & -2.86463400 & 1.2141050 \\
\hline & 0.74727500 & -3.20908700 & -1.29963 \\
\hline
\end{tabular}




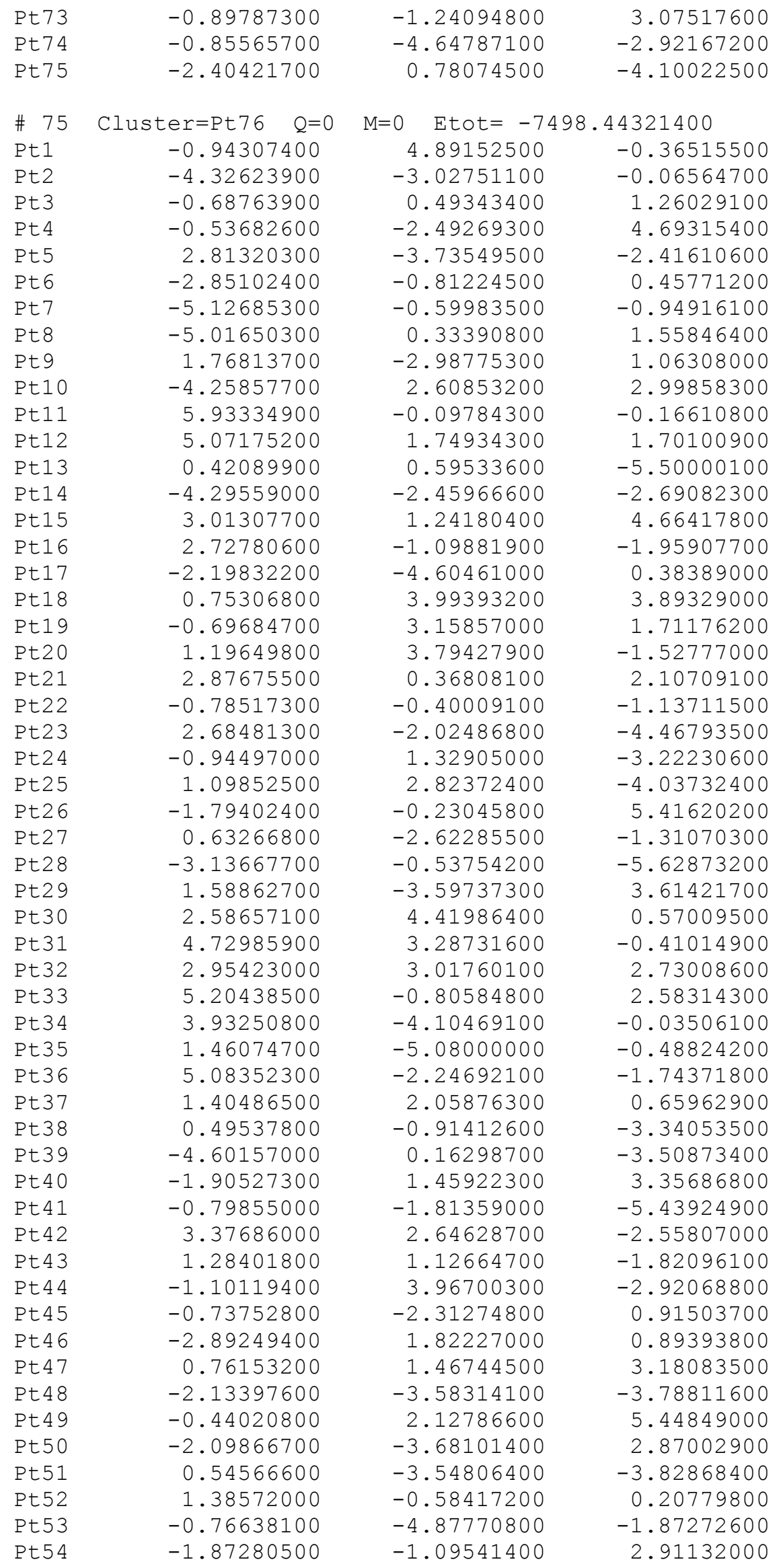




\begin{tabular}{|c|c|c|c|}
\hline Pt5 5 & -2.01071300 & 4.03099500 & 3.86651100 \\
\hline Pt5 6 & 4.88673900 & 0.33761400 & -2.62339500 \\
\hline Pt57 & -2.16451000 & -0.97183900 & -3.26895000 \\
\hline Pt58 & 3.99680700 & -3.24017900 & 2.47662000 \\
\hline Pt59 & -3.37986200 & 2.57074100 & -3.57063200 \\
\hline 60 & 0.87312100 & -0.25090800 & 5.24178900 \\
\hline$=61$ & 3.04268600 & -1.37851600 & 4.14045900 \\
\hline Pt 62 & -0.84035700 & 2.23076800 & -0.74257100 \\
\hline-03 & -2.99487800 & 0.89989500 & -1.56878700 \\
\hline 64 & 0.05134000 & -4.79845600 & 1.77862800 \\
\hline$=6$ & 2.68904300 & 0.60149500 & -4.12413400 \\
\hline 66 & 0.78032700 & -1.15540500 & 2.72017800 \\
\hline 67 & -4.23218000 & -2.12116500 & 2.4016410 \\
\hline t 68 & 0.50222900 & 5.48458000 & 1.73590900 \\
\hline 69 & -3.02387700 & 4.44906900 & 1.39224700 \\
\hline$=70$ & -4.03138700 & 0.03445400 & 4.0076270 \\
\hline$=71$ & -3.16238300 & 3.64270900 & -1.15379300 \\
\hline 72 & 3.50185200 & 0.97634200 & -0.41961100 \\
\hline 73 & -5.17643400 & 2.06092700 & -0.477355 \\
\hline$=74$ & -2.03798300 & -2.64904400 & -1.30247600 \\
\hline$=75$ & -1.91997500 & 1.79067200 & -5.61565400 \\
\hline t76 & 3.81234100 & -1.51244400 & 0.4544530 \\
\hline \# 76 & Cluster $=$ Pt77 $\quad Q=0$ & $=0 \quad$ Etot $=-7611$ & 57273500 \\
\hline Pt 1 & -3.24832100 & -1.17605200 & -0.83560800 \\
\hline Pt2 & 5.00266600 & -1.88011500 & -2.38705900 \\
\hline Pt 3 & -1.00308600 & -2.37941800 & -0.34029200 \\
\hline Pt 4 & 3.91400500 & -3.59456200 & -0.5555170 \\
\hline Pt 5 & -0.37087200 & -3.01827400 & 2.0990230 \\
\hline Pt 6 & -4.95103900 & -0.16033400 & -2.58930300 \\
\hline Pt 7 & 2.83627100 & 1.26945600 & -1.87058100 \\
\hline Pt 8 & -1.46250900 & 5.40803700 & 1.09266600 \\
\hline Pt 9 & -4.65088100 & -0.29020500 & 2.4562640 \\
\hline Pt10 & -2.86356300 & -3.63516100 & 2.69308500 \\
\hline 11 & -0.93458500 & -4.71441900 & -1.86789700 \\
\hline Pt12 & -1.66685600 & 1.73395700 & -2.44875700 \\
\hline Pt13 & -1.04905100 & -3.25697600 & 4.63946800 \\
\hline Pt14 & 1.34334000 & -4.10461700 & 72900 \\
\hline Pt15 & 3.32388000 & -2.24483600 & 4.3665620 \\
\hline Pt16 & -5.25288100 & 2.06319400 & 1.23771600 \\
\hline Pt17 & -1.02081700 & 1.92045500 & -5.05963600 \\
\hline Pt18 & -0.83339500 & -0.81636800 & 5.79285600 \\
\hline Pt & 1.85481900 & 3.74583200 & 0.72978300 \\
\hline Pt20 & 0.70049000 & -2.74918300 & -2.2441290 \\
\hline Pt21 & 2.42885600 & 3.94066700 & -1.8619280 \\
\hline Pt22 & -1.32056000 & 0.42769800 & -0.22522300 \\
\hline Pt23 & -3.43510200 & 3.91891400 & 1.98762800 \\
\hline Pt24 & 2.78797100 & -1.12396200 & 2.05192200 \\
\hline Pt25 & -1.56100100 & -4.85721900 & 0.70441600 \\
\hline Pt26 & 3.79985200 & 2.78693000 & -3.77128500 \\
\hline Pt27 & -5.62363400 & -0.31074300 & -0.0238510 \\
\hline Pt28 & -2.94410400 & -1.40788200 & 4.20899100 \\
\hline Pt29 & -1.77259900 & 3.08896800 & -0.11795800 \\
\hline Pt30 & -5.35360000 & 2.45373000 & -2.43907800 \\
\hline Pt31 & -0.12212200 & 2.01967600 & 1.5681500 \\
\hline Pt32 & 0.03619200 & 1.71257300 & 5.38863600 \\
\hline Pt33 & -1.15755500 & -3.16046000 & -4.05374200 \\
\hline & 4.47928100 & 0.25877500 & -3.9835370 \\
\hline & 5.17785600 & -2.02375 & \\
\hline
\end{tabular}




\begin{tabular}{|c|c|c|c|}
\hline Pt 36 & 2.51801800 & -1.08712700 & -2.98909000 \\
\hline Pt 37 & 0.67524600 & 0.79817900 & -3.29910400 \\
\hline Pt 38 & -2.19380500 & 4.42961900 & -2.37555100 \\
\hline Pt39 & -3.67235000 & 1.38029700 & -0.77884400 \\
\hline Pt 40 & 4.84537400 & 0.60207800 & 1.24174300 \\
\hline Pt 41 & 0.92702500 & -0.46946100 & -1.03898900 \\
\hline Pt 42 & -3.47012300 & -2.26748800 & -3.22241400 \\
\hline Pt 43 & -2.26483700 & -1.20264600 & 1.63339000 \\
\hline Pt 4 & -2.92227400 & 0.16321000 & -4.25961500 \\
\hline Pt 4 & 3.06818800 & -3.66783600 & -3.09114000 \\
\hline t 4 & 0.31018600 & 4.37813600 & 2.70774700 \\
\hline Pt 4 & -1.62125400 & 2.95949200 & 3.70083400 \\
\hline Pt 48 & 2.12789900 & -0.14459100 & -5.39239600 \\
\hline Pt 49 & -3.37173700 & 2.81401000 & -4.12674100 \\
\hline Pt 5 & 2.20073100 & 1.08752000 & 0.69000800 \\
\hline Pt 5 & 1.02746400 & -5.11177100 & 1.35689600 \\
\hline Pt52 & 1.94207300 & 3.25800300 & 4.41369800 \\
\hline Pt53 & 0.18034100 & 3.61557800 & -3.29093600 \\
\hline Pt 54 & 1.66003000 & -4.95192700 & -1.19089600 \\
\hline Pt 55 & 4.12683700 & 0.33762200 & 3.81982900 \\
\hline Pt 56 & 1.55906200 & 0.95446100 & 3.23611300 \\
\hline Pt57 & 0.08662200 & 4.86094800 & -0.94164700 \\
\hline Pt 58 & -3.46561000 & 1.20391900 & 4.32196100 \\
\hline Pt59 & 1.34013300 & -2.63776000 & -4.82006700 \\
\hline Pt 60 & -2.72143200 & 1.41445000 & 1.72489900 \\
\hline Pt 61 & -3.26571700 & -3.76841500 & -1.02075000 \\
\hline Pt 62 & 2.16205000 & -0.08058400 & 5.61227200 \\
\hline Pt 63 & 3.27440800 & -3.74978900 & 2.03531300 \\
\hline Pt 64 & 3.55320000 & 2.61745400 & 2.44464500 \\
\hline Pt 65 & -1.04656600 & 0.34129900 & 3.42764300 \\
\hline Pt 66 & 0.51969900 & 2.16912800 & -1.02220500 \\
\hline Pt 67 & -4.19342900 & 4.01174700 & -0.63314100 \\
\hline Pt 68 & 1.53961600 & -2.67162100 & 0.28404200 \\
\hline Pt 69 & 1.60305000 & 2.42530400 & -5.15060700 \\
\hline Pt70 & -4.54739900 & -2.55422900 & 0.98622400 \\
\hline Pt71 & 5.38600200 & 0.63490200 & -1.37937600 \\
\hline Pt72 & 4.22726400 & 2.80748700 & -0.19094100 \\
\hline Pt73 & 0.82677300 & -1.51720000 & 3.85286500 \\
\hline Pt74 & -1.21258000 & -0.90536400 & -2.51797600 \\
\hline Pt75 & 3.42754000 & -0.95498100 & -0.49054000 \\
\hline Pt 76 & 0.31371500 & -0.62414300 & 1.44366300 \\
\hline Pt77 & -0.54678200 & -0.74222900 & -5.11749700 \\
\hline \# 77 & Cluster $=$ Pt78 $\quad Q=0$ & Etot $=-77$ & 30097000 \\
\hline Pt1 & 3.10082600 & 4.32903500 & 1.86905900 \\
\hline Pt2 & 3.12300900 & -3.96890600 & 3.20468200 \\
\hline Pt3 & -2.25447200 & -4.72730200 & -1.24458800 \\
\hline Pt 4 & -1.89661300 & 4.19903800 & 3.08942300 \\
\hline Pt 5 & -4.94426800 & -0.70328600 & -2.84577900 \\
\hline Pt 6 & -1.01766600 & 4.63094700 & -2.82669300 \\
\hline Pt 7 & -1.80583300 & 0.43472600 & 3.12260300 \\
\hline Pt 8 & -1.66255300 & 1.34976100 & 0.65054600 \\
\hline Pt9 & -2.99048000 & -3.21236500 & -4.52726400 \\
\hline Pt10 & 1.13816200 & -2.59042500 & 2.2505280 \\
\hline Pt11 & 3.02266000 & 0.06077800 & -0.79436300 \\
\hline Pt12 & -3.11627900 & -0.74914500 & 5.00273600 \\
\hline Pt13 & -2.30483100 & -1.26935100 & -2.97712000 \\
\hline Pt14 & -3.42796200 & 3.15018600 & 1.21581500 \\
\hline Pt15 & 4.90811700 & -0.85892300 & 3.2433990 \\
\hline
\end{tabular}




\begin{tabular}{|c|c|c|c|}
\hline Pt16 & 3.62929700 & -0.34072500 & -4.11320600 \\
\hline Pt17 & -1.17064900 & 2.43796100 & 4.91243500 \\
\hline Pt18 & -3.37264900 & -0.52252000 & 1.27237600 \\
\hline Pt19 & 1.48711800 & -0.88550100 & -2.66823100 \\
\hline Pt20 & 1.57850500 & 4.08885500 & -2.86779300 \\
\hline Pt21 & -0.43630900 & -3.59255200 & 0.40893000 \\
\hline Pt22 & -1.02322600 & -4.65780200 & -3.59970100 \\
\hline Pt23 & -4.77850800 & -2.68127100 & 0.50984400 \\
\hline Pt24 & -0.48328400 & -0.02580000 & 5.45980900 \\
\hline Pt25 & -3.16893700 & 4.08368100 & -1.31910000 \\
\hline Pt26 & -3.80002300 & 2.13297800 & -3.05492100 \\
\hline Pt27 & -5.59541400 & -0.31064200 & -0.29021000 \\
\hline Pt28 & -2.80857200 & -4.23755300 & 1.34056400 \\
\hline Pt29 & -0.51164300 & 0.51247400 & -3.65004500 \\
\hline Pt 30 & 0.54997500 & 4.81750200 & 2.11744900 \\
\hline Pt31 & -5.18821700 & 1.25307100 & 1.85995700 \\
\hline Pt 32 & 4.50597800 & 1.61749700 & 4.00943800 \\
\hline Pt33 & -2.18330900 & -2.37834600 & 3.14908200 \\
\hline Pt 34 & 1.33764600 & 3.32546300 & 4.42788100 \\
\hline Pt 35 & -3.19758600 & 0.37422700 & -1.17925300 \\
\hline Pt 36 & 5.16854900 & 0.57909100 & -2.13015200 \\
\hline Pt 37 & 0.73043300 & -0.15448400 & 3.12279400 \\
\hline Pt 38 & 5.24076500 & -0.08059200 & 0.67977800 \\
\hline Pt 39 & 2.84225100 & -0.70790900 & 1.67270900 \\
\hline Pt 40 & 1.31506600 & -1.76778400 & -0.21788900 \\
\hline Pt 41 & -0.58694600 & -4.51041900 & 2.90162900 \\
\hline Pt 42 & 4.80098700 & -1.90620200 & -1.20768300 \\
\hline Pt 43 & 4.85859600 & 3.04582200 & -1.26034100 \\
\hline Pt 44 & -0.31320600 & -2.69558500 & -2.03949900 \\
\hline Pt 45 & 0.87626300 & -2.86870300 & -4.39814500 \\
\hline Pt 46 & -1.66271600 & 5.04823300 & 0.59685600 \\
\hline Pt 47 & -0.63667200 & -0.24730500 & -1.18818000 \\
\hline Pt 48 & -0.00773800 & -5.96757400 & -0.54299400 \\
\hline Pt 49 & -2.89679800 & 0.46025300 & -4.90250300 \\
\hline Pt 50 & -1.56462400 & 2.72668800 & -4.53065600 \\
\hline Pt51 & 0.90171600 & 0.71384800 & 0.62547000 \\
\hline Pt52 & 1.29795900 & -0.34924700 & -5.32708400 \\
\hline Pt53 & -0.82129300 & -1.13923000 & 1.27968300 \\
\hline Pt 54 & 1.16571200 & 1.63339200 & -1.92806800 \\
\hline Pt 55 & 4.51721900 & -2.72186700 & 1.32238100 \\
\hline Pt 56 & -3.61081800 & 2.20704200 & 3.72715200 \\
\hline Pt 57 & -2.39727500 & -2.13730700 & -0.51400500 \\
\hline Pt58 & 3.06920300 & 4.95472300 & -0.77460700 \\
\hline Pt59 & -0.11045600 & 2.29872800 & 2.49322800 \\
\hline Pt 60 & 2.44099300 & 1.77037300 & 2.49886000 \\
\hline Pt 61 & 3.22982900 & -2.80863800 & -3.10999000 \\
\hline Pt 62 & 0.51341500 & 5.53920300 & -0.86393400 \\
\hline Pt 63 & -1.12994600 & -1.44798100 & -5.37657700 \\
\hline Pt 64 & 2.99119100 & -3.72290600 & -0.57263000 \\
\hline Pt 65 & 2.64863000 & 2.51119300 & 0.01203900 \\
\hline Pt 66 & 1.39669700 & -4.67458500 & -2.41100300 \\
\hline Pt 67 & 0.04577800 & -2.47012100 & 4.62726400 \\
\hline Pt 68 & 4.87602700 & 2.38341100 & 1.42527400 \\
\hline Pt 69 & 1.08219100 & 2.26381200 & -4.7357790 \\
\hline Pt 70 & -1.40440200 & 2.20657800 & -1.83306500 \\
\hline Pt71 & 1.64243300 & -5.01873300 & 1.28264700 \\
\hline Pt72 & 2.60785700 & -1.62313100 & 4.3457690 \\
\hline Pt73 & 0.11385700 & 3.14887400 & 0.0468800 \\
\hline & -4.74239500 & -1.72075200 & 3.1026180 \\
\hline
\end{tabular}




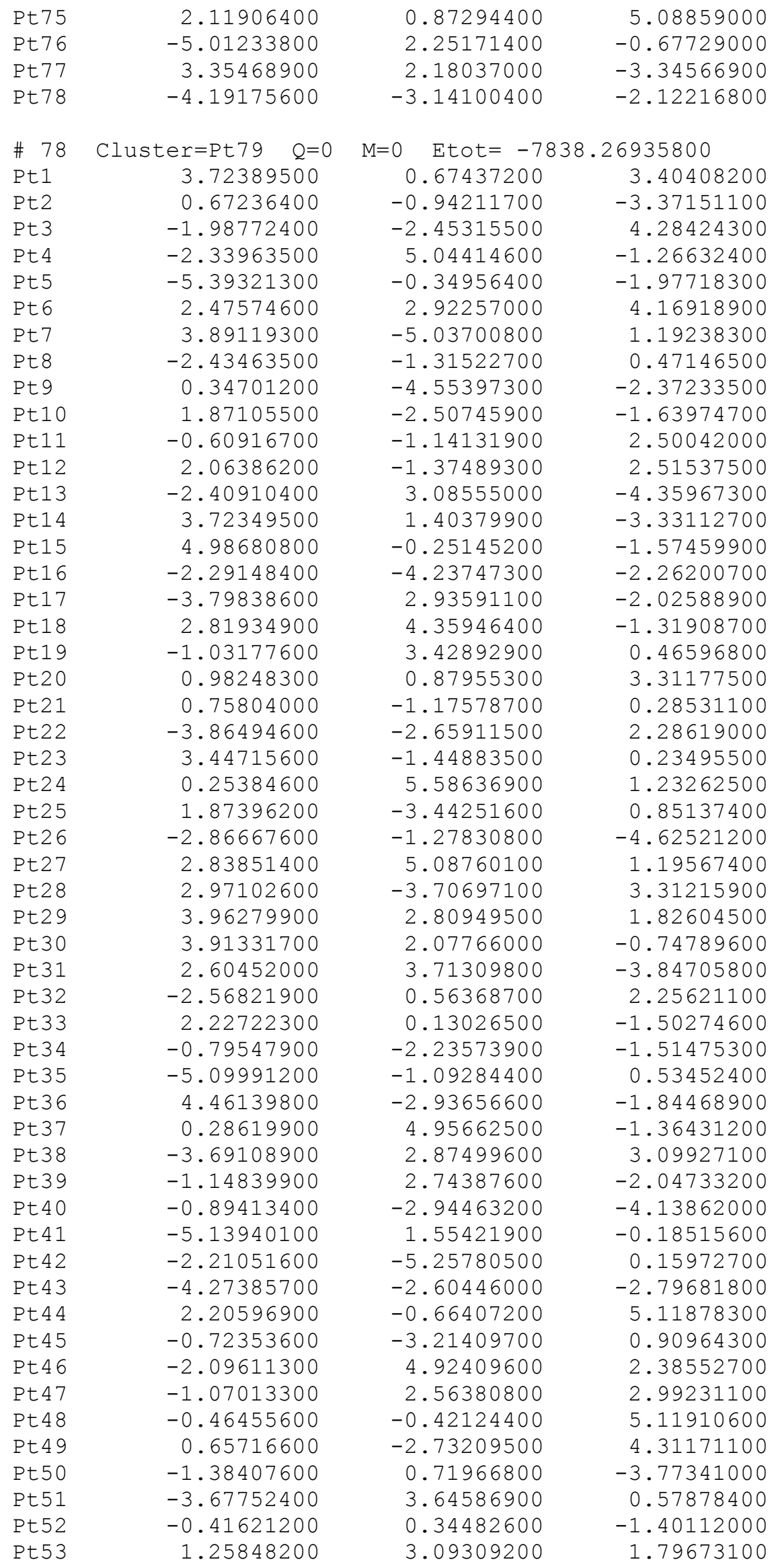




\begin{tabular}{|c|c|c|c|}
\hline Pt5 4 & 2.03772400 & 0.34957900 & -5.27051700 \\
\hline Pt5 5 & -3.98409700 & -3.35902300 & -0.28160800 \\
\hline Pt5 6 & -3.95443300 & -0.72813100 & 4.08699500 \\
\hline Pt57 & -4.01108100 & 0.99532300 & -3.79681200 \\
\hline Pt58 & 0.08170200 & 2.27385700 & 5.35357500 \\
\hline Pt59 & 0.54692400 & -4.80885600 & 2.68484100 \\
\hline Pt 60 & -2.09188900 & -4.56097600 & 2.71622900 \\
\hline Pt 61 & 4.67159400 & -1.70185000 & 2.68519600 \\
\hline Pt 62 & 0.36022600 & 4.59750100 & 3.81540400 \\
\hline Pt 63 & 1.77144200 & -3.21653300 & -4.24560300 \\
\hline Pt 64 & 5.10851900 & 0.46349000 & 1.04835800 \\
\hline Pt 65 & -2.76570700 & -0.59637700 & -1.99979500 \\
\hline Pt 66 & -2.53947400 & 1.31773100 & -0.24133800 \\
\hline Pt 67 & 0.01528600 & 4.18504300 & -3.90851700 \\
\hline Pt 68 & 1.13797200 & 2.41668900 & -0.71152400 \\
\hline Pt 69 & 2.34480900 & 0.82097700 & 1.03131300 \\
\hline Pt70 & 2.76069900 & -4.94245300 & -1.22054000 \\
\hline Pt71 & -0.30185500 & 1.01657200 & 1.06336200 \\
\hline Pt72 & 5.49403800 & -3.04991500 & 0.57638300 \\
\hline Pt73 & -2.42641300 & 1.30784200 & 4.86277200 \\
\hline Pt74 & 0.42627200 & -5.51758200 & 0.08468900 \\
\hline Pt75 & -5.19296300 & 0.82672400 & 2.33834300 \\
\hline Pt76 & 1.02585900 & 1.72664400 & -3.21645000 \\
\hline Pt77 & 3.39967200 & -1.23014300 & -3.58314300 \\
\hline Pt78 & -0.43360900 & -0.75313400 & -5.75175100 \\
\hline Pt79 & -0.07819300 & 2.02218700 & -5.63282600 \\
\hline \# 79 & Cluster $=$ Pt $80 \quad Q=0$ & \multicolumn{2}{|c|}{$\mathrm{M}=0 \quad$ Etot $=-7945.49677500$} \\
\hline Pt 1 & 0.31862700 & -1.47716700 & 0.52566100 \\
\hline Pt2 & 2.60399900 & -3.08789400 & 4.48932300 \\
\hline Pt3 & 1.35908800 & -5.42008200 & -2.15422800 \\
\hline Pt 4 & 4.21703100 & -0.35631100 & -3.33777300 \\
\hline Pt 5 & -2.63770100 & 0.93577800 & -5.03446500 \\
\hline Pt 6 & -5.30126100 & 1.07420700 & -0.73204300 \\
\hline Pt 7 & -2.41200900 & 4.65419700 & -1.70386700 \\
\hline Pt 8 & 5.23919300 & -1.09135500 & 2.35115000 \\
\hline Pt9 & 0.44009700 & -5.70533100 & 1.93670200 \\
\hline Pt10 & -2.27675100 & -1.32376500 & 4.87417700 \\
\hline Pt11 & 3.55072000 & -3.91576000 & 2.16037000 \\
\hline Pt12 & 1.70009900 & 5.33206700 & 1.21780800 \\
\hline Pt13 & -0.59807100 & 2.87945000 & -0.78436300 \\
\hline Pt14 & -0.80783900 & 5.58320300 & 0.20895400 \\
\hline Pt15 & 2.50916200 & 1.09297500 & 2.05710500 \\
\hline Pt16 & -1.06013000 & 4.10370900 & 4.09476900 \\
\hline Pt17 & 1.62484700 & -0.58073700 & -2.83519900 \\
\hline Pt18 & 4.03245300 & 2.25198000 & -2.81502400 \\
\hline Pt19 & -5.10247700 & -1.52520700 & -1.32167300 \\
\hline Pt20 & -3.12972900 & 2.53091800 & 3.66843900 \\
\hline Pt21 & -2.51165300 & -1.17907500 & -1.8380060 \\
\hline Pt22 & -2.62851300 & 4.63129200 & 1.9140220 \\
\hline Pt23 & 4.34328500 & -2.88349000 & -2.55595500 \\
\hline Pt24 & -4.88643600 & 1.82231700 & 1.78451100 \\
\hline Pt25 & -4.19868900 & 0.09887900 & 3.67167400 \\
\hline Pt26 & -4.60471600 & -0.72608100 & 1.1535780 \\
\hline Pt27 & 0.04583800 & -1.20345500 & -6.1253830 \\
\hline Pt28 & 2.35466800 & 1.30322000 & -4.6300030 \\
\hline Pt29 & 2.12212300 & 3.93215800 & -3.68567900 \\
\hline Pt30 & -1.83960100 & -2.94998900 & -0.00137700 \\
\hline Pt31 & 1.54676000 & 4.28508100 & 3.68847500 \\
\hline
\end{tabular}




\begin{tabular}{|c|c|c|c|}
\hline Pt 32 & 1.85229300 & 2.81214500 & 0.18084500 \\
\hline Pt33 & -3.54106600 & -3.59066500 & -2.02196200 \\
\hline Pt 34 & -4.41411200 & -3.30182500 & 0.51585900 \\
\hline Pt 35 & -0.77662800 & -4.27917900 & 3.90918500 \\
\hline Pt36 & -0.59011400 & -3.16061000 & -4.45256900 \\
\hline Pt 37 & -1.48222500 & -5.61377600 & 0.03158900 \\
\hline Pt 38 & -2.29678500 & 2.13872500 & 1.16160300 \\
\hline Pt 39 & 1.23938600 & 0.26640100 & 5.52182100 \\
\hline Pt 40 & 2.35750200 & -2.27471200 & -0.97191300 \\
\hline Pt 41 & -0.36292300 & 0.23894000 & -1.30404900 \\
\hline Pt 42 & -3.03450100 & -2.12058800 & -4.24114300 \\
\hline Pt 43 & 2.80298100 & -1.45905700 & 1.46790400 \\
\hline Pt 44 & 2.50482700 & -1.29299700 & -5.20160400 \\
\hline Pt 45 & 2.12947600 & 0.25827600 & -0.38083800 \\
\hline Pt 46 & -0.13248800 & -2.33408900 & -1.93535700 \\
\hline Pt 47 & -2.63871900 & -4.46940400 & 2.07048000 \\
\hline Pt 48 & -0.36085200 & 3.95004800 & -4.63250100 \\
\hline Pt 49 & -3.46620200 & 2.85684900 & -3.34596600 \\
\hline Pt 50 & -2.75001500 & 1.36782800 & -1.26106500 \\
\hline Pt51 & 0.14305400 & 5.00762600 & -2.21221700 \\
\hline Pt52 & -1.01069100 & 1.93254000 & -3.16692400 \\
\hline Pt53 & 4.40022400 & 3.16409200 & -0.38401000 \\
\hline Pt5 4 & 1.43234400 & 1.92225200 & -2.21998200 \\
\hline Pt 55 & 4.88221300 & -1.94388000 & -0.13722400 \\
\hline Pt 56 & -4.32571400 & 0.26933900 & -3.11772200 \\
\hline Pt 57 & -2.10605100 & -0.40052500 & 0.59389600 \\
\hline Pt58 & -1.28158700 & 1.11927400 & 5.52460400 \\
\hline Pt59 & -1.39908100 & -2.13096900 & 2.43857000 \\
\hline Pt 60 & 3.55709000 & 3.52851500 & 2.13014200 \\
\hline Pt 61 & 2.99398900 & 2.08414700 & 4.44694400 \\
\hline Pt 62 & 3.10628900 & -4.73551400 & -0.28483700 \\
\hline Pt 63 & 0.08133400 & 1.05227900 & 1.10744800 \\
\hline Pt 64 & -0.15924000 & 3.56675200 & 1.70196300 \\
\hline Pt 65 & 2.58359100 & 4.94008100 & -1.22496000 \\
\hline Pt 66 & 0.58989600 & -4.02172000 & -0.05556200 \\
\hline Pt 67 & -1.63928400 & 0.42274000 & 3.01174300 \\
\hline Pt 68 & 0.20975800 & -2.14099500 & 5.18869000 \\
\hline Pt 69 & 0.44643200 & 1.89380900 & 3.61842500 \\
\hline Pt 70 & -0.84012700 & -0.58647600 & -3.77230900 \\
\hline Pt71 & 1.88110000 & -3.20565400 & -3.48591500 \\
\hline Pt72 & 4.65620200 & 0.53602600 & -0.90286700 \\
\hline Pt73 & 5.06768400 & 1.40303100 & 1.54409100 \\
\hline Pt 74 & 1.03301800 & -3.20207800 & 2.37068900 \\
\hline Pt75 & 3.27529900 & -0.57215600 & 4.0104430 \\
\hline Pt76 & -1.21671900 & -4.74414800 & -2.46326800 \\
\hline Pt77 & -3.96366600 & -2.44827600 & 3.07368200 \\
\hline Pt78 & 0.75948300 & -0.67193200 & 3.00667000 \\
\hline Pt79 & -4.13742400 & 3.47677600 & -0.13369500 \\
\hline Pt 80 & -0.07166600 & 1.40700100 & -5.55850500 \\
\hline
\end{tabular}

Table S4. Cartesian coordinates ( $\AA$ ) and binding energies (kcal/mol) of platinum clusters optimized with SCG5A potential.

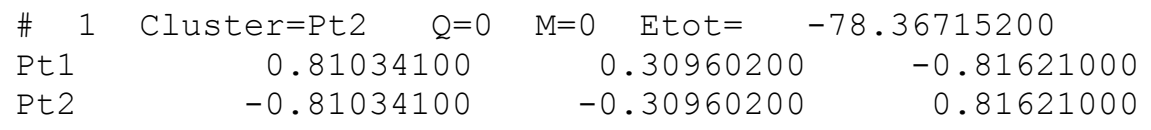

\# 2 Cluster=Pt3 $\quad \mathrm{Q}=0 \quad \mathrm{M}=0 \quad$ Etot $=\quad-143.04994900$ 


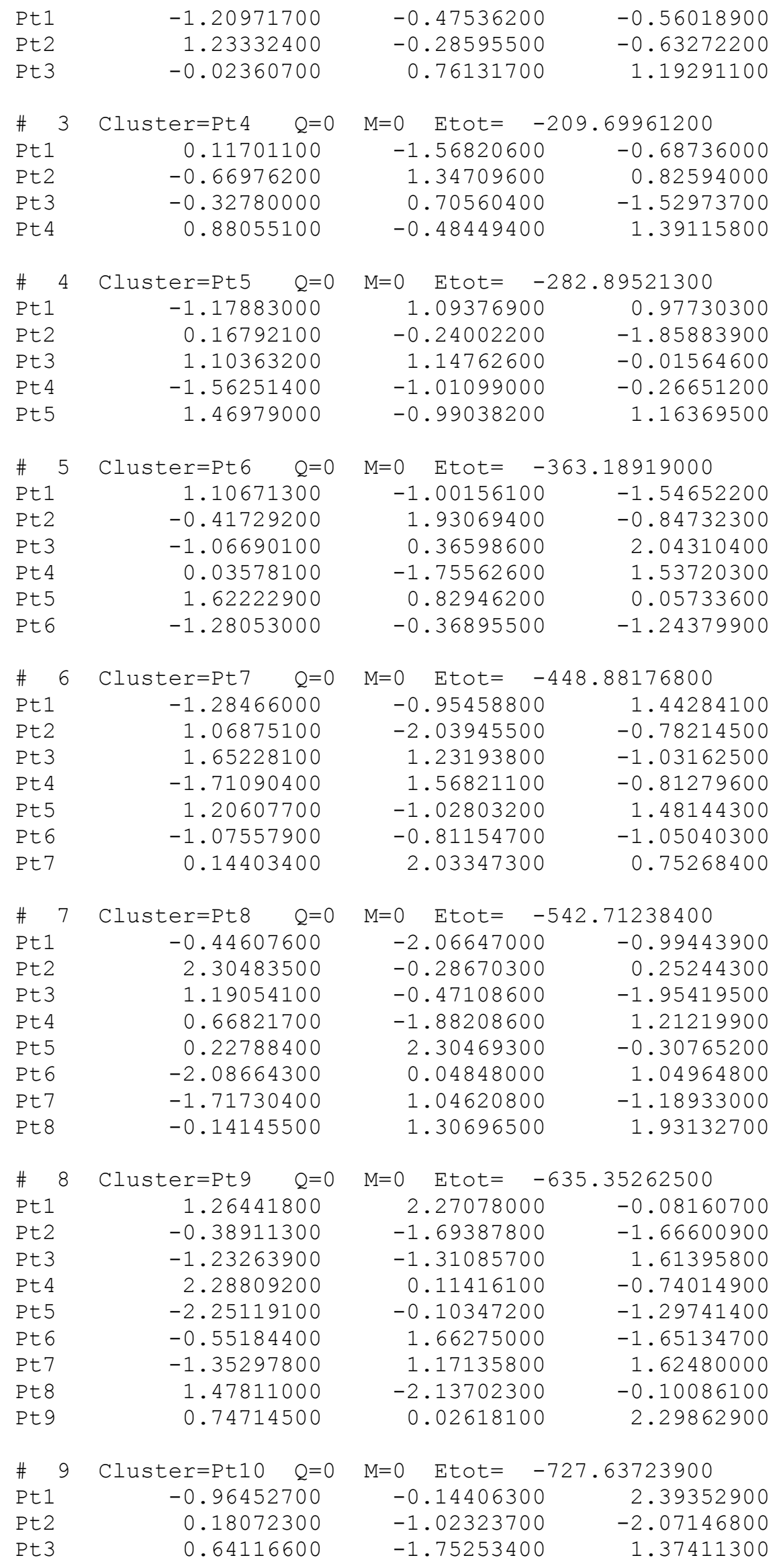




\begin{tabular}{|c|c|c|c|}
\hline Pt 4 & -1.54176800 & 0.77023000 & -1.92607000 \\
\hline Pt5 & 1.78042100 & 1.35126100 & 1.32754700 \\
\hline 6 & -1.12597000 & -2.17707600 & -0.31033600 \\
\hline 7 & 1.45862800 & 1.86095000 & -1.08049000 \\
\hline 8 & -0.27359400 & 2.52153200 & 0.57027200 \\
\hline 9 & -2.29785600 & -0.07803200 & 0.29055300 \\
\hline 10 & 2.14277700 & -1.32903200 & -0.56765100 \\
\hline 10 & uster $=$ Pt 11 & Etot $=$ & 24449100 \\
\hline$=1$ & -1.11818700 & 2.44000400 & -0.04498100 \\
\hline 2 & 0.21056800 & -1.43184600 & -1.93830900 \\
\hline 3 & 2.32293900 & -0.38412100 & -1.01713000 \\
\hline 4 & -1.38786300 & -2.24994300 & -0.17002300 \\
\hline 5 & 2.38797300 & -0.22829900 & 147 \\
\hline 6 & -1.65931400 & 0.17628300 & -2.36175400 \\
\hline 7 & 0.74677000 & 1.61970300 & 36591300 \\
\hline 8 & 1.02388200 & 1.74217200 & -1.14748000 \\
\hline & -0.93534500 & 0.00831800 & 2.3285590 \\
\hline 10 & -2.06779500 & 0.13858600 & 0.102530 \\
\hline 11 & 0.47637200 & -1.83085600 & 1.4 \\
\hline 11 & uster=Pt12 & Etot $=$ & 8553200 \\
\hline 1 & -0.09617400 & 2.80429200 & 0.60783000 \\
\hline 2 & -2.53806400 & 0.58459700 & -0.24599800 \\
\hline 3 & 0.93376100 & -2.54860200 & -0 . \\
\hline 4 & 1.77748500 & -0.28758400 & -1.684 \\
\hline 5 & -1.09372600 & -1.69327400 & 1.66752100 \\
\hline 6 & 1.32054800 & 0.56997500 & 2.48476200 \\
\hline 1 & -1.05198400 & 0.79350300 & 1.73864000 \\
\hline 8 & -0.48296100 & -0.31428400 & -2.81260300 \\
\hline & 300 & 1.4 & 0. \\
\hline 10 & -0.78390000 & 1.78 & -1 \\
\hline 11 & 1.36284800 & -1.60445500 & 74700 \\
\hline 12 & -1.33935600 & -1.52350800 & -0.80264300 \\
\hline 12 & Cluster $=$ Pt $13 \quad Q=0$ & $\mathrm{M}=0 \quad \mathrm{Etot}=-1 \mathrm{C}$ & 1762 \\
\hline 1 & 2.05439800 & 0.97417200 & -0 . \\
\hline 2 & 0.69343900 & -1.560 & 1. \\
\hline 3 & -2.24659500 & -1.06276700 & -0.12307100 \\
\hline$=4$ & 1.27919400 & 0.62103100 & -2.61440200 \\
\hline 5 & 0.44977600 & -1.57648600 & -1.87195100 \\
\hline & 1.50782200 & 0.70122600 & 2 . \\
\hline & 16900 & -1.4 & -0 . \\
\hline & 8500 & 2.7 & -0 . \\
\hline & -1.53327200 & -0.73922000 & 212400 \\
\hline 1( & -2.64203200 & 1.36811200 & -0.07107500 \\
\hline & -0.61692500 & -2.90984900 & -0.09641100 \\
\hline 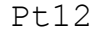 & -0.94275800 & 1.36518800 & -1.85452900 \\
\hline & -0.76010200 & 1.51039700 & 1.54559200 \\
\hline 13 & Cluster=Pt14 $Q=0$ & Etot $=-11$ & 46995700 \\
\hline-1 & 0.91290600 & 2.59760200 & -0.93584 \\
\hline 2 & -1.26146500 & 1.49225700 & -1.37273500 \\
\hline Pt 3 & -2.68673700 & -0.49923900 & -0.99445400 \\
\hline Pt4 & -1.92597400 & -0.37639700 & 1.360690 \\
\hline 5 & -0.02150400 & -0.03003800 & 2.90781200 \\
\hline & 0.02150400 & 0.03003800 & -2.90781200 \\
\hline & -0.63392300 & -1.82593100 & -1.4023720 \\
\hline & 00 & 00 & \\
\hline
\end{tabular}




\begin{tabular}{|c|c|c|c|}
\hline Pt9 & -0.91290600 & -2.59760200 & 0.93584200 \\
\hline Pt10 & 1.26146500 & -1.49225700 & 1.37273500 \\
\hline Pt11 & 1.79533500 & -2.06832400 & -0.97751600 \\
\hline 12 & 1.92597400 & 0.37639700 & -1.36069000 \\
\hline$=13$ & 0.63392300 & 1.82593100 & 1.40237200 \\
\hline 14 & 2.68673700 & 0.49923900 & 0.994454 \\
\hline 14 & Cluster=Pt15 $\mathrm{Q}=0$ & \multicolumn{2}{|c|}{$\mathrm{M}=0 \quad$ Etot $=-1202.37298200$} \\
\hline Pt 1 & -2.31455300 & -0.11375500 & 1.83353100 \\
\hline t2 & 2.36246700 & -0.35640900 & -1.61720700 \\
\hline-3 & -0.79343400 & -1.05796100 & -2.56414100 \\
\hline 4 & -1.22216500 & -2.87356000 & 0.2440800 \\
\hline$=5$ & 0.33164900 & 1.01930200 & -1.86047100 \\
\hline Pt 6 & -2.20861900 & -0.74657500 & 788500 \\
\hline t7 & 0.79458900 & -2.18860000 & -1.02671000 \\
\hline 58 & -0.29275200 & -1.52391000 & 2.10468500 \\
\hline$=9$ & -0.27352600 & 3.42015500 & -1.62228000 \\
\hline 10 & -1.91632000 & 1.66729900 & -0.94841400 \\
\hline 11 & 2.25314900 & 0.24526900 & 0.780877 \\
\hline 2 & 0.82676900 & 0.584 & 2. \\
\hline 3 & 1.91569900 & -2.17601900 & 1.1 \\
\hline$=14$ & 1.29621200 & 2.38143900 & 0.01551300 \\
\hline$=15$ & -0.75916300 & 1.71876800 & 1.25672900 \\
\hline 15 & Cluster=Pt16 $Q=0$ & \multicolumn{2}{|c|}{$\mathrm{M}=0 \quad \mathrm{Etot}=-1291.05156600$} \\
\hline Pt1 & 0.32136500 & 1.37483000 & -1.89880500 \\
\hline et 2 & -0.04301700 & 1.12598700 & 749600 \\
\hline t3 & 2.17773600 & 1.62037300 & -0.31264100 \\
\hline t4 & -0.01533400 & 2.63145900 & 0.25727600 \\
\hline t5 & 2.18344900 & -1.30526400 & 1.3579760 \\
\hline 6 & -2.76366100 & 400 & -1 \\
\hline t7 & -1.15863000 & -1.71478100 & 494000 \\
\hline Pt 8 & 0.28629300 & -1.24699100 & 2.96149200 \\
\hline t9 & 3.02011400 & -0.62476600 & -0.88837300 \\
\hline 10 & -3.37225300 & -0.74030100 & 0.3802490 \\
\hline 1 & 0.78729400 & -2.87671900 & 0.024556 \\
\hline 2 & 42400 & -1.28241000 & -1.3 \\
\hline 13 & -2.05977700 & 1.34 & 0 . \\
\hline$=14$ & 2.45443800 & 1.06439100 & 710700 \\
\hline t15 & 0.74633300 & -1.10616900 & -1.72464300 \\
\hline$=16$ & -0.86392700 & -0.17505700 & -3.40465 \\
\hline \# 16 & Cluster=Pt17 $\quad \mathrm{Q}=0$ & \multicolumn{2}{|c|}{$\mathrm{M}=0 \quad \mathrm{Etot}=-1389.21440600$} \\
\hline Pt1 & 0.58784600 & 1.70812400 & 1.3 \\
\hline Pt2 & 1.81830500 & -2.78797700 & 6600 \\
\hline Pt 3 & -1.73437400 & -0.98469500 & -1.83086400 \\
\hline Pt 4 & 0.42404000 & -1.57866300 & 2.43594700 \\
\hline t 5 & 1.49604100 & -1.15559900 & -2.24761300 \\
\hline Pt 6 & 2.59756800 & -0.56229300 & 381100 \\
\hline Pt 7 & -2.13983600 & -0.13358200 & 0.50723400 \\
\hline Pt 8 & 2.25024200 & 0.12646000 & 2.29092300 \\
\hline Pt9 & -1.54571200 & -2.46603400 & 1.17753600 \\
\hline Pt10 & -0.00560500 & 3.16363700 & -1.45860900 \\
\hline Pt11 & 1.93824200 & 1.75451400 & -0.72633000 \\
\hline Pt12 & 0.16146300 & -0.00184500 & -0.4579610 \\
\hline Pt13 & -2.79004900 & 1.22337200 & -1.52485800 \\
\hline$t \perp 4$ & -1.83281400 & 2.28085500 & 1.24501300 \\
\hline 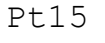 & -0.06472400 & -2.50755100 & -0.8380290 \\
\hline & -0.09666300 & 1.51469200 & \\
\hline
\end{tabular}




$\begin{array}{lrrr}\text { Pt17 } & -1.06397000 & 0.40658500 & 2.70636000 \\ \text { \# 17 } & \text { Cluster=Pt18 } \mathrm{Q}=0 & \mathrm{M}=0 \quad \text { Etot }=-1485.60619200 \\ \text { Pt1 } & -1.15529200 & 1.45912700 & 3.02555100 \\ \text { Pt2 } & 1.14566400 & 2.17471300 & 2.41330900 \\ \text { Pt3 } & -3.12535500 & -0.78062500 & 1.54115300 \\ \text { Pt4 } & 1.85189800 & -1.58260700 & -3.13206200 \\ \text { Pt5 } & 2.27316300 & 0.43699900 & -0.07664600 \\ \text { Pt6 } & -2.11143600 & -1.29328200 & -0.71958300 \\ \text { Pt7 } & 1.42353000 & -0.32491900 & 2.15070100 \\ \text { Pt8 } & -0.48557900 & -3.08214800 & -1.23497200 \\ \text { Pt9 } & -2.44885400 & 1.05641000 & 0.01164000 \\ \text { Pt10 } & -0.57060300 & 1.64381300 & -1.56423100 \\ \text { Pt11 } & 2.48072800 & -1.93647700 & -0.72290500 \\ \text { Pt12 } & -1.26884600 & 3.21549300 & 0.27606100 \\ \text { Pt13 } & -1.32300600 & -0.34032800 & -2.88641700 \\ \text { Pt14 } & -0.17975600 & -0.01719300 & 0.26346300 \\ \text { Pt15 } & 0.59370600 & -2.32756400 & 0.88436400 \\ \text { Pt16 } & 1.62172900 & 0.80004500 & -2.46852100 \\ \text { Pt17 } & -0.36762800 & -1.81014700 & 3.12132600 \\ \text { Pt18 } & 1.64593700 & 2.70868900 & -0.88223300\end{array}$

$\begin{array}{lrrrr}\# 18 & \text { Cluster=Pt19 } Q=0 & M=0 & \text { Etot }=-1583.13762300 \\ \text { Pt1 } & 1.38701100 & -1.88722500 & -0.42569000 \\ \text { Pt2 } & -1.22898800 & 0.09269600 & -3.52806000 \\ \text { Pt3 } & 0.99660500 & -0.51224000 & -2.49649900 \\ \text { Pt4 } & 0.68583500 & -0.59578000 & 3.87556000 \\ \text { Pt5 } & 3.32345700 & -0.30282700 & -0.10053400 \\ \text { Pt6 } & -1.93081500 & 1.03051000 & 0.88773100 \\ \text { Pt7 } & 1.95717900 & 2.57902800 & -1.18048100 \\ \text { Pt8 } & 0.06742100 & 0.19660400 & -0.27822300 \\ \text { Pt9 } & -1.94814000 & -2.33115300 & 0.35842600 \\ \text { Pt10 } & -3.11466900 & -0.47084100 & -0.82960900 \\ \text { Pt11 } & -2.65614600 & 1.92072900 & -1.33380200 \\ \text { Pt12 } & 2.06063100 & -1.18803600 & 1.89308100 \\ \text { Pt13 } & 0.35185800 & 1.90383400 & -2.93592700 \\ \text { Pt14 } & 2.91177100 & 1.89167600 & 1.01967100 \\ \text { Pt15 } & -2.33263100 & -0.93537400 & 2.38048800 \\ \text { Pt16 } & -0.04635900 & 2.60861000 & 0.38244800 \\ \text { Pt17 } & -0.62293300 & -2.48942200 & -1.77181000 \\ \text { Pt18 } & 0.19443600 & -2.89887500 & 1.51918800 \\ \text { Pt19 } & -0.05552300 & 1.38808600 & 2.56404000 \\ & & & & \\ \text { \#19 } & \text { Cluster }=\text { Pt20 } 000 & M=0 & \text { Etot }=-1685.76716300 \\ \text { Pt1 } & 2.38274200 & -0.03885000 & -2.62089800 \\ \text { Pt2 } & 1.75576500 & 0.25080700 & -0.23517800 \\ \text { Pt3 } & -1.52104200 & -2.04213000 & 1.42030300 \\ \text { Pt4 } & -1.39080000 & -0.83996400 & -0.80472100 \\ \text { Pt5 } & -0.29505800 & 0.13960700 & 1.20683400 \\ \text { Pt6 } & 1.66656200 & 1.46657300 & 1.93382500 \\ \text { Pt7 } & 0.34068900 & -2.63288900 & 3.01799400 \\ \text { Pt8 } & 0.11249700 & 0.76863500 & -2.03246000 \\ \text { Pt9 } & 0.71696100 & 3.19196000 & 0.33885400 \\ \text { Pt10 } & -0.65755600 & -3.16783500 & -1.56764200 \\ \text { Pt11 } & 1.11756900 & 3.03812200 & -2.12552700 \\ \text { Pt12 } & -1.97184500 & 1.89037100 & -2.76834000 \\ \text { Pt13 } & -2.81806200 & 0.07217100 & 1.02687700 \\ \text { Pt14 } & -2.49036900 & 2.16053200 & -0.33982700 \\ \text { Pt15 } & -0.88219200 & 0.41624700 & 3.63488700\end{array}$




\begin{tabular}{|c|c|c|c|}
\hline 116 & 2.63760600 & -2.81816400 & -0.88686300 \\
\hline Pt17 & 0.67528200 & -2.96567100 & 0.58087800 \\
\hline t18 & 2.84461000 & $-0.7530230 c$ & 1.74735800 \\
\hline t1 & -1.37925700 & 3.09694100 & 1.66694500 \\
\hline t20 & -0.84410300 & -1.23344100 & -3.19329800 \\
\hline \# 20 & luster $=\mathrm{Pt} 21$ & \multicolumn{2}{|c|}{$\mathrm{M}=0 \quad$ Etot $=-1790.68215400$} \\
\hline Pt1 & -1.61845300 & 3.77989900 & -0.63523200 \\
\hline Pt2 & -0.76365900 & 1.96534200 & 3.1164900 \\
\hline Pt3 & 1.90328500 & 1.36970700 & -0.7023380 \\
\hline t 4 & 0.51094600 & -0.12019200 & 0.8060190 \\
\hline t5 & -1.30971400 & -2.89122900 & 1.48796300 \\
\hline Pt 6 & -1.57890100 & $-1.7753510 c$ & -3.04137500 \\
\hline Pt 7 & 0.60048900 & 3.21550200 & 0.34579200 \\
\hline Pt 8 & -1.87728700 & -0.46440500 & 1.49261400 \\
\hline Pt9 & -1.89148800 & 1.5717610 & -3.15402300 \\
\hline Pt10 & 0.47009600 & 0.9471380 & -3.64338200 \\
\hline t11 & -0.43643600 & 0.7456340 & -1 \\
\hline t12 & 1.65141800 & -1.2321 & -3 \\
\hline t13 & -2.39506400 & 1.546 & 0 . \\
\hline t14 & -0.08348900 & -0.4178580 & 3.2364890 \\
\hline Pt15 & -2.38821700 & -0.7701720 & -0.89680300 \\
\hline Pt16 & 2.78818900 & -0.6654100 & 1.73421000 \\
\hline Pt17 & 01900 & -3.29 & -0 . \\
\hline Pt18 & 1.60708100 & 2.3 & \\
\hline Pt19 & -0.64005400 & -2.58369 & -0.90 \\
\hline Pt20 & 2.32824300 & -1.0507940 & -0.68356100 \\
\hline Pt21 & 1.57399800 & -2.2650860 & 3.2249890 \\
\hline \# 21 & ter $=$ Pt $22 \quad Q=0$ & \multicolumn{2}{|c|}{$\mathrm{M}=0 \quad$ Etot $=-1893.64124200$} \\
\hline Pt 1 & -1.42201800 & 0.4777520 & 371300 \\
\hline Pt2 & 0.89742500 & 1.2901410 & 0.41146400 \\
\hline Pt3 & 2.86258200 & 0.9498030 & 1.91924700 \\
\hline Pt 4 & 3.46933300 & -0.0870930 & -1.25420000 \\
\hline Pt5 & -1.78727100 & 0.6208870 & 2.47535200 \\
\hline Pt 6 & -0.07842900 & 2.42 & 500 \\
\hline Pt 7 & -1.50645200 & -2.8379950 & -0 . \\
\hline Pt 8 & -2.33718600 & -1.5460420 & 1.21963100 \\
\hline Pt9 & 0.26580000 & 3.2949710 & -0.90645100 \\
\hline Pt10 & -0.06030700 & -1.9028510 & 3.70267500 \\
\hline Pt11 & -0.33621900 & 1.8756230 & -2.85823400 \\
\hline Pt12 & 0.25533800 & -1.0969 & -0.9905 \\
\hline Pt13 & 7100 & 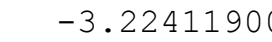 & 1 \\
\hline Pt14 & -2.88290200 & -0.0733950 & -1.98835200 \\
\hline Pt15 & 0.06302200 & -0.7828930 & 1.48143800 \\
\hline Pt16 & 1.88541900 & -2.9215810 & -0.45127200 \\
\hline Pt17 & -3.84307400 & 1.1385640 & -0.05243500 \\
\hline Pt18 & 57500 & 2.2090290 & -1.24295500 \\
\hline Pt19 & -0.75885400 & -0.554 & -3 \\
\hline Pt20 & 2.46683400 & -1.4717370 & 1.54437700 \\
\hline Pt21 & 1.74818100 & -0.6691310 & -2.94085100 \\
\hline Pt22 & -2.09206900 & 2.8851290 & -0.2294550 \\
\hline \# 22 & luster $=$ Pt23 $\quad Q=0$ & \multicolumn{2}{|c|}{$\mathrm{M}=0 \quad$ Etot $=-2001.45554200$} \\
\hline Pt1 & 1.77979800 & -1.3404230 & 1.28345 \\
\hline Pt2 & -2.65673900 & -1.3487860 & 2.60078200 \\
\hline Pt3 & -2.41384000 & -2.1145210 & -0.6142190 \\
\hline Pt 4 & 1.29833700 & 3.2613440 & 1.79629100 \\
\hline 23 & -0.12204700 & -3.0586640 & -1.01831100 \\
\hline
\end{tabular}




\begin{tabular}{|c|c|c|c|}
\hline Pt 6 & -3.70085700 & -0.17509200 & -1.55803400 \\
\hline Pt 7 & 0.57994000 & -0.65652900 & -0.82305200 \\
\hline Pt 8 & 2.33606600 & -2.44656600 & -0.91086500 \\
\hline Pt9 & -0.02603300 & -1.62696400 & -3.08181600 \\
\hline t10 & -0.36344800 & 0.77557600 & -3.63593700 \\
\hline t11 & 2.82825500 & -0.06366900 & -3.15687300 \\
\hline t12 & -1.39321500 & 0.91174200 & -1.33701200 \\
\hline 13 & -0.10120600 & 0.70593100 & 3.63961500 \\
\hline t14 & 0.32144900 & 1.45640400 & 0.44079800 \\
\hline 15 & 1.67804400 & 2.43090000 & -1.41684300 \\
\hline$=16$ & -0.52596500 & 2.99775700 & -2.43359600 \\
\hline 17 & -2.77918800 & 2.56428600 & -0.03943700 \\
\hline$=18$ & 0.55111100 & -1.71040100 & 3.43330900 \\
\hline 19 & -1.60725900 & 1.80932100 & 2.00220700 \\
\hline$=20$ & -1.34060900 & -0.36160900 & 0.79827300 \\
\hline$=21$ & 0.03816800 & -3.14682700 & 1.47113900 \\
\hline$t 22$ & 2.94466600 & 0.48730200 & 2.52811800 \\
\hline$=23$ & 2.67457300 & 0.64948700 & 0.03200600 \\
\hline \# 23 & luster $=\mathrm{Pt} 24$ & $=0 \quad$ Etot $=-21$ & 51677300 \\
\hline Pt 1 & -2.85235700 & 0.10515500 & 2.57551800 \\
\hline Pt2 & 0.79805800 & 0.54346300 & -1.27872300 \\
\hline Pt3 & 3.31806500 & 0.29322100 & 0.78122700 \\
\hline Pt 4 & -0.10298800 & 1.23304800 & 0.95589400 \\
\hline Pt 5 & 2.15088200 & 2.47386400 & 0.97956600 \\
\hline Pt 6 & -3.31345600 & 0.70892900 & -0.56069600 \\
\hline Pt 7 & 1.03394100 & -0.89107100 & 0.76269700 \\
\hline Pt 8 & -1.17668100 & -0.59988200 & -0.32909800 \\
\hline Pt9 & 2.73436700 & 1.67297800 & -2.30243300 \\
\hline Pt10 & 0.94826100 & -3.29539700 & 1.40744900 \\
\hline $\mathrm{P}$ & 3.10690300 & -2.22795400 & 0.66825200 \\
\hline t12 & 0.93454800 & -2.67025600 & -1.93063800 \\
\hline t13 & -0.05980600 & 3.68839800 & 1.20637000 \\
\hline Pt14 & -2.09013400 & 2.38122500 & 1.92376200 \\
\hline Pt15 & 0.58618300 & 2.96234600 & -1.95595700 \\
\hline 6 & -3.34058400 & -1.77016300 & -0.19652300 \\
\hline Pt17 & -0.43408700 & -1.31089300 & -3.56699300 \\
\hline Pt & -1.83194200 & 36900 & -1.4 \\
\hline t19 & -1.30946800 & -2.77742000 & 2.31333600 \\
\hline Pt20 & -1.59405600 & 0.85627400 & -3.36988000 \\
\hline Pt21 & 2.62892000 & -0.85409400 & -2.30307900 \\
\hline Pt 2 & 1.62208200 & 0.53106300 & 3.70681900 \\
\hline Pt23 & -1.30316400 & -3.04689400 & -0.90230500 \\
\hline Pt24 & -0.45348600 & -0.50430800 & 2.8759650 \\
\hline \# 24 & Cluster $=$ Pt $25 \quad Q=0$ & $\mathrm{M}=0 \quad$ Etot $=-22$ & 1302 \\
\hline Pt 1 & -0.59439700 & -3.11680600 & -3.08255400 \\
\hline Pt2 & 1.32881800 & -1.82916300 & 2.78176300 \\
\hline Pt 3 & 0.23762300 & 2.48120700 & 3.62672600 \\
\hline Pt 4 & -0.80539800 & 4.00417800 & 1.99371700 \\
\hline Pt 5 & 2.84494600 & -2.08135700 & -0.22635600 \\
\hline Pt 6 & -0.79704200 & 0.89542500 & -0.77936500 \\
\hline Pt 7 & 1.80772200 & -2.59734000 & -3.42116800 \\
\hline Pt 8 & -2.35927900 & -0.45301400 & -2.29768200 \\
\hline Pt 9 & 0.35916700 & 3.09336900 & -1.03607100 \\
\hline Pt10 & 2.21817600 & 0.60363200 & -2.40773000 \\
\hline & 1.45631300 & -0.00888300 & -0.11997300 \\
\hline & -0.29337300 & -1.44570700 & -1.2487270 \\
\hline & -2.41204100 & 0.96641500 & 2.24221100 \\
\hline
\end{tabular}




\begin{tabular}{|c|c|c|c|}
\hline Pt14 & 0.00690100 & 0.98307600 & 1.61395400 \\
\hline Pt15 & -0.82026800 & -0.66382800 & 3.35487900 \\
\hline Pt16 & -2.80591900 & 2.12917700 & 0.10163600 \\
\hline Pt17 & 3.92375000 & 0.11331500 & -0.67202300 \\
\hline Pt18 & 2.37365000 & 0.35936200 & 2.23611000 \\
\hline 119 & -1.42745300 & -0.90192000 & 0.91513300 \\
\hline$=20$ & -1.86571700 & 2.78529300 & -2.09187200 \\
\hline-21 & -1.35461600 & -3.34140300 & 1.28105300 \\
\hline Pt22 & 0.73384700 & -3.34108700 & -0.08302600 \\
\hline Pt23 & 1.89879700 & 2.32708500 & 0.72003700 \\
\hline Pt24 & -0.09070400 & 0.22639500 & -3.13769300 \\
\hline Pt25 & -3.56350400 & -1.18741700 & -0.26298200 \\
\hline 2 & Cluster=Pt26 & Etot $=-232$ & 8066700 \\
\hline Pt 1 & 2.79394100 & 0.14339000 & -2.88912800 \\
\hline Pt2 & 2.65681700 & -2.00756900 & 1.21170900 \\
\hline Pt 3 & -2.58757900 & 0.71874500 & -2.50133200 \\
\hline Pt 4 & 0.92154600 & -3.76289500 & 0.71621700 \\
\hline t5 & 1.41799100 & -1.72312200 & -1.95137200 \\
\hline Pt 6 & -2.40388700 & -2.72856100 & 0.68425100 \\
\hline Pt 7 & -0.95624800 & 3.24852700 & -1.31862300 \\
\hline Pt 8 & -0.47402100 & 3.67146300 & 1.07214100 \\
\hline Pt9 & -0.53635500 & -2.58022700 & 2.33232500 \\
\hline Pt10 & -4.21018400 & -1.10079900 & 0.07633200 \\
\hline Pt11 & 0.35906900 & 2.34484600 & 2.95716700 \\
\hline$=12$ & -2.92299200 & 1.94649500 & -0.35650600 \\
\hline Pt13 & -1.45388800 & -0.40633100 & 3.06509400 \\
\hline Pt14 & -2.35843600 & -1.56379100 & -1.54797300 \\
\hline Pt15 & 0.42568700 & -1.33246900 & 0.3544270 \\
\hline Pt16 & -1.18716100 & 0.46607800 & 0.7244840 \\
\hline Pt17 & 3.42068000 & 2.08540700 & -0.134525 \\
\hline Pt18 & 1.20554200 & 0.981 & 0.05502300 \\
\hline Pt19 & 1.49456900 & 2.86265600 & -1.59171200 \\
\hline Pt20 & 1.05527200 & -0.00665200 & 3.31692600 \\
\hline Pt21 & -0.38215900 & 0.01188600 & -1.56656800 \\
\hline Pt22 & 3.67795900 & -1.20623000 & -0.9688850 \\
\hline Pt23 & 2.95542400 & 0.456 & 1.76919200 \\
\hline Pt24 & -2.97935600 & 1.3 & 2.1 \\
\hline Pt25 & 0.62180700 & 1.27216900 & -3.43760900 \\
\hline Pt26 & -0.55403700 & -3.18310000 & -2.1844900 \\
\hline$\# 26$ & Cluster $=\operatorname{Pt} 27 \quad \mathrm{Q}=0$ & $\mathrm{M}=0 \quad \mathrm{E}$ tot $=-24$ & 6517 \\
\hline Pt 1 & 3.76448800 & 1.28161000 & -0.80874 \\
\hline Pt2 & 0.55720700 & 2.41 & 2.3987 \\
\hline Pt 3 & 0.44804200 & 1.19528200 & 0.17371800 \\
\hline Pt 4 & 2.04697800 & -2.83937200 & 0.67809600 \\
\hline Pt 5 & -0.03486100 & -2.22200400 & 3.36325200 \\
\hline Pt 6 & -1.71015000 & 3.18881200 & 1.61757400 \\
\hline Pt 7 & 1.09029400 & 2.38727700 & -1.9525000 \\
\hline Pt 8 & -3.44476800 & -0.64758800 & -0.45963300 \\
\hline Pt9 & -1.14075700 & -0.03393300 & -1.25918300 \\
\hline Pt10 & -2.28204200 & 0.38584100 & -3.44400900 \\
\hline Pt11 & -2.51369700 & -2.94585600 & -0.27972400 \\
\hline Pt12 & 0.76039400 & -0.86238400 & 1.48718500 \\
\hline Pt13 & 0.21685100 & -0.63159100 & -3.2259800 \\
\hline Pt14 & -0.16153500 & -3.84619700 & 0.1492650 \\
\hline & -1.28209900 & 3.23719800 & -1.78684500 \\
\hline PLIt & -0.37755800 & 0.30394200 & 3.3599780 \\
\hline te 1 & 2.13744100 & 0.97211300 & -3.7215470 \\
\hline
\end{tabular}




\begin{tabular}{|c|c|c|c|}
\hline Pt18 & 2.82044400 & 1.30772800 & 2.46115000 \\
\hline Pt19 & -1.55775300 & -0.02773600 & 1.16424200 \\
\hline Pt20 & 3.21838500 & -1.73474900 & -2.26799000 \\
\hline t21 & 0.15889600 & -2.90621100 & -2.14874300 \\
\hline 2 & -3.53941500 & 0.58251200 & 2.64965100 \\
\hline$=23$ & 1.41818600 & 3.49219600 & 0.27174100 \\
\hline 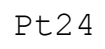 & 1.19281000 & -0.90215300 & -0.94277000 \\
\hline$=2$ & -2.72192300 & 1.74656900 & -0.32096400 \\
\hline 2 & 3.14347200 & -0.58701000 & 0.71203300 \\
\hline 2. & -2.20732700 & -2.31326100 & 2.13200500 \\
\hline 27 & Cluster $=$ Pt28 $\quad Q=0$ & \multicolumn{2}{|c|}{$\mathrm{M}=0 \quad \mathrm{Etot}=-2553.93461900$} \\
\hline t1 & -3.61231200 & -1.95412400 & 1.25810000 \\
\hline Pt2 & -0.76604600 & 1.27619900 & 2.77851500 \\
\hline t3 & -2.12208000 & -0.96591000 & -3.09395500 \\
\hline-4 & -0.75844800 & 2.09264100 & -3.67366600 \\
\hline$=5$ & 3.25184500 & 0.20432400 & -2.09583500 \\
\hline 6 & 3.55759100 & 1.35456400 & 1.06008100 \\
\hline 7 & -1.38661400 & -3.04731800 & 0.99601600 \\
\hline$=8$ & -1.36879100 & -0.99691400 & 3.57038400 \\
\hline$=9$ & 1.96633700 & -0.58099500 & 3.28705500 \\
\hline 10 & -0.28283200 & 3.15633700 & -1.46916300 \\
\hline & -3.09610100 & 1.34258100 & 1.90273500 \\
\hline & 0.93335000 & 0.38552600 & -2.98594700 \\
\hline & -0.52448400 & -0.70803500 & 1.23924800 \\
\hline 4 & -3.24733600 & 1.72701100 & -1.44475400 \\
\hline$P$ & 3.03445600 & 2.67048200 & -1.83498700 \\
\hline 16 & -1.79728200 & -3.31628500 & -2.34064200 \\
\hline 7 & 1.05853500 & -2.08466100 & -3.1857120 \\
\hline & 1.24429500 & 2.73297400 & 3.07128600 \\
\hline & -1.04647900 & 0.95112900 & -0.55890100 \\
\hline & 1.16808900 & -2.93309600 & 3.12075100 \\
\hline-21 & -2.40526300 & 3.57889900 & 1.05487800 \\
\hline Pt2 2 & 2.81710500 & -1.01265700 & 0.98696500 \\
\hline Pt23 & 0.34475300 & -1.07712400 & -1.01640700 \\
\hline 4 & 0.93287300 & 3.30012100 & 0.6632050 \\
\hline 5 & 1.22621000 & 3000 & 0.33606000 \\
\hline$P$ & 3.12646500 & -2.871 & -0.65 \\
\hline 27 & -2.98440900 & -0.64906700 & -0.7795330 \\
\hline$\Omega^{2}$ & 0.73657200 & -3.40914000 & -0.19005700 \\
\hline 28 & Cluster $=$ Pt $29 \quad Q=0$ & $=0 \quad E \operatorname{tot}=-26$ & 0410 \\
\hline Pt 1 & -0.48284100 & -2.29578200 & -2.87887100 \\
\hline 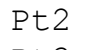 & -0.85152400 & -1.058 & 0.81590 \\
\hline Pt3 & -2.36385200 & 2.31176000 & 2.9973920 \\
\hline Pt 4 & -3.16488900 & -0.13151400 & 0.78840300 \\
\hline Pt 5 & -2.73460000 & -2.08302200 & 2.25452200 \\
\hline Pt 6 & -0.59528500 & 3.44782700 & -0.82364800 \\
\hline Pt 7 & 1.52881200 & -0.29347500 & 0.90917100 \\
\hline Pt 8 & 2.42452900 & 0.954 & -3.41051700 \\
\hline Pt9 & -3.59869800 & 1.87962500 & -1.67043000 \\
\hline F & -1.65083600 & 0.29422900 & -1.15717900 \\
\hline Pt11 & -0.73783000 & 3.65325300 & 1.63568800 \\
\hline & 2.52500900 & 2.20839200 & -1.28219000 \\
\hline & 2.68504700 & -1.16044000 & -2.1247970 \\
\hline & -1.65086600 & -0.17301600 & -3.62531500 \\
\hline & 0.76799400 & 1.03715400 & 2.91107100 \\
\hline & -3.51431700 & 2.34595000 & 0.7804100 \\
\hline & 2.10644000 & 2.16861400 & 1.132323 \\
\hline
\end{tabular}




\begin{tabular}{|c|c|c|c|}
\hline t18 & 0.10313600 & -3.35369200 & 1.28477200 \\
\hline Pt19 & -0.59580700 & 1.94138900 & -2.76751600 \\
\hline t20 & 0.85749100 & -2.06402300 & -0.71880300 \\
\hline Pt2 & -3.37779000 & -1.60299800 & -1.20637100 \\
\hline t22 & 0.82842800 & 0.37340800 & -1.43721600 \\
\hline t23 & -0.23662700 & 1.38272600 & 0.58798700 \\
\hline tet24 & 4.76601600 & 0.25044700 & 0.18689600 \\
\hline t 2 & -0.88967300 & -3.87121800 & -0.9630790 \\
\hline t2 & 1.85782700 & -2.19878600 & 2.5768840 \\
\hline t2 & 3.30613100 & -2.76662800 & -0.33498500 \\
\hline t28 & 3.65446100 & -0.52595100 & 2.25924700 \\
\hline t29 & -0.96588300 & -0.67004400 & 3.28025200 \\
\hline 29 & uster $=\mathrm{Pt} 30 \quad \mathrm{Q}=0$ & \multicolumn{2}{|c|}{$\mathrm{M}=0 \quad$ Etot $=-2758.10096900$} \\
\hline Pt1 & -0.89591500 & -0.03241700 & 0.26454000 \\
\hline Pt2 & -1.87012400 & 3.39977100 & 2.15212700 \\
\hline Pt3 & 1.01643300 & -3.18942400 & 2.15768500 \\
\hline Pt 4 & 0.26345800 & 2.02358600 & -0.41244800 \\
\hline Pt5 & 1.96253100 & -3.25021000 & -0.20104700 \\
\hline Pt 6 & 4.08179600 & -0.60532000 & -0.33297400 \\
\hline Pt7 & -3.03545900 & -1.03277900 & 1.00655400 \\
\hline Pt 8 & 3.11964400 & 2.27717800 & -1.68416200 \\
\hline Pt9 & 1.97408500 & 1.28990000 & 1.25449200 \\
\hline Pt10 & 2.12337600 & 3.56193500 & 390700 \\
\hline Pt11 & -1.03118500 & 1.04293200 & 2.52534200 \\
\hline Pt12 & -1.36198400 & -2.33813100 & 2.43574900 \\
\hline Pt13 & -0.90883600 & -4.52569000 & -1.43154900 \\
\hline Pt14 & 0.88148000 & -0.19784100 & -1.46574900 \\
\hline Pt15 & 1.36675900 & 3.28488800 & 2.62178500 \\
\hline Pt16 & 1.10550500 & -0.07357600 & 3.16678700 \\
\hline Pt17 & 0.13411000 & -0.81910000 & -3.79350500 \\
\hline Pt18 & 2.73069700 & -1.80974700 & -2.09810900 \\
\hline Pt19 & -1.87873700 & -1.58567800 & -2.56426300 \\
\hline Pt20 & -0.78049800 & -0.00205900 & 4.77130800 \\
\hline Pt2 2 & 1.23606700 & -1.05048700 & 0.84081700 \\
\hline Pt22 & -1.07391200 & 8800 & 773800 \\
\hline Pt23 & -0.29013800 & -2.23898300 & -0.72881100 \\
\hline Pt24 & -3.04909100 & 0.47128600 & -3.39587400 \\
\hline Pt25 & -2.74624700 & 1.52687800 & 0.80652100 \\
\hline Pt26 & -3.93757200 & 0.30058100 & -0.99093000 \\
\hline Pt27 & -2.68615600 & -2.92011400 & -0.59599300 \\
\hline Pt28 & 1.60529300 & 1.18967600 & -3.39863400 \\
\hline Pt2 & -1.38328600 & 00 & -2.26089100 \\
\hline Pt 30 & 3.32790400 & -0.62904200 & 2.1150610 \\
\hline \# 30 & Cluster $=$ Pt $31 \quad Q=0$ & $I=0$ & 3739 \\
\hline Pt1 & -2.83098800 & 1.87585000 & 0.22106000 \\
\hline Pt 2 & -2.82569900 & -0.18498300 & 1.62334400 \\
\hline Pt3 & 1.25019100 & 1.92937100 & 2.45772700 \\
\hline Pt 4 & -1.77344100 & 3.05402900 & 2.11433000 \\
\hline Pt5 & -1.70073000 & 1.37878700 & -4.08182300 \\
\hline Pt 6 & -2.39102800 & -0.82774200 & 3.98492600 \\
\hline Pt 7 & -3.29511500 & 0.16267600 & -2.62598100 \\
\hline Pt 8 & 1.36922500 & 0.31951800 & -3.6761850 \\
\hline Pt9 & -1.86379300 & -2.79682900 & -2.0258990 \\
\hline Pt10 & 3.24183600 & -0.16929100 & 0.65546400 \\
\hline Pt11 & 4.12440700 & -0.08406500 & -1.6781530 \\
\hline 2 & 0.43255100 & -2.67489800 & 3.8350480 \\
\hline & -4.34979100 & 0.04923200 & $-0.375929 c$ \\
\hline
\end{tabular}




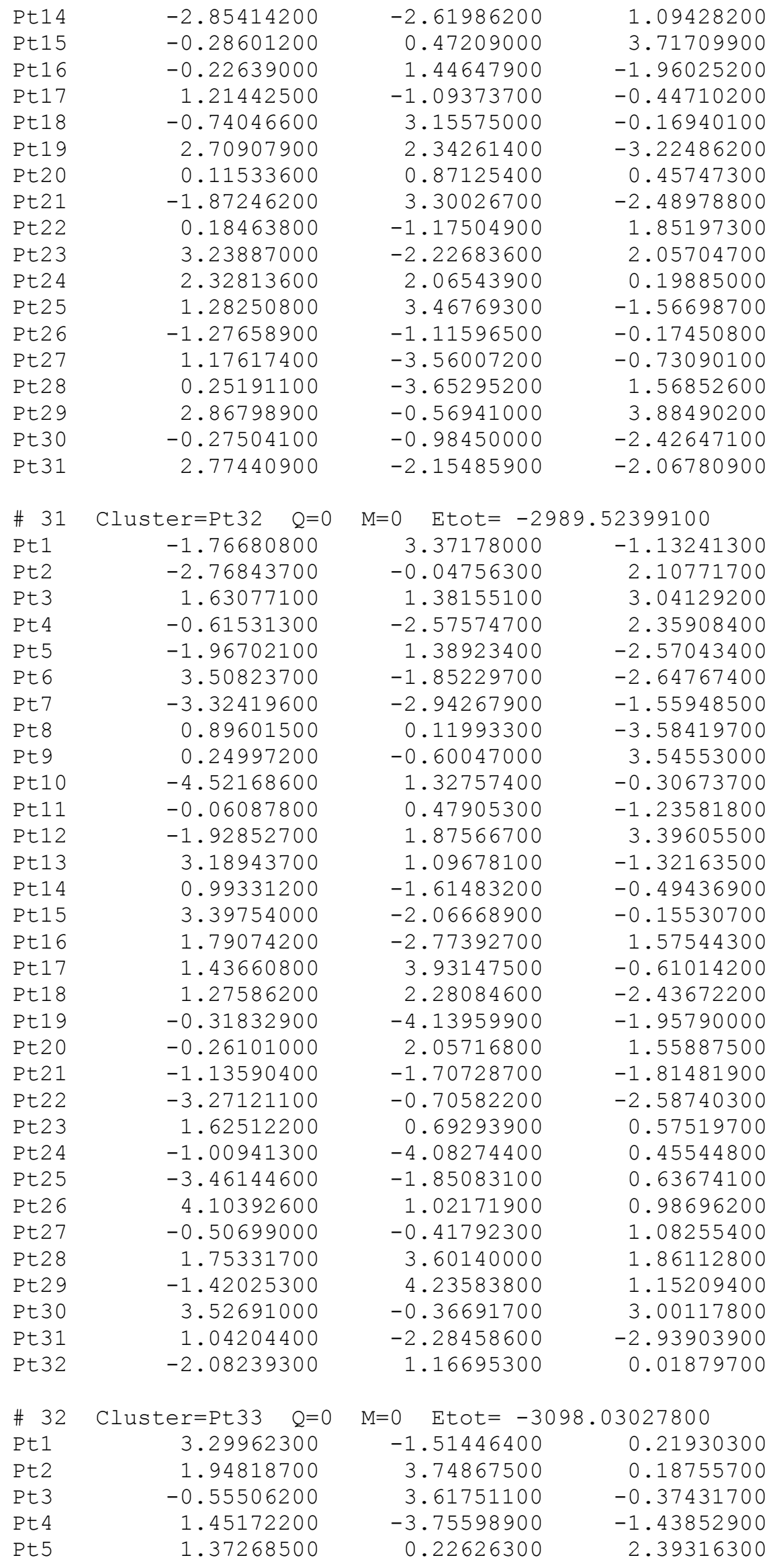




\begin{tabular}{|c|c|c|c|}
\hline Pt 6 & -2.97228800 & -2.33121100 & -2.02196900 \\
\hline Pt 7 & 1.00867000 & 3.30157600 & 3.43539800 \\
\hline Pt 8 & -0.90806200 & -0.61187900 & 1.81824000 \\
\hline Pt9 & 1.86732800 & 1.44845300 & -0.80911700 \\
\hline 10 & 0.89701100 & -0.74516400 & 0.10500400 \\
\hline 11 & -1.78396800 & 0.52432900 & -3.58272900 \\
\hline$=12$ & 1.24627000 & -0.43097400 & -2.31654900 \\
\hline$=13$ & -4.00474900 & -0.62209800 & 0.65723000 \\
\hline 14 & -0.57951700 & -3.85575700 & 1.29438000 \\
\hline 15 & 1.09137800 & -1.17862200 & 4.45502400 \\
\hline 16 & 0.20728200 & -0.59714200 & -4.59489500 \\
\hline 17 & -2.12768100 & 2.32796500 & -1.84467000 \\
\hline 18 & -1.19841000 & 2.37779200 & 2.8138840 \\
\hline$=19$ & -2.46456700 & 2.28220600 & 0.67894900 \\
\hline$=20$ & -0.09935300 & 1.44340000 & 0.74936400 \\
\hline 21 & -1.33617200 & -0.58647300 & 4.26831600 \\
\hline$=22$ & 3.14786900 & 1.67720600 & 1.30681000 \\
\hline 23 & 3.66176500 & -0.00600100 & -1.72849900 \\
\hline 24 & 1.61688400 & -2.71424700 & 1.57706800 \\
\hline$=25$ & -1.21714700 & -1.99868900 & -0.21897000 \\
\hline$=26$ & -1.84609300 & -4.28516400 & -0.90761400 \\
\hline$=27$ & 2.29369500 & 2.73151800 & -2.95808500 \\
\hline$=28$ & -3.42035000 & 0.09870200 & -1.69040400 \\
\hline$=29$ & -2.92028100 & -1.78121000 & 2.59175100 \\
\hline 30 & -0.93348800 & 0.19464600 & -1.2623410 \\
\hline$=31$ & 3.29334400 & 1.04137400 & 3.70564300 \\
\hline$=32$ & -0.12101300 & 2.46382400 & -3.47800700 \\
\hline-33 & 0.08449100 & -2.49035300 & -3.03038800 \\
\hline 3 & Cluster $=$ Pt $34 \quad \mathrm{Q}=0$ & $=0 \quad$ Etot $=-32$ & 82039900 \\
\hline Pt1 & -2.31754700 & 2.73075500 & -1.77762100 \\
\hline Pt2 & -1.11260600 & -3.51469900 & 1.5092190 \\
\hline Pt 3 & 0.55520400 & 0.65477900 & -4.68738700 \\
\hline Pt 4 & 0.89861300 & 0.07307900 & 3.84710600 \\
\hline Pt 5 & -2.44794300 & -0.98184700 & -4.4501290 \\
\hline Pt 6 & 1.00198600 & -2.39300200 & 3.8227930 \\
\hline Pt 7 & 1.48316700 & -3.04193300 & -1.86732500 \\
\hline Pt 8 & -0.73295800 & 0.97460200 & -2.55412900 \\
\hline Pt9 & 3.85597900 & 1.12699800 & -1.8426200 \\
\hline Pt10 & 1.06071200 & -0.92048200 & 0.84543500 \\
\hline 11 & -3.29683300 & -1.36475200 & 2.5829030 \\
\hline Pt12 & -0.76458500 & -3.35960800 & -2.91713300 \\
\hline $\mathrm{P}$ & 3.33389500 & -1.29824500 & 1.77104700 \\
\hline Pt14 & -0.42431900 & 25000 & 2.1996250 \\
\hline Pt15 & -1.02926900 & -0.40119200 & 2.1403450 \\
\hline Pt16 & 2.60458400 & 3.34091600 & 0.82536000 \\
\hline Pt17 & 0.89613900 & -0.63338500 & -1.61846200 \\
\hline Pt18 & 1.00475300 & 2.70056000 & -2.12409200 \\
\hline Pt19 & 2.05051600 & -3.17108700 & 0.60106300 \\
\hline Pt20 & -2.52592700 & -0.69246000 & -1.9927910 \\
\hline Pt21 & 0.07311000 & 1.81402700 & 2.2292830 \\
\hline Pt22 & 1.92329000 & 1.21748300 & -0.30994800 \\
\hline Pt23 & 1.66420900 & 2.43515500 & 4.04866700 \\
\hline Pt24 & -2.40630700 & 0.45293100 & 4.03159700 \\
\hline Pt25 & -4.42079200 & -1.16830600 & -0.4739310 \\
\hline Pt26 & -3.06075200 & 0.93782500 & -0.15785600 \\
\hline 21 & 0.39337200 & 4.07105600 & -0.15122100 \\
\hline 20 & 0.87487600 & -1.68802100 & -3.9040690 \\
\hline & -1.09403500 & -1.63265900 & \\
\hline
\end{tabular}




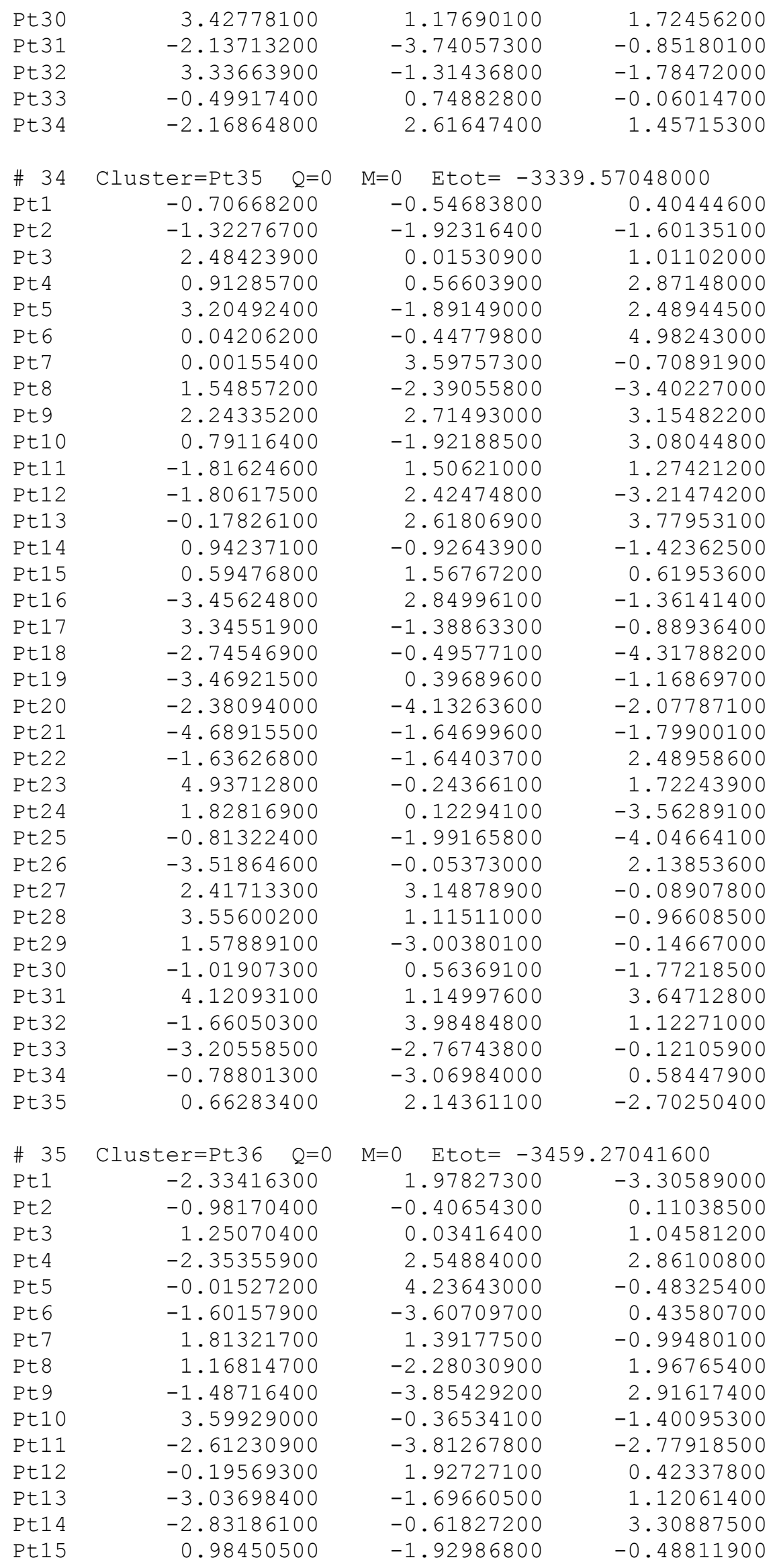




\begin{tabular}{|c|c|c|c|}
\hline Pt16 & -0.62708400 & -4.72738700 & -1.57213800 \\
\hline Pt17 & 2.05411300 & 2.37638900 & 1.49667400 \\
\hline Pt18 & -2.80592100 & 0.64451400 & -1.25115300 \\
\hline Pt19 & -1.94438300 & -0.53155800 & -3.28156500 \\
\hline et20 & 1.98077700 & -2.97608200 & -2.49694600 \\
\hline Pt21 & 3.85297400 & 2.83783100 & -1.50481600 \\
\hline$=22$ & 0.87142900 & -4.25518500 & 0.41914300 \\
\hline Pt2 & 3.66355300 & -0.62930200 & 1.09410200 \\
\hline$=24$ & -2.38059600 & -1.89931000 & -1.24332900 \\
\hline Pt25 & 0.75699700 & 3.07985100 & -2.55399900 \\
\hline Pt26 & 0.80675700 & 2.10950600 & 3.64990700 \\
\hline$=27$ & 0.79567100 & 1.12962200 & -4.10280800 \\
\hline 28 & 3.84319400 & 1.17434900 & 2.8366130 \\
\hline Pt29 & 4.08009000 & 3.70685700 & 0.78167100 \\
\hline$=30$ & 0.01216000 & -2.07159700 & -3.70820900 \\
\hline Pt31 & -0.76875600 & 0.54314600 & 2.46590600 \\
\hline Pt32 & -4.66609100 & -0.03489400 & 0.26719000 \\
\hline$=33$ & 1.13772700 & 4.74869600 & 1.69349100 \\
\hline$=34$ & 0.23562600 & -0.18216800 & -2.06487500 \\
\hline$=35$ & -2.57807800 & 2.57944300 & 0.32100400 \\
\hline Pt36 & 0.31426500 & -1.16847100 & 4.01663000 \\
\hline 36 & Cluster $=$ Pt $37 \quad Q=0$ & $=0 \quad$ Etot $=-35$ & 43431900 \\
\hline Pt1 & 1.61139100 & 1.88207100 & 1.9115630 \\
\hline Pt2 & -2.63999000 & 2.01851400 & -2.0016510 \\
\hline Pt 3 & -0.34776600 & 4.58586600 & 1.04477200 \\
\hline Pt 4 & -3.01746200 & -1.85649300 & 0.07663500 \\
\hline Pt 5 & -0.80293400 & 2.70640100 & 4.02489400 \\
\hline Pt 6 & 3.15702500 & -0.65436600 & 0.3677900 \\
\hline Pt 7 & 0.71014700 & -0.00746700 & 0.56052900 \\
\hline Pt 8 & 1.93331600 & 1.12505800 & -1.2840170 \\
\hline Pt9 & 3.44029300 & -1.32892100 & -2.95714600 \\
\hline Pt10 & 1.43911000 & -1.29235800 & -1.40730100 \\
\hline Pt11 & -0.61580600 & 2.10012700 & 0.76721100 \\
\hline Pt12 & -0.31290300 & 1.28623800 & -2.34773800 \\
\hline Pt13 & 0.45322700 & -2.98334400 & -2.99242500 \\
\hline Pt14 & 1.39186900 & 3.28883300 & -0.17385900 \\
\hline Pt15 & -1.15441100 & 0.091 & 2.1 \\
\hline Pt16 & -4.54856300 & -0.30101000 & -1.0358500 \\
\hline Pt17 & 2.47497800 & -2.84354800 & 1.30749800 \\
\hline Pt18 & 2.93759400 & 0.00098100 & 2.8938000 \\
\hline Pt19 & -2.87682700 & 1.48557100 & 3.27120500 \\
\hline Pt20 & -0.92886200 & -0.88327800 & -3.60354900 \\
\hline Pt2 & 0.02314200 & -1.80276200 & 3.343219 \\
\hline Pt22 & 1.11371100 & 0.00042600 & 4.6361450 \\
\hline Pt23 & -2.84579900 & -2.40185000 & -3.18781600 \\
\hline Pt24 & 1.07794500 & 4.03836500 & 3.04078300 \\
\hline Pt25 & -0.03248800 & 3.74541200 & -2.18415800 \\
\hline Pt26 & 3.79504300 & 2.62146700 & -0.56239600 \\
\hline Pt27 & 0.44528300 & -3.97020000 & 2.2001310 \\
\hline Pt28 & -1.74173800 & -3.93167000 & -0.3569800 \\
\hline Pt29 & -0.97489100 & -0.73363600 & -1.09413400 \\
\hline Pt30 & 0.59687600 & -4.51173500 & -1.00469600 \\
\hline Pt31 & -0.70460000 & -2.07669500 & 0.97267400 \\
\hline Pt 32 & -3.12617800 & -1.05955600 & 3.1478200 \\
\hline Pt33 & 2.67251400 & -3.42171100 & -1.85324400 \\
\hline & 0.07614100 & 0.50192700 & -5.40438400 \\
\hline - & 2.83475900 & 0.98159900 & -3.61317200 \\
\hline 年 & -2.57170700 & 0.68112400 & 0.1457430 \\
\hline
\end{tabular}




\begin{tabular}{|c|c|c|c|}
\hline Pt37 & -2.94143900 & 2.91895400 & 1.20890600 \\
\hline \# 37 & luster $=\operatorname{Pt} 38 \quad Q=0$ & Etot $=-37 c$ & 42632800 \\
\hline Pt1 & 3.80410400 & 0.76440900 & -0.0321070 \\
\hline Pt2 & -4.80942300 & -1.19232800 & 1.56024900 \\
\hline Pt 3 & -4.03340600 & -0.87062300 & -0.79532100 \\
\hline Pt 4 & -1.76099100 & 4.21112600 & 0.89762600 \\
\hline Pt5 & -4.76115400 & 1.30250200 & 1.77326700 \\
\hline Pt 6 & -2.81526400 & 1.09249900 & 0.24239700 \\
\hline Pt 7 & 1.02417800 & 0.84375900 & 3.74489200 \\
\hline Pt 8 & -1.63723200 & -2.05622000 & 0.90945700 \\
\hline Pt 9 & 0.35446300 & -2.31188400 & -0.59342700 \\
\hline Pt10 & 4.84889500 & -2.48441600 & 0.66933500 \\
\hline Pt11 & 1.75019600 & 1.10650100 & 1.33794600 \\
\hline Pt12 & -2.22401000 & 0.10464700 & 3.49883100 \\
\hline Pt13 & 1.59889400 & -0.14048800 & -0.84838400 \\
\hline Pt14 & 0.71830800 & -4.53216500 & 0.52582600 \\
\hline Pt15 & -0.85713000 & -3.00908700 & -3.51204600 \\
\hline Pt16 & -0.83494700 & -0.14551300 & -0.52203600 \\
\hline Pt17 & 0.84290200 & 3.04762800 & 2.59832500 \\
\hline Pt18 & 4.21877800 & -0.1 & 0600 \\
\hline Pt19 & -2.06359200 & -0.82958900 & -3.64299900 \\
\hline Pt20 & 2.16815400 & 0.84450000 & -3.91560000 \\
\hline Pt21 & -1.27131700 & 3.33053200 & -3.55486100 \\
\hline Pt22 & 1.72058400 & -1.41052800 & 1.26446100 \\
\hline Pt23 & 0.85527100 & -3.29833200 & 49500 \\
\hline Pt24 & -3.26382600 & 3.423 & -2.0 \\
\hline Pt25 & -1.84567400 & -3.31515300 & -1.21321500 \\
\hline Pt26 & 2.83575800 & -2.46617000 & -0.76816100 \\
\hline Pt27 & 1.26831300 & 1000 & 0700 \\
\hline Pt28 & 0.09 & 4.2 & -1.67909900 \\
\hline Pt29 & -0.33290300 & -0.16 & 35700 \\
\hline Pt 30 & -2.27039700 & -2.382 & 3.27364400 \\
\hline Pt31 & 2.64196500 & 3.00073100 & -0.00665100 \\
\hline Pt32 & 4.77089400 & -0.34026500 & -2.02436500 \\
\hline Pt33 & -3.0 & 0.9 & $-2.2 \cdot \operatorname{s} \cdot \ln$ \\
\hline Pt34 & 9900 & $-1 \cdot 3$ & 400 \\
\hline Pt 35 & -0.68430800 & 2.30261800 & -0.33760600 \\
\hline Pt36 & 2.31582400 & -2.36089200 & -3.19634300 \\
\hline Pt37 & 0.06146000 & 0.15117200 & -2.83735600 \\
\hline Pt38 & -1.44681900 & 2.04959300 & 2.05537200 \\
\hline \# 38 & ter $=\operatorname{Pt} 39 \quad \mathrm{Q}=0$ & $=0 \quad$ Etot $=-38$ & 432 \\
\hline Pt 1 & -3.75375700 & 2.73170000 & -0.14232700 \\
\hline Pt2 & 3.47409200 & 1.11487800 & 3.01598700 \\
\hline Pt3 & 1.29224300 & -2.89902900 & -3.53062800 \\
\hline Pt 4 & 2.92461000 & 0.87801800 & -1.52550600 \\
\hline Pt 5 & -0.81950800 & 2.94128400 & -3.23065300 \\
\hline Pt 6 & 4600 & 2.75 & 367200 \\
\hline Pt 7 & 68200 & -0.675 & 385500 \\
\hline Pt 8 & -2.02939500 & -2.69610700 & 1.00639100 \\
\hline Pt 9 & 0.37372600 & -2.02970500 & 1.22664400 \\
\hline Pt10 & -2.42906500 & -0.25115600 & 0.76851700 \\
\hline Pt11 & 0.31948600 & 1.34703600 & 1.8241950 \\
\hline Pt12 & 2.24747500 & 3.95980200 & 1.99587900 \\
\hline Pt13 & 2.07179600 & -4.06753300 & -0.55578000 \\
\hline Pt14 & -1.41444800 & 1.98197100 & 0.14226900 \\
\hline Pt15 & -1.54417900 & 0.74802500 & -2.06645900 \\
\hline Pt16 & 1.70528700 & -0.61052200 & 2.73981300 \\
\hline
\end{tabular}




\begin{tabular}{|c|c|c|c|}
\hline Pt17 & 0.95015000 & 2.15939600 & -0.60433700 \\
\hline Pt18 & -0.66214200 & -2.29111900 & -1.03232900 \\
\hline Pt19 & -3.20311500 & 2.56320900 & -2.59468100 \\
\hline Pt20 & -2.27961800 & -3.88791600 & -2.08687300 \\
\hline Pt21 & 1.33115800 & -1.01447300 & -1.91009500 \\
\hline Pt22 & 2.13835800 & -3.06204500 & 2.64919700 \\
\hline Pt23 & -0.10414200 & -0.04436700 & -0.14651300 \\
\hline Pt24 & -4.10111800 & -1.91959100 & -0.10237800 \\
\hline Pt25 & -0.18932300 & -4.36523000 & 0.48801800 \\
\hline Pt26 & 1.05787200 & 1.48724500 & -3.95202200 \\
\hline Pt27 & -0.67350000 & -1.29535600 & -3.30036800 \\
\hline Pt28 & 4.60590700 & -0.09064900 & 1.18400500 \\
\hline Pt29 & -4.83483100 & -0.10900900 & -1.60826800 \\
\hline Pt30 & 1.24137000 & 1.49477200 & 4.09518600 \\
\hline Pt31 & -3.10051000 & -0.83944000 & -3.22254200 \\
\hline Pt32 & -1.09089600 & 4.26439400 & 1.10823600 \\
\hline Pt33 & -0.67308200 & 3.13373300 & 3.30934600 \\
\hline Pt3 4 & -1.48847500 & 0.05324900 & 3.02033800 \\
\hline Pt35 & -4.32568300 & 1.06985000 & 1.62529500 \\
\hline Pt36 & 4.34131800 & -3.27179200 & 0.09681800 \\
\hline Pt37 & 0.46874400 & 4.63738500 & -0.82358900 \\
\hline Pt38 & 3.77147500 & -1.49320700 & -1.61321500 \\
\hline Pt39 & -1.11100800 & -2.40598400 & 3.32990200 \\
\hline \# 39 & Iuster $=$ Pt $40 \quad Q=0$ & $\mathrm{M}=0 \quad \mathrm{Etot}=-39$ & 40782400 \\
\hline Pt1 & -1.30722600 & 3.06049500 & -2.15859700 \\
\hline Pt2 & 1.38037100 & -2.49962900 & -3.04131900 \\
\hline Pt3 & 2.91985600 & 2.03571800 & -3.44910500 \\
\hline Pt 4 & -0.34826900 & -5.61213800 & -1.01776300 \\
\hline Pt 5 & 4.40991900 & -0.38199200 & -0.18098900 \\
\hline Pt 6 & 2.73265000 & -1.50552700 & 1.32061000 \\
\hline Pt 7 & -0.26913800 & 5.84679800 & -0.70022000 \\
\hline Pt 8 & 1.57243900 & -4.20432600 & -0.25262200 \\
\hline Pt 9 & 1.23913100 & 1.04361300 & 3.00763800 \\
\hline Pt10 & -0.50371000 & -4.95901600 & 2.30750300 \\
\hline Pt11 & 0.97514400 & 2.82716100 & 0.31392400 \\
\hline Pt12 & -0.90870200 & -1.51560700 & -3.19903200 \\
\hline Pt13 & -1.57914700 & -2.75003300 & 2.75493000 \\
\hline Pt14 & -3.50069600 & 3.18532200 & -0.98236300 \\
\hline Pt15 & -2.01326700 & 0.69643000 & -2.59987600 \\
\hline Pt16 & 0.05325800 & 4.13937200 & 2.20814000 \\
\hline Pt17 & -3.22047800 & 1.37148000 & 1.73031900 \\
\hline Pt18 & 2.71378200 & -3.90024800 & 1.95720700 \\
\hline Pt19 & -1.02534100 & 1.36552000 & 0.5551200 \\
\hline Pt20 & -2.09944600 & 3.62437100 & 1.05268300 \\
\hline Pt21 & -4.03451500 & -0.04405800 & -0.13794000 \\
\hline Pt22 & 0.73583900 & 4.46261200 & -2.49644500 \\
\hline Pt23 & -1.87929700 & 1.77956500 & 3.78965300 \\
\hline Pt24 & -3.89550600 & -1.89523900 & 2.5492440 \\
\hline Pt25 & -1.74479100 & -0.98230200 & 0.0857030 \\
\hline Pt26 & 2.11073800 & -0.14504900 & -2.59726600 \\
\hline Pt27 & 3.56465300 & -2.06565500 & -1.86281500 \\
\hline Pt28 & -0.80949200 & -3.25792500 & -0.45117500 \\
\hline Pt29 & 1.24731500 & 0.39183700 & 0.5798960 \\
\hline Pt30 & -3.07526500 & -4.15265300 & 0.1204630 \\
\hline Pt31 & 0.28795100 & 1.24679800 & -1.5459820 \\
\hline Pt32 & -0.85792100 & -0.37246200 & 3.1692170 \\
\hline Pt33 & 3.41558400 & 0.86446700 & 1.7811630 \\
\hline & 0.64009700 & -2.22187600 & 3.84273600 \\
\hline
\end{tabular}




\begin{tabular}{|c|c|c|c|}
\hline Pt35 & 0.41735400 & -1.21986700 & -1.08620800 \\
\hline Pt36 & -0.26321900 & 1.71746700 & -4.01723500 \\
\hline t37 & 2.63801800 & 4.67688500 & 0.26912100 \\
\hline$=38$ & 3.13513300 & 2.59850700 & -1.01048800 \\
\hline$=3$ & 0.23025900 & -1.74838800 & 1.34504300 \\
\hline 10 & -3.08406700 & -1.50042700 & -1.95287300 \\
\hline 40 & Cluster $=$ Pt $41 \quad \mathrm{Q}=0$ & \multicolumn{2}{|c|}{$\mathrm{M}=0 \quad$ Etot $=-4050.29797600$} \\
\hline t1 & -2.52242800 & -0.55391900 & 4.20543500 \\
\hline$=2$ & -0.50355900 & -1.87286500 & -1.47968000 \\
\hline$=3$ & 1.06263800 & 4.07281900 & -1.67360100 \\
\hline 4 & -3.34937300 & -0.32925800 & -1.64312000 \\
\hline 5 & -2.20682400 & -2.89111400 & 0.02458800 \\
\hline 6 & 4.36024300 & -0.45430700 & 1.25919200 \\
\hline t 7 & -0.00939300 & -2.25212400 & 0.95532200 \\
\hline$=8$ & -2.60542300 & -1.01500800 & -3.92029200 \\
\hline 9 & -2.89781100 & -3.40573600 & 2.35905800 \\
\hline & 0.06617700 & 1.75521800 & -1.65483300 \\
\hline & -4.39869700 & -0.41784800 & 2.59293500 \\
\hline & -0.43639200 & -2.01141600 & 4.24574500 \\
\hline$=1$. & 3.92781800 & -0.60533500 & -1.20415000 \\
\hline 14 & 2.42062600 & -4.09415700 & 1.22715700 \\
\hline$\perp$ & -0.96290500 & -4.34184800 & -1.56676900 \\
\hline & 1.64352000 & 1.49424600 & 0.27935600 \\
\hline & -1.18056900 & 0.57984400 & 2.40386600 \\
\hline 18 & -0.27424000 & 2.24780900 & -4.06636900 \\
\hline$P$ & 0.81743800 & 2.08852500 & 907400 \\
\hline$=20$ & 3.15598300 & 2.66044000 & -1.39869800 \\
\hline$\angle$ & -2.70750900 & -0.49812200 & 0.74895100 \\
\hline$=22$ & -1.21034700 & 2.52318200 & 3.96029400 \\
\hline 23 & 2.33386900 & -1.66175100 & 0 . \\
\hline 24 & -3.80600600 & 2.73811100 & 58400 \\
\hline P & -0.36937300 & 0.01412200 & 0.12969200 \\
\hline $\mathrm{P}$ & 3.19855100 & 2.54052100 & 1.92618100 \\
\hline$=2$ & 2.66860200 & -1.88545800 & -2.94885000 \\
\hline 28 & 4.14349600 & 1.06625500 & -3.06086800 \\
\hline$P$ & 0.38955900 & 4.43781900 & 769300 \\
\hline & -2.09442200 & 4.08636200 & -0.5 \\
\hline & -0.49797000 & -4.01223300 & 2.70312400 \\
\hline-32 & 0.39548200 & -2.42776400 & -3.80027300 \\
\hline & 1.42394400 & -1.22895600 & 2.71191500 \\
\hline & 1.46667400 & 0.64119100 & -4.7977630 \\
\hline & -2.66856800 & 2.30580900 & -3.39133600 \\
\hline & -0.33714200 & -0.12149700 & -3 \\
\hline & -2.98711800 & 1.75715100 & 933500 \\
\hline & 2.24109500 & -3.62552900 & -1.19913400 \\
\hline & 1.44401100 & -0.29611700 & -1.48021700 \\
\hline Pt 40 & -0.75768300 & 2.37781000 & 0.66531100 \\
\hline Pt 41 & 1.62402800 & 0.61512600 & 4.38333800 \\
\hline$\# 41$ & Cluster=Pt $42 \quad \mathrm{Q}=0$ & Etot $=-42$ & 0268 \\
\hline Pt 1 & 3.24196600 & -1.24986600 & -0.96495800 \\
\hline Pt2 & 5.66031700 & 0.76142900 & 0.11008900 \\
\hline Pt 3 & -3.31403100 & 0.66794500 & 0.78524700 \\
\hline Pt 4 & 0.78634700 & -3.15396200 & -1.9239580 \\
\hline Pt 5 & 3.44362100 & -1.32402400 & 1.53083900 \\
\hline te & -1.25325400 & -0.79006100 & 0.82153800 \\
\hline & -2.22946300 & -3.88625500 & 1.6039350 \\
\hline & 00 & 546000 & \\
\hline
\end{tabular}




\begin{tabular}{|c|c|c|c|}
\hline Pt9 & -1.25931800 & -2.94132200 & -0.49919900 \\
\hline Pt10 & 4.67334400 & 4.94312700 & 1.69135500 \\
\hline Pt11 & 4.36750200 & 1.92450200 & -1.75922800 \\
\hline Pt12 & 1.84901700 & -0.03123300 & -2.64795800 \\
\hline Pt13 & 2.41016400 & 1.09757700 & -0.47181800 \\
\hline Pt14 & -5.77197900 & -1.38219200 & -0.20593300 \\
\hline Pt15 & -5.86281600 & -3.46730300 & -1.61143300 \\
\hline Pt16 & -0.23792900 & 1.37675700 & -2.75083700 \\
\hline Pt17 & 3.59447900 & 3.14132100 & 0.31612900 \\
\hline Pt18 & 6.11013500 & 2.05593300 & 2.21088600 \\
\hline Pt19 & -1.27648700 & -0.59671100 & -1.64071000 \\
\hline Pt20 & -3.26660500 & -1.61927500 & 2.03322100 \\
\hline Pt21 & -0.89172000 & 2.53782500 & 1.73692500 \\
\hline Pt22 & -2.78178200 & -4.70922600 & -1.49272700 \\
\hline Pt23 & 1.62443300 & 5.62748700 & 0.49029700 \\
\hline Pt24 & 2.18788800 & 3.22963700 & -1.79046600 \\
\hline Pt25 & -3.72215800 & -2.69977700 & -2.62885800 \\
\hline Pt26 & -4.98693100 & -5.42751800 & 0.85104700 \\
\hline Pt27 & -4.67183300 & -0.35523400 & -2.20282800 \\
\hline Pt28 & 1.32532500 & 1.28067400 & 1.80532300 \\
\hline Pt29 & 2.58024600 & -0.04967200 & 3.52157700 \\
\hline Pt 30 & -5.38957900 & -3.06964600 & 1.63794700 \\
\hline Pt31 & 1.65528900 & 4.40293000 & 2.69774700 \\
\hline Pt 32 & -3.28920600 & -1.51520000 & -0.45772500 \\
\hline Pt 33 & -2.28201900 & 1.67421900 & -1.26788300 \\
\hline Pt 34 & 0.97081200 & -3.33288100 & 0.58872100 \\
\hline Pt35 & 4.71513100 & 5.09989700 & -0.77353900 \\
\hline Pt 36 & -2.70703400 & -0.66360200 & -3.71363500 \\
\hline Pt 37 & -0.07629700 & 1.17559000 & -0.24904500 \\
\hline Pt 38 & -0.51022200 & -1.89993500 & -3.64760500 \\
\hline Pt 39 & 3.62235100 & 1.97082700 & 2.52964000 \\
\hline Pt 40 & -0.71036500 & 0.361 & 2.97108800 \\
\hline Pt 41 & 1.21359000 & -0.86237000 & 0.47166600 \\
\hline Pt 42 & 0.19060500 & -1.94710900 & 2.53150600 \\
\hline \# 42 & Cluster $=\mathrm{Pt} 43$ & \multicolumn{2}{|c|}{$\mathrm{M}=0 \quad \mathrm{E}$ tot $=-4326.38919800$} \\
\hline Pt 1 & -3.96180400 & -0.79226600 & -3.35222500 \\
\hline Pt2 & 1.73986300 & 1.56885100 & 0.09364600 \\
\hline Pt 3 & -0.77404900 & 0.38230100 & -3.62422400 \\
\hline Pt 4 & -1.78563700 & -0.96228500 & 0.79493300 \\
\hline Pt5 & 2.78644800 & 1.88586900 & 4.58897200 \\
\hline Pt 6 & 2.77896000 & 0.21808600 & 1.91142100 \\
\hline Pt 7 & -1.29924500 & -1.79161300 & -4.73611000 \\
\hline Pt 8 & 0.85558700 & 0.88972000 & 3.28707600 \\
\hline Pt9 & -5.21011900 & 0.66627400 & -5.02646300 \\
\hline Pt10 & 0.05400100 & 3.34045700 & -0.14991200 \\
\hline Pt11 & -3.67555500 & -2.31173700 & -5.28440800 \\
\hline Pt12 & -2.76993200 & 2.14875600 & 0.14306100 \\
\hline Pt13 & 2.56108400 & -1.52805800 & -0.94798400 \\
\hline Pt14 & 2.40241200 & 0.14432700 & -2.73528200 \\
\hline Pt15 & 0.23190100 & -2.64008700 & -2.95104700 \\
\hline Pt16 & 2.17300200 & -2.94926000 & 1.02092700 \\
\hline Pt17 & 0.08426800 & -1.30611300 & -0.83109800 \\
\hline Pt18 & -2.21845200 & 0.34397400 & 2.82440700 \\
\hline Pt19 & 0.73938300 & -0.53143100 & -5.4160150 \\
\hline Pt20 & 0.60257600 & -0.99742300 & 1.6278750 \\
\hline Pt21 & 4.23326600 & -2.32561900 & 3.41604000 \\
\hline Pt22 & -0.20531500 & -1.72716300 & 4.85840600 \\
\hline Pt23 & -3.54300500 & 1.45886200 & -2.22451700 \\
\hline
\end{tabular}




\begin{tabular}{|c|c|c|c|}
\hline Pt24 & -1.49199500 & 2.67190700 & -2.98868800 \\
\hline Pt25 & -2.07882500 & -0.53158400 & -1.71801900 \\
\hline Pt26 & -3.41541400 & 2.33040000 & -4.57819100 \\
\hline Pt27 & 5.02102800 & 0.02718900 & 3.00158600 \\
\hline Pt28 & -0.24780300 & 0.65143100 & 5.59237700 \\
\hline Pt29 & -0.52829000 & 1.19470900 & 1.14192100 \\
\hline Pt30 & -0.26374200 & 1.14560400 & -1.31856100 \\
\hline Pt31 & -2.15055600 & -2.85588800 & -0.78113800 \\
\hline Pt32 & -0.08524100 & -3.38570600 & 1.97247400 \\
\hline Pt33 & 1.59585000 & 3.25729500 & 2.84420200 \\
\hline$t 34$ & 2.05164600 & 0.47103600 & 6.53832900 \\
\hline Pt35 & 2.85280500 & -2.70699800 & 5.53101400 \\
\hline Pt36 & -0.54232500 & 2.76939500 & 4.10486700 \\
\hline Pt 37 & -0.79553400 & 1.35105500 & -5.96670500 \\
\hline$=38$ & -4.49368500 & -0.48043400 & -0.95415300 \\
\hline$=39$ & 4.14780900 & 1.10512600 & 0.04899900 \\
\hline$=40$ & 0.99060700 & 2.15536700 & -3.23289400 \\
\hline Pt41 & 4.19367900 & -0.68151600 & 6.19218400 \\
\hline Pt42 & 1.95456400 & -1.29053800 & 3.70605300 \\
\hline Pt43 & -2.51422000 & -0.38227200 & -6.42313300 \\
\hline$\# 43$ & ter $=\operatorname{Pt} 44$ & $=0 \quad$ Etot $=-4$ & 60778400 \\
\hline Pt1 & 1.37989400 & -5.77960100 & 2.7222870 \\
\hline Pt2 & -1.80454200 & -0.72791800 & 3.3463050 \\
\hline Pt3 & 1.24666900 & 4.87848600 & -3.98789100 \\
\hline Pt 4 & 0.53279700 & -1.40260300 & 3.89376100 \\
\hline Pt5 & 2.66414600 & 3.75777800 & 0.33546800 \\
\hline Pt 6 & -0.05199000 & -2.58686000 & -0.7098790 \\
\hline Pt 7 & -0.04855500 & -4.85857400 & -1.7421980 \\
\hline Pt 8 & 1.68830600 & 2.73610200 & -2.6855640 \\
\hline Pt9 & -0.98975500 & 6.12432800 & -4.01088700 \\
\hline Pt10 & -0.21218200 & -1.48021800 & 1.52225200 \\
\hline Pt11 & -1.80587300 & -3.49876700 & 0.79610500 \\
\hline Pt12 & 1.62115300 & -6.71851100 & 0.4243460 \\
\hline Pt13 & 2.49836400 & 4.92372300 & -1.83163800 \\
\hline Pt14 & -3.17383800 & -1.46732600 & 0.31854500 \\
\hline Pt15 & -2.81139100 & 3.78460200 & -2.45188800 \\
\hline Pt16 & 0.15006000 & -4.74223600 & 4.63554900 \\
\hline Pt17 & -2.10973500 & -5.44774600 & 2.35659600 \\
\hline Pt18 & 1.95604200 & -3.41689200 & 3.42386600 \\
\hline Pt19 & -1.20820300 & 2.76191100 & 3.09804400 \\
\hline Pt20 & -0.89556100 & -6.77167300 & 0.64747200 \\
\hline Pt21 & 1.39411400 & -0.67500200 & -0.1840880 \\
\hline Pt22 & -1.25989000 & 2.08561500 & -1.5709790 \\
\hline Pt23 & 0.54142000 & 0.55117400 & -2.1781070 \\
\hline Pt24 & -0.20274100 & 6.92608100 & -1.78917400 \\
\hline Pt25 & -2.83869600 & 0.91517100 & 0.91840900 \\
\hline Pt26 & 0.10881600 & 0.70725200 & 2.58479700 \\
\hline Pt27 & -2.65852100 & 0.21209200 & -2.47373000 \\
\hline Pt28 & -1.61469400 & 3.09612600 & 0.6542900 \\
\hline Pt29 & 0.15818000 & -1.72201000 & -3.0900260 \\
\hline Pt30 & -1.11895800 & 2.73573900 & -4.00335900 \\
\hline Pt31 & -2.55296400 & -3.02939000 & -1.55783000 \\
\hline Pt32 & -1.11183700 & -0.25408000 & -0.4735560 \\
\hline Pt33 & -2.42457400 & -3.13593900 & 3.1907500 \\
\hline Pt 34 & 2.41644000 & 0.68206500 & 1.6567180 \\
\hline Pt35 & 2.15524100 & -3.74515900 & -1.3603160 \\
\hline Pt36 & 3.46812400 & 0.64044100 & -0.6497540 \\
\hline & 0.59761400 & -3.80333700 & 1.4040930 \\
\hline
\end{tabular}




\begin{tabular}{|c|c|c|c|}
\hline$t 38$ & 0.36494100 & $3.8266360 \mathrm{c}$ & -0.87600700 \\
\hline Pt39 & 0.29345000 & 5.98174900 & 0.47448300 \\
\hline Pt 40 & 3.05299700 & -2.37977700 & 0.51202100 \\
\hline Pt41 & -1.78986100 & 5.25402900 & -0.67656800 \\
\hline Pt 42 & 0.40362400 & $1.5765620 \mathrm{c}$ & 0.24581000 \\
\hline$t 43$ & 2.82524300 & $0.5126760 \mathrm{c}$ & -3.05949300 \\
\hline Pt 44 & 1.16672400 & 2.97328000 & 2.20096400 \\
\hline 44 & ter $=$ Pt 45 & \multicolumn{2}{|c|}{$\mathrm{M}=0 \quad \mathrm{Etot}=-4584.84260600$} \\
\hline Pt1 & 4.63852000 & 0.63369600 & -3.19428000 \\
\hline Pt2 & -3.69708200 & 0.41299200 & 2.30412700 \\
\hline Pt 3 & 2.05076500 & 2.84768500 & -2.52570600 \\
\hline Pt 4 & 2.85704000 & -4.55495200 & -4.80410700 \\
\hline Pt 5 & -1.47294800 & -0.54177400 & 1.62487600 \\
\hline Pt 6 & -0.33211500 & -0.36601900 & 5.56030700 \\
\hline Pt 7 & -4.22876700 & 2.5276170 & 0.93195600 \\
\hline Pt 8 & -1.66921400 & 1.6262420 & 6.30367800 \\
\hline Pt 9 & -2.42885800 & -2.3481080 & -1.03743000 \\
\hline Pt10 & 0.71774700 & -0.4122170 & -5.48395300 \\
\hline Pt11 & -1.28073100 & 3.9999630 & 0.70472300 \\
\hline Pt12 & 3.20304400 & -2.4856020 & -6.10740500 \\
\hline Pt13 & 0.52698400 & -1.5172160 & -2.49772600 \\
\hline Pt14 & 0.18710600 & -2.7358800 & -4.63461100 \\
\hline Pt15 & 3.30215200 & 0.5095860 & 829600 \\
\hline Pt16 & -2.26971900 & -0.7070530 & 852100 \\
\hline Pt17 & -2.84576700 & 3.5526920 & 5.24898400 \\
\hline Pt18 & 3.13635000 & 0.0175430 & -5.99943200 \\
\hline Pt19 & -1.36691200 & -0.1140430 & -1.53889500 \\
\hline Pt20 & -4.35101400 & 0.2413900 & -0.05682900 \\
\hline Pt21 & -1.59123300 & 1.5124070 & 2.95644400 \\
\hline Pt22 & 0.42787300 & -4.0311890 & -2.4999900 \\
\hline Pt23 & 1.26057900 & -0.3140070 & 3.55959500 \\
\hline Pt24 & 4.49867300 & -2.7748400 & -3.95889700 \\
\hline Pt25 & -5.71582700 & 2.4228470 & 3.95768900 \\
\hline Pt26 & -2.25733400 & -0.5549810 & -3.86353200 \\
\hline Pt27 & 0.38343400 & 2.5682450 & 44200 \\
\hline Pt28 & 1.92630800 & -0.0436680 & -1.13221200 \\
\hline Pt29 & -1.20462700 & 1.5260700 & 0.31457700 \\
\hline Pt30 & -0.74671500 & 3.6596820 & 3.91518800 \\
\hline Pt31 & -2.15874000 & -2.7988130 & 2.33071600 \\
\hline Pt32 & 1.85954800 & 2.2906010 & -0.08282300 \\
\hline Pt33 & 0.81856500 & 0.2 & 1.09551700 \\
\hline Pt3 4 & -0.08416500 & 1.656 & 273000 \\
\hline Pt 35 & -4.36940100 & 0.5003140 & 4.75392500 \\
\hline Pt36 & -2.28873100 & 2.1744530 & -1.81721700 \\
\hline Pt37 & 1.35331400 & -2.1021370 & 1.79302900 \\
\hline Pt38 & 1.25544800 & 2.1279780 & 4.25677200 \\
\hline Pt39 & 2.36371100 & -0.2718730 & -3.61067800 \\
\hline Pt 40 & -0.19439200 & -1.7756320 & 254500 \\
\hline Pt 41 & 1.68421000 & -3.4710330 & -0.34923800 \\
\hline Pt 42 & -3.88490600 & 3.8228840 & 2.99633400 \\
\hline Pt 43 & 3.85369400 & -1.3830900 & -1.93831600 \\
\hline Pt 44 & 4.40358000 & -1.6821460 & 0.48375300 \\
\hline Pt 45 & 3.73055200 & -3.9030980 & -1.79289700 \\
\hline \# 45 & Cluster $=$ Pt $46 \quad Q=0$ & Etot $=-$ & 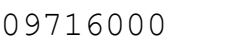 \\
\hline Pt1 & 0.39719300 & 5.8592670 & 2.04348400 \\
\hline Pt2 & -0.71157900 & -6.0872400 & -2.06452400 \\
\hline Pt3 & -1.66029400 & -1.8432690 & -2.596397 \\
\hline
\end{tabular}




\begin{tabular}{|c|c|c|c|}
\hline Pt 4 & -3.87303900 & -2.73944400 & -1.90099300 \\
\hline Pt5 & 0.37828500 & -1.52955100 & -1.24350400 \\
\hline Pt 6 & -2.49660500 & 2.73336300 & 1.76496400 \\
\hline Pt 7 & 1.80599600 & -3.75724500 & -4.99683000 \\
\hline Pt 8 & -1.42302700 & $5.3762850 \mathrm{C}$ & 3.63346500 \\
\hline Pt9 & 3.09721800 & $4.8695940 \mathrm{C}$ & 3.45473300 \\
\hline Pt10 & -2.63408500 & 0.42284000 & -2.46673600 \\
\hline Pt11 & -1.13441300 & -5.28886600 & -5.27724000 \\
\hline t12 & 2.16608300 & -3.07260700 & -0.33763800 \\
\hline t13 & -0.61976700 & -3.45190100 & 1.39885600 \\
\hline t14 & -3.11087000 & -5.99881300 & -2.66318900 \\
\hline t15 & 0.46505800 & 2.61566500 & 0.31761400 \\
\hline t16 & 2.48221500 & 2.79807000 & 4.59579500 \\
\hline t17 & -0.58240800 & 3.22811100 & 5.92333800 \\
\hline 18 & 1.45809400 & 0.31627000 & -0.02125800 \\
\hline t19 & 2.00905300 & 1.12464900 & -2.33053900 \\
\hline t20 & 3.66355100 & 0.13976400 & 2.71693500 \\
\hline t21 & 1.47131300 & -5.35966000 & -3.01361100 \\
\hline Pt22 & 3.77219800 & 3.90380100 & 0.35808300 \\
\hline t23 & -0.22304800 & 3.43907900 & 2.60122100 \\
\hline t24 & -0.33499300 & -0.99672500 & 1.05475500 \\
\hline t25 & 2.89384800 & -1.08934300 & -1.63419600 \\
\hline Pt26 & -1.95560000 & 2.13797400 & 4.08357900 \\
\hline Pt27 & 0.02418300 & 2.56773700 & -2.13201500 \\
\hline Pt28 & -1.86159700 & 3.09951600 & -0.57909000 \\
\hline Pt29 & 3.56922500 & 1.42456700 & 0.59382200 \\
\hline Pt 30 & 1.01278100 & 0.85836400 & 4.65199500 \\
\hline Pt31 & 0.44199300 & 5.42263300 & 5.35640000 \\
\hline Pt 32 & 0.52300300 & 1.08402600 & 2.23355100 \\
\hline Pt 33 & 0.83626100 & 5.03579700 & -0.25771200 \\
\hline Pt 34 & -2.50022400 & -0.54520400 & 2.13545000 \\
\hline Pt35 & -3.29021900 & 0.07807000 & -0.09964800 \\
\hline Pt36 & -0.88998000 & -3.72541300 & -1.07966600 \\
\hline Pt 37 & -2.88083100 & -3.20811100 & 0.36703600 \\
\hline Pt 38 & 0.41912600 & -3.12062700 & -3.07594500 \\
\hline Pt39 & 1.27067900 & -1.29762300 & -4.50776400 \\
\hline Pt 40 & 2.14288700 & -1.54736800 & 1.63316900 \\
\hline t 41 & -2.72450300 & -3.85962000 & -3.88878400 \\
\hline Pt 42 & -1.41568200 & -2.85509100 & -5.72997300 \\
\hline Pt 43 & -0.92107700 & 0.70220000 & -0.66609100 \\
\hline Pt 44 & -0.46827300 & -0.83207900 & 3.56355900 \\
\hline Pt 45 & 2.16140700 & 2.95458800 & 2.08667500 \\
\hline & -0.74953500 & 0.01357000 & -4.00513700 \\
\hline \# 46 & Cluster $=$ Pt $47 \quad Q=0$ & \multicolumn{2}{|c|}{$\mathrm{M}=0 \quad$ Etot $=-4876.28791100$} \\
\hline Pt1 & 1.52466100 & 3.80946200 & 3.23723100 \\
\hline Pt2 & -0.99011800 & 0.19708600 & -0.50861600 \\
\hline Pt3 & -3.81768700 & 2.25000300 & 5.82035000 \\
\hline Pt 4 & 0.23432900 & -5.65771000 & -4.34385400 \\
\hline Pt5 & 1.84521300 & -2.92825200 & -5.56264100 \\
\hline Pt 6 & -0.51868600 & 2.72813200 & 5.53296400 \\
\hline Pt 7 & -1.12227000 & -2.45466200 & 1.40190700 \\
\hline Pt 8 & 3.52884300 & $-1.1065430 c$ & -1.45265200 \\
\hline Pt9 & 2.37159700 & 0.63511600 & 2.7944480 \\
\hline Pt10 & -3.17355700 & 1.43540300 & -0.13003400 \\
\hline Pt11 & -3.99522000 & 3.41287600 & 1.28654500 \\
\hline Pt 12 & -0.51518600 & -1.89266000 & -1.76150300 \\
\hline Pt13 & -1.07568800 & 5.77088800 & 4.10969500 \\
\hline & 3.76045600 & $-3.1637540 c$ & 0.093480 \\
\hline
\end{tabular}




\begin{tabular}{|c|c|c|c|}
\hline Pt15 & 1.13567300 & -0.08213300 & 0.77313700 \\
\hline Pt16 & 4.11536000 & -2.92473000 & -4.44354400 \\
\hline Pt17 & 1.79702500 & -5.43541800 & -1.16251800 \\
\hline Pt18 & 0.14378100 & 4.51321800 & 1.31828500 \\
\hline Pt19 & -1.58378200 & -1.19530900 & 3.56782400 \\
\hline Pt20 & 0.00705600 & 2.06779900 & 0.84498900 \\
\hline Pt21 & -4.85649700 & 3.08384700 & 3.67925300 \\
\hline Pt22 & 3.02000600 & 1.14799100 & -0.34202600 \\
\hline Pt23 & 4.08910200 & -5.42702100 & -2.26103300 \\
\hline Pt24 & 1.37278100 & -3.38522500 & -2.49004100 \\
\hline Pt25 & -1.81693800 & 3.53786300 & -0.05105400 \\
\hline Pt26 & -0.53315200 & -4.37210200 & -1.27226900 \\
\hline Pt27 & -4.14868600 & 0.77158600 & 2.94752100 \\
\hline Pt28 & 1.26941500 & -2.34460800 & 2.12815900 \\
\hline Pt29 & -2.13262700 & -0.25185900 & -2.67837500 \\
\hline Pt 30 & -1.81895300 & 0.69619300 & 1.84804100 \\
\hline Pt31 & 2.39914100 & 1.04962300 & -3.70183200 \\
\hline Pt 32 & -1.03408900 & 2.77857000 & -2.29398600 \\
\hline Pt 33 & 2.57958500 & -4.87307900 & -4.17101900 \\
\hline Pt 34 & 0.15299900 & 1.67464400 & 3.31271800 \\
\hline Pt 35 & -0.13399500 & 0.88556000 & -3.65251400 \\
\hline Pt 36 & 1.23123600 & 2.66287900 & -1.19788900 \\
\hline Pt 37 & -2.44749300 & 4.29932100 & 5.53264600 \\
\hline Pt 38 & 1.18196200 & -0.05268700 & -1.73863100 \\
\hline Pt 39 & -2.05840200 & 1.17239900 & 4.30077400 \\
\hline Pt 40 & -1.70181600 & 3.12181900 & 2.42226300 \\
\hline Pt 41 & 5.49231300 & -2.15329100 & -2.54718100 \\
\hline Pt 42 & -2.89450400 & 5.32011500 & 2.48946200 \\
\hline Pt 43 & 2.28007400 & -1.41873000 & -3.62210700 \\
\hline Pt 44 & 1.53900800 & -2.16104900 & -0.39609000 \\
\hline Pt 45 & -2.96266700 & -1.03317800 & 0.41578200 \\
\hline Pt 46 & 0.09546400 & -1.27644800 & -4.86647300 \\
\hline Pt 47 & -1.83506700 & -3.43194500 & -3.20959300 \\
\hline$\# 47$ & Cluster $=$ Pt $48 \quad \mathrm{Q}=0$ & \multicolumn{2}{|c|}{$\mathrm{M}=0 \quad \mathrm{Etot}=-5040.84683100$} \\
\hline Pt1 & 1.32003200 & -0.00743600 & -0.40547100 \\
\hline Pt2 & 3.55093400 & 0.89160900 & -1.07478000 \\
\hline Pt3 & 2.98620000 & 4.11462600 & -2.06382100 \\
\hline Pt 4 & 1.18815000 & -4.67795900 & 0.44259300 \\
\hline Pt5 & -3.11456200 & -6.28841300 & 0.38207600 \\
\hline Pt 6 & 2.48695000 & -1.75609900 & 0.92497600 \\
\hline Pt7 & -3.03806200 & -0.16812700 & 1.33709400 \\
\hline Pt 8 & -0.92857300 & -0.66461700 & -1.23265800 \\
\hline Pt9 & 3.86104900 & 1.10790900 & 1.42137900 \\
\hline Pt10 & 1.70076900 & 2.53129100 & -0.66044400 \\
\hline Pt11 & -0.92926600 & 1.39123900 & -2.61555600 \\
\hline Pt12 & -1.66229200 & 2.74123100 & 1.82443000 \\
\hline Pt13 & -2.82451800 & 0.96037900 & -0.90673400 \\
\hline Pt14 & 0.83906700 & 2.46413300 & 1.7161920 \\
\hline Pt15 & 1.57280800 & 1.08551000 & -2.67508600 \\
\hline Pt16 & 2.15849300 & -2.07856600 & -1.55047700 \\
\hline Pt17 & -0.08024800 & -2.96795700 & -0.91073000 \\
\hline Pt18 & 1.86593200 & 7.07358800 & -1.48442700 \\
\hline Pt19 & -1.31190600 & -4.55104200 & 0.56138500 \\
\hline Pt20 & 3.09479200 & 3.46372100 & 1.1822510 \\
\hline Pt21 & -0.48239300 & 1.03049600 & 3.2302300 \\
\hline Pt22 & -0.40974700 & 5.65106300 & 2.31279100 \\
\hline Pt23 & -0.26956200 & -7.21228300 & -1.21551800 \\
\hline & 2.74208700 & 2.29324900 & 3.3582910 \\
\hline
\end{tabular}




\begin{tabular}{|c|c|c|c|}
\hline Pt25 & 0.76405800 & -5.06273400 & -1.99036000 \\
\hline Pt26 & -1.87496100 & -1.97570500 & 2.72913200 \\
\hline Pt27 & 1.35635400 & -0.63781000 & 2.82630000 \\
\hline Pt28 & 0.21226500 & -1.86266200 & -3.13029900 \\
\hline Pt29 & -3.22368400 & -4.18529500 & -1.03064300 \\
\hline Pt30 & -2.17380800 & $-2.2419420 c$ & 0.23382800 \\
\hline Pt31 & 4.22993700 & 5.22950000 & -0.14396700 \\
\hline Pt32 & -0.54155800 & -0.48280600 & 1.22216900 \\
\hline Pt33 & -1.39346000 & 3.92164000 & -0.38042300 \\
\hline Pt34 & -1.12096700 & -4.78204000 & -3.58777200 \\
\hline Pt3 & 0.91624900 & 4.58461600 & 0.42377600 \\
\hline Pt3 & -2.27363700 & -1.66734500 & -3.04910900 \\
\hline Pt3 & 1.83622800 & $6.6077090 \mathrm{C}$ & 1.73637500 \\
\hline Pt 3 & -0.55842200 & 1.60087900 & -0.13414000 \\
\hline Pt3 & -2.44967900 & $-6.3132830 c$ & -2.08251400 \\
\hline Pt 4 & -1.22532800 & -6.54026300 & 2.02441900 \\
\hline Pt 4 & -4.14556200 & -1.91475200 & -1.38867900 \\
\hline Pt 4 & 0.97301200 & 4.04259000 & 3.67292200 \\
\hline Pt 4 & -4.38871300 & $-3.1940560 c$ & 0.89843700 \\
\hline Pt 4 & -3.61093700 & -4.58945100 & -3.47215100 \\
\hline Pt 4 & 0.04597200 & -3.54057300 & 2.36941300 \\
\hline Pt 4 & 0.41205300 & 4.23994700 & -2.09172200 \\
\hline Pt4 7 & 3.95637700 & 5.49012700 & 2.34096100 \\
\hline Pt 4 & -0.03792300 & 6.84616600 & 0.10605900 \\
\hline \# 48 & Iuster $=$ Pt $49 \quad \mathrm{Q}=0$ & \multicolumn{2}{|c|}{$\mathrm{M}=0 \quad$ Etot $=-5148.86154700$} \\
\hline Pt 1 & 3.78457500 & -4.90660700 & 2.75863000 \\
\hline Pt2 & -5.35661500 & 3.52554800 & -3.05971600 \\
\hline Pt 3 & 2.85081000 & $-3.5891480 c$ & 0.59891300 \\
\hline Pt 4 & 2.43267100 & -1.92524300 & 2.41654600 \\
\hline Pt 5 & 1.57244000 & 2.30581400 & 0.01282900 \\
\hline Pt 6 & 2.35554800 & 1.31127400 & -2.13825300 \\
\hline Pt 7 & -0.19120800 & -1.91891100 & 0.42595600 \\
\hline Pt 8 & 0.61199500 & 2.31111200 & 2.34846100 \\
\hline Pt9 & -1.52204900 & -1.49156000 & -2.64001900 \\
\hline Pt10 & -1.16022700 & $6.5756820 \mathrm{c}$ & -3.01322600 \\
\hline Pt11 & -4.50913000 & 1.61051300 & -1.73963000 \\
\hline Pt12 & 0.22112900 & $-3.5408840 c$ & -1.43378100 \\
\hline Pt13 & -1.71638300 & 2.69274300 & -0.32268600 \\
\hline Pt14 & -3.34988300 & 3.34269300 & -5.82641400 \\
\hline Pt15 & -3.59222100 & 1.11933600 & 0.56364500 \\
\hline Pt16 & -0.79899100 & $-5.6161070 c$ & 2.58244500 \\
\hline Pt17 & 0.75018500 & -3.66129500 & 1.97841800 \\
\hline Pt18 & 1.50284100 & -1.64100500 & 4.71620600 \\
\hline Pt19 & 4.16217300 & -3.64001400 & 4.87787100 \\
\hline Pt20 & -0.04616200 & -5.29577200 & 0.25463300 \\
\hline Pt21 & -2.01280900 & 3.30916300 & -2.77925700 \\
\hline Pt22 & 1.86878000 & -1.91304900 & -2.27036500 \\
\hline Pt23 & 3.33213600 & 0.90819400 & 1.08561600 \\
\hline Pt24 & -1.20087700 & 0.36183200 & 0.48723700 \\
\hline Pt25 & -2.04133700 & 0.89234200 & -2.02007900 \\
\hline Pt26 & -0.64695700 & 0.75823700 & 3.78813300 \\
\hline Pt27 & -3.73662300 & 0.64381300 & -3.89491300 \\
\hline Pt28 & 5.39438900 & -2.99764400 & 2.8093140 \\
\hline Pt29 & -2.03643600 & 5.23795200 & -0.05967200 \\
\hline Pt30 & 0.07117900 & 4.18419100 & 0.76219300 \\
\hline Pt31 & 4.48298100 & -1.88801400 & -0.2096610 \\
\hline Pt32 & 3.05412800 & -6.0733860 & 0.72036400 \\
\hline & -2.13359600 & -1.54815400 & 1.97015700 \\
\hline
\end{tabular}




\begin{tabular}{|c|c|c|c|}
\hline et 34 & -0.63058500 & -2.51698200 & 3.72023300 \\
\hline Pt 35 & -2.57079500 & -2.00458200 & -0.43759000 \\
\hline Pt 36 & -3.71268200 & 4.92994600 & -1.84795100 \\
\hline Pt 37 & 2.11475100 & -1.14482500 & 0.09002200 \\
\hline Pt 38 & 0.27066800 & -0.20160500 & -1.40226600 \\
\hline Pt 39 & -1.19531900 & 2.42092700 & -4.98973800 \\
\hline Pt 40 & -0.31091000 & 0.40486600 & -3.80115600 \\
\hline Pt 41 & 0.49815100 & 4.93281400 & -3.89491400 \\
\hline Pt 4 & 1.06327000 & -4.09757900 & 4.57090500 \\
\hline Pt 4 & 0.05789600 & 2.20459400 & -2.01996200 \\
\hline Pt 4 & 0.76714200 & -0.14046300 & 1.91986600 \\
\hline Pt 4 & 2.13957400 & -6.26101200 & 4.02844100 \\
\hline Pt 46 & 3.59855700 & -0.45833400 & 4.03856100 \\
\hline Pt 47 & -2.63877300 & 2.88295500 & 2.02671500 \\
\hline Pt 4 & 0.98168700 & 4.42347700 & -1.52584500 \\
\hline Pt 4 & -2.82908800 & 5.18215900 & -4.22521300 \\
\hline \# 49 & Cluster $=$ Pt 50 & $I=0 \quad$ Etot $=-52$ & 99511100 \\
\hline Pt1 & 1.68495500 & 2.71244500 & -2.34587700 \\
\hline Pt2 & 2.89623700 & 0.35416900 & -0.32962600 \\
\hline Pt3 & -3.58366400 & 2.80389000 & 0.51851800 \\
\hline Pt 4 & 2.50415700 & -1.67356800 & -4.26774800 \\
\hline Pt5 & -3.93064500 & -2.95452400 & -0.24480700 \\
\hline Pt 6 & -1.27156200 & -1.22446700 & 1.02593500 \\
\hline Pt 7 & -5.73881400 & -2.93865600 & 1.49994000 \\
\hline Pt 8 & 3.72549100 & 3.42957300 & -0.97404900 \\
\hline Pt 9 & -2.25260800 & -3.45302100 & 1.56549000 \\
\hline Pt10 & 6.11813700 & 2.79021600 & -3.22572600 \\
\hline Pt11 & 5.15342200 & 1.48899400 & -0.17506600 \\
\hline Pt12 & -3.60146700 & -0.56901700 & 0.42586700 \\
\hline Pt13 & -3.17921000 & 1.03968000 & 2.23079300 \\
\hline Pt14 & 1.05982200 & -0.26816900 & 1.25210400 \\
\hline Pt15 & -3.78227700 & -0.87794500 & 5.07715700 \\
\hline Pt16 & 5.91489400 & -2.84362500 & -1.85835100 \\
\hline Pt17 & 2.60069200 & 1.62085100 & 1.81757500 \\
\hline Pt18 & 4.96725000 & -1.50909200 & -4.80481200 \\
\hline Pt19 & -0.23122000 & 0.73488400 & 3.16806100 \\
\hline Pt20 & 7.04437100 & -0.15946100 & -0.40239100 \\
\hline Pt21 & 4.85282700 & -0.64035500 & -1.53459800 \\
\hline Pt22 & 1.56994100 & -0.87658000 & -2.06106600 \\
\hline Pt23 & 3.14084500 & -2.76641500 & 0.34348400 \\
\hline Pt24 & 4.34760900 & -0.16278600 & 1.65250000 \\
\hline Pt25 & -6.01960600 & -0.49779400 & 1.13480800 \\
\hline Pt26 & -2.32683000 & 1.08270400 & 4.61910400 \\
\hline Pt27 & -1.77841200 & 1.07979700 & 0.16889500 \\
\hline Pt28 & 0.56929000 & 1.01304700 & -0.81467600 \\
\hline Pt29 & -1.60999500 & 2.83364900 & 3.01350000 \\
\hline Pt 30 & 3.28505900 & 0.82120000 & -2.72347900 \\
\hline Pt 31 & -3.29435300 & -1.10235200 & -2.00230800 \\
\hline Pt 32 & -3.12502700 & -1.38608100 & 2.71394300 \\
\hline Pt 33 & -3.88250300 & -3.22668000 & 4.27019400 \\
\hline Pt 34 & -0.90976300 & -0.84543800 & -1.40507100 \\
\hline Pt 35 & 6.72708000 & 0.38750400 & -2.83600500 \\
\hline Pt 36 & -0.83341600 & 2.97426500 & -1.2132330 \\
\hline Pt 37 & -6.49451000 & 1.72653200 & 2.23846200 \\
\hline Pt 38 & -5.10631500 & 1.16027900 & -0.55532600 \\
\hline Pt 39 & -6.52367000 & -1.45884300 & 3.3747050 \\
\hline Pt 40 & 4.98732000 & 0.95199400 & -4.56184200 \\
\hline & 0.73417700 & -2.29478800 & -0.1350920 \\
\hline
\end{tabular}




$\begin{array}{lrrr}\text { Pt42 } & 0.16921600 & -1.74696100 & 3.03916100 \\ \text { Pt43 } & -0.87825400 & -3.32710600 & -1.67135600 \\ \text { Pt44 } & 0.80733500 & 2.94389500 & 0.76444500 \\ \text { Pt45 } & -1.11930200 & 1.12757900 & -2.92175900 \\ \text { Pt46 } & -4.89677900 & 3.01678500 & 3.62827900 \\ \text { Pt47 } & 3.02733700 & 2.59208500 & -4.42399600 \\ \text { Pt48 } & 0.65323800 & 0.01542700 & -4.24766500 \\ \text { Pt49 } & 3.41641700 & -2.58735000 & -2.10712900 \\ \text { Pt50 } & -5.58691400 & 0.68963100 & 4.30013600\end{array}$

Table S5. Comparison of global minimum structures for $\mathrm{Pt}_{15}-\mathrm{Pt}_{46}$ clusters found in ref ${ }^{1}$ and in the present work (SCG5 potential). Favorable ("magic") structures are marked with gray color.

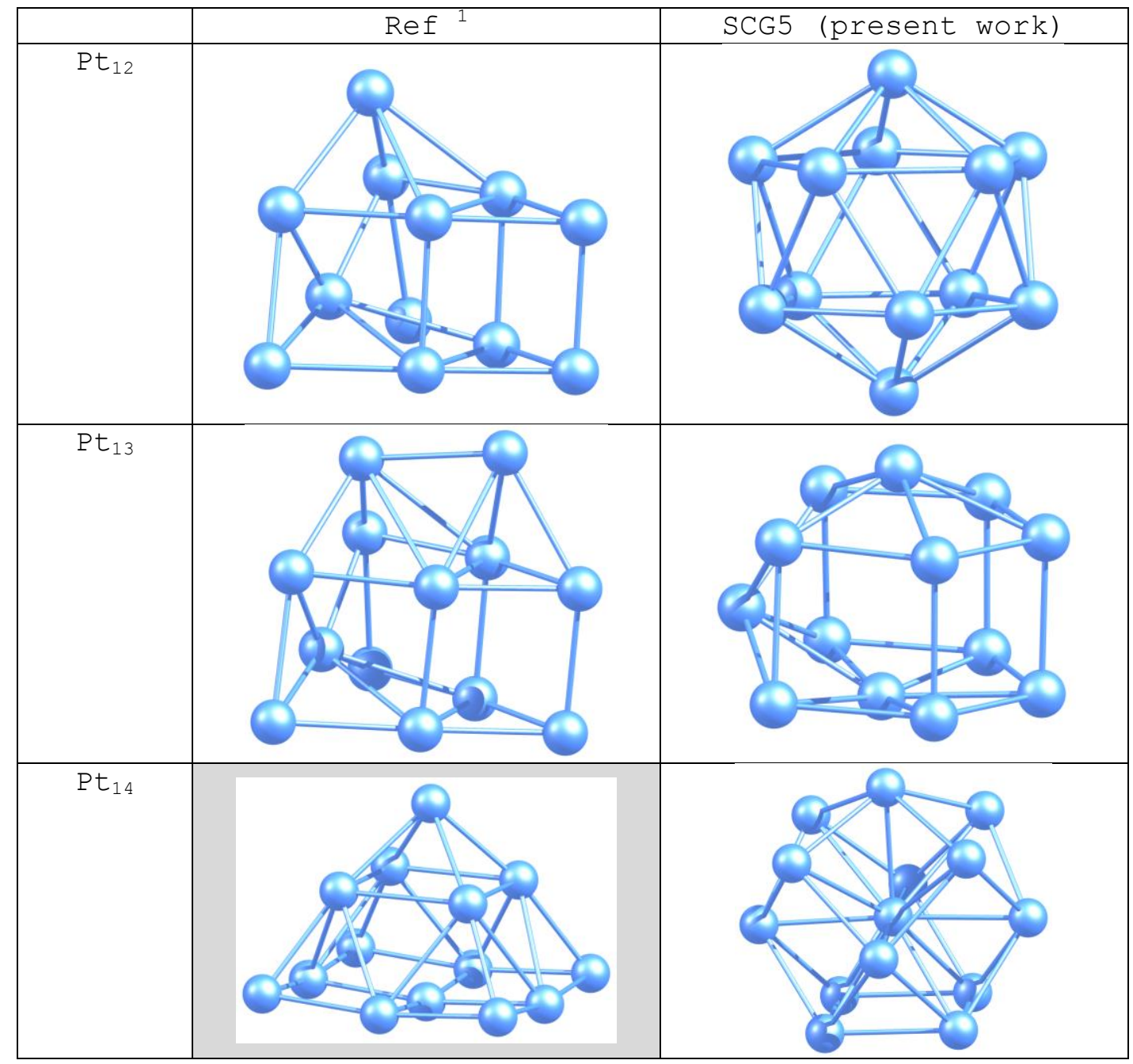




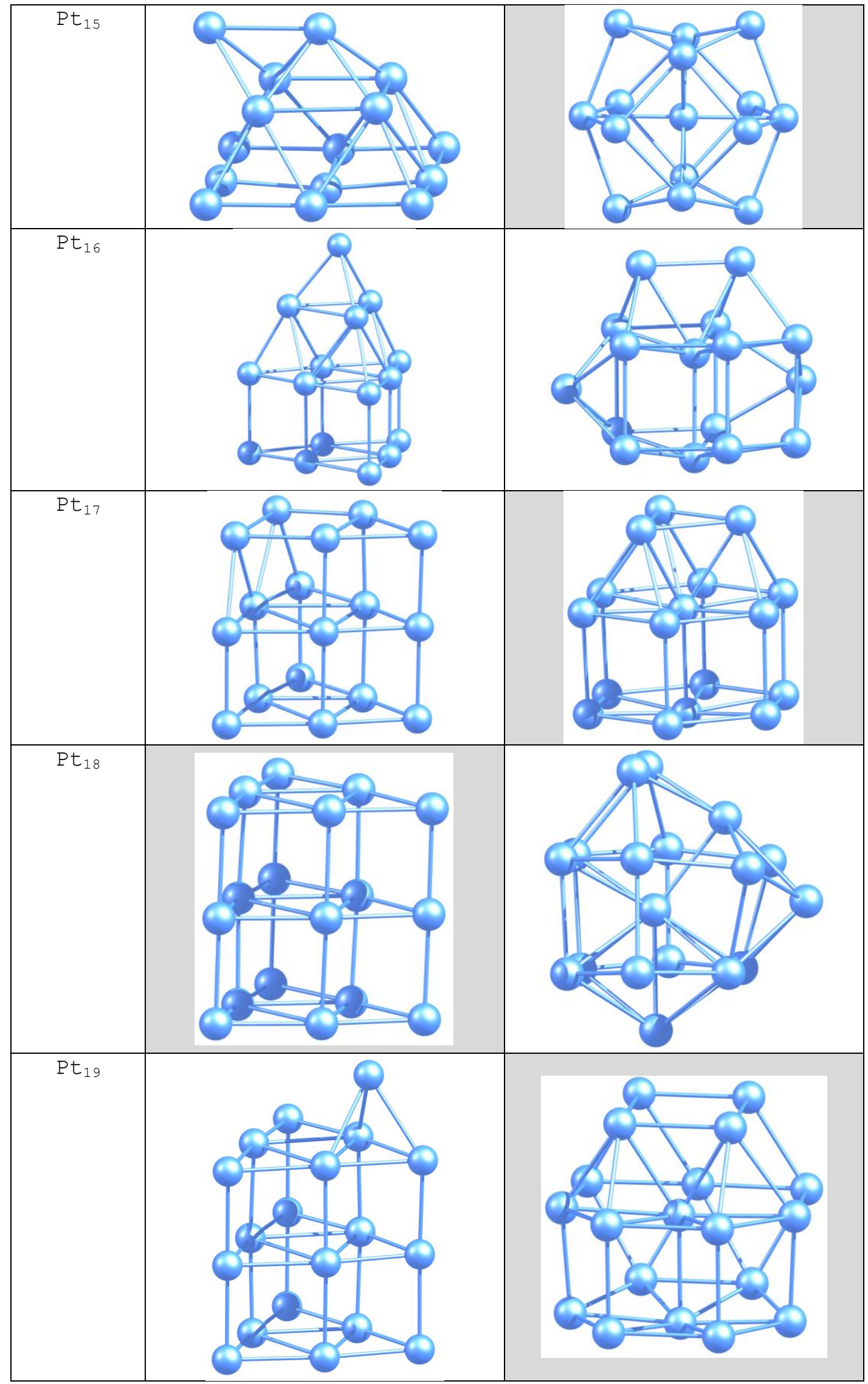




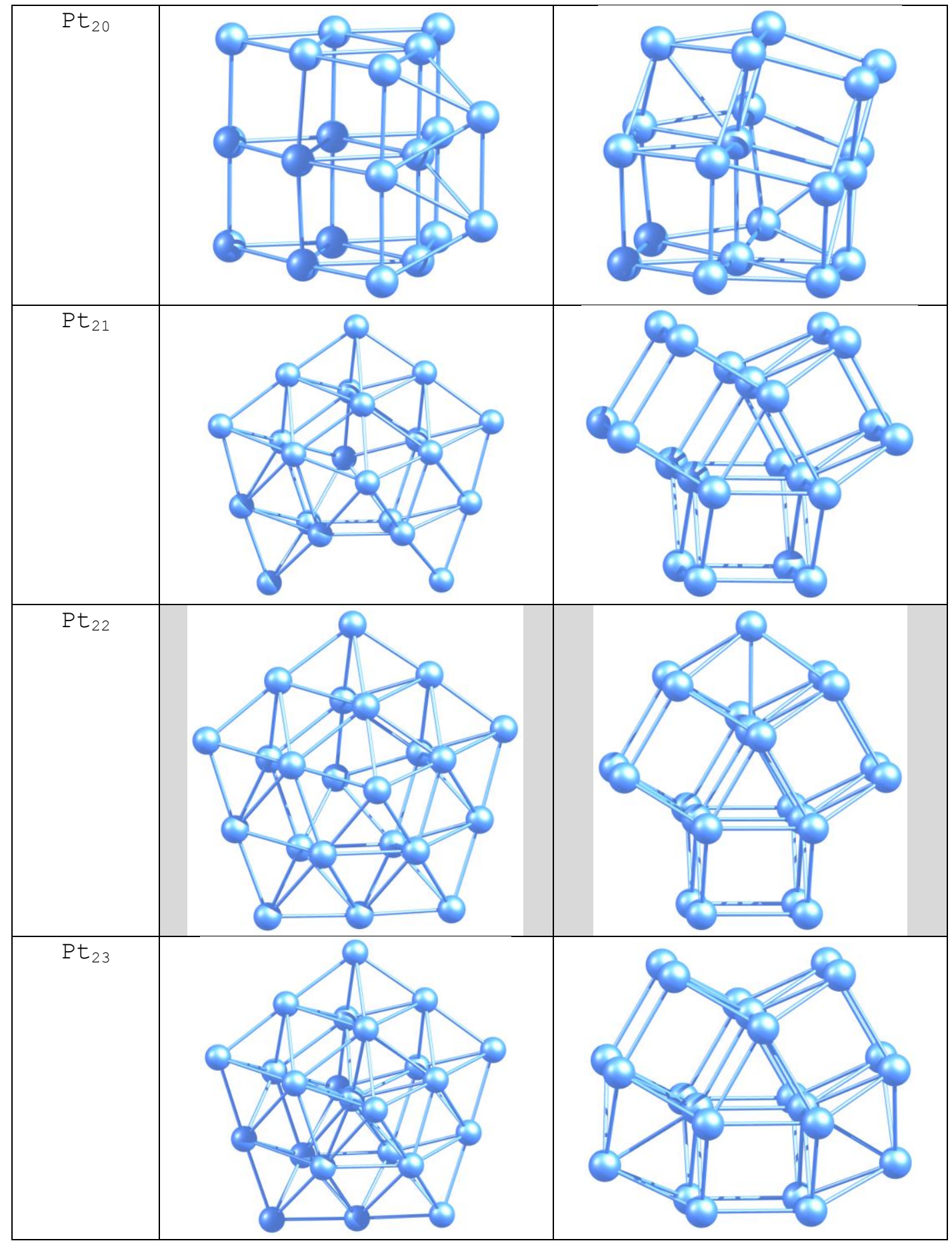




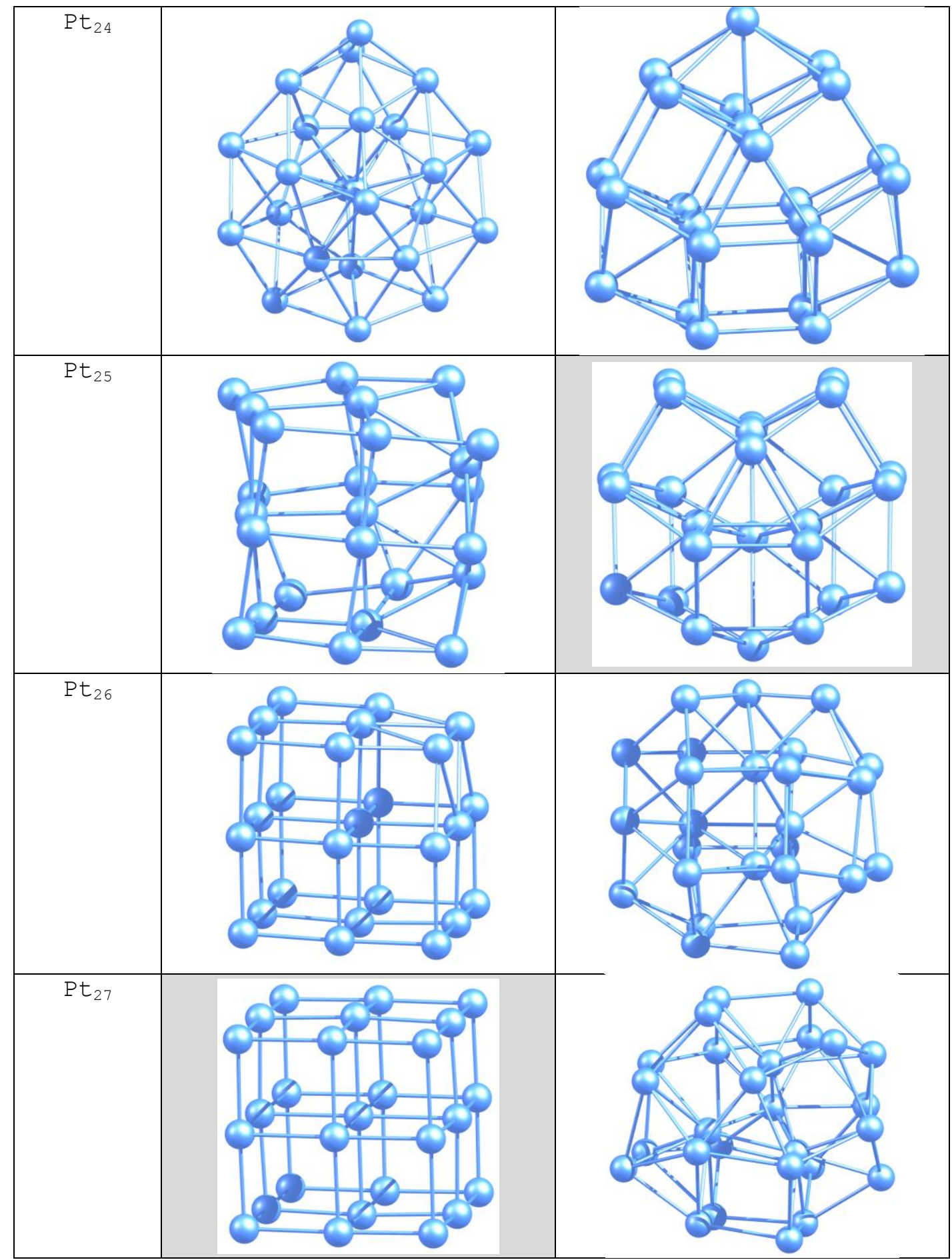




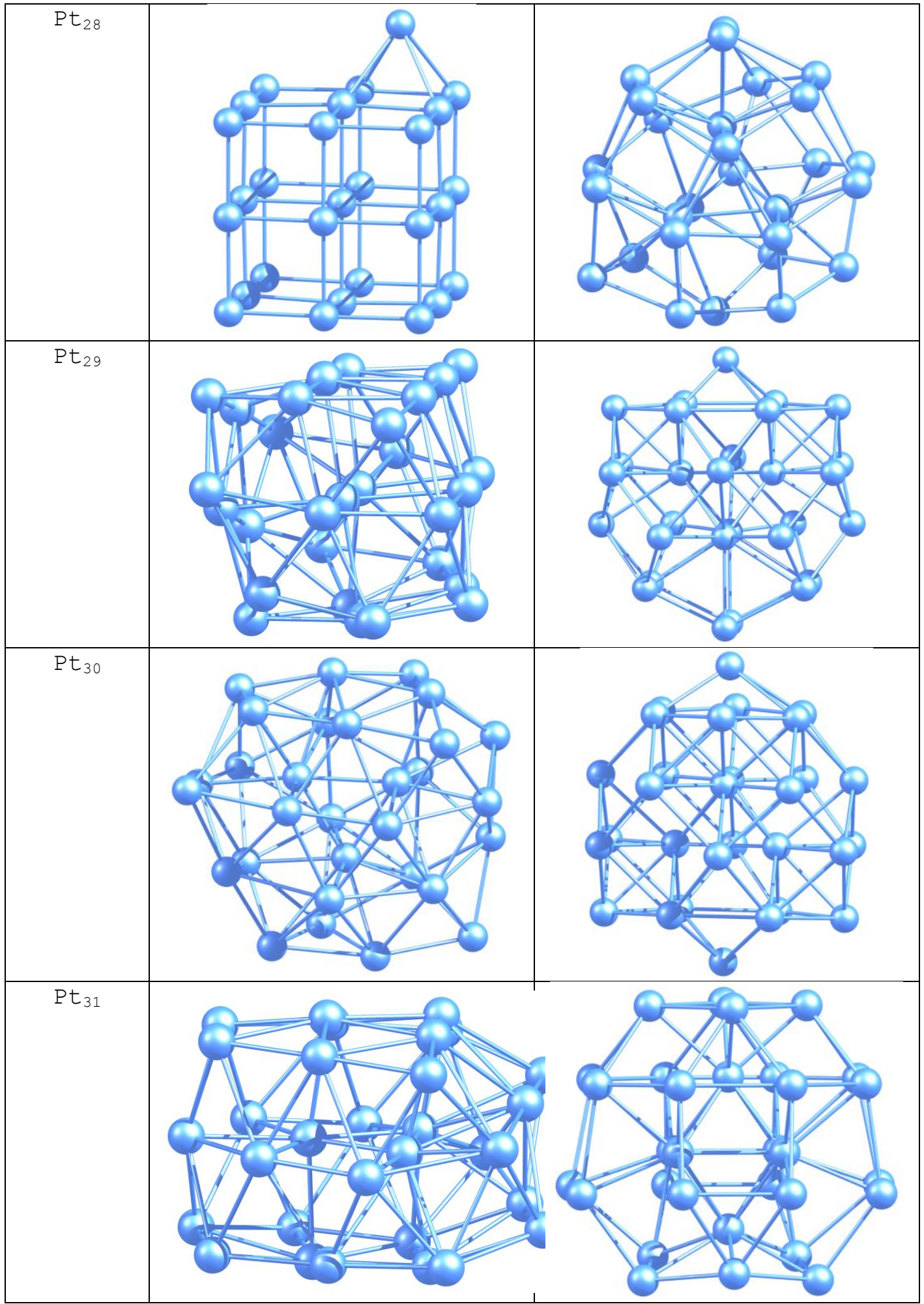




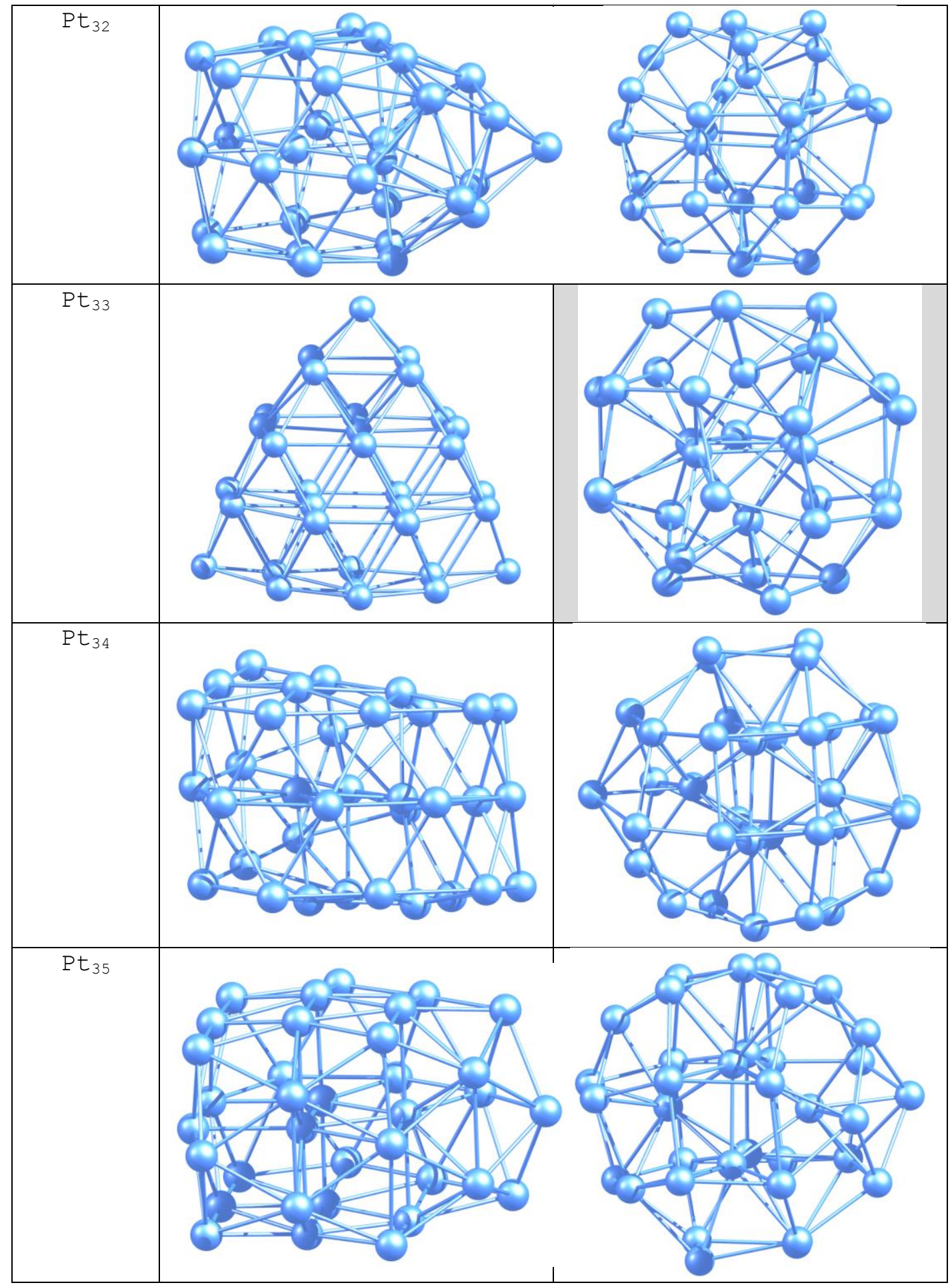




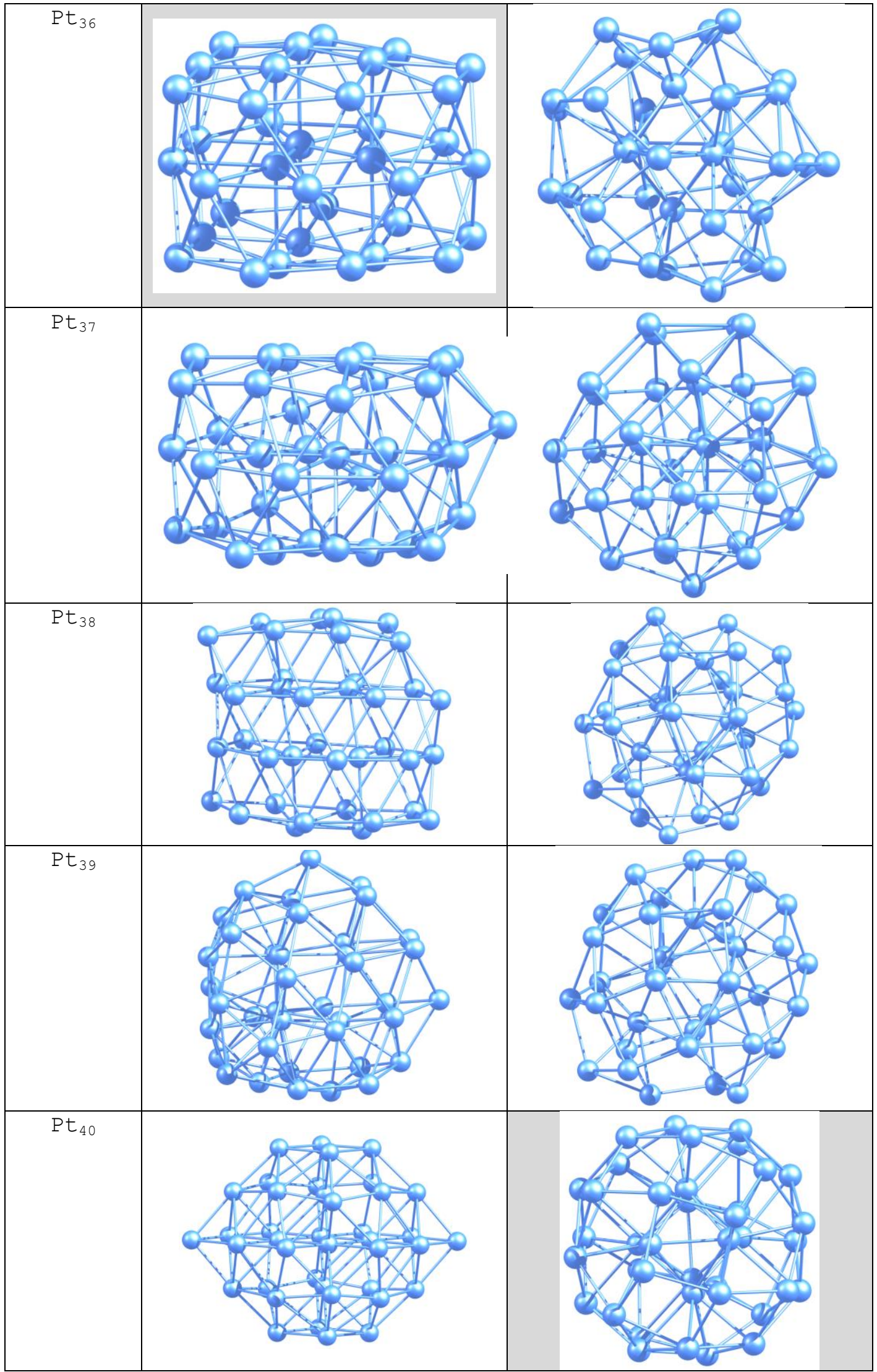




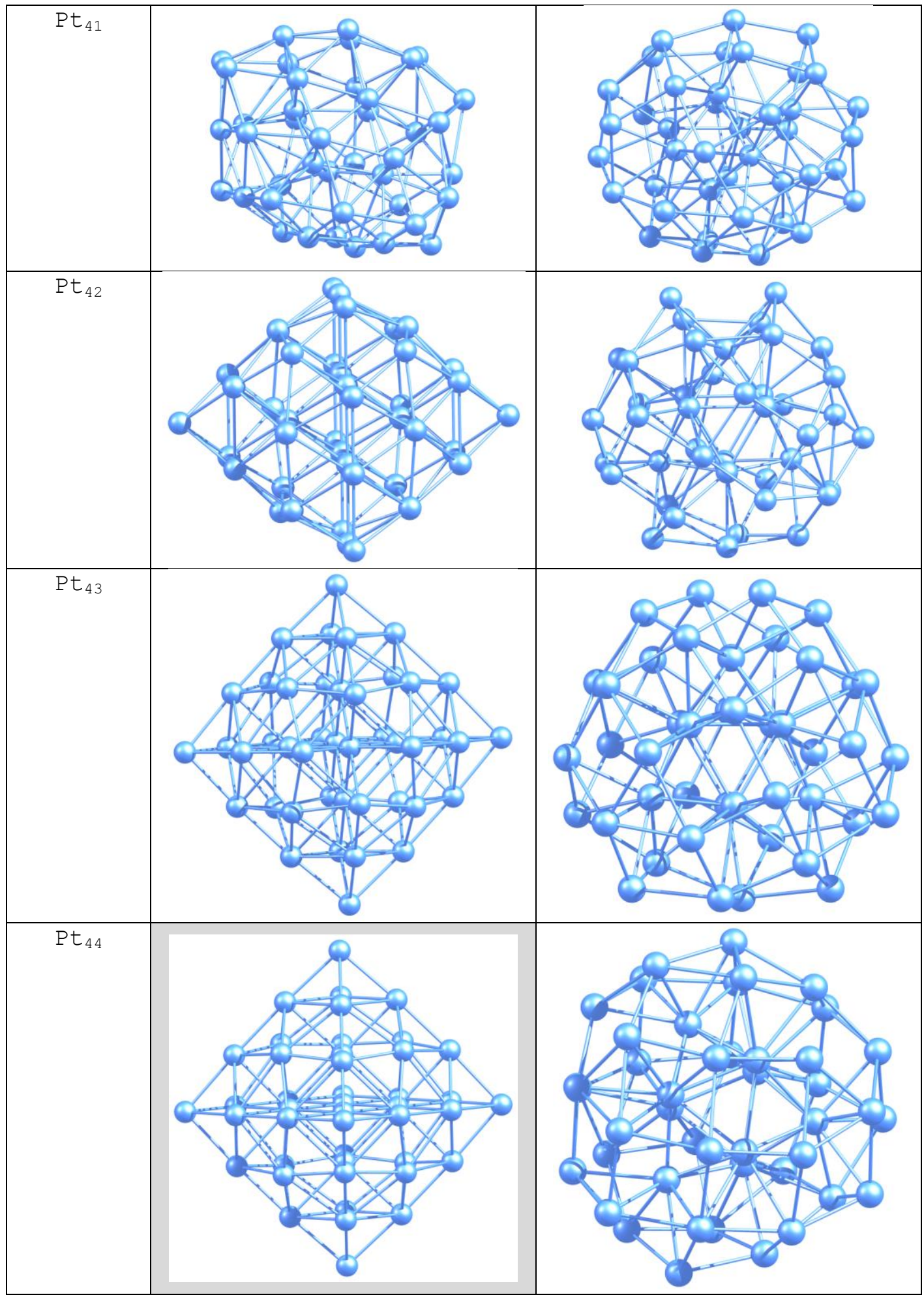




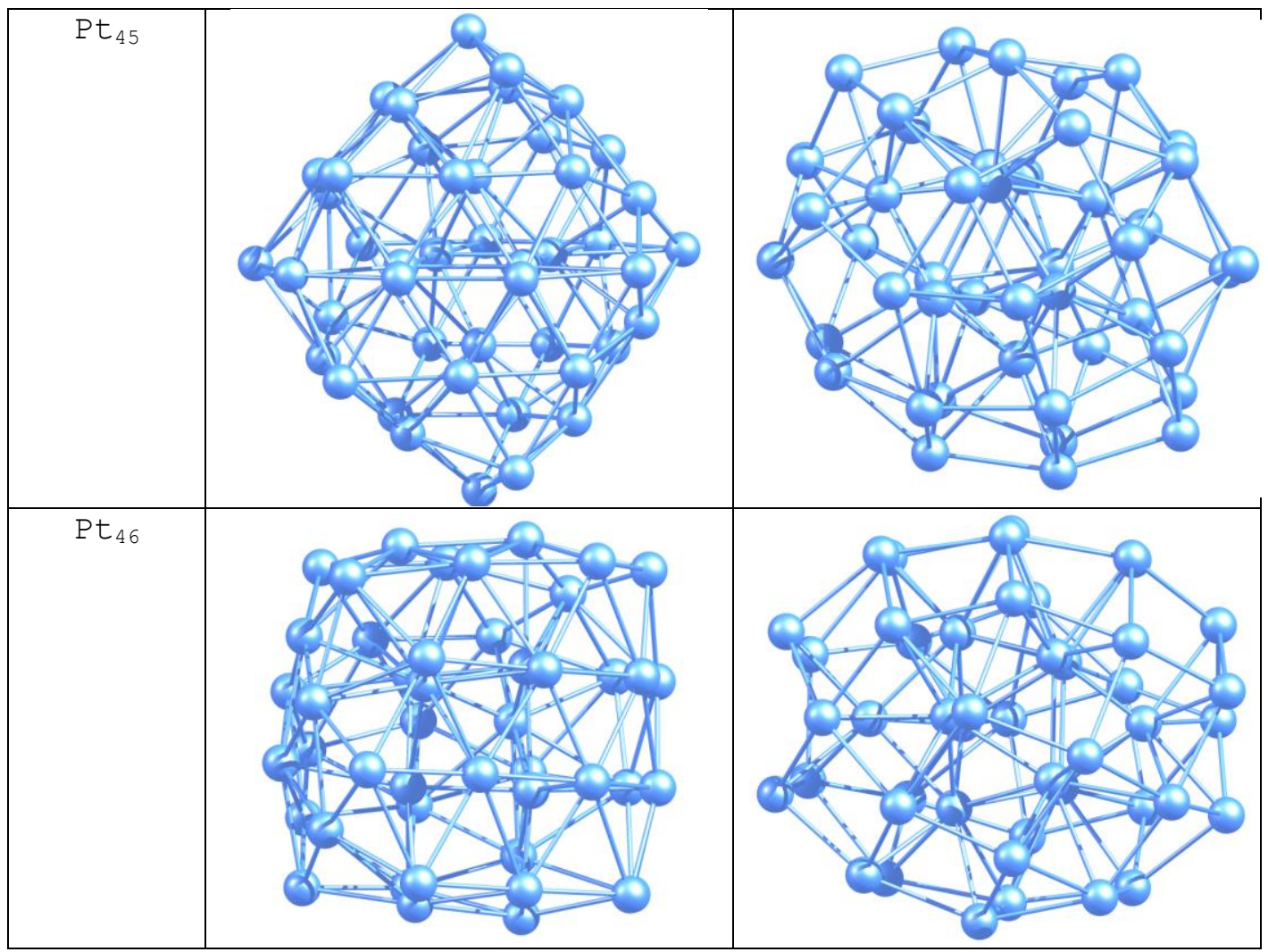

Reference:

1. Wei, G.-F.; Liu, Z.-P., Subnano Pt Particles from a First-Principles Stochastic Surface Walking Global Search. Journal of Chemical Theory and Computation 2016, 12, 4698-4706. 